\title{
Final Elucidation of the Absolute Configuration of Hormaomycin Total Synthesis of Hormaomycin and Analogs
}

\author{
Dissertation \\ zur Erlangung des Doktorgrades \\ der Mathematisch-Naturwissenschaftlichen Fakultäten \\ der Georg-August-Universität zu Göttingen
}

vorgelegt von

Boris Zlatopolski

aus Sankt-Petersburg/Russland 
D 7

Referent:

Prof. Dr. A. de Meijere

Korreferent:

Prof. Dr. A. Zeeck

Tag der mündlichen Prüfung: 05.11.2003 
Meinem Lehrer, Herrn Prof. Dr. A. de Meijere, danke ich herzlich für die interessante Themenstellung, die während dieser Arbeit erwiesene Unterstützung und stete Diskussionsbereitschaft zu Fragen dieser Arbeit. 

Lisa und Anna 



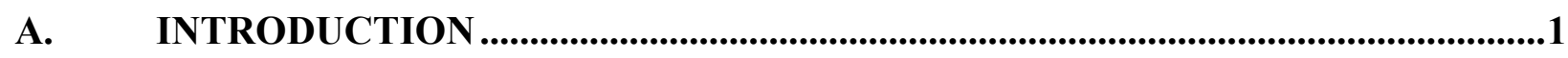

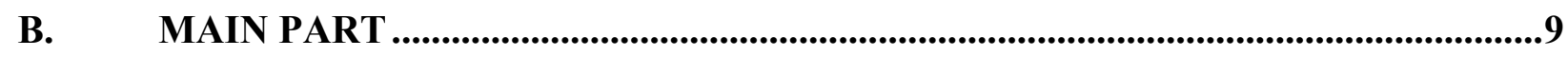

1. Elucidation of the absolute configuration of 4-(Z)-propenylproline residue in hormaomycin. Synthesis of $(2 S, 4 R)-4-(Z)$-propenylproline and $(2 S, 4 R)-4-(Z)-\left(4,1^{\prime}-\right.$ dideutero)propenylproline. 9

1.1. Determination of the relative configuration of $4-(Z)$-propenylproline moiety in hormaomycin. Attempts to prepare pure diastereomers of 4-(Z)-propenylproline via alkylation with (Z)-propenyllithium cuprate.

1.2. Elucidation of the absolute configuration of 4-(Z)-propenylproline moiety in hormaomycin. 13

1.3. Attempts to prepare 4-(Z)-propenylproline starting from pyroglutamic acid 15

1.4. Synthesis of N-Boc protected 4-(Z)-propenylproline and dideutero-4-(Z)propenylproline starting from 4-hydroxyproline

2. Synthesis of $(R)$-allo-threonine and $(2 S, 3 R)-\beta$-Methylphenylalanine.

3. Elucidation of the absolute configuration of trans-(2'-nitrocyclopropyl)alanine residue in hormaomycin. Synthesis of $\left(2 R, 1^{\prime} R, 2^{\prime} R\right)-\left(2^{\prime}\right.$-nitrocyclopropyl)alanine and $\left(2 S, 1^{\prime} R S, 2^{\prime} R S\right)-2$-deutero-(2'-nitrocyclopropyl)alanine. .34

4. Synthesis of $O$-MOM protected 5-chloro-1-hydroxypyrrol-2-carboxylic acid ........43

5. Final assembling of hormaomycin .49

5.1. General remarks

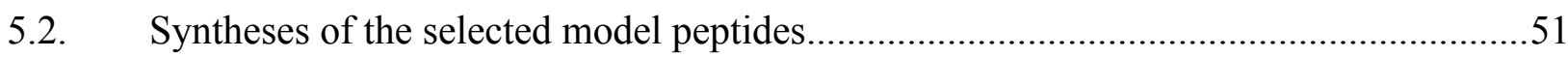

5.3. Synthesis of N-Alloc protected cyclodepsipeptide 171a ..........................................56 
5.4. Comparative study of acidolytic deprotection Boc, $\mathrm{Z}$ and several Z-derived amino protecting groups 59

5.5. Synthesis of N-MeZ protected cyclodepsipeptide 171c ............................................61

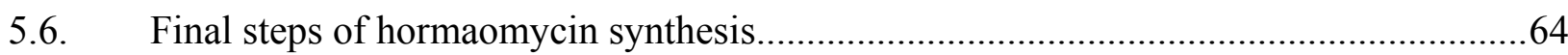

6. Synthesis of hormaomycin analogs. Evaluation of biological activity .....................68

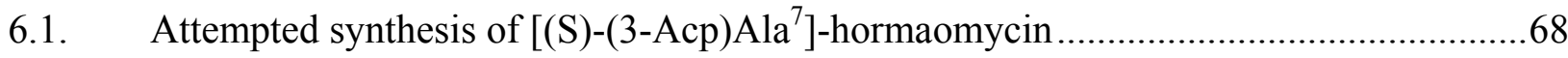

6.2. Synthesis of $\left[(\mathrm{S})-\left(\mathrm{NO}_{2}\right) \mathrm{Nva}^{7}\right]$-hormaomycin. Isolation of $\left[(\mathrm{S})-\left(\mathrm{NO}_{2}\right) \mathrm{Nva}^{3}\right]-$ horamomycin and $\left[(\mathrm{S})-\left(\mathrm{NO}_{2}\right) \mathrm{Nva}^{3},(\mathrm{~S})-\left(\mathrm{NO}_{2}\right) \mathrm{Nva}^{7}\right]$-hormaomycin ............................ 71

6.3. Synthesis of $\left[\mathrm{a}-\mathrm{Dab}^{1}\right]$-hormaomycin and $\left[\mathrm{a}-\mathrm{Dab}^{1}, \mathrm{a}-\mathrm{Il} \mathrm{e}^{5}\right]$-hormaomycin ........................ 74

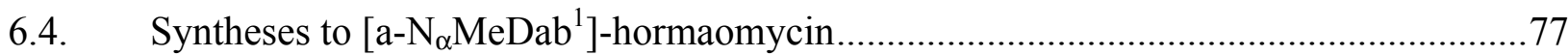

6.5. Evaluation of the biological activity of building blocks and hormaomycin analogs as

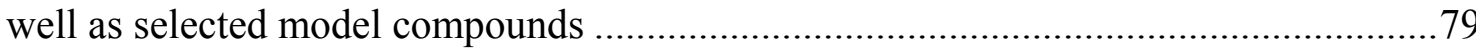

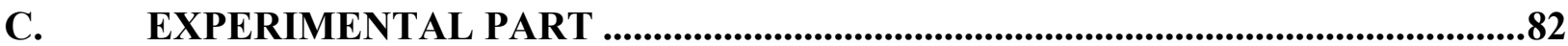

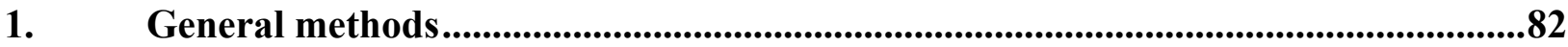

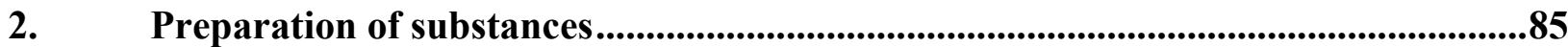

2.1. Determination of the relative configuration of 4-(Z)-propenylproline moiety in hormaomycin. Attempts to prepare pure diastereomers of 4-(Z)-propenylproline via alkylation with (Z)-propenyllithium cuprate.

2.2. Elucidation of the absolute configuration of 4-(Z)-propenylproline moiety in hormaomycin. .93

2.3. Attempts to prepare 4-(Z)-propenylproline starting from pyroglutamic acid .96

2.4. Synthesis of N-Boc protected 4-(Z)-propenylproline and dideutero-4-(Z)propenylproline starting from 4-hydroxyproline 
2.5. Elucidation of the absolute configuration of trans-(2'-nitrocyclopropyl)alanine residue in hormaomycin. Synthesis of (2R,1'R,2'R)-(2'-nitrocyclopropyl)alanine and (2S, 1'RS,2'RS)-2-deutero-(2'-nitrocyclopropyl)alanine 126

2.6. Synthesis of O-MOM protected 5-chloro-1-hydroxypyrrol-2-carboxylic acid.............138

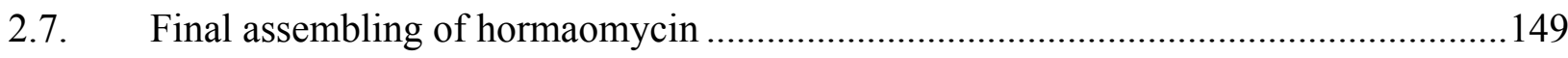

2.8. Synthesis of hormaomycin analogs. Evaluation of biological activity ......................208

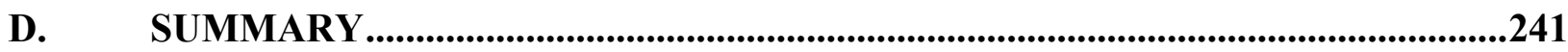

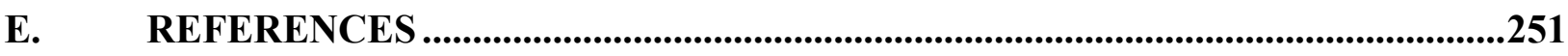

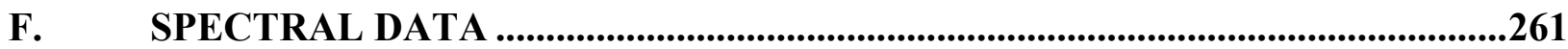

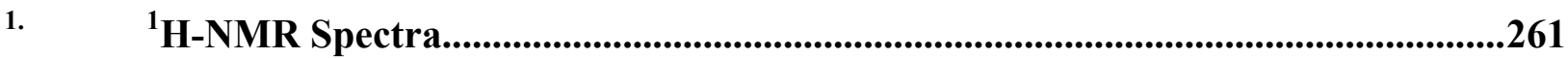

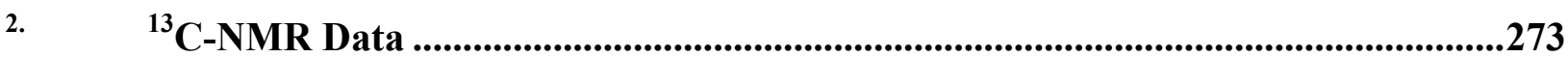





\section{A. INTRODUCTION}

The exploration of microorganisms as sources of medicinally relevant compounds has a much shorter and less well-known history than the use of substances of the plant or animal origin. Nevertheless from the time of the discovery of the antibacterial effect of penicillin by A. Flemming in 1928 and beginning of its widespread application and manufacturing during Second World War, the substances, which are produced by these simplest forms of life, attract more and more attention of scientists. Among other substances, which are "manufactured" on these scarcely small "pharmaceutical factories" the special place is occupied by secondary metabolites, which can be defined as the naturally produced substances, which do not play an explicit role in the internal economy of the organisms that produce them. Microorganisms may have evolved the ability to produce such compounds because of the selection advantages conferred upon them as a result of the interactions of the compounds with specific receptors in other organisms. Although almost 20000 microbial metabolites and approximately 100000 plant products have been described so far, secondary metabolites still appear to be an inexhaustible source of lead structures for new antimicrobials, antiviral, antitumor and immunosupressive drugs, and agricultural and pharmacological agents. In addition, numerous secondary metabolites, such as benzylpenicillin, cephalosporins, erythromycin, strobilurin, etc. were lead structures that later became the basis for synthetic and semisynthetic derivatives with improved pharmacological properties. ${ }^{[1]}$ Some of these compounds play the key role not only in defense mechanisms of microorganisms, but can be used as signal substances for intercellular communication between the cells, executing the function similar to those, that are performed in higher organisms by hormones and pheromones. ${ }^{[2]}$ There are a lot of processes during the life cycle of bacteria, which are regulated by these substances. They regulate, for example, metabolic potential and quorum 
sensing in Gram-negative pathogenic bacteria, competence and sporulation in Bacillus, sporulation, multicellular differentiation and motility in Myxococcus, antibiotic production, morphological differentiation and sporulation in Streptomyces and Erwinia, and gene transfer mechanisms in Enterococcus. ${ }^{[3]}$ It seems to be very attractive to use knowledge about these substances either in terms of controlling cellular proliferation or conversely to increase a production of a particular natural products. The latter possibility was first realized in 1960s, when it was shown, that several substances of actinomycetes could control the production of antibiotics and morphological differentiation (aerial mycelium formation) already in nanomolar concentrations. All these compounds were structurally very similar 2,3-disubstituted $\gamma$ butyrolactones, which nevertheless showed a remarkably different spectrum of the action: Afactor 1 (Khokhlov factor) stimulated the production of streptomycin, IM-type regulators stimulated the production of staphylomycin and VB-type regulators stimulated virginiamycin production in different Streptomyces species. ${ }^{[4]}$ Also it worth to mention the modified homoserine lactones, i.e. $N$-( $(\beta$-ketocaproyl)-(S)-homoserine lactone (KHL) of $V$. fischeri, which can stimulate the carbapenem antibiotic biosynthesis in E. carovora and B-factor of $A$. mediterranei 2, an adenosine derivative, which induces rifamycin $\mathrm{B}$ synthesis in nocardia species. ${ }^{[5]}$
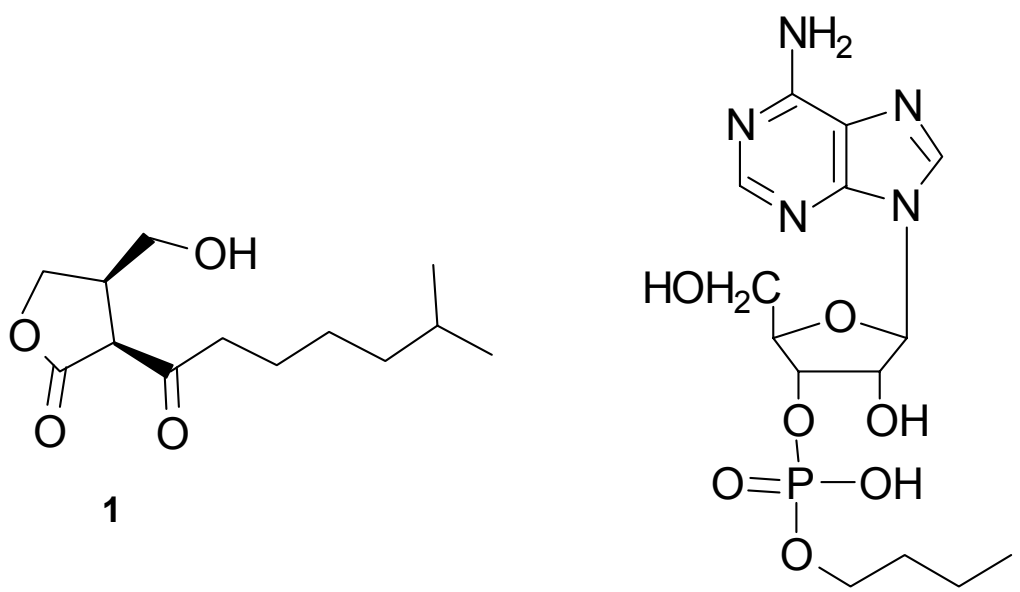
Hormaomycin 3 was isolated from Streptomyces griseoflavus, strain W-384, during the screening of intermolecular signal substances by Anders et al. in 1989. ${ }^{[67]}$ It was the first substance with peptide structure, which inducted the antibiotic production and aerial mycelium formation not only in own producers but also in another Streptomyces species: hydroxistreptomycin in S. flaveolus, streptolin in S. fridae, Tirandamycin in S. griseoflavus, strain 1306, and bafilomycin in S. griseus. The significant increase in the antibiotic production was observed already at $0.05 \mu \mathrm{g} / \mathrm{L}$ concentration of hormaomycin. This substance showed also strong antibiotic activity against coryneform bacteria $(\mathrm{MIC}=0.0005 \mu \mathrm{g} / \mathrm{mL}$ for Arthrobacter oxydans ${ }^{[8]}$

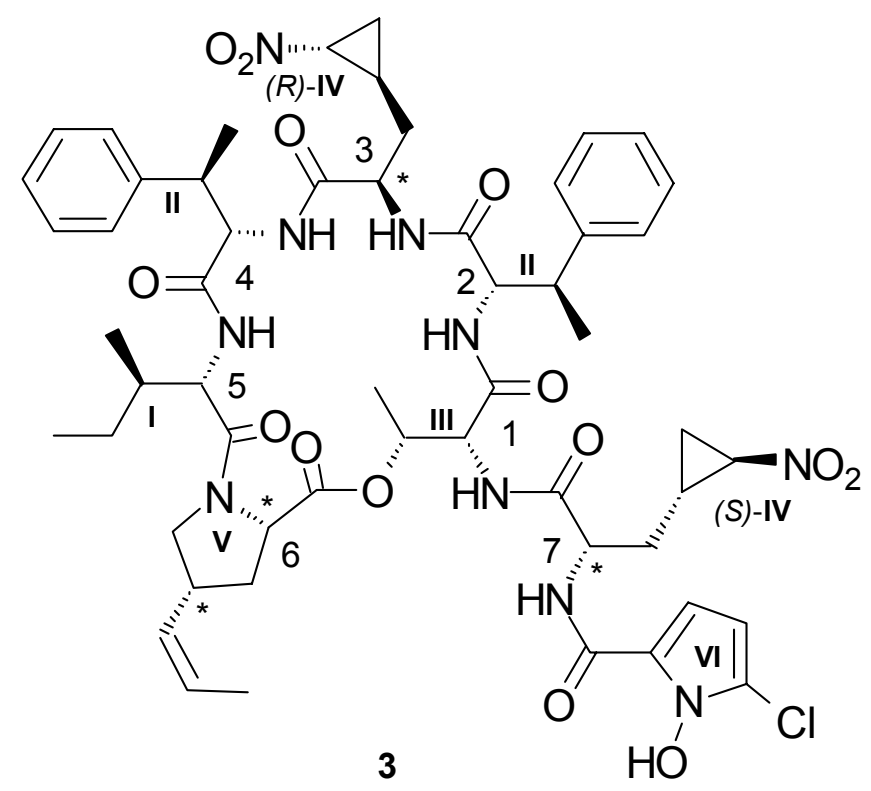

Figure 1. Structure and absolute configuration of Hormaomycin 3. The stereocenters with unknown before absolute configuration are marked with asterisk (*). I (S)-Ile; II (2S,3R)( $\beta \mathrm{Me})$ Phe; III (2R)- $a$-Thr; IV $\left(1^{\prime} R, 2^{\prime} R\right)$-(3-Ncp)Ala; V (2S,4R)-4-(Z)-(4-PE)Pro; VI Chpca.

The constitution of this cyclic depsipeptide showed features unusual even for this structurally flexible class of compounds. Initial structural investigation made by E. Rössner, A. Zeeck et al. ${ }^{[7,8,9]}$ disclosed that besides one residue of the proteinogenic (S)-isoleucine [Ile], hormaomycin contains two units of 3-(2S,3R)-methylphenylalanine [( $\beta \mathrm{Me}) \mathrm{Phe}]$, one of $(2 R)$-allo-threonine $[a-$ 
Thr] as well as two moieties of 3-(trans-2'-nitrocyclopropyl)alanine [(3-Ncp)Ala] and one of 4(Z)-propenylproline [(4-PE)Pro]. The side chain of $\mathbf{3}$ is terminated with the residue of 5-chloro1-hydroxypyrrole-2-carboxylic acid [Chpca] (Figure 1). The latter three elements have never been found in any natural product before. A partial assignment of the configuration of (3Ncp)Ala residues in $\mathbf{3}$ was made later by Zindel and de Meijere. ${ }^{[10]}$ The retention times of the derivatized synthetically prepared enantiomerically pure mixtures of the diastereomers of 3(trans-2'-nitrocyclopropyl)alanine were compared with the derivatized total hydrolyzate of natural hormaomycin. These experiments unambiguously proved that both (3-Ncp)Ala residues in the cyclic depsipeptide $\mathbf{3}$ have the same $\left(1^{\prime} R, 2^{\prime} R\right)$ configuration at the nitrocyclopropyl ring and the opposite configuration at the $\alpha$-carbons. Nevertheless the orientation of the substituents at the $\alpha$-carbons of each (3-Ncp)Ala moiety in $\mathbf{3}$ left unclear. To the clarify situation, the feeding experiments with enantiomerically pure deuterium labelled 3-(trans-2'-nitrocyclopropyl)alanine should be carried out. Recently (2S,1'RS, $\left.2^{\prime} R S\right)-3,3$-dideutero-3-(trans-2'nitrocyclopropyl)alanine was synthesized by $K$. Loscha ${ }^{[11]}$ and the deuterium labelled hormaomycin was successfully obtained after the appropriate feeding experiments, which was carried out by P. Alvermann. ${ }^{[12] ~}{ }^{1} \mathrm{H},{ }^{2} \mathrm{H}$ NMR and MS-ESI spectra of this substance undoubtedly showed that the double insertion of the labelled amino acid took place. The possible explanation for this fact is that the $(2 S)$-isomer initially synthesized in cell can later be fully epimerized by the specific epimerase, before the assembling of the peptide chain of hormaomycin started, or already during this process after binding of the amino acid with multienzyme complex to allow access to the $(2 R)$-isomer necessary to complete the biosynthesis of $\mathbf{3}$. The relative and absolute configuration of 4-(Z)-propenylproline moiety stayed unclear and no attempts to elucidate it had been made.

While several synthetic routes for preparation of D-allo-threonine and (2S,3R)-3phenylmethylalanine were described in the literature, the enantio- and diastereoselective 
synthesis of 3-(trans-2'-nitrocyclopropyl)alanine, which was not suppose before, was intensely investigated in our group. At first, $\left(2 R S, 1^{\prime} S, 2^{\prime} S\right)-,\left(2 R S, 1^{\prime} R, 2^{\prime} R\right)-$ and $\left(2 S, 1^{\prime} R S, 2^{\prime} R S\right)-3-\left(\right.$ trans $-2^{\prime}-$ nitrocyclopropyl)alanines were successfully synthesized. ${ }^{[10,13]}$ Unfortunately, a great number of steps and relatively low overall yield strongly decreased the preparative value of this synthetic route. In fact, this procedure even did not allow to obtain any enantiomerically pure diastereomer of 3-(trans -2 -nitrocyclopropyl)alanine. The improvement of the originally reported procedure was connected with the progress in the preparation of the entiomerically pure $\left(1^{\prime} R, 2^{\prime} R\right)-(2-$ nitrocyclopropyl)methanol $\mathbf{1 0}$ which was the key intermediate for this synthesis and proposed by M. Brandl, A. de Meijere, et al. ${ }^{[14]}$ The optical resolution of trans-(2-nitrocyclopropyl)carboxylic acid via crystallization of the corresponding acyl 4-isopropyl-1,3-oxazolidin-2-ones 9 allowed to prepare the target alcohol only in 5 steps. It was previously obtained via a tedious twelve-step route starting from enantiomerically pure protected glycerine aldehyde. A large accessible quantity of $\mathbf{1 0}$ allowed $\mathrm{K}$. Loscha to synthesize $\left(2 S, 1^{\prime} R, 2^{\prime} R\right)-3-\left(2^{\prime}\right.$-nitrocyclopropyl)alanine successfully, although only $50 \mathrm{mg}$ of this amino acid was prepared. ${ }^{[11]}$ 

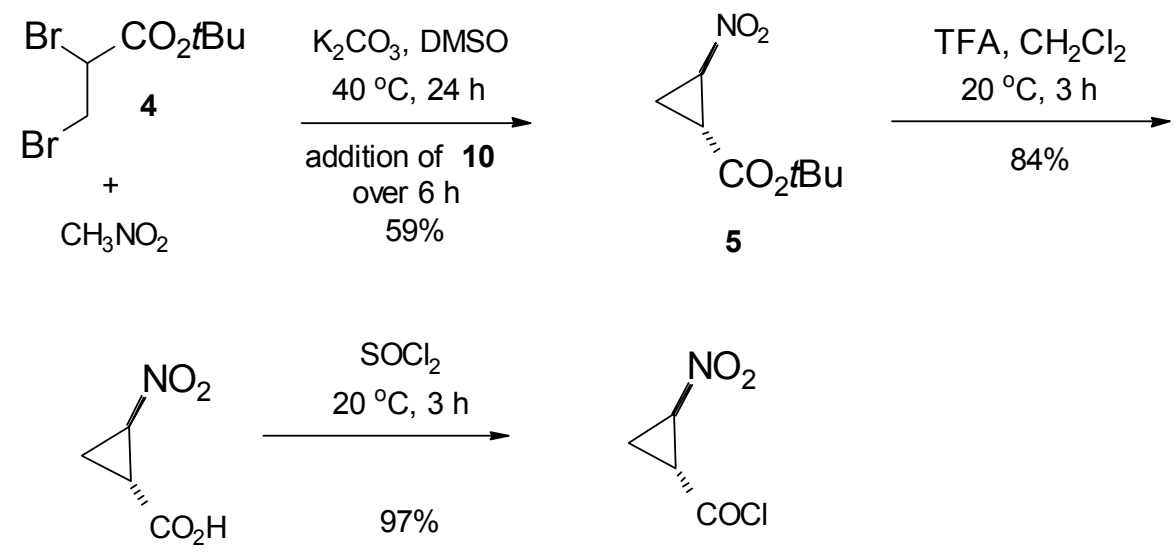

6

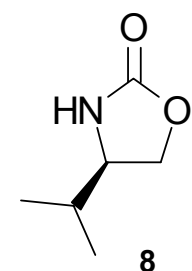

1) BuLi, THF

2) $7,-78^{\circ} \mathrm{C}, 1 \mathrm{~h}$

3) 5 crystallizations from hexane/ $\mathrm{CHCl}_{3}$

$42 \%$<smiles>CC(C)[C@@H]1COC(=O)N1C(=O)[C@@H]1C[C@H]1[N+](=O)[O-]</smiles>

9

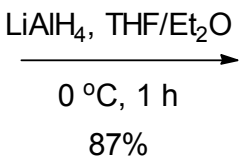

$87 \%$<smiles>O=[N+]([O-])C1C[C@H]1CO</smiles>

10

Scheme 1

The significant progress was also achieved in the preparation of 4-(Z)-propenylproline. This compound was obtained from 5-(2-dimethylaminopropyl)-piperidone-2 already in 1958 but only as a mixture of all four possible isomers of this amino acid together with all possible isomers 4allylproline. $^{[15]}$ The procedure recently proposed by E. Melotto ${ }^{[16]}$ allowed to prepare 4-(Z)propenylproline as an individual compound starting from $\mathrm{N}, \mathrm{O}$-diprotected pyroglutamic acid.

No procedures for the synthesis of $N$-hydroxypyrrolcarboxylic acids or $N$ hydroxypyrrolcarboxamides are described in the literature. An attempt to synthesize 5-chloro-1hydroxypyrrole-2-carboxylic acid, made by Ritzau appeared unsuccessful. ${ }^{[17]}$

Structure-activity relationships for hormaomycin were partially investigated using substances obtained via modification of the natural compound and also several hormaomycin analogs which were isolated after feeding experiments with synthetic amino acids or $\mathrm{NaBr}$. The cleavage of the lactone ring of hormaomycin with $\mathrm{K}_{2} \mathrm{CO}_{3}$ in methanol, which was carried out by Rössner, ${ }^{[8]}$ gave 
only biologically inactive material. The same author performed the hydrogenation of the natural depsipeptide with $\mathrm{Pd} / \mathrm{C}$ catalyst in methanol, caused the reduction of the double bond of 4-(Z)propenylproline moiety together with the water-elimination and the reductive dehalogenation of Chpca fragment and the partial reduction of the nitro-groups in both (3-Ncp)Ala residues. The resultant mixture which contained hormaomycin-like substances did not show any antibiotic activity. Later the fine attenuation conditions of hydrogenation of native depsipeptide allowed Ritzau $^{[17]}$ to successfully prepare the hormaomycin analog containing fragment of 4propylproline instead of original 4-(Z)-propenylproline moiety. This compound initiated aerial mycelium formation in Streptomyces species even stronger than native hormaomycin and showed antibiotic activity against coryneform bacteria, although it was noticeably lower than those of unmodified depsipeptide. Same author prepared analog of hormaomycin, which contained bromine instead of chlorine substituent in Chpca fragment. This substitution caused only little loss of the capability to induce the formation of aerial mycelium and drastic decrease of the antibiotic activity. The feeding experiments with synthetically prepared 2-(trans-2'nitrocyclopropyl)glycine $\mathbf{1 0}^{[18]}$ and 3-(trans-2'-carbomethoxycyclopropyl)alanine $\mathbf{1 1}^{[19]}$ allowed Alvermann ${ }^{[12]}$ to obtain both possible hormaomycin analogs containing 2-(trans-2'nitrocyclopropyl)glycine residue instead of one (3-Ncp)Ala moiety and depsipeptides with both (3-Ncp)Ala fragments substituted either on 2-(trans-2'-nitrocyclopropyl)glycine residues, or on 3-(trans-2'-carbomethoxycyclopropyl)alanine moieties. All these analogs did not display any hormaomycin-like activity.

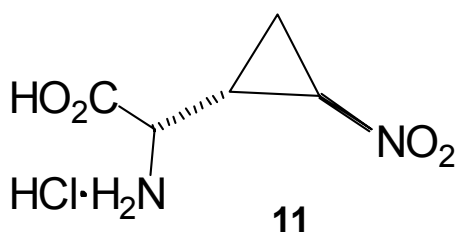

11

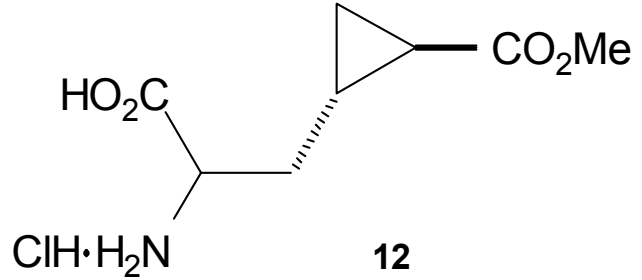

12 
As it was already mentioned, hormaomycin contains two moieties of 3-(trans-2'nitrocyclopropyl)alanine. The aliphatic nitro-compounds occur in nature very seldom. In fact, less than thirty such compounds were isolated till now and among them dipeptide nitropeptine 13, isolated from S. xanthochromogenus, which displayed noticeable antifungal activity. ${ }^{[20]}$ It would be reasonable to suppose that the unique biological activity of hormaomycin is connected with its nitro-group containing fragments. 3-(trans-2'-Nitrocyclopropyl)alanine itself (at least as a mixture of all possible isomers) was already tested and appeared to be inactive, but the inactivity of this amino acid could be connected just with the low capability of many amino acids to permeate across cell wall in the absence of the special transport mechanism because of their low hydrophobicity. Also it was known, that one of the new potent inhibitor of influenza neuraminidase A-315675 14 contains 4-(Z)-propenylproline fragment. ${ }^{21}$ Therefore a more detailed study of the role of this fragment for biological activity of hormaomycin was necessary.

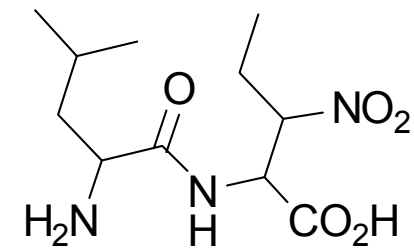

13

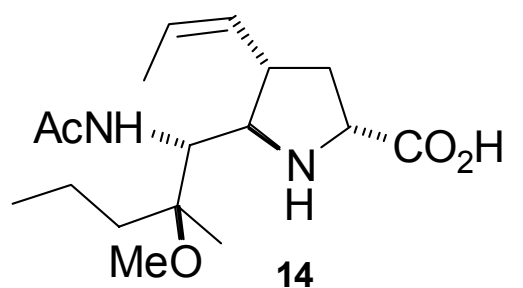

14

The previously achieved progress in the synthesis of the structural units of hormaomycin as well as the investigation of the structure-activity relationships for this compound which was briefly described above allowed formulating the following goals for the presenting work:

- Final elucidation of the absolute configuration of all stereogenic centers of hormaomycin.

- Total synthesis of this depsipeptide.

- Synthesis of analogs of hormaomycin including, possibly, shortened ones, to determine minimal structure units which are responsible for different kinds of activity of hormaomycin. 


\title{
B. MAIN PART
}

\section{Elucidation of the absolute configuration of 4-(Z)-propenylproline residue in hormaomycin. Synthesis of $(2 S, 4 R)-4-(Z)$-propenylproline and $(2 S, 4 R)-4-$ (Z)-(4,1'-dideutero)propenylproline}

\author{
1.1. Determination of the relative configuration of 4-(Z)-propenylproline moiety in \\ hormaomycin. Attempts to prepare pure diastereomers of 4-(Z)-propenylproline via \\ alkylation with (Z)-propenyllithium cuprate
}

Before the synthesis of 4-(Z)-propenylproline could be initiated, it was necessary to determine which from four possible isomers of this amino acid contained the target molecule and therefore should be synthesized. This problem was solved in two steps: first the relative configuration and then the absolute configuration of (4-PE)Pro moiety in hormaomycin were determined.

To solve the first problem, fast and simple access to the propenylproline even as a mixture of isomers was desired. Taking into account a lack of any reasonably effective routes for the preparation H-(4-PE)Pro-OH, the procedure of Thottathil and Moniot ${ }^{[22]}$ (used by authors for the large scale preparation of $N$-Boc protected trans-4-phenylproline) was chosen first starting point (Scheme 2). The tosylate $(2 S, 4 R)-\mathbf{1 5}$, prepared according to known procedure, ${ }^{[22,23]}$ was successfully alkylated with 3 equivalents of (Z)-propenyllithium cuprate ${ }^{24}$ in THF/Et 20 1:2 mixture to give the inseparable by conventional methods mixture of $N$-Boc protected cis- and trans-4-propenyl- $N$-prolines, cis-16 and trans-16 in 1:2.2 ratio according to ${ }^{13} \mathrm{C}$ NMR spectrum and in $84 \%$ yield. The isomerization of the double bond was insignificant and didn't exceed 4 $10 \%$, according to ${ }^{13} \mathrm{C}$ NMR spectrum (determined via comparison of the intensities of signals of 
the methyl groups: $\delta=13.1 \mathrm{ppm}$ for $(Z)$-isomer and $17.8 \mathrm{ppm}$ for $(E)$-isomer). A portion of this mixture was separated by preparative HPLC to give desired diastereomers. ${ }^{[25]}$

The relative configuration of each isomer was unambiguously established by NOESY experiments (Scheme 2). Although NMR spectra of these substances showed the presence of two rotamers, the signals of $2-\mathrm{H}, 3-\mathrm{H}_{\mathrm{a}}, 3-\mathrm{H}_{\mathrm{b}}$ and $4-\mathrm{H}$ were cleanly separated from each other. For the trans-isomer the strong correlations between $2-\mathrm{H}(\delta=4.40 \mathrm{ppm})$ and $3-\mathrm{H}_{\mathrm{a}}(\delta=1.82 \mathrm{ppm})$, and between $4-\mathrm{H}(\delta=3.18-3.33)$ and $3-\mathrm{H}_{\mathrm{b}}(\delta=2.41)$, while no correlations between either $2-\mathrm{H}$ and $3-\mathrm{H}_{\mathrm{b}}$, or $4-\mathrm{H}$ and $3-\mathrm{H}_{\mathrm{a}}$, or between $2-\mathrm{H}$ and $4-\mathrm{H}$ were observed. On the contrary, for the cisisomer the direct correlation between $2-\mathrm{H}(\delta=4.22,4.28 \mathrm{ppm})$ and $4-\mathrm{H}(\delta=2.99-3.16 \mathrm{ppm})$ and also strong correlations between $2-\mathrm{H}$ and $3-\mathrm{H}_{\mathrm{b}}(\delta=2.38,2.44)$, and $4-\mathrm{H}$ and $3-\mathrm{H}_{\mathrm{b}}$ were seen, while no correlation between $2-\mathrm{H}$ and $3-\mathrm{H}_{\mathrm{a}}(\delta=1.75,1.88 \mathrm{ppm})$ and only weak correlation between $4-\mathrm{H}$ and $3-\mathrm{H}_{\mathrm{a}}$ were observed. Both isomers were deprotected with trifluoroacetic acid for 20 min and the appropriate amino acids - cis-17 and trans $\mathbf{- 1 7}$ - were obtained. Whereas trans-16 was deprotected smoothly to give corresponding trifluoroacetate in quantitative yield, the desired amino acid cis-17 was obtained after several recristallizations of the hydrochloride in only $25 \%$ yield. Two samples of each amino acid were taken. One sample of each amino acid was derivatized with $(S)$-FDVA $\left[N_{\alpha}\right.$-2,4-dinitro-5-fluorophenyl- $(S)$-valine amide $]$ reagent $^{[26]}$ and the enantiomeric purity of each isomer was determined according to Marfey method. ${ }^{[27]}$ This experiment showed $e e=69 \%$ for the trans-isomer and almost full racemization in case of the cis-isomer. These data were in good agreement with the data on enantiomeric purities of isomeric 4-phenylprolines obtained according to this procedure. ${ }^{[22]}$ The another samples of 4-(Z)propenylprolines were transformed into dabsyl (4-dimethylaminoazobenzene-4'-sulfonyl) derivatives. $^{[2]}$ That were compared by HPLC with the sample of DABS-(4-PE)Pro-OH obtained after the HPLC separation from the DABS-derivatised total hydrolyzate of natural hormaomycin. 
MS/MS technique was used for the determination of the necessary fraction. These experiments showed that 3 contains $c i s-(4-P E)$ Pro moiety. ${ }^{[12]}$

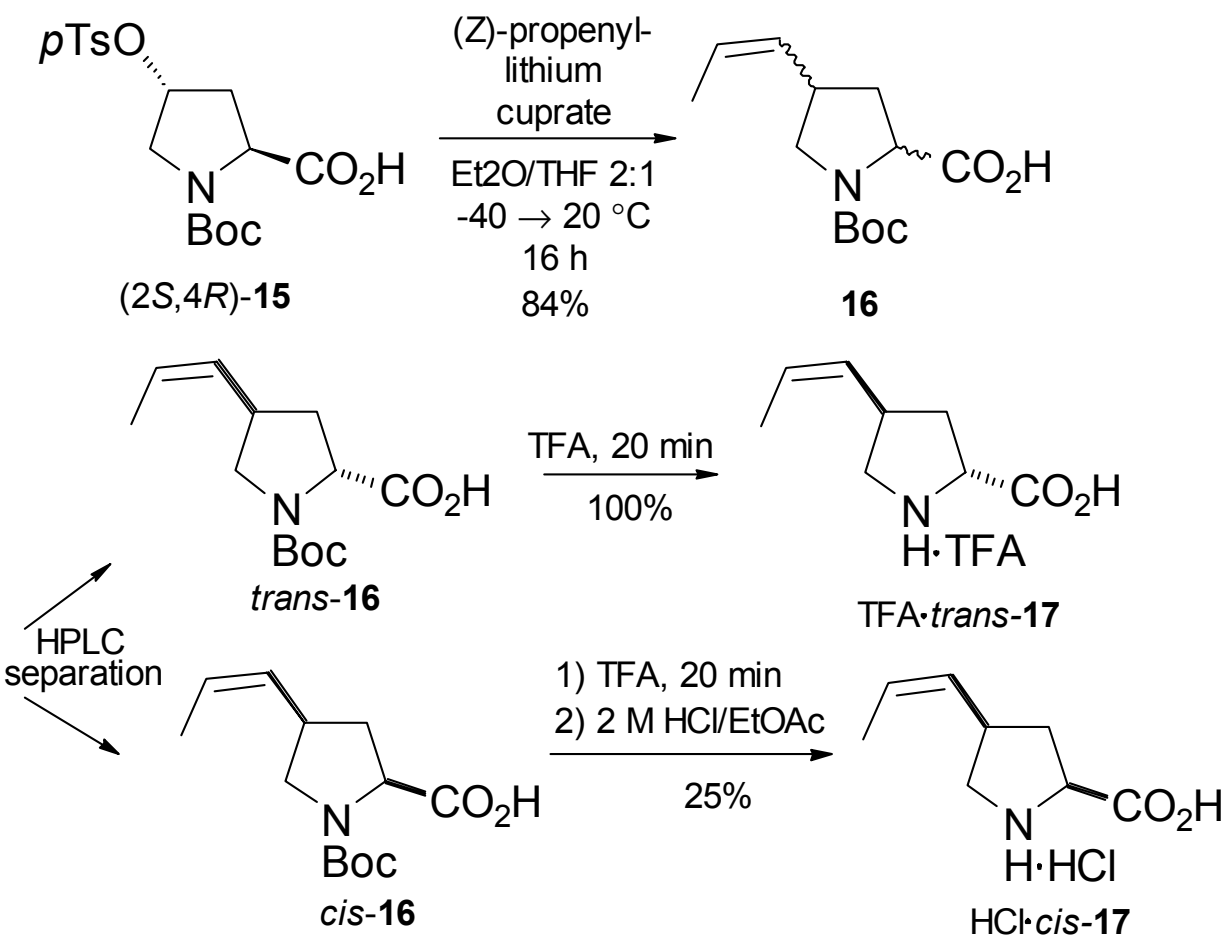

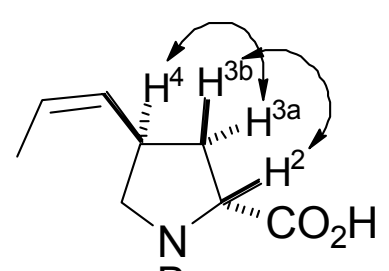

Boc

trans-16

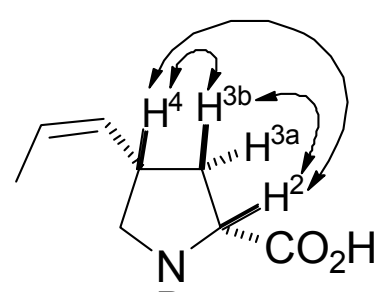

Boc

Scheme 2

To explain a significant degree of the retention of the C-4 configuration, it was supposed that this reaction could mainly proceed through intermediate lactone $(2 S, 4 R)-18{ }^{[29]}$ The enolate of this lactone can not epimerize at C-2 for sterical reasons (Bredt's rule). The tosylate $(2 S, 4 R)-15$ was treated with 2 equivalents of dry $\mathrm{Na}_{2} \mathrm{CO}_{3}$ in boiling butanone- 2 to give this known substance ${ }^{[30]}$ 
in $91 \%$ yield. ${ }^{[31]}$ However, an attempted reaction of $(2 S, 4 R)-18$ with $(Z)$-propenyllithium cuprate gave a complex mixture, which didn't contain desired products (Scheme 3).

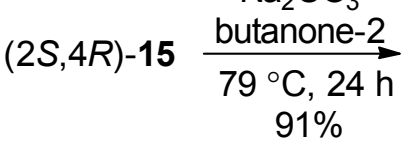

$91 \%$

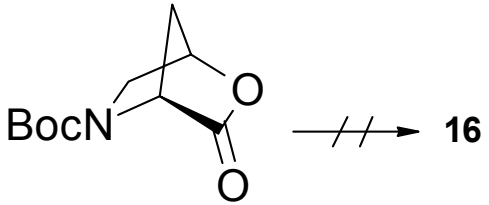

$(S)-18$

Scheme 3

Although the introduction of the $(Z)$-propenyl substituent at forth position of the proline ring via reaction of the tosylate $(2 S, 4 R)-\mathbf{1 5}$ with the corresponding lithium cuprate proceeded smoothly, it gave mainly the undesirable trans-isomer, and ee for the cis-isomer which was obtained as only minor component was unacceptably low. On the other hand, it is known that tosylates and mesylates secondary alcohols (but not iodides) usually give preferentially $\mathrm{S}_{\mathrm{N}} 2$ products in reactions with homocuprates. To study the possibility, if reaction in our case can proceed by $\mathrm{S}_{\mathrm{N}} 2$ mechanism, and at the same time to exclude epimerization at C-2, the compound (2S)-22 was prepared according to protocol of Wuts et al. ${ }^{[32]}$ (Scheme 4).

(2S,4R)-19 was first acylated with phenylchloroformate under Schotten-Baumann conditions using $\mathrm{NaHCO}_{3}$ as a base and, the derivative $(2 S, 4 R)-\mathbf{2 0}$ was reduced with borane in THF to give the $N$-acylprolinol $(2 S, 4 R)-\mathbf{2 1}$ in $74 \%$ yield. Then was $(2 S, 4 R)-\mathbf{2 1}$ cyclizated with $t$ BuOK in THF at $0{ }^{\circ} \mathrm{C}$ to the desired bicyclic oxazolidinone $(2 S, 4 R)-22$ in $83 \%$ yield. Treatment of this compound with tosyl chloride in pyridine gave the corresponding tosylate $(2 S, 4 R)-23$ in $82 \%$ yield, which was further treated with 3 equivalent of (Z)-propenyllithium cuprate in $\mathrm{THF} / \mathrm{Et}_{2} \mathrm{O}$ 1:2 to give the diastereo- and enantiomerically pure product $(2 S, 4 S)-\mathbf{2 4}$ with partially isomerized double bond but only in 5\% (9\% taking conversion into account) yield. All attempts to improve the yield failed. 

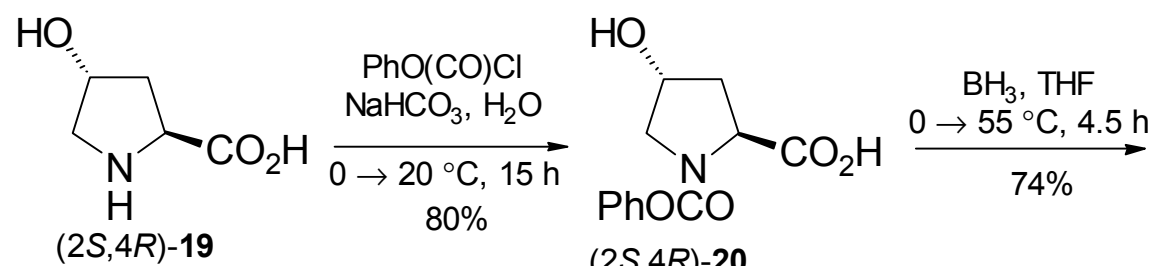

$(2 S, 4 R)-20$

$\mathrm{HO}$

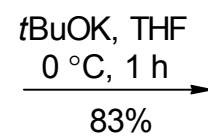

(Z)-propenyllithium

$\underset{\text { Et2O/THF } 2 \cdot 1}{\text { cuprate }}$

$-40 \rightarrow 20^{\circ} \mathrm{C}$

$16 \mathrm{~h}$

$5 \%(9 \%)$

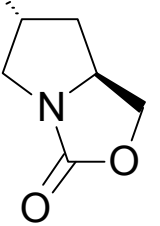

$(2 S, 4 R)-22$

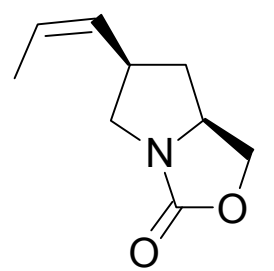

$(2 S, 4 R)-24$

ZIE $=4: 1$

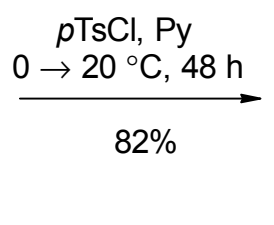

pTsO,<smiles>O=C(O)[C@H]1C[C@@H](O)CN1C(=O)O</smiles>

$(2 S, 4 R)-21$

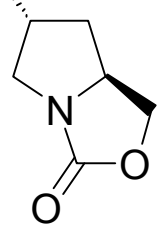

$(2 S, 4 R)-23$

Scheme 4

1.2. Elucidation of the absolute configuration of 4-(Z)-propenylproline moiety in hormaomycin

Once the relative configuration of (4-PE)Pro residue in hormaomycin was elucidated, the absolute configuration of this fragment should be established. At first, several experiments failed, as it was impossible to isolate this amino acid (or its derivatives) from the total hydrolyzate of natural depsipeptide due to instability of 4-(Z)-propenylproline in the presence of strong acids routinely applied for the total hydrolysis of peptides. At the same time, it was known for a long time that the catalytic hydrogenation of $\mathbf{3}$ with palladium or platinum catalysts transforms the (4-PE)Pro moiety into the 4-propylproline residue (and also reduces other functionalities of molecule, see introduction for details). Therefore, both enantiomers of cispropylproline, which was necessary for the determination of the absolute configuration, were prepared (Scheme 5). 


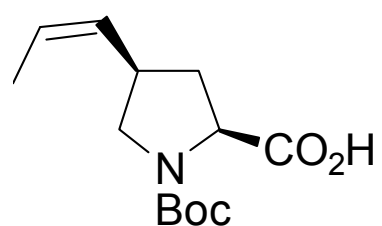

(2S, 4R)-16
1) $\mathrm{H}_{2}, \mathrm{Pd} / \mathrm{C}$

EtOAc, $3 \mathrm{~h}$

2) $2 \mathrm{M} \mathrm{HCl}$ in EtOAc $20^{\circ} \mathrm{C}, 1 \mathrm{~h}$

$79 \%$

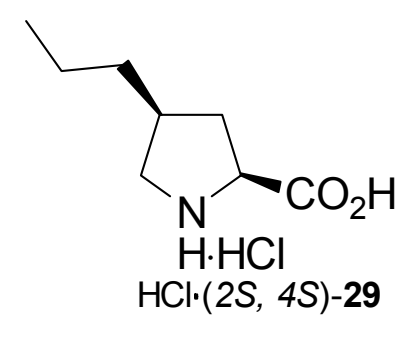

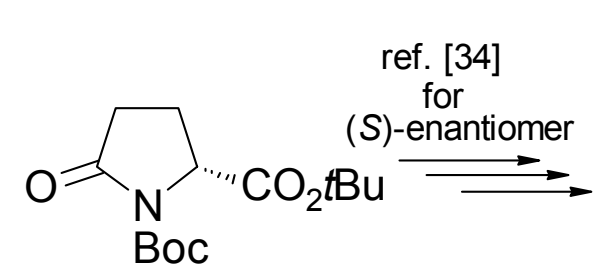

(R)-25<smiles>C=CCC</smiles>

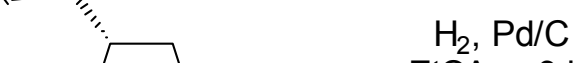<smiles>CCOC(=O)C1CCC(=O)N1</smiles>

Boc

$(2 R, 4 R)-26$

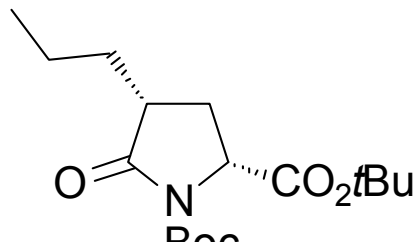

Boc

(2R,4R)-27
1) $\mathrm{LiBEt}_{3} \mathrm{H}$, THF

2) $\underset{\mathrm{Et}_{3} \mathrm{SiH}, \mathrm{BF}_{3} \cdot \mathrm{Et}_{2} \mathrm{O}}{\longrightarrow}$

$\mathrm{CH}_{2} \mathrm{Cl}_{2},-78^{\circ} \mathrm{C}, 2.5 \mathrm{~h}$

$79 \%$

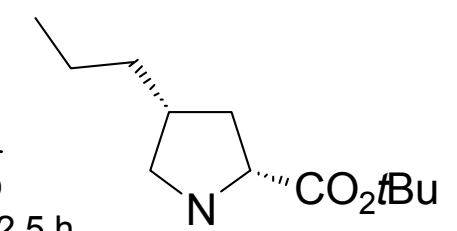

BOC

$(2 R, 4 R)-28$

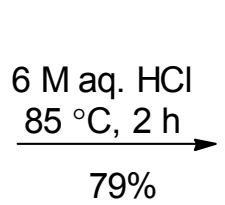

Scheme 5

$\mathrm{HCl} \cdot(2 R, 4 R)-29$

The double bond of the enantiomerically pure $N$-Boc protected 4-(Z)-propenylproline $(2 S, 4 R)-16$ was hydrogenated on $10 \% \mathrm{Pd} / \mathrm{C}$ in ethyl acetate, and then $\mathrm{N}$-Boc protection was removed with 2 $\mathrm{M} \mathrm{HCl}$ in EtOAc to give the known $(2 S, 4 S)-\mathbf{2 9}^{[33]}$ as a hydrochloride in $79 \%$ yield. The $(2 R, 4 R)-$ isomer was obtained after the deprotection of $(2 R, 4 R)-\mathbf{2 8}$. The latter was prepared from the cisallyl derivative $(2 R, 4 R)$-26 which was in turn obtained by alkylation with allyl bromide of the enolate of the $N$-Boc protected tert-butyl- $(R)$-pyroglutamate $(R)-25^{[34]}$ in the same way as it was described for $(2 S, 4 S)$-isomer $(R)-\mathbf{2 6} .^{[35]}$ Hydrogenation of the double bond in $(R)-\mathbf{2 6}$ on $10 \%$ $\mathrm{Pd} / \mathrm{C}$, followed by two step reduction of the lactam cycle of the intermediate $N, O$-protected 4propylpyroglutamic acid $(2 R, 4 R)-27$ according to procedure of $\mathrm{J}$. Ezquerra et al. completed this synthsis. ${ }^{[36]}$ Both enantiomers were transformed to (S)-FDVA derivatives, which were compared with the derivatizated hydrolyzate of the hydrogenated $\mathbf{3}^{[37]}$ using HPLC/MS technique according 
to the advanced Marfey method. ${ }^{[38]}$ These experiments showed that hormaomycin contains $(2 S, 4 R)-(4-P E)$ Pro residue.

\section{3. $\quad$ Attempts to prepare 4-(Z)-propenylproline starting from pyroglutamic acid}

As it was already mentioned, when this work was started, the only reasonable route for the preparation of this amino acid was proposed by E. Melotto who prepared $N$-Boc protected (2S,4RS)-4-(Z)-propenylproline (2S,4RS)-16 starting from pyroglutamic acid with the construction of the double bond via the Wittig reaction. ${ }^{[16]}$ It seemed to be possible further use this scheme for the preparation of the desired compound having in mind further improvement of its diastereoselectivity and/or a separation of diastereomeric mixture. First, it was necessary just to reproduce the already described synthesis of the 4-carbonylproline derivative 36 . Unexpectedly, the preparation of the dimethyl acetals $(S)-32$ from the aldehyde (S)-31 which was in turn prepared from the known enamine $(S)-\mathbf{3 0}^{[39]}$ already required an optimization.

The protection of the aldehyde group should be carried out at $20^{\circ} \mathrm{C}$ instead of initially proposed $60{ }^{\circ} \mathrm{C}$ in order to obtain the desired mixture of the diastereomeric acetals with reproducibly high yield (Scheme 6). This mixture was separated by conventional flash chromatography to give the individual epimers $(2 S, 4 S)-32(53 \%$ yield) and $(2 S, 4 R)-32(22 \%$ yield $)$. The reduction of the lactam ring of the acetals 32 with borane in THF according to the proposed procedure was unselective and repeatedly gave the 4-substituted proline $(2 S, 4 S)-33$ only in low yield together with significant quantity of the "half amidal" $(2 S, 4 S)-34$ and variable quantities of prolinol $(2 S, 4 S)-35$ 
<smiles>CNC=C1CC(C(C)=O)NC1=O</smiles>

(S)-30

$\mathrm{OHC}$<smiles>CC(=O)OC(=O)C1C[C@@H](C)C(=O)N1C(=O)OCc1ccccc1</smiles>

(S)-31

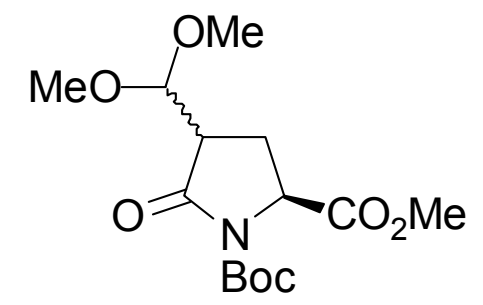

(S)-32

(2S,4S)-32

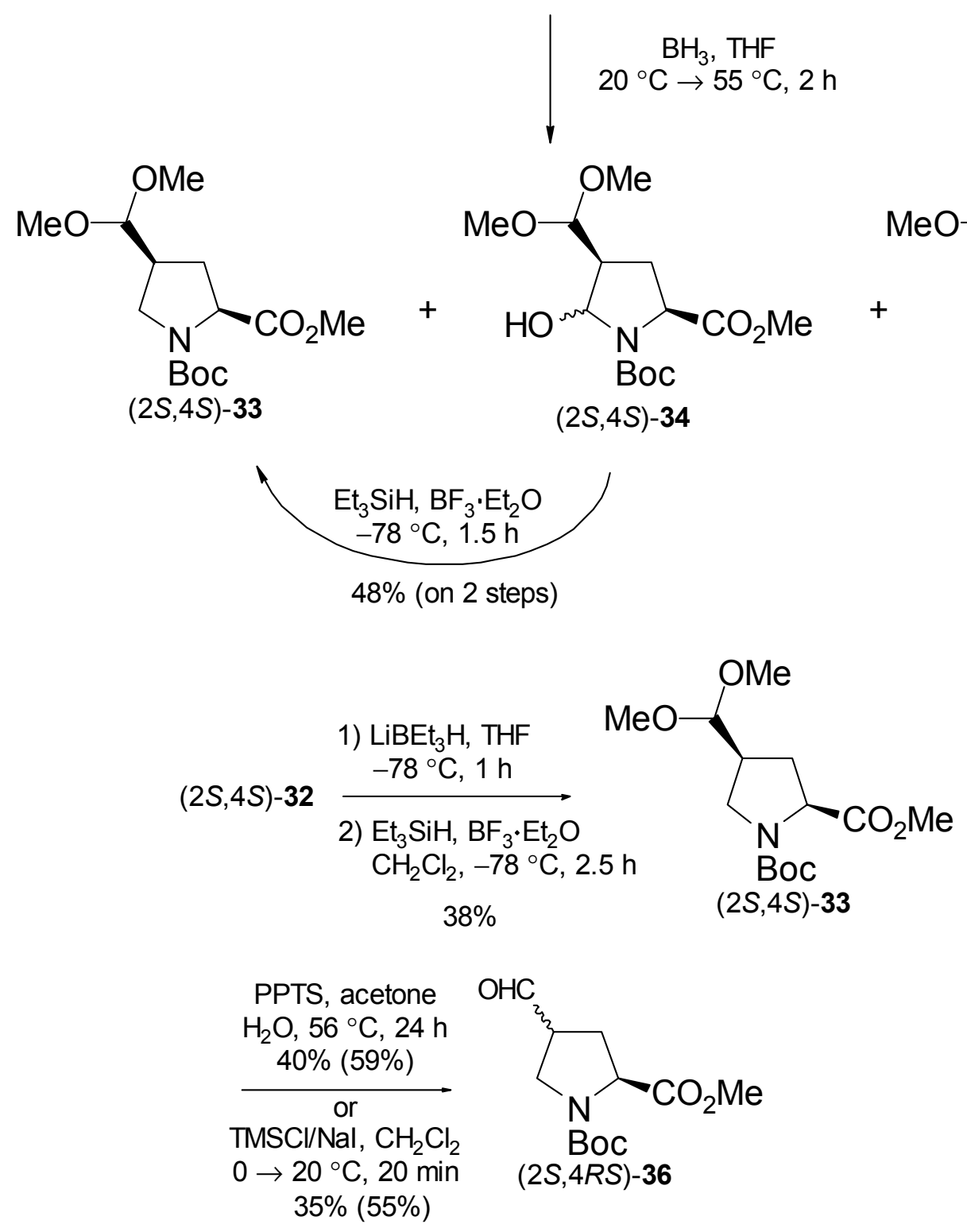

Scheme 6 
After several unsuccessful attempts to increase the yield of the target product by varying temperature, order of addition or excess of $\mathrm{BH}_{3} \cdot \mathrm{THF}$, it was found that the separation of the mixture of the "half amidal" $(2 S, 4 S)-\mathbf{3 4}$ and $(2 S, 4 S)-\mathbf{3 3}$ and further reduction of $(2 S, 4 S)$-34 in (2S,4S)-33 by treatment with $\mathrm{BF}_{3} \cdot \mathrm{Et}_{2} \mathrm{O}$ and triethylsilane in $\mathrm{CH}_{2} \mathrm{Cl}_{2}$ at $-78{ }^{\circ} \mathrm{C}$ allowed to obtain the required $(2 S, 4 S)-33$ in $48 \%$ overall yield over two steps.

The application of the original protocol of J. Ezquerra et al. ${ }^{[36]}$ gave this substance in $38 \%$ yield. The hydrolysis of the dimethyl acetal $(2 S, 4 S)-33$ with TMSI in $\mathrm{CH}_{2} \mathrm{Cl}_{2}$ at $20{ }^{\circ} \mathrm{C}$, as it was originally described by E. Melotto, gave the aldehyde $\mathbf{3 6}$ in 35\% (55\% taking into account only the reacted material). Alternatively, treatment of (2S,4S)-33 with PPTS in refluxing acetone gave the target compound in $40 \%(59 \%)$ yield. Its ${ }^{13} \mathrm{C}$ NMR spectra showed that in both cases significant (up to $30 \%$ ) epimerization at C-4 took place. ${ }^{[40]}$ As the acidity of PPTS is very low and TMSI doesn't have any, it was supposed that the configurational lability the aldehyde $\mathbf{3 6}$ could cause epimerization already during column chromatography on silica gel. Application of eluents containing different concentration of triethylamine didn't improve anything. As the stereochemical efficiency of this route was unacceptably low, other synthetic pathways should be tested.

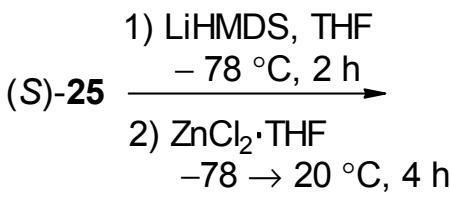

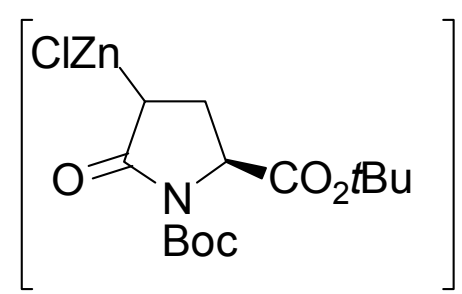

$(S)-37$
1-(Z)-propenyl bromide

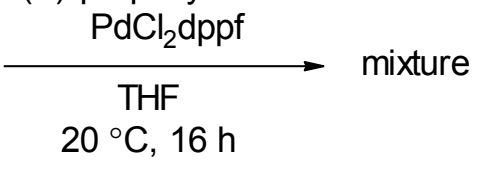

Scheme 7

A direct introduction of the $(\mathrm{Z})$-propenyl fragment at the fourth position of the lactam ring of the pyroglutamic acid via Pd-catalyzed coupling reaction looked as a very attractive way. As it is 
known, reactions of this kind are the most powerful, versatile and popular tools for the formation of the carbon-carbon bonds. In our case the palladium-catalyzed Negishi cross-coupling reaction $^{[41]}$ (reaction of aryl and vinyl halides/triflates with organozinc reagents) seemed to be a method of choice because of the high functional group compatibility of organozinc compounds, that was essentially important in our case. Unfortunately, several attempts to carry out reaction between the organozinc compound $(S)$-37, which was obtained from $(S)-25$ via the corresponding lithium enolate and propenyl bromide, were unsuccessful and gave only complex mixtures (Scheme 7).

Another possibility would be the deconjugative isomerisation of the protected 4propylidenpyroglutamic acid into the corresponding 4-propenylpyroglutamic acid. At least for simple objects studied before, this reaction often proceeded in such a way that the $(E)-\alpha, \beta-$ unsaturated esters or amides give the $(Z)$-deconjugated products, and ,on the contrary $(Z)$ conjugated double bond transforms into $(E)$-double bond with good selectivity. ${ }^{[42]}$ The $N$-Boc protected tert-butyl $(S)-4-(E)$-propilidenepyroglutamate $(S)-39^{[33 b]}$ was prepared, as it was described by J. Ezquerra et al. ${ }^{[43]}$ for the corresponding ethyl ester: $(S)$-25 (Scheme 5) was first treated with LiHMDS in THF at $-78^{\circ} \mathrm{C}$, and then the reaction with propionic aldehyde in the presence of 1.15 equivalent of $\mathrm{BF}_{3} \cdot \mathrm{Et}_{2} \mathrm{O}$ at the same temperature gave the known alcohol $(S)$ $\mathbf{3 8}^{[44]}$ as a partially separable mixture of three from four possible diastereomers (configuration at C-2 retained) in much more better yield (78\% against $28 \%$ ) than it was obtained before (Scheme 8). 
(S)-25

1) LiHMDS, THF

$\frac{-78^{\circ} \mathrm{C}, 1 \mathrm{~h}}{2) \mathrm{EtCHO}, \mathrm{BF}_{3} \cdot \mathrm{Et}_{2} \mathrm{O}}$

$-78{ }^{\circ} \mathrm{C}, 90 \mathrm{~min}$ $78 \%$

1) LiHMDS, HMPT, THF 2) $\underset{2,6 \text {-di-tert-Butylphenol }}{\stackrel{-78}{\circ} \mathrm{C}, 1 \mathrm{~h}}$ $-78{ }^{\circ} \mathrm{C}, 5 \mathrm{~min}$ $56 \%$

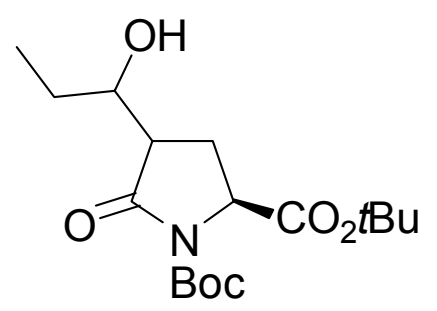

(S)-38

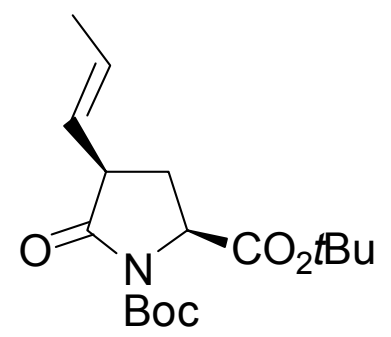

$(2 S, 4 R)-40$

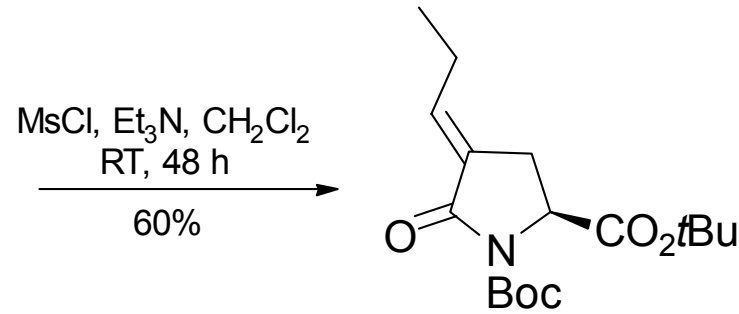

(S)-39

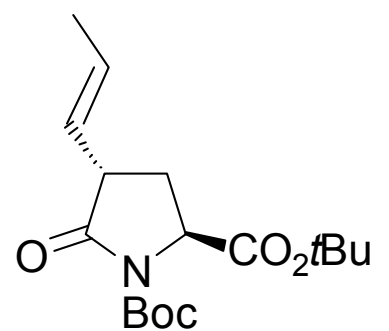

$(2 S, 4 S)-40$

cis/trans $=2: 1$

Scheme 8

The latter was treated with an excess of mesyl chloride and triethylamine in $\mathrm{CH}_{2} \mathrm{Cl}_{2}$, and the mixture of isomeric 4-propylidene derivatives was separated by column chromatography to give the required $(E)$-isomer $(S)$-39 $(60 \%)$ together with small amount of $(Z)$-isomer $(4 \%)$. The configuration of the double bond in the main product was proved by NOESY experiment. The $\mathrm{N}, \mathrm{O}$-protected 4-(Z)-propylidenepyroglutamic acid $(S)$-39 was treated with slight excess of LiHMDS in THF/HMPT mixture at $-78{ }^{\circ} \mathrm{C}$ for $1 \mathrm{~h}$, and the resulting enolate was quenched with 2,6-di-tert-butylphenol as a sterically hindered proton source. This method of enolates quenching is known to give preferentially cis-isomers because of the protonation from the least hindered side. ${ }^{[45]}$ The formed mixture of diastereomers was separated by column chromatography to give the $c i s$-isomer $(2 S, 3 S)-\mathbf{4 0}(37 \%)$ and the trans-isomer $(2 S, 3 R)-\mathbf{4 0}(19 \%)$. Attempt to remove the last traces of the trans-epimer from $(2 S, 3 S)$-40 showed the conformational instability of the latter: ca. $30-40 \%$ conversion of this product in the thermodynamically more stable trans-isomer was observed after usual flash chromatography. Surprisingly, ${ }^{13} \mathrm{C}$ NMR spectra of these products showed that $100 \%$ retention of configuration of the double bond took place. Such kind of deconjugative isomerisation which has not yet been described for such complicated objects makes possible to prepare still unknown 4-alkenyl substituted pyroglutamic acids (and 
consequently alkenyl substituted glutamic acids, prolines and leucines). Nevertheless the wrong configuration of the double bond and the conformational instability of $(2 S, 3 S)-\mathbf{4 0}$ precluded the use of this approach for the synthesis of 4-(Z)-propenylproline.

1.4. Synthesis of $\mathrm{N}$-Boc protected 4-(Z)-propenylproline and dideutero-4-(Z)propenylproline starting from 4-hydroxyproline

As already mentioned before, Negishi reaction looked to be a very attractive way to introduce the $(Z)$-propenyl fragment. To test this reaction for proline derivatives, the known iodide $(2 S)-\mathbf{4 1}$ was obtained from Boc-Hyp-OMe ${ }^{[46]}$ by Mitsunobu reaction according to Dormoy. ${ }^{[47]}$ It was allowed to react with $\mathrm{Zn}$ dust (activated according to Knochel et al ${ }^{[48]}$ ) and then a Pd catalyst and $(Z)$-propenyl bromide were added. The product of the elimination of the intermediate organozinc compound, the $N$-protected allylglycine ester $(S)-42$, was obtained as a sole product in $67 \%$ yield (Scheme 9).

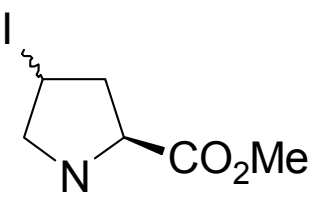

Boc (2S)-41

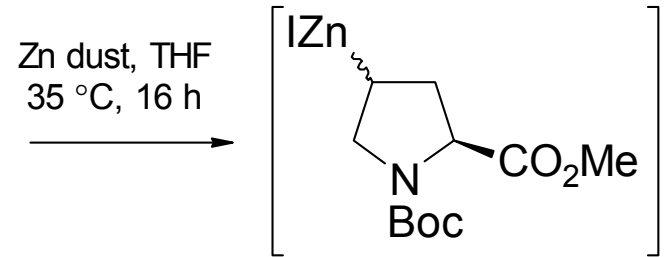

$67 \%$ $\mathrm{BocHN} \mathrm{CO}_{2} \mathrm{Me}$

$(2 S)-42$

Scheme 9

The reproduction of this reaction without redundant components starting from both pure diastereomers of iodide $(S)-\mathbf{4 1}$ or their mixture, gave the allylglicine derivative $(S)-\mathbf{4 2}$ in essentially same yield. The optical purity of $(S)-\mathbf{4 2}$ was not determined intentionally but the optical rotation value of this material was in good agreement with the literature value. ${ }^{[49]}$

Another option would be to try constructing the double bond of the propenyl fragment using Wittig reaction. Towards this goal, the effective route for the diastereoselective introduction of 
the formyl group into forth position of proline without use of either strong bases and acids or chromatographic purification as these reagents or $\mathrm{SiO}_{2}$ might cause epimerization at $\mathrm{C}-4$ in the target aldehyde. The reduction of nitriles with $\mathrm{DIBAH}^{[50]}$ is one of the mildest ways to obtain the aldehyde: this reagent is not basic, the reduction with this reagent often gives aldehydes which are pure enough to be directly used in the Wittig reaction without any chromatographic purification. The work-up procedure without use of strong bases or acids was reported. ${ }^{[51]}$ Moreover, the preparation of several $N, O$-protected 4-cyanoprolines was already described. ${ }^{[52]}$

The $N$-Boc protected 4-hydroxyproline $(2 S, 4 R)-\mathbf{4 3}^{[53]}$ was esterified with large excess of tertbutyl bromide and $\mathrm{K}_{2} \mathrm{CO}_{3}$ in the presence of 1 equivalent of TEBACl in DMA according to procedure Martinez et al. ${ }^{[54]}$ to give the corresponding tert-butyl ester $(2 S, 4 R)-44^{[55]}$ in $65 \%$ yield which was in turn treated with mesyl chloride and triethylamine in $\mathrm{CH}_{2} \mathrm{Cl}_{2}$ to give mesylate $(2 S, 4 R)-\mathbf{4 5}^{[56]}$ in $86 \%$ yield. The latter was transformed into the fully protected derivative of $4-$ cyanoproline $(2 S, 4 S)-\mathbf{4 6}$ by reaction with tetrabutylammonium cyanide in DMF at $60{ }^{\circ} \mathrm{C}$ in $57 \%$ yield (Scheme 10).

$\mathrm{HO}$<smiles>CC1CC(C(=O)O)N(C(=O)OCc2ccccc2)C1</smiles>
$(2 S, 4 R)-43$

$\mathrm{MsO}$<smiles>CCCOC(=O)C1CCCN1</smiles>

Boc

$(2 S, 4 R)-45$ $t \mathrm{BuBr}, \mathrm{K}_{2} \mathrm{CO}_{3}$, TEBACl

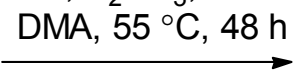

$65 \%$

$(\mathrm{Bu})_{4} \mathrm{~N}^{+} \mathrm{CN}^{-}$, DMF $\underset{57 \%}{\stackrel{6{ }^{\circ} \mathrm{C}, 12 \mathrm{~h}}{\longrightarrow}}$

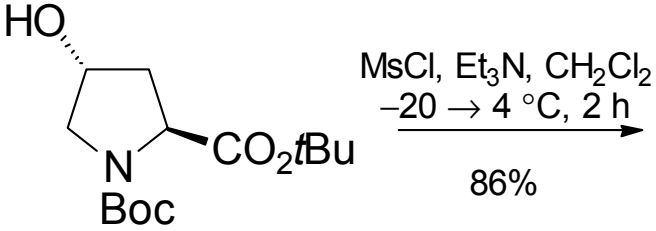

$(2 S, 4 R)-44$

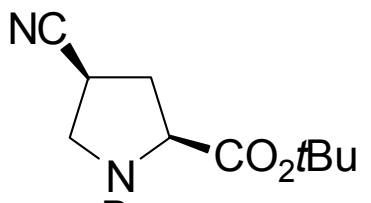

Boc

$(2 S, 4 S)-46$

Scheme 10

Although the selective reduction of the nitrile group with DIBAH in the presence of a tert-butyl ester was described, ${ }^{[56]}$ attempts of the reduction of $(2 S, 4 S)-\mathbf{4 6}$ even with submolar quantities of 
the reducing agent at $-78^{\circ} \mathrm{C}$ always gave the appropriate prolinol as a side product. To exclude this side reaction and risk of epimerization at C-2 during the nitrile insertion or Wittig reaction (when strong base should be used) the reduction of the carboxylic group of hydroxyproline may be used. The mesylate $(2 S, 4 R)-47^{[57]}$ gave on the treatment with tetrabutylammonium cyanide mainly the cyclization product $(S)-\mathbf{4 9}$ and the nitrile $(2 S, 4 S)$-48 was obtained only as a minor product (Scheme 11). Therefore, the compound was first $O$-MOM protected with MOMBr and DIEA in $\mathrm{CH}_{2} \mathrm{Cl}_{2}$ to give the corresponding MOM-ether $(2 S, 4 R)-\mathbf{5 0}$ in $92 \%$ crude yield. The latter was further transformed into the nitrile $(2 S, 4 S)-51$ (41\%) (Scheme 12).

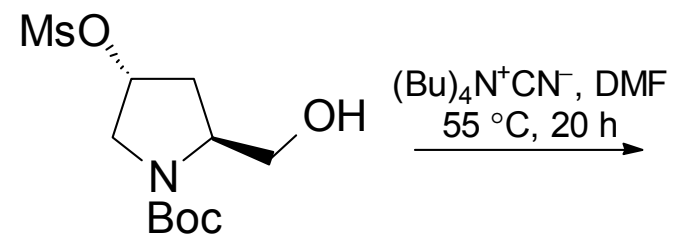

$(2 S, 4 R)-47$

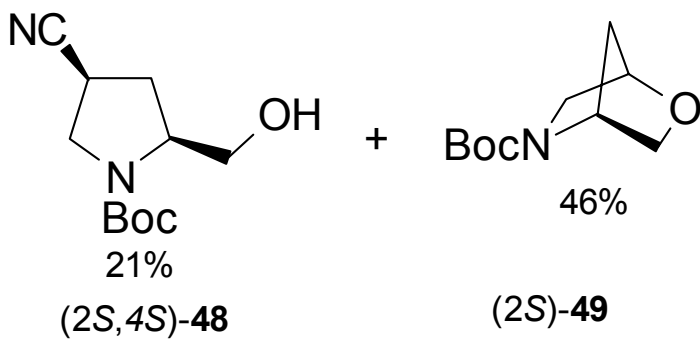

$(2 S, 4 S)-48$

Scheme 11

Compound $(2 S, 4 S)-\mathbf{5 1}$ was smoothly reduced into the aldehyde $(2 S, 4 S)-\mathbf{5 2}$ with DIBAH in $\mathrm{CH}_{2} \mathrm{Cl}_{2}$ at $-30{ }^{\circ} \mathrm{C}$ in $90 \%$ crude yield. The Wittig reaction between this substance and nonstabilized ylide, prepared from ethyltriphenylphosponium bromide according to Bestmann et al. ${ }^{[58]}$ using NaHMDS as a base to increase the $Z$-selectivity of this reaction, gave the desired fully protected 4-(Z)-propenylprolinol $(2 S, 4 R S)-53(38 \%)$ with excellent (Z)-selectivity as a 3:1 mixture of $(2 S, 4 R) /(2 S, 4 S)$-isomers. 

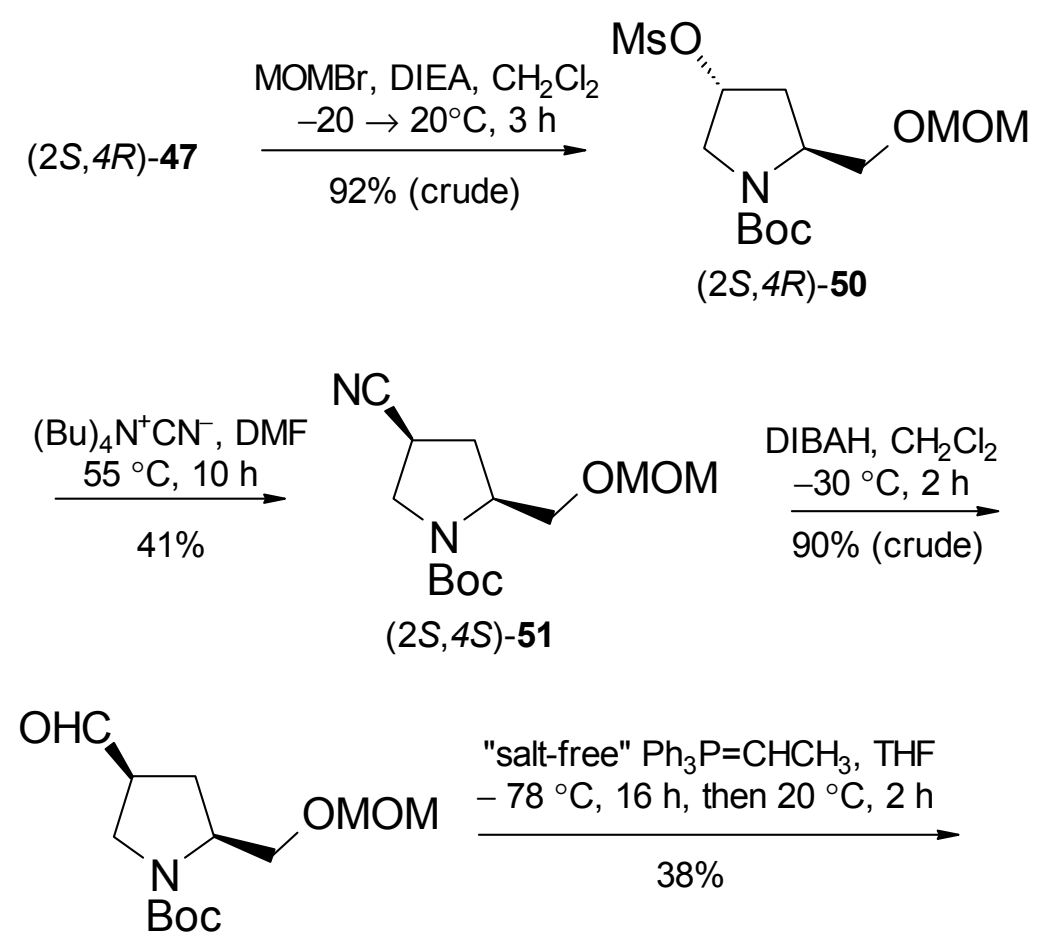

$(2 S, 4 S)-52$

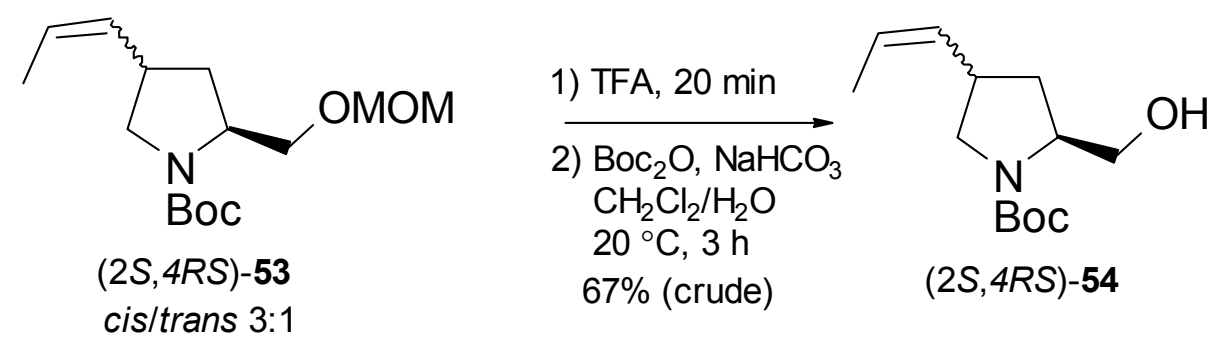

Scheme 12

On the next step MOM-group has to be selectively cleaved in the presence of $N$-Boc group with $p \mathrm{TsOH} \cdot \mathrm{H}_{2} \mathrm{O}$ at the ambient temperature according to Katoh et al. ${ }^{[59]}$ Unexpectedly, MOM-ester was entirely stable under these conditions. In fact, $N$-Boc group in this case was even more acidsensitive than the $O-\mathrm{MOM}$ ether, and its partial cleavage was observed. To obtain the alcohol (2S,4RS)-54 both protecting groups were cleaved by TFA, and the crude amino alcohol was immediately acylated with $\mathrm{Boc}_{2} \mathrm{O}$ to give the required somewhat impure product $(2 S, 4 R)-\mathbf{5 4}$ in $67 \%$ yield as a mixture of diastereomers which could be separated by conventional column chromatography. At this point it was clear that I should concentrate my efforts on further optimization of this route: improve diastereoselectivity of the formation of the double bond and 
try to achieve a higher yield of the alcohol $\mathbf{5 4}$ on the deprotection step.

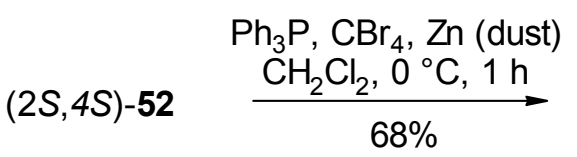

$\frac{\underset{\mathrm{CH}_{2} \mathrm{Cl}_{2}, 0{ }^{\circ} \mathrm{C}, 1 \mathrm{~h}}{68 \%}}{6}$

$\mathrm{Br}$<smiles>COC[C@H]1C[C@@H](/C=C\C(Br)Br)CN1</smiles>

Boc

(2S, 4RS)-55

cis/trans $=1.4: 1$

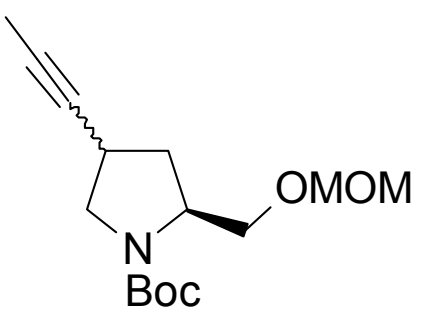

$(2 S, 4 R S)-56$

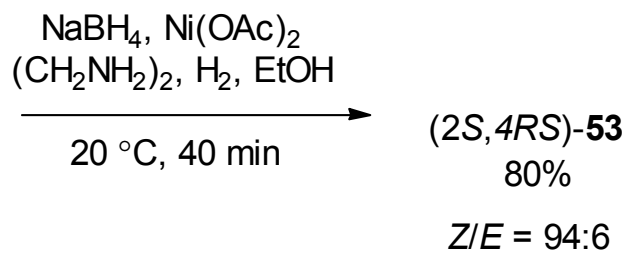

1) $n \mathrm{BuLi}, \mathrm{THF},-78^{\circ} \mathrm{C}, 1 \mathrm{~h}$ then $20^{\circ} \mathrm{C}, 1 \mathrm{~h}$

2) Mel, $-78 \rightarrow 20{ }^{\circ} \mathrm{C}, 20 \mathrm{~h}$

$44 \%$

Scheme 13

As an alternative to the conventional Wittig reaction Corey-Fuchs sequence ${ }^{[60]}$ was tested (Scheme 13). The aldehyde $(2 S, 4 S)-52$ was treated with excess of ilyde, preliminary obtained from $\mathrm{CBr}_{4}$ and $\mathrm{PPh}_{3}$ in the presence of $\mathrm{Zn}$ dust, in $\mathrm{CH}_{2} \mathrm{Cl}_{2}$ to give the vicinal dibromalkene $(2 S, 4 R S)-55$ in $68 \%$ yield and with almost full epimerization (cis/trans ratio 1.4:1). This product was treated first with 2 equivalents of $n \mathrm{BuLi}$ in THF and then with a large excess of methyl iodide to give the fully protected 4-propinylprolinol $(2 S, 4 R S)-56(44 \%)$ which was then hydrogenated on P-2 nickel catalyst ${ }^{[61]}$ in ethanol in the presence of diethylamine with excellent stereoselectivity and the target $(2 S, 4 R S)-54(80 \%)$ together with the overhydrogenation product $(2 S, 4 R S)-57(12 \%)$ was obtained. However an overall efficiency of this sequence was cleanly insufficient, and no attempts to improve this scheme were made.

As the $O$-MOM group could not be selectively cleaved in the presence of the Boc group, it was substituted with the tert-butyldimethylsilyl group (Scheme 14). The $N$-Boc protected 4hydroxyproline $(2 S, 4 R)-\mathbf{4 3}$ was treated with ethyl chloroformate and triethylamine in $\mathrm{CH}_{2} \mathrm{Cl}_{2}$ at $-30{ }^{\circ} \mathrm{C}$, and the resultant mixed anhydride was reduced with aqueous $\mathrm{NaBH}_{4}$ in presence of 
tetra- $n$-butylammonium bromide as a phase transfer catalyst to give the $N$-Boc protected 4 hydroxyprolinol $(2 S, 4 R)-\mathbf{5 8}$ in $82 \%$ yield. A primary hydroxyl group of $\mathbf{5 8}$ was selectively TBDMS-protected, as it was described by Aoyagi and Williams, and $(2 S, 4 R)-\mathbf{5 9}$ was prepared in $84 \%$ yield. ${ }^{[62]}$ The treatment of this substance with mesyl chloride and triethylamine in $\mathrm{CH}_{2} \mathrm{Cl}_{2}$ gave the mesylate $(2 S, 4 R)-\mathbf{6 0}$ in almost quantitative yield, which was in turn transformed into the nitrile $(2 S, 4 S)-61$ on treatment with tetrabutylammonium cyanide in DMF at $65{ }^{\circ} \mathrm{C}$ for $6 \mathrm{~h}$ with reproducible yield in $30-50 \mathrm{~g}$ scale. ${ }^{[63]}$

$(2 S, 4 R)-43$

1) $\mathrm{ClCO}_{2} \mathrm{Et}, \mathrm{Et}_{3} \mathrm{~N}, \mathrm{CH}_{2} \mathrm{Cl}_{2}$ 2) $\underset{\begin{aligned} \mathrm{NaBH}_{4}, \mathrm{Bu}_{4} \mathrm{~N}^{+} \mathrm{Br}^{-} \\ -10 \rightarrow 0{ }^{\circ} \mathrm{C}, 2 \mathrm{~h}\end{aligned}}{-}$ $82 \%$
$\mathrm{HO}$<smiles>O=C(O)[C@@H]1CCCN1</smiles>
$(2 S, 4 R)-58$
TBDMSCI, DMAP, $\mathrm{Et}_{3} \mathrm{~N}$

$\mathrm{CH}_{2} \mathrm{Cl}_{2}, 20^{\circ} \mathrm{C}, 3 \mathrm{~d}$

$82 \%$

$\mathrm{MsO}$

$\overbrace{\mathrm{N}}^{\longrightarrow \text { OTBDMS } \frac{\begin{array}{l}\mathrm{MsCl}, \mathrm{Et}_{3} \mathrm{~N}, \mathrm{CH}_{2} \mathrm{Cl}_{2} \\ -40 \rightarrow 0{ }^{\circ} \mathrm{C}, 3 \mathrm{~h}\end{array}}{98 \%}}$

Boc

(2S, 4R)-59

$\mathrm{NC}$<smiles>CC1CNC(=O)C1CO[Sb]</smiles>

Boc

$(2 S, 4 S)-61$

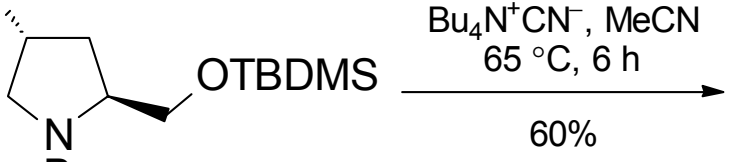

Boc

$(2 S, 4 R)-60$

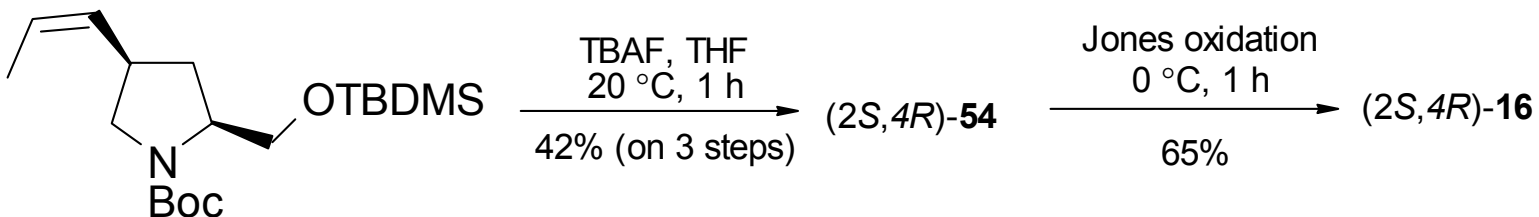

Scheme 14

The latter was reduced to aldehyde $(2 S, 4 S)$-62 with DIBAH in 97\% crude yield, as described above. A slow addition of $(2 S, 4 S)-62$ to $3-4$ equivalents of the ylide, obtained on treatment of ethyltriphenylphosponium bromide with THF solution of the freshly sublimed potassium tertbutoxide (application of this base also allows to improve the $Z$-selectivity of Wittig reaction), ${ }^{[64]}$ 
in THF at $-78^{\circ} \mathrm{C}$ allowed to decrease the epimerization significantly.The fully protected 4-(Z)propenylprolinol $(2 S, 4 R)-63$ with excellent $Z / E$-selectivity (E/Z ratio 1:14-30) and which contained only traces of the $(2 S, 4 R)$-epimer was obtained. The TBDMS group was smoothly removed with tetrabutylammonium fluoride in THF to give the alcohol $(2 S, 4 R)-54$ in $42 \%$ yield over 3 steps. This substance was oxidized with 10 equivalent of Jones reagent in acetone at $4{ }^{\circ} \mathrm{C}$ and the desired $N$-Boc protected 4-(Z)-propenylproline $(2 S, 4 R)-16$ was obtained in $65 \%$ yield $\left(18 \%\right.$ overall yield on 8 steps). ${ }^{[65]}$ The high diastereo- and enantiomeric purity of this product was confirmed by HPLC chromatography ( $d r>98: 1$, ee 97\%). ${ }^{[66]}$

To investigate the biosynthesis of hormaomycin $\mathbf{3}$ as well as to confirm the assigment of the absolute configuration of (4-PE)Pro moiety in $\mathbf{3}$ once more, the deuterium labelled diastereo- and enantiomerically pure 4-(Z)-propenylproline should be synthesized. As the productive synthesis of the $N$-Boc protected 4-(Z)-propenylproline starting from 4-hydroxyproline had already been developed, synthesis of the deuterated H-(4-PE)Pro-OH labelled at C-2 starting from $(2 S, 4 R)-2$ deutero-4-hydroxyproline D-(2S,4R)-19 was first tried (Scheme 15). 

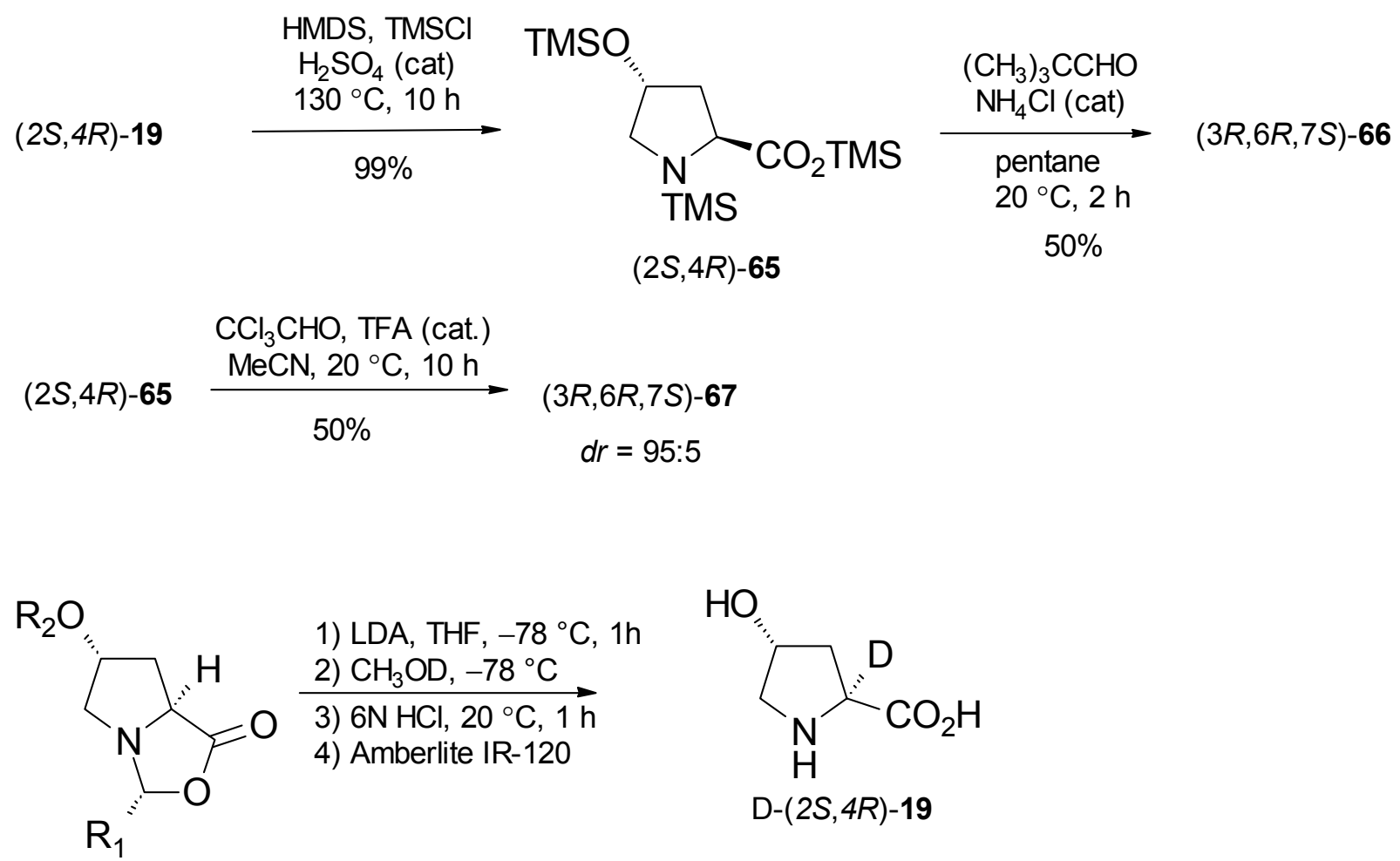

$$
\begin{aligned}
& (3 R, 6 R, 7 S)-64: \mathrm{R}_{1}=t \mathrm{Bu}, \mathrm{R}_{2}=\mathrm{Ac} \\
& (3 R, 6 R, 7 S)-66: \mathrm{R}_{1}=t \mathrm{Bu}, \mathrm{R}_{2}=\mathrm{TMS} \\
& (3 R, 6 R, 7 S)-67: \mathrm{R}_{1}=\mathrm{CCl}_{3}, \mathrm{R}_{2}=\mathrm{TMS}
\end{aligned}
$$

$$
\begin{aligned}
& 15 \%(\% \mathrm{D}=77) \\
& 16 \%(\% \mathrm{D}=87) \\
& 18 \% d r 4: 1
\end{aligned}
$$

\section{Scheme 15}

As the latter was prepared via bicyclic compound $(3 R, 6 R, 7 S)-64$ according to Weber and Seebach $^{[67]}$ only in $15 \%$ yield, two analogs of $\mathbf{6 4}$ were tested in attempt to prepare the desired deuterated product in higher yield. The trisilylated derivative $(2 S, 4 R)-\mathbf{6 5}$ (which was obtained on heating of a suspension of 4-hydroxyproline in HMDS/TMSCl mixture with catalytic quantity of $\mathrm{H}_{2} \mathrm{SO}_{4}$ in almost quantitative yield as it was described for proline ${ }^{[68]}$ ) was allowed to react with 1 equivalent of pivalic aldehyde in pentane in the presence of TFA or $\mathrm{NH}_{4} \mathrm{Cl}$ to give the bicyclic compound $(3 R, 6 R, 7 S)-66(50 \%)$ with variable diastereomeric purity. The enolate obtained after the deprotonation of this substance with LDA in THF at $-78^{\circ} \mathrm{C}$ was quenched with $\mathrm{CH}_{3} \mathrm{OD}$ to give after decomposition of the reaction product with $6 \mathrm{M} \mathrm{HCl}$ at the ambient temperature the diastereomerically pure D-(2S,4R)-19 with improved degree of deuteration (87\% against original $77 \%)$, but again only in $16 \%$ yield. The bicyclic derivative $(3 R, 6 R, 7 S)-67$ with trichloromethyl substituent instead of tert-butyl group was prepared by the reaction between $(2 S, 4 R)$ - $\mathbf{6 5}$ and 
chloral in acetonitrile in the presence of TFA with $d r$ 95:5 in 50\% yield (the similar cyclization product from proline was successfully used by Wang and Germanas for the preparation of 2allyl, benzyl and methyl substituted prolines with good diastereoselectivity ${ }^{[69]}$ ). Possibly, because of the smaller sterical volume of trichloromethyl group compared with tert-butyl group, the deprotonation of $(2 R, 5 S, 7 R)-67$ with LDA followed by quenching of the intermediate enolate with $\mathrm{CH}_{3} \mathrm{OD}$ gave, after decomposition of the reaction product with boiling $6 \mathrm{M} \mathrm{HCl}$, the mixture of epimeric 2-deutero-4-hydroxiprolines D-(2S,4RS)-19 in $(2 S, 4 R) /(2 S, 4 S) 4: 1$ ratio, and again in unacceptably low $18 \%$ yield.

As yields were always unacceptably low, other positions for the introduction of the deuterium label were chosen. It was decided to prepare 4,1'-dideutero-4-(Z)-propenylproline. The deuteration positions were chosen to establish the possible biotransformation of the deuterated amino acid in its isomer: $(R)$-cis or $(S)$-trans. If epimerization of chiral centers of this amino acid in course of the biosynthesis of hormaomycin took place, it should cause the loss of the deuterium label at C-4, and the depsipeptide labelled only at C-1' of (4-PE)Pro fragment would have been obtained after feedings experiments. Additionally, the introduction of the second deuterium label at $\mathrm{C}-1^{\prime}$ allowed to obtain a visible change of ${ }^{1} \mathrm{H}$ NMR spectrum of 3 in its relative empty region (between 5.3 and $6.0 \mathrm{ppm}$ ), as well as to avoid the possible scattering of the deuterium label during the construction of the double bond between $\mathrm{C}-4$ and $\mathrm{C}-1$ ' positions. The nitrile $(2 S, 4 S)-61$ was treated with a small excess of LDA in THF at $-78^{\circ} \mathrm{C}$, and the intermediate enolate was quenched at the same temperature with the saturated solution of $\mathrm{Na}_{2} \mathrm{SO}_{4}$ in $\mathrm{D}_{2} \mathrm{O}$ (to obtain more of the desired cis-isomer according to Takano et al) ${ }^{[70]}$ and thus the separable mixture of epimers at C-4, D- $(2 S, 4 S)-\mathbf{6 1}$ and $\mathrm{D}-(2 S, 4 R)-\mathbf{6 1}$ in 2:1 ratio was obtained (Scheme 16). 


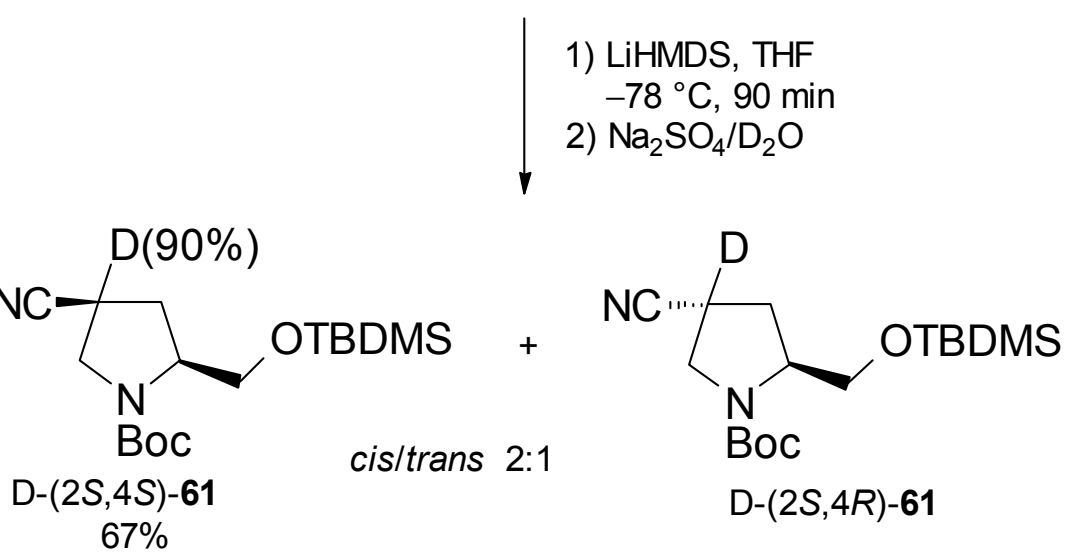

Scheme 16

If $\mathrm{D}_{2} \mathrm{O}$ was used for the quenching of the enolate, the reverse selectivity was observed. The mixture of epimers was separated, and the trans-isomer D-(2S,4R)-61 was deprotonated again and the mixture of epimers in the same ratio as before was obtained after the quenching of the enolate. The required isomer was separated and combined with the first portion to give the deuterated product D-(2S,4S)-61 (90\% deuterium content according to ${ }^{1} \mathrm{H}$ NMR spectrum) in $67 \%$ overall yield. ${ }^{[71]}$ The aldehyde D-(2S,4S)-62, prepared as it was described for the nondeuterated analog, was allowed to react with ylide, obtained from (1,1dideutero)ethyltriphenylphosphonium bromide $^{[72]}$ (1.9 deuterium atoms pro molecule; the nondeuterated compound was unobservable in ${ }^{1} \mathrm{H}$ NMR spectrum) and potassium tert-butoxide, and the dideuterated fully protected prolinol $\mathrm{D}_{2}-(2 S, 4 R)-62$ was then TBDMS-deprotected with tetrabutylammonium fluoride to give the $N$-Boc protected prolinol $\mathrm{D}_{2}-(2 S, 4 R)-54\left(\mathrm{D}_{2}\right.$ and $\mathrm{D}$ contents $65 \%$ and $34 \%$ respectively, as determined by MS) in $45 \%$ overall yield over 3 steps. The latter was oxidized with Jones reagent in acetone to give the dideuterated acid $\mathrm{D}_{2}-(2 S, 4 R)-16$ in $61 \%$. The cleavage of Boc group with TFA gave the necessary product only in $36 \%$ yield. Therefore, several another conditions for the Boc-deprotection were tried (Scheme 17). ${ }^{[73]}$ To avoid this problem, the $p$-nitrobenzyl ester $\mathrm{D}_{2}-(2 S, 4 R)-\mathbf{6 8}$, which was prepared by esterification of $\mathrm{D}_{2}-(2 S, 4 R)-16^{[74]}$ with $p$-nitrobenzyl bromide and $\mathrm{K}_{2} \mathrm{CO}_{3}$ in $\mathrm{MeCN}$ in $88 \%$ yield, was $N$-Boc deprotected with $5 \mathrm{M} \mathrm{HCl}$ in $\mathrm{Et}_{2} \mathrm{O}$ to give the corresponding amino ester as a hydrochloride in $88 \%$ yield. The latter was hydrolyzed with equimolar quantity of $0.1 \mathrm{M} \mathrm{NaOH}$ to give, after 
carefull acidification, the dideuterated amino acid $\mathrm{D}_{2}-(2 S, 4 R)-\mathbf{1 7}$ in almost quantitative yield as a mixture with ca. equimolar quantity of $\mathrm{NaCl}$. This mixture was directly used for the feeding experiment.

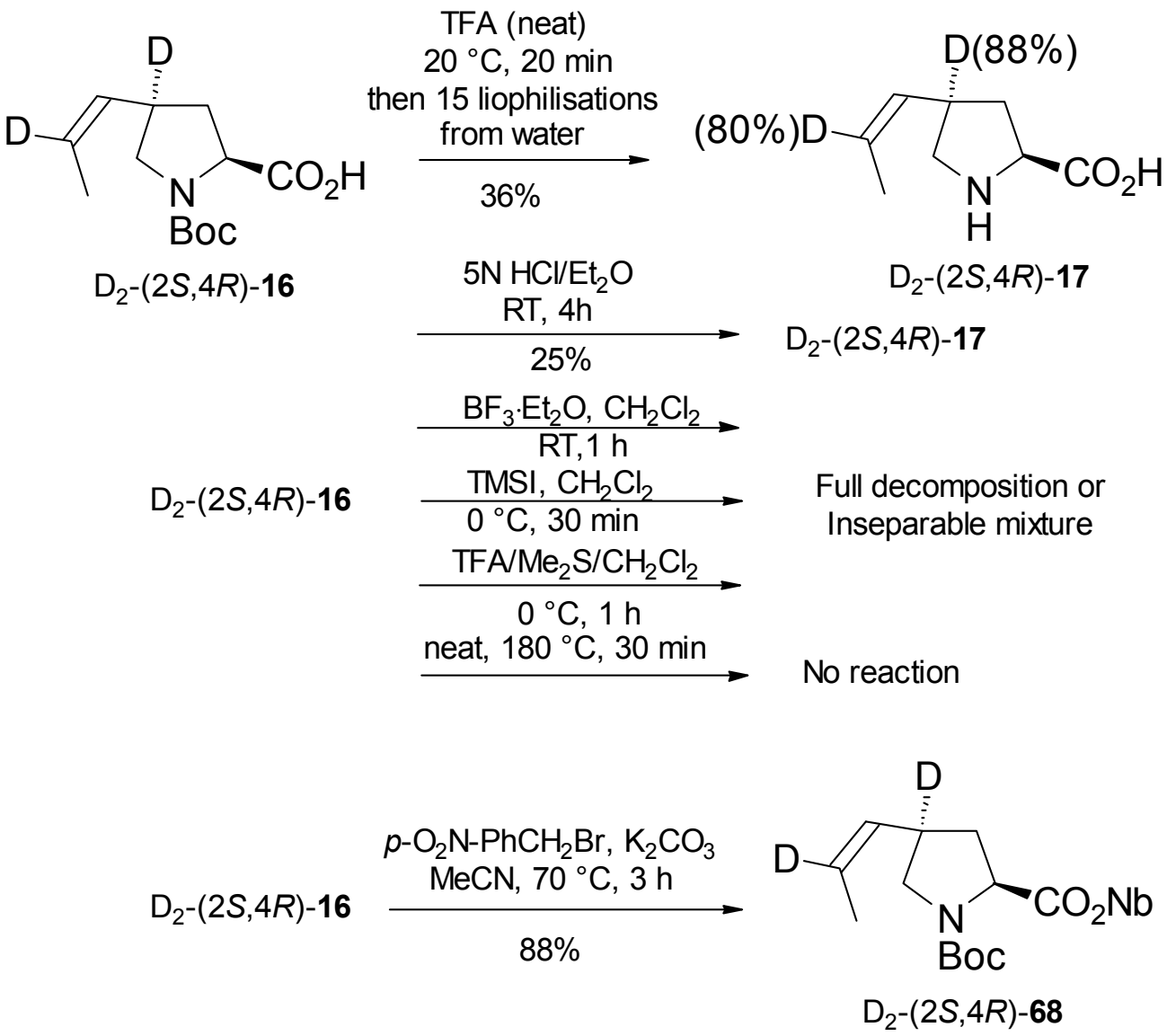

1) $5 \mathrm{~N} \mathrm{HCl} / \mathrm{Et}_{2} \mathrm{O}$

$\mathrm{RT}, 3 \mathrm{~h}$

2) $0.1 \mathrm{~N} \mathrm{NaOH}$

$0^{\circ} \mathrm{C}, 30 \mathrm{~min}$

$\mathrm{D}_{2}-(2 S, 4 R)-17$

then $1 \mathrm{~N} \mathrm{HCl}$

$88 \%$

Scheme 17

No one possible biotransformation occurred and the doubly deuterated 3a was successfully obtained. These experiments also cleanly demonstrated that the biosynthesis of H-(4-PE)Pro-OH takes place before the assembly of the peptide chain of $\mathbf{3}$. 


\section{Synthesis of $(R)$-allo-threonine and $(2 S, 3 R)-\beta$-Methylphenylalanine}

$(R)$-allo-threonine was prepared according to the previously described procedure (Scheme 18). ${ }^{[75]}(R)$-Threonine $(R)-\mathbf{6 9}$ was first transformed in its methyl ester and then acylated with benzoyl chloride to give the fully protected amino acid $(R)-70$ in $74 \%$ yield, which was further underwent cyclization in oxazoline $(2 R, 3 R)-71$ with inversion of the configuration at $\mathrm{C}-3(90 \%)$. The latter was hydrolyzed with $6 \mathrm{~N} \mathrm{HCl}$ to give the desired amino acid $(R)-a-72$ in $52 \%$ yield.

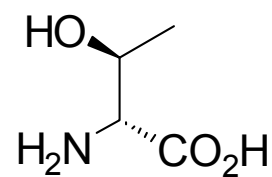

$(R)-69$

1) $\mathrm{SOCl}_{2}, \mathrm{MeOH}$ $-20^{\circ} \mathrm{C} \rightarrow 55^{\circ} \mathrm{C}, 2 \mathrm{~h}$

2) $\mathrm{BZCl}, \mathrm{Et}_{3} \mathrm{~N}, \mathrm{MeOH}$ $20{ }^{\circ} \mathrm{C}, 1 \mathrm{~h}$ $74 \%$<smiles>COC(=O)C(NC(=O)OCc1ccccc1)C(C)O</smiles>

(R)-70

1) $6 \mathrm{~N} \mathrm{HCl}$ $100^{\circ} \mathrm{C}, 5 \mathrm{~h}$

2) Pyridine

$52 \%$

$(2 R, 3 R)-71$<smiles>CC(O)[C@H](N)C(=O)O</smiles>

(R)-a-72

Scheme 18

Among numerous published enantio- and diastereoselective syntheses of $\beta$-methylphenylalanines ${ }^{[76]}$ only approach described by Hruby et al, ${ }^{[77]}$ based on the method of Evans et al., ${ }^{[78]}$ was feasible for preparation of multigram quantities of this amino acid. As the initial procedure required a notorious separation of the enantiomers of $\gamma$-phenylbutyric acid, the modification of this route developed by the same group, was chosen (Scheme 19). ${ }^{[79]}$ The mixed anhydride of $\gamma$ phenylbutyric acid $\mathbf{7 3}$ formed on treatment with triethylamine and pivalic acid in THF was coupled with lithium enolate of $(4 R)$-phenyloxazolidinone-2 $(R)-74^{[80]}$ to yield the epimeric mixture of (4R)-4-phenyl-3-(3'-phenylbutyryl)oxazolidinones-2 (4R)-75. The mixture was separated by crystallization to give the desired $(4 R, 3 ' S)$-isomer in $44 \%$ yield. The same 
compound was obtained as described for its enantiomer in another publication of Hruby et al. ${ }^{81}$ by conjugated addition of phenylmagnesium cuprate to the acyloxazolidinone $(Z)-(R)-76$ [prepared by analogy to $(4 R)-75$ ] in $80 \%$ yield. $\left(4 R, 3^{\prime} S\right)-75$ was converted to a boron enolate by use of dibutylborontriflate in $\mathrm{CH}_{2} \mathrm{Cl}_{2}$.<smiles>CC(CC(=O)O)c1ccccc1</smiles>

73<smiles>Cc1ccccc1</smiles><smiles>C/C=C/C(=O)N1CCOC1=O</smiles>

$(E)-(R)-76$
1) $\mathrm{PivCl}, \mathrm{Et}_{3} \mathrm{~N}, \mathrm{THF}$

$-78 \rightarrow 0^{\circ} \mathrm{C}, 2 \mathrm{~h}$

2) $(R)-74,-78 \rightarrow 20^{\circ} \mathrm{C}, 1 \mathrm{~h}$

3) 8 crystallizations from

EtOAc/Hexane

$44 \%$

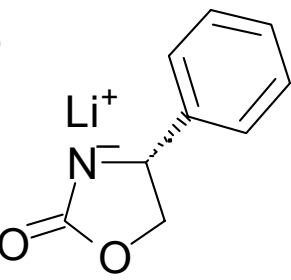

(R)-74

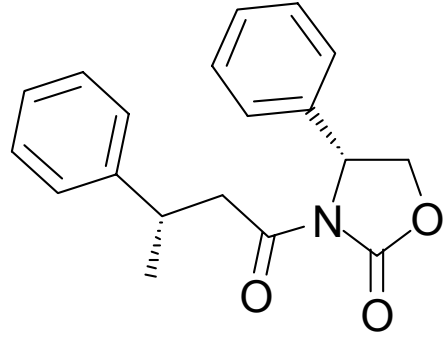

(4R,3'S)-75

$d r>95: 5$

\section{$\mathrm{PhMgBr}, \mathrm{CuBr} / \mathrm{Me}_{2} \mathrm{~S}$ \\ THF, $-15^{\circ} \mathrm{C}, 10 \mathrm{~min}$}

$74 \%$

$\left(4 R, 3^{\prime} S\right)-75$

$d r>95: 5$

$\left(4 R, 3^{\prime} S\right)-75$

1) $\mathrm{Bu}_{2} \mathrm{BOTf}, i-\mathrm{Pr}_{2} \mathrm{NEt}$ $\mathrm{CH}_{2} \mathrm{Cl}_{2},-78 \rightarrow 0{ }^{\circ} \mathrm{C}$

2) NBS, $-78^{\circ} \mathrm{C}$

3) TMGA, MeCN, $20^{\circ} \mathrm{C}, 10 \mathrm{~h}$ $76 \%$<smiles>C[C@@H](C(=O)N1C(=O)OC[C@H]1c1ccccc1)c1ccccc1</smiles>

$\left(4 R, 2^{\prime} S, 3^{\prime} R\right)-77$

$d r>95: 5$

1) $\mathrm{LiOH}, \mathrm{H}_{2} \mathrm{O}_{2}, \mathrm{THF}, 0^{\circ} \mathrm{C}, 45 \mathrm{~min}$

2) $\mathrm{Pd} / \mathrm{C}, \mathrm{H}_{2}, 2.5 \mathrm{bar}, \mathrm{AcOH}, 24 \mathrm{~h}$

3) Dowex 50W

$76 \%$<smiles>C[C@H](c1ccccc1)[C@H](N)C(=O)O</smiles>

$(2 S, 3 R)-78$

Scheme 19

Selective bromination was accomplished using NBS, and $\mathrm{S}_{\mathrm{N}} 2$ displacement of the resulting 
crude bromide gave the azide $\left(4 R, 2^{\prime} S, 3^{\prime} R\right)-77$ with high diastereoselectivity in $76 \%$ yield in two steps. Finally reduction of the azido acid obtained after removal of the chiral auxiliary gave $(2 S, 3 R)-\beta$-methylphenylalanine $(2 S, 3 R)-78$ in $46 \%$ overall yield over 6 steps. 
3. Elucidation of the absolute configuration of trans-(2'nitrocyclopropyl)alanine residue in hormaomycin. Synthesis of $\left(2 R, 1^{\prime} R, 2^{\prime} R\right)-\left(2^{\prime}-\right.$ nitrocyclopropyl)alanine and $\left(2 S, 1^{\prime} R S, 2^{\prime} R S\right)-2$-deutero-(2'nitrocyclopropyl)alanine

Further progress in preparation of trans-(2'-nitrocyclopropyl)alanine was achieved, when this work was already under way. The enantioselective alkylation of the Oppolzer sultam derived glycine equivalent $\mathbf{7 9}^{[82]}$ with trans-(2'-nitrocyclopropyl)methyliodide $\mathbf{8 0}$, which had earlier routinely been applied as a key step in trans-(2'-nitrocyclopropyl)alanines syntheses, often gave the desired alkylation product only in low yield and was not suitable for the preparation of this amino acid in multigramm quantities.
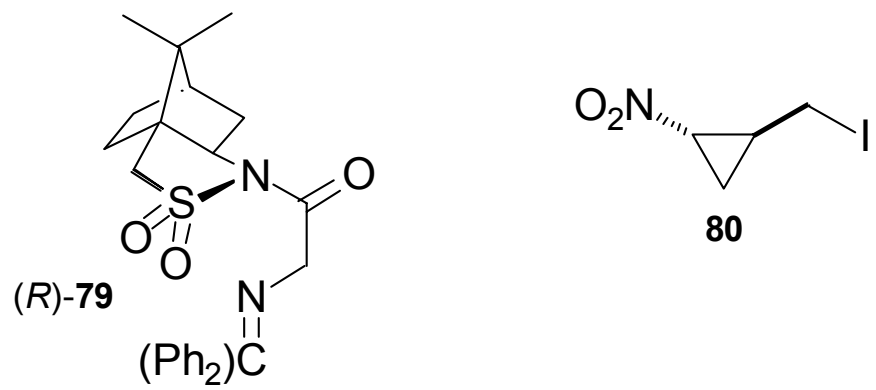

80

Already first experiment with the Belokon complex $\mathbf{8 1}^{[83]}$ instead of this chiral glycine equivalent, allowed to significantly improve yield on this crucially important step. As a further development of this methodology the procedure for productive preparation of all four possible isomers of trans-(2'-nitrocyclopropyl)alanine exploited a noteable difference in solubility between the two diastereomeric products of the enantioselective alkylation $\mathbf{8 2}$ of the complex $\mathbf{8 1}$ with the racemic iodide $\mathbf{8 0}$, which allowed their separation from the same reaction mixture was published by Larionov, de Meijere, et al. [Scheme 20; alkylation only $(S)-\mathbf{8 1}$ showed]. ${ }^{[84]}$

This approach allows to obtain trans-(2'-nitrocyclopropyl)alanines with high diastereo- and enantiomeric purity. Moreover whole $\left(2 S, 1^{\prime} R, 2^{\prime} R\right)-\left(2^{\prime}\right.$-nitrocyclopropyl)alanine, used in course of 
this work, which was generously presented by Oleg V. Larionov, was prepared in this way.

(2S)-81<smiles>O=C1CN2N=C1O[N+]21C(=O)[C@@H]2CCCN2[N+]12C(=O)c1ccccc1N2Cc1ccccc1</smiles>

1) $80, \mathrm{NaH}$

$\mathrm{DMF} / \mathrm{MeCN}(1: 2)$

$-50 \rightarrow 0^{\circ} \mathrm{C}$

40-50 min

2) $60 \%$ aq. $\mathrm{AcOH}$

3) crystallization<smiles>O=C1[C@@H]2CCCN2[N+]23OC(=O)n4c(=O)c5ccccc5n4[N+]2=C(c2ccccc2)c2ccccc2N13</smiles>

(2S, 2'S, 1"S, 2"S)-82

less soluble<smiles>O=C1O[N+]23N(C(=O)[C@@H]4CCCN42)c2ccccc2C(c2ccccc2)=[N+]3[C@@H]1C[C@H]1C[C@@H]1[N+](=O)[O-]</smiles>

(2S, 2'S, 1" $\left.R, 2^{\prime \prime} R\right)-82$

more soluble

Scheme 20

Nevertheless applying this route only 50\% maximal yield of the desired isomer can be achieved. It may be not taking into account if it's necessary to prepare any of $(2 S)$-isomers of this amino acid $[(S)$-proline, which must be used for preparation of the chiral auxiliary $\mathbf{8 1}$ in this case, is cheap] but it can be a problem if it is necessary to prepare a large quantity of any of $(2 R)$-isomer. This disadvantage was overcome when the enantiomerically pure iodide $\mathbf{8 0}$ was applied.

The mixture of the diastereomeric acyloxazolidinones 9 was prepared (Scheme 1). It was 
separated by crystallization to give individual diastereomers, $\left(4 R, 1^{\prime} R, 2^{\prime} R\right)-\mathbf{9}$ and $\left(4 R, 1^{\prime} S, 2^{\prime} S\right)-\mathbf{9}$, after final purification by column chromatography in $70 \%$ and $45 \%$ respectively. As described procedure of their reductive cleavage ${ }^{[14]}$ gave on scaling-up the desired alcohols of reproducibly low quality and low yield, an alternative two step approach was developed (Scheme 21).

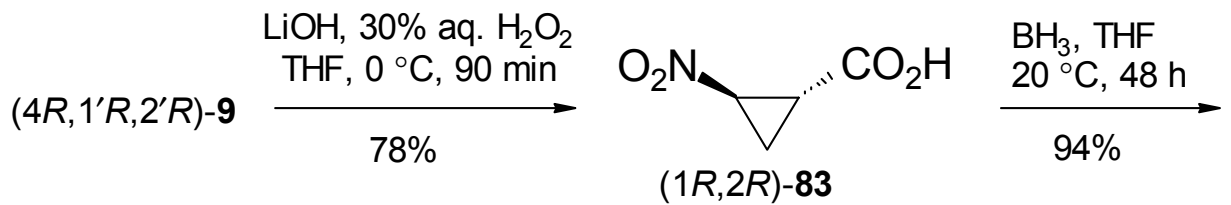

$$
\begin{aligned}
& \begin{array}{c}
\mathrm{P}(\mathrm{Ph})_{3}, \mathrm{I}_{2}, \mathrm{ImH}, \mathrm{CH}_{2} \mathrm{Cl}_{2} \\
\left(1^{\prime} R, 2^{\prime} R\right)-10 \stackrel{0 \rightarrow 20^{\circ} \mathrm{C}, 3 \mathrm{~h}}{\longrightarrow}\left(1^{\prime} R, 2^{\prime} R\right)-80
\end{array}
\end{aligned}
$$

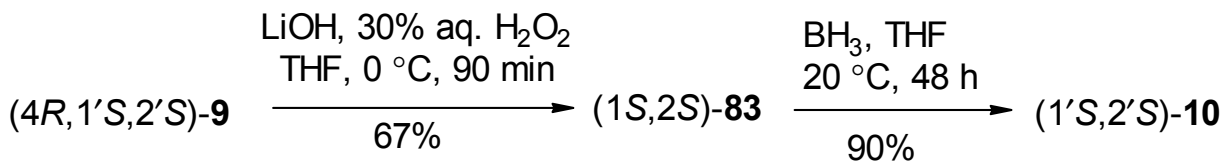

Scheme 21

The chiral auxiliary was removed by hydrolysis with $\mathrm{LiOH}$ in presence of hydrogen peroxide at low temperature and the carboxylic acids, $(1 R, 2 R)-\mathbf{8 3}$ and $(1 S, 2 S)-\mathbf{8 3}$, were obtained in $78 \%$ and $67 \%$ yield respectively. They were further reduced with $\mathrm{BH}_{3} \cdot \mathrm{THF}$ in THF to give the alcohols, $\left(1^{\prime} R, 2^{\prime} R\right)-10$ and $\left(1^{\prime} S, 2^{\prime} S\right)-\mathbf{1 0}$, in $94 \%$ and $90 \%$ yield respectively. On treatment of $\left(1^{\prime} R, 2^{\prime} R\right)-10$ with iodine, triphenylphosphine and imidazol in $\mathrm{CH}_{2} \mathrm{Cl}_{2}$ according to protocol of Gordon and Gottardo $^{[85]}$ iodide $\left(1^{\prime} R, 2^{\prime} R\right)-\mathbf{8 0}$ was obtained in $95 \%$ yield.

It was employed in the alkylation of the chiral glycine equivalent $(R)-\mathbf{8 1}{ }^{[86]}$ and the complex $\left(2 R, 2^{\prime} R, 1^{\prime \prime} R, 2^{\prime \prime} R\right)-82$ was obtained in $74 \%$ yield. The latter was decomposed by treatment with 6 $\mathrm{M} \mathrm{HCl}$ to give amino acid $\left(2 R, 1^{\prime} R, 2^{\prime} R\right)-\mathbf{8 3}$ in $55 \%$ yield together with the recovered amide $(R)-\mathbf{8 4}$ $(84 \%)$, which could be used for the preparation of the new portion of the $N i$-complex $(R)-81$ (Scheme 22). 

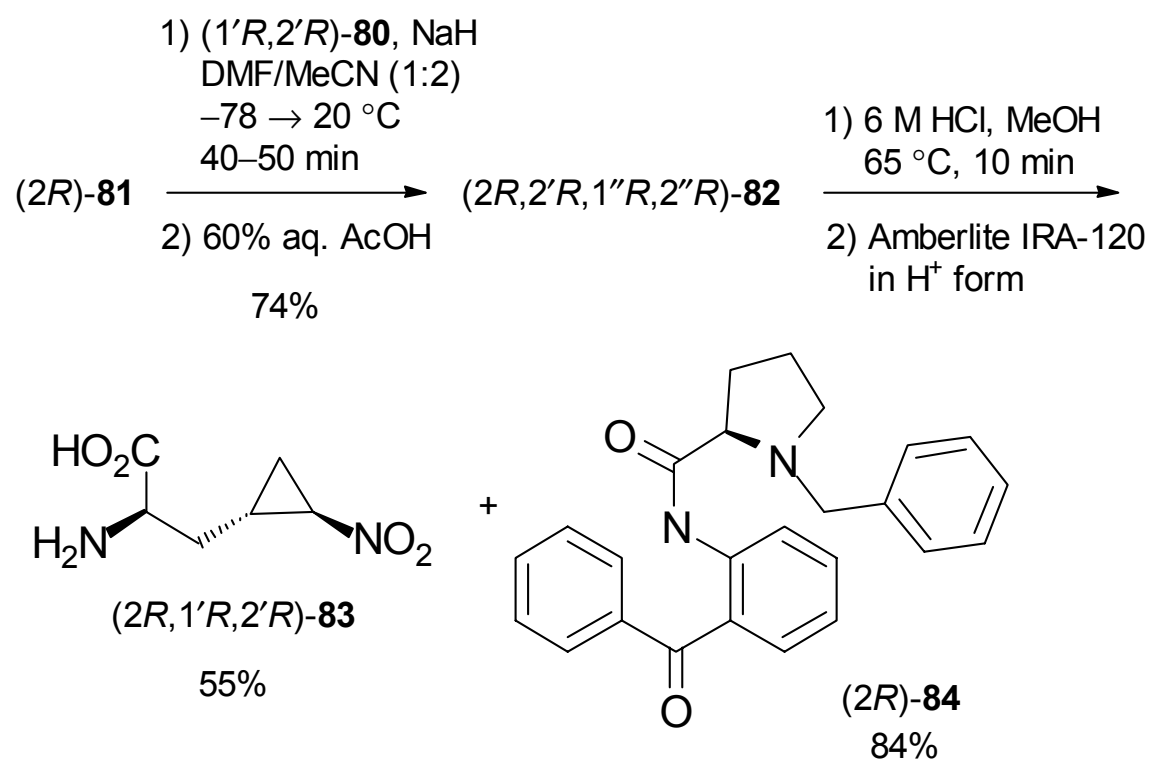

Scheme 22

In order to begin with the assembling of the peptide chain of hormaomycin, the configuration of both (3-Ncp)Ala fragments of the depsipeptide at C-2 should be assigned. The previous unsuccessful attempt to determine it via feeding experiment with the doubly deuterated at C-3 trans-(2'-nitrocyclopropyl)alanine $3-\mathrm{D}_{2}-\left(2 S, 1^{\prime} R S, 2^{\prime} R S\right)-\mathbf{8 3}$ (see introduction) showed that the new feeding experiment with the enantiomerically pure $\mathrm{H}-(3-\mathrm{Ncp}) \mathrm{Ala}-\mathrm{OH}$, labelled at $\mathrm{C}-2$, should be carried out.

$(2 S)-\mathrm{D}_{2} \mathbf{- 8 1}$, which was prepared from unlabeled $(2 S)-\mathbf{8 1}{ }^{[86]}$ by isotope exchange in excellent yield and with excellent deuteration degree (Scheme 23) according to protocol of Ragnarsson and Elemes, ${ }^{[87]}$ was alkylated with the racemic iodide $\mathbf{8 0}$ in $\mathrm{CD}_{3} \mathrm{CN} / \mathrm{DMF}$ mixture ${ }^{[88]}$ using $\mathrm{NaH}$ as a base to give the alkylated $N i$-complex $\left(2 S, 2^{\prime} S, 1^{\prime} R S, 2^{\prime} R S\right)-\mathrm{D}-82$ in $70 \%$ yield, which was in turn cleaved with $6 \mathrm{M} \mathrm{HCl}$ to give after ion-exchange chromatography the desired deuterated amino acid 2-D-(2S,1'RS,2'RS)-83 in $68 \%$ yield (Scheme 24$)$. 


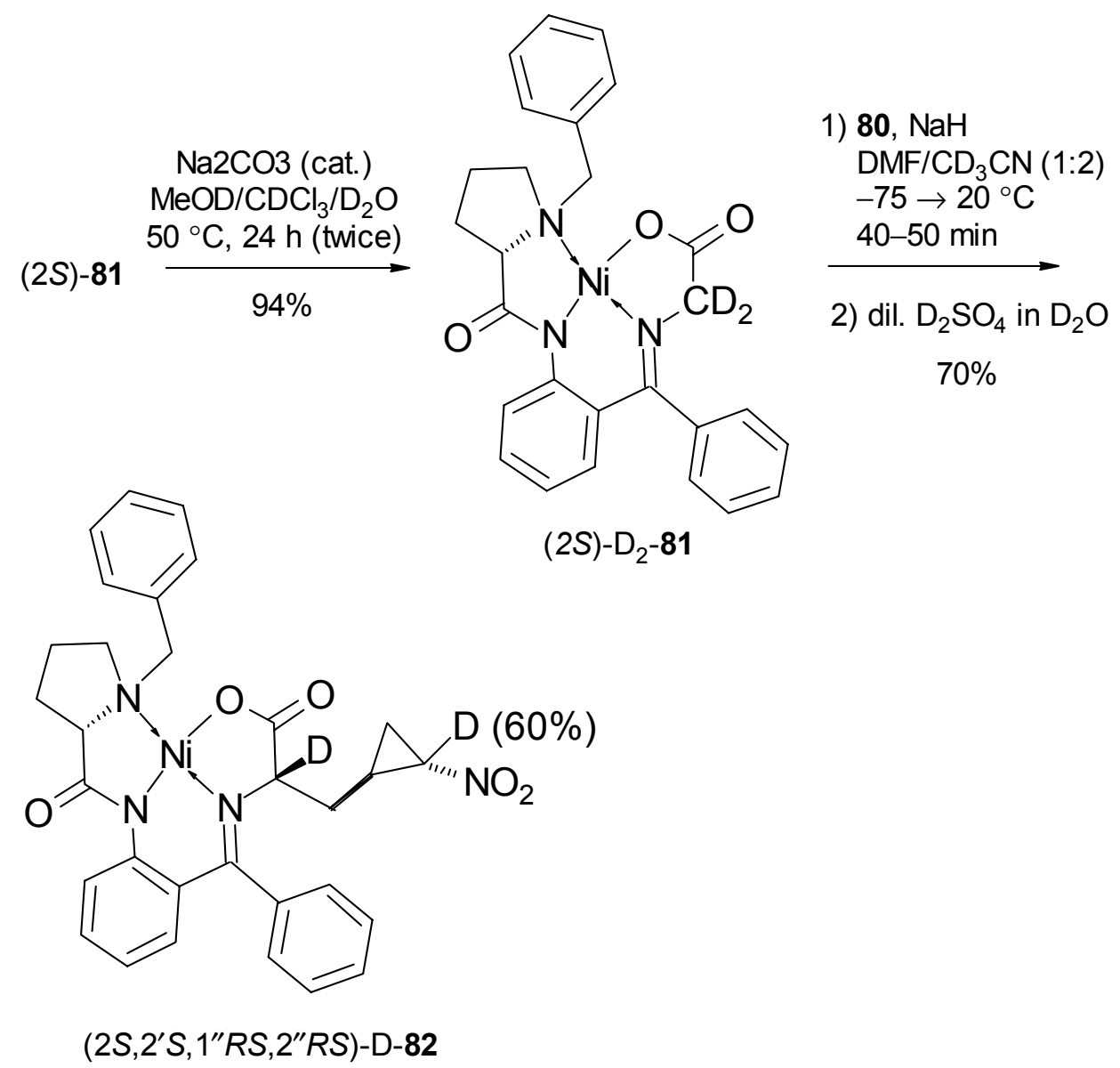

Scheme 23

The MS- and NMR-spectra of this substance showed that the partial enrichment with deuterium at $2^{\prime}$ position of the cyclopropyl ring occurred.
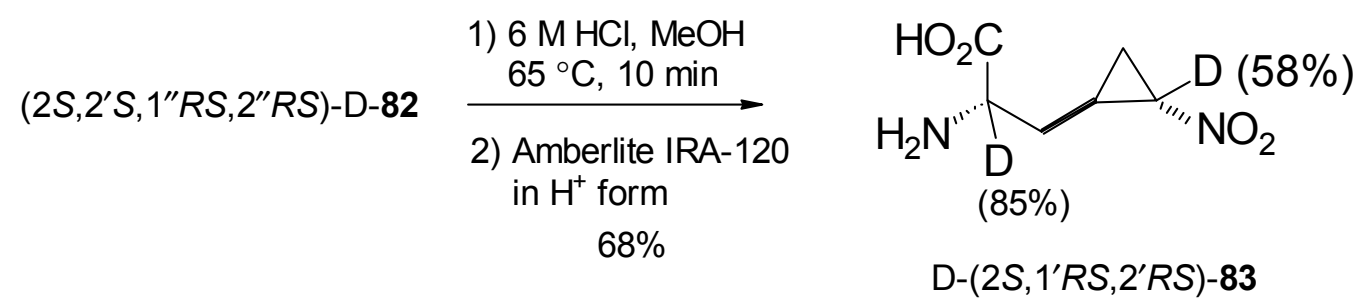

Scheme 24

The feeding experiment with $\mathrm{D}-\left(2 S, 1^{\prime} R S, 2^{\prime} R S\right)-\mathbf{8 3}$ was successful and gave deuterated hormaomycin $\mathbf{3 b}$, which was enriched with deuterium at three positions according to NMR spectra. The deuterium labels were introduced at 2' position of (3-Ncp)Ala moiety in the ring part of $\mathbf{3}$ as well as at C-2 and C-2' of the (3-Ncp)Ala-II residue in the side chain of $\mathbf{3}$ (figure 
2). ${ }^{[89]}$ These observations showed that hormaomycin contains $(2 R)-(3-N c p)$ Ala moiety in the ring part and (2S)-(3-Ncp)Ala in the side chain.

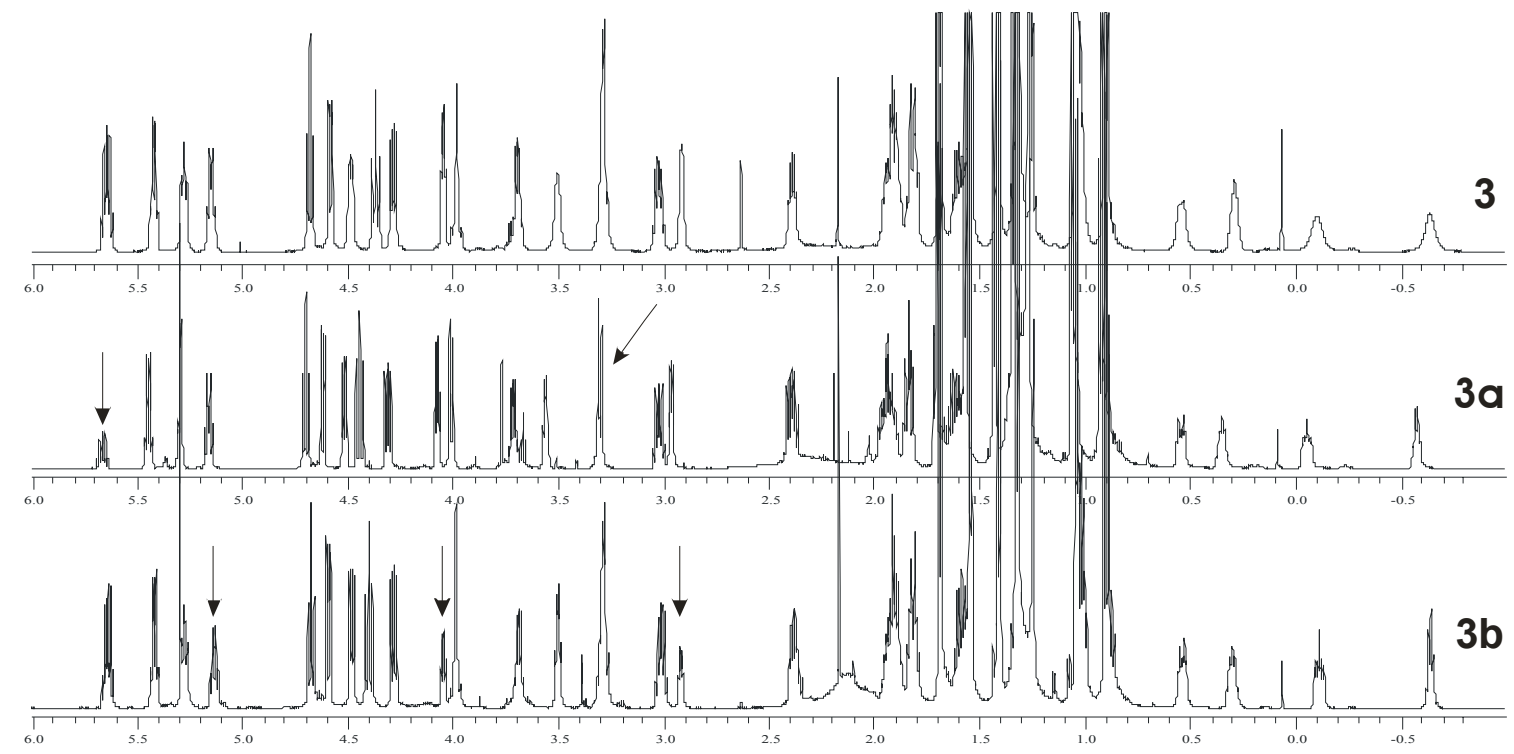

Figure 2. Comparison of ${ }^{1} \mathrm{H} \mathrm{NMR}\left(600 \mathrm{MHz}, \mathrm{CDCl}_{3}\right)$ spectra of hormaomycin 3 (upper trace) and deuterated hormaomycins after feeding experiments with deuterated 4-(Z)-propenylproline 3a (middle trace) and with deuterated 3-(2'-nitrocyclopropyl)alanine (lower trace) hormaomycin $3 \mathbf{b}$.

Also noteworthy, that the deuteration degree at C-2 of (3-Ncp)Ala residue was significantly lower than expected (decrease of the absorption of $\mathrm{H}-2$ of (3-Ncp)Ala moiety in ${ }^{1} \mathrm{H}$ NMR spectrum of $\mathbf{3 b}$ was only $40 \%$ comparing with the corresponding signal in the spectrum of the nondeuterated 3). It indicates that in this case epimerization at the second carbon in $\mathrm{H}-(3-$ Ncp)Ala-OH (at least partially) takes place before the assembling of the peptide backbone of hormaomycin and it goes faster then the latter.

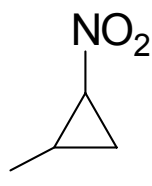

84

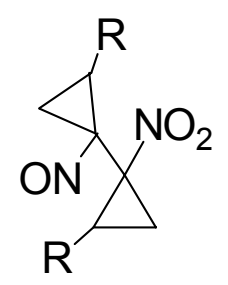

85

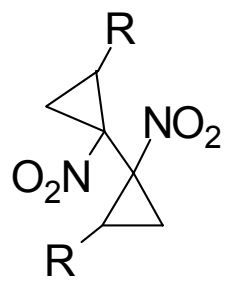

86

Observation, that the partial enrichment with deuterium of at C-2" of the alkylated $\mathrm{Ni}$-complex $\left(2 S, 2^{\prime} S, 1^{\prime} R S, 2^{\prime} R S\right)-\mathrm{D}-82$ (and therefore deprotonation of the nitrocyclopropyl ring) took place, was in remarkable contradiction with the earlier results of D. Seebach et al.. ${ }^{[90]}$ Attempted to 
deprotonate nitrocyclopropane or 2-methylnitrocyclopropane $\mathbf{8 4}$ with different bases and then catch the resulting carbocations with a variety of electrophiles including $\mathrm{D}_{2} \mathrm{O}$, only products of their dimerization 85 or/and oxidative dimerization 86 were obtained. This discrepancy prompted us to briefly reinvestigate the deprotonation of the nitrocyclopropyl ring (Scheme 25).

The $N i$-complex $\left(2 S, 2^{\prime} S, 1^{\prime \prime} R, 2^{\prime \prime} R\right)-\mathbf{8 2}$ was treated in $\mathrm{CD}_{3} \mathrm{CN} / \mathrm{DMF}$ mixture with $\mathrm{NaH}$ to give after quenching with dilute $\mathrm{D}_{2} \mathrm{SO}_{4}$ in $\mathrm{D}_{2} \mathrm{O} N i$-complex of the same absolute configuration in $71 \%$ yield, which contained $55 \%$ and $65 \%$ deuterium at C-2 and C-2' of (3-Ncp)Ala moiety respectively. ${ }^{[91]}$ Under the same conditions the $O-\operatorname{Tr}$ protected $\left(1 ' S, 2^{\prime} S\right)-\left(2^{\prime}-\right.$ nitrocyclopropyl)methanol $(1 ' S, 2 ' S)-87$, which was obtained on treatment of the nondeuterated alcohol 10 with trityl chloride in pyridine using DMAP as a catalyst in $78 \%$ yield, and nitrocyclopropane $\mathbf{8 8}^{[92]}$ gave deuterated analogs in $72 \%$ and $25 \%$ yield respectively. The deuteration degree for these compounds was $>95 \%$. In case of $\left(1^{\prime} S, 2^{\prime} S\right)-87{ }^{1} \mathrm{H}$ NMR spectrum of the crude product showed that the partial (about 5-7\%) epimerization took place.

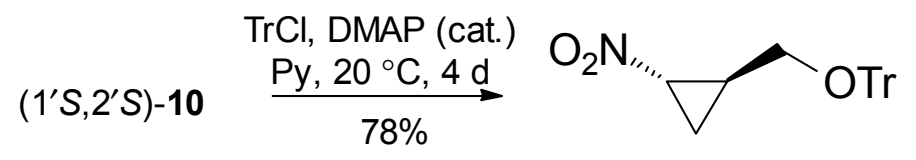

$\left(1^{\prime} S, 2^{\prime} S\right)-87$
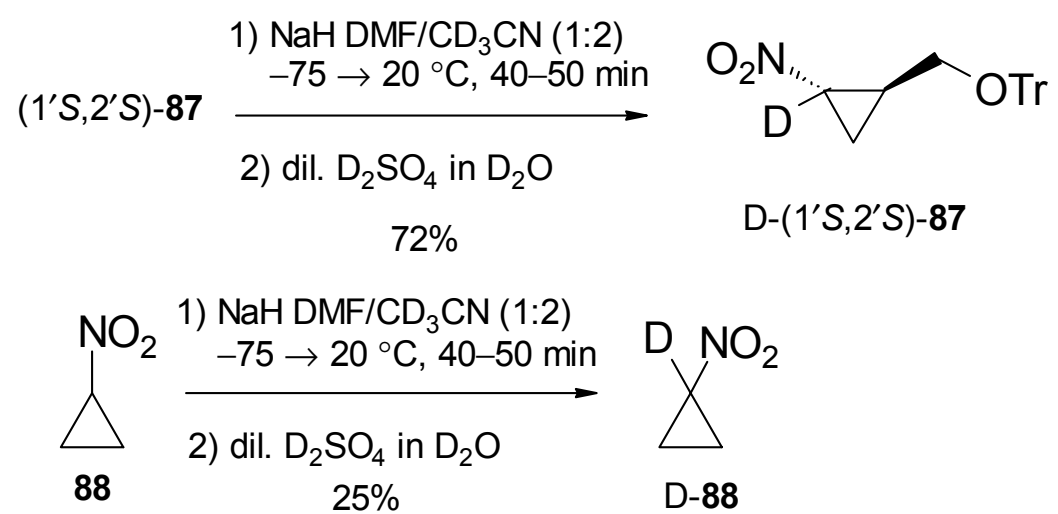

Scheme 25

Once the deuterium labeling of the nitrocyclopropyl ring was achieved, the possibility of its alkylation should be investigated. Two examples of the successful addition of benzaldehyde to 
the gem-disubsituted 2,2-dimethyl-1-nitrocyclopropane $\mathbf{8 9}^{[93]}$ and to the highly substituted trans1,2-dinitrospiropentane $\mathbf{9 0}^{[94]}$ have only been described up-to-date.
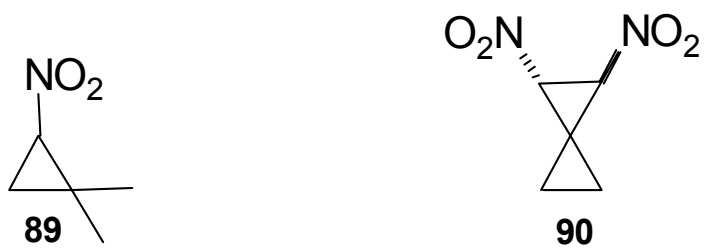

The latter was also iodinated by treatment first with sodium methylate in methanol and followed with iodine. All attempts to obtain another substitution products even in case of these sterically hindered substrates were unsuccessful (the sterical hindrance should have prevented formation of the dimerization and oxidative dimerisation products similar to those that were described before). The tritylated alcohol $\left(1^{\prime} S, 2^{\prime} S\right)$-88 was chosen as a starting material for this set of experiments since enantiomerically pure product of its alkylation (epimerization at C-2' seemed to be hardly possible) could serve for the preparation of amino- and nitrocyclopropyl containing products (i. e., $\beta$ - and $\gamma$-amino acids) in an enantioselective manner. The allylation and benzylation reactions of this substrate using a large excess of $\mathrm{NaH}$ and the corresponding halide in dimethoxymethane gave expected products in moderate yields (Scheme 26).

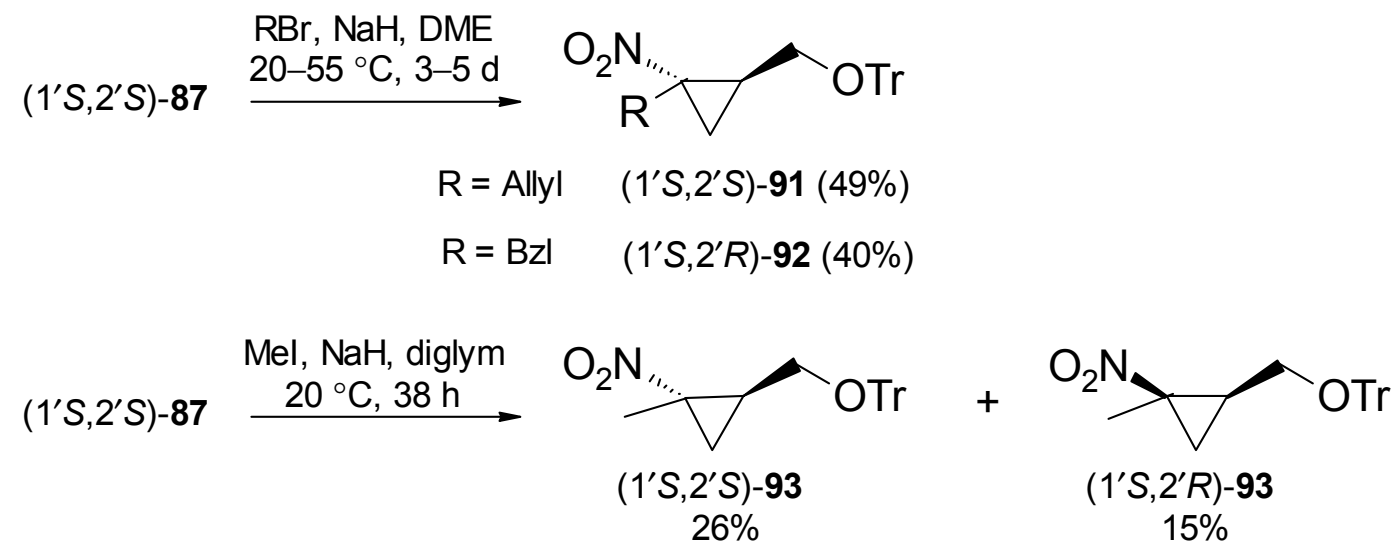

Scheme 26

According to ${ }^{1} \mathrm{H}$ NMR spectra of reaction mixtures, the partial epimerization at $\mathrm{C}-1$ ' took place. The appropriate pure trans-isomers were obtained after column chromatography and/or preparative TLC. The methylation reaction, which was carried out in diglym, gave larger 
epimerization extent compared with experiments in DME (cis/trans ratio for methylation 1:2 against 1:7 for allylation and 1:4 for benzylation). 


\section{Synthesis of O-MOM protected 5-chloro-1-hydroxypyrrol-2-carboxylic acid}

The most straightforward way for the preparation of the protected 5-chloro-1-hydroxypyrrol-2carboxylic acid seemed to be the simultaneous dehydration/chlorination of the fully protected $N$ hydroxypyroglutamic acid ${ }^{[95]}$ with $\mathrm{POCl}_{3}, \mathrm{PCl}_{5}$ or related reagents as it was described for several derivatives of pyroglutamic acid. ${ }^{[96]}$ Nevertheless the obvious instability of such kind of compounds under harsh conditions, which should be applied, sharply diminished the attractiveness of this way. In attempt to prepare $O$-protected 2,5-dichloropyrrol 95, which could be further monometallated and then quenched with $\mathrm{CO}_{2}$ to give the desired acid, 94 ${ }^{[97]}$ was heated with trichlorobenzo-1,2-dioxaphosphole (Scheme 27). This attempt gave complex mixture, which didn't contain any heteroaromatic compounds.

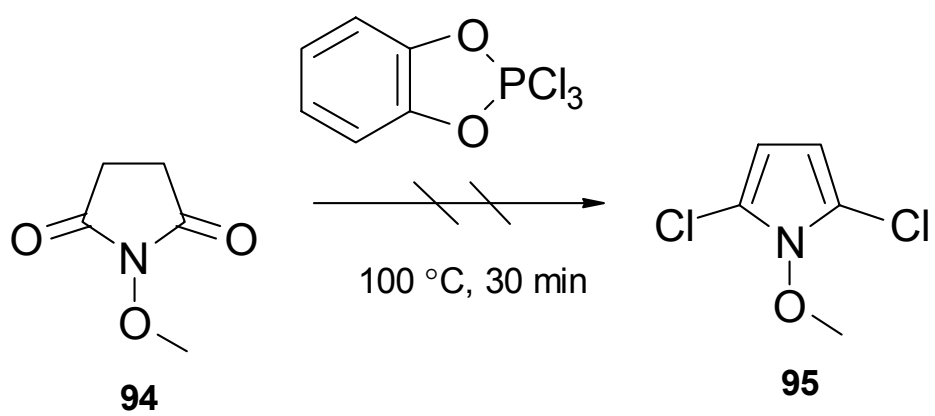

Scheme 27

$\mathrm{N}$-Hydroxy or $\mathrm{N}$-alkoxypyrroles have not attracted much attention of chemists and among relatively poor data about this compound only the publication of Abramovitch et al., ${ }^{[98]}$ who described the preparation of several 2-cyano-1-hydroxypyrroles by the thermal decomposition of the corresponding 3-unsubstituted 2-azidopyridine $\mathrm{N}$-oxides in benzene at $85-95^{\circ} \mathrm{C}$, contained some helpful information. According to the postulated mechanism, this reaction proceeds via elimination of nitrogen with ring opening to give unsaturated nitrile, which in turn undergoes electrocyclic ring closure to give $2 \mathrm{H}$-pyrrole- $\mathrm{N}$-oxide. Finally prototropic shift leads to the aromatic 2-cyano-1-hydroxypyrroles. 
2-Azido-6-chloropyridine- $N$-oxide 102 should be first synthesized to prepare 5-chloro-2-cyano1-hydroxypyrrol 103 according to the abovementioned procedure. This became feasible via diazotization of 2-amino-6-chloropyridine- $N$-oxide $\mathbf{1 0 1}$ followed by addition of sodium azide in $76 \%$ yield. As an attempt of the direct oxidation of 2-amino-6-chloropyridine ${ }^{[99]}$ with $m$ chloroperbenzoic acid (MCPBA) according to Pentimalli's procedure ${ }^{[100]}$ unexpectedly did not give the desired $N$-oxide, two another approaches were applied to prepare the compound $\mathbf{1 0 2}$ (Scheme 28).

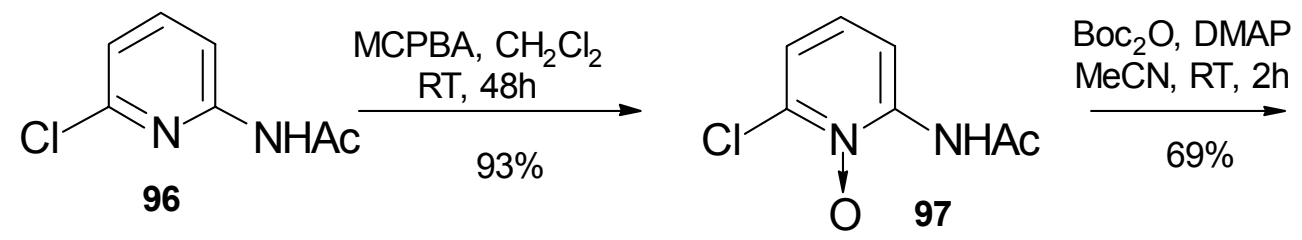<smiles></smiles>

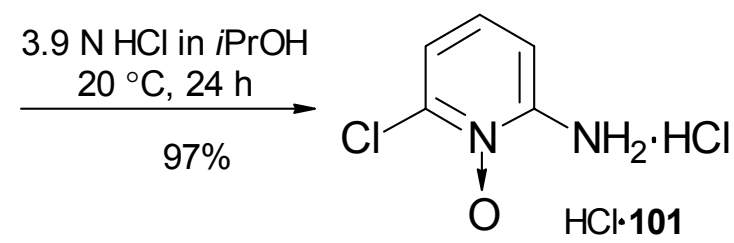

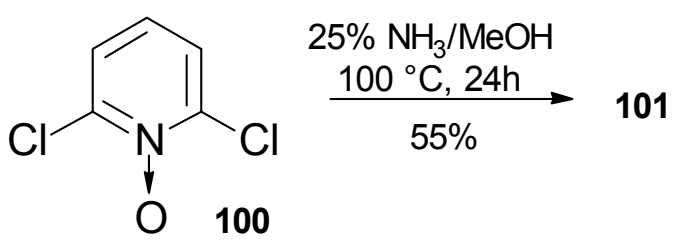<smiles></smiles>

Scheme 28

According to the first one, 2-acetamido-6-chloropyridine $\mathbf{9 6}^{[101]}$ was oxidized with MCPBA in dichloromethane to give the $N$-oxide 97 in $93 \%$, which in turn was $N$-acylated with $\mathrm{Boc}_{2} \mathrm{O}$ to give the diacylated derivative $\mathbf{9 8}$ in $69 \%$ yield. This compound was further deacetylated with $N, N, N^{\prime}, N^{\prime}$-tetramethylguanidine (TMG) in methanol to give the $N$-Boc protected amine 99 in $60 \%$ yield. The deprotection of the latter with $3.9 \mathrm{M} \mathrm{HCl}$ in isopropanol gave the desired amine 
$\mathbf{1 0 1}$ as a hydrochloride in $97 \%$ yield. The treatment of 2,6-dichloropyridine- $N$-oxide $\mathbf{1 0 0}^{[102]}$ with $25 \% \mathrm{NH}_{3}$ in methanol in a sealed tube at $100{ }^{\circ} \mathrm{C}$ for $24 \mathrm{~h}$ gave this compound as a free base in a single step and in 55\% yield.

The thermal decomposition of the azide $\mathbf{1 0 2}$ in degassed benzene gave 5-chloro-2-cyano-1hydroxypyrrol 103 in 49\% yield and the product of its deoxygenation 104 in 11\% yield (Scheme 29). As several attempts to hydrolyze $\mathbf{1 0 3}$ failed, this compound was MOM-protected on the hydroxy group. Hydrolysis of the nitrile group in the intermediate $\mathbf{1 0 5}$ according to protocol Cacchi et al. ${ }^{[103]}$ led to the amide 106 (all attempts to hydrolyze $\mathbf{1 0 5}$ or $\mathbf{1 0 6}$ were unsuccessful) which, in turn, was doubly acylated with $\mathrm{Boc}_{2} \mathrm{O}$ to give the desired triacylated intermediate $\mathbf{1 0 7}$ together with the side product 108. The alkaline hydrolysis of $107^{[104]}$ gave the $O$-MOMprotected Chpca-OH 109 (43\% overall yield over 4 steps from 103).

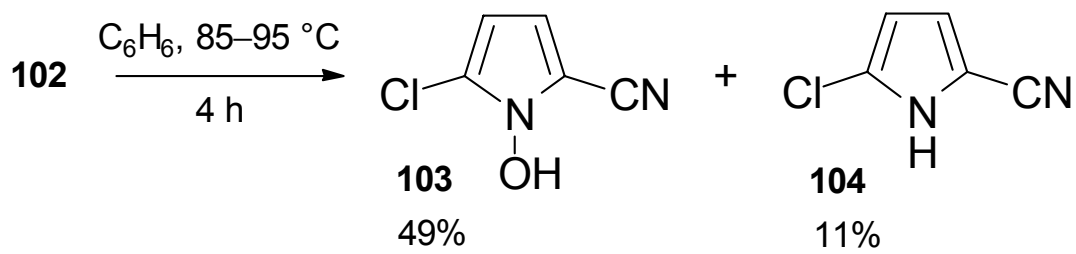

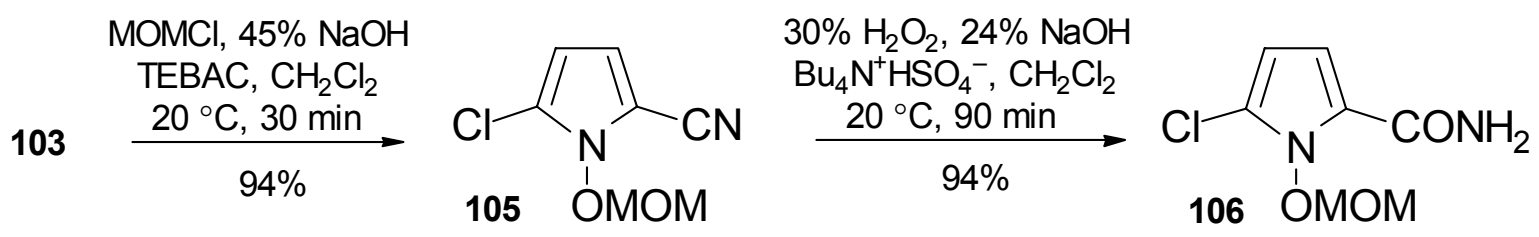

$$
\begin{aligned}
& \underset{\substack{10 \% \\
\mathrm{MeCN}, 20^{\circ} \mathrm{C}, 3 \mathrm{~h}}}{\mathrm{Boc}_{2} \mathrm{O}, \mathrm{DMOM}} \text { (cat) } \\
& 1 \mathrm{~N} \mathrm{NaOH} \\
& \text { Dioxane } \\
& 107 \\
& \underset{60 \%}{\stackrel{55^{\circ} \mathrm{C}, 2 \mathrm{~h}}{\longrightarrow}}
\end{aligned}
$$

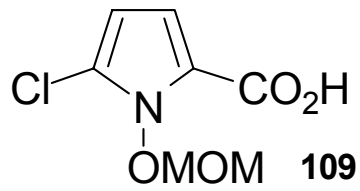

Scheme 29 
To test the cleavage of MOM group from the hydroxyl group of Chpca fragment, attached to some peptide (or $O$-protected amino acid), and applicability of the Chpca-acylated amino acid with free hydroxyl group for peptide synthesis the acyltripeptide 119 was prepared (Scheme 30).

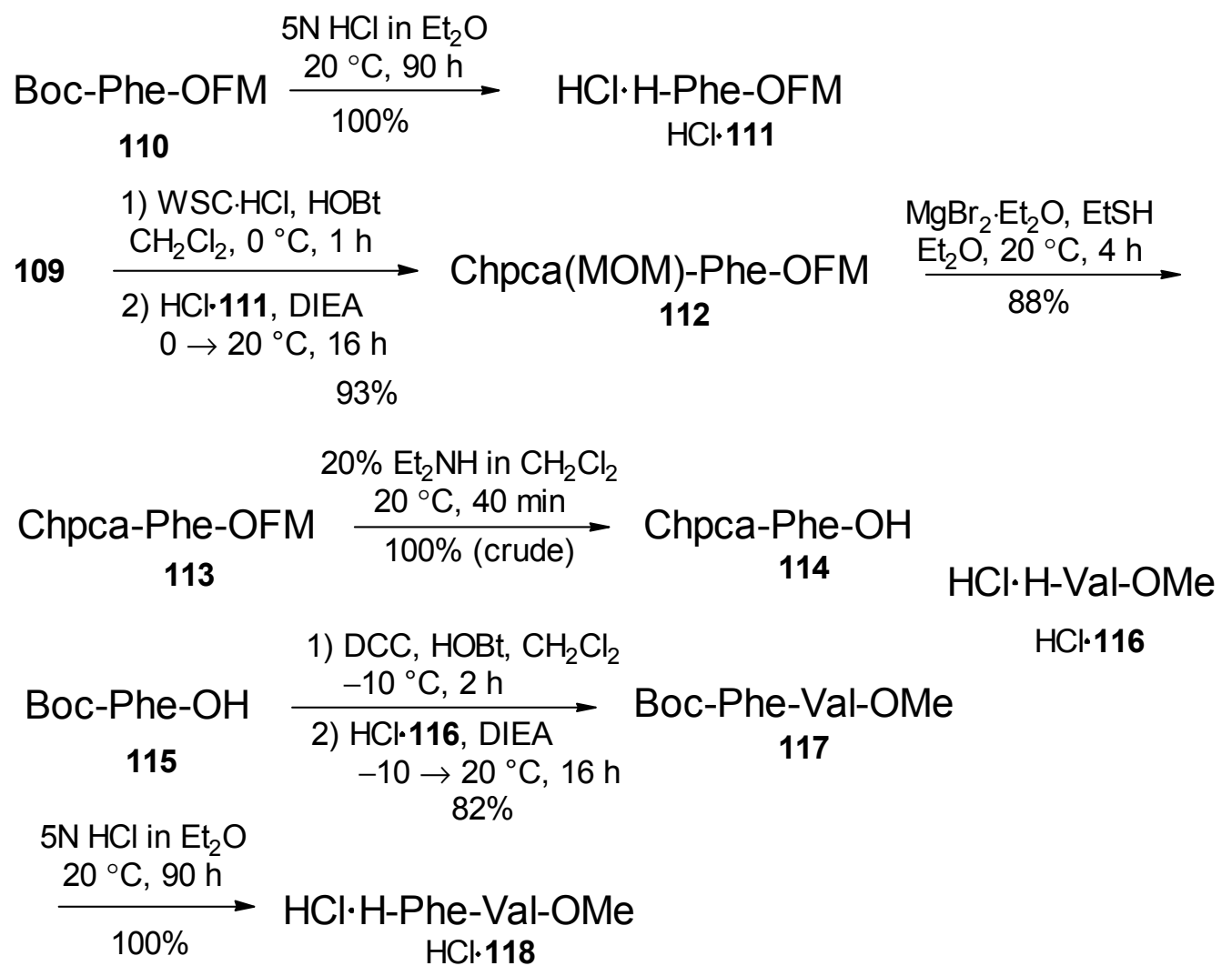

$$
\begin{aligned}
& 114+\mathrm{HCl} \cdot 118 \\
& 23 \% \downarrow \begin{array}{c}
\mathrm{HATU}, \mathrm{DIEA}, \mathrm{CH}_{2} \mathrm{Cl}_{2} \\
20{ }^{\circ} \mathrm{C}, 90 \mathrm{~min}
\end{array}
\end{aligned}
$$

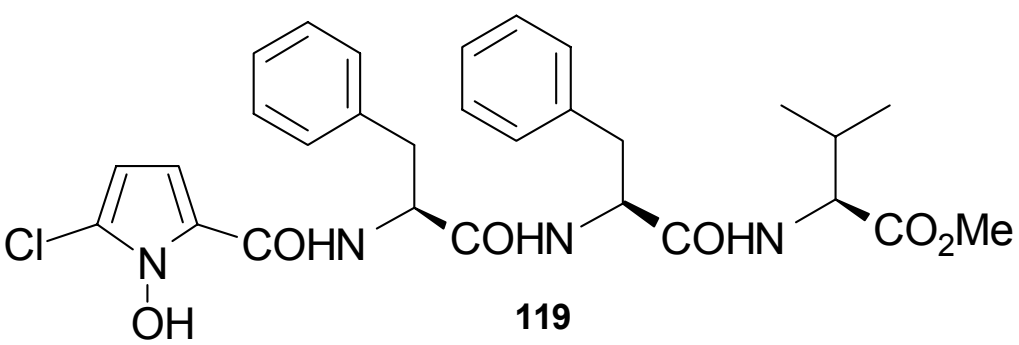

Scheme 30

Phenylalanine 9-fluorenylmethyl ester 111 obtained after Boc-group removal from $N$-termini of $N$-Boc protected amino ester $\mathbf{1 1 0}^{[105]}$ with $5 \mathrm{M} \mathrm{HCl}$ in $\mathrm{Et}_{2} \mathrm{O}$ was coupled with the acid $\mathbf{1 0 9}$ using the combination of $N^{N}$-(3-dimethylaminopropyl)- $N$-ethylcarbodiimide hydrochloride (EDC) and 1-hydroxybenzotriazole (HOBt) to give the acyl amino ester 112 in $93 \%$ yield, which after 
removal of the methoxymethyl group with $\mathrm{MgBr}_{2} \cdot \mathrm{Et}_{2} \mathrm{O}$ and ethyl mercaptan in diethyl ether ${ }^{[106]}$ gave the ester 113 (88\%). The latter was deprotected using $20 \%$ diethylamine in $\mathrm{CH}_{2} \mathrm{Cl}_{2}$ to give the unstable 114 in $100 \%$ crude yield, which was immediately coupled with the dipeptide 118 obtained after deprotection of the peptide 117 with $5 \mathrm{M} \mathrm{HCl}$ in $\mathrm{Et}_{2} \mathrm{O}$, which was previously prepared from $N$-Boc protected phenylalanine 115 and valine methyl ester 116 using dicyclohexylcarbodiimide (DCC) and HOBt in $82 \%$ yield, using the $O$-(7-azabenzotriazole-1yl)- $N, N, N^{\prime}, N^{\prime}$-tetramethyluronium hexafluorophosphate $(\mathrm{HATU})^{[107]}$ to give the desired acyltripeptide 119 in $23 \%$ yield.

One of the possibilities to study the structure-activity relationship for hormaomycin is connected with varying of the halogen substituent at $\mathrm{C}-5$ of Chpca moiety. While the bromine-des-chlorine analog of $\mathbf{3}$ was successfully obtained by varying of the ionic content of the culture media (see introduction), attempts to introduce fluorine or iodine constituent instead of chlorine atom using this methodology failed.

To synthesize 5-fluor-2-cyano-1-hydroxypyrrol 2-amino-6-fluoropyridine- $N$-oxide should be first prepared (Scheme 31). Surprisingly, an attempt to oxidize 2,6-difluoropyridine 120 with $30 \%$ aq. $\mathrm{H}_{2} \mathrm{O}_{2}$ in TFA failed, and only starting material was recovered. The oxidation of 120 with anhydrous trifluoroperacetic acid in $\mathrm{CH}_{2} \mathrm{Cl}_{2}$ at $20^{\circ} \mathrm{C}$ for 4 days gave only traces of the desired product $\mathbf{1 2 1}$, which quickly decomposed at $20^{\circ} \mathrm{C}$.

According to the second approach, successfully used for preparation of 2-amino-6chloropyridine- $N$-oxide 101, 2-acetamido-6-fluoropyridine $\mathbf{1 2 3}$ had to be synthesized. This compound was successfully prepared by diazotization of the 2-acetamido-6-aminopyridine $\mathbf{1 2 2}^{[108]}$ in pyridinium hydrofluoride in $79 \%$ yield. ${ }^{[109]}$ 

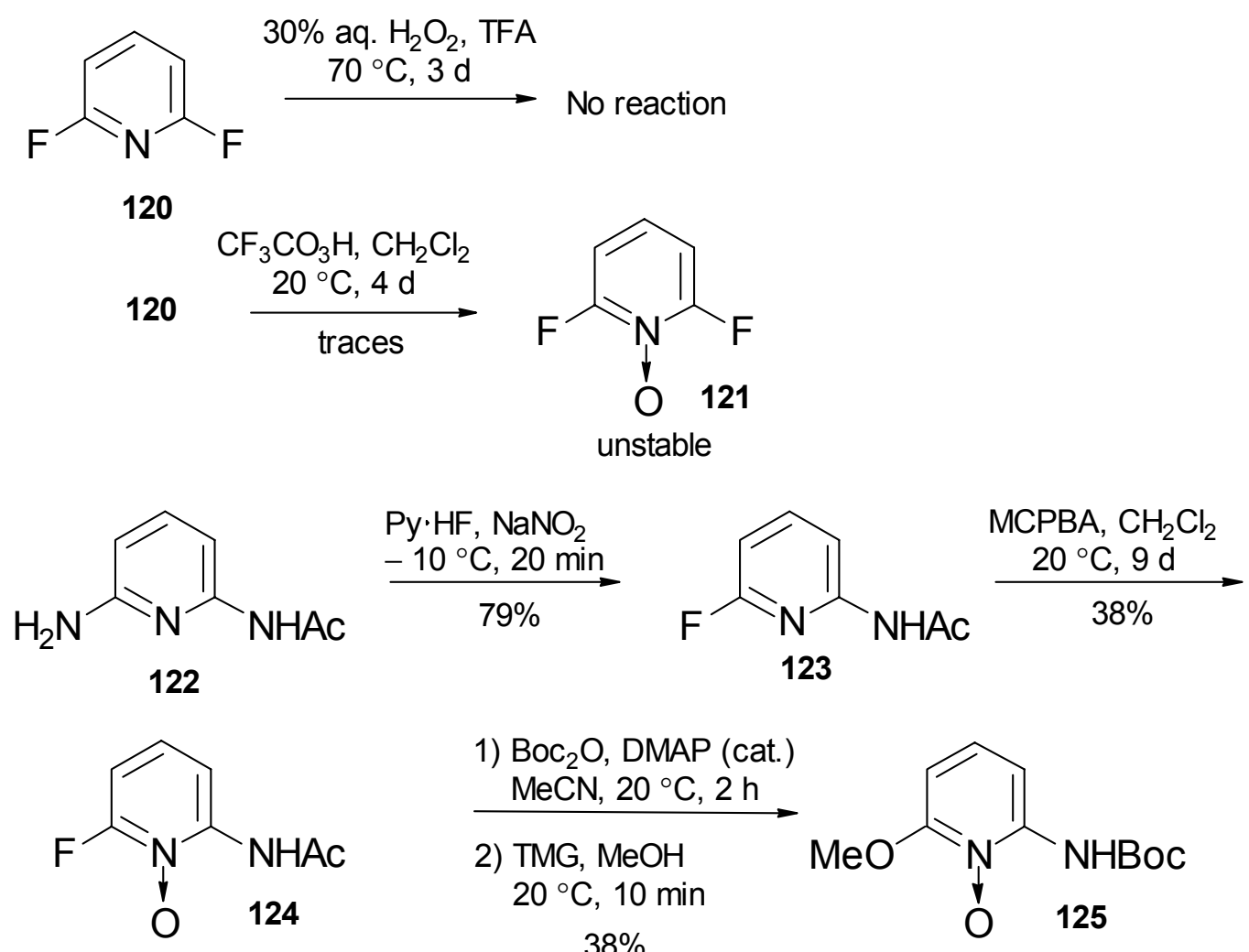

Scheme 31

The amide 123 was converted to the corresponding $\mathrm{N}$-oxide 124 using $\mathrm{MCPBA}$ in $\mathrm{CH}_{2} \mathrm{Cl}_{2}$ in $38 \%$ yield. The latter was N-Boc acylated with $\mathrm{Boc}_{2} \mathrm{O}$ in presence of DMAP in MeCN and the crude diacetylated intermediate was directly treated with 1 eq. of TMG in methanol to give $N$ Boc protected 2-amino-6-methoxypyridine- $N$-oxide $\mathbf{1 2 5}$ as the sole isolable product in $38 \%$ yield. A possibility to introduce fluorine atom into the $N$-hydroxypyrrol ring via fluorination of the $O$-protected 2-cyano-1-hydroxypyrrole or the corresponding derivative of 2-cyano-1hydroxypyrrolecarboxylic acid with a suitable fluorination agent has not been tested yet. 


\section{Final assembling of hormaomycin}

\subsection{General remarks}

Once the absolute configuration of hormaomycin $\mathbf{3}$ had been established and necessary quantities of $\left(2 S, 1^{\prime} S, 2^{\prime} R\right)$ - and (2R, 1'S,2'R)-3-(trans-2-nitrocyclopropyl)alanine, $N$-Boc-protected $(2 S, 4 R)-4$ (Z)-propenylproline, as well as the $O$-MOM protected 5-chloro-1-hydroxypyrrole-2-carboxylic acid and other building blocks were prepared, the assembly of hormaomycin could be initiated.

At first look the peptide synthesis of hormaomycin seems to be not a very complicated problem. The modern "state of art" of peptide coupling ${ }^{[110]}$ allows to prepare peptides, that do not contain extremely hindered fragments such as $\alpha, \alpha$-dialkyl amino acids, $N$-alkyl amino acids or even more challenging $N$-aryl amino acids, provided with a proper choice of the coupling reagent, solvent and other experimental conditions, in high yield and with high optical purity. As almost all amino acids, which comprise hormaomycin, are $\beta$-branched (the only exception is 3-(2'nitrocyclopropyl)alanine) HATU, as well as the combination of EDC and 7-aza-1hydroxybenzotriazole (HOAt) ${ }^{[111]}$ or $\mathrm{HOBt}$ were used on each condensation step to ensure the high-yielding coupling. The most unusual in hormaomycin is the ester bond between the secondary (4-PE)Pro moiety and the hydroxy group of $a$-Thr. Among several methods described in literature the dialkylaminopyridine-promoted carbodiimide-mediated esterification was chosen for its formation. ${ }^{[12]}$

On the other hand the reactivities of a double bond in 4-(Z)-propenylproline and nitro-group in nitrocyclopropylalanine moieties as well as, what is not so obvious, the ester bond between propenylproline and $(R)$-allo-threonine residues make a proper choice of the protecting groups and also the conditions for their deprotection to be a real challenge. Thus, the presence of the 
double bond hampers the application of catalytic hydrogenolysis and $\mathrm{HBr} / \mathrm{AcOH}$ reagent for the deprotection of peptides containing the propenylproline residue. The aliphatic nitro-group in (3Ncp)Ala is not compatible with the reductive cleavage conditions, and the threonine ester bond is sensitive to alkaline and basic conditions. ${ }^{[13]}$ Because of this base sensitivity all attempts to remove the Fmoc-group from the model depsipeptides 126 and $\mathbf{1 2 7}$ gave only complex mixtures (Scheme 32). Furthermore, attempted removal of the $\mathrm{ONb}$ group from the $C$-termini of the depsipeptide 128 with TBAF caused the fission of threonine ester bond and instead of the desired product 129 only dipeptide 130 was obtained in $90 \%$ yield.
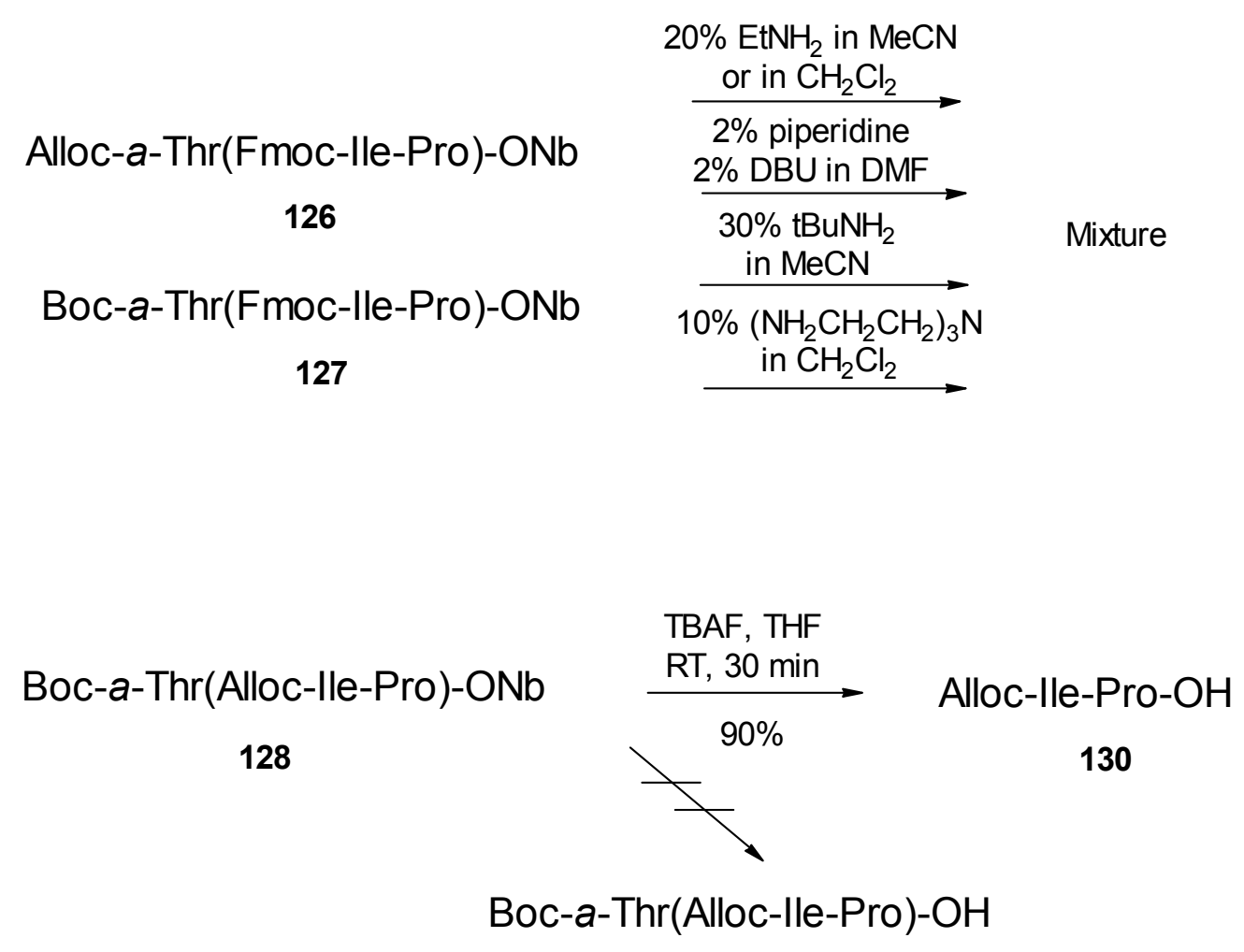

129

Scheme 32

The key step in the synthesis of hormaomycin is the formation of the macrocycle. The greater facility with which amide bonds can be formed, a consequence of the superior nucleophilicity of the amine over the hydroxyl group, makes macrolactamization the preferred mode for ring closure. The amide bond between $(\beta \mathrm{Me}) \mathrm{Phe}$ and Ile seems to be least suitable for cyclization because of the possibility of cyclo-[Ile-(4-PE)Pro] diketopiperazine formation ( $\beta$-position to the ester bond) as well as significant epimerization and low yield, which are connected with the bulk 
side chains of these amino acids. That between $a$-Thr and $(\beta \mathrm{Me})$ Phe is more preferable because of the suppression of racemization by urethane protection and that between $(\beta \mathrm{Me}) \mathrm{Phe}$ and $(\mathrm{R})$ (3-Ncp)Ala since the cyclization tends to proceed faster between residues of the opposite than of the same $\alpha$-center configuration. ${ }^{[114]}$ The ring closure at the amide bond between Ile and (4PE)Pro should give a greater epimerization extent because of the higher basicity of proline relative to primary amino acids and that between $(R)-(3-\mathrm{Ncp}) \mathrm{Ala}$ and $(\beta \mathrm{Me}) \mathrm{Phe}$ is also less preferable since the large side chain of latter shields its amino group.

To complete synthesis the cyclic depsipeptide should be deprotected leaving intact its sensitive elements and then side chain of hormaomycin should be added.

\section{2. $\quad$ Syntheses of the selected model peptides}

Keeping in mind the ring closure between $a$-Thr and ( $\beta \mathrm{Me})$ Phe the applicability of the acid hypersensitive 2-nitrophenylsulfenyl (Nps) group ${ }^{[115]}$ as a temporary protecting group for amino function together with Boc group as a permanent protecting group for amino function and methylthiomethyl (MTM) group, which can be removed in the presence of the Boc-group with $\mathrm{MgBr}_{2} \cdot \mathrm{Et}_{2} \mathrm{O}$ in $\mathrm{Et}_{2} \mathrm{O}$ or $\mathrm{CH}_{2} \mathrm{Cl}_{2}$, as a protecting group for carboxylic function was tested (Scheme 33).

The $N$-Boc protected MTM ester 132, obtained via reaction between MTMI and 131 in MeCN in $77 \%$ yield, was acylated with $N$-Nps protected proline $\mathbf{1 3 3}^{[116]}$ to give the ester $134(85 \%)$, which after removal of $\mathrm{Nps}$ group with 2 eq. $0.1 \mathrm{M} \mathrm{HCl}$ in $\mathrm{Et}_{2} \mathrm{O}$ and $\mathrm{EtSH}$, was coupled with $N$-Nps protected isoleucine $\mathbf{1 3 5}^{[116]}$ to give the depsitripeptide $\mathbf{1 3 6}$ in $80 \%$ yield. Treatment of $\mathbf{1 3 4}$ with $\mathrm{MgBr}_{2} \cdot \mathrm{Et}_{2} \mathrm{O}$ in $\mathrm{CH}_{2} \mathrm{Cl}_{2}$ instead of the selective removal of MTM group unexpectedly caused also the concurrent cleavage of the Nps group and gave only traces of the target 137. An additional 
study showed that 135 completely decomposed in the same conditions within 20 min. Consequently the exhaustive low-yielding chromatographic purification, that was necessary to prepare pure deprotected peptides, precluded further investigation of this protection scheme.

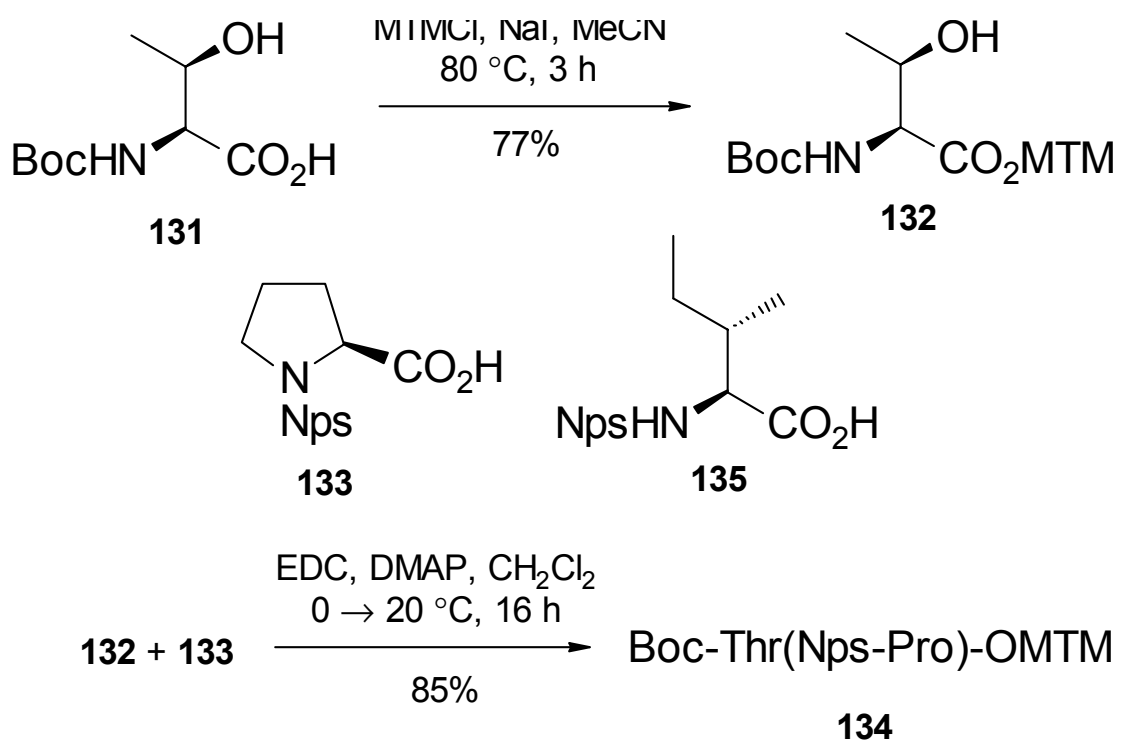

1) $0.1 \mathrm{~N} \mathrm{HCl}$ in $\mathrm{Et}_{2} \mathrm{O}$

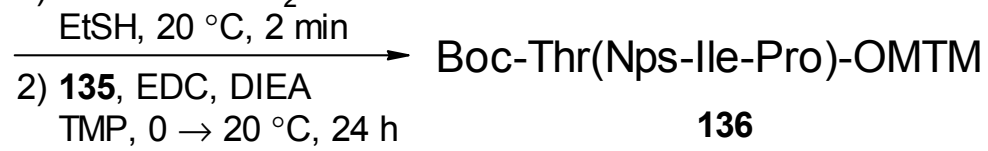

TMP, $0 \rightarrow 20^{\circ} \mathrm{C}, 24 \mathrm{~h}$

136

$80 \%$

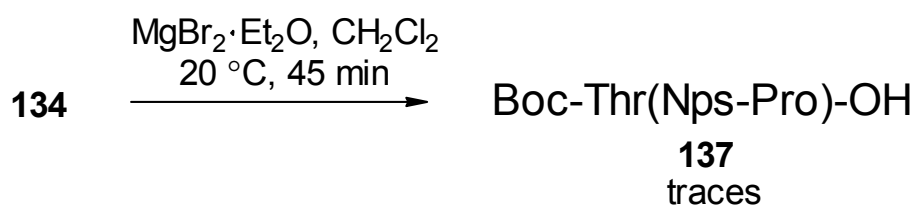

Scheme 33

In order to study the possibility of construction of the linear precursor for the cyclization at the amide bond between ( $\beta \mathrm{Me}) \mathrm{Phe}$ and (3-Ncp)Ala via condensation of already assembled tetra- and dipeptide fragments, depsipeptide 142 was prepared (Scheme 34). The dipeptide 138, ${ }^{[117]}$ after removal of the Boc group from the $N$-termini, was coupled with $N$-Boc protected $\beta$ methylphenylalanine $139^{[76 \mathrm{~b}]}$ to give the tripeptide 140 in $83 \%$ yield, methyl ester function of which was hydrolyzed with triethylbenzylammonium hydroxide (Triton X) to give the acid $\mathbf{1 4 1}$ (77\%). The latter was coupled with the alcohol 132 to give the desired product 142 in a satisfactory $45 \%$ yield. 
1) $2 \mathrm{M} \mathrm{HCl}$ in $\mathrm{MeOH}$

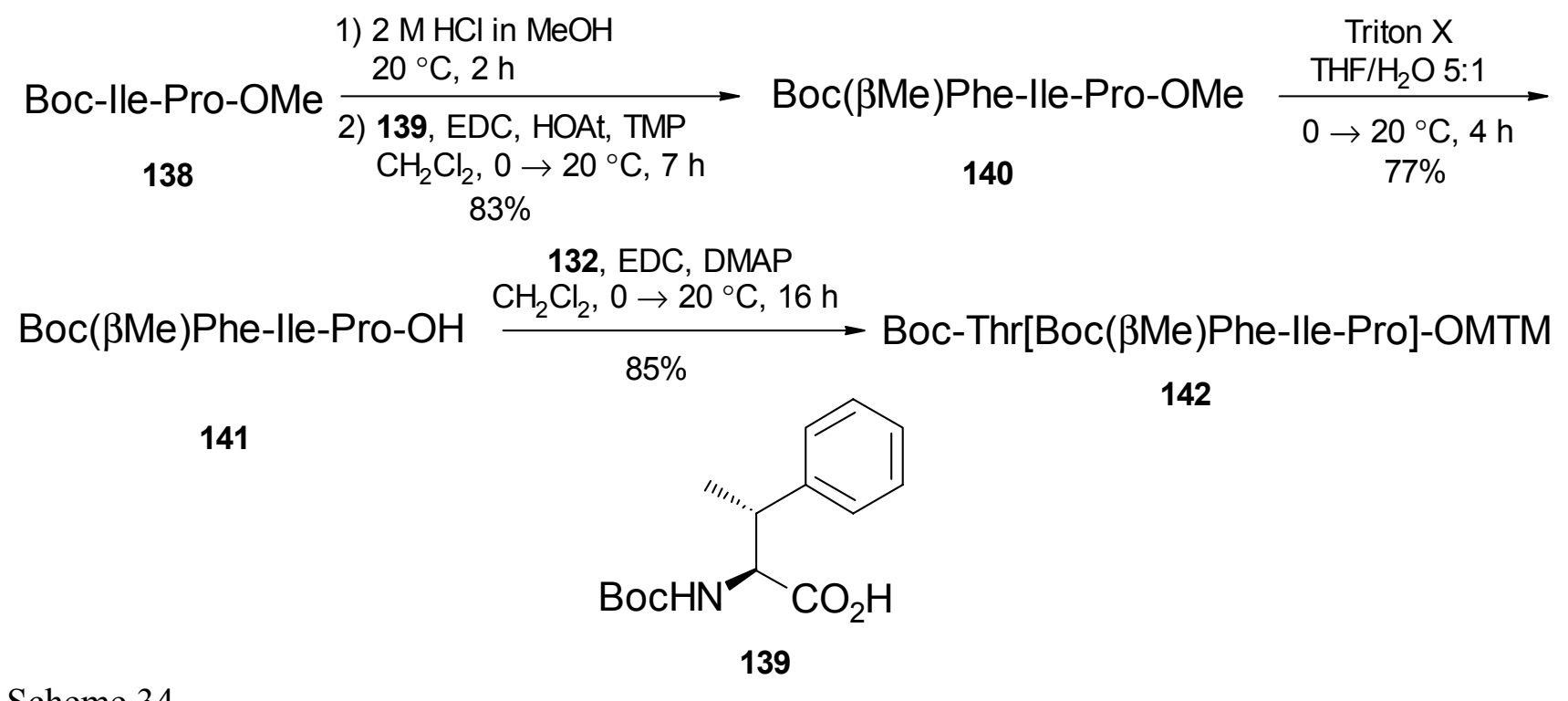

Scheme 34

The synthesis of hexadepsipeptide 150 was further embarked on (Scheme 35). After removal of the Boc group, the tripeptide $\mathbf{1 4 0}$ was coupled with $N$-Boc protected (2S,1'RS,2'RS)-3-(2'nitrocyclopropyl)alanine $\left(2 S, 1^{\prime} R S, 2^{\prime} R S\right)-143$ to give tetrapeptide ester $144(67 \%)$, which was hydrolyzed with tetrabutylammonium hydroxide ${ }^{[118]}$ to give the crude acid $\mathbf{1 4 5}$ in a quantitative yield. $N$-Fmoc protected $(\beta \mathrm{Me})$ Phe-OH $146^{[119]}$ was esterified with 3,4-dimethoxybenzyl alcohol to give the Dmob ester 147 in $57 \%$ yield.

This ester, after removal of the Fmoc group, was coupled with $N$-Alloc protected $a$-Thr-OH 148 , which was obtained from $(R)$-allo-threonine $\mathbf{7 2}$ and pentafluorophenyl allyl carbonate (AllocOPfp), to give the dipeptide 149 in $90 \%$ yield. Several attempts to esterify the acid 145 with the alcohol 149 were unsuccessful and gave in the best case $\mathbf{1 5 0}$ as a component of the mixture inseparable by conventional methods. 


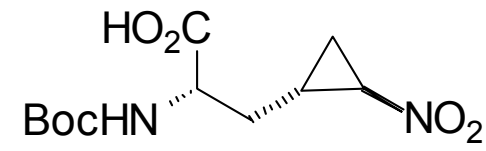

(2S, $\left.1^{\prime} R S, 2^{\prime} R S\right)-143$

1) $2 \mathrm{M} \mathrm{HCl}$ in $\mathrm{MeOH}$

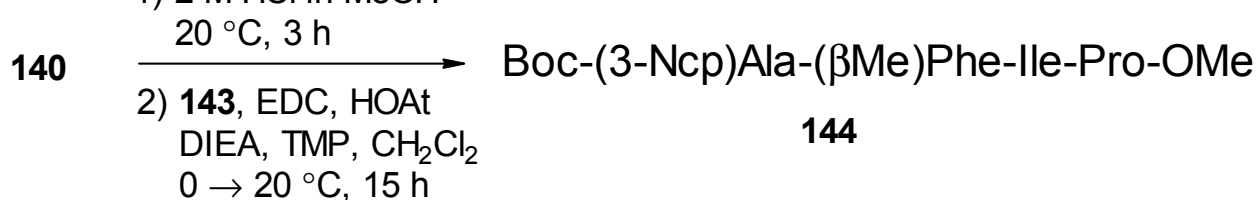

$$
\begin{aligned}
& 67 \%
\end{aligned}
$$

$40 \%$ aq. $(\mathrm{Bu})_{4} \mathrm{~N}^{+} \mathrm{OH}^{-}$

$\underset{100 \% \text { (crude) }}{\stackrel{\mathrm{THF}, 4}{\circ} \mathrm{C}, 3.5 \mathrm{~h}}$ Boc-(3-Ncp)Ala-( $\beta \mathrm{Me})$ Phe-lle-Pro-OH

145
2,4-Bzl(OMe) ${ }_{2} \mathrm{OH}$
EDC, DMAP, $\mathrm{CH}_{2} \mathrm{Cl}_{2}$
Fmoc- $(\beta M e)$ Phe-OH
146 $\frac{0 \rightarrow 20^{\circ} \mathrm{C}, 16 \mathrm{~h}}{57 \%}$ Fmoc- $(\beta M e)$ Phe-ODmob
147

1) $50 \% \mathrm{Et}_{2} \mathrm{NH}, \mathrm{MeCN}$

$20^{\circ} \mathrm{C}, 40 \mathrm{~min}$

2) 148, EDC, HOAt, TMP

$\mathrm{CH}_{2} \mathrm{Cl}_{2} / \mathrm{DMF}$ 1:1

Alloc-a-Thr-( $\beta M e)$ Phe-ODmob

$0 \rightarrow 20^{\circ} \mathrm{C}, 7 \mathrm{~h}$

$90 \%$
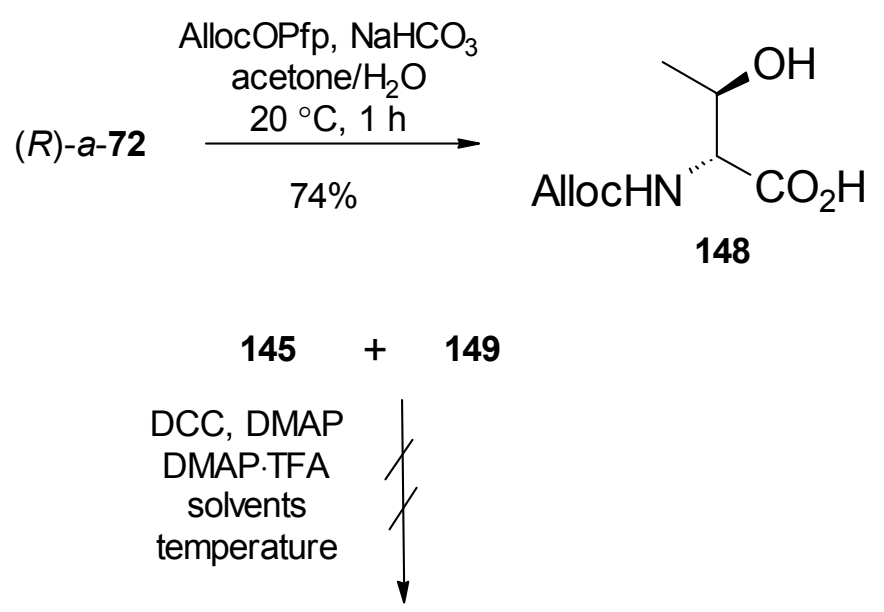

Alloc-a-Thr[Boc-(3-Ncp)Ala-( $\beta M e) P h e-I l e-P r o]-(\beta M e) P h e-O D m o b$ 
In order to evaluate, if the Alloc group can be successfully removed from the amino group of the depsipeptides containing $O$-acylated $a$-Thr moiety as their $N$-terminal amino acid, the ester $\mathbf{1 5 6}$ was prepared (Scheme 36).

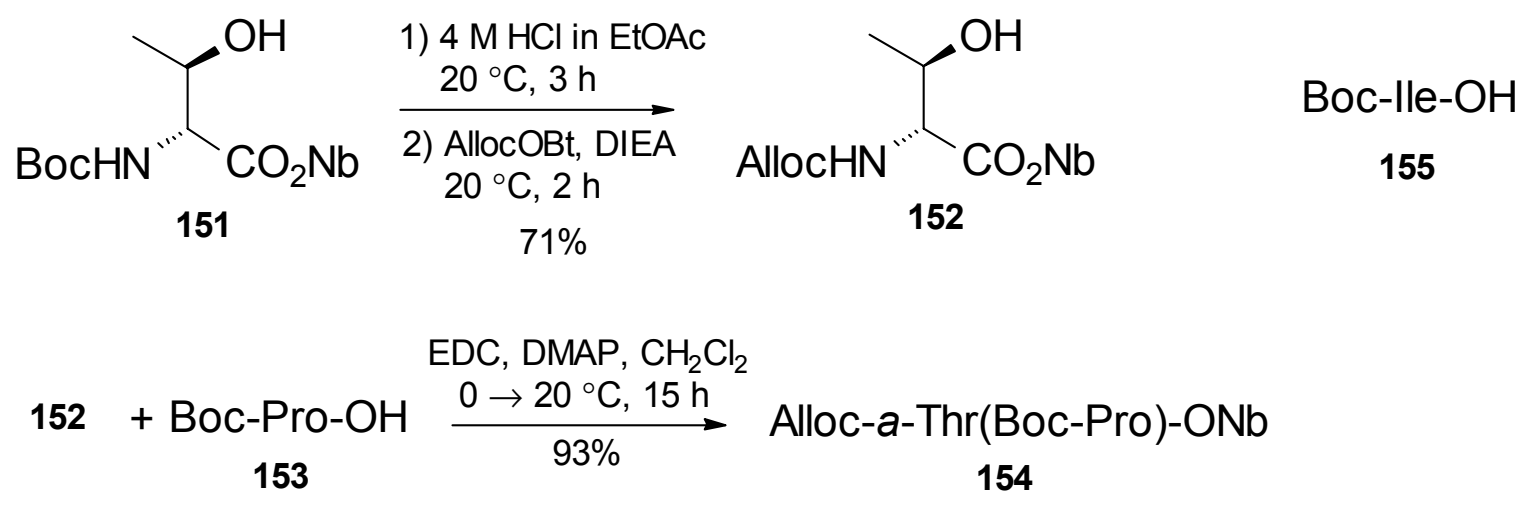

1) TFA, $20^{\circ} \mathrm{C}, 40 \mathrm{~min}$

2) 155 , EDC, HOAt

DIEA, TMP, $\mathrm{CH}_{2} \mathrm{Cl}_{2}$

Alloc-a-Thr(Boc-lle-Pro)-ONb

$0 \rightarrow 20^{\circ} \mathrm{C}, 15 \mathrm{~h}$ 156

$89 \%$

\section{Scheme 36}

The $a$-Thr derivative 151 (prepared as described for $(2 S, 3 R)$-isomer), ${ }^{[120]}$ after removal of the Boc group, was again $N$-protected with $N$-hydroxybenzotriazolyl allyl carbonate (AllocOBt) to give 152 (71\%), which was further $O$-acylated with $N$-Boc protected Pro-OH 153 to give the ester 154 in $93 \%$ yield. The latter, after removal of the Boc group, was coupled with $N$-Boc protected Ile-OH 155 to give the desired product 156 in $89 \%$ yield.

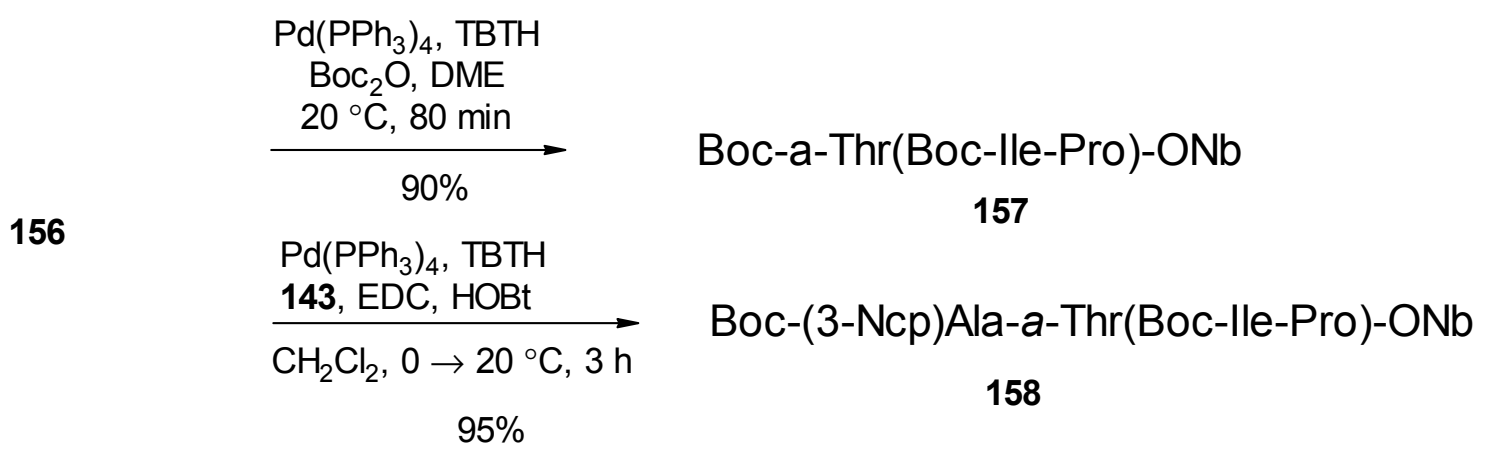

Scheme 37

All attempts to remove the Alloc-group from the $N$-termini of $a$-Thr residue of $\mathbf{1 5 6}$ failed to give a reasonable yield of the required product since the initially formed amino ester rearranged via $O$ 
$\rightarrow N$ migration. Thus, transprotection and transacylation of this depsipeptide according to protocol of Hiemstra et al. ${ }^{[121]}$ were tested (Scheme 37). The palladium-catalyzed deprotection by means of tributyltin hydride (TBTH) as a nucleophile in the presence of $\mathrm{Boc}_{2} \mathrm{O}$ or $\mathrm{HOBt}$ ester, formed in situ from $\left(2 S, 1^{\prime} R S, 2^{\prime} R S\right)$-141, as active acylating agents gave the expected products, 157 and 158, in excellent yields ( $N$-methyl morpholinium $p$-toluene sulfinate was also used as a nuclephile but gave the transprotected product of somewhat lower quality).

\subsection{Synthesis of $\mathrm{N}$-Alloc protected cyclodepsipeptide 171a}

Once reasonably effective route for removal of the Alloc group from the $\mathrm{N}$-termini of $\mathrm{O}$ acetylated $a$-Thr moiety had been established, the synthesis of the linear precursor with the bond linking Ile and (4-PE)Pro as a point for macrocyclization could be commenced. The protection strategy for this synthesis based on use of the Boc-, Z-, Fmoc- and Alloc-groups as an amino protection and 2-trimethylsilylethyl (TMSE) ${ }^{[122]}$ or (2-trimethylsilyl)ethoxymethyl (SEM) ${ }^{[123]}$ groups for the protection of carboxylic functions.

The SEM ester 159, which was applied for this synthesis, was obtained in $58 \%$ yield by treatment of $N$-Alloc protected $a$-Thr-OH 148 with $\mathrm{SEM}-\mathrm{Cl}$ and $\mathrm{Li}_{2} \mathrm{CO}_{3}$ in DMF. $N$-Fmoc protected $\left(2 R, 1^{\prime} R, 2^{\prime} R\right)-(3-\mathrm{Ncp})$ Ala-OH $\mathbf{1 6 0}$ was obtained by acylation of the amino acid $\left(2 R, 1^{\prime} R, 2^{\prime} R\right)-\mathbf{8 3}$ with FmocOSu $(93 \%)$, and amino ester 161 was obtained by esterification of $\mathbf{1 5 5}$

with 2-trimethylsilylethanol, was followed by the selective removal of Boc group and decomposition of the intermediate tosylate (54\% crude yield over 3 steps) (Scheme 38 ). 


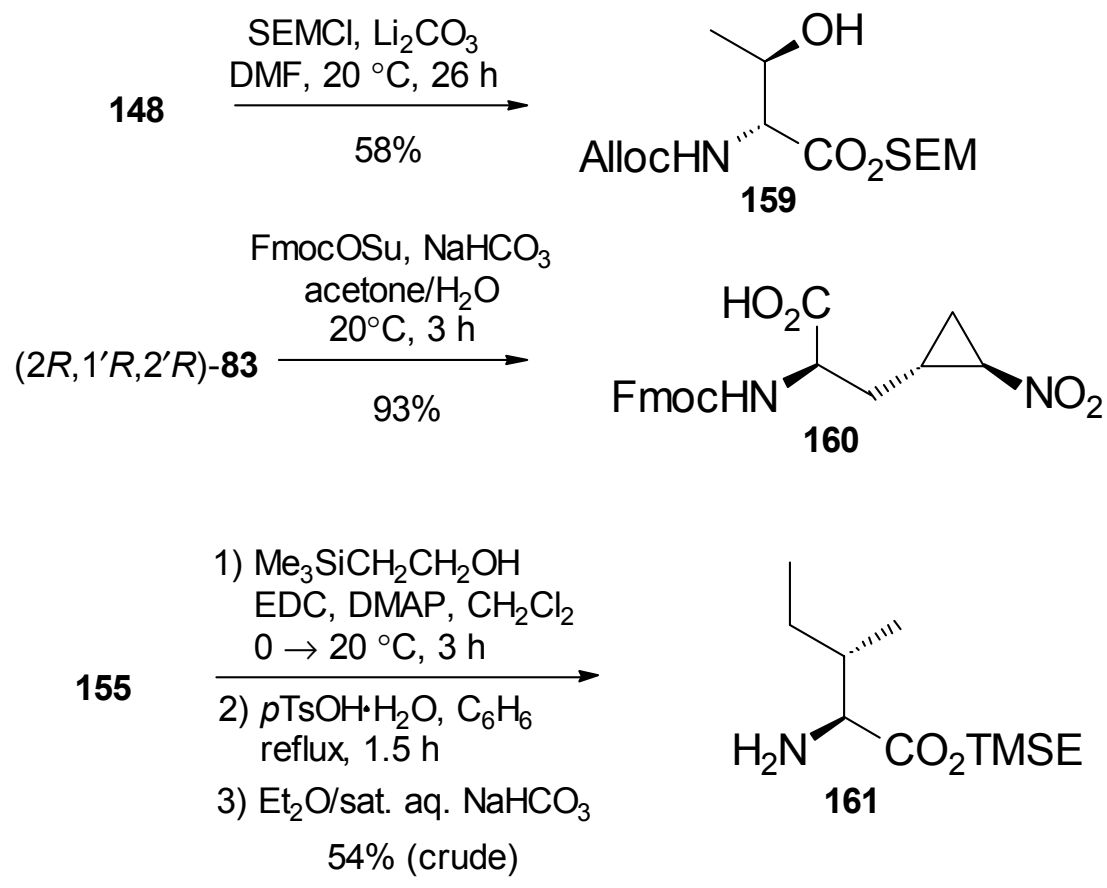

Scheme 38

The prepared amino ester was coupled with $N$-Boc protected $(\beta \mathrm{Me}) \mathrm{Phe}-\mathrm{OH} 139$ to yield dipeptide $162(86 \%)$, which, after removal of the Boc group, by treatment with $3.9 \mathrm{M} \mathrm{HCl}$ in dioxane (78\%), in turn was condensed with the $N$-protected amino acid $\mathbf{1 6 0}$ to give tripeptide 164 in $73 \%$ yield (Scheme 38). This peptide, after removal of the Fmoc group (100\%) was coupled with $N$-Fmoc protected ( $\beta \mathrm{Me})$ Phe-OH 146 to give tetrapeptide 167 (89\%) (schema 39).

Alcohol 159 was esterified with the $N$-Boc protected (4-PE)Pro-OH (2S,3R)-16 using EDC in the presence of DMAP to give 166 (64\%). This, after removal of the SEM group by treatment with $\mathrm{MgBr}_{2} \cdot \mathrm{Et}_{2} \mathrm{O}$ in $\mathrm{CH}_{2} \mathrm{Cl}_{2}(65 \%),{ }^{[124]}$ was directly coupled with tetrapeptide 169 using HATU in the presence of HOAt to give hexadepsipeptide $\mathbf{1 7 0}$ in $80 \%$ yield. 


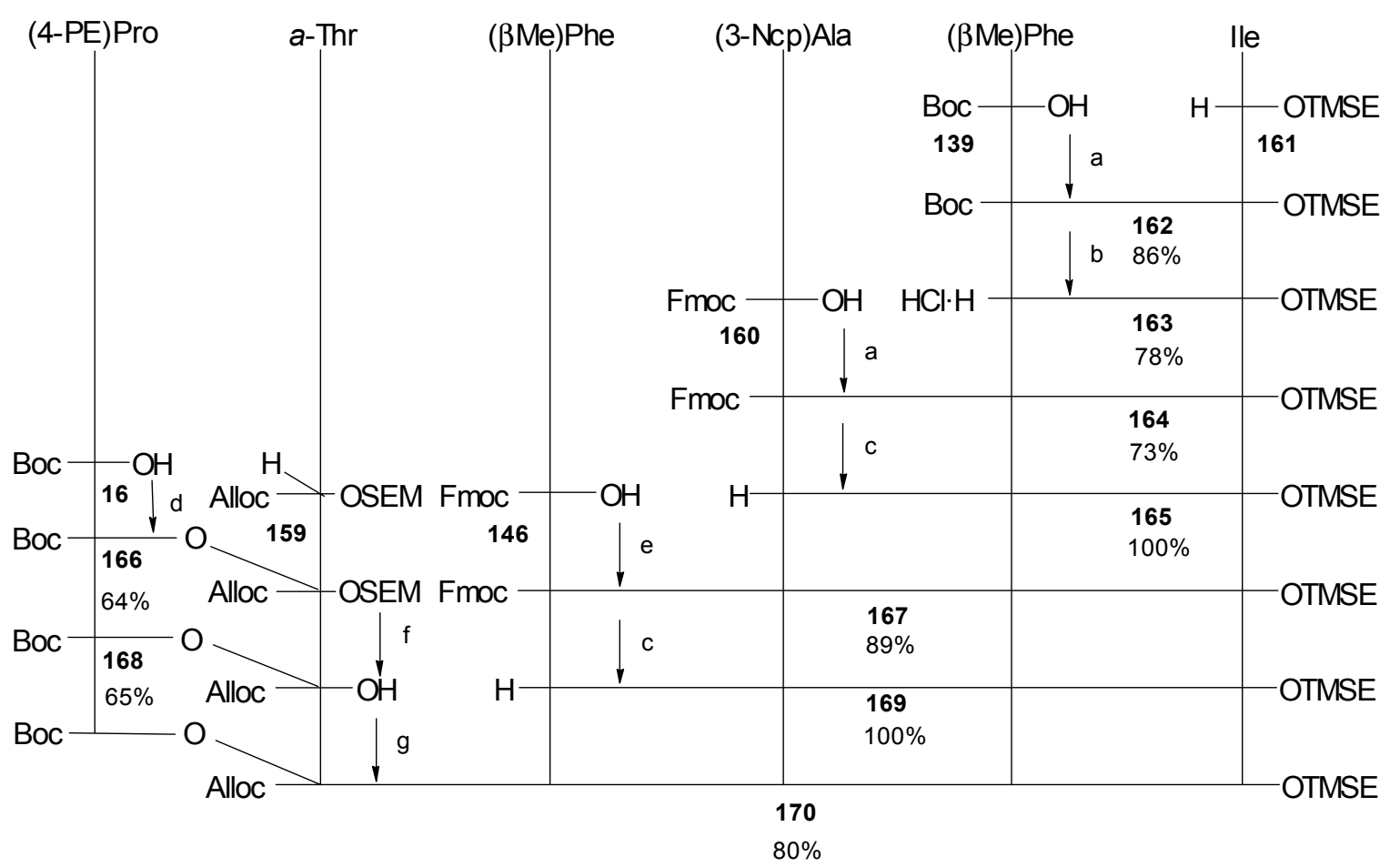

Scheme 39. Reagents and conditions: a) EDC, HOAt, DIEA, 2,4,6-trimethylpyridine, $\mathrm{CH}_{2} \mathrm{Cl}_{2}, 0$ $\rightarrow 20{ }^{\circ} \mathrm{C}, 14$ h. b) $3 \mathrm{M} \mathrm{HCl}$ in dioxane, $20{ }^{\circ} \mathrm{C}, 40$ min. c) $50 \% \mathrm{Et}_{2} \mathrm{NH} / \mathrm{MeCN}, 20{ }^{\circ} \mathrm{C}, 40 \mathrm{~h}$. d) EDC, DMAP, $\mathrm{CH}_{2} \mathrm{Cl}_{2}, 0 \rightarrow 20{ }^{\circ} \mathrm{C}, 24$ h. e) EDC, HOAt, 2,4,6-trimethylpyridine, DMF, $0 \rightarrow 20$ ${ }^{\circ} \mathrm{C}, 14 \mathrm{~h}$. f) $\mathrm{MgBr}_{2} \cdot \mathrm{Et}_{2} \mathrm{O}, \mathrm{CH}_{2} \mathrm{Cl}_{2},-30 \rightarrow 0{ }^{\circ} \mathrm{C}, 2$ h. g) HATU, HOAt, DIEA, 2,4,6trimethylpyridine, DMF, $0 \rightarrow 20^{\circ} \mathrm{C}, 24 \mathrm{~h}$.

The acidolytic removal of the Boc and TMSE groups from the latter occurred quantitatively and was followed by macrocyclization, using HATU in the presence of HOAt under high dilution conditions, ${ }^{[125]}$ which gave macrocycle 171a in 26\% yield after HPLC purification (Scheme 40). Unfortunately, an attempted transprotection with $\mathrm{Boc}_{2} \mathrm{O}$ and $\mathrm{Pd}\left(\mathrm{PPh}_{3}\right)_{4}$ in the presence of TBTH gave an inseparable mixture of unreacted and transprotected peptide, 171a and $\mathbf{1 7 1} \mathbf{b}$, in a low yield, presumably, due to steric hindrance. 

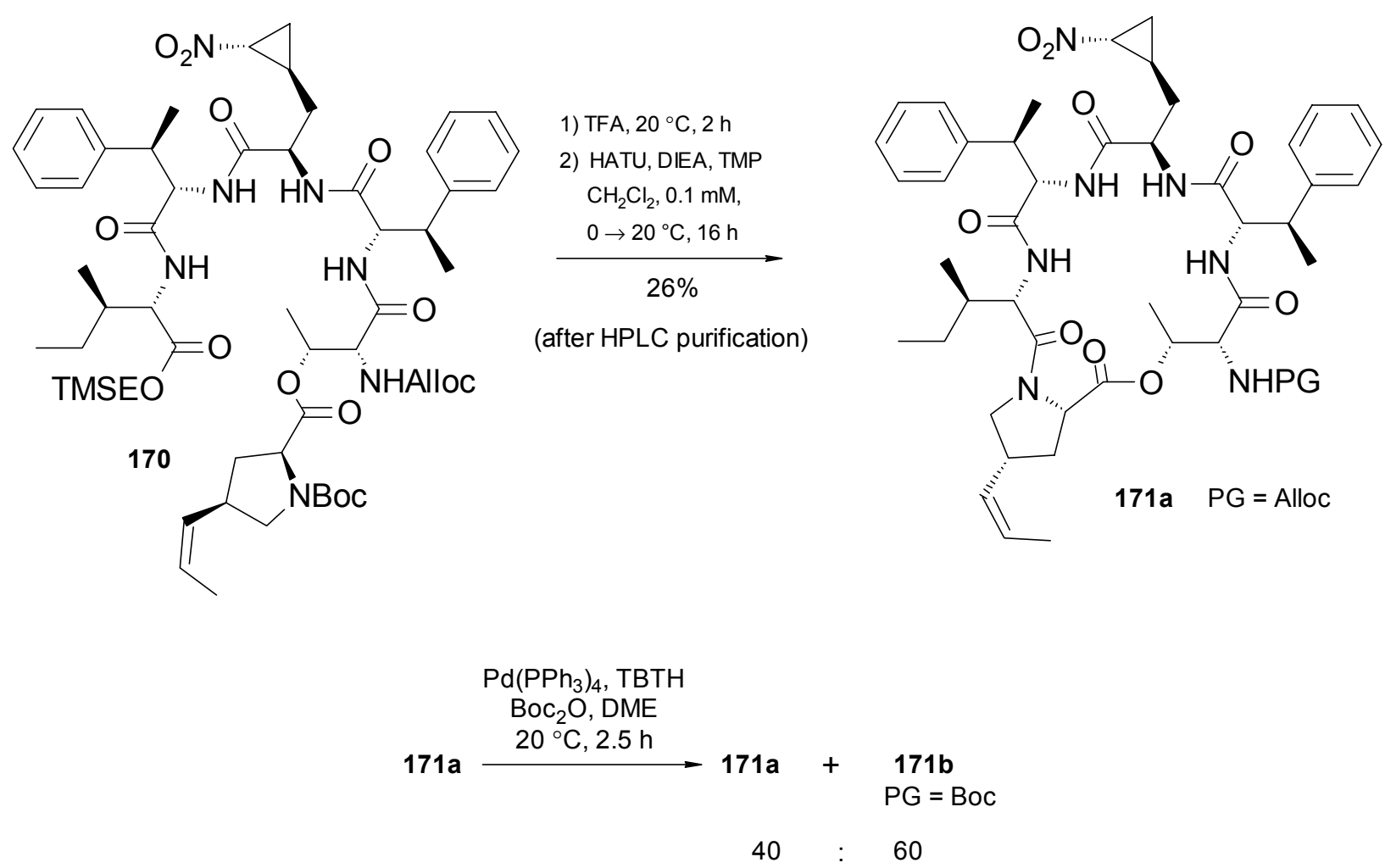

Scheme 40

5.4. Comparative study of acidolytic deprotection Boc, $Z$ and several Z-derived amino protecting groups

As shown by model experiments, peptides with the $O$-acylated threonine moiety on the $N$ termini and free $\alpha$-amino function are unstable in basic, neutral and even weakly acidic conditions supposedly probably due to a fast $\mathrm{O} \rightarrow \mathrm{N}$ acyl shift. Nevertheless this side reaction is completely suppressed under relatively strong acidic conditions. As a means of preserving the Boc group as an amino protecting group on the (4-PE)Pro moiety, first the $\mathrm{Z}$ group as a protecting group for the $\alpha$-amino group of $O$-acetylated threonine was tested (Scheme 41).

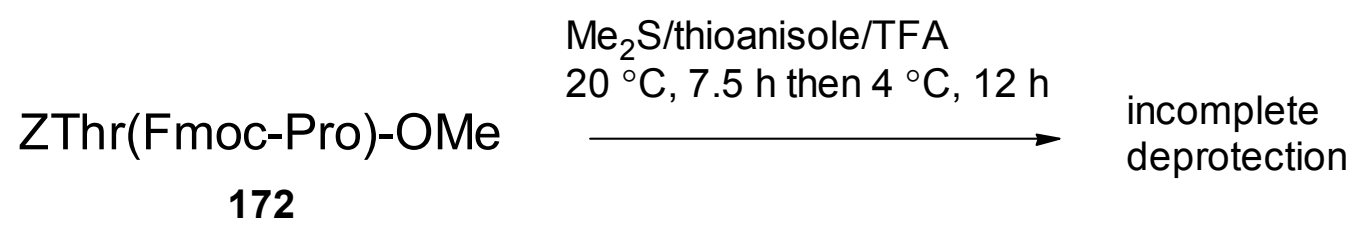

172

Scheme 41 
Unfortunately, the rate of the "push-pull"-based cleavage of the Z-protecting group ${ }^{[126]}$ [more acidic (liquid HF, MSA or TFMSA in TFA, TMSOTf) reagents in this case cause simultaneous cleavage of the ester bond in the threonine moiety] was unacceptably low even in case of the model ester Z-Thr(FmocPro)-OMe. In order to overcome this difficulty the easiness of the acidolytic removal of the Z-group should be finely attenuated.
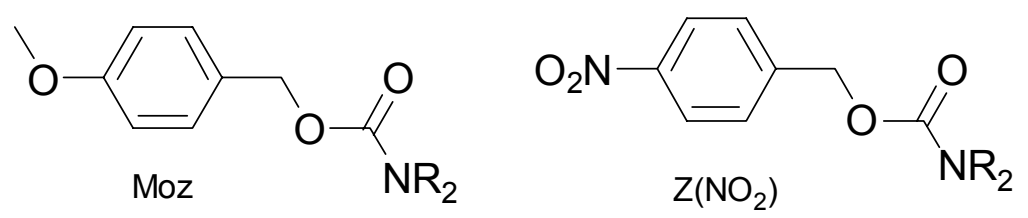

$$
\begin{array}{cccc}
p-\mathrm{MeO} & p-\mathrm{NO}_{2} & p-\mathrm{Me} & m-\mathrm{Me} \\
\sigma^{+}-0.78 & 0.78 & -0.31 & -0.10
\end{array}
$$

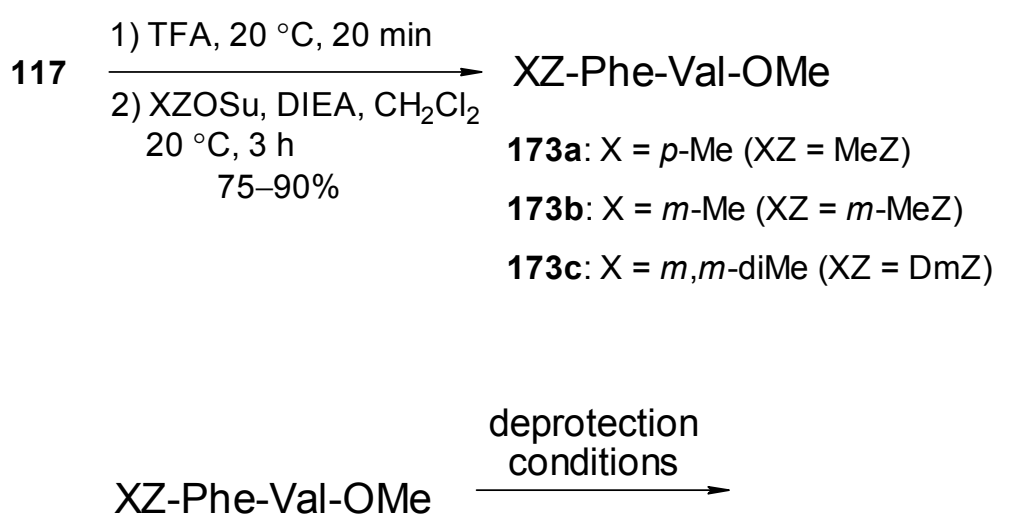


The stability of urethane-type protecting groups under acidic conditions depends on the stability of carbocations formed after their cleavage. This stability in case of the $m$ - and $p$ - substituted benzyl cations correlates with the corresponding $\sigma^{+}$Hammett constants. ${ }^{[127]}$ Whereas the p- $\mathrm{NO}_{2}$ substituted $\mathrm{Z}$ group $\left[\mathrm{Z}\left(\mathrm{NO}_{2}\right)\right], \sigma_{\mathrm{NO} 2}^{+}=0.78$, is noticeably more stable to TFA and $\mathrm{HBr}$ in $\mathrm{AcOH}$, than the original $\mathrm{Z}$ group, the $p$-methoxy substituted $\mathrm{Z}$ group $(\mathrm{MeZ}), \sigma_{\mathrm{OMe}}^{+}=-0.78$, is cleaved with TFA at $0{ }^{\circ} \mathrm{C}$ within several minutes. Cleavage of $p$-methyl $\mathrm{Z}$ (MeZ), $m$-methyl $\mathrm{Z}(m$-MeZ) and $m, m$-dimethyl $\mathrm{Z}$ groups $(\mathrm{DmZ})$ in the model dipeptides $\mathbf{1 7 3}$ was briefly evaluated (Scheme 42). The $\mathrm{MeZ}$ and $\mathrm{DmZ}$ groups were stable to $2 \mathrm{M} \mathrm{HCl}$ in EtOAc for at least $2 \mathrm{~h}$ and the $m$-MeZ group was stable to this reagent, while the Boc group cleaved from hexadepsipeptide $\mathbf{1 7 0}$ within 2 min. Furthermore, the MeZ and DmZ groups were quantitatively cleaved with $10 \%$ anisole in TFA within $1 \mathrm{~h}$ and $m$-MeZ with 20\% thioanisole in TFA for $3.5 \mathrm{~h}$. For a further application the MeZ group was chosen.

\subsection{Synthesis of N-MeZ protected cyclodepsipeptide 171c}

The dicyclopropylmethyl (DCPM) ${ }^{[128]}$ and methoxymethyl (MOM) groups were used in the course of this synthesis for the protection of the carboxylic group. The alkylation of the $N$-MeZ protected amino acid 175, which was obtained by reaction between of $\mathrm{H}-a$-Thr-OH and MezOSu (90\%), with allyl bromide in the presence of $\mathrm{K}_{2} \mathrm{CO}_{3}$ in $\mathrm{MeCN}$ gave 176 in $84 \%$ yield (Scheme 43). 


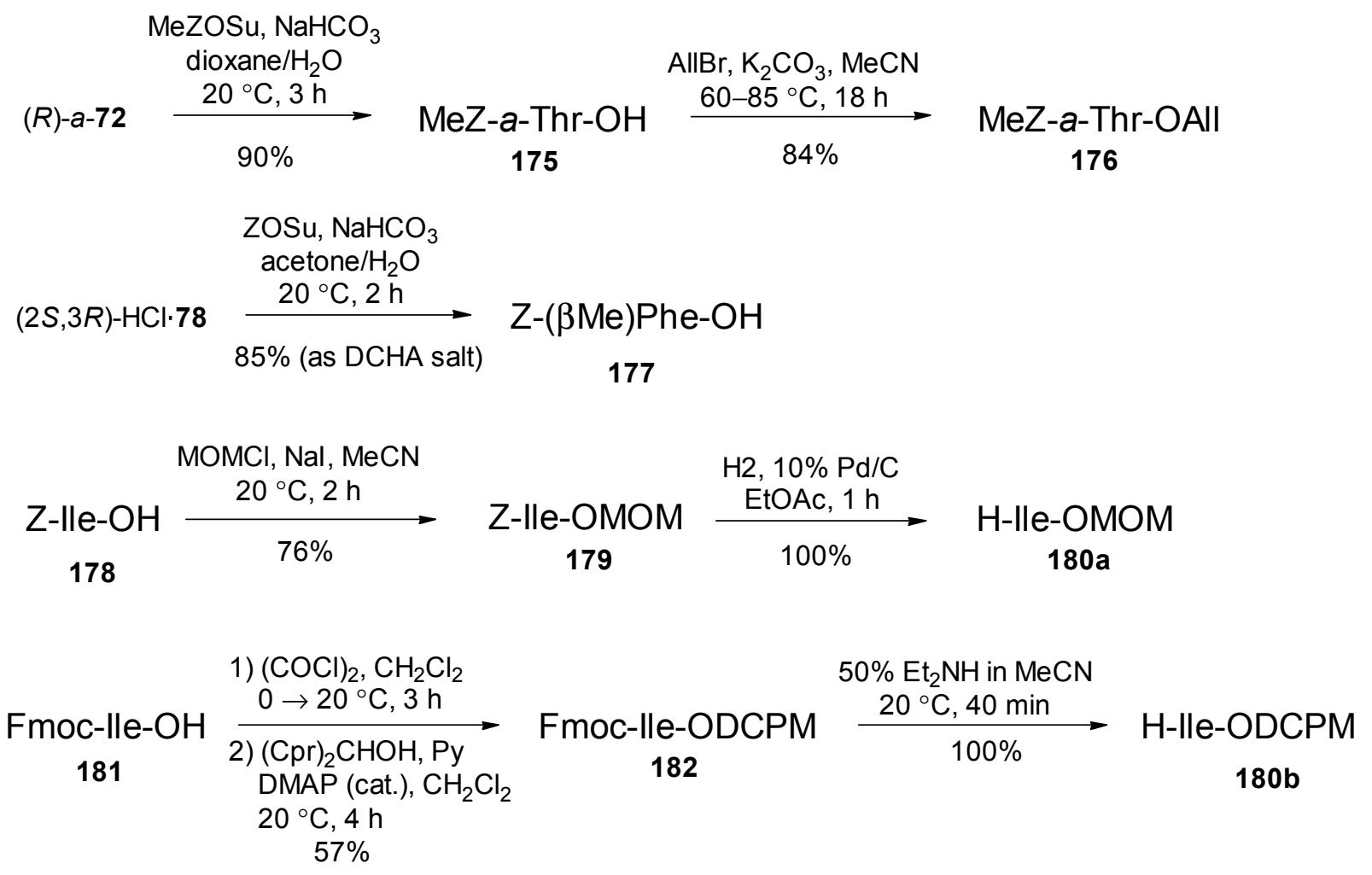

Scheme 43

$N$-Z Protected ( $\beta \mathrm{Me})$ Phe-OH 177 was obtained by acylation of the corresponding amino acid with $\mathrm{ZOSu}$ in $85 \%$ yield. The alkylation of $\mathrm{N}$-Z protected Ile-OH 178 with MOMI (generated in situ) gave MOM-ester 179 in 76\% yield, which was, immediately after removal of the Z-group (100\%), used in the coupling step. The DCPM ester $\mathbf{1 8 0 b}$ was obtained just before the next step by removal of the Fmoc group from the $N$-termini of $182(100 \%)$, which was in turn prepared via esterification reaction of the intermediate acyl chloride Fmoc-Ile-Cl with dicyclopropylmethanol in the presence of pyridine in $57 \%$ yield.

Having in mind to ring-close an acyclic precursor already containing the ester bond between the (4-PE)Pro and the $a$-Thr residues by forming the peptide bond between the Ile and (4-PE)Pro moieties, the methoxymethyl or dicyclopropylmethyl ester of Ile, 180a or 180b, were condensed with $N$-Z-protected ( $\beta \mathrm{Me}) \mathrm{Phe}-\mathrm{OH} 177$ (Scheme 44). After removal of the $\mathrm{Z}$ group from the $N$ - 
termini of the resulting dipeptides, $\mathbf{1 8 3 a}(66 \%)$ and $\mathbf{1 8 3 b}(84 \%)$, by catalytic hydrogenation to give $184 \mathbf{a}(54 \%)$ and $184 \mathbf{b}(100 \%)$, the latter were coupled with $N$-Fmoc-protected-(2R, $\left.1^{\prime} S, 2^{\prime} R\right)$ (3-Ncp)Ala-OH 160 to yield tripeptides 185a (80\%) and 185b (79\%). The relatively low yield of the amino ester 184a must be attributed to the noticeable susceptibility of the MOM ester to nucleophilic attack by the free amino group, especially if a formation of a diketopiperazine ring is possible.

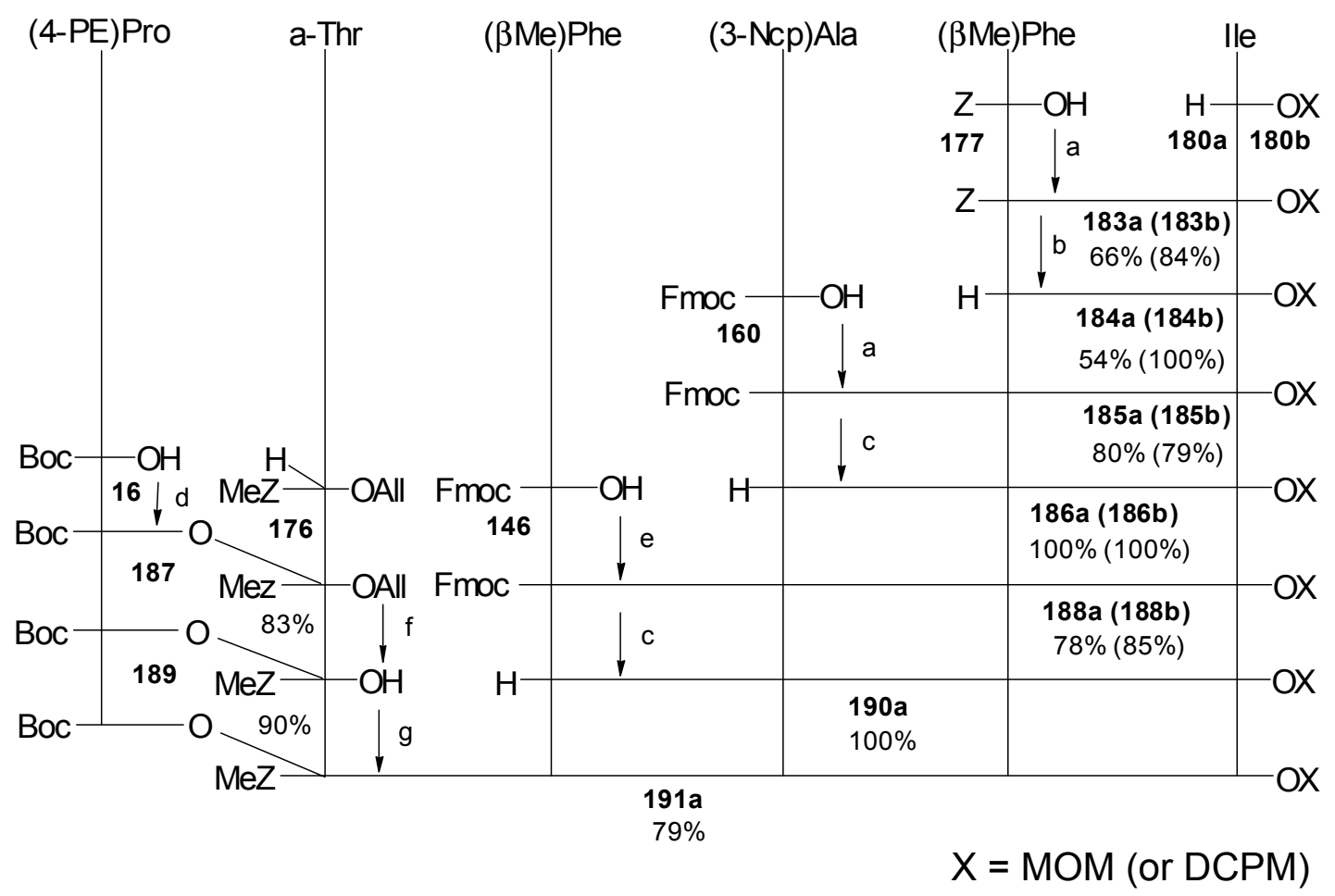

Scheme 44. Reagents and conditions: a) EDC, HOAt, DIEA, 2,4,6-collidine, $\mathrm{CH}_{2} \mathrm{Cl}_{2}, 0 \rightarrow 20{ }^{\circ} \mathrm{C}$, 14 h. b) $\mathrm{H}_{2}, \mathrm{Pd} / \mathrm{C}$, EtOAc, $20{ }^{\circ} \mathrm{C}, 40$ min. c) $50 \% \mathrm{Et}_{2} \mathrm{NH} / \mathrm{MeCN}, 20{ }^{\circ} \mathrm{C}, 1 \mathrm{~h}$. d) EDC, 4pyrrolidinopyridine, $\mathrm{CH}_{2} \mathrm{Cl}_{2}, 0 \rightarrow 20{ }^{\circ} \mathrm{C}, 24 \mathrm{~h}$. e) EDC, HOAt, 2,4,6-collidine, DMF, $0 \rightarrow 20{ }^{\circ} \mathrm{C}$, 14 h. f) $\mathrm{Pd}\left(\mathrm{PPh}_{3}\right)_{4}, \mathrm{~N}$-Methylaniline, DME, $20{ }^{\circ} \mathrm{C}, 1$ h. g) HATU, HOAt, DIEA, 2,4,6-collidine, $\mathrm{CH}_{2} \mathrm{Cl}_{2}, 0 \rightarrow 20^{\circ} \mathrm{C}, 24 \mathrm{~h}$.

Tripeptides 185a and 185b, after removal of the Fmoc-group (100\% yield), were coupled with $N$-Fmoc-protected $(\beta \mathrm{Me})$ Phe-OH 10 to give tetrapeptides $\mathbf{1 8 8 a}(78 \%)$ and $\mathbf{1 8 8 b}(84 \%)$. The latter was used for the successful preparation of hormaomycin analogs (see Chapter 6). 
The 4-pyrrolidinopyridine-catalyzed condensation of the $N$-Boc-protected (4-PE)Pro-OH 16 and $N, C$-protected $a$-Thr 176 gave the ester 187 (83\%), which, after palladium-promoted removal of the allyl group (90\%), was coupled with the tetrapeptide 190a using the HATU reagent in the presence of HOAt to give the hexadepsipeptide 191a in 79\% yield.

The MOM and Boc groups were removed from the termini of the latter (the ESI-MS spectrum showed that the MeZ group stayed intact), and the cyclizing peptide condensation succeeded under high dilution conditions, using the HATU reagent. The cyclodepsipeptide 171c was obtained after HPLC purification in 53\% yield over 2 steps (Scheme 45).

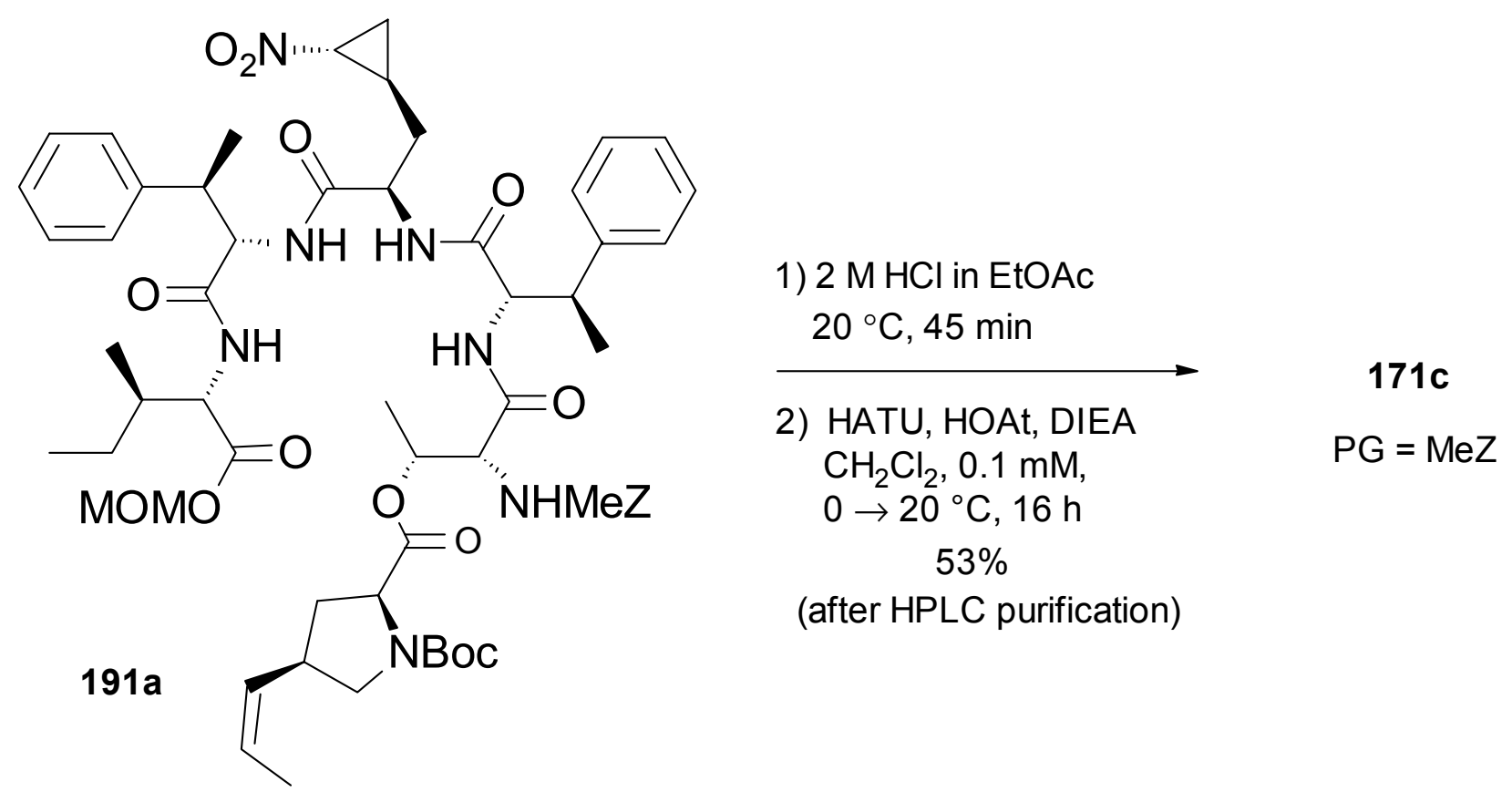

Scheme 45

5.6. Final steps of hormaomycin synthesis

In order to complete the assembling of hormaomycin, the side chain should be attached. N-Boc protected (3-Ncp)Ala-OH $\left(2 S, 1^{\prime} R, 2^{\prime} R\right)-143$ was esterified with 9-fluorenylmethanol (FmOH) using EDC in the presence of 4-pyrrolidinopyridine giving 192 (74\%) (Scheme 46). The latter, after removal of the Boc-group, was coupled with $O-\mathrm{MOM}$ protected $\mathrm{Chpca-OH} \mathbf{1 0 9}$ to give the acyl amino ester 193 in $80 \%$ yield. After removal of the MOM group, by treatment with 
$\mathrm{MgBr}_{2} \cdot \mathrm{Et}_{2} \mathrm{O}$, the resulted $194(78 \%)$ was further transformed into acid 195, which was too unstable (complete polymerization in THF or $\mathrm{CDCl}_{3}$ solution within $30 \mathrm{~min}$ ) to be used on the last coupling step. In order to overcome the difficulty, 193, after removal of the Fm group, was coupled with macrocycle 171c, the MeZ group of which was in turn removed with $10 \%$ anisole in TFA, using the (7-azabenzotriazol-1-yloxy)tris(pyrrolidino)phosphonium hexafluorophosphate reagent $(\mathrm{PyAOP})^{[107]}$ in the presence of HOAt.

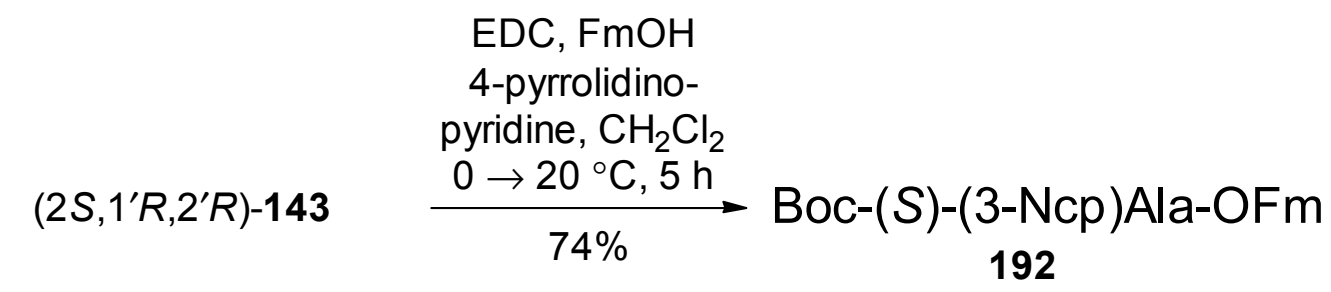

1) $4 \mathrm{M} \mathrm{HCl}$ in $\mathrm{EtOAC}$

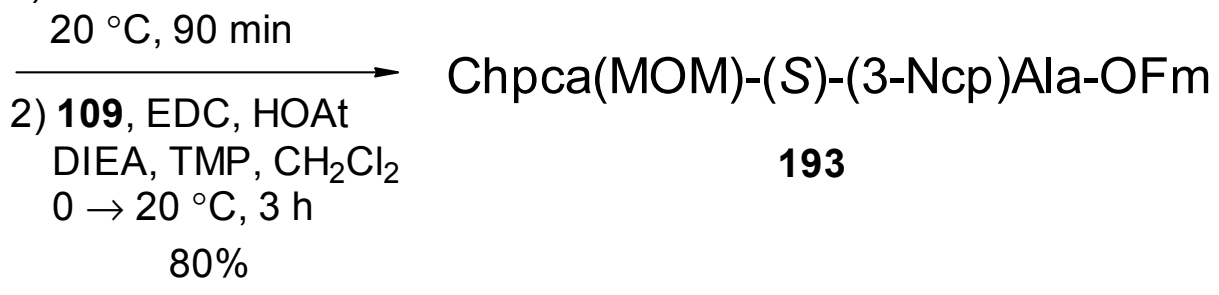

$\mathrm{MgBr}_{2} \cdot \mathrm{Et}_{2} \mathrm{O}, \mathrm{EtSH}$

$\mathrm{CH}_{2} \mathrm{Cl}_{2}, 20^{\circ} \mathrm{C}, 3 \mathrm{~h}$

$78 \%$

Chpca-(S)-(3-Ncp)Ala-OFm

194

$10 \%\left(\mathrm{NH}_{2} \mathrm{CH}_{2} \mathrm{CH}_{2}\right)_{3} \mathrm{~N}$

in $\mathrm{CH}_{2} \mathrm{Cl}_{2}, 20{ }^{\circ} \mathrm{C}, 20$ min

\section{Chpca-(S)-(3-Ncp)Ala-OH}

195

unstable

Scheme 46

This coupling step was unexpectedly accompanied with significant racemization at C-2 of (S)-(3-

Ncp)Ala moiety and separation of the obtained mixture of epimers by preparative TLC gave $O$ MOM protected hormaomycin $O$-MOM-3 (26\%) and diastereomeric epi-O-MOM-3 (16\%) (Scheme 47). 


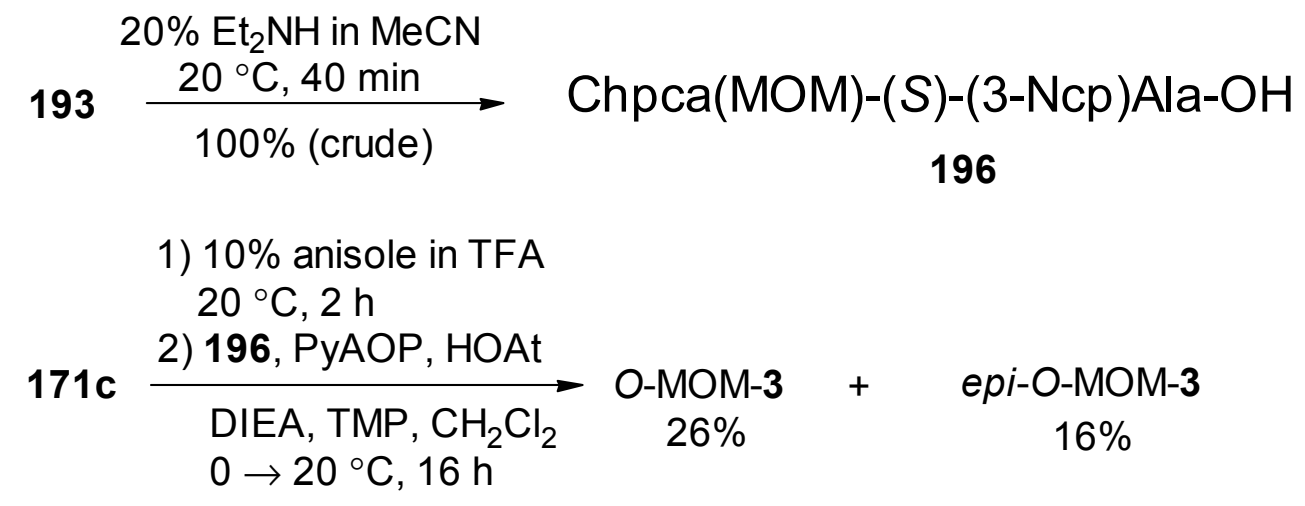

Scheme 47

To complete the assembly of $\mathbf{3}$ in a stepwise manner, the $N$-MeZ protected cyclic intermediate 171c was deprotected and first coupled with $N$-Teoc-protected $\left(2 S, 1^{\prime} R, 2^{\prime} R\right)-(3-\mathrm{Ncp})$ Ala-OH 197 (Scheme 48). After removal of the Teoc-group, the intermediate in turn was coupled with the $O$ MOM-protected 5-chloro-1-hydroxypyrrole-2-carboxylic acid $\mathbf{1 0 9}$.

Finally, the MOM group was removed by treatment with $\mathrm{MgBr}_{2} \cdot \mathrm{Et}_{2} \mathrm{O}$ and $\mathrm{EtSH}$ in dichloromethane to give a substance, which was expected to be identical with the native hormaomycin 3 (51\% on 5 steps).

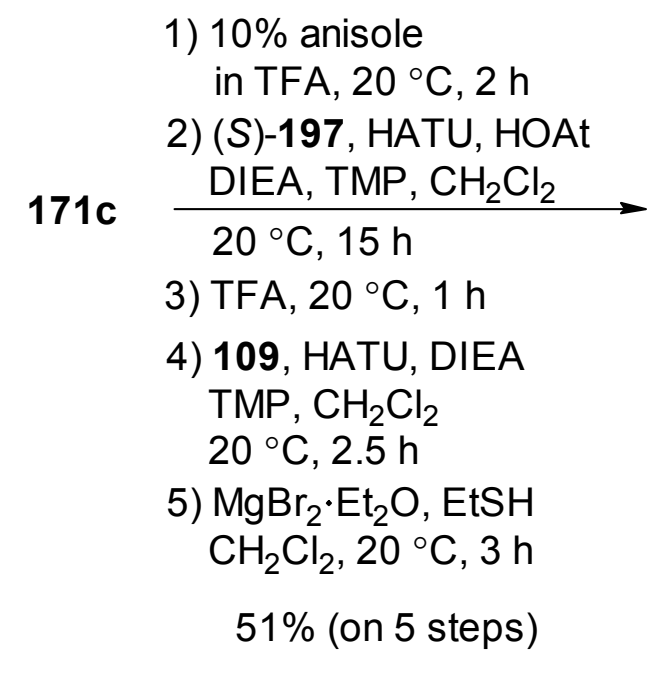

Teoc-(2S, 1'R,2'R)-(3-Ncp)Ala-OH

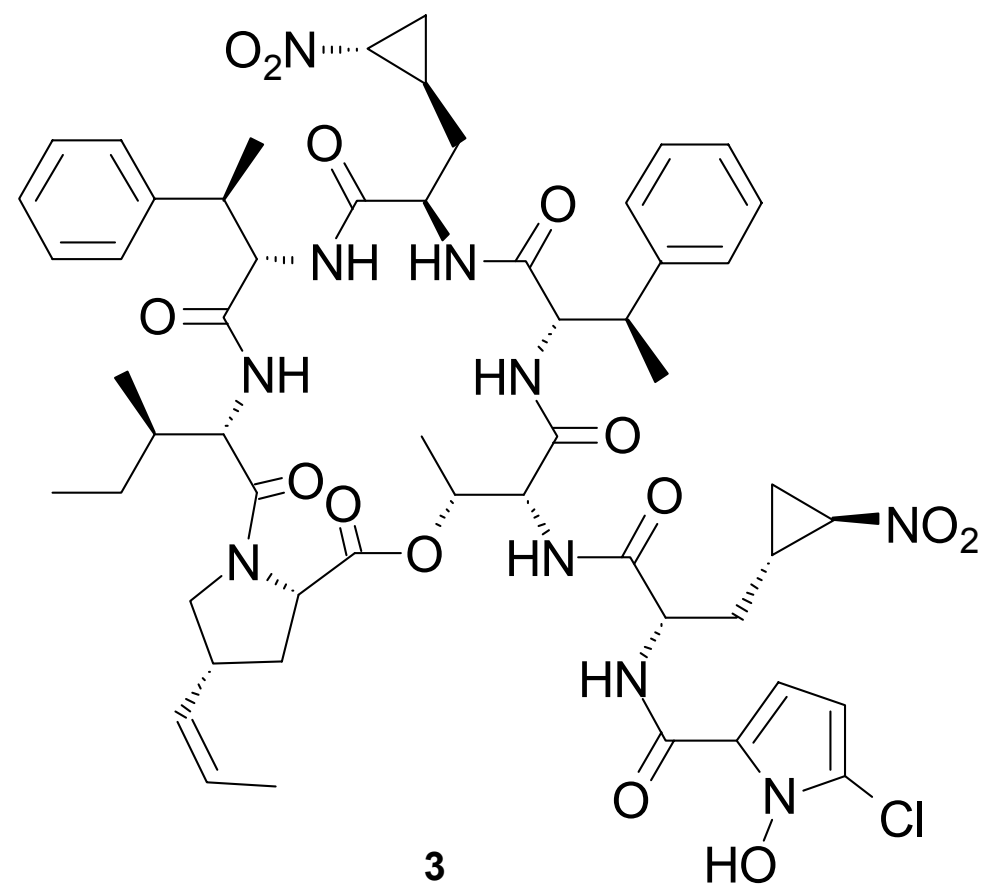

(S)-197

Scheme 48 
It indeed disclose the expected $\left[\mathrm{M}-\mathrm{H}^{+}+2 \mathrm{Na}^{+}\right],\left[\mathrm{M}+\mathrm{Na}^{+}\right]$and $\left[\mathrm{M}-\mathrm{H}^{+}\right]$peaks in its positiveand negative-mode ESI/MS spectra, had the same optical rotation, almost identical CD trace, at least in the wavelength region above $210 \mathrm{~nm}$ where no solvent interference is expected (these spectra were measured in methanol), identical UV spectrum, a very similar ${ }^{13} \mathrm{C}$ NMR spectrum and identical HPLC retention time as an authentic sample of the natural material. The synthetic product also showed the same capability to inhibit the growth of coryneform bacteria as native hormaomycin 3. However, there were some differences in their ${ }^{1} \mathrm{H}$ NMR spectra, measured in $\mathrm{CDCl}_{3}$ and $\mathrm{CD}_{3} \mathrm{OH}$. These discrepancies were initially interpreted to indicate that a diastereomer of the natural product had been synthesized. Yet, this possibility was excluded by the results of LC/MS experiments according to the advanced Marfey method, ${ }^{[38]}$ which rigorously proved that the MePhe, Ile, Ncpa and $a$-Thr residues were not epimerized in any of the peptide coupling and deprotection steps. Moreover prof. W. A. König (Hamburg) kindly repeated some GC separations $^{[9]}$ to determine the absolute configuration of the 3-phenylmethylalanine residue in hormaomycin 3, using pure (2S,3R)-3-methylphenylalanine, provided by us, as one of the reference compounds (previously ${ }^{[9]}$ a mixture of all stereoisomers of 3-methylphenylalanine had been used as a reference). These latest results were in accordance with those obtained before. Furthermore the comparative ${ }^{1} \mathrm{H}-{ }^{1} \mathrm{H}$ COSY experiments in $\mathrm{CD}_{3} \mathrm{OH}$, which were performed by Prof. C. Griesinger and Dr. U. Reinscheid (Göttingen), showed the same correlations for both samples between the four amide protons in 7.87-8.37 ppm region and four $\alpha$-protons in 3.80$4.50 \mathrm{ppm}$ (the two left correlations were undistinguished for each probe). Consequently, the small differences in the ${ }^{1} \mathrm{H}$ NMR spectra of natural and synthetic hormaomycin must be attributed to slightly different conformational dynamics due to traces of different impurities in the two samples. 


\section{Synthesis of hormaomycin analogs. Evaluation of biological activity}

\section{1. $\quad$ Attempted synthesis of $[(S)-(3-A c p)$ Ala $]$-hormaomycin}

Once the synthesis of hormaomycin $\mathbf{3}$ was successfully completed, the synthesis of its analogs was initiated. As a first target for this synthetic investigation analog, contained in the side chain $\left(2 S, 1^{\prime} R, 2^{\prime} R\right)-3-\left(2^{\prime}\right.$-aminocyclopropyl)alanine [(S)-(3-Acp)Ala] instead of $(S)$-(3-Ncp)Ala moiety was chosen. Noteworthy, that the corresponding feeding experiment with H-(3-Acp)Ala-OH failed and no products contained this amino acid were isolated. ${ }^{[12]}$

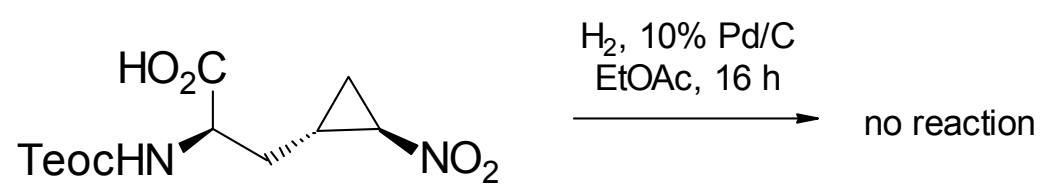

$(S)-197$

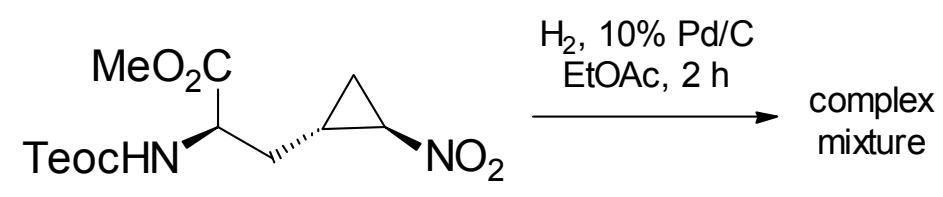

$(S)-198$

Schema 49.

As initial attempts of the reduction of the already $N$-Teoc protected (3-Ncp)Ala-OH $(S)-197$ and its methyl ester $(S)$-198 failed (Scheme 49), the free amino acid $\left(2 S, 1^{\prime} R, 2^{\prime} R\right)-\mathbf{8 3}$ was hydrogenated on $10 \% \mathrm{Pd} / \mathrm{C}$ in methanol according to procedure of Larionov and de Meijere ${ }^{[129]}$ to give the desired diamino acid, which without any purification was further transformed via acylation of the corresponding copper complex with ZOSu in $N_{\omega}$-Z protected H-(3-Acp)Ala(Z)OH 199 in 52\% yield over two steps (Scheme 50). 


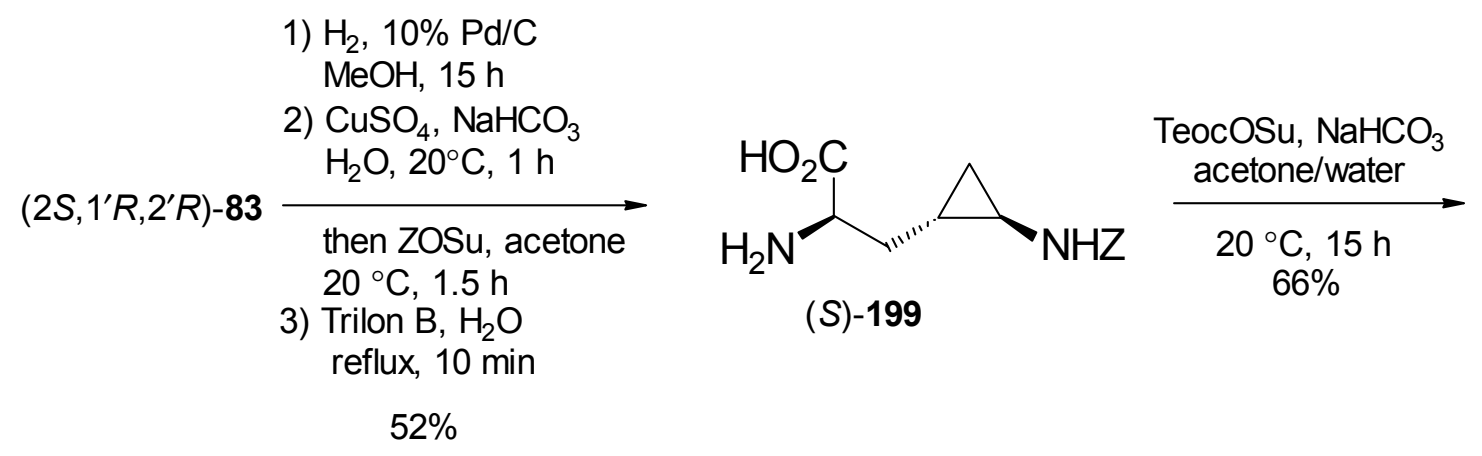

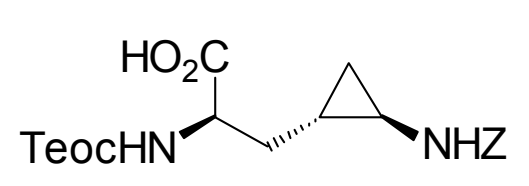

(S)-200
1) $10 \% \mathrm{Pd} / \mathrm{C}, \mathrm{MeOH}$

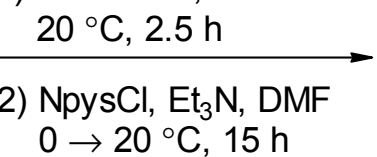

$44 \%$

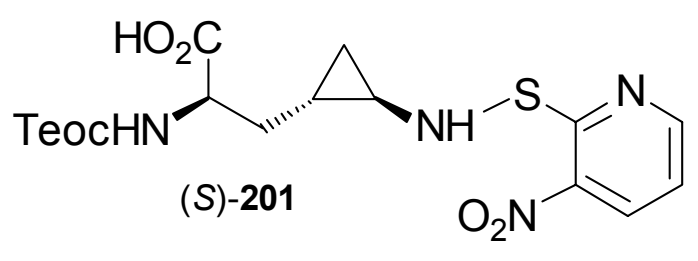

Scheme 50

The latter was further $N_{\alpha}$-protected with Teoc-OSu to give the protected diamino acid 200 (66\%), which, after hydrogenolytic removal of the Z-group, was in turn allowed to react with 3nitro-2-pyridinesulfenyl chloride $(\mathrm{NpysCl})$ to give the orthogonally protected derivative 201 in $44 \%$ yield.

The Npys group, which was proposed for the amino protection by Matsueda et al., ${ }^{[130]}$ is stable to trifluoroacetic acid, but easily removed with very dilute $(0.1-0.2 \mathrm{M}) \mathrm{HCl}$ in organic solvents. It is reported that it can be also cleaved under nearly neutral conditions with triphenylphosphine and a proton source as well as in essentially under neutral conditions with thiols such as 2pyridinethiol $\mathrm{N}$-oxide or 2-mercaptobenzothiazole. To evaluate the deprotection conditions for this group the $N_{\alpha}-\mathrm{Z}, N_{\omega}$-Npys protected ornithine amide 204 was prepared and several deprotection conditions were examined (Scheme 51).

On the contrary to the related Nps group, Npys group was only partially cleaved with $\mathrm{MgBr}_{2} \cdot \mathrm{Et}_{2} \mathrm{O}$ and $\mathrm{EtSH}$ in $\mathrm{CH}_{2} \mathrm{Cl}_{2}$ for $4.5 \mathrm{~h}$. It was rather insensitive to 2- pyridinethiol $N$-oxide and gave, by treatment with this reagent for $5 \mathrm{~h}$, only traces of the deprotected material. Gratifyingly it was completely removed with 3 eq. $0.1 \mathrm{M}$ triphenylphosphine and 3 eq. of $0.1 \mathrm{M}$ 
pyridinium chloride in $\mathrm{CH}_{2} \mathrm{Cl}_{2}$ for 2 min, although the yield of the deprotected amino amide was moderate.

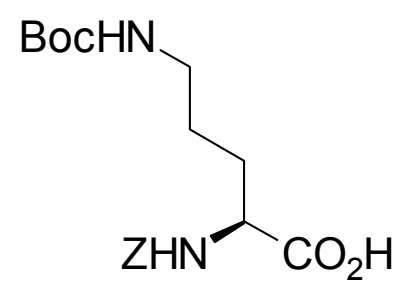

202

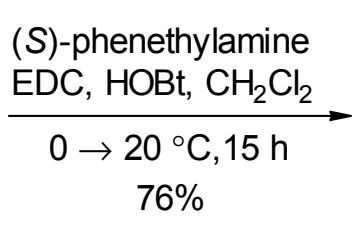

1) TFA, $20^{\circ} \mathrm{C}, 20 \mathrm{~min}$

2) $\mathrm{NpysCl}, \mathrm{Et}_{3} \mathrm{~N}, \mathrm{THF}$

$0 \rightarrow 20^{\circ} \mathrm{C}, 15 \mathrm{~h}$

$59 \%$

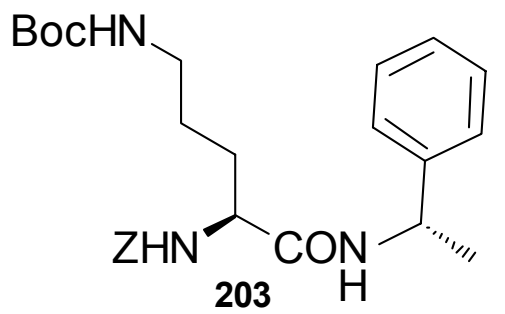

203

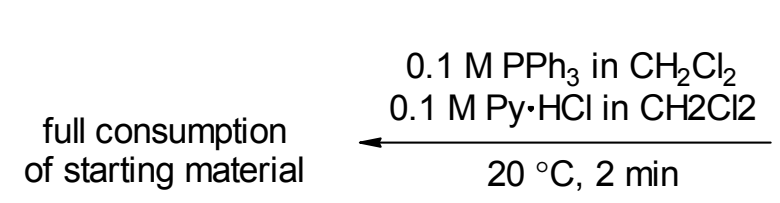

Schema 51.

The cyclic intermediate 171c, after removal of the MeZ group, was acylated with the orthogonally protected diamino acid 201 (Scheme 52). After removal of the Teoc group, the resultant intermediate product was in turn coupled with the $O$-MOM protected Chpca 109 to give the $N$-protected branched acyl depsipeptide [(3-Acp)Ala(Npys) $\left.{ }^{7}\right]$-MOM-3 in $64 \%$ yield over four steps. The latter was treated first with $\mathrm{MgBr}_{2} \cdot \mathrm{Et}_{2} \mathrm{O}$ and $\mathrm{EtSH}$ in $\mathrm{CH}_{2} \mathrm{Cl}_{2}$, and then with $\mathrm{PPh}_{3}$ and $\mathrm{Py} \cdot \mathrm{HCl}$. Although the resulted mixture contained two Erlich reagent active compounds according to TLC, attempt of its separation by exclusion chromatography did not give any isolable compounds, containing pyrrole fragment. 


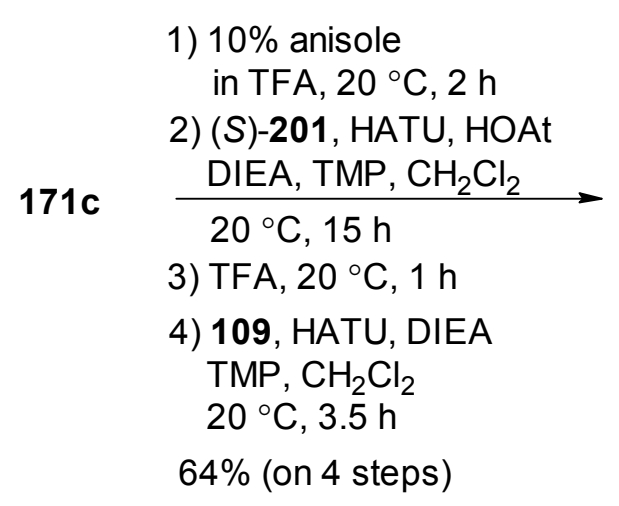

1) $10 \%$ anisole in TFA, $20^{\circ} \mathrm{C}, 2 \mathrm{~h}$

(S)-201, HATU, HOA

3) TFA, $20^{\circ} \mathrm{C}, 1 \mathrm{~h}$

109, HATU, DIEA

TMP, $\mathrm{CH}_{2} \mathrm{Cl}_{2}$

$64 \%$ (on 4 steps)

Scheme 52

6.2. Synthesis of $\left[(\mathrm{S})-\left(\mathrm{NO}_{2}\right) \mathrm{Nva}^{7}\right]$-hormaomycin. Isolation of $\left[(\mathrm{S})-\left(\mathrm{NO}_{2}\right) \mathrm{Nva} a^{3}\right]$-horamomycin and $\left[(\mathrm{S})-\left(\mathrm{NO}_{2}\right) \mathrm{Nva}^{3},(\mathrm{~S})-\left(\mathrm{NO}_{2}\right) \mathrm{Nva}^{7}\right]$-hormaomycin

From previous experiments it was already known that the presence of the $N$-hydroxy group at Chpca moiety is crucially important for both kinds of biological activity of hormaomycin. The fact, that analogs with transformed or substituted (3-Ncp)Ala moieties, obtained before, do not possess the hormaomycin-like biological activity, point out the significance of this structural elements of 3. Nevertheless the importance of each (3-Ncp)Ala residue was absolute unclear and the question, could any analogs without one or two nitrocyclopropyl fragments retain some biological activity yet, stayed still open. To clarify situation the synthesis of hormaomycin analog with 5-nitronorvaline [( $\left.\left.\mathrm{NO}_{2}\right) \mathrm{Nva}\right]$ moiety (methylen group instead of cyclopropyl ring) in place of (3-Ncp)Ala residue in the side chain was initiated. For the preparation of $[(R)$ $\left.\left(\mathrm{NO}_{2}\right) \mathrm{Nva}^{3},(S)-\left(\mathrm{NO}_{2}\right) \mathrm{Nva}^{7}\right]$ - and $\left[(R)-\left(\mathrm{NO}_{2}\right) \mathrm{Nva}^{3}\right]$-analogs feeding experiment with $\mathrm{H}-\mathrm{Nva}-\mathrm{OH}$ was used. 
The $N_{\alpha}$-Boc, $N_{\omega}$-Z protected ornithine 205 was esterified to give the corresponding tert-butyl ester $\mathbf{2 0 6}^{[131]}$ in 94\% yield, which, after removal of the Z-group, was transformed in 207 (58\%) via oxidation of the amino group with MCPBA in refluxing 1,2-dichloroethane according to protocol of Gilbert and Borden. ${ }^{[132]}$

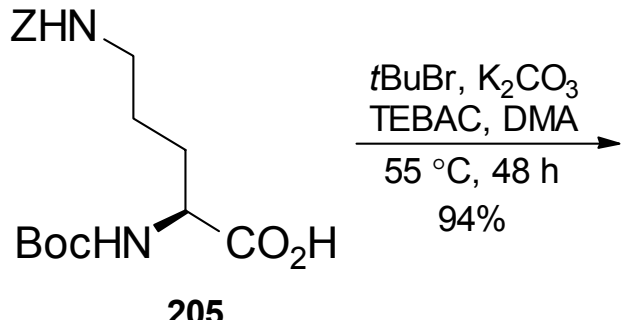

205
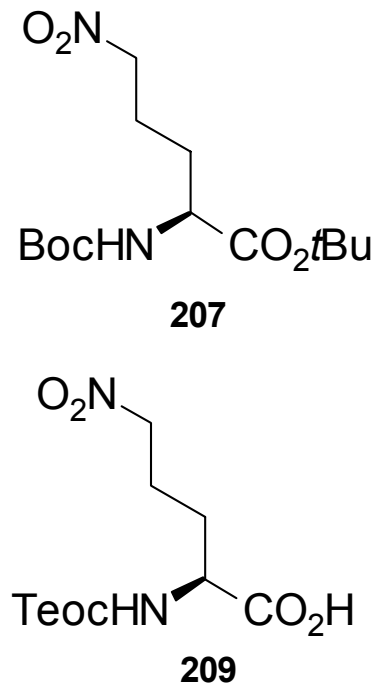

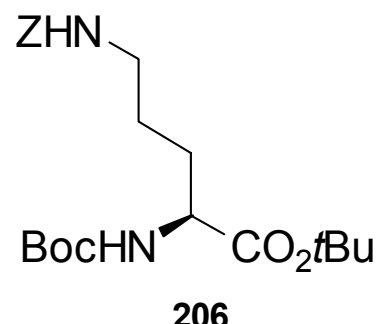

1) $\mathrm{H}_{2}, 10 \% \mathrm{Pd} / \mathrm{C}$ EtOAc, $3 \mathrm{~h}$

2) MCPBA, $\mathrm{ClCH}_{2} \mathrm{CH}_{2} \mathrm{Cl}$ reflux, $20 \mathrm{~min}$

$58 \%$

206 $\underset{56 \%}{\text { 1) } \mathrm{TFA}, 20^{\circ} \mathrm{C}, 1 \mathrm{~h}}$
$\underset{5 \mathrm{M} \mathrm{HCl} \text { in EtOAC }}{\longrightarrow}$

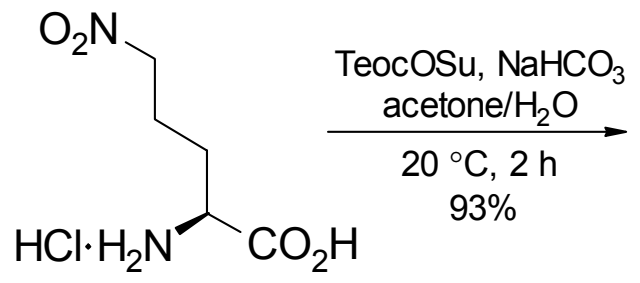

$\mathrm{HCl} \cdot 208$

Scheme 53

The simultaneous removal of the both protecting group gave amino acid as a hydrochloride $\mathbf{2 0 8}$ $(56 \%)$, which was further treated with TeocOSu to give the $N$-protected $\left(\mathrm{NO}_{2}\right) \mathrm{Nva}-\mathrm{OH} 209$ in 93\% yield (schema 53). This derivative was also prepared from $N_{\alpha}-\mathrm{Z}, N_{\omega}$-Boc protected ornithine 202 but in noticeably lower yield.

To evaluate applicability of 5-nitronorvaline for peptide synthesis the acylated tripeptide $\mathbf{2 1 1}$ was prepared (Scheme 54). Dipeptide 117, after removal of the Boc, was coupled with 209 to give tripeptide $\mathbf{2 1 0}$ in $59 \%$ yield. Sequential deprotection of its $N$-termini was followed by acylation with Chpca(MOM)-OH 109 and gave 211 (79\%). 


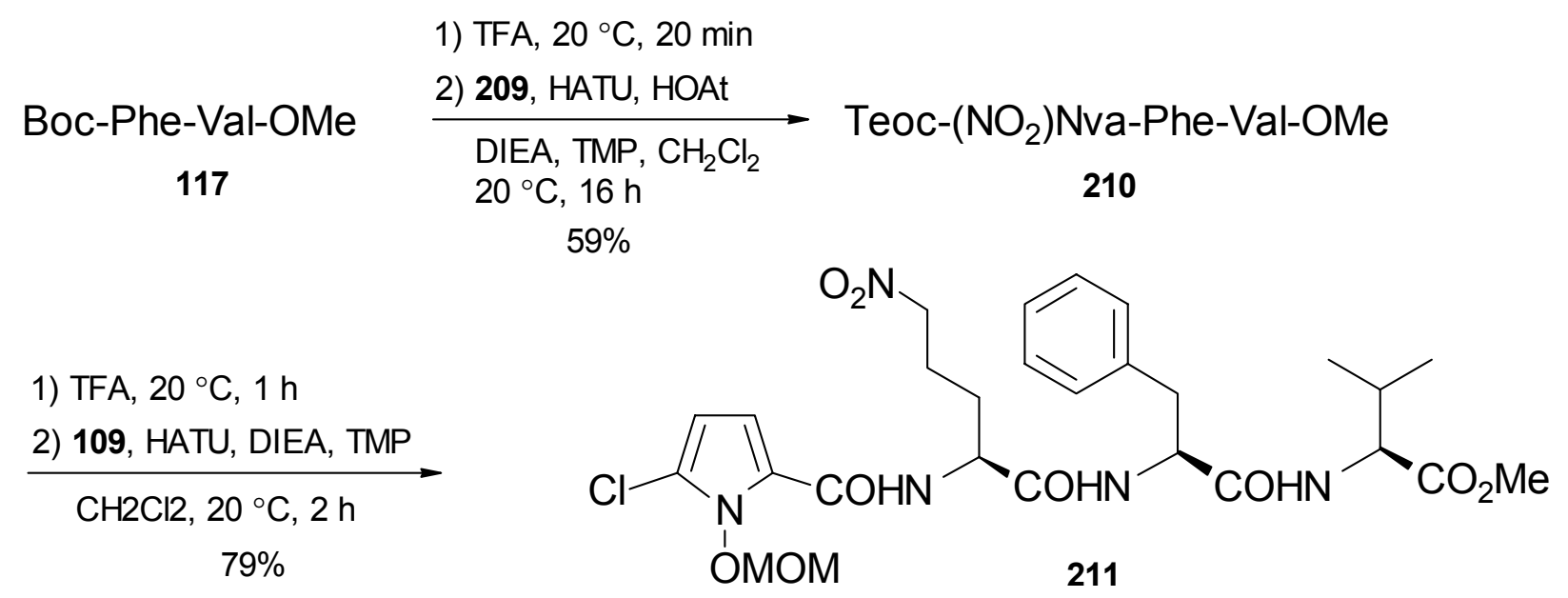

Scheme 54

After that, the assembling of analog $\left[(S)-\left(\mathrm{NO}_{2}\right) \mathrm{Nva}^{7}\right]-3$ could be initiated. The cyclic intermediate 171c, after removal of the MeZ group, was coupled with the $N$-Teoc protected amino acid 209. After removal of the Teoc group the resulted intermediate was acylated with Chpca(MOM)-OH 109. Finally the desired analog was obtained after removal of the MOM group in overall $60 \%$ yield over 5 steps (schema 55 ).

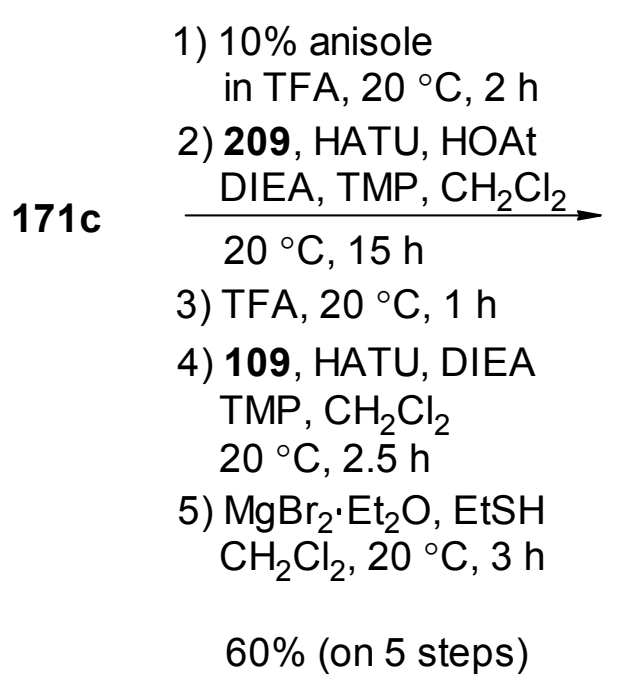

Scheme 55

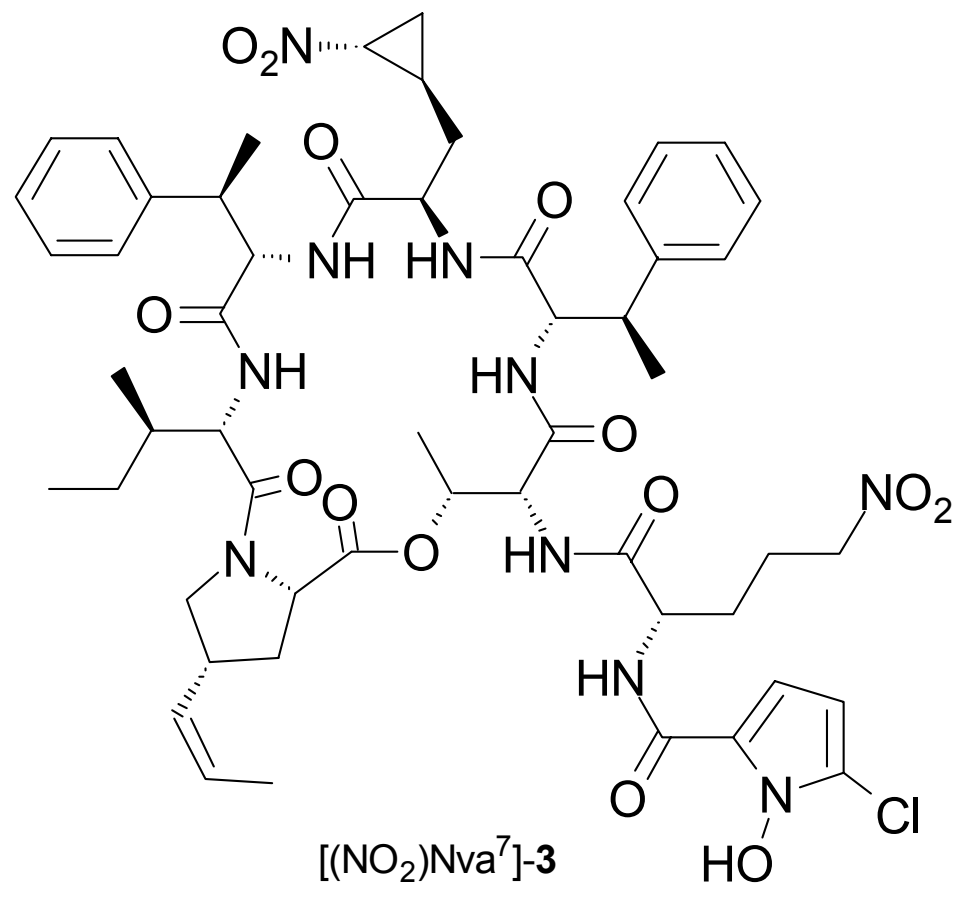

$\left[\left(\mathrm{NO}_{2}\right) \mathrm{Nva}^{7}\right]-3$ 
which, according to ESI-MS spectra, contained besides $3\left(\mathrm{~m} / \mathrm{z}=1151\left[\mathrm{M}+\mathrm{Na}^{+}\right] ; \mathrm{m} / \mathrm{z}=1127[\mathrm{M}\right.$ $\left.\left.-\mathrm{H}^{+}\right]\right)$analogs with one (3-Ncp)Ala residue substituted on $\left(\mathrm{NO}_{2}\right) \mathrm{Nva}$ moiety $(\mathrm{m} / \mathrm{z}=1139[\mathrm{M}+$ $\left.\left.\mathrm{Na}^{+}\right] ; \mathrm{m} / \mathrm{z}=1115\left[\mathrm{M}-\mathrm{H}^{+}\right]\right)$and analog with two $\left(\mathrm{NO}_{2}\right)$ Nva residues instead of two (3-Ncp)Ala moieties $\left(\mathrm{m} / \mathrm{z}=1127\left[\mathrm{M}+\mathrm{Na}^{+}\right] ; \mathrm{m} / \mathrm{z}=1103\left[\mathrm{M}-\mathrm{H}^{+}\right]\right)$. HPLC analysis of this mixture showed four main peaks with hormaomycin-like UV spectra in ratio ca. 2:1:1:0.5. The preparative HPLC separation of mixture allowed to isolate chromatographically pure $\left[(S)-\left(\mathrm{NO}_{2}\right) \mathrm{Nva}^{3}\right]-3$ and $[(S)$ $\left.\left(\mathrm{NO}_{2}\right) \mathrm{Nva}^{3},(S)-\left(\mathrm{NO}_{2}\right) \mathrm{Nva}^{7}\right]-3$. The careful evaluation of spectral and biological properties of these substances is continued.

\subsection{Synthesis of [a-Dab $\left.{ }^{1}\right]$-hormaomycin and $\left[a-D a b^{1}, a-I l e^{5}\right]$-hormaomycin}

Hormaomycin analog with the amide bond instead of the ester bond in the ring part became next task of this investigation. This insignificant change could show how strong affects the conformation of the ring part of hormaomycin on its biological properties as the presence of the odd amide bond could have the significant influence on the intramolecular hydrogen bonds. Furthermore amide bonds are usually much more stable to enzymatic cleavage and it could cause a prolonged action of the aza-analog comparing with native hormaomycin $\mathbf{3}$.<smiles>CC=CC(=O)O[Mg]Br</smiles>

212

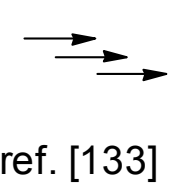

ref. [133]

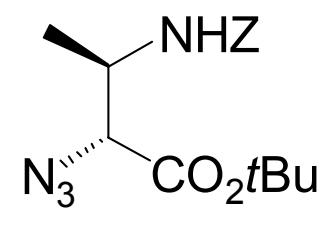

213

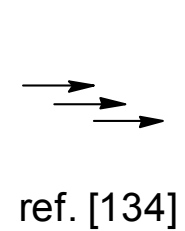

ref. [134]

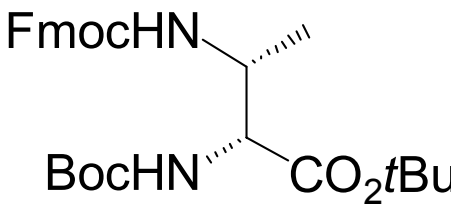

214

1) TFA, $20^{\circ} \mathrm{C}, 1 \mathrm{~h}$

2) $\mathrm{SOCl}_{2}, \mathrm{MeOH}$ $-20 \rightarrow 50^{\circ} \mathrm{C}, 21 \mathrm{~h}$

3) $\mathrm{MeZOSu}, \mathrm{NaHCO}_{3}$ acetone $/ \mathrm{H}_{2} \mathrm{O}$ $20^{\circ} \mathrm{C}, 1.5 \mathrm{~h}$<smiles>CCCN[C@@H](C(=O)OC)[C@H](C)NC(C)C</smiles>
$69 \%$ 
To prepare this analog the orthogonally protected $(2 R, 3 R)$-diaminobutyric acid ( $a$-Dab) should be first synthesized (Scheme 56). The tert-butyl ester 213, which was prepared according to published procedure ${ }^{[133]}$ with Sharpless asymmetric aminohydroxylation as a key step followed by stereospecific azidation, was transformed in fully protected $a$-Dab derivative $\mathbf{2 1 4}$ as it was described by Orgel et al.. ${ }^{[134]}$ The latter, after simultaneous removal of the $N$-Boc and $O$-tertbutyl groups with trifluoroacetic acid, was esterified with methanol and the resulted $N_{\beta}$-protected diamino methyl ester was acetylated with MeZOSu to give 215 in 69\% yield over three steps.

After removal of the Fmoc group diamino ester 215 was coupled with the $N$-Boc protected (4PE)Pro-OH 16 to give methyl ester 216 in 83\% yield, which was in turn saponified using tetrabutylammonium hydroxide (Scheme 57).

\author{
1) $50 \% \mathrm{Et}_{2} \mathrm{NH}$ in $\mathrm{MeCN}$ \\ $20{ }^{\circ} \mathrm{C}, 40 \mathrm{~min}$ \\ $215 \underset{\mathrm{CH}_{2} \mathrm{Cl}_{2}, 0 \rightarrow 20^{\circ} \mathrm{C}, 6 \mathrm{~h}}{\stackrel{2)}{\longrightarrow}}$ MeZ-a-Dab[(4-PE)Pro]-OMe \\ $83 \%$ \\ 216
}

$40 \%$ aq. $\mathrm{Bu}_{4} \mathrm{~N}^{+} \mathrm{OH}^{-}$

$\mathrm{THF}, 0^{\circ} \mathrm{C}, 45 \mathrm{~min}$

$85 \%$

\title{
MeZ-a-Dab[(4-PE)Pro]-OH
}

217

Scheme 57

Intermediate $217(85 \%)$ was coupled with tetrapeptide $\mathbf{1 8 8 b}$, after removal of the Fmoc group from the $N$-termini of the latter, to yield the branched hexapeptide 218 (80\%) (Scheme 58).

The acidolytic removal of the Boc and DCPM groups from 218 occurred quantitatively and was followed by macrocyclization, using HATU in the presence of HOAt under high dilution conditions, which gave the epimeric macrocycles, 219a and 219b, in $28 \%$ and $19 \%$ yield respectively after HPLC separation (Scheme 59). 


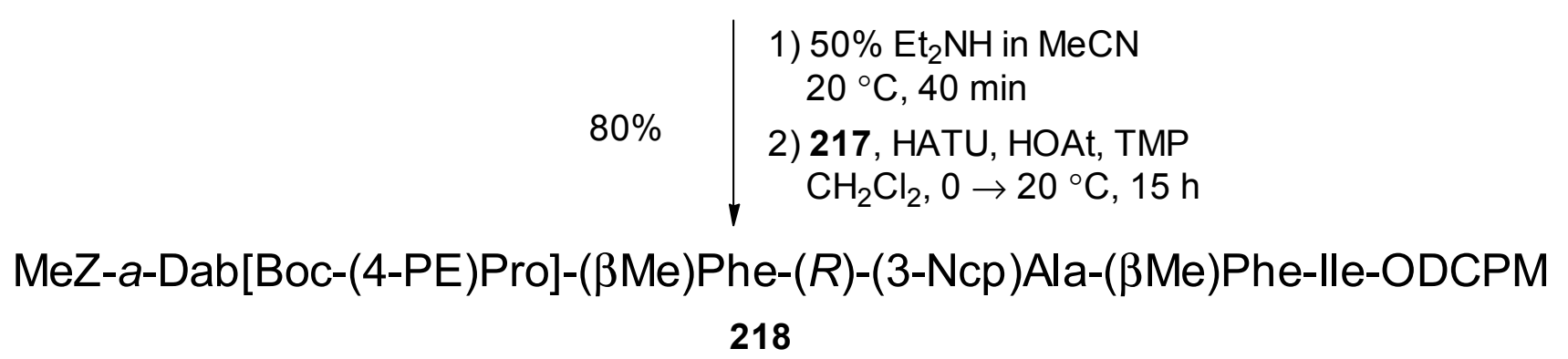

Scheme 58

Investigation of the amino acid content of these products according to the advanced Marfey method $^{[38]}$ showed that $\mathbf{2 1 9 b}$ contained instead of Ile residue $(R)$ - $a$-Ile [ $a$-Ile] moiety.

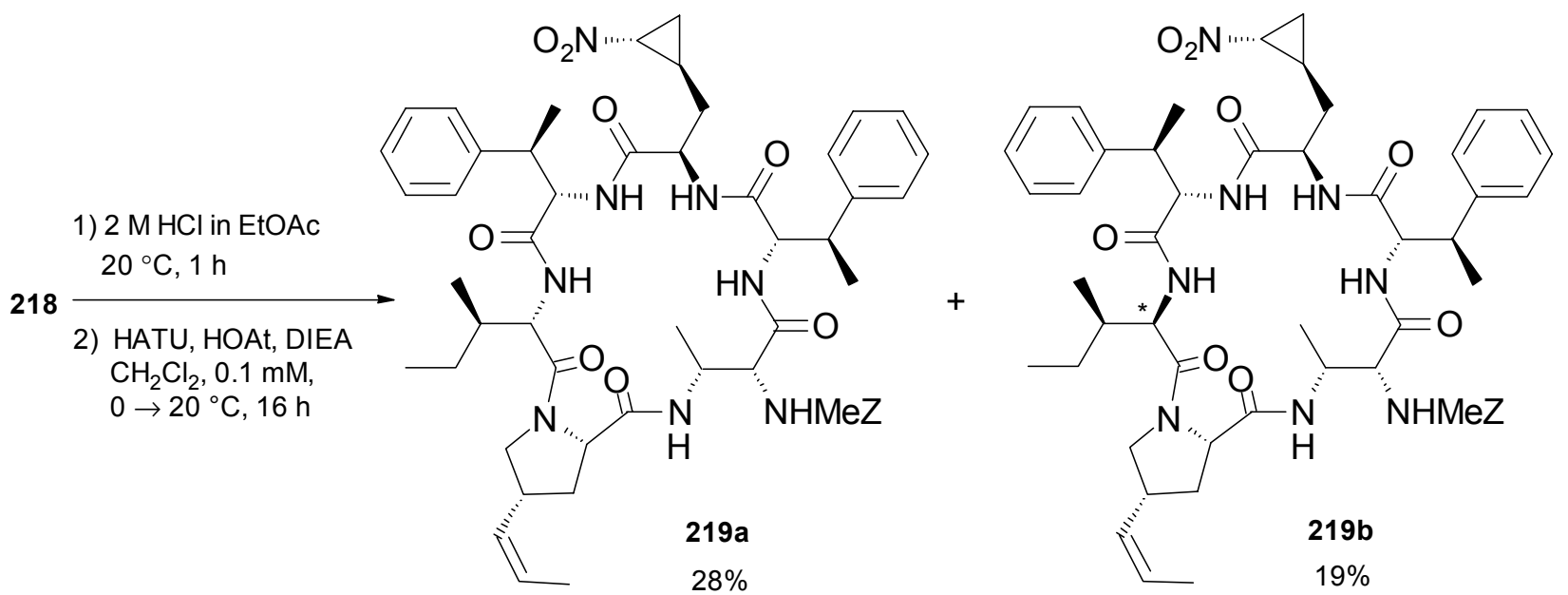

Scheme 59

The cyclic branched peptide 219a, after removal of the MeZ group, was acylated with the $N$ Teoc protected $\left(2 S, 1^{\prime} R, 2^{\prime} R\right)-(3-\mathrm{Ncp})$ Ala-OH 197 (Scheme 60). The resultant intermediate, after removal of the Teoc group, was coupled with acid 109. $\mathrm{MgBr}_{2} \cdot \mathrm{Et}_{2} \mathrm{O} / \mathrm{EtSH}$-mediated removal of the MOM group completed this synthesis and gave the desired [a-Dab $\left.{ }^{1}\right]-3$ in $68 \%$ yield on five steps. The likeness of the ${ }^{1} \mathrm{H}$-spectra of this substance and those of native hormaomycin 3 indicated that the expected remarkable conformational changes did not take place. 


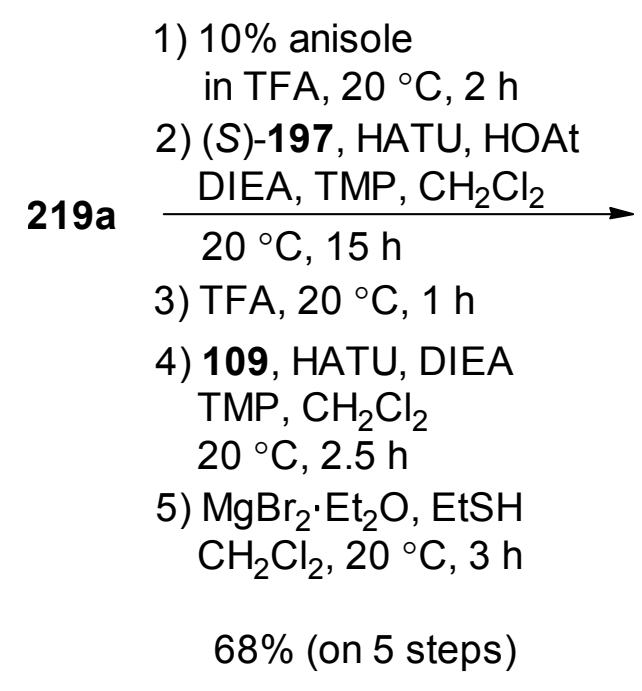

1) $10 \%$ anisole

in TFA, $20^{\circ} \mathrm{C}, 2 \mathrm{~h}$

TFA, $20^{\circ} \mathrm{C}, 1 \mathrm{~h}$

4) 109, HATU, DIEA

TMP, $\mathrm{CH}_{2} \mathrm{Cl}_{2}$

$20^{\circ} \mathrm{C}, 2.5 \mathrm{~h}$

$\mathrm{MgBr}_{2} \cdot \mathrm{Et}_{2} \mathrm{O}$, EtSH

$68 \%$ (on 5 steps)

Scheme 60

Similarly [a-Dab $\left.{ }^{1}, a-\mathrm{Ile}^{6}\right]-\mathbf{3}$ was prepared from intermediate $\mathbf{2 1 9 b}$ in $39 \%$ yield over five steps after HPLC purification. Its ${ }^{1} \mathrm{H}$-spectrum differed sharply from ${ }^{1} \mathrm{H}$-spectrum of $\mathbf{3}$ and showed (at least in $\mathrm{CDCl}_{3}$ ) on the presence of several conformers.

\subsection{Syntheses to $\left[a-N_{\alpha M} M e D a b^{1}\right]$-hormaomycin}

For a further synthetic investigation of $\mathbf{3}$ analog, contained $(2 R, 3 R)-N_{\beta}$-methyl diaminobutyric acid $\left[a-\mathrm{N}_{\beta} \mathrm{MeDab}\right]$ moiety instead of $a-\mathrm{Thr}$ residue, was chosen.

The fully protected amino acid $\mathbf{2 2 2}$ was synthesized starting from the known $\mathbf{2 2 0}^{[135]}$ (Scheme 61). After removal of the TBDMS group followed by removal of the tert-butyl ester, the $\mathrm{N}-\mathrm{Z}$ protected isothreonine $\mathbf{2 2 0}$ was esterified with diazomethane to give intermediate, which was further converted into the corresponding mesylate. 


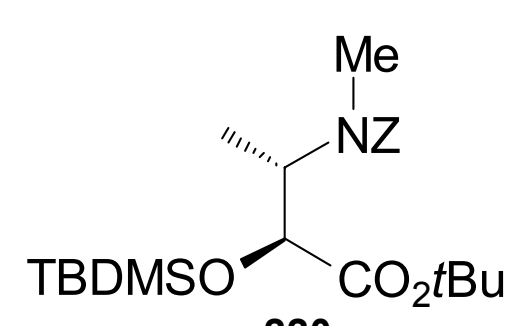

220

ref. [133]

1) $5 \%$ aq. $\mathrm{HF}, \mathrm{MeCN}$

$0 \rightarrow 20^{\circ} \mathrm{C}, 4 \mathrm{~h}$

2) TFA, $20^{\circ} \mathrm{C}, 1 \mathrm{~h}$

3) $\mathrm{CH}_{2} \mathrm{~N}_{2}, \mathrm{Et}_{2} \mathrm{O} / \mathrm{MeOH}$

4) $\mathrm{MsCl}, \mathrm{Et}_{3} \mathrm{~N}, \mathrm{CH}_{2} \mathrm{Cl}_{2}$ $-30 \rightarrow 20^{\circ} \mathrm{C}, 5 \mathrm{~h}$

5) $\mathrm{NaN}_{3}, \mathrm{DMF}, 70^{\circ} \mathrm{C}, 15 \mathrm{~h}$

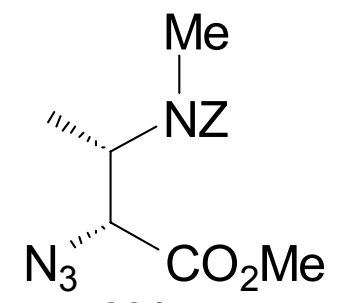

$35 \%$

1) $\mathrm{Ph}_{3} \mathrm{P}, \mathrm{THF} / \mathrm{H}_{2} \mathrm{O}, 20^{\circ} \mathrm{C}, 24 \mathrm{~h}$

then $\mathrm{Boc}_{2} \mathrm{O}, 20^{\circ} \mathrm{C}, 24 \mathrm{~h}$

2) $\mathrm{H}_{2}, 10 \% \mathrm{Pd} / \mathrm{C}$, EtOAc, $3 \mathrm{~h}$

3) FmocOPfp, HOAt (cat.)

TMP, EtOAc, $20^{\circ} \mathrm{C}, 15 \mathrm{~h}$

4) $2 \mathrm{M} \mathrm{HCl}$ in EtOAc, $20^{\circ} \mathrm{C}, 1 \mathrm{~h}$

5) MeZOSu, DIEA, TMP

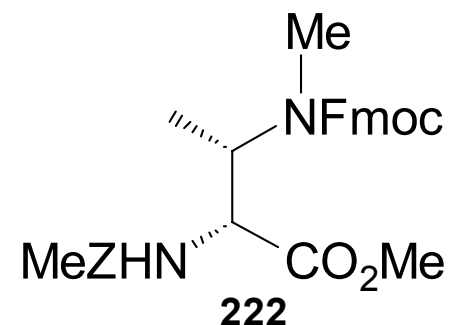

$\mathrm{MeCN}, 20^{\circ} \mathrm{C}, 16 \mathrm{~h}$

222

$37 \%$

Scheme 61

This transformation was followed by displacement of the mesylate with $\mathrm{NaN}_{3}$ in $\mathrm{DMF}$ to give azide 221, which was further transformed in the $N_{\alpha}$-Boc, $N_{\beta}-\mathrm{Z}$ protected $a-N_{\beta} \mathrm{MeDab}$ methyl ester, by treatment first with triphenylphosphine and water, and then with $\mathrm{Boc}_{2} \mathrm{O}$. Further removal of the $\mathrm{Z}$ group by hydrogenolysis was followed by introduction of the Fmoc group to give the intermediate $\mathrm{N}_{\alpha}$-Boc, $\mathrm{N}_{\beta}$-Fmoc protected $a$ - $\mathrm{N}_{\beta}$ MeDab methyl ester, which, after removal of the Boc group, was finally acylated with MeZOSu to give 222 in $13 \%$ yield over ten steps.

This diamino acid, after removal of the Fmoc group, was coupled with the $N$-Boc protected (4PE)Pro-OH 16 giving dipeptide 223 (79\%) (Scheme 62). Noteworthy, that NMR-spectra of 223 showed on the presence of at least four conformers (instead of usual two) and the complicated temperature dependence (on heating till $100^{\circ} \mathrm{C}$ the coalescence point was almost reached but spectra, measured at $125^{\circ} \mathrm{C}$, showed again on the presence of several conformers; as at this 
temperature substance already noticeably decomposed, no attempts to measure spectra at higher temperature has been made).

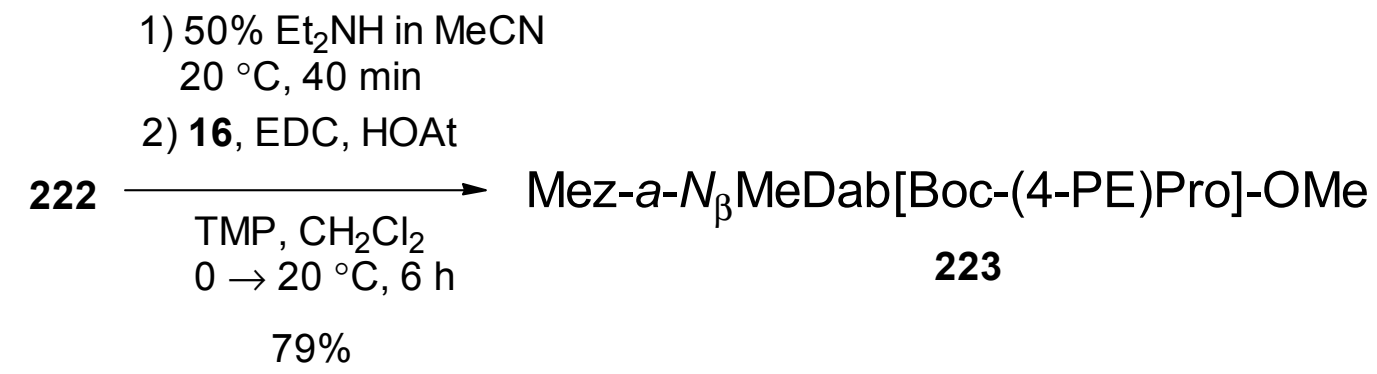

Scheme 62

6.5. Evaluation of the biological activity of building blocks and hormaomycin analogs as well as selected model compounds

All biological tests were performed by Ms. M. Klingebiel and Mr. H.-P. Kroll in prof. A. Zeeck's group (Göttingen).

To evaluate the possible antibiotic activity the plate diffusion tests on bacteriostatic activity against test strains of E. coli, B. subtilis, S. aureus, C. albicans, as well as against highly sensitive to hormaomycin Arthrobacter oxydans with two isomers of 3-(2'nitrocyclopropyl)alanine, $\left(2 S, 1^{\prime} R, 2^{\prime} R\right)-\mathbf{8 3}$ and $\left(2 R, 1^{\prime} R, 2^{\prime} R\right)-\mathbf{8 3}, 4-(Z)$-propenylproline $(2 S, 4 R)-\mathbf{1 6}$, 1-hydroxy-2-chloro-5-cyanopyrrole 103, and with the model acyl tripeptide 119 were carried out. In these experiments all tested compounds did not show any noticeable activity with the only exception for $\mathbf{1 0 3}$, which displayed significant activity against $S$. aureus.

As the evaluation of the morphogen activity was impossible due to the technical reasons only the antibiotic activity against Arthrobacter species was tested (table 1). 


\begin{tabular}{|c|c|}
\hline Compound & Relative activity $(\%)$ \\
\hline 119 & 0 \\
\hline 193 & 0 \\
\hline 194 & 19 \\
\hline$O$-MOM-3 & 19 \\
\hline epi-O-MOM-3 & 13 \\
\hline Synthetic 3 & 113 \\
\hline 211 & 39 \\
\hline 219a & 0 \\
\hline$\left[\left(\mathrm{NO}_{2}\right) \mathrm{Nva}^{7}\right]-3$ & 90 \\
\hline$\left[a-\mathrm{Dab}^{1}\right]-\mathbf{3}$ & 103 \\
\hline$\left[a-\mathrm{Dab}^{1}, a-\mathrm{Ile}^{5}\right]-\mathbf{3}$ & 6 \\
\hline
\end{tabular}

Table 1. Relative bacteriocidal activity of several compounds in plate diffusion tests against Arthrobacter crystallopoites (strain 20119): plates diameter $9 \mathrm{~mm}$, thickness $0.5 \mathrm{~mm} ; 0.05 \mathrm{mg}$ substance pro plate. Relative activity $=(\varnothing$ of inhibition zone $-9 / \varnothing$ of inhibition zone of $\mathbf{3}-9)$ $\times 100(\%)$.

For the most potent compound $\left[\left(\mathrm{NO}_{2}\right) \mathrm{Nva}^{7}\right]-3,\left[a-\mathrm{Dab}^{1}\right]-\mathbf{3}$ and 211 the dilution row plate diffusion tests were also performed (table 2).

\begin{tabular}{|ccccc|}
\hline Compound mg pro plate & $5 \times 10^{-2}$ & $5 \times 10^{-3}$ & $5 \times 10^{-4}$ & $5 \times 10^{-5}$ \\
\hline $\mathbf{3}$ & 100 & 94 & 71 & 39 \\
{$\left[\left(\mathrm{NO}_{2}\right) \mathrm{Nva}^{7}\right]-\mathbf{3}$} & 90 & 84 & 58 & 26 \\
{$\left[a-\mathrm{Dab}^{1}\right]-\mathbf{3}$} & 103 & 90 & 68 & 35 \\
$\mathbf{2 1 1}$ & 39 & 23 & 2 & 0 \\
\hline
\end{tabular}

Table 2. Relative bactericidal activity of several compounds in dilution row plate diffusion tests against Arthrobacter crystallopoites (strain 20119) (estimated relative to activity of hormaomycin at $5 \times 10^{-2} \mathrm{mg}$ pro plate) 
Even these preliminary tests allowed making some conclusions about SAR for the antibiotic activity of hormaomycin 3:

1) side chain of hormaomycin causes its antibiotic activity;

2) lack of the aliphatic nitro-group in the side chain of amino acid, acetylated with Chpca, causes complete loss of the bactericidal activity (109 vs. 211);

3) substitution of the cyclopropyl ring in the side chain of amino acid, acetylated with Chpca, at least with the methylene group, causes only insignificant loss activity $\left(\mathbf{3}\right.$ vs. $\left.\left[\left(\mathrm{NO}_{2}\right) \mathrm{Nva}^{7}\right]-\mathbf{3}\right)$;

4) presence of the free $N$-hydroxy group on Chpca moiety is very important; its alkylation causes significant decrease of activity (3 vs. $O$-MOM-3, 193 vs. 194);

5) cyclic part of hormaomycin 3 does not have any intrinsic antibiotic activity (219), nevertheless the significant changes of its conformations can give inactive compounds ([ $a$ $\left.\left.\mathrm{Dab}^{1}, a-\mathrm{Ile}^{5}\right]-3\right)$

6) generally, group or substituent, connected with the fragment, which is responsible for antibiotic action, can noticeably effect the antibiotic activity (194 vs. 211).

Further, it seems to be very possible, that the cyclic part of $\mathbf{3}$ diminishes its antibiotic activity $(O-$ MOM-3 vs. 211). Nevertheless additional test with 211, after removal of the MOM group, should be made. Consequently, if the resultant compound has similar or higher activity than hormaomycin 3, it will mean that the postulated mechanism of the antibiotic action of hormaomycin 3 (perturbation of the cell membrane) is wrong. 


\section{Experimental Part}

\section{General methods}

${ }^{1}$ H NMR spectra: Bruker AM 250 (250 MHz), AMX 300 (300 MHz) or Varian Unity 300 (300 MHz), Inova 500 (500 MHz), Inova 600 (600 MHz). Proton chemical shifts are reported in ppm relative to residual peak of deuterated solvent or tetramethylsilane: $\delta(\mathrm{ppm})=0$ for TMS, 2.49 for $\left[\mathrm{D}_{5}\right] \mathrm{DMSO}, 4.65$ for $\mathrm{HOD}$ in $\mathrm{D}_{2} \mathrm{O}, 7.26$ for $\mathrm{CHCl}_{3}, 1.73$ and 3.55 for $\left[\mathrm{D}_{7}\right] \mathrm{THF}, 3.35$ for $\mathrm{CHD}_{2} \mathrm{OD}, 2.04$ for $\left[\mathrm{D}_{5}\right]$ acetone. Higher order NMR spectra were approximately interpreted as first-order spectra, if possible. For the characterization of the observed signal multiplicities the following abbreviations were applied: $\mathrm{s}=$ singlet, $\mathrm{d}=$ doublet, $\mathrm{t}=$ triplet, $\mathrm{q}=$ quartet, quin $=$ quintet, $\mathrm{m}=$ multiplet, as well as $\mathrm{br}=$ broad, $\mathrm{Ar}-\mathrm{H}=$ aryl-H. ${ }^{13} \mathrm{C}$ NMR spectra [additional DEPT (Distortionless Enhancement by Polarization transfer) or APT (Attached Proton Test)]: Bruker AM 250 (62.9 MHz), AMX 300 (75.5 MHz) or Varian Unity 300 (75.5 MHz), Inova 500 (125.7 $\mathrm{MHz})$, Inova $600(125.7 \mathrm{MHz})$ instruments. ${ }^{13} \mathrm{C}$ chemical shifts are reported relative to peak of solvent or tetramethylsilane: 0 for TMS, 39.5 for $\left[\mathrm{D}_{5}\right] \mathrm{DMSO}, 77.0$ for $\mathrm{CDCl}_{3}, 25.5$ and 3.55 for $\left[\mathrm{D}_{7}\right] \mathrm{THF}, 3.35$ for $\mathrm{CHD}_{2} \mathrm{OD}, 29.8$ for $\left[\mathrm{D}_{5}\right]$ acetone or to dioxane in $\mathrm{D}_{2} \mathrm{O}(\delta=67.2 \mathrm{ppm})$. The following abbreviations were applied: DEPT: $+=$ primary or tertiary (positive signal in DEPT), $-=$ secondary (negative signal in DEPT), $\mathrm{C}_{\text {quat }}=$ quaternary (null signal in DEPT); APT: $+=$ primary or tertiary (positive signal in DEPT), $-=$ secondary or quaternary (negative signal in APT); if it was necessary and possible HMBS (Heteronuclear Multiple Bond Connectivity) and/or HMQS (Heteronuclear Multiple Quantum Coherence) were also measured. IR-spectra: Bruker IFS 66 (FT-IR) spectrometer as $\mathrm{KBr}$ pellets or oils between $\mathrm{KBr}$ plates. MS: EI-MS: Finnigan MAT 95, $70 \mathrm{eV}$, high resolution EI-MS spectra with perfluorkerosene as reference substance; DCI-MS: Finnigan MAT 95, $200 \mathrm{eV}$, reactant gas $\mathrm{NH}_{3}$; ESI-MS: Finnigan LCQ. HPLC-MS: pump: Flux Instruments Rheos 4000; degasser: Flux Instruments ERC 3415 $\alpha$; detector: Linear UVIS-205; data system: Flux Instruments Janeiro; ESI: Finnigan LCQ, positive 
and negative mode; data system: Finnigan LCQ Xcalibur; column:Crom Superspher 100 RP-18 endcapped (4 $\mu \mathrm{m}, 2 \times 100 \mathrm{~mm})$; HPLC conditions: eluent $\mathrm{A}: \mathrm{H}_{2} \mathrm{O}(0.05 \% \mathrm{HCOOH})$, eluent B: $90 \% \mathrm{MeCN}(0.05 \% \mathrm{HCOOH}), 30 \rightarrow 70 \% \mathrm{~B}$ for $30 \mathrm{~min}$, flow rate: $300 \mu \mathrm{L} / \mathrm{min}$. HPLC: pump: Kontron 322 system, detector: Kontron DAD 440, mixer: Kontron HPLC 360, data system:

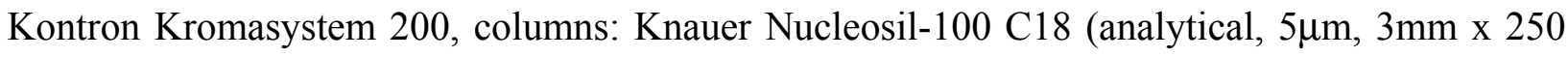
$\mathrm{mm}$ ), Knauer Nucleosil-100 C18 (preparative, $5 \mu \mathrm{m}, 8 \mathrm{~mm}$ x $250 \mathrm{~mm}$ ). Optical rotations: PerkinElmer 241 digital polarimeter, 1-dm cell; optical rotation values are given in $10^{-1} \mathrm{deg} \mathrm{cm}^{2} \mathrm{~g}^{-1}$; concentrations $(c)$ are given in $\mathrm{g} / 100 \mathrm{~mL}$. Circular dichroism: Jasco J 500A. Molar ellipticities $(\Theta)$ are given in grad $\mathrm{cm}^{2} 10^{-1} \mathrm{~mol}^{-1}$. M.p.: Büchi 510 capillary melting point apparatus, uncorrected values. TLC: Macherey-Nagel precoated sheets, $0.25 \mathrm{~mm}$ Sil G/UV 254 . The chromatograms were viewed under UV light and/or by treatment with phosphomolibdic acid ( $10 \%$ in ethanol), or ninhidrin $(0.2 \%$ in ethanol), or Ehrlich's reagent (freshly prepared solution of $1 \mathrm{~g}$ of 4-dimethylaminobenzaldehyde in $25 \mathrm{~mL} \mathrm{36 \%} \mathrm{HCl}$ and $75 \mathrm{~mL}$ methanol). Column chromatography: Merck silica gel, grade 60, 230-400 mesh and Baker silica gel, 40-140 mesh. Preparative TLC: Macherey-Nagel, silica gel SIL G/UV $\mathrm{UV}_{254}$, layer thickness $0.25 \mathrm{~mm}(100 \times 200$ $\mathrm{mm}$ or $200 \times 200 \mathrm{~mm}$ ). Elemental analyses: Mikroanalytisches Laboratorium des Instituts für Organische Chemie der Universität Göttingen. Starting materials: Anhydrous solvents were prepared according to standard methods by distillation over drying agents and were stored under argon. All other solvents were distilled before use. All reactions were carried out with magnetic stirring and, if air or moister sensitive, in flame-dried glassware under argon or nitrogen. 
The following compounds were prepared according to literature procedures:

$(2 S, 4 R)-4$-Tosyloxi- $N$-Boc-proline, ${ }^{[22]}$ tert-butyl $(R)-N$-(tert-butyloxycarbonyl)pyroglutamate [as it was described for $(S)$-isomer], ${ }^{[34]}$ tert-butyl $(2 R, 4 R)$-( $N$-tert-butyloxycarbonyl)-4-allylpyroglutamate $[$ as it was described for $(2 S, 4 S)$-isomer $],{ }^{[35]}$ methyl $(2 S, 4 R)-(N$-tertbutyloxycarbonyl)-4-hydroxyprolinate, ${ }^{[46]}$ methyl $\quad(2 S, 4 R S)$-(N-tert-butyloxycarbonyl)-4iodopro-linate, ${ }^{[47]} \quad(2 S, 4 R)-\left(N\right.$-tert-butyloxycarbonyl)-4-hydroxyproline, ${ }^{[53]}, \quad(2 S, 4 R)-(N$-tertbutyloxy-carbonyl)-4-mesyloxyprolinol, ${ }^{[57]}$ (2S,4R)-(N-tert-butyloxycarbonyl)-4-hydroxyprolixnol tert-butyldimetylsilyl ester, ${ }^{[62]}$ adduct of $(2 S, 4 R)-4$-hydroxyproline and pivalic aldehyde, ${ }^{[64]}$ rac-(trans-2'-nitrocyclopropyl)methanol, ${ }^{[84]} \quad$ (1,1-dideutero)ethyltriphenylphosphonium bromide,${ }^{[72]} \quad(R)$-allo-threonine,${ }^{[75]} \quad(4 R)$-phenyloxazolidinone-2, ${ }^{[80]} \quad(4 R)-4$-phenyl-3-(3'phenylbutyryl)oxazo-lidinone-2, ${ }^{[79]}(4 R, 3 ' S)-4$-phenyl-3-(3'-phenylbutyryl)oxazolidinone-2 [as it was described for $\left(4 S, 3^{\prime} R\right)$-isomer $],{ }^{[81]} 1,1,3,3$-tetramethyl guanidinium azide, ${ }^{[136]}$ rac- $\beta$ methylphenylalanine ${ }^{[137]}(2 \mathrm{~S}, 3 \mathrm{R})-\beta$-methylphenylalanine ${ }^{[79]}(S)$ and $(R)$ Belokon's chiral glycine equivalents, ${ }^{[86]}$ (4R)-4-isopropyl-1,3-trans-(2'-nitrocyclopropyl)carbonyloxazolidinones$2,^{[14]} \operatorname{trans}-\left(2^{\prime}\right.$-nitrocyclopro-pyl)methyl iodide, ${ }^{[80]}$ 1-methoxysuccinimide, ${ }^{[97]}$ 2-amino-6chloropyridine, ${ }^{[99]} \quad 2$-acetamido-6-chloropyridine, ${ }^{[101]}$ 2,6-dichloropyridine- $N$-oxide, ${ }^{[102]}$ 9flourenylmethyl $(S)$-(N-tert-butyloxycarbonyl)phenylalaninate, ${ }^{[105]}$ 2-acetamino-6-aminopyridine ${ }^{[107]}(S)-(2-n i t r o p h e n y l) s u l f e n y l p r o l i n e,{ }^{[116]}(S)-(2-n i t r o p h e n y l)$ sulfenylisoleucine, ${ }^{[16]}$ methyl (N-tert-butyloxycarbonyl)-isoleucyl-prolinate, ${ }^{[117]} p$-nitrobenzyl $(R)$-allo-( $N$-tert-butyloxycarbonyl)threoninate [as it was described for $(S)$-threo-isomer], ${ }^{[120]}(2 S, 3 R)-(N-9$-fluorenylmethyloxycarbonyl)- $\beta$-methylphenylalanine, ${ }^{[119]}$ tert-butyl $(2 R, 3 R)-2$-azido-3- (benzyloxycarbonylamino)butanoate, ${ }^{[133]}$ tert-butyl (2R,3R)-2-(tert-butyloxycarbonylamino)-3-(9-fluorenylmethyloxycarbonylamino)butanoate ${ }^{[134]} \quad$ tert-butyl $\quad(2 R, 3 R)-3$-(benzyloxycarbonylmethylamino)-2-tertbutyldimethylsilyloxybutanoate ${ }^{[135]}$ tetrabutylammonium cyanide ${ }^{[138]}$ 9-fluorenylmethyl $N$ hydroxysuccinyl carbonate, ${ }^{[139]}$ nitrophenylsulfenyl chloride, ${ }^{[139]}$ 3-nitropyridine-2-sulfenyl chloride ${ }^{[140]}$ 1-hydroxy-7-aza-benzotriazol, ${ }^{[141]} \quad$ (7-azabenzotriazol-1-yloxy)tris(pyrrolidino)- 
phosphonium hexafluorophosphate, ${ }^{[141]}$ 2-(trimethylsilyl)ethyl $N$-succinyl carbonate. ${ }^{[142]}$ $\left(2 S, 1^{\prime} R, 2^{\prime} R\right)-3-\left(2^{\prime}-\right.$ Nitrocyclopropyl)alanine and nitrocyclopropane were kindly provided by Oleg V. Larionov (Göttingen).

\section{Preparation of substances}

2.1. Determination of the relative configuration of 4-(Z)-propenylproline moiety in hormaomycin. Attempts to prepare pure diastereomers of 4-(Z)-propenylproline via alkylation with (Z)-propenyllithium cuprate

$(2 S, 4 R)-4-T o s y l o x i-N-B o c-p r o l i n e ~(2 S, 4 R)-(15)::^{[22]}$ M.p. $138-139{ }^{\circ} \mathrm{C} ; R_{\mathrm{f}}=0.19$ (hexanes/EtOAc<smiles>O=C(O)C1C[C@@H](O[SeH])CN1</smiles>
$1: 2(1.5 \% \mathrm{AcOH}) ;[\alpha]_{20}^{D}-62.7\left(c=0.33, \mathrm{CHCl}_{3}\right) ;{ }^{1} \mathrm{H}$ NMR $(250$

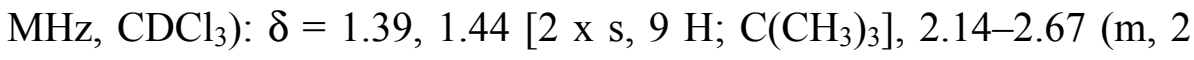
$\left.\mathrm{H} ; 3-\mathrm{H}_{\mathrm{A}}, 5-\mathrm{H}_{\mathrm{A}}\right), 2.46\left(\mathrm{~s}, 3 \mathrm{H} ; \mathrm{CH}_{3}\right), 3.45-3.81\left(\mathrm{~m}, 2 \mathrm{H}, 3-\mathrm{H}_{\mathrm{B}}, 5-\mathrm{H}_{\mathrm{B}}\right)$, 4.39, $4.42(2 \mathrm{x} \mathrm{t}, J=8 \mathrm{~Hz}, 1 \mathrm{H} ; 2-\mathrm{H}), 5.02(\mathrm{~m}, 1 \mathrm{H}, 4-\mathrm{H}), 7.37$ (d, $\left.J=8.25 \mathrm{~Hz}, 2 \mathrm{H}, 3^{\prime}-\mathrm{H}\right), 7.79$ $\left(\mathrm{d}, J=8.25 \mathrm{~Hz}, 2 \mathrm{H}, 2{ }^{\prime}-\mathrm{H}\right) ;{ }^{13} \mathrm{C} \mathrm{NMR}\left(62.9 \mathrm{MHz}, \mathrm{CDCl}_{3}\right): \delta=21.6\left(+, \mathrm{CH}_{3}\right), 28.0,28.2[+$, $\left.\mathrm{C}\left(\mathrm{CH}_{3}\right)_{3}\right], 35.3,37.0(-, \mathrm{C}-3), 51.8,52.3(-, \mathrm{C}-5), 51.2(+, \mathrm{C}-2), 78.2,78.6\left[\mathrm{C}_{\text {quat }}, \mathrm{C}\left(\mathrm{CH}_{3}\right)_{3}\right]$, 81.2, $81.8(+, \mathrm{C}-4), 127.7\left(+, \mathrm{C}-3^{\prime}\right), 130.1\left(+, \mathrm{C}-2^{\prime}\right), 133.1,133.24\left(\mathrm{C}_{\text {quat }}, \mathrm{C}-4^{\prime}\right), 145.3\left(\mathrm{C}_{\text {quat }}, \mathrm{C}-\right.$ $\left.1^{\prime}\right)$, 153.2, $155.0\left(\mathrm{C}_{\text {quat }}, \mathrm{NCO}_{2}\right), 175.2,177.5\left(\mathrm{C}_{\text {quat }}, \mathrm{C}-1\right)$; IR $(\mathrm{KBr}): \mathrm{nu}($ tilde $)=3062 \mathrm{~cm}^{-1}, 2985$, 2956, 1754, 1635, 1433, 1171; elemental analysis calcd (\%) for $\mathrm{C}_{17} \mathrm{H}_{23} \mathrm{NO}_{7} \mathrm{~S}$ (385.4): C 52.98, $\mathrm{H}$ 6.01, N 3.63; found C 52.67, H 6.19, N 3.55.

trans- and cys-N-Boc-4-(Z)-Propenylprolines cis-(16) and trans-(16): To a Li span (0.087g, $12.53 \mathrm{mmol})$ in anhydrous diethyl ester $(7 \mathrm{~mL})$ under stirring was added a solution of cis-1- 
bromopropene $(0.3 \mathrm{~mL}, 5.01 \mathrm{mmol})$ in diethyl ether $(3 \mathrm{~mL})$ at $-10{ }^{\circ} \mathrm{C}$ over $5 \mathrm{~min}$ under argon (Ar). After this, the reaction mixture was stirred at the same temperature for additional $1 \mathrm{~h}$ and added to a suspension of $\mathrm{CuBr} \cdot \mathrm{Me}_{2} \mathrm{~S}(0.529 \mathrm{~g}, 2.26 \mathrm{mmol})$ in diethyl ester $(15 \mathrm{~mL})$ at $-45-40{ }^{\circ} \mathrm{C}$ (internal temperature) for $10 \mathrm{~min}$. After $1 \mathrm{~h}$, a solution of $(2 S, 4 R)-15(0.29 \mathrm{~g}, 0.75 \mathrm{mmol})$ in THF (10.5 mL) was added to obtained almost clear green-yellowish solution of lithium dipropenylcuprate at the same temperature over $15 \mathrm{~min}$ and the reaction mixture was allowed to warm to $20^{\circ} \mathrm{C}$ over a period of $16 \mathrm{~h}$. The dark-colored reaction mixture was cooled in water-ice bath and treated with saturated $\mathrm{NH}_{4} \mathrm{Cl}(5-10 \mathrm{~mL})$. Sodium hydroxide $(10 \%)$ was added to bring the $\mathrm{pH}$ to 10 and the organic layer separated. The aqueous phase was washed with ether $(3 \times 10$ $\mathrm{mL})$, acidificated with $4 \mathrm{~N} \mathrm{H}_{2} \mathrm{SO}_{4}$ to $\mathrm{pH} 2-3$ and then extracted with ethyl acetate $(2 \times 20 \mathrm{~mL})$. The combined organic layers were washed with water $(2 \times 10 \mathrm{~mL})$, then with brine $(2 \times 5 \mathrm{~mL})$, dried and concentrated under reduced pressure The residue was filtered through pad of silica gel $(6 \mathrm{~cm})$ in $\mathrm{MeOH} / \mathrm{CHCl}_{3}$ 1:10 to give after evaporation of solvent the propenylproline $(0.155 \mathrm{~g}$, $81 \%$ ) as an inseparable mixture of diastereomers in ratio $2.2: 1$ (according to ${ }^{13} \mathrm{C}$ spectrum). The portion $(25 \mathrm{mg}$ ) of this mixture was separated by HPLC (conditions A, flow rate $3 \mathrm{~mL} / \mathrm{min}$ ) to give trans-isomer trans-16 $\left(13.8 \mathrm{mg}, \mathrm{t}_{\mathrm{R}}=6.84 \mathrm{~min}\right)$, cis-isomer cis-16 $\left(5.4 \mathrm{mg}, \mathrm{t}_{\mathrm{R}}=6.35 \mathrm{~min}\right)$ in pure form.

trans-16: M.p. $41-45{ }^{\circ} \mathrm{C} ;{ }^{1} \mathrm{H}$ NMR $\left(500 \mathrm{MHz}, \mathrm{CDCl}_{3}\right): \delta=1.42,1.48\left[2 \times \mathrm{s}, 9 \mathrm{H}, \mathrm{C}\left(\mathrm{CH}_{3}\right)_{3}\right], 1.66$

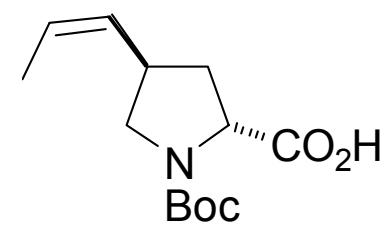
$\left(\mathrm{d}, J=6.0 \mathrm{~Hz}, 3 \mathrm{H} ; 3^{\prime}-\mathrm{H}\right), 1.82(\mathrm{ddd}, J=11.9,11.9,9.1 \mathrm{~Hz}, 0.7 \mathrm{H}, 3-$ $\mathrm{H}_{\mathrm{a}}$ ), 2.01 (ddd, $\left.J=11.9,11.9,10.3 \mathrm{~Hz}, 0.3 \mathrm{H}, 3-\mathrm{H}_{\mathrm{a}}\right), 2.11-2.24(\mathrm{~m}$, $\left.0.3 \mathrm{H} ; 3-\mathrm{H}_{\mathrm{b}}\right), 2.41\left(\mathrm{dd}, J=12.6,6.2 \mathrm{~Hz}, 0.7 \mathrm{H} ; 3-\mathrm{H}_{\mathrm{b}}\right), 2.97(\mathrm{dd}, J=$ $\left.10.0 \mathrm{~Hz}, 0.7 \mathrm{H}, 5-\mathrm{H}_{\mathrm{a}}\right), 3.05\left(\mathrm{dd}, J=9.5 \mathrm{~Hz}, 0.3 \mathrm{H}, 5-\mathrm{H}_{\mathrm{a}}\right), 3.18-3.33(\mathrm{~m}, 1 \mathrm{H}, 4-\mathrm{H}), 3.58(\mathrm{dd}, J=$ 8.5, 8.5 Hz, 0.7 H, 5- $\left.\mathrm{H}_{\mathrm{b}}\right), 3.76\left(\mathrm{dd}, J=9.3,9.3 \mathrm{~Hz}, 0.3 \mathrm{H}, 5-\mathrm{H}_{\mathrm{b}}\right), 4.31$ ( d, $J=9.5 \mathrm{~Hz}, 0.3 \mathrm{H}, 2-$ H), $4.40(\mathrm{~d}, J=8.5 \mathrm{~Hz}, 0.7 \mathrm{H}, 2-\mathrm{H}), 5.25\left(\mathrm{dd}, J=9.8,9.8 \mathrm{~Hz}, 1 \mathrm{H}, 1^{\prime}-\mathrm{H}\right), 5.52(\mathrm{dq}, J=9.8$, $\left.7.0 \mathrm{~Hz} 1 \mathrm{H}, 2^{\prime}-\mathrm{H}\right), 6.40-7.50\left(\mathrm{br}, 1 \mathrm{H}, \mathrm{CO}_{2} \mathrm{H}\right) ;{ }^{13} \mathrm{C} \mathrm{NMR}\left(125.7 \mathrm{MHz}, \mathrm{CDCl}_{3}\right): \delta=13.2(+$, $\left.\mathrm{CH}_{3}\right), 28.2,28.3\left[+, \mathrm{C}\left(\mathrm{CH}_{3}\right)_{3}\right], 34.2,35.2(+, \mathrm{C}-4), 35.1,37.0(-, \mathrm{C}-3), 51.4,51.9(-, \mathrm{C}-5), 58.9$, 
$59.2(+, \mathrm{C}-2), 80.3,81.3\left[\mathrm{C}_{\text {quat }}, \mathrm{C}\left(\mathrm{CH}_{3}\right)_{3}\right], 126.7,126.9\left(+, \mathrm{C}-2^{\prime}\right), 129.2,129.6\left(+, \mathrm{C}-1^{\prime}\right), 153.8$, $156.0\left(\mathrm{C}_{\text {quat }}, \mathrm{NCO}_{2}\right), 175.4,178.4\left(\mathrm{C}_{\text {quat }}, \mathrm{C}-1\right) . \mathrm{IR}(\mathrm{KBr}): \mathrm{nu}($ tilde $)=3023 \mathrm{~cm}^{-1}, 2981,2880$, 1722, 1650, 1404, 1227, 1159; MS (EI, $70 \mathrm{eV}), m / z(\%): 255(1)\left[\mathrm{M}^{+}\right], 210(8)\left[\mathrm{M}^{+}-\mathrm{CHO}_{2}\right], 182$ (2) $\left[\mathrm{M}^{+}-\mathrm{C}_{4} \mathrm{H}_{9} \mathrm{O}\right], 154(100)\left[\mathrm{M}^{+}-\mathrm{C}_{5} \mathrm{H}_{9} \mathrm{O}_{2}\right], 110(70)\left[\mathrm{C}_{7} \mathrm{H}_{12} \mathrm{~N}^{+}\right], 87$ (8), 57 (80) $\left[\mathrm{C}_{4} \mathrm{H}_{9}{ }^{+}\right]$; HRMS (EI): calcd for $\mathrm{C}_{13} \mathrm{H}_{21} \mathrm{NO}_{4}$ : 255.1471; found 255.1471.

cis-16: M.p. $65-68{ }^{\circ} \mathrm{C} ;{ }^{1} \mathrm{H}$ NMR $\left(500 \mathrm{MHz}, \mathrm{CDCl}_{3}\right): \delta=1.41,1.45\left[2 \times \mathrm{s}, 9 \mathrm{H}, \mathrm{C}\left(\mathrm{CH}_{3}\right)_{3}\right], 1.65$

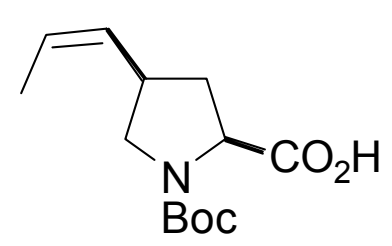
(d, $\left.J=6.5 \mathrm{~Hz}, 3 \mathrm{H} ; 3^{\prime}-\mathrm{H}\right), 1.75$ (ddd, $J=12.5,10.3,10.3 \mathrm{~Hz}, 0.5 \mathrm{H}, 3-$ $\left.\mathrm{H}_{\mathrm{a}}\right), 1.88\left(\mathrm{ddd}, J=12.0,10,10 \mathrm{~Hz}, 0.5 \mathrm{H}, 3-\mathrm{H}_{\mathrm{a}}\right), 2.38(\mathrm{dddd}, J=13.0$, 7.0, 7.0, 7.0 Hz, 0.5 H; 3- $\mathrm{H}_{\mathrm{b}}$ ), 2.44 (dddd, $J=12.5,6.5,6.5,6.5 \mathrm{~Hz}$, $\left.0.5 \mathrm{H} ; 3-\mathrm{H}_{\mathrm{b}}\right), 2.99-3.16\left(\mathrm{~m}, 2 \mathrm{H}, 4-\mathrm{H}, 5-\mathrm{H}_{\mathrm{a}}\right), 3.67\left(\mathrm{dd}, J=9.3,7.3 \mathrm{~Hz}, 0.5 \mathrm{H}, 5-\mathrm{H}_{\mathrm{b}}\right), 3.69-3.78$ (m, $\left.0.5 \mathrm{H}, 5-\mathrm{H}_{\mathrm{b}}\right), 4.22(\mathrm{dd}, J=8.3,8.3 \mathrm{~Hz}, 0.5 \mathrm{H}, 2-\mathrm{H}), 4.28(\mathrm{dd}, J=8.3,8.3 \mathrm{~Hz}, 0.5 \mathrm{H}, 2-\mathrm{H})$, 5.23 (ddq, $\left.J=10.8,10.8,1.8 \mathrm{~Hz}, 1 \mathrm{H}, 1^{\prime}-\mathrm{H}\right), 5.52$ (dq, $\left.J=10.8,6.5 \mathrm{~Hz}, 1 \mathrm{H}, 2^{\prime}-\mathrm{H}\right), 6.82-7.57$ (br, $\left.1 \mathrm{H}, \mathrm{CO}_{2} \mathrm{H}\right) ;{ }^{13} \mathrm{C} \mathrm{NMR}\left(125.7 \mathrm{MHz}, \mathrm{CDCl}_{3}\right): \delta=13.2\left(+, \mathrm{CH}_{3}\right), 28.2,28.4\left[+, \mathrm{C}\left(\mathrm{CH}_{3}\right)_{3}\right]$, 35.8, $36.0(+, \mathrm{C}-4), 35.9,37.4(-, \mathrm{C}-3), 51.5,52.1(-, \mathrm{C}-5), 59.3,59.6(+, \mathrm{C}-2), 80.4,81.0\left[\mathrm{C}_{\text {quat }}\right.$, $\left.\mathrm{C}\left(\mathrm{CH}_{3}\right)_{3}\right], 126.6,126.9\left(+, \mathrm{C}-2^{\prime}\right), 129.2,129.3\left(+, \mathrm{C}^{\prime} 1^{\prime}\right), 153.6,155.6\left(\mathrm{C}_{\text {quat }}, \mathrm{NCO}_{2}\right), 176.3,178.6$ $\left(\mathrm{C}_{\text {quat }}, \mathrm{C}-1\right)$; IR $(\mathrm{KBr}): \mathrm{nu}(\mathrm{tilde})=3020 \mathrm{~cm}^{-1}, 2975,2943,1736,1633,1441,1252,1168 ; \mathrm{MS}$ (EI, $70 \mathrm{eV}), m / z(\%): 255(1)\left[\mathrm{M}^{+}\right], 210(8)\left[\mathrm{M}^{+}-\mathrm{CHO}_{2}\right], 182(2)\left[\mathrm{M}^{+}-\mathrm{C}_{4} \mathrm{H}_{9} \mathrm{O}\right], 154(100)\left[\mathrm{M}^{+}-\right.$ $\left.\mathrm{C}_{5} \mathrm{H}_{9} \mathrm{O}_{2}\right], 110(70)\left[\mathrm{C}_{7} \mathrm{H}_{12} \mathrm{~N}^{+}\right], 87$ (8), 57 (80) $\left[\mathrm{C}_{4} \mathrm{H}_{9}{ }^{+}\right]$; HRMS (EI): calcd for $\mathrm{C}_{13} \mathrm{H}_{21} \mathrm{NO}_{4}$ : 255.1471; found 255.1471.

trans-4-(Z)-propenylproline trifluoroacetate TFA.trans-(17): Compound trans-17 (13.8 mg,

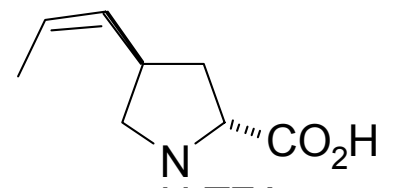

$\mathrm{H} \cdot$ TFA $0.054 \mathrm{mmol})$ was deprotected with TFA $(1 \mathrm{~mL})$ at $20^{\circ} \mathrm{C}$ for $20 \mathrm{~min}$. The resulting solution was concentrated under reduced pressure to give trifluoroacetate trans-17 (13.9 $\mathrm{mg}, 100 \%)$ as a hydroscopic faint rose solid. ${ }^{1} \mathrm{H}$ NMR $\left(250 \mathrm{MHz}, \mathrm{CD}_{3} \mathrm{OD}\right): \delta=1.72\left(\mathrm{dd}, J=7.0,1.8 \mathrm{~Hz}, 3 \mathrm{H}, 3^{\prime}-\mathrm{H}\right), 2.11-2.29$ (m, $\left.1 \mathrm{H}, 3-\mathrm{H}_{\mathrm{a}}\right), 2.35-2.53(\mathrm{~m}, 1 \mathrm{H}, 4-\mathrm{H}), 3.02\left(\mathrm{dd} J=9.5,11 \mathrm{~Hz}, 1 \mathrm{H}, 3-\mathrm{H}_{\mathrm{b}}\right), 3.60(\mathrm{dd}, J=7.5$, 
$\left.11.1 \mathrm{~Hz}, 5-\mathrm{H}_{\mathrm{b}}\right), 4.55$ (dd, $\left.J=4.7,9.6 \mathrm{~Hz}, 2-\mathrm{H}\right), 5.35$ (ddq, $\left.J=9.8,9.8,1.8 \mathrm{~Hz}, 1 \mathrm{H}, 1^{\prime}-\mathrm{H}\right), 5.74$ (ddq, $J=9.8,1.0,7.0 \mathrm{~Hz}, 1 \mathrm{H}, 2^{\prime}-\mathrm{H}$ ); the residual peak of $\mathrm{CD}_{2} \mathrm{HOD}$ masked absorption for 5- $\mathrm{H}_{\mathrm{a}}$; ${ }^{13} \mathrm{C}$ NMR (62.9 MHz, CD $\left.3 \mathrm{OD}\right): \delta=13.5\left(+, \mathrm{C}-3^{\prime}\right), 36.2(-, \mathrm{C}-3), 36.2(+, \mathrm{C}-4), 51.9(-, \mathrm{C}-5)$, $60.7(+, \mathrm{C}-2), 117.9\left(\mathrm{q}, J=289 \mathrm{~Hz}, \mathrm{CF}_{3}\right), 128.9\left(+, \mathrm{C}-2^{\prime}\right), 129.7\left(+, \mathrm{C}-1^{\prime}\right), 162.1(\mathrm{q}, J=37.4 \mathrm{~Hz}$ $\left.\mathrm{CF}_{3} \underline{\mathrm{CO}}\right), 171.9\left(\mathrm{C}_{\text {quat }}, \mathrm{C}-1\right)$; IR (film): nu(tilde) $=3250-1950 \mathrm{~cm}^{-1}, 1679,1631,1410,1203$, 1137; MS (EI, $70 \mathrm{eV}), m / z(\%): 155(3)\left[\mathrm{M}^{+}\right], 110(100)\left[\mathrm{M}^{+}-\mathrm{CHO}_{2}\right], 87(16), 69$ (43) $\left[\mathrm{CF}_{3}^{+}\right]$, 51 (16), 45 (45) $\left[\mathrm{CHO}_{2}^{+}\right], 41$ (15) $\left[\mathrm{C}_{3} \mathrm{H}_{5}^{+}\right]$; HRMS (EI): calcd for $\mathrm{C}_{8} \mathrm{H}_{13} \mathrm{NO}_{2} \cdot \mathrm{C}_{2} \mathrm{HF}_{3} \mathrm{O}_{2}$ : 155.0946·113.9928; found 155.0946.

cis-4-(Z)-propenylproline hydrochloride HCl-cis-(17): Compound cis-16 (10.2 mg, 0.040 mmol)

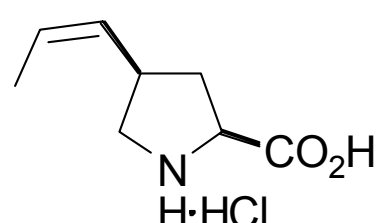
$\mathrm{H} \cdot \mathrm{HCl}$ was deprotected with TFA $(1 \mathrm{~mL})$ at $20{ }^{\circ} \mathrm{C}$ for $20 \mathrm{~min}$. The resulting solution was concentrated under reduced pressure. The residue was taken up with $2 \mathrm{M} \mathrm{HCl} / \mathrm{EtOAc}(3 \times 2 \mathrm{~mL})$ and then concentrated under reduced pressure. The residue was recrystallized four times from $\mathrm{CH}_{2} \mathrm{Cl}_{2} / \mathrm{Et}_{2} \mathrm{O}$ to give after prolonged drying at 0.01 Torr hydrochloride cis-17 (2.0 $\mathrm{mg}, 26 \%)$ as a hydroscopic brownish semisolid. ${ }^{1} \mathrm{H}$ NMR $\left(250 \mathrm{MHz}, \mathrm{D}_{2} \mathrm{O}\right): \delta=1.47\left(\mathrm{dd}, J=6.9,1.9 \mathrm{~Hz}, 3 \mathrm{H}, 3^{\prime}-\mathrm{H}\right), 1.61-1.81(\mathrm{~m}$, $\left.1 \mathrm{H}, 3-\mathrm{H}_{\mathrm{a}}\right), 2.45\left(\mathrm{ddd}, J=13.0,7.1,7.1 \mathrm{~Hz}, 1 \mathrm{H}, 3-\mathrm{H}_{\mathrm{b}}\right), 2.90\left(\mathrm{dd} J=10.1,10.1 \mathrm{~Hz}, 1 \mathrm{H}, 5-\mathrm{H}_{\mathrm{a}}\right)$, 3.24 (ddddd, $J=9.0,9.0,9.0,9.0,9.0 \mathrm{~Hz}, 4-\mathrm{H}), 3.31-3.42\left(\mathrm{~m}, 5-\mathrm{H}_{\mathrm{b}}\right), 4.25(\mathrm{dd} J=8.9 \mathrm{~Hz}, 2-\mathrm{H})$, $5.11\left(\mathrm{ddq}, J=8.8,8.8,1.9 \mathrm{~Hz}, 1 \mathrm{H}, 1^{\prime}-\mathrm{H}\right), 5.44-5.58\left(\mathrm{~m}, 2^{\prime}-\mathrm{H}\right) ;{ }^{13} \mathrm{C} \mathrm{NMR}\left(62.9 \mathrm{MHz}, \mathrm{D}_{2} \mathrm{O}\right): \delta=$ $13.1\left(+, \mathrm{C}-3^{\prime}\right), 35.6(-, \mathrm{C}-3), 36.5(+, \mathrm{C}-4), 50.8(-, \mathrm{C}-5), 60.5(+, \mathrm{C}-2), 127.7\left(+, \mathrm{C}-2^{\prime}\right), 129.6(+$, C-1'), 172.7 (C $\left.\mathrm{C}_{\text {quat }}, \mathrm{C}-1\right)$; MS (ESI), positive $m / z(\%): 178(6)\left[\mathrm{M}+\mathrm{Na}^{+}\right], 156(100)\left[\mathrm{M}+\mathrm{H}^{+}\right]$.

tert-butyl (2)-3-oxo-2-oxa-5-azabicyclo[2.2.1]heptane-5-carboxylate (2S)-(22): A suspension of

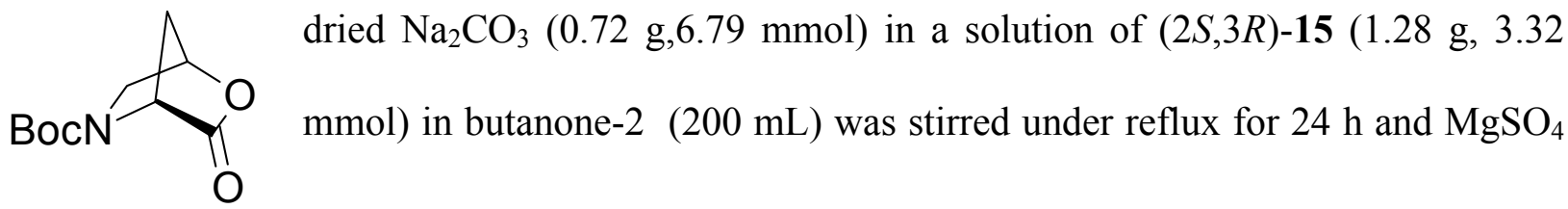


(one spoon) was then added. After $5 \mathrm{~min}$ the mixture was cooled to $20^{\circ} \mathrm{C}$ and filtered. The filtrate was concentrated under reduced pressure to give yellow solid, which was recrystallized from EtOAc/hexanes 1:1 to give $(2 S)-22(0.645 \mathrm{~g}, 91 \%)$ as a colorless cubic crystals. M.p. 106.5-107 ${ }^{\circ} \mathrm{C}$ (lit.: ${ }^{[30]}$ m.p. $\left.109-111^{\circ} \mathrm{C}\right) ;[\alpha]_{20}^{D}-48.3\left(c=1.04, \mathrm{CHCl}_{3}\right)\left[\right.$ lit.: ${ }^{[30]}[\alpha]_{24}^{\mathrm{D}}-49.6(c=$ $\left.\left.1.40, \mathrm{CHCl}_{3}\right)\right]$.

N-Carbophenoxy-(2S,4R)-4-hydroxyproline (2S,4R)-(20): Phenyl chloroformate (11.5 mL, 91.37

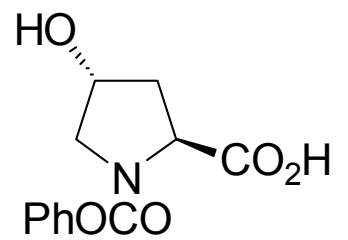
mmol) was added to a vigorously stirred ice-cold solution of 4hydroxyproline $(2 S, 4 R)-19(10 \mathrm{~g}, 76.26 \mathrm{mmol})$ and $\mathrm{NaHCO}_{3}(19.22 \mathrm{~g}$, $228.8 \mathrm{mmol})$ in water $(75 \mathrm{~mL})$ for $5 \mathrm{~min}$. The mixture was allowed to warm to $20^{\circ} \mathrm{C}$ and stirring continued for a further $15 \mathrm{~h}$. The mixture was filtered, the $\mathrm{pH}$ was adjusted to $0-1$ with concentrated $\mathrm{HCl}$ and the resultant suspension or emulsion was extracted with THF $(3 \times 60 \mathrm{~mL})$ under salting-out conditions. The organic layer was dried, filtered and concentrated under reduced pressure. The resulting solid was extracted with a big volume of boiling EtOAc. The resultant solution was concentrated to ca. $30 \mathrm{~mL}$ and the precipitate formed was filtered off, recrystallized from $\mathrm{THF} / \mathrm{Et}_{2} \mathrm{O} /$ hexanes and dried to give $(2 S, 4 R)-20(15.30 \mathrm{~g}$, $80 \%)$ as a colorless pulver. $[\alpha]_{20}^{D}-45.8(c=0.86, \mathrm{THF}) ;{ }^{1} \mathrm{H}$ NMR $\left(250 \mathrm{MHz},\left[\mathrm{D}_{6}\right] \mathrm{DMSO}\right): \delta=$ 1.91-2.11 (m, $\left.1 \mathrm{H}, 3-\mathrm{H}_{\mathrm{a}}\right), 2.16-2.34\left(\mathrm{~m}, 1 \mathrm{H}, 3-\mathrm{H}_{\mathrm{b}}\right), 3.34-3.68(\mathrm{~m}, 2 \mathrm{H}, 5-\mathrm{H}), 4.20-4.35(\mathrm{~m}, 1 \mathrm{H}$, 4-H), 4.25, $4.44(2 \times \mathrm{dd}, J=8.0 \mathrm{~Hz}, 1 \mathrm{H}, 2-\mathrm{H}), 5.00-5.40$ (br, $1 \mathrm{H}, \mathrm{OH}), 7.01-7.42$ (m, $5 \mathrm{H}, \mathrm{Ar}-$ $\mathrm{H}) ;{ }^{13} \mathrm{C}$ NMR (62.9 MHz, [D 6 DMSO): $\delta=38.3,39.1(-, \mathrm{C}-3), 55.3,55.4(-, \mathrm{C}-5)$, 55.3, $55.4(+$, C-2), 68.1, 68.9 (+, C-4), 121.9, 122.0 (+, Ar-C), 125.56, 126.00 (+, Ar-C), 129.6 (+, Ar-C), 151.2, $151.3\left(\mathrm{C}_{\text {quat }}, \mathrm{Ar}-\mathrm{C}\right), 152.6,152.9\left(\mathrm{C}_{\text {quat }}, \mathrm{NCO}_{2}\right), 173.6,174.2\left(\mathrm{C}_{\text {quat }}, \mathrm{C}-1\right)$; IR (KBr): $\mathrm{nu}(\mathrm{tilde})=3250-2750 \mathrm{~cm}^{-1}, 2990,2975,2953,2894,1744,1677,1414,1200,1074 ;$ MS (EI, 70 $\mathrm{eV}), m / z(\%)=251(0.3)\left[\mathrm{M}^{+}\right], 130(8), 94(100)\left[\mathrm{C}_{6} \mathrm{H}_{6} \mathrm{O}^{+}\right], 77(11)\left[\mathrm{C}_{6} \mathrm{H}_{5}^{+}\right], 68(28)\left[\mathrm{C}_{4} \mathrm{H}_{6} \mathrm{~N}^{+}\right]$, $41(13)$. 
N-Carbophenoxy-(2S,4R)-4-hydroxyprolinol (2S,4R)-(21): A 1 M solution of $\mathrm{BH}_{3}$ in THF (84

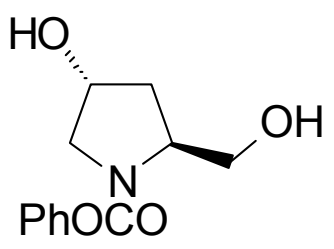

$\mathrm{mL})$ was added dropwise to a stirred ice-cold solution of the acid (2S,4R)-20 (4.00 g, $15.92 \mathrm{mmol})$ in THF $(80 \mathrm{~mL})$ for $2 \mathrm{~h}$, the mixture was allowed to warm to $20^{\circ} \mathrm{C}$ and stirred at this temperature for a further $2 \mathrm{~h}$, and finally refluxed for $30 \mathrm{~min}$. The reaction flask was then cooled in a water/ice bath and $1 \mathrm{M} \mathrm{HCl}$ in $\mathrm{MeOH}(90 \mathrm{~mL})$ was carefully added for $1 \mathrm{~h}$. All volatiles were then removed, the residue was redissolved in EtOAc $(20 \mathrm{~mL})$, cooled, filtered and the resultant clear solution was concentrated again. The oily residue was purified by column chromatography $\left(\mathrm{MeOH} / \mathrm{CHCl}_{3} 1: 7, R_{\mathrm{f}}=0.44\right)$ to give $(2 S, 4 R)-21(2.80 \mathrm{~g}$, max. $74 \%)$ as a viscous colorless oil which contained ca. $4 \%$ of diphenyl carbonate according to ${ }^{1} \mathrm{H}$ NMR spectrum and was used without additional purification for the next step. ${ }^{1} \mathrm{H}$ NMR $\left(250 \mathrm{MHz}, \mathrm{CDCl}_{3}\right): \delta=1.67-1.89(\mathrm{~m}$, $\left.1 \mathrm{H}, 3-\mathrm{H}_{\mathrm{a}}\right), 2.10$ (dddd, $\left.J=13.5,7.0,2.0,2.0,1 \mathrm{H}, 3-\mathrm{H}_{\mathrm{b}}\right), 2.15-2.42(\mathrm{br}, 1 \mathrm{H}, \mathrm{OH}), 3.59-3.72(\mathrm{~m}$, $\left.2 \mathrm{H}, 5-\mathrm{H}_{\mathrm{a}}, 1-\mathrm{H}_{\mathrm{a}}\right), 3.72-3.86\left(\mathrm{~m}, 2 \mathrm{H}, 5-\mathrm{H}_{\mathrm{b}}, 1-\mathrm{H}_{\mathrm{b}}\right), 4.15-4.35(\mathrm{~m}, 1 \mathrm{H}, 4-\mathrm{H}), 4.38-4.54$ (br, $1 \mathrm{H}$, OH), 7.09-7.29 (m, $4 \mathrm{H}, \mathrm{Ar}-\mathrm{H}), 7.30-7.43(\mathrm{~m}, 1 \mathrm{H}, \mathrm{Ar}-\mathrm{H})$; The peak of 2-H was masked by absorption of $\mathrm{OH} ;{ }^{13} \mathrm{C} \mathrm{NMR}\left(62.9 \mathrm{MHz}, \mathrm{CDCl}_{3}\right): \delta=36.7,37.0(-, \mathrm{C}-3), 55.5,55.7$ (-, C-5), 57.9, $59.2(+$, C-2), 63.2, 65.0 (-, C-1), 68.8 (+, C-4), $121.6(+$, Ar-C), 125.5 (+, Ar-C), 129.2, $129.3(+, \mathrm{Ar}-\mathrm{C}), 150.7\left(\mathrm{C}_{\text {quat }}, \mathrm{Ar}-\mathrm{C}\right), 155.3\left(\mathrm{C}_{\text {quat, }}, \mathrm{NCO}_{2}\right)$.

(6R,7aS)-6-Hydroxyperhydro-1H-pyrrolo[1,2-c][1,3]oxazolone-3 (2S,4R)-(22): A $1.6 \mathrm{M} t \mathrm{BuOK}$

$\mathrm{HO}$<smiles>O=C1OC[C@@H]2CCCN12</smiles>
in $(10.5 \mathrm{~mL}, 16.80 \mathrm{mmol})$ was added dropwise to a stirred ice-cold solution of alcohol $(2 S, 4 R)-21(4.00 \mathrm{~g}, 16.86 \mathrm{mmol})$ in THF $(50 \mathrm{~mL})$ for $15 \mathrm{~min}$ and stirring continued at the same temperature for $1 \mathrm{~h}$. The $\mathrm{pH}$ was then carefully adjusted to 5-6 with $1 \mathrm{M} \mathrm{HCl}$ in $\mathrm{MeOH}$ and the mixture was filtered and concentrated under reduced pressure. The residue was purified by column chromatography $\left(\mathrm{CHCl}_{3} / \mathrm{MeOH} 15: 1, R_{\mathrm{f}}=0.21\right)$ and then crystallized from EtOAc/hexanes to give $(2 S, 4 R)-\mathbf{2 1}$ $(2.00 \mathrm{~g}, 83 \%)$ as a colorless solid. M.p. $66-69^{\circ} \mathrm{C} ;[\alpha]_{20}^{D}-41.0\left(c=1.03, \mathrm{CHCl}_{3}\right) ;{ }^{1} \mathrm{H}$ NMR (500 
$\left.\mathrm{MHz}, \mathrm{CDCl}_{3}\right): \delta=1.54\left(\mathrm{ddd}, J=13.0,10.7,5.4 \mathrm{~Hz}, 1 \mathrm{H}, 3-\mathrm{H}_{\mathrm{a}}\right), 2.03(\mathrm{dd}, J=13.0,5.5 \mathrm{~Hz}, 1 \mathrm{H}$, 3- $\left.\mathrm{H}_{\mathrm{b}}\right), 3.05\left(\mathrm{ddd}, J=12.5,1.5,0.5 \mathrm{~Hz}, 1 \mathrm{H}, 5-\mathrm{H}_{\mathrm{a}}\right), 3.21-3.25(\mathrm{br}, 1 \mathrm{H}, \mathrm{OH}), 3.79$ (dd, $J=12.5$, $\left.5.5 \mathrm{~Hz}, 1 \mathrm{H}, 5-\mathrm{H}_{\mathrm{b}}\right), 4.13\left(\mathrm{dd}, J=8.9,3.4 \mathrm{~Hz}, 1 \mathrm{H}, 1-\mathrm{H}_{\mathrm{a}}\right), 4.17-4.24(\mathrm{~m}, 1 \mathrm{H}, 2-\mathrm{H}), 4.51(\mathrm{dd}, J=$ 7.7, $\left.8.9 \mathrm{~Hz}, 1 \mathrm{H}, 1-\mathrm{H}_{\mathrm{b}}\right), 4.57(\mathrm{ddd}, J=5.4,1.0,1.0 \mathrm{~Hz}, 1 \mathrm{H}, 4-\mathrm{H}) ;{ }^{13} \mathrm{C}$ NMR $(125.7 \mathrm{MHz}$, $\left.\mathrm{CDCl}_{3}\right): \delta=40.3(-, \mathrm{C}-3), 55.4(-, \mathrm{C}-5), 57.8(+, \mathrm{C}-2), 67.5(-, \mathrm{C}-1), 72.1(+, \mathrm{C}-4), 161.8\left(\mathrm{C}_{\text {quat }}\right.$, $\mathrm{NCO}_{2}$ ); IR (KBr): nu(tilde) $=3441 \mathrm{~cm}^{-1}, 2989,2878,2862,1720,1476,1464,1407,1253,1175$, 1011; MS (EI, $70 \mathrm{eV}), m / z(\%)=143(40)\left[\mathrm{M}^{+}\right], 115(20)\left[\mathrm{M}^{+}-\mathrm{CO}\right], 99(100)\left[\mathrm{M}^{+}-\mathrm{CO}_{2}\right], 55$ (24) $\left[\mathrm{CHO}_{2}^{+}\right]$; HRMS (EI): calcd for $\mathrm{C}_{6} \mathrm{H}_{9} \mathrm{NO}_{3}$ : 143.0582; found 143.0582; elemental analysis (\%) for $\mathrm{C}_{6} \mathrm{H}_{9} \mathrm{NO}_{3}$ (143.1): calcd. C 50.35, H 6.34, N 9.79; found C 50.66, H 6.28, N 9.67.

(6R,7aS)-6-Tosyloxyperhydro-1H-pyrrolo[1,2-c][1,3]oxazolone-3 (2S,4R)-(23): Pyridine (7.2 pTsO,

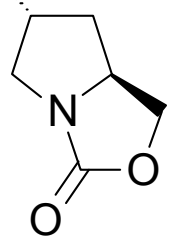
$\mathrm{mL})$ was added to a stirring mixture of $(2 S, 4 R)-22(1.03 \mathrm{~g}, 7.20 \mathrm{mmol})$ and tosyl chloride $(2.74 \mathrm{~g}, 14.37 \mathrm{mmol})$ at the $4{ }^{\circ} \mathrm{C}$ for $10 \mathrm{~min}$. The reaction flask was left at the same temperature for 4 days and the mixture was then stirred at $20^{\circ} \mathrm{C}$ for $4 \mathrm{~h}$. After this, the mixture was diluted with EtOAc (100 $\mathrm{mL})$ and washed with ice-cold $1 \mathrm{M} \mathrm{H}_{2} \mathrm{SO}_{4}(5 \times 40 \mathrm{~mL})$, water $(2 \times 20 \mathrm{~mL})$, brine $(2 \times 20 \mathrm{~mL})$, dried, filtered and concentrated under reduced pressure. The residue was triturated with hexanes to give the crude product which was recrystallized from EtOAc/hexanes to give $(2 S, 4 R)-22(1.78$ $\mathrm{g}, 82 \%)$ as a colorless crystalline solid. $R_{\mathrm{f}}=0.07$ EtOAc/hexanes $1: 2.5 ;[\alpha]_{20}^{D}-53.8(c=1.56$, $\left.\mathrm{CHCl}_{3}\right) ;{ }^{1} \mathrm{H} \mathrm{NMR}\left(250 \mathrm{MHz}, \mathrm{CDCl}_{3}\right): \delta=1.43-1.73\left(\mathrm{~m}, 1 \mathrm{H}, 3-\mathrm{H}_{\mathrm{a}}\right), 2.32(\mathrm{dd}, J=14.0,5.0 \mathrm{~Hz}$, $\left.1 \mathrm{H}, 3-\mathrm{H}_{\mathrm{b}}\right), 2.47(\mathrm{~s}, 3 \mathrm{H}, \mathrm{Me}), 3.17\left(\mathrm{dd}, J=13.8,1.8 \mathrm{~Hz}, 1 \mathrm{H}, 5-\mathrm{H}_{\mathrm{a}}\right), 3.91(\mathrm{dd}, J=13.8,6.0 \mathrm{~Hz}$, $\left.1 \mathrm{H}, 5-\mathrm{H}_{\mathrm{b}}\right), 4.07-4.15(\mathrm{~m}, 1 \mathrm{H}, 2-\mathrm{H}), 4.16\left(\mathrm{dd}, J=9.4,2.5 \mathrm{~Hz}, 1 \mathrm{H}, 1-\mathrm{H}_{\mathrm{a}}\right), 4.53(\mathrm{dd}, J=9.4$, $\left.8.9 \mathrm{~Hz}, 1 \mathrm{H}, 1-\mathrm{H}_{\mathrm{b}}\right), 5.11-5.17(\mathrm{~m}, 1 \mathrm{H}, 4-\mathrm{H}), 7.38$ (d, J=8.3 Hz, $\left.2 \mathrm{H}, \mathrm{Ar}-\mathrm{H}\right), 7.79$ (d, J=8.3 Hz, $2 \mathrm{H}, \mathrm{Ar}-\mathrm{H}) ;{ }^{13} \mathrm{C}$ NMR $\left(125.7 \mathrm{MHz}, \mathrm{CDCl}_{3}\right): \delta=21.4(+, \mathrm{Me}), 37.6(-, \mathrm{C}-3), 52.7(-, \mathrm{C}-5), 57.4$ (+, C-2), 66.5 (-, C-1), 81.3 (+, C-4), 127.5, 130.0, 132.8 (+, Ar-C), $145.3\left(\mathrm{C}_{\text {quat }}, \mathrm{Ar}-\mathrm{H}\right), 160.9$ 
$\left(\mathrm{C}_{\text {quat }}, \mathrm{NCO}_{2}\right)$; IR $(\mathrm{KBr}): \mathrm{nu}(\mathrm{tilde})=3157 \mathrm{~cm}^{-1}, 3068,3058,3024,3003,2980,2960,2918$, 2895, 2803, 1759, 1597, 1405, 1328, 1320, 1262, 1185, 1175, 1092, 1060.

Attempt of the preparation of (6R,7aS)-6-(Z)-propenylperhydro-1H-pyrrolo[1,2-

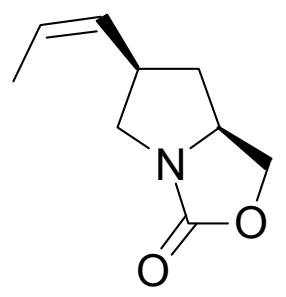
c][1,3]oxazolone-3 (2S,4R)-(24): A solution of $(2 S, 4 R)-23(0.25 \mathrm{~g}, 0.84$ mmol) in THF $(5 \mathrm{~mL})$ was added dropwise to a solution of $(Z)$ propenyllithium cuprate in $\mathrm{Et}_{2} \mathrm{O}(10 \mathrm{~mL})$ prepared as described for the preparation of $N$-Boc protected 4-(Z)-propenylprolines from propenyllithium which was in turn prepared from lithium (90 $\mathrm{mg}, 11.76 \mathrm{mmol})$ and $1-(Z)-$ propenyl bromide $(0.50 \mathrm{~mL}, 0.59 \mathrm{mmol})$ in $\mathrm{Et}_{2} \mathrm{O}(5 \mathrm{~mL})$ and suspension of $\mathrm{CuBr}_{2} \cdot \mathrm{Me}_{2} \mathrm{~S}(0.544$ $\mathrm{g}, 2.65 \mathrm{mmol})$ in $\mathrm{Et}_{2} \mathrm{O}(5 \mathrm{~mL})$ at $-40{ }^{\circ} \mathrm{C}$ for $30 \mathrm{~min}$ and stirring continued for a further $3 \mathrm{~h}$. The reaction mixture was then allowed to warm to $0{ }^{\circ} \mathrm{C}$ and stirring continued for an additional $16 \mathrm{~h}$. The reaction was then quenched with saturated aqueous $\mathrm{NH}_{4} \mathrm{Cl}(4 \mathrm{~mL})$ and the mixture was diluted with $\mathrm{Et}_{2} \mathrm{O}(40 \mathrm{~mL})$, the organic layer was separated, washed with $1 \mathrm{M} \mathrm{H}_{2} \mathrm{SO}_{4}(3 \times 10$ $\mathrm{mL})$, water $(3 \times 10 \mathrm{~mL})$, saturated $\mathrm{NaHCO}_{3}(3 \times 10 \mathrm{~mL})$, water $(3 \times 10 \mathrm{~mL})$, brine $(2 \times 5 \mathrm{~mL})$, dried, filtered and concentrated under reduced pressure. The residue $(120 \mathrm{mg})$ was separated by column chromatography (EtOAc/hexanes $\left.1: 2.5, R_{\mathrm{f}}=0.22\right)$ to give $(2 S, 4 R)-\mathbf{2 4}(11 \mathrm{mg}, 5 \%)$ as a colorless oil and starting material $(90 \mathrm{mg})$. According to NMR spectra $Z / E$ ratio of this product was ca. 4:1. $[\alpha]_{20}^{D}-66.8\left(c=0.38, \mathrm{CHCl}_{3}\right) ;{ }^{1} \mathrm{H} \mathrm{NMR}\left(250 \mathrm{MHz}, \mathrm{CDCl}_{3}\right): \delta=1.20-1.43(\mathrm{~m}, 1 \mathrm{H}$, 3- $\left.\mathrm{H}_{\mathrm{a}}\right), 1.65\left(\mathrm{dd}, J=7.0,2.0 \mathrm{~Hz}, 3 \mathrm{H}, 3^{\prime}-\mathrm{H}\right), 2.11$ (ddd, $\left.J=12.0,5.5 \mathrm{~Hz}, 5.5 \mathrm{~Hz}, 1 \mathrm{H}, 3-\mathrm{H}_{\mathrm{b}}\right), 3.14-$ $3.46(\mathrm{~m}, 3 \mathrm{H}, 4-\mathrm{H}, 5-\mathrm{H}), 3.90-4.05(\mathrm{~m}, 1 \mathrm{H}, 2-\mathrm{H}), 4.20\left(\mathrm{dd}, J=9.0,3.5 \mathrm{~Hz}, 1 \mathrm{H}, 1-\mathrm{H}_{\mathrm{a}}\right), 4.49$ (dd, $J=9.0,8.3 \mathrm{~Hz}, 1 \mathrm{H}, 2-\mathrm{H}), 5.23-5.40\left(\mathrm{~m}, 1 \mathrm{H}, 1^{\prime}-\mathrm{H}\right), 5.53\left(\mathrm{dq}, J=10.5,7.5 \mathrm{~Hz}, 1 \mathrm{H}, 2^{\prime}-\mathrm{H}\right) ;{ }^{13} \mathrm{C}$ $\operatorname{NMR}\left(62.9 \mathrm{MHz}, \mathrm{CDCl}_{3}\right)$ : only spectrum for $(Z)$-isomer is described $\delta=13.0\left(+, \mathrm{C}-3^{\prime}\right), 38.4(-$, C-3), $38.5(+, \mathrm{C}-4), 51.3(-, \mathrm{C}-5), 59.2(+, \mathrm{C}-2), 67.3(-, \mathrm{C}-1), 126.1\left(+, \mathrm{C}-2^{\prime}\right), 130.5\left(+, \mathrm{C}-1^{\prime}\right)$, $158.3\left(\mathrm{C}_{\text {quat }}, \mathrm{NCO}_{2}\right)$; IR $(\mathrm{KBr}): \mathrm{nu}(\mathrm{tilde})=3013 \mathrm{~cm}^{-1}, 2974,2935,2917,2887,2859,1753$, 1393, 1328, 1252, 1230, 1206, 1077, 1061; MS (EI, $70 \mathrm{eV}), m / z(\%)=167(30)\left[\mathrm{M}^{+}\right], 99(34)$ 
$\left[\mathrm{C}_{5} \mathrm{H}_{9} \mathrm{NO}^{+}\right], 82(18)\left[\mathrm{C}_{5} \mathrm{H}_{8} \mathrm{~N}^{+}\right], 67(23), 55(100)\left[\mathrm{CHO}_{2}^{+}\right], 41(23)\left[\mathrm{C}_{3} \mathrm{H}_{5}^{+}\right]$; HRMS (EI): calcd for $\mathrm{C}_{6} \mathrm{H}_{9} \mathrm{NO}_{3}$ : 167.0946; found 167.0946.

2.2. Elucidation of the absolute configuration of 4-(Z)-propenylproline moiety in hormaomycin

tert-Butyl (2R,4R)-N-Boc-4-propylpyroglutamate (2R,4R)-(27): $(2 R, 4 R)-26(0.22 \mathrm{~g}, 0.676 \mathrm{mmol})$

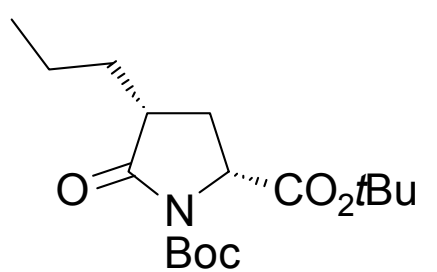
was hydrogenated on $10 \% \mathrm{Pd} / \mathrm{C}(90 \mathrm{mg})$ in EtOAC $(10 \mathrm{~mL})$ at 20 ${ }^{\circ} \mathrm{C}$ for $15 \mathrm{~h}$. The reaction mixture was filtered, solvent was removed under reduced pressure and the product was purified by column chromography (hexanes/EtOAc 4:1, $\left.R_{\mathrm{f}}=0.46\right)$ to give $(2 R, 4 R)-27$ as a colorless oil $(0.198 \mathrm{~g}, 90 \%) .{ }^{1} \mathrm{H}$ NMR $\left(250 \mathrm{MHz}, \mathrm{CDCl}_{3}\right): \delta=0.89$ (t, $\left.J=7.1 \mathrm{~Hz}, 3 \mathrm{H}, 3^{\prime}-\mathrm{H}\right)$, $1.28-1.50\left(\mathrm{~m}, 3 \mathrm{H}, 1^{\prime}-\mathrm{H}_{\mathrm{a}}, 2^{\prime}-\mathrm{H}\right), 1.46\left[\mathrm{~s}, 9 \mathrm{H}, \mathrm{C}\left(\mathrm{CH}_{3}\right)_{3}\right], 1.48\left[\mathrm{~s}, 9 \mathrm{H}, \mathrm{C}\left(\mathrm{CH}_{3}\right)_{3}\right], 1.52-1.67(\mathrm{~m}$, $\left.1 \mathrm{H}, 3-\mathrm{H}_{\mathrm{a}}\right), 1.72-1.91\left(\mathrm{~m}, 1 \mathrm{H}, 1^{\prime}-\mathrm{H}_{\mathrm{b}}\right), 2.42-2.61\left(\mathrm{~m}, 2 \mathrm{H}, 3-\mathrm{H}_{\mathrm{a}}, 4-\mathrm{H}\right), 4.36(\mathrm{dd}, J=8.8,6.3 \mathrm{~Hz}, 1$ $\mathrm{H}, 2-\mathrm{H}) ;{ }^{13} \mathrm{C}$ NMR $\left(62.9 \mathrm{MHz}, \mathrm{CDCl}_{3}\right): \delta=13.6\left(+, \mathrm{C}-3^{\prime}\right), 20.1\left(-, \mathrm{C}-2^{\prime}\right), 27.7\left(-, \mathrm{C}-1^{\prime}\right), 27.7[+$, $\left.2 \times \mathrm{C}\left(\mathrm{CH}_{3}\right)_{3}\right], 33.0(-, \mathrm{C}-3), 42.2(+, \mathrm{C}-4), 57.9(+, \mathrm{C}-2), 81.8\left[\mathrm{C}_{\text {quat }}, \mathrm{C}\left(\mathrm{CH}_{3}\right)_{3}\right], 83.0\left[\mathrm{C}_{\text {quat }}\right.$, $\left.\mathrm{C}\left(\mathrm{CH}_{3}\right)_{3}\right], 149.3\left(\mathrm{C}_{\text {quat }}, \mathrm{NCO}_{2}\right), 170.4\left(\mathrm{C}_{\text {quat }}, \mathrm{C}-1\right), 175.4\left(\mathrm{C}_{\text {quat }}, \mathrm{C}-5\right)$; IR (film): nu(tilde) $=2978$ $\mathrm{cm}^{-1}, 2934,2874,1792,1744,1719,1316,1158 ; \mathrm{MS}(\mathrm{CI}), \mathrm{m} / z(\%): 362(8)\left[\mathrm{M}+2 \mathrm{NH}_{4}^{+}-\mathrm{H}^{+}\right]$, $345(88)\left[\mathrm{M}+\mathrm{NH}_{4}^{+}\right], 328(6)\left[\mathrm{M}+\mathrm{H}^{+}\right]$.

tert-Butyl (2R,4R)-N-Boc-4-propylprolinate $(2 R, 4 R)-(\mathbf{2 8}): \quad \mathrm{A} \quad 1 \quad \mathrm{M}$ solution of lithium

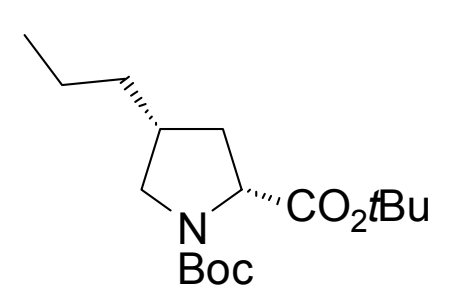
triethylborohydride in THF $(0.8 \mathrm{~mL})$ was added to a solution of $(2 R, 4 R)-(27)(0.198 \mathrm{~g}, 0.605 \mathrm{mmol})$ in $\mathrm{THF}(5 \mathrm{~mL})$ at $-78{ }^{\circ} \mathrm{C}$ under nitrogen atmosphere. After $1 \mathrm{~h}$ the reaction mixture was quenched with saturated aqueous $\mathrm{NaHCO}_{3}(2 \mathrm{~mL})$ and warmed to $0{ }^{\circ} \mathrm{C} .30 \%$ $\mathrm{H}_{2} \mathrm{O}_{2}$ (3 drops) was added and the mixture was stirred at $0{ }^{\circ} \mathrm{C}$. After 20 min the organic solvent 
was removed under reduced pressure, and the aqueous layer was extracted with $\mathrm{CH}_{2} \mathrm{Cl}_{2}(3 \times 10$ $\mathrm{mL}$ ). The combined organic layers were dried, filtered and concentrated. The residue was dried at 0.01 Torr for $3 \mathrm{~h}$, then dissolved in anhydrous $\mathrm{CH}_{2} \mathrm{Cl}_{2}(5 \mathrm{~mL})$ containing triethylsilane $(0.1$ $\mathrm{mL}, 0.622 \mathrm{mmol}$ ) and was cooled to $-78{ }^{\circ} \mathrm{C}$ under nitrogen atmosphere. Boron trifluoride etherate $(0.1 \mathrm{~g}, 0.705 \mathrm{mmol})$ was then added to the reaction mixture for $10 \mathrm{~min}$. After $30 \mathrm{~min}$, triethylsilane $(0.1 \mathrm{~mL}, 0.622 \mathrm{mmol})$ and boron trifluoride etherate $(0.1 \mathrm{~g}, 0.705 \mathrm{mmol})$ were added and the reaction mixture was stirred for $2 \mathrm{~h}$ at the same temperature. Saturated aqueous $\mathrm{NaHCO}_{3}(2 \mathrm{~mL})$ was added and the reaction mixture was extracted with $\mathrm{CH}_{2} \mathrm{Cl}_{2}(3 \times 10 \mathrm{~mL})$, dried, concentrated under reduced pressure and purified by column chromatography $\left(\right.$ EtOAc/hexanes $\left.1: 1, R_{\mathrm{f}}=0.56\right)$ to give $(2 R, 4 R)-\mathbf{2 8}(0.150 \mathrm{~g}, 79 \%)$ as a colorless oil. $[\alpha]_{24}^{\mathrm{D}} 75.4$ $\left(c=0.98, \mathrm{CHCl}_{3}\right)$ (lit. for $(S)$-isomer: $\left.{ }^{[33 \mathrm{~b}]}[\alpha]_{24}^{\mathrm{D}}-52\left(c=1.1, \mathrm{CHCl}_{3}\right)\right) ;{ }^{1} \mathrm{H} \mathrm{NMR}(250 \mathrm{MHz}$, $\left.\mathrm{CDCl}_{3}\right): \delta=0.89\left(\mathrm{t}, J=6.9 \mathrm{~Hz}, 3 \mathrm{H}, 3^{\prime}-\mathrm{H}\right), 1.22-1.38\left(\mathrm{~m}, 5 \mathrm{H}, 3-\mathrm{H}_{\mathrm{a}}, 1^{\prime}-\mathrm{H}, 2^{\prime}-\mathrm{H}\right), 1.42[\mathrm{~s}, 9 \mathrm{H}$, $\left.\mathrm{C}\left(\mathrm{CH}_{3}\right)_{3}\right], 1.42,1.43\left[2 \times \mathrm{s}, 9 \mathrm{H}, \mathrm{C}\left(\mathrm{CH}_{3}\right)_{3}\right], 1.97-2.21(\mathrm{~m}, 1 \mathrm{H}, 4-\mathrm{H}), 2.37$ (ddd, $J=8.1,8.1,8.1$ $\left.\mathrm{Hz}, 0.5 \mathrm{H}, 3-\mathrm{H}_{\mathrm{b}}\right), 2.42\left(\mathrm{ddd}, J=7.8,7.8,7.8 \mathrm{~Hz}, 0.5 \mathrm{H}, 3-\mathrm{H}_{\mathrm{b}}\right), 2.94(\mathrm{dd}, J=10.1,10.1 \mathrm{~Hz}, 1 \mathrm{H}$, 5- $\left.\mathrm{H}_{\mathrm{a}}\right), 3.62\left(\mathrm{dd}, J=10.1,7.3 \mathrm{~Hz}, 0.3 \mathrm{H}, 5-\mathrm{H}_{\mathrm{b}}\right), 3.74\left(\mathrm{dd}, J=10.1,7.3 \mathrm{~Hz}, 0.7 \mathrm{H}, 5-\mathrm{H}_{\mathrm{b}}\right), 4.06$ (dd, $J=8.1,8.1 \mathrm{~Hz}, 0.7 \mathrm{H}, 2-\mathrm{H}), 4.11(\mathrm{dd}, J=8.1,8.1 \mathrm{~Hz}, 0.3 \mathrm{H}, 2-\mathrm{H}) ;{ }^{13} \mathrm{C} \mathrm{NMR}(62.9 \mathrm{MHz}$, $\left.\mathrm{CDCl}_{3}\right): \delta=14.1\left(+, \mathrm{C}-3^{\prime}\right), 21.3\left(-, \mathrm{C}-2^{\prime}\right), 27.9,28.0\left[+, \mathrm{C}\left(\mathrm{CH}_{3}\right)_{3}\right], 28.3,28.4\left[+, \mathrm{C}\left(\mathrm{CH}_{3}\right)_{3}\right], 35.0$, $36.2\left(-, \mathrm{C}^{\prime} \mathbf{1}^{\prime}\right), 37.3(-, \mathrm{C}-3), 37.6,38.3(+, \mathrm{C}-4), 52.1,52.4(-, \mathrm{C}-5), 59.8(+, \mathrm{C}-2), 79.4,79.6$ $\left[\mathrm{C}_{\text {quat }}, \mathrm{C}\left(\mathrm{CH}_{3}\right)_{3}\right], 80.7\left[\mathrm{C}_{\text {quat }}, \mathrm{C}\left(\mathrm{CH}_{3}\right)_{3}\right], 153.8\left(\mathrm{C}_{\text {quat }}, \mathrm{NCO}_{2}\right), 172.4\left(\mathrm{C}_{\text {quat }}, \mathrm{C}-1\right)$; IR (film): nu(tilde) $=2980 \mathrm{~cm}^{-1}, 2931,2871,1717,1703,1398,1367,1174,1152 ; \mathrm{MS}(\mathrm{CI}), \mathrm{m} / z(\%): 332$ (12) $\left[\mathrm{M}+\mathrm{NH}_{4}^{+}\right], 314(100)\left[\mathrm{M}+\mathrm{H}^{+}\right]$. 
(2S,4S)-Propylproline hydrochloride $\mathrm{HCl} \cdot(2 S, 4 S)-(29):(2 S, 4 R)-16(20 \mathrm{mg}, 0.078 \mathrm{mmol})$ was

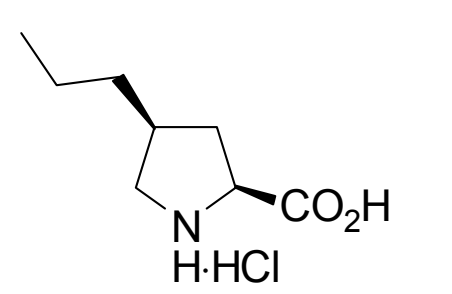
hydrogenated on $10 \% \mathrm{Pd} / \mathrm{C}(5 \mathrm{mg})$ in EtOAC $(2 \mathrm{~mL})$ at $20{ }^{\circ} \mathrm{C}$ under ambient pressure of hydrogen for $15 \mathrm{~h}$. The reaction mixture was filtered, solvent was removed under reduced pressure and the residue was treated with $2 \mathrm{~N} \mathrm{HCl}$ in EtOAc at $20^{\circ} \mathrm{C}$ for $1 \mathrm{~h}$. Solvent was removed and $(2 S, 4 S)-29$ was obtained as a an extremely hygroscopic colorless solid $(12 \mathrm{mg}, .79 \%) .[\alpha]_{20}^{D}-7.8(c=1.0$, $\left.\mathrm{CHCl}_{3}\right)$.

(2R,4R)-4-propylproline hydrochloride (2R,4R)-(29): Compound $(2 R, 4 R)-9 \quad(0.07$ g, 0.223 mmol) was heated with $6 \mathrm{M} \mathrm{HCl}(2 \mathrm{~mL})$ at $85^{\circ} \mathrm{C}$ for $2 \mathrm{~h}$. The reaction mixture was concentrated under reduced pressure. The traces of water was removed by codistillation with toluene $(2 \times 10$ $\mathrm{mL}$ ) and the residue was recrystallized thrice from $\mathrm{CH}_{2} \mathrm{Cl}_{2} / \mathrm{Et}_{2} \mathrm{O}$ to give after prolonged drying at 0.01 Torr $(2 R, 4 R)-(6)(0.034 \mathrm{~g}, 79 \%)$ as an extremely hygroscopic white solid. $[\alpha]_{24}^{\mathrm{D}} 7.7(c=$ $\left.0.88, \mathrm{CHCl}_{3}\right) ;{ }^{1} \mathrm{H}$ NMR $\left(250 \mathrm{MHz}, \mathrm{D}_{2} \mathrm{O}\right): \delta=0.83\left(\mathrm{~m}, 3 \mathrm{H}, 3^{\prime}-\mathrm{H}\right), 1.19-1.54\left(\mathrm{~m}, 4 \mathrm{H}, 1^{\prime}-\mathrm{H}, 2^{\prime}-\right.$ H), 1.70 (dddd, $J=12.8,12.8,10.0,10.0 \mathrm{~Hz}, 1 \mathrm{H} ; 4-\mathrm{H}), 2.26-2.49$ (m, $\left.1 \mathrm{H}, 3-\mathrm{H}_{\mathrm{a}}\right), 2.50-2.68$ (m, $\left.1 \mathrm{H}, 3-\mathrm{H}_{\mathrm{b}}\right), 2.95\left(\mathrm{dd}, J=10.9,10.9 \mathrm{~Hz}, 1 \mathrm{H}, 5-\mathrm{H}_{\mathrm{a}}\right), 3.50\left(\mathrm{dd}, J=10.9,8.3 \mathrm{~Hz}, 1 \mathrm{H}, 5-\mathrm{H}_{\mathrm{b}}\right), 4.35$ (dd, $J=8.9,8.9 \mathrm{~Hz}, 1 \mathrm{H}, 2-\mathrm{H}) ;{ }^{1} \mathrm{H}$ NMR $\left(250 \mathrm{MHz}, \mathrm{CD}_{3} \mathrm{OD}\right): \delta=0.99$ (t, $\left.J=7 \mathrm{~Hz}, 3 \mathrm{H}, 3^{\prime}-\mathrm{H}\right)$, 1.30-1.62 (m, 4 H, 1'-H, 2'-H), 1.74 (dddd, $J=11.7,11.7,10.2,10.2 \mathrm{~Hz}, 1 \mathrm{H} ; 4-\mathrm{H}), 2.33-2.68$ $\left(\mathrm{m}, 1 \mathrm{H}, 3-\mathrm{H}_{\mathrm{a}}\right), 2.65\left(\mathrm{ddd}, J=12.2,6.7,6.7 \mathrm{~Hz}, 1 \mathrm{H}, 3-\mathrm{H}_{\mathrm{b}}\right), 2.98(\mathrm{dd}, J=10.2,10.2 \mathrm{~Hz}, 1 \mathrm{H}, 5-$ $\left.\mathrm{H}_{\mathrm{a}}\right), 3.53\left(\mathrm{dd}, J=11.7,7.8 \mathrm{~Hz}, 1 \mathrm{H}, 5-\mathrm{H}_{\mathrm{b}}\right), 4.37(\mathrm{dd}, J=8.6,8.6 \mathrm{~Hz}, 1 \mathrm{H}, 2-\mathrm{H}) ;{ }^{13} \mathrm{C}$ NMR $(62.9$ $\left.\mathrm{MHz}, \mathrm{D}_{2} \mathrm{O}\right): \delta=14.1\left(+, \mathrm{C}^{\prime} 3^{\prime}\right), 21.5\left(-, \mathrm{C}^{\prime} 2^{\prime}\right), 34.6\left(-, \mathrm{C}^{\prime} 1^{\prime}\right), 35.3(-, \mathrm{C}-3), 38.8(+, \mathrm{C}-4), 51.6(-$,

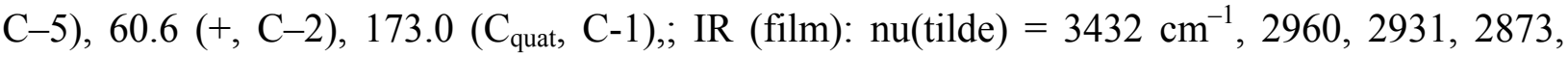
1736, 1389, 1252; MS (EI, $70 \mathrm{eV}), m / z(\%): 157(1)\left[\mathrm{M}^{+}\right], 112(100)\left[\mathrm{M}^{+}-\mathrm{HCO}_{2}\right], 87$ (4), $70(5)$ $\left[\mathrm{C}_{4} \mathrm{H}_{8} \mathrm{~N}^{+}\right], 69$ (11), 43 (15) $\left[\mathrm{C}_{3} \mathrm{H}_{7}^{+}\right], 41$ (18) $\left[\mathrm{C}_{3} \mathrm{H}_{5}{ }^{+}\right]$; HRMS (EI): calcd for $\mathrm{C}_{8} \mathrm{H}_{15} \mathrm{NO}_{2}$ : 157.1103; found 157.1103. 
Total hydrolysis of peptides (GP 1): Peptides were hydrolyzed with $6 \mathrm{M} \mathrm{HCl} / \mathrm{AcOH}(1: 1,0.5 \mathrm{~mL}$ per $1 \mathrm{mg}$ peptide) in sealed tube at $105^{\circ} \mathrm{C}$ over $24 \mathrm{~h}$. The solvent was then removed under reduced pressure and the residue was used for further derivatization.

Derivatisation with (S)-FDVA (GP 2): $0.4 \mathrm{mg}$ probe was dissolved in $0.5 \mathrm{~mL} \mathrm{H} \mathrm{H}_{2} \mathrm{O}, \mathrm{pH}$ of the solution was adjusted to 9 with $1 \mathrm{M} \mathrm{NaHCO}_{3}$ and $1 \%$ acetone solution of $(S)$-FDVA $(0.1 \mathrm{~mL})$ was added. The reaction mixture was stirred at $40{ }^{\circ} \mathrm{C}$ for $1 \mathrm{~h}$ and then $\mathrm{pH}$ was adjusted to $6-7$ with $0.1 \mathrm{M} \mathrm{HCl}$. After this, the reaction mixture was diluted with $1 \mathrm{~mL}$ of $\mathrm{MeCN}$ and was directly used for HPLC/MS experiments.

Determination of the absolute configuration of (4-PE)Pro residue in hormaomycin 3: The hydrogenated hormaomycin (ca. $0.5 \mathrm{mg}$ ) was hydrolyzed and, after derivatisation with $(S)$ FDVA, was used for HPLC/MS experiments. Detection: UV: $340 \mathrm{~nm}$, ESI-MS (positive) m/z: 437.7 - 438.7, ESI-MS (negative) $\mathrm{m} / \mathrm{z}: 435.7$ - 436.7; $(S)$-FDVA-(2S,4S)-29: $t_{\mathrm{R}}=14.77 \mathrm{~min}$, mix probe -1 peak; $(S)$-FDVA- $(2 R, 4 R)-29: t_{\mathrm{R}}=17.98$ min, mix probe -2 peaks.

\section{3. $\quad$ Attempts to prepare 4-(Z)-propenylproline starting from pyroglutamic acid}

N-Boc tert-Butyl (2S)-4-(1'-hydroxypropyl)pyroglutamate (S)-(38): A solution of LiHMDS in

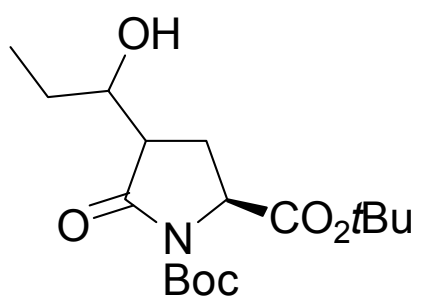

$\mathrm{g}, 5.01 \mathrm{mmol})$ in THF $(40 \mathrm{~mL})$ at $-78^{\circ} \mathrm{C}$ and the resultant mixture was stirred at the same temperature for a further $1 \mathrm{~h}$. A solution of propionaldehyde $(0.42 \mathrm{~mL}, 5.79 \mathrm{mmol})$ and $\mathrm{BF}_{3} \cdot \mathrm{Et}_{2} \mathrm{O}$ $(0.73 \mathrm{~mL}, 5.76 \mathrm{mmol})$ in THF $(18 \mathrm{~mL})$ was then added and stirring continued at the same 
temperature for $75 \mathrm{~min}$. The reaction was then quenched with saturated $\mathrm{NH}_{4} \mathrm{Cl}(30 \mathrm{~mL})$ and the mixture was diluted with $\mathrm{Et}_{2} \mathrm{O}(250 \mathrm{~mL})$. The organic layer was separated, washed with water (3 $\times 40 \mathrm{~mL}), 0.5 \mathrm{M} \mathrm{H}_{2} \mathrm{SO}_{4}(3 \times 40 \mathrm{~mL})$, water $(3 \times 40 \mathrm{~mL})$, brine $(2 \times 30 \mathrm{~mL})$, dried, filtered and concentrated under reduced pressure. The residue was separated by column chromatography (EtOAc/hexanes 1:3) to give one pure possible diastereomer $(0.785 \mathrm{~g}, 46 \%)$ as a colorless oil and the inseparable mixture of two another diastereomers in ratio $1: 3[0.55 \mathrm{~g}, 78 \%$ overall yield of (S)-38]. No attempts to elucidate the configuration of these products were made. The pure diastereomer: $R_{\mathrm{f}}=0.26$ EtOAc/hexanes $1: 3 ;{ }^{1} \mathrm{H}$ NMR $\left(250 \mathrm{MHz}, \mathrm{CDCl}_{3}\right): \delta=0.98(\mathrm{t}, J=$ $\left.7.3 \mathrm{~Hz}, 3 \mathrm{H}, 3^{\prime}-\mathrm{H}\right), 1.48$ [s, $\left.9 \mathrm{H}, \mathrm{C}\left(\mathrm{CH}_{3}\right)_{3}\right], 1.50$ [s, $\left.9 \mathrm{H}, \mathrm{C}\left(\mathrm{CH}_{3}\right)_{3}\right], 1.84-2.12(\mathrm{~m}, 2 \mathrm{H}, 3-\mathrm{H}), 2.63$ (dt, $J=12.2,8.7 \mathrm{~Hz}, 1 \mathrm{H}, 4-\mathrm{H}), 3.59-3.70\left(\mathrm{~m}, 1 \mathrm{H}, 1^{\prime}-\mathrm{H}\right), 4.24(\mathrm{~s}, \mathrm{OH}), 4.44(\mathrm{~d}, J=8.0 \mathrm{~Hz}, 1 \mathrm{H}$, 2-H); The signal of 2 '- $\mathrm{H}$ was masked by absorptions of $\mathrm{C}\left(\mathrm{CH}_{3}\right)_{3} ;{ }^{13} \mathrm{C} \mathrm{NMR}\left(62.9 \mathrm{MHz}, \mathrm{CDCl}_{3}\right)$ : $\delta=8.8\left(+, \mathrm{C}^{\prime} 3^{\prime}\right), 24.9\left(-, \mathrm{C}-2^{\prime}\right), 26.7(-, \mathrm{C}-3), 27.4\left[+, \mathrm{C}\left(\mathrm{CH}_{3}\right)_{3}\right], 27.5\left[+, \mathrm{C}\left(\mathrm{CH}_{3}\right)_{3}\right], 45.5(+, \mathrm{C}-$ 4), $57.4(+, \mathrm{C}-2), 72.7\left(+, \mathrm{C}-1^{\prime}\right), 82.1\left[\mathrm{C}_{\text {quat }}, \mathrm{C}\left(\mathrm{CH}_{3}\right)_{3}\right], 83.2\left[\mathrm{C}_{\text {quat }}, \mathrm{C}\left(\mathrm{CH}_{3}\right)_{3}\right], 148.5\left(\mathrm{C}_{\text {quat }}, \mathrm{NCO}_{2}\right)$, 169.6 ( $\left.\mathrm{C}_{\text {quat }}, \mathrm{C}-1\right), 176.1\left(\mathrm{C}_{\text {quat }}, \mathrm{C}-5\right)$. The mixture of diastereomers: $R_{\mathrm{f}}=0.26$ EtOAc/hexanes 1:3; ${ }^{1} \mathrm{H}$ NMR $\left(250 \mathrm{MHz}, \mathrm{CDCl}_{3}\right): \delta=0.99\left(\mathrm{t}, J=7.3 \mathrm{~Hz}, 3 \mathrm{H}, 3^{\prime}-\mathrm{H}\right), 1.15-1.30\left(\mathrm{~m}, 0.4 \mathrm{H}, 2^{\prime}-\right.$ $\left.\mathrm{H}_{\mathrm{a}}\right), 1.48\left[\mathrm{~s}, 9 \mathrm{H}, \mathrm{C}\left(\mathrm{CH}_{3}\right)_{3}\right], 1.50\left[\mathrm{~s}, 9 \mathrm{H}, \mathrm{C}\left(\mathrm{CH}_{3}\right)_{3}\right], 1.85-2.10(\mathrm{~m}, 0.6 \mathrm{H}, 3-\mathrm{H}), 2.31-2.80(\mathrm{~m}$, $2 \mathrm{H}, 3-\mathrm{H}, 4-\mathrm{H}), 3.49-3.70\left(\mathrm{~m}, 1 \mathrm{H}, 1^{\prime}-\mathrm{H}\right), 4.12(\mathrm{~s}, \mathrm{OH}), 4.35$ (dd, $\left.J=8.0,8.0 \mathrm{~Hz}, 0.7 \mathrm{H}, 2-\mathrm{H}\right)$, $4.43(\mathrm{dd} ; J=1.2,10.4 \mathrm{~Hz}, 0.3 \mathrm{H}, 2-\mathrm{H})$; The signal of 2'-H was partially masked by absorptions of $\mathrm{C}\left(\mathrm{CH}_{3}\right)_{3} ;{ }^{13} \mathrm{C} \mathrm{NMR}\left(62.9 \mathrm{MHz}, \mathrm{CDCl}_{3}\right): \delta=8.5,9.0\left(+, \mathrm{C}-3^{\prime}\right), 18.5,21.6\left(-, \mathrm{C}-2^{\prime}\right), 24.6(-, \mathrm{C}-3)$, 27.0, $27.1\left[+, \mathrm{C}\left(\mathrm{CH}_{3}\right)_{3}\right], 27.7,27.8\left[+, \mathrm{C}\left(\mathrm{CH}_{3}\right)_{3}\right], 46.7,47.2(+, \mathrm{C}-4), 57.8,57.9(+, \mathrm{C}-2), 70.0$, $72.9\left(+, \mathrm{C}^{-1}{ }^{\prime}\right), 82.1,82.3\left[\mathrm{C}_{\text {quat }}, \mathrm{C}\left(\mathrm{CH}_{3}\right)_{3}\right], 83.2,83.8\left[\mathrm{C}_{\text {quat }}, \mathrm{C}\left(\mathrm{CH}_{3}\right)_{3}\right], 148.9,149.0\left(\mathrm{C}_{\text {quat }}\right.$, $\left.\mathrm{NCO}_{2}\right), 169.8,170.5\left(\mathrm{C}_{\text {quat }}, \mathrm{C}-1\right), 174.6,175.9$ ( $\left.\mathrm{C}_{\text {quat }}, \mathrm{C}-5\right)$. 
tert-Butyl N-Boc 2-(S)-4-(E)-propylidenepyroglutamate (S)-(39): $\mathrm{Et}_{3} \mathrm{~N}$ (4.00 mL, $28.56 \mathrm{mmol}$ ) followed by mesyl chloride $(0.23 \mathrm{~mL}, 2.97 \mathrm{mmol})$ were added

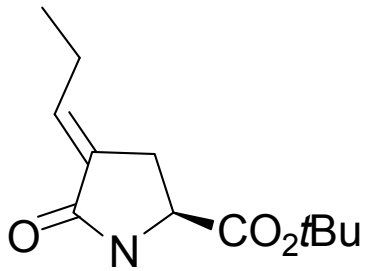

Boc dropwise to a stirred ice-cold solution of the mixture of diastereomers of $(S)-38(0.55 \mathrm{~g}, 1.60 \mathrm{mmol})$ in $\mathrm{CH}_{2} \mathrm{Cl}_{2}(20 \mathrm{~mL})$ and the mixture was then allowed to warm to $20^{\circ} \mathrm{C}$. Stirring continued for a further $48 \mathrm{~h}$ and the reaction was then quenched with water $(3 \mathrm{~mL})$. EtOAc $(80 \mathrm{~mL})$ was added and the organic layer was washed with water $(4 \times 20 \mathrm{~mL})$ and brine $(2 \times 20 \mathrm{~mL})$, dried, filtered and concentrated under reduced pressure. The resultant oily residue was identical with the one obtained by reaction of the pure diastereomer of $(S)-38(0.785 \mathrm{~g}, 2.29 \mathrm{mmol})$ with $\mathrm{Et}_{3} \mathrm{~N}(5.8 \mathrm{~mL}, 41.44 \mathrm{mmol})$ and mesyl chloride $(0.32 \mathrm{~mL}, 4.13 \mathrm{mmol})$ in $\mathrm{CH}_{2} \mathrm{Cl}_{2}(20 \mathrm{~mL})$. Both portions were combined and separated by column chromatography (twice: first EtOAc/hexanes 3:7, then EtOAc/hexanes 1:3) to give $(E)-2(0.76 \mathrm{~g}, 60 \%)$ as a faint tan solid and $(Z)$-isomer of (S)-39 (55 mg, 4\%). The configuration of the main product was confirmed with NOESY experiment.

$(S)-39: R_{\mathrm{f}}=0.55 \mathrm{EtOAc} /$ hexanes 3:7; ${ }^{1} \mathrm{H} \mathrm{NMR}\left(250 \mathrm{MHz}, \mathrm{CDCl}_{3}\right): \delta=1.01(\mathrm{t}, J=7.5 \mathrm{~Hz}, 3 \mathrm{H}$, 3'-H), $1.42\left[\mathrm{~s}, 9 \mathrm{H}, \mathrm{C}\left(\mathrm{CH}_{3}\right)_{3}\right], 1.45\left[\mathrm{~s}, 9 \mathrm{H}, \mathrm{C}\left(\mathrm{CH}_{3}\right)_{3}\right], 2.11$ (dddd, $J=7.5,7.5,7.5,7.5,7.5 \mathrm{~Hz}$, $\left.2 \mathrm{H}, 2^{\prime}-\mathrm{H}\right), 2.42-2.54\left(\mathrm{~m}, 1 \mathrm{H}, 3-\mathrm{H}_{\mathrm{a}}\right), 2.89-3.09\left(\mathrm{~m}, 1 \mathrm{H}, 3-\mathrm{H}_{\mathrm{b}}\right), 4.45(\mathrm{dd}, J=7.8,3.5 \mathrm{~Hz}, 1 \mathrm{H}, 2-$ $\mathrm{H}), 6.57-6.70\left(\mathrm{~m}, 1 \mathrm{H}, 1^{\prime}-\mathrm{H}\right) ;{ }^{13} \mathrm{C} \mathrm{NMR}\left(62.9 \mathrm{MHz}, \mathrm{CDCl}_{3}\right): \delta=12.6\left(+, \mathrm{C}-3^{\prime}\right), 22.6\left(-, \mathrm{C}-2^{\prime}\right)$, $25.5(-, \mathrm{C}-3), 27.7\left[+, \mathrm{C}\left(\mathrm{CH}_{3}\right)_{3}\right], 27.8\left[+, \mathrm{C}\left(\mathrm{CH}_{3}\right)_{3}\right], 56.4(+, \mathrm{C}-2), 82.0\left[\mathrm{C}_{\text {quat }}, \mathrm{C}\left(\mathrm{CH}_{3}\right)_{3}\right], 83.0$ $\left[\mathrm{C}_{\text {quat }}, \mathrm{C}\left(\mathrm{CH}_{3}\right)_{3}\right], 127.9\left(\mathrm{C}_{\text {quat }}, \mathrm{C}-4\right), 140.2\left(+, \mathrm{C}-1^{\prime}\right), 149.8\left(\mathrm{C}_{\text {quat }}, \mathrm{NCO}_{2}\right), 166.2\left(\mathrm{C}_{\text {quat }}, \mathrm{C}-1\right)$, $170.2\left(\mathrm{C}_{\text {quat }}, \mathrm{C}-5\right)$.

(Z)-isomer: $R_{\mathrm{f}}=0.75$ EtOAc/hexanes $3: 7 ; \delta=1.01\left(\mathrm{t}, J=7.5 \mathrm{~Hz}, 3 \mathrm{H}, 3^{\prime}-\mathrm{H}\right), 1.45[\mathrm{~s}, 9 \mathrm{H}$, $\left.\mathrm{C}\left(\mathrm{CH}_{3}\right)_{3}\right], 1.51$ [s, $\left.9 \mathrm{H}, \mathrm{C}\left(\mathrm{CH}_{3}\right)_{3}\right], 2.43-2.58\left(\mathrm{~m}, 1 \mathrm{H}, 3-\mathrm{H}_{\mathrm{a}}\right), 2.75$ (dddd, $J=7.5,7.5,7.5,7.5$, $\left.7.5 \mathrm{~Hz}, 2 \mathrm{H}, 2^{\prime}-\mathrm{H}\right), 2.89-3.09$ (m, $\left.1 \mathrm{H}, 3-\mathrm{H}_{\mathrm{b}}\right), 4.42$ (dd, J= 7.8, 3.5 Hz, $\left.1 \mathrm{H}, 2-\mathrm{H}\right), 5.92-6.06$ (m, $\left.1 \mathrm{H}, 1^{\prime}-\mathrm{H}\right) ;{ }^{13} \mathrm{C} \mathrm{NMR}\left(62.9 \mathrm{MHz}, \mathrm{CDCl}_{3}\right): \delta=13.7\left(+, \mathrm{C}-3^{\prime}\right), 20.6\left(-, \mathrm{C}-2^{\prime}\right), 27.8\left[+, \mathrm{C}^{\prime}\left(\mathrm{CH}_{3}\right)_{3}\right]$, 
$27.9\left[+, \mathrm{C}\left(\mathrm{CH}_{3}\right)_{3}\right], 29.1(-, \mathrm{C}-3), 56.5(+, \mathrm{C}-2), 82.0\left[\mathrm{C}_{\text {quat }}, \mathrm{C}\left(\mathrm{CH}_{3}\right)_{3}\right], 83.0\left[\mathrm{C}_{\text {quat }}, \mathrm{C}\left(\mathrm{CH}_{3}\right)_{3}\right]$, $126.0\left(\mathrm{C}_{\text {quat }}, \mathrm{C}-4\right), 144.8\left(+, \mathrm{C}-1^{\prime}\right), 150.1\left(\mathrm{C}_{\text {quat }}, \mathrm{NCO}_{2}\right), 165.9\left(\mathrm{C}_{\text {quat }}, \mathrm{C}-1\right), 170.2\left(\mathrm{C}_{\text {quat }}, \mathrm{C}-5\right)$.

N-Boc tert-Butyl (2S,4S)-4-(E)-(1'-propenyl)pyroglutamate $(2 S, 4 S)-(\mathbf{4 0})$ and $N$-Boc tert-Butyl (2S,4R)-4-(E)-(1'-propenyl)pyroglutamate $(2 S, 4 R)-(\mathbf{4 0})$ : A solution of LiHMDS in THF/HMPT was obtained by addition of a $2.47 \mathrm{M} \mathrm{BuLi}$ solution in hexanes $(0.54 \mathrm{~mL}, 1.33 \mathrm{mmol})$ to a solution of HMDS $(0.33 \mathrm{~mL}, 1.56 \mathrm{mmol})$ and HMPT $(0.356 \mathrm{~g}, 1.99 \mathrm{mmol})$ in THF $(5 \mathrm{~mL})$ at $78^{\circ} \mathrm{C}$ followed by stirring of the resultant mixture at $4{ }^{\circ} \mathrm{C}$ for $15 \mathrm{~min}$. It was then added dropwise to a solution of $(E)-2(0.375 \mathrm{~g}, 1.15 \mathrm{mmol})$ in THF $(5 \mathrm{~mL})$ at $-78{ }^{\circ} \mathrm{C}$ within $20 \mathrm{~min}$ and the mixture was stirred at the same temperature for a further $1 \mathrm{~h}$. A solution of 2,6-di-tertbutylphenol $(0.713 \mathrm{~g}, 3.46 \mathrm{mmol})$ in THF $(1 \mathrm{~mL})$ was then added for $10 \mathrm{~min}$. After that, a saturated aqueous $\mathrm{NH}_{4} \mathrm{Cl}$ (ca. $8 \mathrm{~mL}$ ) was added and the mixture was allowed to warm to $20{ }^{\circ} \mathrm{C}$ and diluted with $\mathrm{Et}_{2} \mathrm{O}(50 \mathrm{~mL})$. The organic layer was separated, washed with water $(3 \times 40$ $\mathrm{mL}), 0.5 \mathrm{M} \mathrm{H}_{2} \mathrm{SO}_{4}(3 \times 40 \mathrm{~mL})$, water $(3 \times 40 \mathrm{~mL})$, brine $(2 \times 30 \mathrm{~mL})$, dried, filtered and concentrated under reduced pressure. The residue containing the mixture of diastereomers and 2,6-di-tert-butylphenol was separated by column chromatography (twice, EtOAc/hexanes 1:3) to give $(2 S, 4 S)-\mathbf{4 0}(0.140 \mathrm{~g}, 37 \%)$ as a faint yellow oil and $(2 S, 4 R)-\mathbf{4 0}(70 \mathrm{mg}, 19 \%)$ as a colorless solid. (2S,4S)-40 contained a little impurity of diastereomer (according to TLC; it was unobservable in ${ }^{1} \mathrm{H}$ NMR spectrum) and attempt to purify it by additional column chromatography was made. As the $(2 S, 4 S)$-isomer isomerized to another diastereomer only material with essentially the same quality $(70 \mathrm{mg})$ together with $(2 S, 4 R)-40(40 \mathrm{mg})$ and the mixed fraction $(25 \mathrm{mg})$ were obtained. 
$(2 S, 4 R)-40:[\alpha]_{24}^{\mathrm{D}} 7.1\left(c=0.31, \mathrm{CHCl}_{3}\right) ; R_{\mathrm{f}}=0.42$ EtOAc/hexanes $1: 3 ;{ }^{1} \mathrm{H}$ NMR $(250 \mathrm{MHz}$,

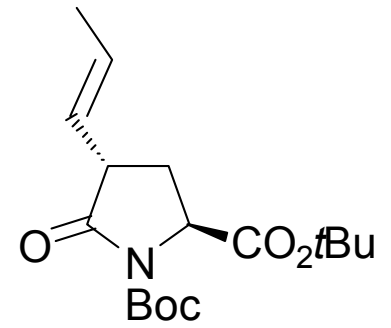

$(2 S, 4 S)-40$ $\left.\mathrm{CDCl}_{3}\right): \delta=1.48\left[\mathrm{~s}, 9 \mathrm{H}, \mathrm{C}\left(\mathrm{CH}_{3}\right)_{3}\right], 1.50\left[\mathrm{~s}, 9 \mathrm{H}, \mathrm{C}\left(\mathrm{CH}_{3}\right)_{3}\right], 1.71(\mathrm{dd}, J$ = 6.3, $\left.1.3 \mathrm{~Hz}, 3 \mathrm{H}, 3^{\prime}-\mathrm{H}\right), 2.04-2.31(\mathrm{~m}, 2 \mathrm{H}, 3-\mathrm{H}), 3.19-3.24(\mathrm{~m}, 1 \mathrm{H}$, 4-H), 4.44 (dd, $J=9.0,1.8 \mathrm{~Hz}, 1 \mathrm{H}, 2-\mathrm{H}), 5.47(\mathrm{dd}, J=9.0,1.3 \mathrm{~Hz}$, $\left.1 \mathrm{H}, 1^{\prime}-\mathrm{H}\right), 5.63\left(\mathrm{dq}, J=1.3,6.3 \mathrm{~Hz}, 1 \mathrm{H}, 2{ }^{\prime}-\mathrm{H}\right) ;{ }^{13} \mathrm{C} \mathrm{NMR}(62.9$ $\left.\mathrm{MHz}, \mathrm{CDCl}_{3}\right): \delta=17.9\left(+, \mathrm{C}-3^{\prime}\right), 27.77\left[+, \mathrm{C}\left(\mathrm{CH}_{3}\right)_{3}\right], 27.79\left[+, \mathrm{C}\left(\mathrm{CH}_{3}\right)_{3}\right], 28.7(-, \mathrm{C}-3), 44.7(+$, C-4), $57.5(+, \mathrm{C}-2), 82.2\left[\mathrm{C}_{\text {quat }}, \mathrm{C}\left(\mathrm{CH}_{3}\right)_{3}\right], 83.1\left[\mathrm{C}_{\text {quat }}, \mathrm{C}\left(\mathrm{CH}_{3}\right)_{3}\right], 126.0\left(+, \mathrm{C}-2^{\prime}\right), 129.7\left(+, \mathrm{C}^{\prime} 1^{\prime}\right)$, 149.4 ( $\left.\mathrm{C}_{\text {quat }}, \mathrm{NCO}_{2}\right), 170.1\left(\mathrm{C}_{\text {quat }}, \mathrm{C}-1\right), 173.6\left(\mathrm{C}_{\text {quat }}, \mathrm{C}-5\right)$; IR $(\mathrm{KBr}): \mathrm{nu}($ tilde $)=2979 \mathrm{~cm}^{-1}, 2934$, $1781,1743,1708,1457,1367,1296,1221,1155 ;$ MS $(\mathrm{ESI})$, positive $m / z(\%)=348(75)[\mathrm{M}+$ $\left.\mathrm{Na}^{+}\right]$.

$(2 S, 4 S)-40: R_{\mathrm{f}}=0.38 \mathrm{EtOAc} /$ hexanes $1: 3 ;{ }^{1} \mathrm{H} \mathrm{NMR}\left(250 \mathrm{MHz}, \mathrm{CDCl}_{3}\right): \delta=1.47[\mathrm{~s}, 9 \mathrm{H}$,

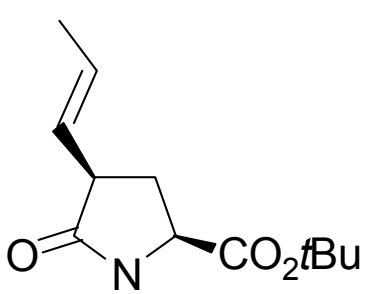

Boc $\left.\mathrm{C}\left(\mathrm{CH}_{3}\right)_{3}\right], 1.50\left[\mathrm{~s}, 9 \mathrm{H}, \mathrm{C}\left(\mathrm{CH}_{3}\right)_{3}\right], 1.66\left(\mathrm{dd}, J=5.0,1.3 \mathrm{~Hz}, 3 \mathrm{H}, 3^{\prime}-\mathrm{H}\right)$, $1.86\left(\mathrm{dd}, J=12.5,6.5 \mathrm{~Hz}, 1 \mathrm{H}, 3-\mathrm{H}_{\mathrm{a}}\right), 2.53(\mathrm{ddd}, J=13.3,6.5 \mathrm{~Hz}, 1 \mathrm{H}, 3-$ $\left.\mathrm{H}_{\mathrm{b}}\right), 3.09-3.23(\mathrm{~m}, 1 \mathrm{H}, 4-\mathrm{H}), 4.38$ (dd, $\left.J=9.0,5.8 \mathrm{~Hz}, 1 \mathrm{H}, 2-\mathrm{H}\right), 5.30$ $5.52\left(\mathrm{~m}, 1 \mathrm{H}, 1^{\prime}-\mathrm{H}\right), 5.52-5.72\left(\mathrm{~m}, 1 \mathrm{H}, 2^{\prime}-\mathrm{H}\right) ;{ }^{13} \mathrm{C}$ NMR $(62.9 \mathrm{MHz}$, $\left.\mathrm{CDCl}_{3}\right): \delta=17.8\left(+, \mathrm{C}^{\prime} 3^{\prime}\right), 27.7\left[+, 2 \times \mathrm{C}\left(\mathrm{CH}_{3}\right)_{3}\right], 28.6(-, \mathrm{C}-3), 45.7(+, \mathrm{C}-4), 58.0(+, \mathrm{C}-2)$, $82.0\left[\mathrm{C}_{\text {quat }}, \mathrm{C}\left(\mathrm{CH}_{3}\right)_{3}\right], 83.1\left[\mathrm{C}_{\text {quat }}, \mathrm{C}\left(\mathrm{CH}_{3}\right)_{3}\right], 126.3\left(+, \mathrm{C}-2^{\prime}\right), 129.3\left(+, \mathrm{C}-1^{\prime}\right), 149.4\left(\mathrm{C}_{\text {quat }}, \mathrm{NCO}_{2}\right)$, $170.1\left(\mathrm{C}_{\text {quat }}, \mathrm{C}-1\right), 173.6\left(\mathrm{C}_{\text {quat }}, \mathrm{C}-5\right)$. 
2.4. Synthesis of $\mathrm{N}-\mathrm{Boc}$ protected 4-(Z)-propenylproline and dideutero-4-(Z)propenylproline starting from 4-hydroxyproline

N-Boc Methyl (S)-allylglicinate (2S)-42: A vigorously stirred suspension of zinc dust (0.926 g,

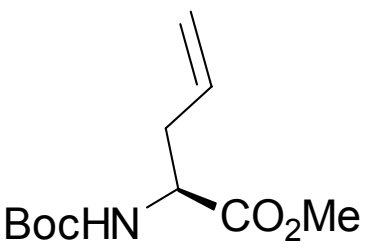
$14.16 \mathrm{mmol})$ in anhydrous THF $(2 \mathrm{~mL})$ contained $\mathrm{C}_{2} \mathrm{H}_{4} \mathrm{Br}_{2}(0.09 \mathrm{~mL}$, $1.04 \mathrm{mmol}$ ) was heated with heat gun till boiling started and then cooled to $20^{\circ} \mathrm{C}$. After this, $\mathrm{TMSCl}(0.1 \mathrm{~mL})$ was added and the suspension was stirred for a further $10 \mathrm{~min}$. A solution of $N$-Boc protected $4-(2 S, 4 R S)$-iodoproline $(2 S)-\mathbf{4 1}(1.30$ $\mathrm{g}, 3.66 \mathrm{mmol})$ in THF $(2.5 \mathrm{~mL})$ was then added to the mixture, the temperature was increased to $30-35^{\circ} \mathrm{C}$ and stirring continued at this temperature for $16 \mathrm{~h}$. After this, the mixture was filtered, concentrated under reduced pressure and distributed between $0.5 \mathrm{M} \mathrm{H}_{2} \mathrm{SO}_{4}(20 \mathrm{~mL})$ and $\mathrm{Et}_{2} \mathrm{O}(40$ $\mathrm{mL})$. The organic layer was separated, washed with water $(3 \times 10 \mathrm{~mL})$, brine $(2 \times 10 \mathrm{~mL})$, dried, filtered and concentrated under reduced pressure. The residue was purified by column chromatography (EtOAc/hexanes $\left.1: 4, R_{\mathrm{f}}=0.30\right)$ to give the title compound $(0.56 \mathrm{~g}, 67 \%)$ as a colorless liquid. The spectral data were in accordance with literature. $[\alpha]_{20}^{D} 19.1(c=0.98$, $\left.\mathrm{CHCl}_{3}\right)\left[\right.$ lit.. $\left.{ }^{[49]}[\alpha]_{20}^{D} 18.8\left(c=1.0, \mathrm{CHCl}_{3}\right)\right]$.

N-Boc tert-Bityl (2S,3R)-4-hydroxyprolinate $(2 S, 4 R)-(44)$ : Triethylbenzylammonium chloride $\mathrm{HO}$<smiles>CCCCC1CCCN1</smiles>
Boc (7.78 g, $34.16 \mathrm{mmol})$ was added to a vigorously stirred suspension of dried $\mathrm{K}_{2} \mathrm{CO}_{3}(123.0 \mathrm{~g}, \quad 0.888 \mathrm{~mol})$ in a solution of $N$-Boc protected hydroxyproline $(2 S, 4 R)-43(7.9 \mathrm{~g}, 34.16 \mathrm{mmol})$ and tert-butyl bromide $(185 \mathrm{~mL}, 1.639 \mathrm{~mol})$ in DMA $(256 \mathrm{~mL})$ and the mixture was stirred at $55-60{ }^{\circ} \mathrm{C}$ for $48 \mathrm{~h}$. It was then poured in water $(2 \mathrm{~L})$ and the resultant suspension was extracted with $\mathrm{Et}_{2} \mathrm{O}(500 \mathrm{~mL})$. The organic layer was washed with water $(8 \times 100 \mathrm{~mL})$, brine $(2 \times 50 \mathrm{~mL})$, dried, filtered and concentrated under reduced pressure. The residue was purified by column chromatography (EtOAc/hexanes 1:2, $R_{\mathrm{f}}=0.32$ ) and finally by two recrystallizations from hexanes to give 
$(2 S, 4 R)-44(6.40 \mathrm{~g}, 65 \%)$ as a colorless solid. M.p. $64-67^{\circ} \mathrm{C}\left(\right.$ lit.: ${ }^{[55]}$ m.p. $\left.62-64{ }^{\circ} \mathrm{C}\right) ;[\alpha]_{20}^{D}=-$ $58.0\left(c=0.84, \mathrm{CHCl}_{3}\right)\left[\right.$ lit.: $\left.{ }^{[55]}[\alpha]_{20}^{D}=-64.8\left(c=1.3, \mathrm{CHCl}_{3}\right)\right]$. The spectral data were in accordance with literature.

N-Boc tert-Bityl (2S,3R)-4-mesyloxyprolinate (2S,3R)-(45): Mesyl chloride (1.97 mL, 25.40 mmol) was added to a solution of $(2 S, 3 R)-44(5.60 \mathrm{~g}, 19.49 \mathrm{mmol})$ and $\mathrm{MsO}$

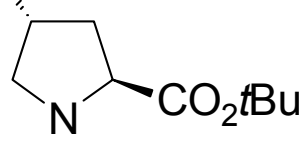

Boc TEA (4.08 $\mathrm{mL}, 29.15 \mathrm{mmol})$ in $\mathrm{CH}_{2} \mathrm{Cl}_{2}(40 \mathrm{~mL})$ at $-20{ }^{\circ} \mathrm{C}$ for $5 \mathrm{~min}$ and the mixture was allowed to warm to $4{ }^{\circ} \mathrm{C}$ for $2 \mathrm{~h}$. After this, the reaction was quenched with saturated $\mathrm{NaHCO}_{3}$ (ca. $20 \mathrm{~mL}$ ) and the mixture was diluted with $\mathrm{Et}_{2} \mathrm{O}(200 \mathrm{~mL})$. The organic layer was separated and washed with saturated $\mathrm{NaHCO}_{3}(4 \times 40 \mathrm{~mL})$, water $(2 \times 40 \mathrm{~mL})$, brine $(2 \times 40 \mathrm{~mL})$, dried, filtered and concentrated under reduced pressure. The residue was recrystallized from $\mathrm{Et}_{2} \mathrm{O}$ /pentane to give $(2 S, 3 R)-45(6.09 \mathrm{~g}, 86 \%)$ as a colorless solid. $R_{\mathrm{f}}=0.53$ EtOAc/hexanes 1:2. M.p. $67-69^{\circ} \mathrm{C}$ (lit.: ${ }^{[56]}$ m.p. $\left.78-79{ }^{\circ} \mathrm{C}\right) ;[\alpha]_{20}^{D}-48.3\left(c=1.04, \mathrm{CHCl}_{3}\right)\left[\right.$ lit.. $\left.{ }^{[56]}[\alpha]_{20}^{D}-49.6(c=1.40, \mathrm{MeOH})\right]$; ${ }^{1} \mathrm{H} \mathrm{NMR}\left(250 \mathrm{MHz}, \mathrm{CDCl}_{3}\right): \delta=1.43\left[\mathrm{~s}, 9 \mathrm{H}, \mathrm{C}\left(\mathrm{CH}_{3}\right)_{3}\right], 1.44\left[\mathrm{~s}, 9 \mathrm{H}, \mathrm{C}\left(\mathrm{CH}_{3}\right)_{3}\right], 2.19(\mathrm{ddd}, J=$ $\left.14.3,5.8,5.8 \mathrm{~Hz}, 1 \mathrm{H}, 3-\mathrm{H}_{\mathrm{a}}\right), 2.43-2.71\left(\mathrm{~m}, 1 \mathrm{H}, 3-\mathrm{H}_{\mathrm{b}}\right), 3.03(\mathrm{~s}, 3 \mathrm{H}, \mathrm{Me}), 3.62-3.91(\mathrm{~m}, 2 \mathrm{H}, 5-$ $\mathrm{H}), 4.18-4.38(\mathrm{~m}, 1 \mathrm{H}, 2-\mathrm{H}), 5.15-5.23(\mathrm{~m}, 1 \mathrm{H}, 4-\mathrm{H}) ;{ }^{13} \mathrm{C}$ NMR $\left(62.9 \mathrm{MHz}, \mathrm{CDCl}_{3}\right): \delta=27.9$ $\left[+, \mathrm{C}\left(\mathrm{CH}_{3}\right)_{3}\right], 28.2\left[+, \mathrm{C}\left(\mathrm{CH}_{3}\right)_{3}\right], 36.2,37.5(-, \mathrm{C}-3), 38.7\left(+, \mathrm{SO}_{2} \mathrm{Me}\right), 52.1,52.4(-, \mathrm{C}-5), 57.9$ (+, C-2), 77.9, $78.2(+, \mathrm{C}-4), 80.5,80.6\left[\mathrm{C}_{\text {quat }}, \mathrm{C}\left(\mathrm{CH}_{3}\right)_{3}\right], 81.7\left[\mathrm{C}_{\text {quat }}, \mathrm{C}\left(\mathrm{CH}_{3}\right)_{3}\right], 153.5\left(\mathrm{C}_{\text {quat }}\right.$, $\left.\mathrm{NCO}_{2}\right), 171.2\left(\mathrm{C}_{\text {quat }}, \mathrm{C}-1\right)$.

$N$-Boc tert-Bityl (2S,3S)-4-cyanoprolinate $(2 S, 4 S)-(\mathbf{4 6})$ : A solution of the mesylate $(2 S, 4 R)-45$<smiles>CCCCOC(=O)C1C[C@@H](C#N)CN1</smiles>

(5.50 g, $15.05 \mathrm{mmol})$ and tetrabutylammonium cyanide $(13.30 \mathrm{~g}, 49.55$ mmol) in DMF $(25 \mathrm{~mL})$ was heated at $60-65^{\circ} \mathrm{C}$ for $12 \mathrm{~h}$. The mixture was then diluted with $\mathrm{Et}_{2} \mathrm{O}(150 \mathrm{~mL})$ and washed with water $(7 \times 40 \mathrm{~mL})$, 
brine $(2 \times 10 \mathrm{~mL})$, dried and concentrated under reduced pressure. The residue was purified by column chromatography (EtOAc/hexanes $\left.1: 2.5, R_{\mathrm{f}}=0.65\right)$ to give oil which was triturated with pentane to give $(2 S, 4 S)-46(2.52 \mathrm{~g}, 57 \%)$ as a colorless solid. M.p. $62-64{ }^{\circ} \mathrm{C} ;{ }^{1} \mathrm{H}$ NMR $(250$ $\left.\mathrm{MHz}, \mathrm{CDCl}_{3}\right): \delta=1.42,1.45\left[\mathrm{~s}, 9 \mathrm{H}, \mathrm{C}\left(\mathrm{CH}_{3}\right)_{3}\right], 1.47,1.49\left[\mathrm{~s}, 9 \mathrm{H}, \mathrm{C}\left(\mathrm{CH}_{3}\right)_{3}\right], 2.19(\mathrm{ddd}, J=13.8$, 5.8, 5.8 Hz, $\left.1 \mathrm{H}, 3-\mathrm{H}_{\mathrm{a}}\right), 2.53-2.75\left(\mathrm{~m}, 1 \mathrm{H}, 3-\mathrm{H}_{\mathrm{b}}\right), 3.08\left(\mathrm{ddd}, J=7.5,7.5,7.5 \mathrm{~Hz}, 1 \mathrm{H}, 5-\mathrm{H}_{\mathrm{a}}\right)$, 3.55-3.70 (m, $\left.1 \mathrm{H}, 5-\mathrm{H}_{\mathrm{b}}\right), 3.87-4.01(\mathrm{~m}, 1 \mathrm{H}, 4-\mathrm{H}), 4.19,4.22(2 \times \mathrm{dd}, J=11.0,5.8 \mathrm{~Hz}, 1 \mathrm{H}, 2-$

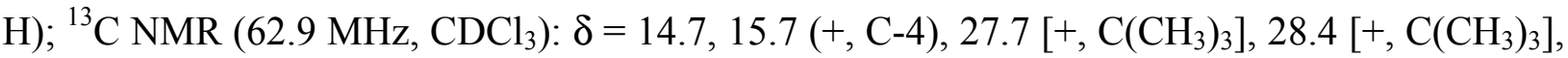

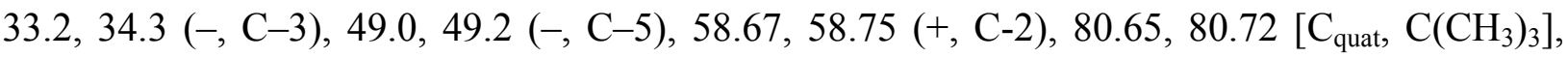
$81.2\left[\mathrm{C}_{\text {quat }}, \mathrm{C}\left(\mathrm{CH}_{3}\right)_{3}\right], 119.3\left(\mathrm{C}_{\text {quat }}, \mathrm{CN}\right), 153.0,153.2\left(\mathrm{C}_{\text {quat }}, \mathrm{NCO}_{2}\right), 170.1,170.3\left(\mathrm{C}_{\text {quat }}, \mathrm{C}-1\right)$; MS (CI), $m / z(\%): 314(25)\left[\mathrm{M}+\mathrm{NH}_{4}^{+}\right], 297(6)\left[\mathrm{M}+\mathrm{H}^{+}\right]$.

$N$-Boc (2S,3R)-4-mesyloxiprolinol methoxymethyl ether (2S,4R)-(50): Methoxymethyl bromide

$\mathrm{MsO}$

(4.10 $\mathrm{mL}, 50.22 \mathrm{mmol})$ was added dropwise to a stirred solution of<smiles>COC[C@@H]1CCCN1C(=O)O</smiles>
$N$-Boc protected 4-mesyloxiprolinol $(2 S, 4 R)-47$ (5.3 g, $17.94 \mathrm{mmol})$ and DIEA $(9.20 \mathrm{~mL}, 51.50 \mathrm{mmol})$ in $\mathrm{CH}_{2} \mathrm{Cl}_{2}(30 \mathrm{~mL})$ at $-20{ }^{\circ} \mathrm{C}$ for $10 \mathrm{~min}$. The temperature was allowed to reach $20^{\circ} \mathrm{C}$ and stirring continued for a further $3 \mathrm{~h}$. The reaction was then diluted with water $(150 \mathrm{~mL})$ and $\mathrm{Et}_{2} \mathrm{O}(150 \mathrm{~mL})$, the organic layer was separated and washed with water $(3 \times 30 \mathrm{~mL}), 1 \mathrm{M} \mathrm{H}_{2} \mathrm{SO}_{4}(3 \times 30 \mathrm{~mL})$, water $(3 \times 30 \mathrm{~mL})$, brine $(2 \times 15 \mathrm{~mL})$, dried and concentrated under reduced pressure to give $(2 S, 4 R)-50(5.60 \mathrm{~g}, 92 \%$ crude) as a faint turbid oil which was used for the next step without additional purification. ${ }^{1} \mathrm{H}$ $\operatorname{NMR}\left(250 \mathrm{MHz}, \mathrm{CDCl}_{3}\right): \delta=1.46\left[\mathrm{~s}, 9 \mathrm{H}, \mathrm{C}\left(\mathrm{CH}_{3}\right)_{3}\right], 2.23-2.47(\mathrm{~m}, 2 \mathrm{H}, 3-\mathrm{H}), 3.03,3.04(2 \times \mathrm{s}$, $3 \mathrm{H}, \mathrm{Me}), 3.32,3.33(2 \times \mathrm{s}, 3 \mathrm{H}, \mathrm{OMe}), 3.43-3.65\left(\mathrm{~m}, 2 \mathrm{H}, 1-\mathrm{H}_{\mathrm{a}}, 5-\mathrm{H}_{\mathrm{a}}\right), 3.65-3.91\left(\mathrm{~m}, 2 \mathrm{H}, 1-\mathrm{H}_{\mathrm{b}}\right.$, 5- $\left.\mathrm{H}_{\mathrm{b}}\right), 3.99-4.23(\mathrm{~m}, 1 \mathrm{H}, 2-\mathrm{H}), 4.59$ (s, $\left.2 \mathrm{H}, \mathrm{OCH}_{2} \mathrm{O}\right), 5.14-5.19$ (m, $\left.1 \mathrm{H}, 4-\mathrm{H}\right) ;{ }^{13} \mathrm{C}$ NMR $(62.9$ $\left.\mathrm{MHz}, \mathrm{CDCl}_{3}\right): \delta=28.3\left[+, \mathrm{C}\left(\mathrm{CH}_{3}\right)_{3}\right], 34.9,35.0(-, \mathrm{C}-3), 38.6(+, \mathrm{Me}), 52.2,52.7(-, \mathrm{C}-5)$, 55.1, $55.3(+, \mathrm{OMe}), 67.6,68.1(-, \mathrm{C}-1), 78.7(+, \mathrm{C}-4), 80.1\left[\mathrm{C}_{\text {quat }}, \mathrm{C}\left(\mathrm{CH}_{3}\right)_{3}\right], 96.4\left(-, \mathrm{OCH}_{2}\right)$, $153.9\left(\mathrm{C}_{\text {quat }}, \mathrm{NCO}_{2}\right)$; The absorptions of OMe masked the signals of $\mathrm{C}-2$. 
$N$-Boc (2S,3S)-4-cyanoprolinol methoxymethyl ether $(2 S, 4 S)-(\mathbf{5 1})$ : A solution of the mesylate

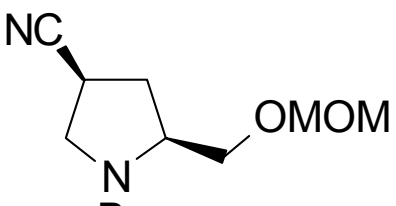

Boc $(2 S, 4 S)-50(1.63 \mathrm{~g}, 4.80 \mathrm{mmol})$ and tetrabutylammonium cyanide $(2.60$ g, $9.68 \mathrm{mmol})$ in DMF $(15 \mathrm{~mL})$ was heated at $60-65^{\circ} \mathrm{C}$ for $10 \mathrm{~h}$. The mixture was then diluted with $\mathrm{Et}_{2} \mathrm{O}(150 \mathrm{~mL})$ and washed with water $(20 \times 40 \mathrm{~mL})$, brine $(2 \times 10 \mathrm{~mL})$, dried and concentrated under reduced pressure. The residue was purified by column chromatography (alumina, EtOAc/petrol ether 1:3, $R_{\mathrm{f}}=0.26$ ) to give $(2 S, 4 S)-51(0.53 \mathrm{~g}, 41 \%)$ as an yellow oil. $R_{\mathrm{f}}=0.43$ EtOAc/hexanes $1: 1.1\left(1 \%\right.$ TEA); ${ }^{1} \mathrm{H}$ NMR $\left(250 \mathrm{MHz}, \mathrm{CDCl}_{3}\right): \delta=1.46\left[\mathrm{~s}, 9 \mathrm{H}, \mathrm{C}\left(\mathrm{CH}_{3}\right)_{3}\right], 2.23-2.55(\mathrm{~m}, 2 \mathrm{H}, 3-\mathrm{H}), 3.02(\mathrm{dddd}, J=8.0,8.0$, 8.0, 8.0 Hz, $1 \mathrm{H}, 4-\mathrm{H}), 3.36$ (s, OMe), $3.46\left(\mathrm{dd}, J=11.0,7.5 \mathrm{~Hz}, 1 \mathrm{H}, 1-\mathrm{H}_{\mathrm{a}}\right), 3.57-3.83$ (m, $2 \mathrm{H}$, $\left.1-\mathrm{H}_{\mathrm{b}}, 5-\mathrm{H}_{\mathrm{a}}\right), 3.85-4.18\left(\mathrm{~m}, 2 \mathrm{H}, 2-\mathrm{H}, 5-\mathrm{H}_{\mathrm{b}}\right), 4.63\left(\mathrm{~s}, 2 \mathrm{H}, \mathrm{OCH}_{2} \mathrm{O}\right) ;{ }^{13} \mathrm{C} \mathrm{NMR}(62.9 \mathrm{MHz}$, $\left.\mathrm{CDCl}_{3}\right): \delta=26.1,26.8(+, \mathrm{C}-4), 28.2,28.3\left[+, \mathrm{C}\left(\mathrm{CH}_{3}\right)_{3}\right], 31.8,32.7(-, \mathrm{C}-3), 49.5(-, \mathrm{C}-5), 55.2$, $56.1(+, \mathrm{OMe}), 67.2,67.8(-, \mathrm{C}-1), 80.3\left[\mathrm{C}_{\text {quat }}, \mathrm{C}\left(\mathrm{CH}_{3}\right)_{3}\right], 96.3\left(-, \mathrm{OCH}_{2} \mathrm{O}\right), 120.1\left(\mathrm{C}_{\text {quat }}, \mathrm{CN}\right)$, 153.4 $\left(\mathrm{C}_{\text {quat }}, \mathrm{NCO}_{2}\right)$; The absorptions of OMe masked the signals of C-2; $\mathrm{MS}(\mathrm{CI}), \mathrm{m} / \mathrm{z}(\%): 288$ (100) $\left[\mathrm{M}+\mathrm{NH}_{4}^{+}\right], 271(55)\left[\mathrm{M}+\mathrm{H}^{+}\right]$; MS (EI, $\left.70 \mathrm{eV}\right): m / z(\%)=270(4)\left[\mathrm{M}^{+}\right], 224(3)\left[\mathrm{M}^{+}-\right.$ $\left.\mathrm{C}_{2} \mathrm{H}_{5} \mathrm{O}\right], 214(6)\left[\mathrm{M}^{+}-\mathrm{C}_{4} \mathrm{H}_{8}\right], 208(23)\left[\mathrm{M}^{+}-\mathrm{C}_{2} \mathrm{H}_{6} \mathrm{O}_{2}\right], 195(41)\left[\mathrm{M}^{+}-\mathrm{C}_{3} \mathrm{H}_{7} \mathrm{O}_{2}\right], 183\left[\mathrm{M}^{+}-\right.$ $\left.\mathrm{C}_{4} \mathrm{H}_{7} \mathrm{O}_{2}\right], 167$ (18), 139 (27) $\left[\mathrm{C}_{7} \mathrm{H}_{11} \mathrm{~N}_{2} \mathrm{O}^{+}\right], 110$ (10) $\left[\mathrm{C}_{7} \mathrm{H}_{12} \mathrm{~N}^{+}\right], 95$ (91) $\left[\mathrm{C}_{5} \mathrm{H}_{7} \mathrm{~N}_{2}^{+}\right], 57$ (100) $\left[\mathrm{C}_{4} \mathrm{H}_{9}^{+}\right], 45$ (25) $\left[\mathrm{C}_{2} \mathrm{H}_{5} \mathrm{O}^{+}\right], 41$ (10); HRMS: calcd for $\mathrm{C}_{13} \mathrm{H}_{22} \mathrm{~N}_{2} \mathrm{O}_{4}$ : 270.1580; found: 270.1580 .

$N$-Boc (2S,3S)-4-formylprolinol methoxymethyl ether (2S,4S)-(52): A 1 M DIBAH in hexanes

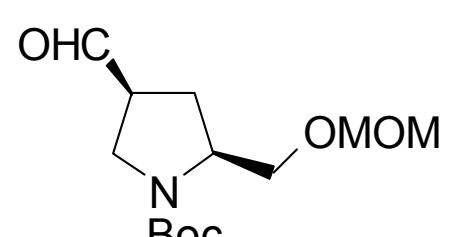

Boc (2.6 $\mathrm{mL}, 2.60 \mathrm{mmol})$ was added dropwise at $-78{ }^{\circ} \mathrm{C}$ over $10 \mathrm{~min}$ to a stirred solution of the cyanide $(2 S, 4 S)-51(0.53 \mathrm{~g}, 1.96 \mathrm{mmol})$ in $\mathrm{CH}_{2} \mathrm{Cl}_{2}(12 \mathrm{~mL})$. The reaction mixture was stirred at $-25--20^{\circ} \mathrm{C}$ for $2 \mathrm{~h}$, whereafter methanol $(1 \mathrm{~mL})$ was added dropwise at $0{ }^{\circ} \mathrm{C}$ for $3 \mathrm{~min}$ and stirring was continued at the same temperature for 20 min. A saturated aqueous $\mathrm{NH}_{4} \mathrm{Cl}(10 \mathrm{~mL})$ was then added and the mixture was allowed to warm to $20^{\circ} \mathrm{C}$. After $20 \mathrm{~min}$ the reaction mixture was diluted with $\mathrm{Et}_{2} \mathrm{O}(100 \mathrm{~mL})$ and saturated aqueous potassium sodium tartrate $(20 \mathrm{~mL})$ and 
vigorous stirring continued for an additional hour. The phases were separated and the organic fraction was washed with $2 \%$ citric acid $(3 \times 25 \mathrm{~mL})$, water $(7 \times 30 \mathrm{~mL})$, brine $(2 \times 20 \mathrm{~mL})$, the aqueous layer was back extracted with $\mathrm{Et}_{2} \mathrm{O}(3 \times 40 \mathrm{~mL})$. The combined organic layers were dried, filtered and concentrated under reduced pressure to give aldehyde $(2 S, 4 S)-52(0.48 \mathrm{~g}, 90 \%$ crude yield) as an opaque oil, which was used for the next step without further purification. $R_{\mathrm{f}}=$ 0.54, EtOAc/hexanes 1:1.1 (0.1\% TEA); ${ }^{1} \mathrm{H}$ NMR $\left(250 \mathrm{MHz}, \mathrm{CDCl}_{3}\right): \delta=1.44[\mathrm{~s}, 9 \mathrm{H}$, $\left.\mathrm{C}\left(\mathrm{CH}_{3}\right)_{3}\right], 2.15-2.42(\mathrm{~m}, 2 \mathrm{H}, 3-\mathrm{H}), 2.77-2.91(\mathrm{~m}, 1 \mathrm{H}, 4-\mathrm{H}), 3.27,3.32(2 \times \mathrm{s}, \mathrm{OMe}), 3.41-3.83$ (m, 4 H, 1-H, 5-H), 3.81-4.13 (m, 1 H, 2-H), 4.33-4.64 (m, 2 H, OCH $2 \mathrm{O}), 9.63$ (s, $1 \mathrm{H}, \mathrm{CHO})$; ${ }^{13} \mathrm{C} \mathrm{NMR}\left(62.9 \mathrm{MHz}, \mathrm{CDCl}_{3}\right): \delta=28.2\left[+, \mathrm{C}\left(\mathrm{CH}_{3}\right)_{3}\right], 28.6,29.4(-, \mathrm{C}-3), 45.9(-, \mathrm{C}-5), 48.4$,

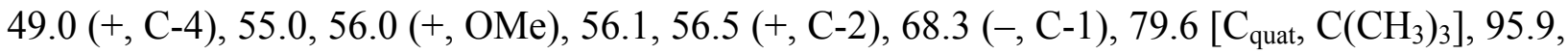
$96.3\left(-, \mathrm{OCH}_{2} \mathrm{O}\right), 153.7\left(\mathrm{C}_{\text {quat }}, \mathrm{NCO}_{2}\right), 200.4,201.5(+, \mathrm{CHO})$.

$N-B o c$ (2S,4RS)-4-(Z)-propenylprolinol methoxymethyl ether (2S,4RS)-(53): A suspension of NaHMDS (0.176 g, $0.96 \mathrm{mmol})$ and ethyltriphenylphosphonium

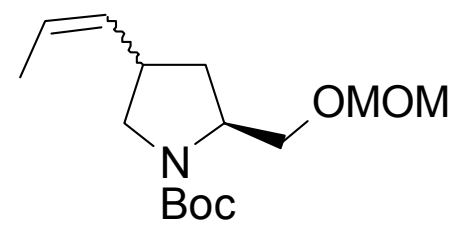
bromide $(0.356 \mathrm{~g}, 0.96 \mathrm{mmol})$ in hexanes was vigorously stirred under reflux for $3.5 \mathrm{~h}$. The mixture was then cooled to $-20{ }^{\circ} \mathrm{C}$, filtered in dry conditions through dried glass wool and the resultant turbid solution was concentrated under reduced pressure in dry conditions. The residue was then taken up with anhydrous THF $(10 \mathrm{~mL})$, cooled to $-78{ }^{\circ} \mathrm{C}$, and a solution of $(2 S, 4 S)-52(0.24 \mathrm{~g}, 0.89 \mathrm{mmol})$ in THF ( $5 \mathrm{~mL}$ ) was added dropwise within $10 \mathrm{~min}$. Stirring continued at the same temperature for a further $16 \mathrm{~h}$, then the mixture was allowed to warm to $20^{\circ} \mathrm{C}$ and stirred at this temperature for an additional $2 \mathrm{~h}$. The reaction mixture was concentrated under reduced pressure, the residue was taken up with EtOAc/hexanes 1:5 (20 mL), filtered through Celite pad $(1 \mathrm{~cm})$, concentrated, dissolved in $\mathrm{Et}_{2} \mathrm{O}(20 \mathrm{~mL})$, concentrated, dissolved in $\mathrm{Et}_{2} \mathrm{O} /$ hexanes 1:1 $(20 \mathrm{~mL})$, filtered, concentrated under reduced pressure. The residue was purified by column chromatography (alumina, EtOAc/hexanes 1:6) to give $(2 S, 4 R S)-53(95 \mathrm{mg}, 38 \%)$ as a colorless oil, gradually 
solidified in a colorless solid. The ratio of $(2 S, 4 R) /(2 S, 4 S)$ was ca. $3 / 1$ according to ${ }^{1} \mathrm{H}$ NMR spectrum. The signals of $(E)$-isomer couldn't be observed due to low content of it. $R_{\mathrm{f}}=0.43$ EtOAc/hexanes 1:5 (0.1\% TEA); ${ }^{1} \mathrm{H}$ NMR $\left(250 \mathrm{MHz}, \mathrm{CDCl}_{3}\right): \delta=1.43\left[\mathrm{~s}, 9 \mathrm{H}, \mathrm{C}\left(\mathrm{CH}_{3}\right)_{3}\right], 1.63$ $\left(\mathrm{d}, J=6.8 \mathrm{~Hz}, 3 \mathrm{H}, 3^{\prime}-\mathrm{H}\right), 1.63-1.90\left(\mathrm{~m}, 1 \mathrm{H}, 3-\mathrm{H}_{\mathrm{a}}\right), 1.93-2.09\left(\mathrm{~m}, 0.25 \mathrm{H}, 3-\mathrm{H}_{\mathrm{b}}\right.$, trans-isomer), 2.01-2.35 (m, 0.75 H, 3-H $\mathrm{H}_{\mathrm{b}}$, cis-isomer), 2.86 (ddd, $\left.J=10.0,10.0,10.0 \mathrm{~Hz}, 1 \mathrm{H}, 4-\mathrm{H}\right), 2.87-3.05$ $\left(\mathrm{m}, 1 \mathrm{H}, 5-\mathrm{H}_{\mathrm{b}}\right), 3.33,3.35(2 \times \mathrm{s}, \mathrm{OMe}), 3.37-4.07\left(\mathrm{~m}, 4 \mathrm{H}, 1-\mathrm{H}, 2-\mathrm{H}, 5-\mathrm{H}_{\mathrm{b}}\right), 4.53,4.54(2 \times \mathrm{s}$, $\left.2 \mathrm{H}, \mathrm{OCH}_{2} \mathrm{O}\right), 5.24$ (ddq, $\left.J=10.8,10.8,1.8 \mathrm{~Hz}, 1 \mathrm{H}, 1^{\prime}-\mathrm{H}\right), 5.46$ (ddq, $J=1.0,10.8,6.8 \mathrm{~Hz}, 1 \mathrm{H}$, $\left.1^{\prime}-\mathrm{H}\right) ;{ }^{13} \mathrm{C} \mathrm{NMR}\left(62.9 \mathrm{MHz}, \mathrm{CDCl}_{3}\right): \delta=13.1\left(+, \mathrm{C}-3^{\prime}\right), 28.4\left[+, \mathrm{C}\left(\mathrm{CH}_{3}\right)_{3}\right], 35.5(+, \mathrm{C}-4), 35.6$, 36.6 (-, C-3), 51.9, 52.4 (-, C-5), 55.1 (+, OMe), 56.7 (+, C-2), 68.4, 69.3 (-, C-1), 79.3 [C $\mathrm{C}_{\text {quat, }}$ $\left.\mathrm{C}\left(\mathrm{CH}_{3}\right)_{3}\right], 96.5\left(-, \mathrm{OCH}_{2} \mathrm{O}\right), 125.8\left(+, \mathrm{C}^{\prime} 1^{\prime}\right), 130.5,130.8\left(+, \mathrm{C}-2^{\prime}\right), 154.2\left(\mathrm{C}_{\text {quat }}, \mathrm{NCO}_{2}\right)$; $\mathrm{MS}$ (CI), $m / z(\%): 303(16)\left[\mathrm{M}+\mathrm{NH}_{4}{ }^{+}\right], 286(100)\left[\mathrm{M}+\mathrm{H}^{+}\right]$; MS (EI, $\left.70 \mathrm{eV}\right): m / z(\%)=285(3)$ $\left[\mathrm{M}^{+}\right], 223(3)\left[\mathrm{M}^{+}-\mathrm{C}_{2} \mathrm{H}_{6} \mathrm{O}\right], 210(53)\left[\mathrm{M}^{+}-\mathrm{C}_{3} \mathrm{H}_{7} \mathrm{O}_{2}\right], 208(23)\left[\mathrm{M}^{+}-\mathrm{C}_{2} \mathrm{H}_{6} \mathrm{O}_{2}\right], 154$ (100) $\left[\mathrm{C}_{9} \mathrm{H}_{16} \mathrm{NO}^{+}\right], 110(45)\left[\mathrm{C}_{7} \mathrm{H}_{12} \mathrm{~N}^{+}\right], 57(30)\left[\mathrm{C}_{4} \mathrm{H}_{9}{ }^{+}\right], 45(8)\left[\mathrm{C}_{2} \mathrm{H}_{5} \mathrm{O}^{+}\right], 41$ (4) $\left[\mathrm{C}_{3} \mathrm{H}_{5}^{+}\right]$; HRMS: calcd for $\mathrm{C}_{15} \mathrm{H}_{27} \mathrm{NO}_{4}$ : 285.1940; found: 285.1940 .

$N-B o c(2 S, 4 R S)-4-(Z)-p r o p e n y l p r o l i n o l ~(2 S, 4 R S)-(54):$ The protected alcohol $(2 S, 4 R S)-53(54$

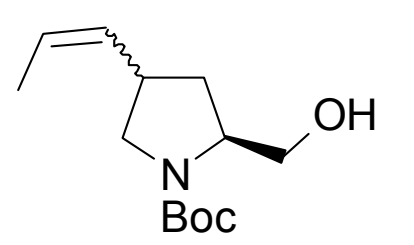
$\mathrm{mg}, 0.19 \mathrm{mmol})$ was deprotected with TFA $(0.5 \mathrm{~mL})$ for $20 \mathrm{~min}$. All volatiles was then removed under reduced pressure to leave the deprotected amino alcohol as a brown semisolid which was taken up with $\mathrm{CH}_{2} \mathrm{Cl}_{2}(4 \mathrm{~mL})$ and water $(4 \mathrm{~mL}) . \mathrm{NaHCO}_{3}(88 \mathrm{mg}, 1.05 \mathrm{mmol})$ and then $\mathrm{Boc}_{2} \mathrm{O}(100 \mathrm{mg}$, $0.46 \mathrm{mmol}$ ) were added to this vigorously stirred biphasic system and vigorous stirring continued for a further $3 \mathrm{~h}$. $N, N$-dimethylaminopropylamine (30 $\mathrm{mg}, 0.29 \mathrm{mmol}$ ) was then added and after 10 min the mixture was taken up with $\mathrm{Et}_{2} \mathrm{O}(50 \mathrm{~mL})$. The organic layer was washed with $1 \mathrm{M}$ $\mathrm{H}_{2} \mathrm{SO}_{4}(3 \times 10 \mathrm{~mL})$, water $(3 \times 10 \mathrm{~mL})$, brine $(2 \times 10 \mathrm{~mL})$, dried, filtered and concentrated under reduced pressure. The residue was purified by column chromatography (EtOAc/hexanes 1:1, $R_{\mathrm{f}}$ $=0.74)$ to give somewhat impure mixture of diasteromers $(2 S, 4 R S)-54(31 \mathrm{mg}, 67 \%)$ as a faint 
yellow oil. This mixture could be separated by column chromatography (for details of this separation and spectral data of individual diastereomers see deprotection of the $\mathrm{N}$-Boc, $\mathrm{O}$ TBDMS protected 4-(Z)-propenylprolinol).

$N$-Boc (2S,4RS)-4-(2',2'-dibromovinyl)prolinol methoxymethyl ether (2S,4RS)-(55): To a stirred

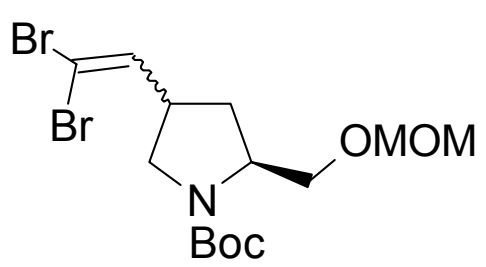
ice-cold mixture of $\mathrm{PPh}_{3}(2.016 \mathrm{~g}, 7.69 \mathrm{mmol}), \mathrm{CBr}_{4}(1.70 \mathrm{~g}, 5.13$ mmol) and activated $\mathrm{Zn}$ dust $(0.34 \mathrm{~g}, 5.20 \mathrm{mmol})$ was added $\mathrm{CH}_{2} \mathrm{Cl}_{2}(5 \mathrm{~mL})$, the temperature was allowed to reach $20^{\circ} \mathrm{C}$ and the mixture was stirred for $24 \mathrm{~h}$. The reaction flask was then immersed with a water/ice bath and a solution of the aldehyde $(2 S, 4 S)-52(0.60 \mathrm{~g}, 2.20 \mathrm{mmol})$ in $\mathrm{CH}_{2} \mathrm{Cl}_{2}(5 \mathrm{~mL})$ was added dropwise to the resultant beige suspension for $10 \mathrm{~min}$. The mixture was stirred at the same temperature for $2 \mathrm{~h}$, the temperature was then allowed to increase to $20{ }^{\circ} \mathrm{C}$ and stirring continued for a further $4 \mathrm{~h}$. The mixture was then filtered through alumina/silica gel pad $(0.5 / 1 \mathrm{~cm})$, concentrated under reduced pressure, taken up with $\mathrm{CH}_{2} \mathrm{Cl}_{2} / \mathrm{EtOAc} /$ hexanes 1:0.7:0.3 $(15 \mathrm{~mL})$, filtered, concentrated under reduced pressure, taken up with $\mathrm{CH}_{2} \mathrm{Cl}_{2} / \mathrm{EtOAc} /$ hexanes 1:0.5:0.5 (15 mL), filtered and concentrated under reduced pressure, dissolved in EtOAc/hexanes 1:1 (15 mL), filtered and concentrated under reduced pressure, dissolved in EtOAc/hexanes 1:2.5, filtered and concentrated under reduced pressure again. The residue was purified by column chromatography (EtOAc/hexanes 1:2.5, $\left.R_{\mathrm{f}}=0.34\right)$ to give $(2 S, 4 R S)-55(6.36 \mathrm{~g}, 68 \%)$ as a colorless viscous oil. The ratio of $(2 S, 4 R) /(2 S, 4 S)$-isomers was $1 / 1.6$ according to ${ }^{1} \mathrm{H}$ NMR spectrum. ${ }^{1} \mathrm{H}$ NMR $\left(250 \mathrm{MHz}, \mathrm{CDCl}_{3}\right): \delta=1.45[\mathrm{~s}, 9 \mathrm{H}$, $\left.\mathrm{C}\left(\mathrm{CH}_{3}\right)_{3}\right], 1.71-1.93\left(\mathrm{~m}, 1 \mathrm{H}, 3-\mathrm{H}_{\mathrm{a}}\right), 2.09-2.21\left(\mathrm{~m}, 0.4 \mathrm{H}, 3-\mathrm{H}_{\mathrm{b}}\right.$, trans-isomer), 2.32 (ddd, $J$ $=11.0,6.6,6.6 \mathrm{~Hz}, 0.6 \mathrm{H}, 3-\mathrm{H}_{\mathrm{b}}$, cis-isomer $), 2.91-3.22\left(\mathrm{~m}, 2 \mathrm{H}, 4-\mathrm{H}, 5-\mathrm{H}_{\mathrm{a}}\right), 3.34,3.35(2 \times \mathrm{s}$, OMe), 3.50-4.11 (m, $\left.4 \mathrm{H}, 1-\mathrm{H}, 2-\mathrm{H}, 5-\mathrm{H}_{\mathrm{b}}\right), 4.60,4.61\left(2 \times \mathrm{s}, 2 \mathrm{H}, \mathrm{OCH}_{2} \mathrm{O}\right), 6.29(\mathrm{~d}, J=8.5 \mathrm{~Hz}$, $0.4 \mathrm{H}, 1^{\prime}-\mathrm{H}$, trans-isomer), 6.37 (d, $J=8.5 \mathrm{~Hz}, 0.4 \mathrm{H}, 11^{\prime}-\mathrm{H}$, cis-isomer); ${ }^{13} \mathrm{C}$ NMR (62.9 MHz, $\left.\mathrm{CDCl}_{3}\right): \delta=28.2\left[+, \mathrm{C}\left(\mathrm{CH}_{3}\right)_{3}\right], 33.4,34.0,34.3(-, \mathrm{C}-3), 39.9,40.8,41.2(+, \mathrm{C}-4), 50.0,50.1$, 


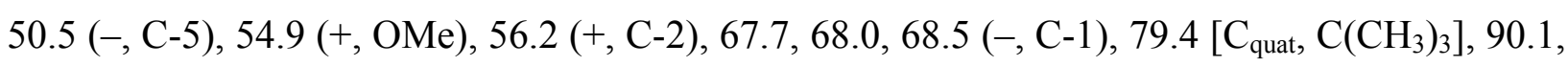
$90.2\left(\mathrm{C}_{\text {quat }}, \mathrm{C}-2^{\prime}\right), 96.2\left(-, \mathrm{OCH}_{2} \mathrm{O}\right), 138.7\left(+, \mathrm{C}-1^{\prime}\right), 153.7\left(\mathrm{C}_{\text {quat }}, \mathrm{NCO}_{2}\right) ; \mathrm{MS}(\mathrm{CI}), \mathrm{m} / z(\%): 447$ (20) $\left[\mathrm{M}+\mathrm{NH}_{4}^{+}\right], 430(100)\left[\mathrm{M}+\mathrm{H}^{+}\right]$.

$N-B o c$ (2S,4RS)-4-propynylprolinol methoxymethyl ether (2S,4RS)-(56): A $2.34 \mathrm{M}$ solution of $n \mathrm{BuLi}$ in hexanes $(1.61 \mathrm{~mL}, 3.77 \mathrm{mmol})$ was added to a solution of

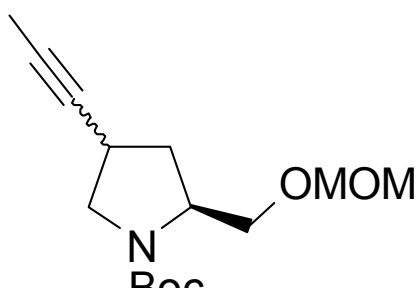
Boc $(2 S, 4 R S)-55(0.734 \mathrm{~g}, 1.71 \mathrm{mmol})$ in anhydrous THF $(12 \mathrm{~mL})$ at $78{ }^{\circ} \mathrm{C}$ for $7 \mathrm{~min}$ and stirring continued at the same temperature for a further $2 \mathrm{~h}$. The temperature was then allowed to increase to $20{ }^{\circ} \mathrm{C}$ and the mixture was stirred at this temperature for an additional $1 \mathrm{~h}$. The reaction flask was immersed with dry ice/acetone bath and methyl iodide $(0.47 \mathrm{~mL}, 7.55 \mathrm{mmol})$ was then added for $20 \mathrm{~min}$. The mixture was allowed to warm to $20^{\circ} \mathrm{C}$ for $20 \mathrm{~h}$ and then stirred at this temperature for an additional $1 \mathrm{~h}$. After this, all volatiles were removed under reduced pressure and the residue was purified by column chromatography (EtOAc/hexanes $1: 4, R_{\mathrm{f}}=0.37$ ) to give $(2 S, 4 R S)-55(0.215 \mathrm{~g}, 44 \%)$ as a colorless oil which completely polymerized at the ambient temperature in the presence of oxygen in several weeks. During the chromatographic purification on this step the undesirable $(2 S, 4 S)$-isomer was partially separated. The ratio of $(2 S, 4 S) /(2 S$, $4 R$ )-isomers was 1:6 according to ${ }^{1} \mathrm{H}$ NMR spectrum. The signals of only main diastereomer

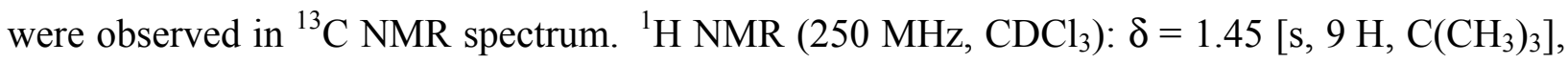
1.87-2.09 (m, $\left.1 \mathrm{H}, 3-\mathrm{H}_{\mathrm{a}}\right), 2.07-2.18\left(\mathrm{~m}, 0.15 \mathrm{H}, 3-\mathrm{H}_{\mathrm{b}}\right.$, trans-isomer), 2.30 (ddd, $J=11.0,8.3$, 8.3 Hz, 0.85 H, 3-H $\mathrm{H}_{\mathrm{b}}$ cis-isomer), 2.72-2.89 (m, $\left.1 \mathrm{H}, 4-\mathrm{H}\right), 3.12$ (dd, $J=10.6,8.6 \mathrm{~Hz}, 1 \mathrm{H}, 5-$ $\left.\mathrm{H}_{\mathrm{a}}\right), 3.33,3.35(2 \times \mathrm{s}, \mathrm{OMe}), 3.49-4.03\left(\mathrm{~m}, 4 \mathrm{H}, 1-\mathrm{H}, 2-\mathrm{H}, 5-\mathrm{H}_{\mathrm{b}}\right) ;{ }^{13} \mathrm{C} \mathrm{NMR}\left(62.9 \mathrm{MHz}, \mathrm{CDCl}_{3}\right)$ : $\delta=3.2(+, \mathrm{C}-4), 28.1\left[+, \mathrm{C}\left(\mathrm{CH}_{3}\right)_{3}\right], 35.2,36.1(-, \mathrm{C}-3), 52.1,52.6(-, \mathrm{C}-5), 54.8(+, \mathrm{OMe}), 56.1$, $56.3(+, \mathrm{C}-2), 67.7,68.5(-, \mathrm{C}-1), 77.2\left(\mathrm{C}_{\text {quat }}, \mathrm{C}-2^{\prime}\right), 79.0\left(\mathrm{C}_{\text {quat }}, \mathrm{C}-1^{\prime}\right), 79.4\left[\mathrm{C}_{\text {quat }}, \mathrm{C}\left(\mathrm{CH}_{3}\right)_{3}\right], 96.2$ $\left(-, \mathrm{OCH}_{2} \mathrm{O}\right), 154.0\left(\mathrm{C}_{\text {quat }}, \mathrm{NCO}_{2}\right)$; IR (film): nu(tilde) $=2978 \mathrm{~cm}^{-1}, 2924,2882,1699,1479$, 1454, 1394, 1365, 1169, 1151, 1112, 1039; MS (EI, $70 \mathrm{eV}): m / z(\%)=283(0.04)\left[\mathrm{M}^{+}\right], 251(8)$ 
$\left[\mathrm{M}^{+}-\mathrm{CH}_{4} \mathrm{O}\right], 227(6)\left[\mathrm{M}^{+}-\mathrm{C}_{4} \mathrm{H}_{8}\right], 208(59)\left[\mathrm{M}^{+}-\mathrm{C}_{3} \mathrm{H}_{7} \mathrm{O}_{2}\right], 180$ (6), 152 (100) $\left[\mathrm{C}_{9} \mathrm{H}_{14} \mathrm{NO}^{+}\right]$, $108(93)\left[\mathrm{C}_{7} \mathrm{H}_{10} \mathrm{~N}^{+}\right], 57(52)\left[\mathrm{C}_{4} \mathrm{H}_{9}^{+}\right], 45(15)\left[\mathrm{C}_{2} \mathrm{H}_{5} \mathrm{O}^{+}\right], 41(6)$.

Attempt of the partial reduction of the triple bond on nickel catalyst in (2S,4RS)-56: To a vigorously stirred suspension of $\mathrm{Ni}(\mathrm{OAc})_{2} \cdot 5 \mathrm{H}_{2} \mathrm{O}(25 \mathrm{mg}, 0.094 \mathrm{mmol})$ in $\mathrm{EtOH}(1 \mathrm{~mL})$ in hydrogen atmosphere was added $\mathrm{NaBH}_{4}(7 \mathrm{mg}, 0.18 \mathrm{mmol})$. Ethylendiamine (3 drops) and then a solution of substance $(2 S, 4 R S)-53(0.204 \mathrm{~g}, 0.72 \mathrm{mmol})$ in EtOH $(2 \mathrm{~mL})$ was added to the resultant thick black suspension and stirring continued for a further $1 \mathrm{~h}$. The mixture diluted with EtOAc/hexanes 1:1 (3 mL) and filtered through silica gel pad $(1 \mathrm{~cm})$. The solvents were removed under reduced pressure and the residue was taken up with EtOAc/hexanes 1:2.5 (10 $\mathrm{mL}$ ), filtered to remove the last traces of the catalyst and concentrated under reduced pressure to give colorless oil $(0.190 \mathrm{~g})$ of $(2 S, 4 R S)-53$, which contained (according to ${ }^{1} \mathrm{H}$ NMR spectrum) ca. $12-13 \%$ of $N$-Boc, $O$-MOM protected 4-propylprolinol.

(2S,4R)-N-Boc-4-Hydroxyprolinol $(2 S, 4 R)-(\mathbf{5 8})$ : To a solution of $(2 S, 4 R)-43(57.5 \mathrm{~g}, 248 \mathrm{mmol})$

$\mathrm{HO}$ and triethylamine $(38.4 \mathrm{~mL}, 273 \mathrm{mmol})$ in $\mathrm{CH}_{2} \mathrm{Cl}_{2}(1000 \mathrm{~mL})$ at $-30{ }^{\circ} \mathrm{C}$,<smiles>OC[C@@H]1CCCN1</smiles>
Boc ethyl chloroformate $(25 \mathrm{~mL}, 261 \mathrm{mmol})$ was added and the mixture was stirred for $40 \mathrm{~min}$. To this mixture tetra- $n$-butylammonium bromide $(8.5 \mathrm{~g}$, $26.4 \mathrm{mmol})$ and then suspension of $\mathrm{NaBH}_{4}(40 \mathrm{~g}, 1057 \mathrm{mmol})$ in ice-cold water $(50 \mathrm{~mL})$ (carefully, by small portions) were added and the reaction mixture allowed to warm to $-10{ }^{\circ} \mathrm{C}$ and stirred for $1 \mathrm{~h}$. The temperature of the mixture was further increased to $0^{\circ} \mathrm{C}$ and stirring continued at this temperature for $1 \mathrm{~h}$. $\mathrm{pH}$ of the aqueous layer was then carefully adjusted to 5 6 with $50 \%$ acetic acid and the mixture was filtered through Celite. The organic layer was separated and aqueous layer was extracted with $\mathrm{CH}_{2} \mathrm{Cl}_{2}(3 \times 100 \mathrm{~mL})$. The aqueous layer was discarded and the combined organic fraction was dried, concentrated under reduced pressure and the residue was purified by column chromatography (EtOAc/hexanes $7: 3, R_{\mathrm{f}}=0.24$ ) to give 
$(2 S, 4 R)-58(44.23 \mathrm{~g}, 82 \%)$ as a white solid. $[\alpha]_{24}^{\mathrm{D}}-58.8(c=1.05$, EtOH$)\left(\right.$ lit.: ${ }^{[143]}[\alpha]_{24}^{\mathrm{D}}-$ 58.87, $c=1.009, \mathrm{EtOH}) ;{ }^{1} \mathrm{H} \mathrm{NMR}\left(250 \mathrm{MHz}, \mathrm{CDCl}_{3}\right): \delta=1.47\left[\mathrm{~s}, 9 \mathrm{H}, \mathrm{C}\left(\mathrm{CH}_{3}\right)_{3}\right], 1.57-1.80(\mathrm{~m}$, $\left.1 \mathrm{H}, 3-\mathrm{H}_{\mathrm{a}}\right), 1.89-1.98(\mathrm{br}, 1 \mathrm{H}, \mathrm{OH}), 1.98-2.11\left(\mathrm{~m}, 1 \mathrm{H}, 3-\mathrm{H}_{\mathrm{b}}\right), 3.35-3.63$ (m, $\left.3 \mathrm{H}, 2-\mathrm{H}, 5-\mathrm{H}\right)$, $3.70\left(\mathrm{dd}, J=9.4,9.4 \mathrm{~Hz}, 1 \mathrm{H}, 1-\mathrm{H}_{\mathrm{a}}\right), 4.04-4.25\left(\mathrm{~m}, 1 \mathrm{H}, 1-\mathrm{H}_{\mathrm{b}}\right), 4.29-4.45(\mathrm{~m}, 1 \mathrm{H}, 4-\mathrm{H}), 4.95-$ 5.09 (br, $1 \mathrm{H}, \mathrm{OH}) ;{ }^{13} \mathrm{C} \mathrm{NMR}\left(62.9 \mathrm{MHz}, \mathrm{CDCl}_{3}\right): \delta=28.3\left[+, \mathrm{C}\left(\mathrm{CH}_{3}\right)_{3}\right], 37.3(-, \mathrm{C}-3), 54.9$, $55.6(-, \mathrm{C}-5), 57.7,58.6(+, \mathrm{C}-2), 63.8,66.4(-, \mathrm{C}-1), 68.8(+, \mathrm{C}-4), 80.4\left[\mathrm{C}_{\text {quat }}, \mathrm{C}\left(\mathrm{CH}_{3}\right)_{3}\right], 155.0$, $156.9\left(\mathrm{C}_{\text {quat }}, \mathrm{NCO}_{2}\right)$; IR (film): nu(tilde $)=3381 \mathrm{~cm}^{-1}, 2981,2942,2899,1653,1412,1160,1041$.

(2S,4R)-N-Boc-O-TBDMS-4-Mesyloxyprolinol $(2 S, 4 R)-(\mathbf{6 0})$ : To a solution of substance $(2 S, 4 R)-$ $\mathrm{MsO}$ 59 (48.14 g, $145 \mathrm{mmol})$ and triethylamine $(30.4 \mathrm{~mL}, 218 \mathrm{mmol})$ in<smiles>C[AsH3-][OH+]C[C@H]1CCCN1</smiles>
Boc

$\mathrm{CH}_{2} \mathrm{Cl}_{2}(120 \mathrm{~mL})$ at $-40^{\circ} \mathrm{C}$, mesyl chloride $(15.2 \mathrm{~mL}, 196 \mathrm{mmol})$ was added for $10 \mathrm{~min}$. The mixture was allowed to warm to $0{ }^{\circ} \mathrm{C}$ for $3 \mathrm{~h}$ and saturated $\mathrm{NaHCO}_{3}(100 \mathrm{~mL})$ was added. The reaction mixture was taken up with $\mathrm{Et}_{2} \mathrm{O}(500 \mathrm{~mL})$, the organic layer was washed with $\mathrm{H}_{2} \mathrm{O}(3 \times 100 \mathrm{~mL}), 1 \mathrm{M} \mathrm{NaHSO}{ }_{4}(3 \times 100$ $\mathrm{mL}), \mathrm{H}_{2} \mathrm{O}(2 \times 100 \mathrm{~mL})$, brine $(2 \times 100 \mathrm{~mL})$ and dried. Concentration under reduced pressure gave $(2 S, 4 R)-60(58.2 \mathrm{~g}, 98 \%)$ as a light yellow oil. $[\alpha]_{24}^{\mathrm{D}}-38.5\left(c=0.55, \mathrm{CHCl}_{3}\right) ; R_{\mathrm{f}}=0.53$ (EtOAc/hexanes 1:2.3); ${ }^{1} \mathrm{H}$ NMR (250 MHz, $\left.\mathrm{CDCl}_{3}\right): \delta=0.01\left[\mathrm{~s}, 6 \mathrm{H}, \mathrm{Si}\left(\mathrm{CH}_{3}\right)_{2}\right], 0.85$ [s, $9 \mathrm{H}$, $\left.\mathrm{SiC}\left(\mathrm{CH}_{3}\right)_{3}\right], 1.44$ [s, $\left.9 \mathrm{H}, \mathrm{C}\left(\mathrm{CH}_{3}\right)_{3}\right], 2.30-2.49(\mathrm{~m}, 2 \mathrm{H}, 3-\mathrm{H}), 3.02\left(\mathrm{~s}, 3 \mathrm{H}, \mathrm{CH}_{3}\right), 3.44-3.63(\mathrm{~m}$, $2 \mathrm{H}, 5-\mathrm{H}), 3.63-4.15(\mathrm{~m}, 3 \mathrm{H}, 1-\mathrm{H}, 2-\mathrm{H}), 5.25-5.33(\mathrm{~m}, 1 \mathrm{H}, 4-\mathrm{H}) ;{ }^{13} \mathrm{C} \mathrm{NMR}(75.5 \mathrm{MHz}$, $\left.\mathrm{C}_{2} \mathrm{D}_{2} \mathrm{Cl}_{4}, 353 \mathrm{~K}\right): \delta=-5.7\left[+, \mathrm{Si}\left(\mathrm{CH}_{3}\right)_{2}\right], 17.8\left(\mathrm{C}_{\text {quat }}, \mathrm{SiC}\right), 25.6\left[+, \mathrm{SiC}\left(\mathrm{CH}_{3}\right)_{3}\right], 28.3$ $\left[+, \mathrm{C}\left(\mathrm{CH}_{3}\right)_{3}\right], 34.8(-, \mathrm{C}-3), 38.4\left(+, \mathrm{SO}_{2} \mathrm{Me}\right), 52.4(-, \mathrm{C}-5), 57.0(+, \mathrm{C}-2), 63.4(-, \mathrm{C}-1), 78.9(+$, C-4), $79.6\left[\mathrm{C}_{\text {quat }}, \mathrm{C}\left(\mathrm{CH}_{3}\right)_{3}\right], 153.6\left(\mathrm{C}_{\text {quat }}, \mathrm{NCO}_{2}\right)$; IR (film): nu(tilde) $=3381 \mathrm{~cm}^{-1}, 2930,2858$, 1698, 1473, 1404, 1366, 1257, 1174, 1120; MS (ESI), (positive) $m / z(\%): 432(91)\left[\mathrm{M}+\mathrm{Na}^{+}\right]$. 
(2S,4S)-N-Boc-O-TBDMS-4-Cyanoprolinol (2S,4S)-(61): A sealed flask containing a solution of

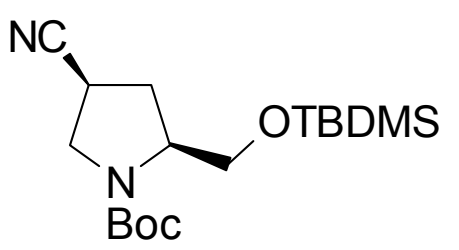
$(2 S, 4 S)-60(52.8 \mathrm{~g}, 129 \mathrm{mmol})$ and tetra- $n$-butylammonium cyanide $(72.0 \mathrm{~g}, 268 \mathrm{mmol})$ in anhydrous $\mathrm{MeCN}(50 \mathrm{~mL})$ was placed into the heated to $65-68{ }^{\circ} \mathrm{C}$ oil-bath. After stirring for $6 \mathrm{~h}$, the mixture was taken up with EtOAc/hexanes 1:4 $(500 \mathrm{~mL})$, washed with water $(8 \times 100 \mathrm{~mL})$ and brine $(2 \times 50$ $\mathrm{mL})$, dried and filtered through a pad of silica gel $(5 \mathrm{~cm})$. The solvents were removed under reduced pressure and the residue was purified by column chromatography (EtOAc/hexanes 1:3, $\left.R_{\mathrm{f}}=0.50\right)$ to give $(2 S, 4 S)-61(26.50 \mathrm{~g}, 60 \%)$ as a yellowish oil solidified by seeding into a white solid. M.p. $55-58{ }^{\circ} \mathrm{C} ;[\alpha]_{24}^{\mathrm{D}}-25.9\left(c=0.9, \mathrm{CHCl}_{3}\right) ;{ }^{1} \mathrm{H} \mathrm{NMR}\left(300 \mathrm{MHz}, \mathrm{C}_{2} \mathrm{D}_{2} \mathrm{Cl}_{4}, 358 \mathrm{~K}\right): \delta=$ $0.10\left[\mathrm{~s}, 6 \mathrm{H}, \mathrm{Si}\left(\mathrm{CH}_{3}\right)_{2}\right], 0.94\left[\mathrm{~s}, 9 \mathrm{H}, \mathrm{SiC}\left(\mathrm{CH}_{3}\right)_{3}\right], 1.48\left[\mathrm{~s}, 9 \mathrm{H}, \mathrm{C}\left(\mathrm{CH}_{3}\right)_{3}\right], 2.29-2.46(\mathrm{~m}, 2 \mathrm{H}, 3-$ H), 2.96 (dddd, $J=8.1,8.1,8.1,8.1 \mathrm{~Hz}, 1 \mathrm{H}, 4-\mathrm{H}), 3.42$ (dd, $J=8.4,10.5 \mathrm{~Hz}, 1 \mathrm{H}, 5-\mathrm{H}_{\mathrm{a}}$ ), 3.74 (dd, $\left.J=3.0,9.8 \mathrm{~Hz}, 1 \mathrm{H}, 5-\mathrm{H}_{\mathrm{b}}\right), 3.79-3.95\left(\mathrm{~m}, 2 \mathrm{H}, 1-\mathrm{H}_{\mathrm{a}}, 2-\mathrm{H}\right), 3.96(\mathrm{dd}, J=7.8,10.8 \mathrm{~Hz}, 1 \mathrm{H}$, $\left.1-\mathrm{H}_{\mathrm{b}}\right) ;{ }^{13} \mathrm{C} \mathrm{NMR}\left(75.5 \mathrm{MHz}, \mathrm{C}_{2} \mathrm{D}_{2} \mathrm{Cl}_{4}, 358 \mathrm{~K}\right): \delta=-5.6\left[+, \mathrm{Si}\left(\mathrm{CH}_{3}\right)_{2}\right], 17.9\left(\mathrm{C}_{\text {quat, }}, \mathrm{SiC}\right), 25.7[+$, $\left.\mathrm{SiC}\left(\mathrm{CH}_{3}\right)_{3}\right], 26.3(+, \mathrm{C}-4), 28.2\left[+, \mathrm{C}\left(\mathrm{CH}_{3}\right)_{3}\right], 31.9(-, \mathrm{C}-3), 49.8(-, \mathrm{C}-5), 57.8(+, \mathrm{C}-2), 62.9(-$, C-1), $80.0\left[\mathrm{C}_{\text {quat }}, \mathrm{C}\left(\mathrm{CH}_{3}\right)_{3}\right], 119.8\left(\mathrm{C}_{\text {quat, }}, \mathrm{CN}\right), 153.2\left(\mathrm{C}_{\text {quat, }}, \mathrm{NCO}_{2}\right)$; IR (film): nu(tilde $)=3381$ $\mathrm{cm}^{-1}, 2952,2895,2855,2240,1696,1471,1400,1259,1172,1099$; MS (CI), m/z (\%):358 (8) $\left[\mathrm{M}+\mathrm{NH}_{4}{ }^{+}\right], 341(100)\left[\mathrm{M}+\mathrm{H}^{+}\right]$; elemental analysis calcd (\%) for $\mathrm{C}_{17} \mathrm{H}_{32} \mathrm{~N}_{2} \mathrm{O}_{3} \mathrm{Si}$ (340.22): $\mathrm{C}$ 59.96, H 9.47, N 8.23; found C 60.29, H 9.55, N 8.04.

$(2 S, 4 S)$ - and $(2 S, 4 R)-N$-Boc- $O$-TBDMS-4-deutero-4-cyanoprolinols $(2 S, 4 S)$-D-(61) and $(2 S, 4 R)$ D-(61): To a solution of nitrile $(2 S, 4 S)-61(9.5 \mathrm{~g}, 27.90 \mathrm{mmol})$ in $\mathrm{THF}(50 \mathrm{~mL})$ at $-78{ }^{\circ} \mathrm{C}$ was added dropwise a solution of ice-cold LDA, prepared from $n \mathrm{BuLi}(15 \mathrm{~mL}$ of $2.44 \mathrm{M}$ solution in hexanes, $36.27 \mathrm{mmol}$ ) and diisopropylamine $(5.9 \mathrm{~mL}, 41.85 \mathrm{mmol})$ in THF $(30 \mathrm{~mL})$, for $40 \mathrm{~min}$. Stirring was continued for an additional $90 \mathrm{~min}$ and then the reaction was quenched with saturated solution of $\mathrm{Na}_{2} \mathrm{SO}_{4}$ in $\mathrm{D}_{2} \mathrm{O}(10 \mathrm{~mL}$; under vigorous stirring $)$ at the same temperature. The mixture was allowed to warm to $20^{\circ} \mathrm{C}$ and it was then concentrated under reduced pressure, 
taken up with $\mathrm{Et}_{2} \mathrm{O}(70 \mathrm{~mL})$, filtered through Celite, concentrated again and separated by column chromatography (EtOAc/hexanes 1:3.7, $R_{\mathrm{f}}=0.38$, trans-isomer $\left.R_{\mathrm{f}}=0.54\right)$ to give $(2 S, 4 S)$-D-61 $(4.21 \mathrm{~g})$ and a mixed fraction enriched with $(2 S, 4 R)-\mathrm{D}-61$ (3.62 $\mathrm{g}, 10.63 \mathrm{mmol})$, which was dissolved in THF $(20 \mathrm{~mL})$, again treated with solution of LDA, prepared from $n \mathrm{BuLi}(5.7 \mathrm{~mL}$, $13.91 \mathrm{mmol})$ and diisopropylamine $(2.23 \mathrm{~mL}, 15.91 \mathrm{mmol})$ in THF $(15 \mathrm{~mL})$ and quenched with saturated solution of $\mathrm{Na}_{2} \mathrm{SO}_{4}$ in $\mathrm{D}_{2} \mathrm{O}(3 \mathrm{~mL})$ as it was described above. Separation of the mixture by column chromatography gave second crop of cis-nitrile $(2 S, 4 S)$-D-61 (2.40 g). It was combined with the first fraction and finally purified by column chromatography as it was described above to give (2S,4S)-D-61 (6.39 g, 67\%, 90\% D according to ${ }^{1} \mathrm{H}$ NMR spectrum) as a coloress oil, gradually solidified in a white solid.

(2S,4S)-D-61: m.p. $53-55^{\circ} \mathrm{C} ;[\alpha]_{24}^{\mathrm{D}}-25.9\left(c=1.1, \mathrm{CHCl}_{3}\right) ;{ }^{1} \mathrm{H}$ NMR $\left(250 \mathrm{MHz}, \mathrm{CDCl}_{3}\right): \delta=$ $0.01\left[\mathrm{~s}, 6 \mathrm{H}, \mathrm{Si}\left(\mathrm{CH}_{3}\right)_{2}\right], 0.88\left[\mathrm{~s}, 9 \mathrm{H}, \mathrm{SiC}\left(\mathrm{CH}_{3}\right)_{3}\right], 1.41[\mathrm{~s}, 9 \mathrm{H}$,
$\mathrm{C}(90 \%)$
$0.1 \mathrm{H}, 4-\mathrm{H}), 3.35\left(\mathrm{~d}, J=11.3 \mathrm{~Hz}, 1 \mathrm{H}, 5-\mathrm{H}_{\mathrm{a}}\right), 3.53-4.05(\mathrm{~m}, 4 \mathrm{H}, 1-$ $\left.\mathrm{H}, 2-\mathrm{H}, 5-\mathrm{H}_{\mathrm{b}}\right) ;{ }^{13} \mathrm{C} \mathrm{NMR}\left(62.9 \mathrm{MHz}, \mathrm{CDCl}_{3}\right): \delta=-5.6\left[+, \mathrm{Si}\left(\mathrm{CH}_{3}\right)_{2}\right], 18.0\left(\mathrm{C}_{\text {quat }}, \mathrm{SiC}\right), 25.7[+$, $\left.\mathrm{SiC}\left(\mathrm{CH}_{3}\right)_{3}\right), 26.2(\mathrm{t}, J=21.7 \mathrm{~Hz}, \mathrm{C}-4), 28.2\left[+, \mathrm{C}\left(\mathrm{CH}_{3}\right)_{3}\right], 30.9,32.1(-, \mathrm{C}-3), 49.5,49.9(-, \mathrm{C}-$ 5), $57.7(+, \mathrm{C}-2), 62.1,63.3(-, \mathrm{C}-1), 80.0\left[\mathrm{C}_{\text {quat }}, \mathrm{C}\left(\mathrm{CH}_{3}\right)_{3}\right], 119.9\left(\mathrm{C}_{\text {quat }}, \mathrm{CN}\right), 153.3\left(\mathrm{C}_{\text {quat }}\right.$, $\mathrm{NCO}_{2}$ ); IR (film): nu(tilde) $=2952 \mathrm{~cm}^{-1}, 2895,2858,2241,1700,1471,1405,1258,1177,1101$; MS (CI), $m / z(\%): 359(1)\left[\mathrm{M}+\mathrm{NH}_{4}^{+}\right], 342(100)\left[\mathrm{M}+\mathrm{H}^{+}\right]$; elemental analysis (\%) for $\mathrm{C}_{17} \mathrm{H}_{31} \mathrm{DN}_{2} \mathrm{O}_{3} \mathrm{Si}$ (341.23): C 59.78, H 9.74, N 8.20; found C 59.54, H 9.60, N 8.03.

The mixed fractions enriched with trans-epimer were purified by column chromatography as it

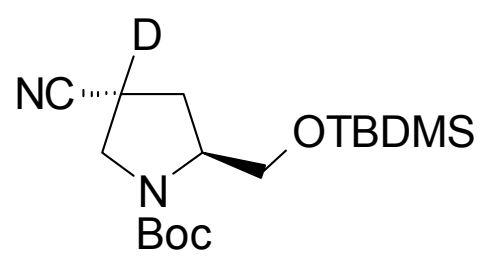
was described before to give $(2 S, 4 R)$-D-61 $(0.56 \mathrm{~g}, 5.9 \%, 82 \% \mathrm{D}$ according to ESI-MS spectrum) as a colorless oil. $[\alpha]_{24}^{\mathrm{D}}-60.2(c=$ $\left.0.48, \mathrm{CHCl}_{3}\right) ;{ }^{1} \mathrm{H}$ NMR $\left(250 \mathrm{MHz}, \mathrm{CDCl}_{3}\right): \delta=0.01[\mathrm{~s}, 6 \mathrm{H}$, $\left.\mathrm{Si}\left(\mathrm{CH}_{3}\right)_{2}\right], 0.85$ [s, $\left.9 \mathrm{H}, \mathrm{SiC}\left(\mathrm{CH}_{3}\right)_{3}\right], 1.44$ [s, $\left.9 \mathrm{H}, \mathrm{C}\left(\mathrm{CH}_{3}\right)_{3}\right], 2.15-2.46$ (m, $\left.2 \mathrm{H}, 3-\mathrm{H}\right), 3.24-3.47$ (m, 0.2 H, 4-H), 3.41-3.80 (m, $3.5 \mathrm{H}, 0.51-\mathrm{H}, 2-\mathrm{H}, 5-\mathrm{H}), 3.87-4.07(\mathrm{~m}, 1.5 \mathrm{H}, 1.51-\mathrm{H}) ;{ }^{13} \mathrm{C}$ 
$\operatorname{NMR}\left(62.9 \mathrm{MHz}, \mathrm{CDCl}_{3}\right): \delta=-5.7\left[+, \mathrm{Si}\left(\mathrm{CH}_{3}\right)_{2}\right], 18.0\left(\mathrm{C}_{\text {quat }}, \mathrm{SiC}\right), 25.7\left[+, \mathrm{SiC}\left(\mathrm{CH}_{3}\right)_{3}\right], 26.5(\mathrm{t}$, $J=15.7 \mathrm{~Hz}, \mathrm{C}-4), 28.2\left[+, \mathrm{C}\left(\mathrm{CH}_{3}\right)_{3}\right], 32.4,33.2(-, \mathrm{C}-3), 49.4,49.5(-, \mathrm{C}-5), 57.5$ (+, C-2), 63.5, $64.0(-, \mathrm{C}-1), 80.0\left[\mathrm{C}_{\text {quat }}, \mathrm{C}\left(\mathrm{CH}_{3}\right)_{3}\right], 120.0\left(\mathrm{C}_{\text {quat }}, \mathrm{CN}\right), 153.2\left(\mathrm{C}_{\text {quat }}, \mathrm{NCO}_{2}\right)$; IR (film): nu(tilde) = $2955 \mathrm{~cm}^{-1}, 2930,2885,2858,2245,1702,1473,1393,1257,1177,1121$; MS (ESI), (positive) $m / z(\%): 364(48)\left[\mathrm{M}+\mathrm{Na}^{+}\right]$.

(2S,4S)-N-Boc-O-TBDMS-4-Formylprolinol (2S,4R)-(62): A $1 \mathrm{M}$ DIBAH in hexanes $(36.1 \mathrm{~mL}$,

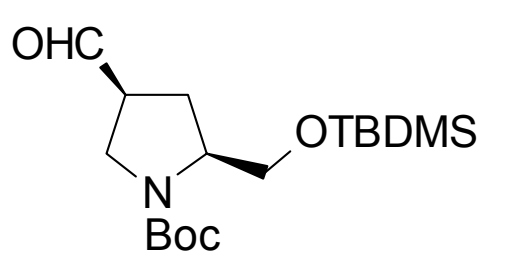
$36.10 \mathrm{mmol}$ ) was added dropwise at $-30{ }^{\circ} \mathrm{C}$ over $10 \mathrm{~min}$ to a stirred solution of the cyanide $(2 S, 4 S)-61(9.10 \mathrm{~g}, 26.72 \mathrm{mmol})$ in $\mathrm{CH}_{2} \mathrm{Cl}_{2}(90 \mathrm{~mL})$. The reaction mixture was stirred at $-30--20{ }^{\circ} \mathrm{C}$ for $2 \mathrm{~h}$, whereafter methanol $(2.1 \mathrm{~mL})$ was added dropwise at $0{ }^{\circ} \mathrm{C}$ for 3 min and stirring was continued at the same temperature for $15 \mathrm{~min}$. A saturated aqueous $\mathrm{NH}_{4} \mathrm{Cl}(8.4 \mathrm{~mL})$ was then added and the mixture was allowed to warm to $20^{\circ} \mathrm{C}$. After $45 \mathrm{~min}$ the reaction mixture was diluted with $\mathrm{Et}_{2} \mathrm{O}(80 \mathrm{~mL})$ and saturated aqueous potassium sodium tartrate $(14 \mathrm{~mL})$ and vigorous stirring continued for an additional hour. The phases were separated and the organic fraction was washed twice with solution of citric acid $(5.14 \mathrm{~g}, 26.72 \mathrm{mmol})$ in water $(120 \mathrm{~mL})$, with water $(5 \times 50 \mathrm{~mL})$, brine $(2 \times 20 \mathrm{~mL})$, dried, filtered and concentrated under reduced pressure. The residue was taken up with hexanes $(30 \mathrm{~mL})$ filtered through Celite pad and concentrated under reduced pressure to give the aldehyde $(2 S, 4 S)-62(9.0 \mathrm{~g}, 98 \%$ crude) as a colorless oil, which was used for the next step without further purification. $R_{\mathrm{f}}=0.37$, EtOAc/hexanes 1:4; ${ }^{1} \mathrm{H}$ NMR $\left(250 \mathrm{MHz}, \mathrm{CDCl}_{3}\right): \delta=0.03\left[\mathrm{~s}, 6 \mathrm{H}, \mathrm{Si}\left(\mathrm{CH}_{3}\right)_{2}\right], 0.86[\mathrm{~s}, 9 \mathrm{H}$, $\left.\mathrm{SiC}\left(\mathrm{CH}_{3}\right)_{3}\right], 1.43\left[\mathrm{~s}, 9 \mathrm{H}, \mathrm{C}\left(\mathrm{CH}_{3}\right)_{3}\right], 1.78-2.20\left(\mathrm{~m}, 1 \mathrm{H}, 3-\mathrm{H}_{\mathrm{a}}\right), 2.22-2.41\left(\mathrm{~m}, 1 \mathrm{H}, 3-\mathrm{H}_{\mathrm{b}}\right), 2.78-$ 3.12 (m, $1 \mathrm{H}, 4-\mathrm{H}), 3.45-4.02$ (m, 5 H, 1-H, 2-H, 5-H), 9.63 (s, $1 \mathrm{H}, \mathrm{CHO})$.

(2S,4S)-N-Boc-O-TBDMS-4-Deutero-4-formylprolinol (2S,4S)-D-(62): A 1 M DIBAH in hexanes $(22.6 \mathrm{~mL}, 22.6 \mathrm{mmol})$ was added dropwise at $-30^{\circ} \mathrm{C}$ over $10 \mathrm{~min}$ to a stirred solution 
of the cyanide $(2 S, 4 S)$-D-61 $(5.72 \mathrm{~g}, 16.74 \mathrm{mmol})$ in $\mathrm{CH}_{2} \mathrm{Cl}_{2}(50 \mathrm{~mL})$. The reaction mixture was stirred at $-30--20{ }^{\circ} \mathrm{C}$ for $2 \mathrm{~h}$, whereafter $\mathrm{CH}_{3} \mathrm{OD}(1 \mathrm{~mL})$ was added dropwise at $0{ }^{\circ} \mathrm{C}$ for 3 min and stirring was continued at the same temperature for $15 \mathrm{~min}$. A saturated aqueous $\mathrm{NH}_{4} \mathrm{Cl}(5.5$ $\mathrm{mL}$ ) was then added and the mixture was allowed to warm to $20^{\circ} \mathrm{C}$. After $45 \mathrm{~min}$ the reaction mixture was diluted with $\mathrm{Et}_{2} \mathrm{O}(80 \mathrm{~mL})$ and saturated aqueous potassium sodium tartrate $(14 \mathrm{~mL})$ and vigorous stirring continued for an additional hour. The phases were separated and the organic fraction was washed twice with solution of citric acid (3.21 g, $16.74 \mathrm{mmol})$ in water (65 $\mathrm{mL})$, with water $(5 \times 50 \mathrm{~mL})$, brine $(2 \times 20 \mathrm{~mL})$, dried and concentrated under reduced pressure. The residue was taken up with hexanes $(30 \mathrm{~mL})$ filtered through Celite pad and concentrated under reduced pressure to give aldehyde $(2 S, 4 S)$-D-62 (5.52 g, 97\% crude) as a colorless oil, which was used for next step without further purification. $R_{\mathrm{f}}=0.37$, EtOAc/hexanes $1: 4 ;{ }^{1} \mathrm{H}$ $\operatorname{NMR}\left(250 \mathrm{MHz}, \mathrm{CDCl}_{3}\right): \delta=0.02\left[\mathrm{~s}, 6 \mathrm{H}, \mathrm{Si}\left(\mathrm{CH}_{3}\right)_{2}\right], 0.87\left[\mathrm{~s}, 9 \mathrm{H}, \mathrm{SiC}\left(\mathrm{CH}_{3}\right)_{3}\right], 1.45[\mathrm{~s}, 9 \mathrm{H}$, $\left.\mathrm{C}\left(\mathrm{CH}_{3}\right)_{3}\right], 2.18-2.31(\mathrm{~m}, 2 \mathrm{H}, 3-\mathrm{H}), 3.43-4.03$ (m, $\left.5 \mathrm{H}, 1-\mathrm{H}, 2-\mathrm{H}, 5-\mathrm{H}\right), 9.65(\mathrm{~s}, 1 \mathrm{H}, \mathrm{CHO}) ;{ }^{13} \mathrm{C}$ $\operatorname{NMR}\left(62.9 \mathrm{MHz}, \mathrm{CDCl}_{3}\right): \delta=-5.5\left[+, \mathrm{Si}\left(\mathrm{CH}_{3}\right)_{2}\right], 18.3\left(\mathrm{C}_{\text {quat }}, \mathrm{SiC}\right), 25.9\left[+, \mathrm{SiC}\left(\mathrm{CH}_{3}\right)_{3}\right], 28.4$ $\left[+, \mathrm{C}\left(\mathrm{CH}_{3}\right)_{3}\right], 46.2,46.5(-, \mathrm{C}-3), 48.7(\mathrm{t}, J=19.7 \mathrm{~Hz}, \mathrm{C}-4), 57.8(+, \mathrm{C}-2), 63.5,64.2(-, \mathrm{C}-1)$, $79.4\left[\mathrm{C}_{\text {quat }}, \mathrm{C}\left(\mathrm{CH}_{3}\right)_{3}\right], 153.9\left(\mathrm{C}_{\text {quat }}, \mathrm{NCO}_{2}\right), 201.7(+, \mathrm{CHO})$.

(2S,4R)-N-Boc-4-(Z)-Propenylprolinol (2S,4R)-(54): A freshly prepared $0.85 \mathrm{M}$ solution of $t$ BuOK in THF (108 mL, $91.68 \mathrm{mmol})$ was added to a suspension of ethyltriphenylphosponium bromide $(44.00 \mathrm{~g}, 118.52 \mathrm{mmol})$ in THF $(50 \mathrm{~mL})$ at $0{ }^{\circ} \mathrm{C}$. The cooling bath was removed and stirring continued for an additional $2 \mathrm{~h}$. The mixture was then cooled to $-78^{\circ} \mathrm{C}$ and a solution of $(2 S, 4 S)-62(9.00 \mathrm{~g}, 26.20 \mathrm{mmol})$ in THF $(30 \mathrm{~mL})$ was added dropwise within $2 \mathrm{~h}$. Stirring continued at the same temperature for a further $24 \mathrm{~h}$, and then the mixture was allowed to warm to $20{ }^{\circ} \mathrm{C}$ for $24 \mathrm{~h}$. After $48 \mathrm{~h}$, the reaction flask was immersed into an ice/water bath and a saturated aqueous $\mathrm{Na}_{2} \mathrm{SO}_{4}(50 \mathrm{~mL})$ was added. The reaction mixture was concentrated under reduced pressure, the residue was taken up with $\mathrm{CH}_{2} \mathrm{Cl}_{2} / \mathrm{Et}_{2} \mathrm{O}$ 1:4 (200 mL), filtered through a 
silica gel pad $(10 \mathrm{~cm})$, concentrated, dissolved in $\mathrm{Et}_{2} \mathrm{O}(20 \mathrm{~mL})$, filtered, concentrated, dissolved in $\mathrm{Et}_{2} \mathrm{O} /$ hexanes 1:1 $(20 \mathrm{~mL})$, filtered, concentrated, dissolved in hexanes $(20 \mathrm{~mL})$, filtered, concentrated, dissolved in pentane $(20 \mathrm{~mL})$, filtered, concentrated and finally purified by column chromatography (EtOAc/hexanes $\left.1: 8, R_{\mathrm{f}}=0.51\right)$ to give the impure $(2 S, 4 R)$ - $N$-Boc- $O$-TBDMS4-(Z)-propenylprolinol $(2 S, 4 S)-63(5.3 \mathrm{~g})$ which was used for the next step without further purification. TBAF $3 \mathrm{H}_{2} \mathrm{O}(8.86 \mathrm{~g}, 28.12 \mathrm{mmol})$ was added to a stirring solution of crude alkene (5.0 g, max. $14.06 \mathrm{mmol})$ in THF $(15 \mathrm{~mL})$ at $20^{\circ} \mathrm{C}$. After $1.5 \mathrm{~h}$ the mixture was taken up with $\mathrm{Et}_{2} \mathrm{O}(100 \mathrm{~mL})$, washed with water $(5 \times 20 \mathrm{~mL})$, brine $(2 \times 20 \mathrm{~mL})$, dried, concentrated under reduced pressure and purified by column chromatography (EtOAc/hexanes 1:2.5, $\left.R_{\mathrm{f}}=0.32\right)$ to give $(2 S, 4 R)-54$ [2.69 g, 42\% over 3 steps from $(2 S, 4 S)-61]$ as a colorless oil solidified by seeding into a white solid and $(2 S, 4 S)$-epimer $(2 S, 4 S)-54(0.12$ g, 2\%). (2S,4S)-54: m.p. $41-43$ ${ }^{\circ} \mathrm{C} ;[\alpha]_{24}^{\mathrm{D}}-47.9\left(c=0.97, \mathrm{CHCl}_{3}\right) ;{ }^{1} \mathrm{H} \mathrm{NMR}\left(250 \mathrm{MHz}, \mathrm{CDCl}_{3}\right): \delta=1.23(\mathrm{ddd}, J=11,11$, $\left.11 \mathrm{~Hz}, 1 \mathrm{H}, 3-\mathrm{H}_{\mathrm{a}}\right), 1.46\left[\mathrm{~s}, 9 \mathrm{H}, \mathrm{C}\left(\mathrm{CH}_{3}\right)_{3}\right], 1.65\left(\mathrm{dd}, J=6.9,0.8 \mathrm{~Hz}, 3 \mathrm{H}, 3^{\prime}-\mathrm{H}\right), 2.13$ (ddd, $J=$ 6.7, 6.1, $\left.6.1 \mathrm{~Hz}, 1 \mathrm{H}, 3-\mathrm{H}_{\mathrm{b}}\right), 2.90\left(\mathrm{dddd}, J=10.4,10.4,10.4,10.4 \mathrm{~Hz}, 1 \mathrm{H}, 5-\mathrm{H}_{\mathrm{a}}\right), 2.85-3.10(\mathrm{~m}$, $1 \mathrm{H}, 4-\mathrm{H}), 3.52-3.77\left(\mathrm{~m}, 3 \mathrm{H}, 5-\mathrm{H}_{\mathrm{b}}, 2-\mathrm{H}, 1-\mathrm{H}_{\mathrm{a}}\right), 3.96\left(\mathrm{dd}, J=7.6,14.9 \mathrm{~Hz}, 1 \mathrm{H}, 1-\mathrm{H}_{\mathrm{b}}\right), 5.18(\mathrm{t}, J$ $\left.=9.8 \mathrm{~Hz}, 1 \mathrm{H}, 1^{\prime}-\mathrm{H}\right), 5.30(\mathrm{dd}, J=8.9,1.8 \mathrm{~Hz}, 1 \mathrm{H}, \mathrm{OH}), 5.52\left(\mathrm{dq}, J=9.8,6.9 \mathrm{~Hz}, 1 \mathrm{H}, 2^{\prime}-\mathrm{H}\right)$; ${ }^{13} \mathrm{C}$ NMR $\left(62.9 \mathrm{MHz}, \mathrm{CDCl}_{3}\right): \delta=13.2\left(+, \mathrm{CH}_{3}\right), 28.4\left[+, \mathrm{C}\left(\mathrm{CH}_{3}\right)_{3}\right], 35.2(+, \mathrm{C}-4), 35.8(-, \mathrm{C}-3)$, $52.7(-, \mathrm{C}-5), 61.1(+, \mathrm{C}-2), 67.6(-, \mathrm{C}-1), 80.4\left[\mathrm{C}_{\text {quat }}, \mathrm{C}\left(\mathrm{CH}_{3}\right)_{3}\right], 126.3\left(+, \mathrm{C}-2^{\prime}\right), 129.8\left(+, \mathrm{C}-1^{\prime}\right)$, $156.8\left(\mathrm{C}_{\text {quat }}, \mathrm{NCO}_{2}\right)$; IR (film): nu(tilde $)=3398 \mathrm{~cm}^{-1}, 2976,2932,2871,1696,1402,1164$; MS (EI): $m / z(\%)=241(1)\left[\mathrm{M}^{+}\right], 210(35)\left[\mathrm{M}^{+}-\mathrm{CH}_{3} \mathrm{O}\right], 168(9), 154(100)\left[\mathrm{M}^{+}-\mathrm{C}_{5} \mathrm{H}_{11} \mathrm{O}\right], 110(96)$ $\left[\mathrm{C}_{7} \mathrm{H}_{12} \mathrm{~N}^{+}\right], 67$ (5), $57(89)\left[\mathrm{C}_{4} \mathrm{H}_{9}^{+}\right], 41(15)\left[\mathrm{C}_{3} \mathrm{H}_{5}^{+}\right]$; HRMS: $\mathrm{C}_{13} \mathrm{H}_{23} \mathrm{NO}_{3}$ : 241.1678; elemental analysis (\%) for $\mathrm{C}_{13} \mathrm{H}_{23} \mathrm{NO}_{3}$ (241.3): calcd. C 64.70, H 9.61, N 5.80; found C 64.83, H 9.74, N 5.64

$(2 S, 4 R)-54: R_{\mathrm{f}}=0.28$ EtOAc/hexanes $1: 2.5 ;{ }^{1} \mathrm{H}$ NMR $\left(250 \mathrm{MHz}, \mathrm{CDCl}_{3}\right): \delta=1.44[\mathrm{~s}, 9 \mathrm{H}$, $\left.\mathrm{C}\left(\mathrm{CH}_{3}\right)_{3}\right], 1.60\left(\mathrm{dd}, J=6.8,1.8 \mathrm{~Hz}, 3 \mathrm{H}, 3^{\prime}-\mathrm{H}\right), 1.68-1.85(\mathrm{~m}, 2 \mathrm{H}, 3-\mathrm{H}), 3.01$ (ddd, $J=9.5,9.5$, $9.5 \mathrm{~Hz}, 1 \mathrm{H}, 5-\mathrm{H}_{\mathrm{a}}$ ), 3.13 (ddddd, $\left.J=8.3,8.3,8.3,8.3,8.3 \mathrm{~Hz}, 1 \mathrm{H}, 4-\mathrm{H}\right), 3.48$ (dd, $J=6.9$, 
$\left.10.1 \mathrm{~Hz}, 1 \mathrm{H}, 5-\mathrm{H}_{\mathrm{b}}\right), 3.54-3.59\left(\mathrm{~m}, 2 \mathrm{H}, 1-\mathrm{H}_{\mathrm{a}}, 2-\mathrm{H}\right), 3.77-4.12\left(\mathrm{~m}, 1 \mathrm{H}, 1-\mathrm{H}_{\mathrm{b}}\right), 4.40$ (br, $1 \mathrm{H}$, $\mathrm{OH}), 5.21\left(\mathrm{dd}, J=10.9,10.9 \mathrm{~Hz}, 1 \mathrm{H}, 1^{\prime}-\mathrm{H}\right), 5.48\left(\mathrm{dq}, J=10.9,6.8 \mathrm{~Hz}, 1 \mathrm{H}, 2^{\prime}-\mathrm{H}\right) ;{ }^{13} \mathrm{C} \mathrm{NMR}$ $\left(62.9 \mathrm{MHz}, \mathrm{CDCl}_{3}\right): \delta=13.2\left(+, \mathrm{CH}_{3}\right), 28.4\left[+, \mathrm{C}\left(\mathrm{CH}_{3}\right)_{3}\right], 34.8(+, \mathrm{C}-4), 35.1(-, \mathrm{C}-3), 52.7(-$, C-5), $59.6(+, \mathrm{C}-2), 67.7(-, \mathrm{C}-1), 80.2\left[\mathrm{C}_{\text {quat }}, \mathrm{C}\left(\mathrm{CH}_{3}\right)_{3}\right], 125.9\left(+, \mathrm{C}-2^{\prime}\right), 130.4\left(+, \mathrm{C}-1^{\prime}\right), 158.3$ $\left(\mathrm{C}_{\text {quat }}, \mathrm{NCO}_{2}\right)$.

$(2 S, 4 R)-N-B o c-4, I^{\prime}-$ Dideutero-4-(Z)-propenylprolinol $(2 S, 4 R)-\mathrm{D}_{2}-(\mathbf{5 4})$ : A freshly prepared 1.08 M solution of $t \mathrm{BuOK}$ in THF $(45.0 \mathrm{~mL}, 41.67 \mathrm{mmol})$ was added to a suspension of $(1,1-$ dideuteroethyl)triphenylphosponium bromide $(18.5 \mathrm{~g}, 49.56 \mathrm{mmol})$ in $\mathrm{THF}(45 \mathrm{~mL})$ at $0{ }^{\circ} \mathrm{C}$, the cooling bath was removed and stirring continued for an additional $2 \mathrm{~h}$. The mixture was then cooled to $-78{ }^{\circ} \mathrm{C}$ and a solution of $(2 S, 4 S)$-D-62 (5.52 g, $\left.16.02 \mathrm{mmol}\right)$ in THF $(30 \mathrm{~mL})$ was added dropwise for $2 \mathrm{~h}$. Stirring continued at the same temperature for further $24 \mathrm{~h}$, and then the mixture was allowed to warm to $20^{\circ} \mathrm{C}$ for $24 \mathrm{~h}$. After $48 \mathrm{~h}$, the reaction flask was immersed into an ice/water bath and a saturated aqueous $\mathrm{Na}_{2} \mathrm{SO}_{4}(50 \mathrm{~mL})$ was added. The reaction mixture was concentrated under reduced pressure, taken up with $\mathrm{CH}_{2} \mathrm{Cl}_{2} / \mathrm{Et}_{2} \mathrm{O}(120 \mathrm{~mL})$, filtered through silica gel pad $(10 \mathrm{~cm})$, concentrated, dissolved in $\mathrm{Et}_{2} \mathrm{O} /$ hexanes $1: 1(50 \mathrm{~mL})$, filtered, concentrated, dissolved in hexanes $(50 \mathrm{~mL})$, filtered, dissolved in pentane $(50 \mathrm{~mL})$, filtered, concentrated and finally purified by column chromatography (EtOAc/hexanes 1:10, $\left.R_{\mathrm{f}}=0.39\right)$ to give impure $(2 S, 4 R)-\mathrm{D}_{2}-\mathbf{6 3}$ (3.87 g) which was used for next step without further purification. TBAF. $3 \mathrm{H}_{2} \mathrm{O}(10.25 \mathrm{~g}, 32.48 \mathrm{mmol})$ was added to a stirring solution of crude alkene $(3.87 \mathrm{~g}$, max. $10.82 \mathrm{mmol})$ in THF $(40 \mathrm{~mL})$ at $20^{\circ} \mathrm{C}$. After $1.5 \mathrm{~h}$ the mixture was taken up with $\mathrm{Et}_{2} \mathrm{O}$ $(250 \mathrm{~mL})$, washed with water $(5 \times 50 \mathrm{~mL})$, brine $(2 \times 30 \mathrm{~mL})$, dried, concentrated under reduced pressure and purified by column chromatography (EtOAc/hexanes 3:7, $R_{\mathrm{f}}=0.34$ ) to give $(2 S, 4 R)-\mathrm{D}_{2}-54\left(1.84 \mathrm{~g}, 45 \%\right.$ on 3 steps from $\mathbf{6}, \mathrm{D}_{2}$ and $\mathrm{D}$ contents $65 \%$ and $34 \%$ respectively, as determined by MS) as a colorless oil solidified by seeding into a white solid. M.p. $35-37{ }^{\circ} \mathrm{C}$; $[\alpha]_{24}^{\mathrm{D}}-46.9\left(c=1.0, \mathrm{CHCl}_{3}\right) ;{ }^{1} \mathrm{H} \mathrm{NMR}\left(300 \mathrm{MHz}, \mathrm{C}_{2} \mathrm{D}_{2} \mathrm{Cl}_{4}, 373 \mathrm{~K}\right)$ : the mixture of 
isotopomers: $\delta=1.36\left(\mathrm{dd}, J=12 \mathrm{~Hz}, 12 \mathrm{~Hz}, 1 \mathrm{H}, 3-\mathrm{H}_{\mathrm{a}}\right), 1.51\left[\mathrm{~s}, 9 \mathrm{H}, \mathrm{C}\left(\mathrm{CH}_{3}\right)_{3}\right], 1.68(\mathrm{dd}, J=$ $\left.0.8 \mathrm{~Hz}, 3 \mathrm{H}, 3^{\prime}-\mathrm{H}\right), 2.20\left(\mathrm{dd}, J=7.1,12.8 \mathrm{~Hz}, 1 \mathrm{H}, 3-\mathrm{H}_{\mathrm{b}}\right), 2.93\left(\mathrm{~d}, J=11 \mathrm{~Hz}, 1 \mathrm{H}, 5-\mathrm{H}_{\mathrm{a}}\right), 2.95-$ $3.05(\mathrm{~m}, 0.1 \mathrm{H}, 4-\mathrm{H}), 3.58-3.78\left(\mathrm{~m}, 3 \mathrm{H}, 2-\mathrm{H}, 1-\mathrm{H}_{\mathrm{a}}, 5-\mathrm{H}_{\mathrm{b}}\right), 3.84-3.99$ (m, $\left.1 \mathrm{H}, 1-\mathrm{H}_{\mathrm{b}}\right), 5.19-5.26$ $\left(\mathrm{m}, 1 \mathrm{H}, 1^{\prime}-\mathrm{H}\right), 5.33(\mathrm{~d}, J=7.8 \mathrm{~Hz}, \mathrm{OH}), 5.58\left(\mathrm{dq}, J=10.5,6.0 \mathrm{~Hz}, 0.2 \mathrm{H}, 2^{\prime}-\mathrm{H}\right) ;{ }^{13} \mathrm{C} \mathrm{NMR}(62.9$ $\left.\mathrm{MHz}, \mathrm{CDCl}_{3}\right): \delta=13.0,13.1\left(+, \mathrm{CH}_{3}\right), 28.4\left[+, \mathrm{C}\left(\mathrm{CH}_{3}\right)_{3}\right], 34.7(\mathrm{t}, J=18.6 \mathrm{~Hz}, \mathrm{C}-4), 35.7(-, \mathrm{C}-$ 3), $52.5(-, \mathrm{C}-5), 61.0(+, \mathrm{C}-2), 67.5(-, \mathrm{C}-1), 80.3\left[\mathrm{C}_{\text {quat }}, \mathrm{C}\left(\mathrm{CH}_{3}\right)_{3}\right], 125.3\left(\mathrm{t}, J=23.2 \mathrm{~Hz}, \mathrm{C}-2^{\prime}\right)$, $126.2\left(+, \mathrm{C}-2^{\prime}\right), 129.5,129.7\left(+, \mathrm{C}-1^{\prime}\right), 156.8\left(\mathrm{C}_{\text {quat }}, \mathrm{NCO}_{2}\right)$; IR (film): nu(tilde) $=3416 \mathrm{~cm}^{-1}$, 2976, 2932, 2870, 1696, 1411, 1174; MS (EI, $70 \mathrm{eV}), m / z(\%)=243(1)\left[\mathrm{M}^{+}\right], 212(50)\left[\mathrm{M}^{+}-\right.$ $\left.\mathrm{CH}_{3} \mathrm{O}\right], 170$ (9), $156(100)\left[\mathrm{M}^{+}-\mathrm{C}_{5} \mathrm{H}_{11} \mathrm{O}\right], 112(80)\left[\mathrm{C}_{7} \mathrm{H}_{10} \mathrm{D}_{2} \mathrm{~N}^{+}\right], 83$ (6), 69 (5), 57 (92) $\left[\mathrm{C}_{4} \mathrm{H}_{9}{ }^{+}\right]$; HRMS (EI): calcd for $\mathrm{C}_{13} \mathrm{H}_{21} \mathrm{D}_{2} \mathrm{NO}_{3}$ : 243.1803; found 243.1803; elemental analysis (\%) for $\mathrm{C}_{13} \mathrm{H}_{22} \mathrm{DNO}_{3}$ (242.3): calcd. C 64.43, H 9.98, N 5.78; found C 64.79, H 9.64, N 5.60.

(2S,4R)-N-Boc-4-(Z)-Propenylproline (2S,4R)-(16): A $2.67 \mathrm{M}$ Jones reagent $(36.5 \mathrm{~mL}, 97.46$ mmol) was added to a solution of alcohol $(2 S, 4 R)-54(2.35 \mathrm{~g}, 9.74 \mathrm{mmol})$ in acetone $(800 \mathrm{~mL})$ at $4{ }^{\circ} \mathrm{C}$ for $1 \mathrm{~h}$ and the mixture was stirred at the same temperature for an additional $2 \mathrm{~h}$. Isopropanol $(5 \mathrm{~mL})$ was then added dropwise within $10 \mathrm{~min}$ and the mixture was allowed to warm to $20{ }^{\circ} \mathrm{C}$. The reaction mixture was concentrated to $200 \mathrm{~mL}$ under reduced pressure at the bath temperature not higher then $30^{\circ} \mathrm{C}$, taken up with $\mathrm{Et}_{2} \mathrm{O}(500 \mathrm{~mL})$ and washed with water $(3$ $\times 100 \mathrm{~mL})$. The aqueous fraction was back extracted with ether $(3 \times 50 \mathrm{~mL})$ and the organic layers were combined, washed with brine $(2 \times 50 \mathrm{~mL})$, dried, filtered, concentrated to $100 \mathrm{~mL}$ under reduced pressure and extracted with saturated aqueous $\mathrm{NaHCO}_{3}(5 \times 40 \mathrm{~mL})$. The combined aqueous fractions were washed with $\mathrm{Et}_{2} \mathrm{O}(2 \times 50 \mathrm{~mL}), \mathrm{pH}$ of the aqueous fractions was carefully adjusted to 2.5-3 with solid $\mathrm{NaHSO}_{4}$, a formed emulsion was extracted with $\mathrm{Et}_{2} \mathrm{O}$ $(2 \times 100 \mathrm{~mL})$ and the organic fraction was washed with $1 \mathrm{M} \mathrm{NaHSO}_{4}(3 \times 50 \mathrm{~mL})$, water $(3 \times 50$ $\mathrm{mL})$, brine $(2 \times 20 \mathrm{~mL})$, dried, filtered and concentrated under reduced pressure. The residue was 
recrystallized twice from hexanes and finally purified by column chromatography (EtOAc/hexanes 1:3 (2\% AcOH), $\left.R_{\mathrm{f}}=0.27\right)$ to give $(2 S, 4 R)-16(1.63 \mathrm{~g}, 65 \%)$ as a white solid. HPLC: detection: $200 \mathrm{~nm}, t_{\mathrm{R}}=18.58 \mathrm{~min}$, gradient: $20 \rightarrow 50 \% \mathrm{MeCN}$ in $\mathrm{H}_{2} \mathrm{O}(0.1 \% \mathrm{TFA})$ for 30 min, purity $>99 \%$; m.p. $84-85^{\circ} \mathrm{C} ;[\alpha]_{24}^{\mathrm{D}}-84.4\left(c=0.86, \mathrm{CHCl}_{3}\right) ;{ }^{1} \mathrm{H} \mathrm{NMR}(250 \mathrm{MHz}$, $\left.\mathrm{CDCl}_{3}\right): \delta=1.42,1.48\left[2 \mathrm{~s}, 9 \mathrm{H}, \mathrm{C}\left(\mathrm{CH}_{3}\right)_{3}\right], 1.66\left(\mathrm{~d}, J=6.8 \mathrm{~Hz}, 3 \mathrm{H}, 3^{\prime}-\mathrm{H}\right), 1.72-1.84,1.93-2.12$ (m, $\left.1 \mathrm{H}, 3-\mathrm{H}_{\mathrm{a}}\right), 2.27-2.54\left(\mathrm{~m}, 1 \mathrm{H}, 3-\mathrm{H}_{\mathrm{b}}\right), 3.06$ (ddddd, $\left.J=9.9,9.9,9.9,9.9,9.9 \mathrm{~Hz}, 1 \mathrm{H}, 4-\mathrm{H}\right)$, 2.98-3.20 (m, $\left.1 \mathrm{H}, 5-\mathrm{H}_{\mathrm{a}}\right), 3.64-3.86\left(\mathrm{~m}, 1 \mathrm{H}, 5-\mathrm{H}_{\mathrm{b}}\right), 4.25,4.35(2 \mathrm{dd}, J=8.3,8.3 \mathrm{~Hz}, 1 \mathrm{H}, 2-\mathrm{H})$, $5.26\left(\mathrm{ddq}, J=8.5,8.5,1.8 \mathrm{~Hz}, 1 \mathrm{H}, 1^{\prime}-\mathrm{H}\right), 5.52\left(\mathrm{dq}, J=8.5,6.8 \mathrm{~Hz}, 1 \mathrm{H}, 2^{\prime}-\mathrm{H}\right) ;{ }^{13} \mathrm{C}$ NMR $(62.9$ $\left.\mathrm{MHz}, \mathrm{CDCl}_{3}\right): \delta=13.1\left(+, \mathrm{CH}_{3}\right), 28.1,28.3\left[+, \mathrm{C}\left(\mathrm{CH}_{3}\right)_{3}\right], 35.7,36.1(+, \mathrm{C}-4), 36.1,37.3(-, \mathrm{C}-$ 3), 51.4, $51.9(-, \mathrm{C}-4), 58.9,59.1(+, \mathrm{C}-2), 80.6\left[\mathrm{C}_{\text {quat }}, \mathrm{C}\left(\mathrm{CH}_{3}\right)_{3}\right], 126.6,126.9\left(+, \mathrm{C}-2^{\prime}\right), 129.0$, $129.2\left(+, \mathrm{C}^{\prime} 1^{\prime}\right), 153.6,154.8\left(\mathrm{C}_{\text {quat }}, \mathrm{NCO}_{2}\right), 177.3,178.4\left(\mathrm{C}_{\text {quat }}, \mathrm{C}-1\right)$; IR $(\mathrm{KBr})$ : nu(tilde) $=3020$, 2975, 2943, 2880, 2625, 1736, 1633, 1441, 1369, 1252, 1168; MS (ESI), positive $m / z(\%)=300$ (35) $\left[\mathrm{M}-\mathrm{H}+2 \mathrm{Na}^{+}\right], 278(16)\left[\mathrm{M}+\mathrm{Na}^{+}\right]$; negative $m / z=254(100)\left[\mathrm{M}-\mathrm{H}^{-}\right]$; HRMS (EI): calcd for $\mathrm{C}_{13} \mathrm{H}_{21} \mathrm{NO}_{4}$ : 255.1471; found 255.1471; elemental analysis (\%) for $\mathrm{C}_{13} \mathrm{H}_{21} \mathrm{NO}_{4}$ (255.1): calcd. C 61.16, H 8.29, N 5.49; found C 61.16, H 8.23, N 5.31.

(2S,4R)-N-Boc-4,1'-Dideutero-4-(Z)-propenylproline $(2 S, 4 R)-\mathrm{D}_{2}-(\mathbf{1 6})$ : A $2.67 \mathrm{M}$ Jones reagent (28.4 $\mathrm{mL}, 75.83 \mathrm{mmol})$ was added to a solution of alcohol $(2 S, 4 R)$ -

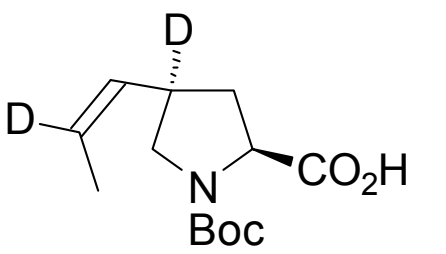
$\mathrm{D}_{2}-54(1.844 \mathrm{~g}, 7.58 \mathrm{mmol})$ in acetone $(600 \mathrm{~mL})$ at $4{ }^{\circ} \mathrm{C}$ within $1 \mathrm{~h}$ and the mixture was stirred at the same temperature for an additional 2h. Isopropanol $(4 \mathrm{~mL})$ was then added dropwise within $10 \mathrm{~min}$ and the mixture was allowed to warm to $20{ }^{\circ} \mathrm{C}$. The reaction mixture was concentrated to $150 \mathrm{~mL}$ under reduced pressure at the bath temperature not higher then $30{ }^{\circ} \mathrm{C}$ and was taken up with $\mathrm{Et}_{2} \mathrm{O}$ $(350 \mathrm{~mL})$ and washed with water $(3 \times 100 \mathrm{~mL})$. The aqueous fraction was back extracted with ether $(3 \times 50 \mathrm{~mL})$ and the organic layers were combined, washed with brine $(2 \times 50 \mathrm{~mL})$, dried, 
filtered, concentrated to $75 \mathrm{~mL}$ under reduced pressure and extracted with saturated aqueous $\mathrm{NaHCO}_{3}(5 \times 40 \mathrm{~mL})$. The combined aqueous fractions were washed with $\mathrm{Et}_{2} \mathrm{O}(2 \times 50 \mathrm{~mL}), \mathrm{pH}$ of the aqueous fractions was carefully adjusted to 2.5-3 with solid $\mathrm{NaHSO}_{4}$ and a formed emulsion was extracted with $\mathrm{Et}_{2} \mathrm{O}(2 \times 75 \mathrm{~mL})$. The organic fraction was washed with $1 \mathrm{M}$ $\mathrm{NaHSO}_{4}(3 \times 30 \mathrm{~mL})$, water $(3 \times 30 \mathrm{~mL})$, brine $(2 \times 10 \mathrm{~mL})$, dried, filtered and concentrated under reduced pressure. The residue was recrystallized twice from hexanes and finally purified by column chromatography (EtOAc/hexanes $\left.1: 3(2 \% \mathrm{AcOH}), R_{\mathrm{f}}=0.27\right)$ to give $(2 S, 4 R)-\mathrm{D}_{2}-16$ $\left(1.18 \mathrm{~g}, 61 \%, \mathrm{D}_{2}\right.$ and $\mathrm{D}$ contents $67 \%$ and $33 \%$ respectively, as determined by ESI-MS) as a white solid. HPLC: detection: $200 \mathrm{~nm}, t_{\mathrm{R}}=18.54 \mathrm{~min}$, gradient: $20 \rightarrow 50 \% \mathrm{MeCN}$ in $\mathrm{H}_{2} \mathrm{O}(0.1 \%$ TFA) for $30 \mathrm{~min}$, purity $>99 \%$; $[\alpha]_{24}^{\mathrm{D}}-68.6\left(c=1.05, \mathrm{CHCl}_{3}\right) ;{ }^{1} \mathrm{H} \mathrm{NMR}\left(250 \mathrm{MHz}, \mathrm{CDCl}_{3}\right)$ : mixture of isotopo- and rotamers: $\delta=1.41,1.46\left[2 \mathrm{~s}, 9 \mathrm{H}, \mathrm{C}\left(\mathrm{CH}_{3}\right)_{3}\right], 1.64\left(\mathrm{~s}, 3 \mathrm{H}, 3^{\prime}-\mathrm{H}\right), 1.72-$ $1.93\left(\mathrm{~m}, 1 \mathrm{H}, 3-\mathrm{H}_{\mathrm{a}}\right), 2.38,2.46\left(2 \mathrm{dd}, J=7.5,7.5 \mathrm{~Hz}, 1 \mathrm{H}, 3-\mathrm{H}_{\mathrm{b}}\right), 3.03(\mathrm{~d}, J=11.5 \mathrm{~Hz}, 0.5 \mathrm{H}, 5-$ $\left.\mathrm{H}_{\mathrm{a}}\right), 3.08\left(\mathrm{~d}, J=10.0 \mathrm{~Hz}, 0.5 \mathrm{H}, 5-\mathrm{H}_{\mathrm{a}}\right), 3.68,3.76\left(2 \mathrm{~d}, J=10.5 \mathrm{~Hz}, 1 \mathrm{H}, 5-\mathrm{H}_{\mathrm{b}}\right), 4.23,4.33(2 \mathrm{dd}$, $J=8.4,8.4 \mathrm{~Hz}, 1 \mathrm{H}, 2-\mathrm{H}), 5.25\left(\mathrm{~m}, 1^{\prime}-\mathrm{H}\right), 5.52\left(\mathrm{dq}, J=8.5,6.8 \mathrm{~Hz}, 0.15 \mathrm{H}, 2^{\prime}-\mathrm{H}\right), 9.00-10.50$ (br, $\left.1 \mathrm{H}, \mathrm{CO}_{2} \mathrm{H}\right) ;{ }^{13} \mathrm{C} \mathrm{NMR}\left(62.9 \mathrm{MHz}, \mathrm{CDCl}_{3}\right): \delta=12.8,12.9\left(+, \mathrm{CH}_{3}\right), 28.0,28.1\left[+, \mathrm{C}\left(\mathrm{CH}_{3}\right)_{3}\right]$, $35.5(t, \mathrm{C}-4), 35.5(+, \mathrm{C}-4), 36.0,37.0(-, \mathrm{C}-3), 51.2,51.7(-, \mathrm{C}-5), 58.8,59.0(+, \mathrm{C}-2), 80.4$ $\left[\mathrm{C}_{\text {quat }}, \mathrm{C}\left(\mathrm{CH}_{3}\right)_{3}\right], 126.4\left(\mathrm{t}, \mathrm{C}-2^{\prime}\right), 126.4,126.7\left(+, \mathrm{C}^{\prime} 2^{\prime}\right), 128.7,128.9\left(+, \mathrm{C}^{\prime} 1^{\prime}\right), 153.5,154.6$ $\left(\mathrm{C}_{\text {quat }}, \mathrm{NCO}_{2}\right), 177.0,177.9\left(\mathrm{C}_{\text {quat }}, \mathrm{C}-1\right)$; IR $(\mathrm{KBr}): \mathrm{nu}($ tilde $)=3020,2975,2943,2880,2623$, 1736, 1632, 1440, 1369, 1253, 1163; MS (ESI), positive $m / z(\%)=302(14)\left[\mathrm{M}-\mathrm{H}+2 \mathrm{Na}^{+}\right]$, 280 (100) $\left[\mathrm{M}+\mathrm{Na}^{+}\right]$; negative $m / z=256(100)\left[\mathrm{M}-\mathrm{H}^{-}\right]$; HRMS (ESI): calcd for $\left[\mathrm{C}_{13} \mathrm{H}_{19} \mathrm{D}_{2} \mathrm{NO}_{4} \mathrm{Na}^{+}\right]: 280.14883$; found 280.14911 . 
$(2 S, 4 R)-4,1^{\prime}$-Dideutero-4-(Z)-propenylproline $(2 S, 4 R)-\mathrm{D}_{2}-(\mathbf{1 7})$ : The $N$-protected acid $\mathrm{D}_{2}-(2 S, 4 R)-$

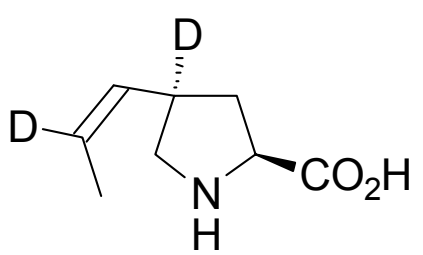

$\mathrm{D}_{2}-16(0.155 \mathrm{~g}, 0.602 \mathrm{mmol})$ was dissolved in trifluoroacetic acid (2 $\mathrm{mL})$ at $20{ }^{\circ} \mathrm{C}$. After $20 \mathrm{~min}$, all volatiles were removed under reduced

pressure to leave yellow oil, which was taken up with bidistilled water $(5 \mathrm{~mL})$. The solution was then concentrated under reduced pressure (this operation was repeated 15 times). The residue was recrystallized thrice from $\mathrm{MeOH} / \mathrm{Et}_{2} \mathrm{O}$ to give $(2 S, 4 R)-\mathrm{D}_{2}-17$ (34 mg, $36 \%)$ as a white solid. $[\alpha]_{24}^{\mathrm{D}}-4.2(c=0.31, \mathrm{MeOH}) ;{ }^{1} \mathrm{H}$ NMR $\left(250 \mathrm{MHz}, \mathrm{D}_{2} \mathrm{O}\right)$ : mixture of isotopomers: $\delta=1.63\left(\mathrm{~s}, 3 \mathrm{H}, 3^{\prime}-\mathrm{H}\right), 1.75\left(\mathrm{dd}, J=11.3 \mathrm{~Hz}, 1 \mathrm{H}, 3-\mathrm{H}_{\mathrm{a}}\right), 2.54(\mathrm{dd}, J=8.3,12.6 \mathrm{~Hz}$, $\left.1 \mathrm{H}, 3-\mathrm{H}_{\mathrm{b}}\right), 3.03$ (d, $J=11.5 \mathrm{~Hz}, 1 \mathrm{H}, 5-\mathrm{H}_{\mathrm{a}}$ ), 3.36 (ddddd, $J=9.3,9.3,9.3,9.3,9.3 \mathrm{~Hz}, 0.15 \mathrm{H}, 4-$ H), $3.48\left(\mathrm{~d}, J=11.5 \mathrm{~Hz}, 1 \mathrm{H}, 5-\mathrm{H}_{\mathrm{b}}\right), 4.16(\mathrm{dd}, J=8.3 \mathrm{~Hz}, 1 \mathrm{H}, 2-\mathrm{H}), 5.27\left(\mathrm{~m}, 1^{\prime}-\mathrm{H}\right), 5.52(\mathrm{~m}$, $\left.0.15 \mathrm{H}, 2^{\prime}-\mathrm{H}\right) ;{ }^{13} \mathrm{C}$ NMR $\left(62.9 \mathrm{MHz}, \mathrm{D}_{2} \mathrm{O}\right): \delta=12.3,12.4\left(+, \mathrm{CH}_{3}\right), 35.5(-, \mathrm{C}-3), 35.8,(\mathrm{t}, \mathrm{C}-4)$, $36.1(+, \mathrm{C}-4), 50.0(-, \mathrm{C}-5), 61.2(+, \mathrm{C}-2), 127.3,127.4\left(+, \mathrm{C}-1^{\prime}\right), 127.8\left(\mathrm{t}, J=23.0 \mathrm{~Hz}, \mathrm{C}-2^{\prime}\right)$, $128.5\left(+, \mathrm{C}-2^{\prime}\right), 174.2\left(\mathrm{C}_{\text {quat }}, \mathrm{C}-1\right)$; IR $(\mathrm{KBr}): \mathrm{nu}($ tilde $)=3101,3008,2969,2915,2856,2361$, 1626, 1388, 1315; MS (EI, $70 \mathrm{eV}), m / z(\%): 157(1)\left[\mathrm{M}^{+}\right], 112(100)\left[\mathrm{M}^{+}-\mathrm{CHO}_{2}\right], 87(16), 69$ (43) $\left[\mathrm{CF}_{3}^{+}\right], 54(7), 41(36)\left[\mathrm{C}_{3} \mathrm{H}_{5}^{+}\right]$; MS (ESI), positive $m / z(\%)=202(16)\left[\mathrm{M}-\mathrm{H}+2 \mathrm{Na}^{+}\right], 180$ (6) $\left[\mathrm{M}+\mathrm{Na}^{+}\right]$; negative $m / z=156(100)\left[\mathrm{M}-\mathrm{H}^{-}\right]$; HRMS (EI): calcd for $\left[\mathrm{C}_{8} \mathrm{H}_{11} \mathrm{D}_{2} \mathrm{NO}_{2}\right]$ : 157.1072; found 157.1072.

p-Nitrobenzyl (2S,4R)-N-Boc-4-(Z)-propenylprolinate $(2 S, 4 R)-(\mathbf{6 8})$ : A suspension of $\mathrm{K}_{2} \mathrm{CO}_{3}(54$ $\mathrm{mg}, 0.391 \mathrm{mmol})$ in a solution of $(2 S, 4 R)-16(0.100 \mathrm{~g}, 0.392 \mathrm{mmol})$ and p-nitrobenzylbromide (89 mg, $0.412 \mathrm{mmol})$ in $\mathrm{MeCN}(1.5 \mathrm{~mL})$ was stirred in a sealed tube at $70{ }^{\circ} \mathrm{C}$ for $3 \mathrm{~h}$. The mixture was then cooled, diluted with $\mathrm{Et}_{2} \mathrm{O}(10 \mathrm{~mL})$, filtered, concentrated under reduced pressure and purified by column chromatography (EtOAc/hexanes 1:3, $R_{\mathrm{f}}=0.43$ ) to give $(2 S, 4 R)-68(0.143 \mathrm{~g}, 88 \%)$ as a colorless glass. ${ }^{1} \mathrm{H} \mathrm{NMR}\left(250 \mathrm{MHz}, \mathrm{CDCl}_{3}\right): \delta=1.34,1.44[2 \mathrm{~s}$, $\left.9 \mathrm{H}, \mathrm{C}\left(\mathrm{CH}_{3}\right)_{3}\right], 1.64\left(\mathrm{~d}, J=6.8 \mathrm{~Hz}, 3 \mathrm{H}, 3^{\prime}-\mathrm{H}\right), 1.63-1.80\left(\mathrm{~m}, 1 \mathrm{H}, 3-\mathrm{H}_{\mathrm{a}}\right), 2.37-2.54(\mathrm{~m}, 1 \mathrm{H}, 3-$ $\left.\mathrm{H}_{\mathrm{b}}\right), 2.98-3.26\left(\mathrm{~m}, 2 \mathrm{H}, 4-\mathrm{H}, 5-\mathrm{H}_{\mathrm{a}}\right), 3.61-3.89\left(\mathrm{~m}, 1 \mathrm{H}, 5-\mathrm{H}_{\mathrm{b}}\right), 4.30,4.37$ (2 dd, $J=8.3,8.3 \mathrm{~Hz}$, 
$1 \mathrm{H}, 2-\mathrm{H}), 5.19\left(\mathrm{dd}, J=13.5 \mathrm{~Hz}, 1 \mathrm{H}, \mathrm{Bzl}-\mathrm{H}_{\mathrm{a}}\right) 5.16-5.30\left(\mathrm{~m}, 1 \mathrm{H}, 1^{\prime}-\mathrm{H}\right), 5.35(\mathrm{~d}, J=13.5 \mathrm{~Hz}$, $\left.1 \mathrm{H}, \mathrm{Bzl}-\mathrm{H}_{\mathrm{b}}\right), 5.43-5.61\left(\mathrm{~m}, 1 \mathrm{H}, 2^{\prime}-\mathrm{H}\right), 7.52(\mathrm{~d}, J=8.3 \mathrm{~Hz}, 2 \mathrm{H}, \mathrm{Ar}-\mathrm{H}), 8.21$ (dd, $J=7.5,7.5 \mathrm{~Hz}$,

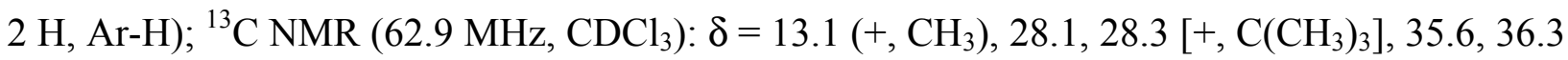
$(+, \mathrm{C}-4), 36.5,37.4(-, \mathrm{C}-3), 51.4,51.9(-, \mathrm{C}-5), 59.0,59.1(+, \mathrm{C}-2), 65.0$ (-, C-Bzl), 80.0 [C $\mathrm{C}_{\text {quat }}$, $\left.\mathrm{C}\left(\mathrm{CH}_{3}\right)_{3}\right], 123.5,123.7$ (+, C-Ar), 126.7, $126.9\left(+, \mathrm{C}-2^{\prime}\right), 128.1,128.4(+, \mathrm{C}-\mathrm{Ar}), 129.0,129.1(+$, C-1'), 142.7, 143.1 ( $\left.\mathrm{C}_{\text {quat }}, \mathrm{C}-\mathrm{Ar}\right), 147.4,147.6$ (C $\left.\mathrm{C}_{\text {quat }}, \mathrm{C}-\mathrm{Ar}\right), 153.3,154.1\left(\mathrm{C}_{\text {quat, }}, \mathrm{NCO}_{2}\right), 172.4$, 172.5 (C $\left.\mathrm{C}_{\text {quat }}, \mathrm{C}-1\right)$; IR (film): nu(tilde) $=3082 \mathrm{~cm}^{-1}, 2976,2932,2869,2623,2241,1754,1699$, 1524, 1400, 1347, 1258, 1174; MS (EI, $70 \mathrm{eV}), m / z(\%)=390$ (1) $\left[\mathrm{M}^{+}\right], 289$ (12) $\left[\mathrm{M}^{+}-\right.$ $\left.\mathrm{C}_{5} \mathrm{H}_{9} \mathrm{O}_{2}\right], 245$ (4), $210(56)\left[\mathrm{M}^{+}-\mathrm{C}_{8} \mathrm{H}_{6} \mathrm{NO}_{4}\right], 154(100)\left[\mathrm{C}_{8} \mathrm{H}_{12} \mathrm{NO}_{2}^{+}\right], 110(90)\left[\mathrm{C}_{7} \mathrm{H}_{12} \mathrm{~N}^{+}\right], 57$ (92) $\left[\mathrm{C}_{4} \mathrm{H}_{9}{ }^{+}\right]$; HRMS (EI): calcd for $\mathrm{C}_{20} \mathrm{H}_{26} \mathrm{~N}_{2} \mathrm{O}_{6}$ : 390.1791; found 390.1791; elemental analysis (\%) for $\mathrm{C}_{20} \mathrm{H}_{26} \mathrm{~N}_{2} \mathrm{O}_{6}$ (390.4): calcd. C 61.53, H 6.71, N 7.17; found C 61.20, H 6.61, N 6.99.

p-Nitrobenzyl $\quad(2 S, 4 R)-N-B o c-4,1^{\prime}$-dideutero-4-(Z)-propenylprolinate $\quad(2 S, 4 R)-\mathrm{D}_{2}-(\mathbf{6 8}): \quad \mathrm{A}$

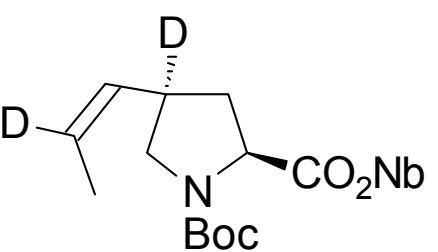

Boc suspension of $\mathrm{K}_{2} \mathrm{CO}_{3}(0.467 \mathrm{~g}, 3.379 \mathrm{mmol})$ in a solution of $(2 S, 4 R)-\mathbf{D}_{\mathbf{2}}-\mathbf{1 6}(0.87 \mathrm{~g}, 3.381 \mathrm{mmol})$ and p-nitrobenzylbromide (0.767 $\mathrm{g}, 3.550 \mathrm{mmol})$ in $\mathrm{MeCN}(5 \mathrm{~mL})$ was stirred in a sealed tube at $70{ }^{\circ} \mathrm{C}$ for $3 \mathrm{~h}$. The mixture was then cooled, diluted with $\mathrm{Et}_{2} \mathrm{O}(10 \mathrm{~mL})$, filtered, concentrated under reduced pressure and purified by column chromatography (EtOAc/hexanes $\left.1: 3, R_{\mathrm{f}}=0.43\right)$ to give $(2 S, 4 R)-\mathrm{D}_{2}-68(1.23 \mathrm{~g}, 88 \%)$ as a colorless glass. $[\alpha]_{24}^{\mathrm{D}}-37.0(c=0.86$, $\left.\mathrm{CHCl}_{3}\right) ;{ }^{1} \mathrm{H} \mathrm{NMR}\left(250 \mathrm{MHz}, \mathrm{CDCl}_{3}\right)$ : mixture of isotopomers: $\delta=1.34,1.44[2 \mathrm{~s}, 9 \mathrm{H}$, $\left.\mathrm{C}\left(\mathrm{CH}_{3}\right)_{3}\right], 1.63\left(\mathrm{~s}, 3 \mathrm{H}, 3^{\prime}-\mathrm{H}\right), 1.63-1.80\left(\mathrm{~m}, 1 \mathrm{H}, 3-\mathrm{H}_{\mathrm{a}}\right), 2.37-2.51\left(\mathrm{~m}, 1 \mathrm{H}, 3-\mathrm{H}_{\mathrm{b}}\right), 3.06(\mathrm{~d}, J=$ $\left.10.5 \mathrm{~Hz}, 1 \mathrm{H}, 5-\mathrm{H}_{\mathrm{a}}\right), 3.67,3.78\left(2 \mathrm{~d}, J=10.5 \mathrm{~Hz}, 1 \mathrm{H}, 5-\mathrm{H}_{\mathrm{b}}\right), 4.30,4.37(2 \mathrm{dd}, J=8.8,8.8 \mathrm{~Hz}$, $1 \mathrm{H}, 2-\mathrm{H}), 5.19\left(\mathrm{dd}, J=13.8 \mathrm{~Hz}, 1 \mathrm{H}, \mathrm{Bzl}-\mathrm{H}_{\mathrm{a}}\right) 5.20-5.26\left(\mathrm{~m}, 1 \mathrm{H}, 1^{\prime}-\mathrm{H}\right), 5.35(\mathrm{~d}, J=13.8 \mathrm{~Hz}$, $1 \mathrm{H}$, Bzl-H $\mathrm{H}_{\mathrm{b}}$, 5.49-5.59 (m, $\left.0.2 \mathrm{H}, 2^{\prime}-\mathrm{H}\right), 7.52(\mathrm{~d}, J=8.5 \mathrm{~Hz}, 2 \mathrm{H}, \mathrm{Ar}-\mathrm{H}), 8.20$ (dd, $J=7.8$, $7.8 \mathrm{~Hz}, 2 \mathrm{H}, \mathrm{Ar}-\mathrm{H}) ;{ }^{13} \mathrm{C} \mathrm{NMR}\left(62.9 \mathrm{MHz}, \mathrm{CDCl}_{3}\right): \delta=12.9,13.0\left(+, \mathrm{CH}_{3}\right), 28.0,28.2[+$, 
$\left.\mathrm{C}\left(\mathrm{CH}_{3}\right)_{3}\right], 35.1(\mathrm{t}, J=23.3 \mathrm{~Hz}, \mathrm{C}-4), 36.3(+, \mathrm{C}-4), 36.4,37.3$ (-, C-3), 51.3, 51.7 (-, C-5), 58.9, $59.0(+, \mathrm{C}-2), 64.9$ (-, C-Bzl), 79.9 [C $\left.\mathrm{C}_{\text {quat }}, \mathrm{C}\left(\mathrm{CH}_{3}\right)_{3}\right], 123.5,123.6(+, \mathrm{C}-\mathrm{Ar}), 126.7,126.8(+, \mathrm{C}-$ $\left.2^{\prime}\right), 128.0,128.3(+, \mathrm{C}-\mathrm{Ar}), 128.9,129.0\left(+, \mathrm{C}-1^{\prime}\right), 142.7,143.1\left(\mathrm{C}_{\text {quat }}, \mathrm{C}-\mathrm{Ar}\right), 147.4,147.6\left(\mathrm{C}_{\text {quat }}\right.$, C-Ar), 153.2, $154.0\left(\mathrm{C}_{\text {quat }}, \mathrm{NCO}_{2}\right), 172.3,172.5\left(\mathrm{C}_{\text {quat }}, \mathrm{C}-1\right)$; $\mathrm{MS}(\mathrm{EI}, 70 \mathrm{eV}), \mathrm{m} / z(\%)=392(1)$ $\left[\mathrm{M}^{+}\right], 291(12)\left[\mathrm{M}^{+}-\mathrm{C}_{5} \mathrm{H}_{9} \mathrm{O}_{2}\right], 247$ (4), $212(50)\left[\mathrm{M}^{+}-\mathrm{C}_{8} \mathrm{H}_{6} \mathrm{NO}_{4}\right], 156(100)\left[\mathrm{C}_{8} \mathrm{H}_{12} \mathrm{NO}_{2}^{+}\right], 112$ (80) $\left[\mathrm{C}_{7} \mathrm{H}_{12} \mathrm{~N}^{+}\right], 57$ (92) $\left[\mathrm{C}_{4} \mathrm{H}_{9}{ }^{+}\right]$; HRMS (EI): calcd for $\mathrm{C}_{20} \mathrm{H}_{24} \mathrm{D}_{2} \mathrm{~N}_{2} \mathrm{O}_{6}$ : 392.1916; found 392.1916; elemental analysis (\%) for $\mathrm{C}_{20} \mathrm{H}_{25} \mathrm{DN}_{2} \mathrm{O}_{6}$ (391.8): calcd. C 61.37, H 6.95, N 7.16; found C 61.41, H 6.66, N 7.08.

(2S,4R)-4,1'-Dideutero-4-(Z)-propenylproline $(2 S, 4 R)-\mathrm{D}_{2}-(\mathbf{1 7}):$ To a solution of compound $(2 S, 4 R)-\mathrm{D}_{2}-68(1.108 \mathrm{~g}, 2.679 \mathrm{mmol})$ in $\mathrm{Et}_{2} \mathrm{O}(5 \mathrm{~mL})$ a $5 \mathrm{M} \mathrm{HCl}$ solution in $\mathrm{Et}_{2} \mathrm{O}(20 \mathrm{~mL})$ was added and stirring continued in dark for $2 \mathrm{~h}$. The mixture was then filtered and a fresh $5 \mathrm{M} \mathrm{HCl}$ solution in $\mathrm{Et}_{2} \mathrm{O}(10 \mathrm{~mL})$ was added. After $1 \mathrm{~h}$, the mixture was diluted with hexanes $(10 \mathrm{~mL})$ and a formed precipitate was separated, washed with $\mathrm{Et}_{2} \mathrm{O}$ and dried to give p-nitrobenzyl $(2 S, 4 R)-4,1^{\prime}$-dideutero-4-(Z)-propenylprolinate hydrochloride $(0.775 \mathrm{~g}, 88 \%)$. MS (ESI), positive $m / z(\%): 293(100)\left[\mathrm{M}+\mathrm{H}^{+}\right]$. A $1 \mathrm{M} \mathrm{NaOH}(4.89 \mathrm{~mL})$ was added dropwise to an ice-cold solution of p-nitrobenzyl $(2 S, 4 R)-4,1^{\prime}$-dideutero-4-(Z)-propenylprolinate hydrochloride $(0.775 \mathrm{~g}$, $2.357 \mathrm{mmol})$ in $\mathrm{MeOH}(5 \mathrm{~mL})$ for $10 \mathrm{~min}$. Stirring continued at the same temperature for an additional $30 \mathrm{~min}$, after that mixture was diluted with water $(20 \mathrm{~mL})$, methanol was removed under reduced pressure and the reaction mixture was extracted with $\mathrm{Et}_{2} \mathrm{O}(10 \times 10 \mathrm{~mL})$. $\mathrm{pH}$ of the water fraction was carefully adjusted to $6.5-7.5$ with $1 \mathrm{M} \mathrm{HCl}$ (ca. $2.40 \mathrm{~mL}$ ) and water was removed under reduced pressure to give after prolonged drying at 0.01 Torr a mixture of $(2 S, 4 R)-\mathrm{D}_{2}-\mathbf{6 8}$ and $\mathrm{NaCl}(0.655 \mathrm{~g}, 0.292 \mathrm{~g}$ of $\mathrm{NaCl}, 99 \%)$ as a white solid which had the same spectral characteristics as described above and which was directly used for feeding experiments. 
Trimethylsilyl $\quad(2 S, 4 R)-1$-trimethylsilyl-4-trimethylsilyloxyprolinate $\quad(2 S, 4 R)-(\mathbf{6 5}): \quad$ To $\quad$ a

TMSO,

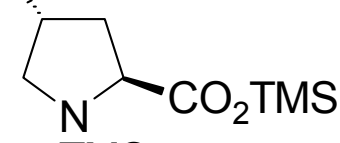

suspension of hydroxyproline $(2 S, 4 R)-19(13.1 \mathrm{~g}, 100 \mathrm{mmol})$ in HMDS $(140 \mathrm{~mL})$ was added $\mathrm{H}_{2} \mathrm{SO}_{4}$ (3 drops) and the mixture was refluxed till almost clear solution was obtained and then for a

further $2 \mathrm{~h}$. TMSCl (32 mL, $252 \mathrm{mmol})$ was then added and the mixture was refluxed for an additional $16 \mathrm{~h}$. All volatiles were removed under reduced pressure (ca. 4 Torr, bath temperature not higher than $100^{\circ} \mathrm{C}$ ) to give the residue which was purified by bulb-to-bulb distillation to give $(2 S, 4 R)-65(34.4 \mathrm{~g}, 99 \%)$ as a turbid very sensitive to moisture liquid. ${ }^{1} \mathrm{H}$ NMR $(250 \mathrm{MHz}$, $\left.\mathrm{CDCl}_{3}\right): \delta=0.03\left[\mathrm{~s}, 9 \mathrm{H}, \mathrm{Si}\left(\mathrm{CH}_{3}\right)_{3}\right], 0.09\left[\mathrm{~s}, 9 \mathrm{H}, \mathrm{Si}\left(\mathrm{CH}_{3}\right)_{3}\right], 0.25\left[\mathrm{~s}, 9 \mathrm{H}, \mathrm{Si}\left(\mathrm{CH}_{3}\right)_{3}\right], 2.00(\mathrm{dd}, \mathrm{J}=$ 8.6, 8.6 Hz, $2 \mathrm{H}, 3-\mathrm{H}), 2.84\left(\mathrm{dd}, J=9.5,3.8 \mathrm{~Hz}, 1 \mathrm{H}, 5-\mathrm{H}_{\mathrm{a}}\right), 3.21\left(\mathrm{dd}, J=9.5,5.0 \mathrm{~Hz}, 1 \mathrm{H}, 5-\mathrm{H}_{\mathrm{b}}\right)$, $3.90(\mathrm{dd}, J=6.5,6.5 \mathrm{~Hz}, 1 \mathrm{H}, 2-\mathrm{H}), 4.36$ (dddd, $J=4.0,4.0,4.0,4.0 \mathrm{~Hz}, 1 \mathrm{H}, 4-\mathrm{H}) ;{ }^{13} \mathrm{C} \mathrm{NMR}$ $\left(62.9 \mathrm{MHz}, \mathrm{CDCl}_{3}\right): \delta=-0.98\left[+, \mathrm{Si}\left(\mathrm{CH}_{3}\right)_{3}\right],-0.36\left[+, \mathrm{Si}\left(\mathrm{CH}_{3}\right)_{3}\right],-0.04\left[+, \mathrm{Si}\left(\mathrm{CH}_{3}\right)_{3}\right], 40.4(-$, C-3), $55.1\left(-\right.$, C-5), $60.5(+, C-2), 72.2(+, C-4), 177.0\left(C_{\text {quat }}, \mathrm{C}-1\right)$.

(3R,6R,7aS)-3-tert-Butyl-6-[(trimethylsilyl)oxy]perhydro-1H-pyrrolo[1,2-c][1,3]oxazolone-1

TMSO<smiles>CC1CC2C(=O)OCN2C1</smiles>

$\mathrm{tBu}$

$(3 R, 6 R, 7 a S)-(66)$ : To a solution of $(2 S, 4 R)-65(13.16 \mathrm{~g}, 37.85 \mathrm{mmol})$ in pentane $(15 \mathrm{~mL})$ pivalic aldehyde $(4.11 \mathrm{~mL}, 37.84 \mathrm{mmol})$ was added dropwise within $10 \mathrm{~min}$ and then $\mathrm{NH}_{4} \mathrm{Cl}$ (ca. $0.05 \mathrm{~g}$ ) was added (if exothermic reaction didn't occur, more $\mathrm{NH}_{4} \mathrm{Cl}$ was added; in some runs

TFA (several drops) were used instead of $\mathrm{NH}_{4} \mathrm{Cl}$ ). After $2 \mathrm{~h}$, the mixture was diluted with pentane $(25 \mathrm{~mL})$, cooled to $-5{ }^{\circ} \mathrm{C}$ and filtered while cold under anhydrous conditions. The resultant almost clear solution was concentrated under reduced pressure to give $(3 R, 6 R, 7 a S)-66$ $(5.18 \mathrm{~g}, 50 \%)$ as a faint turbid very sensitive to moisture oil. The diastereomeric purity of this product varied strongly from run to run and was $80-99 \%$ according to ${ }^{1} \mathrm{H}$ NMR spectra. ${ }^{1} \mathrm{H}$ $\operatorname{NMR}\left(250 \mathrm{MHz}, \mathrm{CDCl}_{3}\right): \delta=0.11\left[\mathrm{~s}, 9 \mathrm{H}, \mathrm{Si}\left(\mathrm{CH}_{3}\right)_{3}\right], 0.92\left[\mathrm{~s}, 9 \mathrm{H}, \mathrm{C}\left(\mathrm{CH}_{3}\right)_{3}\right], 2.04-2.27(\mathrm{~m}, 2 \mathrm{H}$, 3-H), $2.89\left(\mathrm{dd}, J=11.0,8.0 \mathrm{~Hz}, 1 \mathrm{H}, 5-\mathrm{H}_{\mathrm{a}}\right), 3.13\left(\mathrm{dd}, J=11.0,5.0 \mathrm{~Hz}, 1 \mathrm{H}, 5-\mathrm{H}_{\mathrm{b}}\right), 4.27(\mathrm{dd}, J=$ 
11.3, 5.5 Hz, $1 \mathrm{H}, 2-\mathrm{H}), 4.26$ (dddd, $J=5.5,5.5,5.5,5.5 \mathrm{~Hz}, 1 \mathrm{H}, 4-\mathrm{H}), 4.46\left(\mathrm{~s}, 1 \mathrm{H}, 1{ }^{\prime}-\mathrm{H}\right) ;{ }^{13} \mathrm{C}$

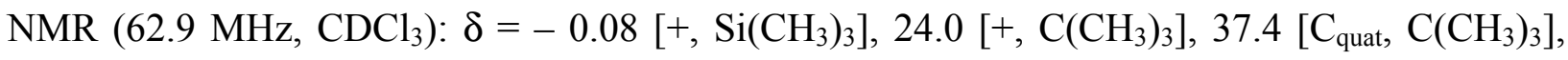
38.0 (-, C-3), $61.3(-, \mathrm{C}-5), 64.9(+, \mathrm{C}-2), 70.7(+, \mathrm{C}-4), 108.2\left(+, \mathrm{C}-1^{\prime}\right), 177.3\left(\mathrm{C}_{\text {quat }}, \mathrm{C}-1\right)$.

(2S,4R)-2-deuterohydroxyproline (2S,4R)-D-(19): To a solution of diisopropylamine $(2.94 \mathrm{~mL}$, $20.98 \mathrm{mmol})$ in THF $(50 \mathrm{~mL})$ was added a $2.34 \mathrm{M} n \mathrm{BuLi}$ in hexanes $(8.9 \mathrm{~mL}, 20.83 \mathrm{mmol})$ at $78^{\circ} \mathrm{C}$ for $5 \mathrm{~min}$. The temperature was then allowed to reach $4{ }^{\circ} \mathrm{C}$ and stirring continued at this temperature for a further $25 \mathrm{~min}$. The resultant LDA solution was transferred through Teflon tube in a dropping funnel immersed with an ice/water bath and added dropwise to a solution of $(3 R, 6 R, 7 a S)-66(5.18 \mathrm{~g}, 19.08 \mathrm{mmol}, d r>95: 5)$ in THF $(80 \mathrm{~mL})$ at $-78{ }^{\circ} \mathrm{C}$ for $1 \mathrm{~h}$. After this, stirring continued at the same temperature for a further $30 \mathrm{~min}$. A solution of $2.34 \mathrm{M} \mathrm{BuLi}$ in hexanes $(8.9 \mathrm{~mL}, 20.83 \mathrm{mmol})$ in THF $(50 \mathrm{~mL})$ was then added for $30 \mathrm{~min}$ and the mixture was stirred for an additional $1 \mathrm{~h}$. The reaction was quenched with $\mathrm{CH}_{3} \mathrm{OD}(4 \mathrm{~mL})$ and the temperature was allowed to reach $20{ }^{\circ} \mathrm{C} .6 \mathrm{M} \mathrm{HCl}(50 \mathrm{~mL})$ was then added and the mixture was stirred for $2 \mathrm{~h}$. The mixture was then concentrated under reduced pressure and the residue was dissolved in water and passed through column with Amberlite IR-120 in $\mathrm{H}^{+}$-form (ca. $250 \mathrm{~mL}$ ). The column was washed with water till neutral $\mathrm{pH}$ of the eluat and then with $10 \%$ aqueous $\mathrm{NH}_{3}$ till the eluat didn't contain ninhidrine active compounds according to TLC. These washings were combined and concentrated to ca. $200 \mathrm{~mL}$. The resultant brown solution was extracted with $n$ butanol saturated with water $(3 \times 50 \mathrm{~mL})$. The aqueous fraction was then concentrated under reduced pressure and the residue was taken up with $\mathrm{EtOH}(15 \mathrm{~mL})$. The precipitate formed was filtered off, dried and precipitated from water with acetone to give $(2 S, 4 R)-\mathrm{D}-19(0.40 \mathrm{~g}, 16 \%)$ as an off-white solid. The spectral data of this compound were in accordance with literature. The deuteration degree was ca. $87 \%$ according to ${ }^{1} \mathrm{H}$ NMR spectrum. $[\alpha]_{20}^{D}-73.4\left(c=0.95, \mathrm{CHCl}_{3}\right)$ $\left[\right.$ lit.. $\left.{ }^{[67]}[\alpha]_{20}^{D}-72.1\left(c=1.0, \mathrm{CHCl}_{3}\right)\right]$. 
(3R,6R,7aS)-3-(Trichloromethyl)-6-[(trimethylsilyl)oxy]perhydro-1H-pyrrolo[1,2-c][1,3]oxazolone-1 (3R,6R,7aS)-(67): A mixture of (2S,4R)-65 (34.01 g, $97.8 \mathrm{mmol})$ and chloral (10 mL,

TMSO<smiles>CC1C[C@@H]2C(=O)OC(C(F)(Cl)Cl)N2C1</smiles>
$102.6 \mathrm{mmol}$ ) was stirred at $20^{\circ} \mathrm{C}$ for $16 \mathrm{~h}$. As according to the ${ }^{1} \mathrm{H}$ NMR spectrum of the reaction mixture the conversion was very low $(<10 \%)$, anhydrous $\mathrm{MeCN}(20 \mathrm{~mL})$, chloral $(10 \mathrm{~mL}, 102.6 \mathrm{mmol})$ and TFA (3 drops) were added. The temperature of the mixture started to increase and the reaction flask was then immersed with a water/ice bath. The mixture was allowed to warm to $20^{\circ} \mathrm{C}$ for $2 \mathrm{~h}$ and stirring continued at this temperature for an additional $1 \mathrm{~h}$. All volatiles were removed under reduced pressure (first 4 Torr, then 0.02 Torr) and the black solid residue was extracted with anhydrous $\mathrm{Et}_{2} \mathrm{O}(3 \times 75 \mathrm{~mL})$. The ethereal solution was concentrated under reduced pressure and the residue (ca. $28.5 \mathrm{~g}$ ) was triturated with anhydrous hexanes (ca. $150 \mathrm{~mL}$ ), the resultant solution was decanted and concentrated under reduced pressure. The residue was taken up with pentane $(200 \mathrm{~mL})$, the solution was filtered and cooled to $-78^{\circ} \mathrm{C}$. The mother liquor was then decanted, the residual solid was taken up again with pentane $(200 \mathrm{~mL})$ and cooled to $-78^{\circ} \mathrm{C}$. The mother liquor was decanted and the residual solid was washed with cold pentane $\left(-78^{\circ} \mathrm{C}\right)$ and dried to give $(3 R, 6 R, 7 a S)-67(16.23 \mathrm{~g}, 50 \%)$ as a colorless reasonably stable to the atmospheric moisture solid. According to ${ }^{1} \mathrm{H}$ NMR spectrum, $d r$ was $>94: 6$. $[\alpha]_{20}^{D}-$ $5.8(c=1.32, \mathrm{THF}) ;{ }^{1} \mathrm{H} \mathrm{NMR}\left(250 \mathrm{MHz}, \mathrm{CDCl}_{3}\right): \delta=0.13\left[\mathrm{~s}, 9 \mathrm{H}, \mathrm{Si}\left(\mathrm{CH}_{3}\right)_{3}\right], 2.17(\mathrm{dd}, J=6.3$, $6.3 \mathrm{~Hz}, 2 \mathrm{H}, 3-\mathrm{H}), 3.18$ (dd, $J=11.3,5.5 \mathrm{~Hz}, 1 \mathrm{H}, 5-\mathrm{H}_{\mathrm{a}}$ ), 3.33 (dd, $J=11.3,5.3 \mathrm{~Hz}, 1 \mathrm{H}, 5-\mathrm{H}_{\mathrm{b}}$ ), 4.25 (dd, $J=7.3,7.3 \mathrm{~Hz}, 1 \mathrm{H}, 2-\mathrm{H}), 4.35$ (dddd, $J=5.5,5.5,5.5,5.5 \mathrm{~Hz}, 1 \mathrm{H}, 4-\mathrm{H}), 5.11$ (s, $1 \mathrm{H}$, $\left.1^{\prime}-\mathrm{H}\right) ;{ }^{13} \mathrm{C}$ NMR $\left(62.9 \mathrm{MHz}, \mathrm{CDCl}_{3}\right): \delta=-0.05\left[+, \mathrm{Si}\left(\mathrm{CH}_{3}\right)_{3}\right], 38.1(-, \mathrm{C}-3), 61.0(+, \mathrm{C}-2), 64.1$ (-, C-5), 70.7 (+, C-4), $100.6\left(\mathrm{C}_{\text {quat, }}, \mathrm{CCl}_{3}\right), 103.6$ (+, C-1'), 175.1 (C quat, $\left.\mathrm{C}-1\right)$; IR (KBr): nu(tilde) $=2960 \mathrm{~cm}^{-1}, 2912,2884,1783,1389,1263,1259,1189,1126,997$; MS (CI), m/z (\%): $355 / 353 / 351(6 / 18 / 18)\left[\mathrm{M}+\mathrm{NH}_{4}^{+}\right], 336 / 334 / 332(31 / 96 / 100)\left[\mathrm{M}+\mathrm{H}^{+}\right]$. 
2.5. Elucidation of the absolute configuration of trans-(2'-nitrocyclopropyl)alanine residue in hormaomycin. Synthesis of $\left(2 \mathrm{R}, 1^{\prime} \mathrm{R}, 2^{\prime} \mathrm{R}\right)-\left(2^{\prime}\right.$-nitrocyclopropyl)alanine and (2S, 1 'RS, 2'RS)-2-deutero-(2'-nitrocyclopropyl)alanine

$(1 R, 2 R)$-2-Nitrocyclopropanecarboxylic acid $(1 R, 2 R)-(\mathbf{8 3})$ : A $30 \%$ aqueous $\mathrm{H}_{2} \mathrm{O}_{2}(8.5 \mathrm{~mL})$ and $\mathrm{O}_{2} \mathrm{~N}{ }^{. . .} \mathrm{CO}_{2} \mathrm{H}$ then a $0.8 \mathrm{M}$ aqueous $\mathrm{LiOH}(137 \mathrm{~mL})$ (the latter dropwise for $50 \mathrm{~min}$ ) were added to an effectively stirred solution of $\left(2 R, 1^{\prime} R, 2^{\prime} R\right)-83(16.55 \mathrm{~g}$, $68.61 \mathrm{mmol})$ in undistilled $\mathrm{THF}(400 \mathrm{~mL})$ at $-2-0{ }^{\circ} \mathrm{C}$ (internal temperature) and stirring continued at the same temperature for additional $40 \mathrm{~min}$. A solution of $\mathrm{NaHSO}_{3}(34.59 \mathrm{~g}, 274.44$ $\mathrm{mmol})$ in water $(70 \mathrm{~mL})$ was carefully added by portions at the same temperature. THF was removed under reduced pressure at the bath temperature $0-10{ }^{\circ} \mathrm{C}$ and the resultant suspension was diluted with ice-cold water $(500 \mathrm{~mL})$ and extracted with cold $\mathrm{CH}_{2} \mathrm{Cl}_{2}(5 \times 100 \mathrm{~mL})$. The organic layers were then pooled, washed with water $(3 \times 100 \mathrm{~mL})$, brine $(2 \times 50 \mathrm{~mL})$, dried, filtered and concentrated under reduced pressure to give $(R)$-4-isopropyloxazolidin-2-one (6.15 g, 69\%). The $\mathrm{pH}$ of the aqueous layer was carefully adjusted to ca. 1 with solid $\mathrm{NaHSO}_{4}$ and the resulting emulsion was extracted with EtOAc $(5 \times 200 \mathrm{~mL})$ under salting-out conditions. The organic fractions were pooled, washed with brine $(3 \times 200 \mathrm{~mL})$, dried, filtered and concentrated under reduced pressure to ca. $40 \mathrm{~mL}$. Hexanes (ca. $100 \mathrm{~mL}$ ) were then added, the mixture was left at $4{ }^{\circ} \mathrm{C}$ for $16 \mathrm{~h}$ and then the precipitate was filtered off. This crude product was finally purified from $\mathrm{CH}_{2} \mathrm{Cl}_{2} /$ hexanes to give the acid $(1 R, 2 R)-83(7.03 \mathrm{~g}, 78 \%)$ as a colorless solid. The spectral data of this compound were in accordance with those for the racemic acid. $[\alpha]_{20}^{D}-223.9$ $(c=0.81, \mathrm{THF})$.

(1S,2S)-2-Nitrocyclopropanecarboxylic acid (1S,2S)-(83): A 30\% aqueous $\mathrm{H}_{2} \mathrm{O}_{2}(1.95 \mathrm{~mL})$ and then a $0.8 \mathrm{M}$ aqueous $\mathrm{LiOH}(31.5 \mathrm{~mL})$ (the latter dropwise for $50 \mathrm{~min}$ ) were added to an 
effectively stirred solution of $\left(2 R, 1^{\prime} S, 2 ' S\right)-9(3.80 \mathrm{~g}, 15.75 \mathrm{mmol})$ in undistilled THF $(92 \mathrm{~mL})$ at $-3-0{ }^{\circ} \mathrm{C}$ (internal temperature) and stirring continued at the same temperature for additional 50 min. A solution of $\mathrm{NaHSO}_{3}(13.97 \mathrm{~g}, 110.84 \mathrm{mmol})$ in water $(50 \mathrm{~mL})$ was then carefully added by portions at the same temperature. THF was removed under reduced pressure at the bath temperature $0-10{ }^{\circ} \mathrm{C}$ and the resultant suspension was diluted with ice-cold water $(250 \mathrm{~mL})$ and extracted with cold $\mathrm{CH}_{2} \mathrm{Cl}_{2}(4 \times 50 \mathrm{~mL})$. The organic layers were then pooled, washed with water $(3 \times 50 \mathrm{~mL})$, brine $(2 \times 30 \mathrm{~mL})$, dried, filtered and concentrated under reduced pressure to give $(R)$-4-isopropyloxazolidin-2-one $(1.36 \mathrm{~g}, 67 \%)$. The $\mathrm{pH}$ of the aqueous layer was carefully adjusted to ca. 1 with solid $\mathrm{NaHSO}_{4}$ and the resulting emulsion was extracted with THF $(3 \times 100$ $\mathrm{mL})$ under salting-out conditions. The organic fractions were pooled, washed with brine $(3 \times 50$ $\mathrm{mL}$ ), dried, filtered and concentrated under reduced pressure. The residue was recrystallized from $\mathrm{CH}_{2} \mathrm{Cl}_{2} /$ hexanes to give the acid $(1 S, 2 S)-\mathbf{8 3}(1.27 \mathrm{~g}, 62 \%)$ as a colorless solid. The spectral data of this compound were in accordance with those for the racemic acid. $[\alpha]_{20}^{D} 204.3(c=0.79$, THF).

(1'S,2'S)-(2'-Nitrocyclopropyl)methanol (1'S,2'S)-(10) and (1'R,2'R)-(2'-Nitrocyclopropyl)methanol $\left(1^{\prime} R, 2^{\prime} R\right)-(\mathbf{1 0})$ : A 1 M solution of $\mathrm{BH}_{3}$ THF in THF $(12.4 \mathrm{~mL})$ was added to a solution of $(1 S, 2 S)-83(1.25 \mathrm{~g}, 9.54 \mathrm{mmol})$ in anhydrous THF $(12 \mathrm{~mL})$ at $4{ }^{\circ} \mathrm{C}$ for $50 \mathrm{~min}$. The cooling bath was then removed and stirring continued for a further $48 \mathrm{~h}$. Methanol $(15 \mathrm{~mL})$ was then added dropwise for $30 \mathrm{~min}$ and the mixture was concentrated under reduced pressure. The residue was taken up with $\mathrm{MeOH}(20 \mathrm{~mL})$ and the resultant solution was refluxed for $4 \mathrm{~h}$. All volatiles were then removed under reduced pressure and the residue was purified by column chromatography (EtOAc/hexanes $2: 3, R_{\mathrm{f}}=0.27$ ) and then by bulb-to-bulb distillation at the bath temperature $100-120^{\circ} \mathrm{C}$ at 0.002 Torr to give $\left(1^{\prime} S, 2^{\prime} S\right)-10(1.00 \mathrm{~g}, 90 \%)$ as a colorless oil. The spectral data of this compound were in accordance with literature. $[\alpha]_{20}^{D} 100.9\left(c=1.03, \mathrm{CHCl}_{3}\right)\left[\right.$ lit.: ${ }^{[13]}[\alpha]_{20}^{D}$ 
$\left.97.8\left(c=1.60, \mathrm{CHCl}_{3}\right)\right] .\left(1^{\prime} R, 2^{\prime} R\right)$-Isomer was also prepared in the same manner in $94 \%$ yield.

The spectral data of this compound were in accordance with literature. $[\alpha]_{20}^{D}-92.6(c=1.01$, $\left.\mathrm{CHCl}_{3}\right)\left[\right.$ lit.: $\left.{ }^{[13]}[\alpha]_{20}{ }^{\mathrm{D}}-95.1\left(c=1.10, \mathrm{CHCl}_{3}\right)\right]$.

Alkylation of (R)-Nickel complex (2R)-81 with (1'R,2'R)-1-(iodomethyl)-2-nitrocyclopropane $\left(1^{\prime} R, 2^{\prime} R\right)-80$. Preparation of $\left(2 R, 2^{\prime} R, 1^{\prime \prime} R, 2^{\prime \prime} R\right)-82$ : A suspension of $(2 R)-81$ (5.50 g, $\left.11.00 \mathrm{mmol}\right)$ in a mixture of DMF $(5.5 \mathrm{~mL})$ and $\mathrm{MeCN}(11 \mathrm{~mL})$ was degassed with three freeze-pump-thaw cycles at $-70{ }^{\circ} \mathrm{C} . \mathrm{NaH}(0.616 \mathrm{~g}, 60 \%$ in oil, $15.40 \mathrm{mmol})$ and iodide $\left(1^{\prime} R, 2^{\prime} R\right)-80$ (2.585 $\mathrm{g}, 11.39$ mmol) were then added. The cooling bath was removed, and the reaction mixture was vigorously stirred for $40 \mathrm{~min}$. The reaction flask was then immersed with ice/water bath and $60 \% \mathrm{AcOH}$ $(1.2 \mathrm{~mL})$ was carefully added. The precipitated solid was filtered off, washed with cold $\left(-35^{\circ} \mathrm{C}\right)$ $\mathrm{MeCN}$ and $\mathrm{Et}_{2} \mathrm{O}$. After this, it was dissolved in $\mathrm{CHCl}_{3}$ and filtered from the tar. The solution was then concentrated under reduced pressure to ca. $10 \mathrm{~mL}$ and $\mathrm{Et}_{2} \mathrm{O}(40 \mathrm{~mL})$ was added. The precipitated solid was filtered off and dried to give $\left(2 R, 2^{\prime} R, 1 " R, 2^{\prime \prime} R\right)-82(4.85 \mathrm{~g}, 74 \%)$ as a red solid. The spectral data of this compound were in accordance with literature. The isotopic pattern $[\mathrm{MS}(\mathrm{EI}, 70 \mathrm{eV})]$ was in accordance with the calculated one. $[\alpha]_{20}^{D}-2335(c=0.055, \mathrm{MeOH})$ $\left[\right.$ lit.: $\left.^{[84]}[\alpha]_{20}^{D}-2406\left(c=0.1, \mathrm{CHCl}_{3}\right)\right]$; HRMS (EI): calcd for $\mathrm{C}_{31} \mathrm{H}_{30} \mathrm{~N}_{4} \mathrm{NiO}_{5}$ : 596.1570; found 596.1570.

$\left(2 R, 1^{\prime} R, 2^{\prime} R\right)-3-\left(2^{\prime}-n i t r o c y c l o p r o p y l\right)$ alanine $\left(2 R, 1^{\prime} R, 2^{\prime} R\right)-(\mathbf{8 3}): 6 \mathrm{M} \mathrm{HCl}(40 \mathrm{~mL})$ was added to<smiles>N[C@@H](C[C@@H]1C[C@H]1[N+](=O)[O-])C(=O)O</smiles>
the suspension of finely grounded $\left(2 R, 2^{\prime} R, 1 " R, 2^{\prime \prime} R\right)-82(4.85 \mathrm{~g}, 8.12$ mmol) in refluxing methanol $(20 \mathrm{~mL})$. The mixture was gently refluxed for $10 \mathrm{~min}$. The resulting green syrup was then concentrated, was taken up with ice-cold water $(50 \mathrm{~mL})$, the precipitate was filtered off and washed with cold water $(20 \mathrm{~mL})$ to give after drying $(R)$-BPB· $\mathrm{HCl} \mathrm{HCl} \cdot(2 R)-84(2.50 \mathrm{~g}, 74 \%$ recovery) as a white solid. The filtrate was combined with washings, $\mathrm{pH}$ was carefully adjusted to 6 with aqueous ammonia and extracted 
with $\mathrm{CHCl}_{3}(3 \times 50 \mathrm{~mL})$. The organic layer was dried, filtered and concentrated under reduced pressure to give another portion of $(R)$-BPB (0.32 g, 84\% overall recovery). Preswallowed Amberlite IRA-120 in $\mathrm{H}^{+}$form $(110 \mathrm{~mL})$ was added to the water fraction and the mixture was stirred for $15 \mathrm{~h}$. The ion-exchange resin was filtered, washed with water to $\mathrm{pH} 5$ and treated with $10 \%$ aqueous $\mathrm{NH}_{3}(2 \times 250 \mathrm{~mL})$ for $2 \mathrm{~h}$. The combined filtrates were concentrated under reduced pressure to give white solid, which was dissolved in hot water $(7 \mathrm{~mL})$. The solution was filtered and diluted with EtOH $(15 \mathrm{~mL})$. The formed precipitate was filtered off and dried. This material $(0.88 \mathrm{~g})$ was additionally purified by recrystallization from water $(4 \mathrm{~mL})$, the precipitated crystals were filtered off, washed with cold $\mathrm{EtOH}: \mathrm{H}_{2} \mathrm{O} 2: 1$ and dried at $50{ }^{\circ} \mathrm{C}$ and 0.002 Torr for $16 \mathrm{~h}$ to give $\left(2 R, 1^{\prime} R, 2^{\prime} R\right)-83(0.77 \mathrm{~g}, 55 \%)$ as a colorless solid. The spectral data of this compound were in agreement with literature. $[\alpha]_{20}^{D}-81.0\left(c=0.33, \mathrm{H}_{2} \mathrm{O}\right)\left[1\right.$ lit.. ${ }^{[84]}[\alpha]_{20}^{D}-72.2(c$ $\left.\left.=0.32, \mathrm{H}_{2} \mathrm{O}\right)\right] ;$ MS (ESI), positive $m / z(\%)=219(40)\left[\mathrm{M}-\mathrm{H}^{+}+2 \mathrm{Na}^{+}\right], 175(14)\left[\mathrm{M}+\mathrm{H}^{+}\right]$; negative $m / z(\%)=369(92)\left[2 \mathrm{M}-2 \mathrm{H}^{+}+\mathrm{Na}^{+}\right], 347(62)\left[2 \mathrm{M}-\mathrm{H}^{+}\right], 173(18)\left[\mathrm{M}-\mathrm{H}^{+}\right]$.

2-Dideuterated Nickel complex $(2 S)-\mathrm{D}_{2}-\mathbf{8 1}$ : Unlabelled (2S)-81 (6 g, $\left.12.043 \mathrm{mmol}\right)$ was

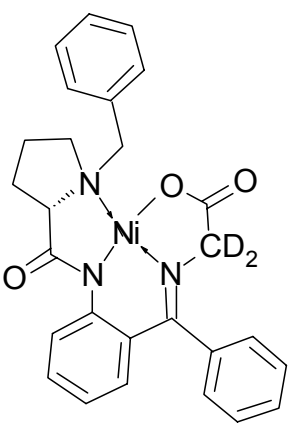
dissolved in a mixture of $\mathrm{CH}_{3} \mathrm{OD}(10 \mathrm{~mL}), \mathrm{D}_{2} \mathrm{O}(7 \mathrm{~mL})$ and $\mathrm{CDCl}_{3}(5 \mathrm{~mL})$ and $\mathrm{Na}_{2} \mathrm{CO}_{3}(0.2 \mathrm{~g}, 2.410 \mathrm{mmol})$ was added. The mixture was stirred at 50 ${ }^{\circ} \mathrm{C}$ for $24 \mathrm{~h}$ in sealed flask, concentrated under reduced pressure, $\mathrm{CH}_{3} \mathrm{OD}$ $(10 \mathrm{~mL}), \mathrm{D}_{2} \mathrm{O}(7 \mathrm{~mL})$ and $\mathrm{CDCl}_{3}(5 \mathrm{~mL})$ was added again and the resultant biphasic reaction mixture was stirred at $50{ }^{\circ} \mathrm{C}$ for an additional $24 \mathrm{~h}$. The reaction mixture was then cooled, diluted with $\mathrm{CHCl}_{3}(30 \mathrm{~mL})$, organic phase was separated, washed with $\mathrm{H}_{2} \mathrm{O}(2 \times 30 \mathrm{~mL})$, dried and concentrated to give after recrystallization from $\mathrm{CHCl}_{3} / \mathrm{Et}_{2} \mathrm{O} /$ hexanes $(2 \mathrm{~S})-\mathrm{D}_{2}-\mathbf{8 1}\left(5.66 \mathrm{~g}, 94 \% ; \mathrm{D}_{2}\right.$ and $\mathrm{D}$ contents $>97 \%$ and $<3 \%$ respectively, as determined by MS) as a dark red solid. M.p. $210-213{ }^{\circ} \mathrm{C} ;{ }^{1} \mathrm{H}$ NMR $(250 \mathrm{MHz}$, $\left.\mathrm{CDCl}_{3}\right): \delta=1.97-2.19(\mathrm{~m}, 2 \mathrm{H}, 4-\mathrm{H}), 2.28-2.46\left(\mathrm{~m}, 1 \mathrm{H}, 3-\mathrm{H}_{\mathrm{a}}\right), 2.46-2.63\left(\mathrm{~m}, 1 \mathrm{H}, 3-\mathrm{H}_{\mathrm{b}}\right), 3.21-$ $3.41\left(\mathrm{~m}, 1 \mathrm{H}, 5-\mathrm{H}_{\mathrm{a}}\right), 3.45\left(\mathrm{dd}, J=10.9,5.6 \mathrm{~Hz}, 1 \mathrm{H}, 5-\mathrm{H}_{\mathrm{b}}\right), 3.59-3.72(\mathrm{~m}, 1 \mathrm{H}, 2-\mathrm{H}), 3.68(\mathrm{~d}, J=$ 
$\left.25.0 \mathrm{~Hz}, 1 \mathrm{H}, \mathrm{Bzl}-\mathrm{H}_{\mathrm{a}}\right), 4.46$ (d, $\left.J=25 \mathrm{~Hz}, 1 \mathrm{H}, \mathrm{Bzl}-\mathrm{H}_{\mathrm{b}}\right), 6.63-6.72$ (m, $\left.1 \mathrm{H}, \mathrm{Ar}-\mathrm{H}\right), 6.74-6.81$ (m, $1 \mathrm{H}, \mathrm{Ar}-\mathrm{H}), 6.88-7.01(\mathrm{~m}, 1 \mathrm{H}, \mathrm{Ar}-\mathrm{H}), 7.03-7.12(\mathrm{~m}, 1 \mathrm{H}, \mathrm{Ar}-\mathrm{H}), 7.14-7.24(\mathrm{~m}, 1 \mathrm{H}, \mathrm{Ar}-\mathrm{H})$, 7.24-7.33 (m, $1 \mathrm{H}, \mathrm{Ar}-\mathrm{H}), 7.35-7.46$ (m, $2 \mathrm{H}, \mathrm{Ar}-\mathrm{H}), 7.44-7.58$ (m, $3 \mathrm{H}, \mathrm{Ar}-\mathrm{H}), 8.04$ (d, $J=8$ $\mathrm{Hz}, 2 \mathrm{H}, \mathrm{Ar}-\mathrm{H}), 8.26(\mathrm{~d}, J=8.5 \mathrm{~Hz}, 1 \mathrm{H}, \mathrm{Ar}-\mathrm{H}) ;{ }^{13} \mathrm{C} \mathrm{NMR}\left(62.9 \mathrm{MHz}, \mathrm{CDCl}_{3}\right): \delta=23.4(-, \mathrm{C}-$ 4), 30.3 (-,C-3), 57.2 (-,C-5), 62.8 (-, C-Bzl), 69.5 (+, C-2), 120.4, 123.9, 125.3, 125.9, 128.5, $128.7,129.0,129.2,129.4,131.4,131.8$ and $132.8(+, A r-C), 124.8,133.0,134.2,142.2\left(\mathrm{C}_{\text {quat }}\right.$, $\operatorname{Ar}-\mathrm{C}), 171.3\left(\mathrm{C}_{\text {quat }}, \mathrm{C}=\mathrm{N}\right), 176.9\left(\mathrm{C}_{\text {quat }}, \mathrm{C}=\mathrm{O}\right), 181.0\left(\mathrm{C}_{\text {quat }}, \mathrm{CO}-\mathrm{N}\right)$; the signals of $\mathrm{CD}_{2}$ carbon could not be detected because of their low intensity; IR $(\mathrm{KBr})$ : nu(tilde) $=2973 \mathrm{~cm}^{-}$ ${ }^{1}, 2869,1673,1642,1589,1442,1336,1264,1176 ;$ MS (EI, $\left.70 \mathrm{eV}\right), m / z(\%): 499(40)\left[\mathrm{M}^{+}\right]$, $455(100)\left[\mathrm{M}^{+}-\mathrm{CO}_{2}\right], 363(8), 267(10), 265$ (17), 217 (41), 160 (41), 91 (78) $\left[\mathrm{C}_{7} \mathrm{H}_{7}^{+}\right]$; HRMS (EI): calcd for $\mathrm{C}_{27} \mathrm{H}_{23} \mathrm{D}_{2} \mathrm{~N}_{3} \mathrm{NiO}_{3}$ : 499.1375; found 499.1375; elemental analysis calcd (\%) for $\mathrm{C}_{27} \mathrm{H}_{23} \mathrm{D}_{2} \mathrm{~N}_{3} \mathrm{NiO}_{3}$ (500.2): C 64.83, H 5.44, N 8.40; found C 65.03, H 5.77, N 8.32.

Alkylation of 2-Dideuterated Nickel complex (2S)- $\mathrm{D}_{2}-\mathbf{8 1}$ with rac-trans-1-(iodomethyl)-2nitrocyclopropane 80. Preparation of $\left(2 S, 2^{\prime} S, 1 " R S, 2 " R S\right)-\mathrm{D}-\mathbf{8 2}$ : A suspension of $(2 S)-\mathrm{D}_{2}-\mathbf{8 1}(4.6$

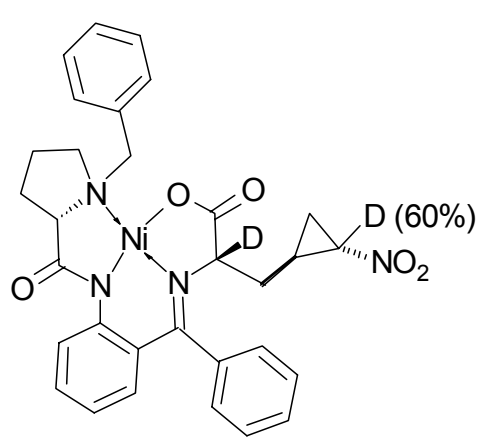
g, $9.196 \mathrm{mmol})$ in a mixture of DMF $(4.0 \mathrm{~mL})$ and $\mathrm{CD}_{3} \mathrm{CN}(8$ $\mathrm{mL}$ ) was degassed with three freeze-pump-thaw cycles at $70{ }^{\circ} \mathrm{C} . \mathrm{NaH}(0.504 \mathrm{~g}, 60 \%$ in oil, $12.595 \mathrm{mmol})$ and iodide 80 (2.12 g, $9.334 \mathrm{mmol})$ were then added. The cooling bath was removed, and the reaction mixture was vigorously stirred for 40 min. The reaction flask was then immersed with ice/water bath and $\mathrm{D}_{2} \mathrm{O}(2 \mathrm{~mL})$ and followed by $\mathrm{D}_{2} \mathrm{SO}_{4}(1.26 \mathrm{~g}, 12.6 \mathrm{mmol})$ in $\mathrm{D}_{2} \mathrm{O}(5 \mathrm{~mL})$ were carefully added. After this, water $(70 \mathrm{~mL})$ was added and the separated dark red oil was triturated with ether to give red-brown solid $(5.7 \mathrm{~g})$ which was further purified by column chromatography $\left(\mathrm{CHCl}_{3} /\right.$ acetone $6: 1, R_{\mathrm{f}}=$ $0.37)$ and then by recrystallization from $\mathrm{CHCl}_{3} / \mathrm{MeOH}$ to give $(2 S, 2 ' S, 1 " R S, 2 " R S)-\mathrm{D}-\mathbf{8 2}(3.87 \mathrm{~g}$, $70 \%$ ) as a dark red solid. According to ${ }^{1} \mathrm{H}$ NMR spectrum deuterium content was $85 \%$ at $\mathrm{C}-2^{\prime}$ 
and $60 \%$ at C-2" respectively. M.p. $>260{ }^{\circ} \mathrm{C},[\alpha]_{20}^{D} 1708(c=0.05, \mathrm{MeOH}) ;{ }^{1} \mathrm{H}$ NMR $(250$ $\left.\mathrm{MHz}, \mathrm{CDCl}_{3}\right)$ : mixture of diastereo- and isotopomers: $\delta=0.46-0.58\left(\mathrm{~m}, 0.5 \mathrm{H}, 3^{\prime \prime}-\mathrm{H}_{\mathrm{a}}\right), 0.85-$ $0.96\left(\mathrm{~m}, 0.5 \mathrm{H}, 3^{\prime \prime}-\mathrm{H}_{\mathrm{a}}\right), 1.05-1.28\left(\mathrm{~m}, 0.5 \mathrm{H}, 3^{\prime}-\mathrm{H}_{\mathrm{a}}\right), 1.45\left(\mathrm{dd}, J=8.4,14.4 \mathrm{~Hz}, 0.5 \mathrm{H}, 3^{\prime}-\mathrm{H}_{\mathrm{a}}\right)$, 1.73-1.84 (m, 0.5 H, 3"'- $\left.\mathrm{H}_{\mathrm{b}}\right), 1.84-1.96\left(\mathrm{~m}, 0.5 \mathrm{H}, 3^{\prime \prime}-\mathrm{H}_{\mathrm{b}}\right), 1.97-2.32\left(\mathrm{~m}, 2.5 \mathrm{H}, 4-\mathrm{H}, 0.53^{\prime}-\mathrm{H}_{\mathrm{b}}\right)$, 2.38-2.58 (m, $\left.2 \mathrm{H}, 3-\mathrm{H}_{\mathrm{a}}, 1^{\prime \prime}-\mathrm{H}\right), 2.58-2.83\left(\mathrm{~m}, 1.5 \mathrm{H}, 3-\mathrm{H}_{\mathrm{a}}, 0.53^{\prime}-\mathrm{H}_{\mathrm{b}}\right), 3.33-3.41\left(\mathrm{~m}, 0.15 \mathrm{H}, 2^{\prime}-\right.$ H), 3.40-3.73 (m, 3 H, 2-H, 5-H), 3.56 (dd, $J=2.3,12.8$ Hz, $\left.1 \mathrm{H}, \mathrm{Bzl}-\mathrm{H}_{\mathrm{a}}\right), 3.92$ (ddd, $J=3.4$, 3.4, 7.0 Hz, 0.30 H, 2" $\left.-\mathrm{H}, 2^{\prime}-\mathrm{H}\right), 4.00\left(\mathrm{dd}, J=14.6,3.9 \mathrm{~Hz}, 0.13 \mathrm{H}, 2^{\prime \prime}-\mathrm{H}\right), 4.44(\mathrm{~d}, J=12.8 \mathrm{~Hz}$, $\left.1 \mathrm{H}, \mathrm{Bzl}-\mathrm{H}_{\mathrm{b}}\right), 6.56-6.73$ (m, $\left.2 \mathrm{H}, \mathrm{Ar}-\mathrm{H}\right), 6.88$ (t, $\left.J=8 \mathrm{~Hz}, 1 \mathrm{H}, \mathrm{Ar}-\mathrm{H}\right), 7.08-7.24$ (m, $\left.2 \mathrm{H}, \mathrm{Ar}-\mathrm{H}\right)$, 7.29-7.59 (m, 5 H, Ar-H), 7.59-7.65 (m, $1 \mathrm{H}$, Ar-H), 8.07 (dt, $J=7.8,1.5 \mathrm{~Hz}, 2 \mathrm{H}, \mathrm{Ar}-\mathrm{H}), 8.13$ $(\mathrm{dd}, J=3.3,8.5 \mathrm{~Hz}, 1 \mathrm{H}, \mathrm{Ar}-\mathrm{H}) ;{ }^{13} \mathrm{C} \mathrm{NMR}\left(62.9 \mathrm{MHz}, \mathrm{CDCl}_{3}\right): \delta=17.7,17.8,18.2,18.4(-, \mathrm{C}-$ $\left.3^{\prime \prime}\right), 21.5,21.6,21.6,21.7\left(+, C-1^{\prime \prime}\right), 23.8(-, C-4), 30.6(-, C-3), 36.1,36.4\left(-, C-3^{\prime}\right), 57.2(-, C-$ 5), $58.5(+, \mathrm{C}-2), 63.1,63.2(-, \mathrm{C}-\mathrm{Bzl}), 68.3\left(\mathrm{t}, J=19.3 \mathrm{~Hz}, \mathrm{C}-2^{\prime}\right), 70.0,70.0\left(+, \mathrm{C}-2^{\prime \prime}\right), 120.6$, $123.5,123.6,127.1,127.1,127.2,127.2,128.7,128.7,128.8,129.0,129.3,129.8,130.0,131.3$, 132.3 and $133.0\left(+\right.$, Ar-C), 125.9, 133.2, 133.4, 133.4, $142.3\left(\mathrm{C}_{\text {quat }}, \mathrm{Ar}-\mathrm{C}\right), 170.9,171.0\left(\mathrm{C}_{\text {quat }}\right.$, $\mathrm{C}=\mathrm{N}), 178.1,178.2\left(\mathrm{C}_{\text {quat }}, \mathrm{C}=\mathrm{O}\right), 180.4\left(\mathrm{C}_{\text {quat }}, \mathrm{CO}-\mathrm{N}\right)$; IR $(\mathrm{KBr}): \mathrm{nu}($ tilde $)=3071 \mathrm{~cm}^{-1}, 2972$, 2922, 2866, 1667, 1624, 1589, 1537, 1436, 1365, 1339, 1257, 1166; MS (EI, 70 eV), m/z (\%): $598(7)\left[\mathrm{M}^{+}\right], \quad 553(15)\left[\mathrm{M}^{+}-\mathrm{CO}_{2}\right], 439(9), 348(7), 217(22), 160(100), 91$ (23) $\left[\mathrm{C}_{7} \mathrm{H}_{7}^{+}\right]$; HRMS (EI): calcd for $\mathrm{C}_{31} \mathrm{H}_{29} \mathrm{DN}_{4} \mathrm{NiO}_{5}$ : 597.1632; found 597.1632; elemental analysis (\%) for $\mathrm{C}_{31} \mathrm{H}_{29} \mathrm{DN}_{4} \mathrm{NiO}_{5}$ (598.3): C 62.23, H 5.22, N 9.36; found C 61.94, H 5.12, N 9.22.

(2S, I'RS, 2'RS)-2-Deutero-3-(2'-nitrocyclopropyl)alanine (2S,1'RS,2'RS)-D-(83): $6 \mathrm{M} \mathrm{HCl}(32$

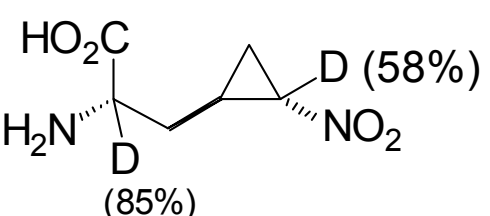
$(85 \%)$
$\mathrm{mL}$ ) was added to the suspension of finely grounded $(2 S, 2 ' S, 1 " R S, 2 " R S)-\mathrm{D}-82(3.87 \mathrm{~g}, 6.472 \mathrm{mmol})$ in refluxing methanol $(16 \mathrm{~mL})$. The mixture was gently refluxed for $10 \mathrm{~min}$.

The resulting green syrup was then concentrated, taken up with ice-cold water $(50 \mathrm{~mL})$, the precipitate was filtered off and washed with cold water $(20 \mathrm{~mL})$. The filtrate was combined with 
washings, $\mathrm{pH}$ was carefully adjusted to 6 with aqueous ammonia and the resultant emulsion was extracted with $\mathrm{CHCl}_{3}(3 \times 50 \mathrm{~mL})$. Preswallowed Amberlite IRA-120 in $\mathrm{H}^{+}$form $(90 \mathrm{~mL})$ was added to the water fraction and the mixture was stirred for $15 \mathrm{~h}$. The ion-exchange resin was filtered off, washed with water to $\mathrm{pH} 5$ and treated with $10 \%$ aqueous $\mathrm{NH}_{3}(2 \times 150 \mathrm{~mL})$ for $2 \mathrm{~h}$. The combined basic filtrates were concentrated under reduced pressure to give white solid, which was dissolved in hot water $(10 \mathrm{~mL})$. The solution was filtered and diluted with EtOH (20 $\mathrm{mL})$. The formed precipitate was filtered off to give $\left(2 S, 1^{\prime} R S, 2^{\prime} R S\right)-\mathrm{D}-\mathbf{8 3}(0.77 \mathrm{~g}, 68 \% ; 60 \%$ of $\left(2 S, 1^{\prime} S, 2^{\prime} R\right)$-isomer according to ${ }^{13} \mathrm{C}$ NMR spectrum) as a white solid. ${ }^{1} \mathrm{H}$ spectrum showed $88 \%$ content of deuterium at $\mathrm{C}-2$ and $58 \%$ at C-2' respectively. According to MS-ESI spectrum $\mathrm{D}_{2}$ and $\mathrm{D}$ contents were $52 \%$ and $42 \%$ respectively. M.p. $193-195^{\circ} \mathrm{C}($ decomp. $) ;[\alpha]_{20}^{D} 3.3(c=0.3$, $\left.\mathrm{H}_{2} \mathrm{O}\right) ;{ }^{1} \mathrm{H}$ NMR $\left(250 \mathrm{MHz}, \mathrm{D}_{2} \mathrm{O}\right)$ : mixture of diastereo- and isotopomers: $\delta=1.24-1.39(\mathrm{~m}, 1 \mathrm{H}$, $\left.3^{\prime}-\mathrm{H}\right), 1.73-2.25\left(\mathrm{~m}, 4 \mathrm{H}, 3-\mathrm{H}, 1^{\prime}-\mathrm{H}, 3^{\prime}-\mathrm{H}\right), 3.8(\mathrm{dd}, J=5.9,5.9 \mathrm{~Hz}, 1 \mathrm{H}, 2-\mathrm{H}), 4.18-4.26(\mathrm{~m}$, $\left.1 \mathrm{H}, 2^{\prime}-\mathrm{H}\right) ;{ }^{13} \mathrm{C}$ NMR $\left(62.9 \mathrm{MHz}, \mathrm{D}_{2} \mathrm{O}\right): \delta=20.4,20.5,20.7,20.9$ (-,C-3'), 24.3, 24.4, 24.6, 24.7 $\left(+, \mathrm{C}^{\prime} 1^{\prime}\right), 33.7,33.8(-, \mathrm{C}-3), 55.9(\mathrm{t}, J=23.4 \mathrm{~Hz}, \mathrm{C}-2), 61.6,61.7\left(+, \mathrm{C}-2^{\prime}\right), 176.0,176.1\left(\mathrm{C}_{\text {quat }}\right.$, C-1); IR (film): nu(tilde) $=3418 \mathrm{~cm}^{-1}, 3034,2102,1598,1530,1440,1394,1365$; MS (ESI), positive $m / z$ (\%): 220/221 (30/40) $\left[\mathrm{M}+2 \mathrm{Na}^{+}-\mathrm{H}^{+}\right]$; negative $m / z(\%): 175 / 176(3 / 3)\left[\mathrm{M}-\mathrm{H}^{-}\right]$; elemental analysis calcd (\%) for $\mathrm{C}_{6} \mathrm{H}_{9} \mathrm{DN}_{2} \mathrm{O}_{4}$ (175.2): C 41.14, H 6.33, N 15.99; found C 40.98, H 6.29, N 15.65.

(1'S,2'S)-Triphenylmethoxy-(2'-Nitrocyclopropyl)methan (1'S,2'S)-(87): Trityl chloride (1.166 g, $4.18 \mathrm{mmol})$ was added to an ice-cold solution of the alcohol (1'S,2'S)-10 (0.50 g, $4.27 \mathrm{mmol})$ and DMAP (ca. $10 \mathrm{mg}$ ) in anhydrous pyridine $\left(8 \mathrm{~mL}\right.$ ). The mixture was allowed to warm to $20{ }^{\circ} \mathrm{C}$ and stirring continued for a further 4 days. Methanol $(3 \mathrm{~mL})$ was then added and the mixture was stirred for an additional $3 \mathrm{~h}$. All volatiles were then removed and the residue was partitioned between $\mathrm{Et}_{2} \mathrm{O}$ and water $(40 \mathrm{~mL}$ of each). The organic layer was washed with water $(2 \times 10 \mathrm{~mL})$, $1 \mathrm{M} \mathrm{NaHSO}_{4}(3 \times 10 \mathrm{~mL})$, water $(3 \times 10 \mathrm{~mL})$, brine $(2 \times 10 \mathrm{~mL})$, dried, filtered and concentrated 
under reduced pressure. The resultant crude product was recrystallized from hexanes to give $(1 ' S, 2 ' S)-87(1.20 \mathrm{~g}, 78 \%)$ as a colorless solid. The spectral data of this compound were in accordance with literature. ${ }^{[13]}$

Deuterium exchange experiments (GP 3): To a stirred solution or suspension of the corresponding nitro-compound $(0.3 \mathrm{mmol})$ in anhydrous $\mathrm{CD}_{3} \mathrm{CN} / \mathrm{DMF} 2: 1(1.5 \mathrm{~mL}), \mathrm{NaH}(0.6$ mmol, $60 \%$ suspension in oil) was added at $-25^{\circ} \mathrm{C}$. The mixture was allowed to warm to $20{ }^{\circ} \mathrm{C}$ and stirring continued for an additional $50 \mathrm{~min}$. The reaction quenched with $0.5 \mathrm{M} \mathrm{D}_{2} \mathrm{SO}_{4}$ in $\mathrm{D}_{2} \mathrm{O}$ $(1.2 \mathrm{~mL})$ and diluted then with water $(15 \mathrm{~mL})$. The crude products were separated by filtration or extraction with $\mathrm{Et}_{2} \mathrm{O}$ and purified by recrystallization, column chromatography or distillation.

Deuteration of $\left(2 S, 2^{\prime} S, 1^{\prime \prime} R, 2^{\prime \prime} R\right)-82$ : $N i$-complex $\left(2 S, 2^{\prime} S, 1 " R, 2^{\prime \prime} R\right)-82(0.25 \mathrm{~g}, 0.42 \mathrm{mmol})$ was treated with $\mathrm{NaH}(0.038 \mathrm{~g}, 60 \%$ suspension in oil, $0.95 \mathrm{mmol})$ in $\mathrm{CD}_{3} \mathrm{CN} / \mathrm{DMF} 2: 1(1.5 \mathrm{~mL})$ according to GP 3 and the crude product was recrystallized from $\mathrm{CHCl}_{3} / \mathrm{Et}_{2} \mathrm{O}$ to give deuterated product $\left(0.181 \mathrm{~g}, 71 \% ; 55 \%\right.$ and $65 \%$ inclusion of the deuterium label at the 2 and $2^{\prime}$ positions of the (3-Ncp)Ala fragment respectively according to ${ }^{1} \mathrm{H}$ NMR spectrum).

Deuteration of (1'S,2'S)-87: Compound (1'S,2'S)-87 (0.30 g, $0.835 \mathrm{mmol})$ was treated with $\mathrm{NaH}$ $\mathrm{O}_{2} \mathrm{~N}$ (0.040 $\mathrm{g}, 60 \%$ suspension in oil, $1.00 \mathrm{mmol})$ in $\mathrm{CD}_{3} \mathrm{CN} / \mathrm{DMF} 2: 1(1.5$<smiles>[2H]C12C[C@H]1C[C@H]2[OH2+]</smiles>
$\mathrm{mL}$ ) according to GP 3 and the crude product was purified by column chromatography (EtOAc/hexanes $\left.1: 14, R_{\mathrm{f}}=0.41\right)$ to give deuterated product $\left(1 ' S, 2^{\prime} S\right)$-D-87 $\left(0.215 \mathrm{~g}, 72 \%\right.$; D content $>99 \%$, as determined by MS) as a white solid. $[\alpha]_{20}^{D} 63.4(c=0.61$, $\left.\mathrm{CHCl}_{3}\right)$ [lit. for $\left.17::^{[13]}[\alpha]_{20}^{D} 66.2\left(c=1.00, \mathrm{CHCl}_{3}\right)\right] ;{ }^{1} \mathrm{H} \mathrm{NMR}\left(250 \mathrm{MHz}, \mathrm{CDCl}_{3}\right): \delta=1.31(\mathrm{dd}$, $\left.J=6.0,7.5 \mathrm{~Hz}, 1 \mathrm{H}, 3^{\prime}-\mathrm{H}_{\mathrm{a}}\right), 1.84\left(\mathrm{dd}, J=5.8,10.5 \mathrm{~Hz}, 1 \mathrm{H}, 3^{\prime}-\mathrm{H}_{\mathrm{b}}\right), 2.17-2.31\left(\mathrm{~m}, 1 \mathrm{H}, 1^{\prime}-\mathrm{H}\right)$, $3.08(\mathrm{dd}, J=5.6,10.1 \mathrm{~Hz}, 1 \mathrm{H}, 1-\mathrm{H}), 3.30$ (dd, $J=4.5,10.3 \mathrm{H}, 1-\mathrm{H}), 7.22-7.43$ (m, $15 \mathrm{H}, \mathrm{Ar}-\mathrm{H})$; ${ }^{13} \mathrm{C}$ NMR (62.9 MHz, $\left.\mathrm{CDCl}_{3}\right): \delta=15.3\left(-, \mathrm{C}-3^{\prime}\right), 25.2\left(+, \mathrm{C}-1^{\prime}\right), 57.3\left(\mathrm{t}, J=27.7 \mathrm{~Hz}, \mathrm{C}-2^{\prime}\right), 61.6$ (-, C-1), $86.7\left(\mathrm{C}_{\text {quat }}, \mathrm{CPh}_{3}\right), 127.1,127.9,128.4$ (+, $\left.\mathrm{Ar}-\mathrm{C}\right), 143.4\left(\mathrm{C}_{\text {quat }}, \mathrm{Ar}-\mathrm{C}\right)$; IR (KBr): 
$\mathrm{nu}($ tilde $)=3111 \mathrm{~cm}^{-1}, 3089,3062,3029,2969,2942,2893,2871,1524,1355,1218,1115,1033$; MS (EI, $70 \mathrm{eV}), \quad m / z(\%): 360(26)\left[\mathrm{M}^{+}\right], 283(12)\left[\mathrm{M}^{+}-\mathrm{C}_{6} \mathrm{H}_{5}\right], 243(100)\left[\mathrm{CPh}_{3}{ }^{+}\right], 165(44)$ $\left[\mathrm{CPh}_{2}{ }^{+}\right]$; HRMS (EI): calcd for $\mathrm{C}_{23} \mathrm{H}_{20} \mathrm{DNO}_{3}$ : 360.1584; found 360.1584; elemental analysis calcd (\%) for $\mathrm{C}_{23} \mathrm{H}_{20} \mathrm{DNO}_{3}$ (360.4): C 76.65, H 6.15, N 3.89; found C 76.47, H 6.06, N 3.72.

Deuteration of nitrocyclopropane 88: Nitrocyclopropane 88 (0.255 g, $2.928 \mathrm{mmol})$ was treated with $\mathrm{NaH}(0.152 \mathrm{~g}, 60 \%$ suspension in oil, $3.800 \mathrm{mmol})$ in $\mathrm{CD}_{3} \mathrm{CN} / \mathrm{DMF} 2: 1(2.4 \mathrm{~mL})$ according to GP 3. The reaction was quenched with $5 \%$ acetic acid in $\mathrm{D}_{2} \mathrm{O}(3 \mathrm{~mL})$ and the mixture was extracted with $\mathrm{Et}_{2} \mathrm{O}(3 \times 10 \mathrm{~mL})$. The combined organic layers were washed with water $(3 \times 10$ $\mathrm{mL})$, brine $(2 \times 10 \mathrm{~mL})$, dried, filtrated through short $(5 \mathrm{~cm})$ column with alumina and concentrated under atmospheric pressure at bath temperature $<45^{\circ} \mathrm{C}$. The residue was subjected to bulb-to-bulb distillation under reduced pressure (125 Torr) and the fraction passed at the bath temperature $>50{ }^{\circ} \mathrm{C}$ was collected to give D-88 $(72 \mathrm{mg}, 90 \%$ purity, $25 \%$; D content $>97 \%$, as determined by ${ }^{1} \mathrm{H}$ NMR) as a colorless liquid. ${ }^{1} \mathrm{H}$ NMR $\left(250 \mathrm{MHz}, \mathrm{CDCl}_{3}\right): \delta=1.12-1.22(\mathrm{~m}$, $2 \mathrm{H}), 1.62(\mathrm{dd}, J=5.3,5.3 \mathrm{~Hz}, 2 \mathrm{H}) ;{ }^{13} \mathrm{C} \mathrm{NMR}\left(62.9 \mathrm{MHz}, \mathrm{CDCl}_{3}\right): \delta=11.4(-), 53.8(\mathrm{t}, J=$ $28.3 \mathrm{~Hz})$; MS (EI, $70 \mathrm{eV}), m / z(\%): 46(38)\left[\mathrm{NO}_{2}{ }^{+}\right], 42(100)\left[\mathrm{C}_{3} \mathrm{H}_{4} \mathrm{D}^{+}\right]$.

(1'S,2'S)-(2-allyl-2-nitrocyclopropyl)methyl trityl ether (1'S,2'S)-(91): $\mathrm{NaH}(0.156 \mathrm{~g}, \quad 60 \%$

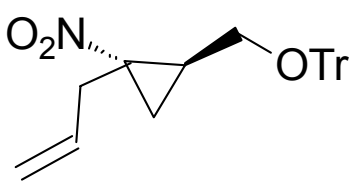
suspension in mineral oil, $3.90 \mathrm{mmol}$ ) was added to a degassed solution of (1'S,2'S)-87 (0.200 g, $0.56 \mathrm{mmol})$ and allyl bromide $(0.67 \mathrm{~g}, 55.38$ $\mathrm{mmol})$ in anhydrous DME $(7 \mathrm{~mL})$ and stirring continued for a further $36 \mathrm{~h}$. The mixture was then heated to $55-60^{\circ} \mathrm{C}$ and stirred for an additional $3 \mathrm{~h}$. After this, the reaction flask was cooled in ice/water bath and water $(3 \mathrm{~mL})$ was carefully added. The mixture was diluted with $\mathrm{Et}_{2} \mathrm{O}(40$ $\mathrm{mL})$ washed with water $(2 \times 10 \mathrm{~mL}), 1 \mathrm{M} \mathrm{NaHSO}_{4}(3 \times 10 \mathrm{~mL})$, again water $(3 \times 10 \mathrm{~mL})$, brine $(2 \times 10 \mathrm{~mL})$, dried, filtered and concentrated under reduced pressure. The residue was purified by column chromatography $\left(\right.$ EtOAc/hexanes $\left.1: 30, R_{\mathrm{f}}=0.11\right)$ to give the crude product $(0.104 \mathrm{~g})$ 
which was according to ${ }^{1} \mathrm{H}$ NMR spectrum impure mixture of cis- and trans-isomers in 1:7. This material was purified by preparative TLC ( 3 plates $200 \times 200 \mathrm{~mm}$, EtOAc/hexanes 1:30, 3 runs $)$ to give (1'S,2'S)-91 (72 mg, 49\%) as a turbid oil which solidified in a colorless solid during prolonged drying at $65^{\circ} \mathrm{C}$ and at 0.002 Torr. M.p. $79-80{ }^{\circ} \mathrm{C} ;[\alpha]_{20}^{D}-33.0\left(c=0.33, \mathrm{CHCl}_{3}\right) ;{ }^{1} \mathrm{H}$ $\operatorname{NMR}\left(250 \mathrm{MHz}, \mathrm{CDCl}_{3}\right): \delta=1.31\left(\mathrm{dd}, J=9.0,6.0 \mathrm{~Hz}, 1 \mathrm{H}, 3^{\prime}-\mathrm{H}_{\mathrm{a}}\right), 1.84(\mathrm{dddd}, J=5.5,9.0,9.0$, 9.0, $\left.9.0 \mathrm{~Hz}, 1 \mathrm{H}, 3^{\prime}-\mathrm{H}_{\mathrm{b}}\right), 1.85$ (dddd, $\left.J=9.0,7.5,6.0,1.2 \mathrm{~Hz}, 1 \mathrm{H}, 1^{\prime}-\mathrm{H}\right), 2.44$ (dddd, $J=13.0$, 6.5, 1.2, 1.2 Hz, $\left.1 \mathrm{H}, 1^{\prime \prime}-\mathrm{H}_{\mathrm{a}}\right), 3.09-3.23\left(\mathrm{~m}, 2 \mathrm{H}, 1-\mathrm{H}_{\mathrm{a}}, 1^{\prime \prime}-\mathrm{H}_{\mathrm{b}}\right), 3.51(\mathrm{dd}, J=10.5,5.5 \mathrm{~Hz}, 1 \mathrm{H}, 1-$ $\left.\mathrm{H}_{\mathrm{b}}\right), 5.11-5.18\left(\mathrm{~m}, 1 \mathrm{H}\right.$, cis-2"-H), 5.18 (dddd, $J=17.4,1.4,1.4,1.4 \mathrm{~Hz}$, trans $\left.-2^{\prime \prime}-\mathrm{H}\right), 5.74-5.92$ (m, $\left.1 \mathrm{H}, 33^{\prime \prime}-\mathrm{H}\right), 7.17-7.43(\mathrm{~m}, 15 \mathrm{H}, \mathrm{Ar}-\mathrm{H}) ;{ }^{13} \mathrm{C} \mathrm{NMR}\left(62.9 \mathrm{MHz}, \mathrm{CDCl}_{3}\right): \delta=18.5\left(-, \mathrm{C}-3^{\prime}\right)$, $29.1\left(+, \mathrm{C}-1^{\prime}\right), 38.2\left(-, \mathrm{C}-1^{\prime \prime}\right), 61.0(-, \mathrm{C}-1), 68.2\left(\mathrm{C}_{\text {quat }}, \mathrm{C}-2^{\prime}\right), 86.6\left(\mathrm{C}_{\text {quat }}, \mathrm{CPh}_{3}\right), 118.7\left(-, \mathrm{C}-3^{\prime \prime}\right)$, 127.0, 127.8, $128.5(+, \quad \mathrm{Ar}-\mathrm{C}), 132.1 \quad\left(+, \mathrm{C}-2^{\prime \prime}\right), 143.8 \quad\left(\mathrm{C}_{\text {quat }}, \mathrm{Ar}-\mathrm{C}\right) ; \quad \mathrm{IR}$ (film): $\mathrm{nu}($ tilde $)=3088 \mathrm{~cm}^{-1}, 3059,3028,2983,2926,2882,1960,1644,1598,1535,1492,1448,1361$, 1344, 1222, 1070; $\mathrm{MS}(\mathrm{EI}, 70 \mathrm{eV}), \quad m / z(\%): 399(9)\left[\mathrm{M}^{+}\right], 322(4)\left[\mathrm{M}^{+}-\mathrm{C}_{6} \mathrm{H}_{5}\right], 243(100)$ $\left[\mathrm{CPh}_{3}{ }^{+}\right], 165(15)\left[\mathrm{CPh}_{2}{ }^{+}\right], 140$ (37) $\left[\mathrm{C}_{7} \mathrm{H}_{10} \mathrm{NO}_{2}{ }^{+}\right], 105$ (33); HRMS (EI): calcd for $\mathrm{C}_{26} \mathrm{H}_{25} \mathrm{NO}_{3}$ : 399.1834; found 399.1834; elemental analysis calcd (\%) for $\mathrm{C}_{26} \mathrm{H}_{25} \mathrm{NO}_{3}$ (399.5): C 78.17, $\mathrm{H}$ 6.31, N 3.51; found C 77.87, H 6.31, N 3.29.

(1'S,2'R)-(2-benzyl-2-nitrocyclopropyl)methyl trityl ether (1'S,2'R)-(92): $\mathrm{NaH}(0.111 \mathrm{~g}, 60 \%$

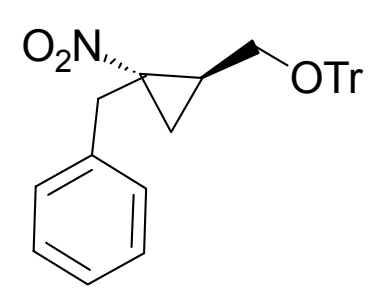
suspension in mineral oil, $2.78 \mathrm{mmol}$ ) was added to a degassed solution of (1'S,2'S)-87 (0.100 g, $0.28 \mathrm{mmol})$ and benzyl bromide $(0.33 \mathrm{~mL}, 2.78$ mmol) in anhydrous DME $(5 \mathrm{~mL})$ and stirring continued for a further 5 days. After this, the reaction flask was cooled in ice/water bath and water $(3 \mathrm{~mL})$ was carefully added. The mixture was diluted with $\mathrm{Et}_{2} \mathrm{O}(40 \mathrm{~mL})$ washed with water $(2 \times 10 \mathrm{~mL}), 1 \mathrm{M} \mathrm{NaHSO}_{4}(3 \times 10 \mathrm{~mL})$, water $(3 \times 10 \mathrm{~mL})$, brine $(2 \times 10 \mathrm{~mL})$, dried, filtered and concentrated under reduced pressure. All volatiles were then removed at $180{ }^{\circ} \mathrm{C}$ (bath temperature) and at 0.002 Torr by bulb-to-bulb distillation. The residual oil was triturated 
with pentane and then purified by preparative TLC $(200 \times 200 \mathrm{~mm}$, EtOAc/hexanes 1:30, 3 runs $)$ to give $\left(1^{\prime} S, 2^{\prime} R\right)-92(50 \mathrm{mg}, 40 \%)$ as a turbid oil. The fraction contained mainly somehow more polar $\left(1 ' S, 2^{\prime} S\right)$-isomer $(15 \mathrm{mg}, 12 \%)$ also was separated. ${ }^{1} \mathrm{H}$ NMR $\left(250 \mathrm{MHz}, \mathrm{CDCl}_{3}\right): \delta=1.24$ $\left(\mathrm{dd}, J=9.5,6.5 \mathrm{~Hz}, 1 \mathrm{H}, 3^{\prime}-\mathrm{H}_{\mathrm{a}}\right), 1.40-1.58\left(\mathrm{~m}, 1 \mathrm{H}, 3^{\prime}-\mathrm{H}_{\mathrm{b}}\right), 1.91$ (dddd, $J=8.0,6.5,6.5,1.3 \mathrm{~Hz}$, $\left.1 \mathrm{H}, 1^{\prime}-\mathrm{H}\right), 3.08\left(\mathrm{~d}, J=15.8 \mathrm{~Hz}, 1 \mathrm{H}, \mathrm{H}_{\mathrm{a}}, \mathrm{Bzl}-\mathrm{H}\right), 3.18\left(\mathrm{dd}, J=11.0,8.5 \mathrm{~Hz}, 1 \mathrm{H}, 1-\mathrm{H}_{\mathrm{a}}\right), 3.52$ (dd, $\left.J=11.0,5.5 \mathrm{~Hz}, 1 \mathrm{H}, 1-\mathrm{H}_{\mathrm{b}}\right), 3.84\left(\mathrm{~d}, J=15.8 \mathrm{~Hz}, 1 \mathrm{H}, \mathrm{H}_{\mathrm{b}}\right.$, Bzl-H), 7.17-7.44 (m, $\left.20 \mathrm{H}, \mathrm{Ar}-\mathrm{H}\right)$; ${ }^{13} \mathrm{C}$ NMR (62.9 MHz, $\left.\mathrm{CDCl}_{3}\right): \delta=18.4\left(-, \mathrm{C}-3^{\prime}\right), 29.1\left(+, \mathrm{C}-1^{\prime}\right), 39.5\left(-, \mathrm{C}-1^{\prime \prime}\right), 61.1(-, \mathrm{C}-1)$, $69.2\left(\mathrm{C}_{\text {quat }}, \mathrm{C}-2^{\prime}\right), 86.7\left(\mathrm{C}_{\text {quat }}, \mathrm{CPh}_{3}\right), 127.0,127.1,127.8,127.9,128.5,129.1(+, \mathrm{Ar}-\mathrm{C}), 136.1$, $143.8\left(\mathrm{C}_{\text {quat }}, \mathrm{Ar}-\mathrm{C}\right)$; IR (film): nu(tilde $)=3088 \mathrm{~cm}^{-1}, 3060,3028,2930,2880,1955,1600,1533$, 1493, 1448, 1362, 1323, 1221, 1071; MS (EI, $70 \mathrm{eV}), \mathrm{m} / z(\%): 449(9)\left[\mathrm{M}^{+}\right], 372(4)\left[\mathrm{M}^{+}-\right.$ $\left.\mathrm{C}_{6} \mathrm{H}_{5}\right], 259$ (36), $243(100)\left[\mathrm{CPh}_{3}{ }^{+}\right], 190(16)\left[\mathrm{C}_{11} \mathrm{H}_{12} \mathrm{NO}_{2}{ }^{+}\right], 165(15)\left[\mathrm{CPh}_{2}{ }^{+}\right], 105$ (33); $\mathrm{HRMS}$ (EI): calcd for $\mathrm{C}_{30} \mathrm{H}_{27} \mathrm{NO}_{3}$ : 449.1991; found 449.1991.

$\left(1^{\prime} S, 2\right.$ 'S)-92: ${ }^{1} \mathrm{H}$ NMR (250 MHz, $\left.\mathrm{CDCl}_{3}\right): \delta=1.06\left(\mathrm{dd}, J=7.8,6.0 \mathrm{~Hz}, 1 \mathrm{H}, 3^{\prime}-\mathrm{H}_{\mathrm{a}}\right.$ ), 2.12 (dddd, $\left.J=10.0,6.0,4.5,1.5 \mathrm{~Hz}, 1 \mathrm{H}, 3^{\prime}-\mathrm{H}_{\mathrm{b}}\right), 2.32-2.47\left(\mathrm{~m}, 1 \mathrm{H}, 1^{\prime}-\mathrm{H}\right), 2.65\left(\mathrm{~d}, J=16.5 \mathrm{~Hz}, 1 \mathrm{H}, \mathrm{H}_{\mathrm{a}}\right.$, Bzl-H), 2.90 (dd, $\left.J=10.8,8.5 \mathrm{~Hz}, 1 \mathrm{H}, 1-\mathrm{H}_{\mathrm{a}}\right), 3.56\left(\mathrm{dd}, J=10.8,5.0 \mathrm{~Hz}, 1 \mathrm{H}, 1-\mathrm{H}_{\mathrm{b}}\right), 3.85(\mathrm{~d}, J=$ 16.5 Hz, $\left.1 \mathrm{H}, \mathrm{H}_{\mathrm{b}}, \mathrm{Bzl}-\mathrm{H}\right), 7.05-7.35$ (m, $\left.15 \mathrm{H}, \mathrm{Ar}-\mathrm{H}\right)$, 7.37-7.46 (m, 5 H, Ar-H).

(1'S,2'S)-(2-methyl-2-nitrocyclopropyl)methyl trityl ether (1'S,2'S)-(93) and (1'S, 2'R)-(2-methyl2-nitrocyclopropyl)methyl trityl ether (1'S,2'R)-(93): $\mathrm{NaH}(0.111 \mathrm{~g}, 60 \%$ suspension in mineral oil, $2.78 \mathrm{mmol})$ was added to a degassed solution of (1'S,2'S)-87 (0.100 $\mathrm{g}, 0.28 \mathrm{mmol})$ and methyl iodide $(0.39 \mathrm{~g}, 2.75 \mathrm{mmol})$ in anhydrous diglym $(5 \mathrm{~mL})$ and stirring continued for $10 \mathrm{~h}$. The second portions of $\mathrm{NaH}(0.111 \mathrm{~g}, 60 \%$ suspension in mineral oil, $2.78 \mathrm{mmol})$ and methyl iodide $(0.39 \mathrm{~g}, 2.75 \mathrm{mmol})$ were added and the mixture was stirred for a further $12 \mathrm{~h}$. The third portions of $\mathrm{NaH}(0.111 \mathrm{~g}, 60 \%$ suspension in mineral oil, $2.78 \mathrm{mmol})$ and methyl iodide $(0.39 \mathrm{~g}$, $2.75 \mathrm{mmol}$ ) were added and stirring continued for an additional $16 \mathrm{~h}$. After this, the reaction flask was cooled in ice/water bath and water $(3 \mathrm{~mL})$ was carefully added. The mixture was 
diluted with $\mathrm{Et}_{2} \mathrm{O}(40 \mathrm{~mL})$ washed with water $(2 \times 10 \mathrm{~mL}), 1 \mathrm{M} \mathrm{NaHSO}_{4}(3 \times 10 \mathrm{~mL})$, water $(3 \times$ $10 \mathrm{~mL})$, brine $(2 \times 10 \mathrm{~mL})$, dried, filtered and concentrated under reduced pressure. The residue was separated by column chromatography (EtOAc/hexanes 1:30) to give the trans-isomer $\left(1^{\prime} S, 2^{\prime} S\right)-93(27 \mathrm{mg}, 26 \%)$ as a viscous colorless oil and the cis-isomer $\left(1^{\prime} S, 2^{\prime} R\right)-93(15 \mathrm{mg}$, $15 \%)$ as an yellow solid. Also their mixture (5 mg) was obtained.

$(1 ' S, 2 ' S)-93: R_{\mathrm{f}}=0.13 \mathrm{EtOAc} /$ hexanes $1: 30 ;{ }^{1} \mathrm{H}$ NMR $\left(250 \mathrm{MHz}, \mathrm{CDCl}_{3}\right): \delta=1.07(\mathrm{dd}, J=9.3$,

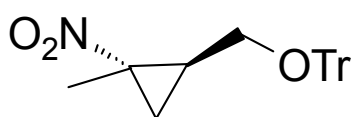
$\left.6.3 \mathrm{~Hz}, 1 \mathrm{H}, 3^{\prime}-\mathrm{H}_{\mathrm{a}}\right), 1.40-1.57\left(\mathrm{~m}, 1 \mathrm{H}, 3^{\prime}-\mathrm{H}_{\mathrm{b}}\right), 1.74(\mathrm{~s}, 3 \mathrm{H}, \mathrm{Me}), 1.82$ $\left(\mathrm{dd}, J=8.8,6.3 \mathrm{~Hz}, 1 \mathrm{H}, 1^{\prime}-\mathrm{H}\right), 3.13\left(\mathrm{dd}, J=11.0,8.8 \mathrm{~Hz}, 1 \mathrm{H}, 1-\mathrm{H}_{\mathrm{a}}\right)$, $3.48\left(\mathrm{dd}, J=11.0,5.8 \mathrm{~Hz}, 1 \mathrm{H}, 1-\mathrm{H}_{\mathrm{b}}\right), 7.10-7.41(\mathrm{~m}, 15 \mathrm{H}, \mathrm{Ar}-\mathrm{H}) ;{ }^{13} \mathrm{C} \mathrm{NMR}(62.9 \mathrm{MHz}$, $\left.\mathrm{CDCl}_{3}\right): \delta=20.7\left(-, \mathrm{C}^{\prime} 3^{\prime}\right), 21.1(+, \mathrm{Me}), 30.9\left(+, \mathrm{C}-1^{\prime}\right), 61.1(-, \mathrm{C}-1), 64.5\left(\mathrm{C}_{\text {quat }}, \mathrm{C}-2^{\prime}\right), 86.6$ $\left(\mathrm{C}_{\text {quat }}, \mathrm{CPh}_{3}\right), 127.0,127.8,128.5(+, \mathrm{Ar}-\mathrm{C}), 143.8\left(\mathrm{C}_{\text {quat }}, \mathrm{Ar}-\mathrm{C}\right)$; IR (film): nu(tilde) $=3088 \mathrm{~cm}^{-}$ ${ }^{1}, 3060,3027,2983,2939,2882,1960,1598,1539,1492,1445,1388,1364,1340,1221,1070$, 1031; MS (EI, $70 \mathrm{eV}), \quad m / z(\%): 373(11)\left[\mathrm{M}^{+}\right], \quad 296(5)\left[\mathrm{M}^{+}-\mathrm{C}_{6} \mathrm{H}_{5}\right], 259 \quad(27), \quad 243(100)$ $\left[\mathrm{CPh}_{3}{ }^{+}\right], 165(53)\left[\mathrm{CPh}_{2}{ }^{+}\right], 114(38)\left[\mathrm{C}_{5} \mathrm{H}_{8} \mathrm{NO}_{2}{ }^{+}\right], 105$ (43), 77 (12) $\left[\mathrm{C}_{6} \mathrm{H}_{5}^{+}\right]$; HRMS (EI): calcd for $\mathrm{C}_{24} \mathrm{H}_{23} \mathrm{NO}_{3}$ : 373.1678 ; found 373.1678.

$\left(1^{\prime} S, 2^{\prime} R\right)-93: R_{\mathrm{f}}=0.16 \mathrm{EtOAc} /$ hexanes $1: 30 ;{ }^{1} \mathrm{H} \mathrm{NMR}\left(250 \mathrm{MHz}, \mathrm{CDCl}_{3}\right): \delta=0.83(\mathrm{dd}, J=7.5$, $\left.5.5 \mathrm{~Hz}, 1 \mathrm{H}, 3^{\prime}-\mathrm{H}_{\mathrm{a}}\right), 1.58(\mathrm{~s}, 3 \mathrm{H}, \mathrm{Me}), 1.94\left(\mathrm{dd}, J=10.5,5.5 \mathrm{~Hz}, 1 \mathrm{H}, 3^{\prime}-\mathrm{H}_{\mathrm{b}}\right), 2.25-2.43(\mathrm{~m}, 1 \mathrm{H}$, $\left.1^{\prime}-\mathrm{H}\right), 2.76\left(\mathrm{dd}, J=10.8,8.8 \mathrm{~Hz}, 1 \mathrm{H}, 1-\mathrm{H}_{\mathrm{a}}\right), 3.48\left(\mathrm{dd}, J=10.8,5.3 \mathrm{~Hz}, 1 \mathrm{H}, 1-\mathrm{H}_{\mathrm{b}}\right), 7.15-7.45$ (m, $15 \mathrm{H}, \mathrm{Ar}-\mathrm{H}) ;{ }^{13} \mathrm{C}$ NMR $\left(62.9 \mathrm{MHz}, \mathrm{CDCl}_{3}\right): \delta=14.7(+, \mathrm{Me}), 22.4\left(-, \mathrm{C}-3^{\prime}\right), 29.7\left(+, \mathrm{C}-1^{\prime}\right)$, $62.4(-, \mathrm{C}-1), 64.0\left(\mathrm{C}_{\text {quat }}, \mathrm{C}-2^{\prime}\right), 86.8\left(\mathrm{C}_{\text {quat }}, \mathrm{CPh}_{3}\right), 127.1,127.9,128.5(+, \mathrm{Ar}-\mathrm{C}), 143.7\left(\mathrm{C}_{\text {quat }}\right.$, Ar-C); IR (KBr): nu(tilde) $=3086 \mathrm{~cm}^{-1}, 3056,3025,2926,2887,1817,1597,1530,1489,1446$, $1390,1364,1340,1226,1182,1070,1030$. 
2.6. Synthesis of O-MOM protected 5-chloro-1-hydroxypyrrol-2-carboxylic acid

2-Acetamido-6-chloro-pyridine-N-oxide (97): m-Chloroperbenzoic acid (11.97 g, 90\% purity,<smiles>CNc1cccc(Cl)[n+]1[O-]</smiles>
$62.44 \mathrm{mmol}$ ) was added to a solution of 2-acetamido-6-chloro-pyridine 96 (8.24 g, $48.30 \mathrm{mmol})$ in $\mathrm{CH}_{2} \mathrm{Cl}_{2}(100 \mathrm{~mL})$ and stirring continued for an additional $60 \mathrm{~h} . \mathrm{K}_{2} \mathrm{CO}_{3}(6.5 \mathrm{~g})$ was then added to the mixture and after stirring for $1 \mathrm{~h}$ the mixture was filtered, concentrated under reduced pressure and the residue was recrystallized from $\mathrm{CH}_{2} \mathrm{Cl}_{2} /$ hexanes to give $97(8.35 \mathrm{~g}, 93 \%)$ as a white solid. ${ }^{1} \mathrm{H}$ $\operatorname{NMR}\left(250 \mathrm{MHz}, \mathrm{CDCl}_{3}\right): \delta=2.29\left(\mathrm{~s}, 3 \mathrm{H}, \mathrm{CH}_{3}\right), 7.15-7.31(\mathrm{~m}, 2 \mathrm{H}, 4-\mathrm{H}, 5-\mathrm{H}), 8.35(\mathrm{dd}, J=$ 2.5, $10.0 \mathrm{~Hz}, 1 \mathrm{H}, 3-\mathrm{H}), 9.80-10.20(\mathrm{br}, 1 \mathrm{H}, \mathrm{NH}) ;{ }^{13} \mathrm{C} \mathrm{NMR}\left(62.9 \mathrm{MHz}, \mathrm{CDCl}_{3}\right): \delta=25.0(+$, $\left.\mathrm{CH}_{3}\right), 112.1(+, \mathrm{C}-3), 119.4(+, \mathrm{C}-5), 127.2(+, \mathrm{C}-4), 140.0\left(\mathrm{C}_{\text {quat }}, \mathrm{C}-6\right), 145.4\left(\mathrm{C}_{\text {quat }}, \mathrm{C}-2\right), 169.0$ $\left(\mathrm{C}_{\text {quat }}, \mathrm{CH}_{3} \mathrm{CO}\right)$; $\mathrm{MS}(\mathrm{EI}, 70 \mathrm{eV}): m / z(\%)=188 / 186(30 / 100)\left[\mathrm{M}^{+}\right], 146 / 144(30 / 100)\left[\mathrm{M}^{+}-\right.$ $\left.\mathrm{C}_{2} \mathrm{H}_{2} \mathrm{O}\right], 130 / 128(3 / 8), 43(78)\left[\mathrm{C}_{2} \mathrm{H}_{3} \mathrm{O}^{+}\right]$; HRMS (EI): calcd for $\mathrm{C}_{7} \mathrm{H}_{7} \mathrm{ClN}_{2} \mathrm{O}_{2}$ : 186.0196; found 186.0196.

tert-Butyl acetyl(6-chloropyridin-2-yl)carbamate-N-oxide (98): DMAP (0.54 g, $4.42 \mathrm{mmol})$ was<smiles>CC(=Nc1cccc(Cl)[n+]1[O-])C(=O)O</smiles>
added to a solution of $97(8.35 \mathrm{~g}, 44.75 \mathrm{mmol})$ and $\mathrm{Boc}_{2} \mathrm{O}(11.09 \mathrm{~g}$, $50.81 \mathrm{mmol})$ in acetonitrile $(60 \mathrm{~mL})$ and stirring continued for an additional $12 \mathrm{~h}$. The reaction mixture diluted with EtOAc $(250 \mathrm{~mL})$ and washed with $1 \mathrm{M} \mathrm{NaHSO}_{4}(3 \times 40 \mathrm{~mL})$, saturated aqueous $\mathrm{NaHCO}_{3}(3 \times 40 \mathrm{~mL}), \mathrm{H}_{2} \mathrm{O}(3 \times$ $40 \mathrm{~mL})$, brine $(2 \times 20 \mathrm{~mL})$, dried, filtered and concentrated under reduced pressure. The crude product was recrystallized from EtOAc/hexanes to give $98(8.31 \mathrm{~g}, 69 \%)$ as a white solid. $R_{\mathrm{f}}=$ 0.59, EtOAc/hexanes 1:1; ${ }^{1} \mathrm{H}$ NMR (250 $\left.\mathrm{MHz}, \mathrm{CDCl}_{3}\right): \delta=1.35\left[\mathrm{~s}, 9 \mathrm{H}, \mathrm{C}\left(\mathrm{CH}_{3}\right)_{3}\right], 2.58(\mathrm{~s}, 3 \mathrm{H}$, $\left.\mathrm{CH}_{3}\right), 7.08-7.22(\mathrm{~m}, 1 \mathrm{H}, 3-\mathrm{H}, 5-\mathrm{H}), 7.41-7.49(\mathrm{~m}, 1 \mathrm{H}, 4-\mathrm{H}) ;{ }^{13} \mathrm{C} \mathrm{NMR}\left(62.9 \mathrm{MHz}, \mathrm{CDCl}_{3}\right): \delta=$ $25.5\left(+, \mathrm{CH}_{3}\right), 27.5\left[+, \mathrm{C}\left(\mathrm{CH}_{3}\right)_{3}\right], 84.5\left[\mathrm{C}_{\text {quat }}, \mathrm{C}\left(\mathrm{CH}_{3}\right)_{3}\right], 124.0(+, \mathrm{C}-3), 124.5(+, \mathrm{C}-5), 125.8(+$, C-4), $141.9\left(\mathrm{C}_{\text {quat }}, \mathrm{C}-6\right), 145.8\left(\mathrm{C}_{\text {quat }}, \mathrm{C}-2\right), 150.0\left(\mathrm{C}_{\text {quat }}, \mathrm{NCO}_{2}\right), 171.8\left(\mathrm{C}_{\text {quat }}, \mathrm{CH}_{3} \mathrm{CO}\right)$; $\mathrm{MS}(\mathrm{EI}$, 
$70 \mathrm{eV}): m / z(\%)=288 / 286(5 / 14)\left[\mathrm{M}^{+}\right], 172 / 170(30 / 100)\left[\mathrm{M}^{+}-\mathrm{C}_{6} \mathrm{H}_{12} \mathrm{O}_{2}\right], 146 / 144(30 / 90)\left[\mathrm{M}^{+}\right.$ $\left.-\mathrm{C}_{6} \mathrm{H}_{12} \mathrm{O}_{3}\right], 57$ (93) $\left[\mathrm{C}_{4} \mathrm{H}_{9}^{+}\right], 43$ (32) $\left[\mathrm{C}_{2} \mathrm{H}_{3} \mathrm{O}^{+}\right]$; HRMS (EI): calcd for $\mathrm{C}_{7} \mathrm{H}_{7} \mathrm{ClN}_{2} \mathrm{O}_{2}$ : 286.0720; found 286.0720 .

2-tert-butyloxycarbonylamino-6-chloro-pyridine-N-oxide (99): To a solution of substance 98<smiles></smiles>
(8.31 g, $28.98 \mathrm{mmol})$ in methanol $(70 \mathrm{~mL})$ 1,1,3,3-tetramethylguanidine (4.53 mL, $36.10 \mathrm{mmol}$ ) was added and stirring continued for an additional $40 \mathrm{~min}$. The mixture was concentrated under reduced pressure to ca. $30 \mathrm{~mL}$, was diluted with $\mathrm{Et}_{2} \mathrm{O}(100 \mathrm{~mL})$, washed with $1 \mathrm{M} \mathrm{NaHCO}_{3}(2 \times 20 \mathrm{~mL})$, brine $(2 \times 20 \mathrm{~mL})$, dried, filtered and concentrated under reduced pressure to leave a solid residue which was recrystallized from octane to give 99 (4.25 g, 60\%) as a white solid. ${ }^{1} \mathrm{H}$ NMR $\left(250 \mathrm{MHz}, \mathrm{CDCl}_{3}\right): \delta=1.50\left[\mathrm{~s}, 9 \mathrm{H}, \mathrm{C}\left(\mathrm{CH}_{3}\right)_{3}\right], 7.09(\mathrm{dd}, J=2.0,8.3 \mathrm{~Hz}, 1 \mathrm{H}, 3-\mathrm{H}), 7.19(\mathrm{dd}, J=$ 8.3, 8.3 Hz, $1 \mathrm{H}, 4-\mathrm{H}), 8.06(\mathrm{dd}, J=2.0,8.3 \mathrm{~Hz}, 1 \mathrm{H}, 5-\mathrm{H}), 9.13-9.31$ (br, $1 \mathrm{H}, \mathrm{NH}) ;{ }^{13} \mathrm{C} \mathrm{NMR}$ $\left(62.9 \mathrm{MHz}, \mathrm{CDCl}_{3}\right): \delta=28.0\left[+, \mathrm{C}\left(\mathrm{CH}_{3}\right)_{3}\right], 82.5\left[\mathrm{C}_{\text {quat }}, \mathrm{C}\left(\mathrm{CH}_{3}\right)_{3}\right], 110.6(+, \mathrm{C}-3), 118.2(+, \mathrm{C}-5)$, $126.9(+, \mathrm{C}-4), 140.0\left(\mathrm{C}_{\text {quat }}, \mathrm{C}-6\right), 146.0\left(\mathrm{C}_{\text {quat }}, \mathrm{C}-2\right), 151.2\left(\mathrm{C}_{\text {quat }}, \mathrm{NCO}_{2}\right)$; IR $(\mathrm{KBr}):$ nu(tilde $)=$ $3297 \mathrm{~cm}^{-1}, 3122,2980,1728,1608,1560,1500,1377,1275,1257,1182,1159$; MS (EI, $\left.70 \mathrm{eV}\right)$ : $m / z(\%)=246 / 244(6 / 20)\left[\mathrm{M}^{+}\right], 190 / 188(3 / 8)\left[\mathrm{M}^{+}-\mathrm{C}_{4} \mathrm{H}_{8} \mathrm{O}\right], 173 / 171(5 / 17)\left[\mathrm{M}^{+}-\mathrm{C}_{4} \mathrm{H}_{9} \mathrm{O}_{2}\right]$, 146/144 (11/36) $\left[\mathrm{C}_{7} \mathrm{H}_{7} \mathrm{ClN}_{2} \mathrm{O}_{2}^{+}\right], 57$ (100) $\left[\mathrm{C}_{4} \mathrm{H}_{9}^{+}\right]$, 41 (8); HRMS (EI): calcd for $\mathrm{C}_{10} \mathrm{H}_{13} \mathrm{ClN}_{2} \mathrm{O}_{3}$ : 244.0615; found 244.0615 .

2-Amino-6-chloro-pyridine-N-oxide hydrochloride HCl-(101): Amide 99 (4.22 g, 17.30 mmol) was deprotected with $3.9 \mathrm{M} \mathrm{HCl}$ in dioxane $(100 \mathrm{~mL})$ for $24 \mathrm{~h}$. All volatiles were then removed and the residue was triturated with ether to give $\mathrm{HCl} \cdot 101(3.03 \mathrm{~g}, 97 \%)$ as a colorless solid. 
2-Amino-6-chloropyridine-N-oxide (101): 2,6-Dicholoropyridine $N$-oxide 100 (6.30 g, 38.4<smiles>Nc1cccc(Cl)[n+]1[O-]</smiles>
mmol) was heated with $25 \% \mathrm{NH}_{3}$ in $\mathrm{MeOH}(100 \mathrm{~mL})$ in a sealed tube at $105^{\circ} \mathrm{C}$ for $26 \mathrm{~h}$ (TLC control). The tube was cooled in a water-ice bath, then opened and the reaction mixture was filtered and concentrated under reduced pressure. The residue was dissolved in $\mathrm{MeOH} / \mathrm{CHCl}_{3} 1: 4$, the solution was filtered again and concentrated to give a dark-red oil, which was purified by column chromatography (silica gel, $\left.\mathrm{MeOH} / \mathrm{CHCl}_{3} 1: 4, R_{\mathrm{f}}=0.50\right)$ and then by recrystallization $\left(\mathrm{CHCl}_{3} /\right.$ hexanes $)$ to give the title compound $(3.04 \mathrm{~g}, 55 \%)$ as a colorless solid. $R_{\mathrm{f}}=0.23$ EtOAc/hexanes 1:1; m.p. $133-135{ }^{\circ} \mathrm{C} ;{ }^{1} \mathrm{H}$ $\operatorname{NMR}\left(250 \mathrm{MHz}, \mathrm{CDCl}_{3}\right): \delta=5.03\left(\mathrm{br}, 2 \mathrm{H}, \mathrm{NH}_{2}\right), 6.31$ (dd, $\left.J=0.5,8.3 \mathrm{~Hz}, 1 \mathrm{H}, 3-\mathrm{H}\right), 6.57$ (dd, $J=0.5,7.5 \mathrm{~Hz}, 1 \mathrm{H}, 5-\mathrm{H}), 7.29(\mathrm{dd}, J=7.8 \mathrm{~Hz}, 1 \mathrm{H}, 4-\mathrm{H}) ;{ }^{13} \mathrm{C} \mathrm{NMR}\left(62.9 \mathrm{MHz}, \mathrm{CDCl}_{3}\right): \delta=$ $106.4(+, \mathrm{C}-3), 112.5(+, \mathrm{C}-5), 139.9(+, \mathrm{C}-4), 149.1\left(\mathrm{C}_{\text {quat }}, \mathrm{C}-6\right), 158.7\left(\mathrm{C}_{\text {quat }}, \mathrm{C}-2\right)$; IR $(\mathrm{KBr})$ : $\mathrm{nu}(\mathrm{tilde})=3400-2500 \mathrm{~cm}^{-1}, 3192,3147,1653,1628,1550,1502,1412,1216,1188 ; \mathrm{MS}(\mathrm{EI}, 70$ $\mathrm{eV}): m / z(\%)=146 / 144(30 / 100)\left[\mathrm{M}^{+}\right], 128 / 126(10 / 30)\left[\mathrm{M}^{+}-\mathrm{H}_{2} \mathrm{O}\right], 119 / 117(5 / 16)\left[\mathrm{M}^{+}-\right.$ $\mathrm{HCN}], 109$ (2) [M+ $-\mathrm{Cl}], 100$ (7), 99 (10), 92 (17), 93/91 (5/16), 81 (7); HRMS: $\mathrm{C}_{5} \mathrm{H}_{5} \mathrm{ClN}_{2} \mathrm{O}$ : calcd for $\mathrm{C}_{5} \mathrm{H}_{5} \mathrm{ClN}_{2} \mathrm{O}_{2}$ : 144.0090; found: 144.0090; elemental analysis calcd (\%) for $\mathrm{C}_{5} \mathrm{H}_{5} \mathrm{ClN}_{2} \mathrm{O}$ (144.6): C 41.54, H 3.49, N 19.38; found C 41.41, H 3.54, N 19.46.

2-Azido-6-chloropyridine- $N$-oxide (102): A $2.5 \mathrm{M}$ aqueous $\mathrm{NaNO}_{2}(11.3 \mathrm{ml})$ was added<smiles>Nc1cccc(Cl)[n+]1O</smiles>
dropwise to an ice-cold solution of 2-Amino-6-chloropyridine- $N$-oxide 101 (3.80 g, $26.3 \mathrm{mmol}$ ) in $85 \mathrm{ml} \mathrm{10 \%} \mathrm{HCl}$ so that the internal temperature was not higher than $5{ }^{\circ} \mathrm{C}$. The reaction mixture was stirred for $10 \mathrm{~min}$, then a $2.5 \mathrm{M} \mathrm{NaN}_{3}(11.3 \mathrm{ml})$ was added dropwise so that the internal temperature was not higher than 5 ${ }^{\circ} \mathrm{C}$ and stirring continued at the same temperature for an additional $1 \mathrm{~h}$. The reaction mixture was extracted with $\mathrm{CH}_{2} \mathrm{Cl}_{2}(8 \times 50 \mathrm{ml})$, dried and filtered through short column with silica gel using first $\mathrm{CH}_{2} \mathrm{Cl}_{2}\left(\begin{array}{ll}1 & 1\end{array}\right)$ and then $\mathrm{CH}_{2} \mathrm{Cl}_{2} / \mathrm{Et}_{2} \mathrm{O}$ 1:1 (1 l) as an eluent. The combined fractions were concentrated to ca. $15 \mathrm{ml}$ under reduced pressure and hexanes $(150 \mathrm{ml})$ were added. The 
precipitate was separated by filtration and washed with $\mathrm{CH}_{2} \mathrm{Cl}_{2} /$ hexanes 1:10 to give after drying the title compound $(3.4 \mathrm{~g}, 76 \%)$ as a faint yellow solid. ${ }^{1} \mathrm{H}$ NMR $\left(250 \mathrm{MHz}, \mathrm{CDCl}_{3}\right): 6.84(\mathrm{dd}, J$ $=2.0,8.3 \mathrm{~Hz}, 1 \mathrm{H}, 3-\mathrm{H}), 7.11(\mathrm{dd}, J=8.3,8.3 \mathrm{~Hz}, 1 \mathrm{H}, 4-\mathrm{H}), 7.24(\mathrm{dd}, J=2.0,8.6 \mathrm{~Hz}, 1 \mathrm{H}, 5-$ $\mathrm{H}) ;{ }^{13} \mathrm{C}$ NMR $\left(62.9 \mathrm{MHz}, \mathrm{CDCl}_{3}\right): \delta=114.5(+, \mathrm{C}-3), 121.5(+, \mathrm{C}-5), 126.2(+, \mathrm{C}-4), 145.4$ $\left(\mathrm{C}_{\text {quat }}, \mathrm{C}-6\right), 156.8\left(\mathrm{C}_{\text {quat }}, \mathrm{C}-2\right)$; IR $(\mathrm{KBr}):$ nu(tilde $)=3126 \mathrm{~cm}^{-1}, 3077,2130,1597,1470,1390$, 1212; MS (EI, $70 \mathrm{eV}): m / z(\%)=172 / 170(14 / 44)\left[\mathrm{M}^{+}\right], 144 / 142(5 / 16)\left[\mathrm{M}^{+}-\mathrm{N}_{2}\right], 114 / 112$ (18/56), 87/85 (6/18), 76 (100); HRMS (EI): calcd for $\mathrm{C}_{5} \mathrm{H}_{3} \mathrm{ClN}_{4} \mathrm{O}$ : 169.9995; found: 169.9995; elemental analysis (\%) for $\mathrm{C}_{5} \mathrm{H}_{3} \mathrm{ClN}_{4} \mathrm{O}$ (144.6): C 35.21, $\mathrm{H} 1.77, \mathrm{~N} 32.85$; found $\mathrm{C} 35.35, \mathrm{H}$ $2.01, \mathrm{~N} 32.66$.

5-Chloro-1-hydroxypyrrole-2-carbonitrile (103) and 5-chloro-2-carbonitrile (104): A solution of 2-Azido-6-chloropyridine- $N$-oxide $102(1.27 \mathrm{~g}, 7.45 \mathrm{mmol})$ in anhydrous benzene $(32 \mathrm{~mL})$ under argon was sealed in a thick-walled tube. The tube was heated first at $90^{\circ} \mathrm{C}$, then the temperature was allowed to increase to $105^{\circ} \mathrm{C}$, and the reaction mixture was left at this temperature for 15 min. Then the temperature was decreased to $85^{\circ} \mathrm{C}$, and stirring continued for an additional $4 \mathrm{~h}$. After this, the reaction mixture was allowed to cool to $20^{\circ} \mathrm{C}$ and was concentrated under reduced pressure at a temperature not higher than $35^{\circ} \mathrm{C}$ to give a red solid, which was purified by column chromatography (silica gel, EtOAc/hexanes 1:4) to give $\mathbf{1 0 3}\left(0.517 \mathrm{~g}, 49 \%, R_{\mathrm{f}}=0.37\right)$ as a light tan solid and $109\left(0.12 \mathrm{~g}, 11 \%, R_{\mathrm{f}}=0.49\right)$ as a white solid.

103: $R_{\mathrm{f}}=0.52$, EtOAc/Hexanes $=1: 3$; m.p. $101-102{ }^{\circ} \mathrm{C} ;{ }^{1} \mathrm{H}$ NMR $\left(250 \mathrm{MHz}, \mathrm{CDCl}_{3}\right): \delta=$<smiles>N#Cc1ccc(Cl)n1O</smiles>
$6.03(\mathrm{~d}, J=5.0 \mathrm{~Hz}, 1 \mathrm{H}, 4-\mathrm{H}), 6.67(\mathrm{~d}, J=5.0 \mathrm{~Hz}, 1 \mathrm{H}, 3-\mathrm{H}), 8.96$ (br, $1 \mathrm{H}$, $\mathrm{OH}) ;{ }^{13} \mathrm{C}$ NMR $\left(62.9 \mathrm{MHz}, \mathrm{CDCl}_{3}\right): \delta=100.5\left(\mathrm{C}_{\text {quat }}, \mathrm{C}-2\right), 104.9(+, \mathrm{C}-4)$, $111.0\left(\mathrm{C}_{\text {quat }}, \mathrm{CN}\right), 116.0(+, \mathrm{C}-3), 131.9\left(\mathrm{C}_{\text {quat }}, \mathrm{C}-5\right)$; IR $(\mathrm{KBr}):$ nu(tilde $)=$ $3139 \mathrm{~cm}^{-1}, 3127,3007,2900,2241,1516,1425,1403,1378$; MS (EI, $\left.70 \mathrm{eV}\right): \mathrm{m} / z(\%)=144 / 142$ (30/100) $\left[\mathrm{M}^{+}\right], 127 / 125(17 / 57)\left[\mathrm{M}^{+}-\mathrm{OH}\right], 107(2)\left[\mathrm{M}^{+}-\mathrm{Cl}\right], 89(3), 80$ (49), 73 (9), 64 (26), 52 
(9); HRMS (EI): calcd for $\mathrm{C}_{5} \mathrm{H}_{3} \mathrm{ClN}_{2} \mathrm{O}$ : 141.9934, found: 141.9934; elemental analysis (\%) for $\mathrm{C}_{5} \mathrm{H}_{3} \mathrm{ClN}_{2} \mathrm{O}$ (142.5): C 42.13, H 2.12, N 19.65; found C 42.03, H 2.35, N 19.44.

104: ${ }^{1} \mathrm{H}$ NMR $\left(250 \mathrm{MHz}, \mathrm{CDCl}_{3}\right): \delta=6.03(\mathrm{~d}, J=7.5 \mathrm{~Hz}, 1 \mathrm{H}, 4-\mathrm{H}), 6.67(\mathrm{~d}, J=7.5 \mathrm{~Hz}, 1 \mathrm{H}, 3-$

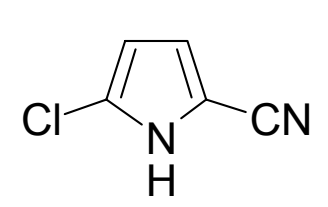

$\mathrm{H}), 8.96(\mathrm{bs}, 1 \mathrm{H}, \mathrm{NH}) ;{ }^{13} \mathrm{C} \mathrm{NMR}\left(62.9 \mathrm{MHz}, \mathrm{CDCl}_{3}\right): \delta=100.0\left(\mathrm{C}_{\text {quat }}, \mathrm{C}-\right.$ 2), $108.6(+, \mathrm{C}-4), 113.6\left(\mathrm{C}_{\text {quat }}, \mathrm{CN}\right), 120.8\left(\mathrm{C}_{\text {quat }}, \mathrm{C}-5\right), 121.5(+, \mathrm{C}-3)$; $\mathrm{MS}$ $(\mathrm{EI}, 70 \mathrm{eV}): m / z(\%)=128 / 126(30 / 100)\left[\mathrm{M}^{+}\right], 101 / 99(1 / 3)\left[\mathrm{M}^{+}-\mathrm{HCN}\right]$,

91 (4) $\left[\mathrm{M}^{+}-\mathrm{Cl}\right], 77 / 75$ (1/4), 64 (16); HRMS (EI): calcd for $\mathrm{C}_{5} \mathrm{H}_{3} \mathrm{ClN}_{2}$ : 125.9985, found: 125.9985 .

5-Chloro-1-methoxymethoxypyrrole-2-carbonitrile (105): To a vigorously stirred emulsion of a<smiles>COn1c(Cl)ccc1C#N</smiles>
$24 \%$ aqueous $\mathrm{NaOH}(0.89 \mathrm{~g}, 5.34 \mathrm{mmol})$ in a solution of 5-Chloro-1hydroxypyrrole-2-carbonitrile $\mathbf{1 0 3}(0.25 \mathrm{~g}, 1.75 \mathrm{mmol})$ and $\mathrm{TBACl} \cdot \mathrm{H}_{2} \mathrm{O}$ (0.05 g, $0.18 \mathrm{mmol})$ in $\mathrm{CH}_{2} \mathrm{Cl}_{2}$ was added $\mathrm{MOMCl}(1.07 \mathrm{ml}, 14.09 \mathrm{mmol})$ and vigorous stirring continued for an additional $30 \mathrm{~min}$. The organic layer was then separated and the aqueous fraction was extracted with $\mathrm{CH}_{2} \mathrm{Cl}_{2}(3 \times 10 \mathrm{ml})$. Combined organic layers were then concentrated under reduced pressure and the crude product was purified first by column chromatography (EtOAc/hexanes 1:7) and finally by bulb-to-bulb distillation at the bath temperature $60-100{ }^{\circ} \mathrm{C}$ and at 0.02 Torr to give the title compound $(0.310 \mathrm{~g}, 94 \%)$ as a colorless liquid which solidified in a freezer in a colorless solid. $R_{\mathrm{f}}=0.55$, EtOAc/hexanes $=1: 4 ;{ }^{1} \mathrm{H}$ $\operatorname{NMR}\left(250 \mathrm{MHz}, \mathrm{CDCl}_{3}\right): \delta=3.73\left(\mathrm{~s}, 3 \mathrm{H}, \mathrm{CH}_{3}\right), 5.18\left(\mathrm{~s}, 2 \mathrm{H}, \mathrm{CH}_{2}\right), 6.05(\mathrm{~d}, J=4.8 \mathrm{~Hz}, 1 \mathrm{H}, 4-$ $\mathrm{H}), 6.65(\mathrm{~d}, J=4.8 \mathrm{~Hz}, 1 \mathrm{H}, 3-\mathrm{H}) ;{ }^{13} \mathrm{C} \mathrm{NMR}\left(62.9 \mathrm{MHz}, \mathrm{CDCl}_{3}\right): \delta=58.9\left(+, \mathrm{CH}_{3}\right), 102.1\left(\mathrm{C}_{\text {quat }}\right.$, C-2), $104.2\left(-, \mathrm{CH}_{2}\right), 105.1(+, \mathrm{C}-4), 111.6\left(\mathrm{C}_{\text {quat }}, \mathrm{CN}\right), 115.5(+, \mathrm{C}-3), 118.6\left(\mathrm{C}_{\text {quat }}, \mathrm{C}-5\right)$; IR (film): nu(tilde) $=3136 \mathrm{~cm}^{-1}, 2945,2839,2227,1430,1218,1167,1091 ; \mathrm{MS}(\mathrm{EI}, 70 \mathrm{eV}): \mathrm{m} / \mathrm{z}$ $(\%)=188 / 186(3 / 8)\left[\mathrm{M}^{+}\right], 127 / 125(2 / 5)\left[\mathrm{M}^{+}-\mathrm{C}_{2} \mathrm{H}_{5} \mathrm{O}_{2}\right], 64(3), 45(100)\left[\mathrm{C}_{2} \mathrm{H}_{5} \mathrm{O}^{+}\right]$; HRMS (EI): calcd for $\mathrm{C}_{7} \mathrm{H}_{7} \mathrm{ClN}_{2} \mathrm{O}_{2}$ : 186.0196, found: 186.0196; elemental analysis calcd (\%) for $\mathrm{C}_{7} \mathrm{H}_{7} \mathrm{ClN}_{2} \mathrm{O}_{2}$ (186.6): C 45.06, H 3.78, N 15.01; found C 44.87, H 3.79, N 14.81. 
5-Chloro-1-methoxymethoxypyrrole-2-carboxamide (106): To a vigorously stirred solution of 5-

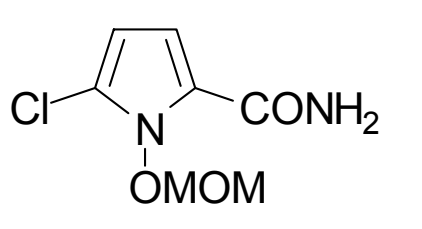

Chloro-1-methoxymethoxypyrrole-2-carbonitrile $\mathbf{1 0 5}$ (0.63 g, 2.30

mmol) and tetra- $n$-butylammonium hydrogen sulfate $(0.16 \mathrm{~g}, 0.47$ mmol) in $\mathrm{CH}_{2} \mathrm{Cl}_{2}(4.3 \mathrm{ml})$ a $20 \%$ aqueous $\mathrm{NaOH}(0.86 \mathrm{~g}, 4.30 \mathrm{mmol})$ and $30 \% \mathrm{H}_{2} \mathrm{O}_{2}(1.64 \mathrm{ml}, 16.22 \mathrm{mmol})$ were added and vigorous stirring continued for an additional $90 \mathrm{~min}$. The layers were separated and the water layer was additionally extracted with $\mathrm{CH}_{2} \mathrm{Cl}_{2}(4 \times 5 \mathrm{ml})$ under salt-out conditions. The combined organic layers were concentrated under reduced pressure and the residue was purified by column chromatography (EtOAc/hexaness $\left.1: 1, R_{\mathrm{f}}=0.29\right)$ to give the title compound $(0.445 \mathrm{~g}, 94 \%)$ as a colorless oil. ${ }^{1} \mathrm{H}$ NMR (250 MHz, $\left.\mathrm{CDCl}_{3}\right): \delta=3.55\left(\mathrm{~s}, 3 \mathrm{H}, \mathrm{CH}_{3}\right), 5.25\left(\mathrm{~s}, 2 \mathrm{H}, \mathrm{CH}_{2}\right), 5.50-6.50\left(\mathrm{br}, 2 \mathrm{H}, \mathrm{NH}_{2}\right)$ $6.05(\mathrm{~d}, J=4.8 \mathrm{~Hz}, 1 \mathrm{H}, 4-\mathrm{H}), 6.73(\mathrm{~d}, J=4.8 \mathrm{~Hz}, 1 \mathrm{H}, 3-\mathrm{H}) ;{ }^{13} \mathrm{C} \mathrm{NMR}\left(62.9 \mathrm{MHz}, \mathrm{CDCl}_{3}\right): \delta=$ $59.5\left(+, \mathrm{CH}_{3}\right), 104.2(+, \mathrm{C}-4), 104.8\left(-, \mathrm{CH}_{2}\right), 111.4(+, \mathrm{C}-3), 118.6\left(\mathrm{C}_{\text {quat }}, \mathrm{C}-2\right), 122.1\left(\mathrm{C}_{\text {quat }}, \mathrm{C}-\right.$ 5), $160.4\left(\mathrm{C}_{\text {quat }}, \mathrm{CONH}_{2}\right)$; IR (film): nu(tilde) $=3466 \mathrm{~cm}^{-1}, 3339,3192,2943,2838,1658,1606$, 1440, 1166, 1085; MS (EI, $70 \mathrm{eV}): m / z(\%)=206 / 204(11 / 32)\left[\mathrm{M}^{+}\right], 174 / 172(23 / 70)\left[\mathrm{M}^{+}-\right.$ $\left.\mathrm{CH}_{4} \mathrm{O}\right], 145 / 143(4 / 13)\left[\mathrm{M}^{+}-\mathrm{C}_{2} \mathrm{H}_{5} \mathrm{O}_{2}\right], 90 / 88(2 / 6), 45$ (100) $\left[\mathrm{C}_{2} \mathrm{H}_{5} \mathrm{O}^{+}\right]$; HRMS (EI): calcd for $\mathrm{C}_{7} \mathrm{H}_{9} \mathrm{ClN}_{2} \mathrm{O}_{2}$ : 204.0302, found: 204.0302; elemental analysis calcd (\%) for $\mathrm{C}_{7} \mathrm{H}_{9} \mathrm{ClN}_{2} \mathrm{O}_{2}$ (204.6): C 41.09, H 4.43, N 13.69; found C 41.32, H 4.21, N 13.47.

Di-tert-butyl [5-chloro-1-(methoxymethoxy)-pyrrol-2-yl]carbonylimidodicarbomate (107) and tert-butyl bis-[5-chloro-1-(methoxymethoxy)-pyrrol-2-yl]carbonylcarbamate (108): To a solution of 5-Chloro-1-methoxymethoxypyrrole-2-carboxamide $(0.41 \mathrm{~g}, 2.00 \mathrm{mmol})$ and $\mathrm{Boc}_{2} \mathrm{O}$ $(2.11 \mathrm{~g}, 9.67 \mathrm{mmol})$ in anhydrous $\mathrm{MeCN}$ (10 ml) 4-pyrrolidinopyridine (50 $\mathrm{mg}, 0.34 \mathrm{mmol})$ was added and stirring continued for an additional $2.5 \mathrm{~h}$. The mixture was then carefully heated with a heat-gun to ca. $50^{\circ} \mathrm{C}$, cooled, concentrated under reduced pressure and the crude product was purified by column chromatography (EtOAc/hexanes 1:8) to give the desired doubly N-Boc- 
acylated product $107\left(0.665 \mathrm{~g}, 82 \%, \mathrm{R}_{\mathrm{f}}=0.13\right)$ as a colorless solid and the by-product $\mathbf{1 0 8}(50$ $\mathrm{mg}, 5 \%, R_{\mathrm{f}}=0.07$ ) as a viscous opaque oil.

107: M.p. $67-68{ }^{\circ} \mathrm{C} ;{ }^{1} \mathrm{H}$ NMR $\left(250 \mathrm{MHz}, \mathrm{CDCl}_{3}\right): \delta=1.42\left[\mathrm{~s}, 18 \mathrm{H}, 2 \times \mathrm{C}\left(\mathrm{CH}_{3}\right)_{3}\right], 3.68(\mathrm{~s}, 3 \mathrm{H}$,

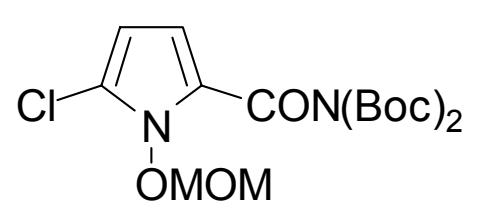

$\left.\mathrm{CH}_{3}\right), 5.22\left(\mathrm{~s}, 2 \mathrm{H}, \mathrm{CH}_{2}\right), 6.07(\mathrm{~d}, J=4.9 \mathrm{~Hz}, 1 \mathrm{H}, 4-\mathrm{H}), 6.75(\mathrm{~d}, J$ )$\left._{2}=4.9 \mathrm{~Hz}, 1 \mathrm{H}, 3-\mathrm{H}\right) ;{ }^{13} \mathrm{C} \mathrm{NMR}\left(62.9 \mathrm{MHz}, \mathrm{CDCl}_{3}\right): \delta=27.7[+, 2$ $\left.\times \mathrm{C}\left(\mathrm{CH}_{3}\right)_{3}\right], 58.8\left(+, \mathrm{CH}_{3}\right), 84.0\left[\mathrm{C}_{\text {quat }}, 2 \times \mathrm{C}\left(\mathrm{CH}_{3}\right)_{3}\right], 104.5(-$, $\left.\mathrm{CH}_{2}\right), 104.9(+, \mathrm{C}-4), 116.0(+, \mathrm{C}-3), 122.3\left(\mathrm{C}_{\text {quat }}, \mathrm{C}-2\right), 124.2\left(\mathrm{C}_{\text {quat }}, \mathrm{C}-5\right), 149.3\left(\mathrm{C}_{\text {quat }}, \mathrm{NCO}_{2}\right)$, $157.0\left(\mathrm{C}_{\text {quat }}, \mathrm{CON}\right)$; IR (KBr): nu(tilde $)=3123 \mathrm{~cm}^{-1}, 3012,2971,2946,1793,1696,1431,1391$, 1371, 1275, 1255, 1156, 1102; MS (EI, $70 \mathrm{eV}): m / z(\%)=406 / 404(2 / 6)\left[\mathrm{M}^{+}\right], 350 / 348(2 / 5)\left[\mathrm{M}^{+}\right.$ $\left.-\mathrm{C}_{4} \mathrm{H}_{8}\right], 294 / 292(2 / 6)\left[\mathrm{M}^{+}-\mathrm{C}_{8} \mathrm{H}_{16}\right], 250 / 248(11 / 35)\left[\mathrm{M}^{+}-\mathrm{C}_{9} \mathrm{H}_{17} \mathrm{O}_{2}\right], 190 / 188(11 / 35)$, 174/172 (30/100) $\left[\mathrm{C}_{7} \mathrm{H}_{7} \mathrm{NO}_{2} \mathrm{Cl}^{+}\right] 143(31), 57\left[\mathrm{C}_{4} \mathrm{H}_{9}{ }^{+}\right](100), 45$ (58) $\left[\mathrm{C}_{2} \mathrm{H}_{5} \mathrm{O}^{+}\right]$; HRMS (EI): calcd for $\mathrm{C}_{17} \mathrm{H}_{25} \mathrm{ClN}_{2} \mathrm{O}_{7}$ : 404.1350, found: 404.1350; elemental analysis calcd (\%) for $\mathrm{C}_{17} \mathrm{H}_{25} \mathrm{ClN}_{2} \mathrm{O}_{7}$ (404.9): C 50.44, H 6.22, N 6.92; found C 50.26, H 5.99, N 6.85.

108: ${ }^{1} \mathrm{H}$ NMR $\left(250 \mathrm{MHz}, \mathrm{CDCl}_{3}\right): \delta=1.39\left[\mathrm{~s}, 9 \mathrm{H}, \mathrm{C}\left(\mathrm{CH}_{3}\right)_{3}\right], 3.64\left(\mathrm{~s}, 3 \mathrm{H}, \mathrm{CH}_{3}\right), 5.24(\mathrm{~s}, 2 \mathrm{H}$,<smiles>COn1c(Cl)ccc1C(=O)NC(=O)c1ccc(Cl)n1OC</smiles>
$\left.\mathrm{CH}_{2}\right), 6.03(\mathrm{~d}, J=4.8 \mathrm{~Hz}, 2 \mathrm{H}, 2 \times 4-\mathrm{H}), 6.74(\mathrm{~d}, J=$ $4.8 \mathrm{~Hz}, 2 \mathrm{H}, 2 \times 3-\mathrm{H}) ;{ }^{13} \mathrm{C} \mathrm{NMR}\left(62.9 \mathrm{MHz}, \mathrm{CDCl}_{3}\right): \delta=$ $27.5\left[+, \mathrm{C}\left(\mathrm{CH}_{3}\right)_{3}\right], 58.9\left(+, \mathrm{CH}_{3}\right), 84.5\left[\mathrm{C}_{\text {quat }}, \mathrm{C}\left(\mathrm{CH}_{3}\right)_{3}\right]$, $104.8\left(-, 2 \times \mathrm{CH}_{2}\right), 104.8(+, 2 \times \mathrm{C}-4), 115.2(+, 2 \times \mathrm{C}-3), 122.5\left(\mathrm{C}_{\text {quat }}, 2 \times \mathrm{C}-2\right), 123.6\left(\mathrm{C}_{\text {quat }}, 2 \times\right.$ C-5), $151.1\left(\mathrm{C}_{\text {quat }}, \mathrm{NCO}_{2}\right), 158.9\left(\mathrm{C}_{\text {quat }}, 2 \times \mathrm{CON}\right)$; IR (film): nu(tilde $)=3131 \mathrm{~cm}^{-1}, 2979,2940$, 2946, 2840, 1751, 1700, 1429, 1395, 1370, 1256, 1149, 1087; MS (CI), m/z (\%):511/509 (33/52) $\left[\mathrm{M}+\mathrm{NH}_{4}^{+}\right], 494 / 492(9 / 14)\left[\mathrm{M}+\mathrm{H}^{+}\right]$. 
5-Chloro-1-methoxymethoxypyrrole-2-carboxylic acid (109): To a solution of the doubly $N$-Boc-

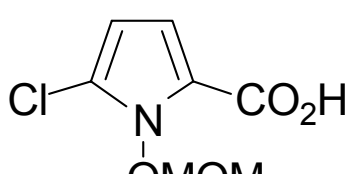

OMOM acylated derivative $107(0.630 \mathrm{~g}, 1.55 \mathrm{mmol})$ in dioxane $(8 \mathrm{~mL})$ was added $1 \mathrm{~N} \mathrm{NaOH}(2.02 \mathrm{~mL})$ and the mixture was stirred at $55^{\circ} \mathrm{C}$ for 2 h. Then, all volatiles were removed under reduced pressure, the residue was taken up with $\mathrm{H}_{2} \mathrm{O}(20 \mathrm{~mL})$ and washed with $\mathrm{CH}_{2} \mathrm{Cl}_{2}(5 \times 5 \mathrm{~mL})$. The $\mathrm{pH}$ of the water layer was adjusted with $1 \mathrm{~N} \mathrm{NaHSO}_{4}$ to 3 , and the product was extracted with $\mathrm{CH}_{2} \mathrm{Cl}_{2}(4 \times 10 \mathrm{~mL})$ under salting-out conditions. The organic phase was dried and concentrated to give after recrystallization from $\mathrm{CH}_{2} \mathrm{Cl}_{2} /$ hexanes $109(193 \mathrm{mg}, 60 \%)$ as a colorless solid. $R_{\mathrm{f}}=0.36$, EtOAc/hexanes = 1:2.5 (5\% AcOH); m.p. $120-122{ }^{\circ} \mathrm{C}($ decomp. $) ;{ }^{1} \mathrm{H}$ NMR $\left(250 \mathrm{MHz}, \mathrm{CDCl}_{3}\right)$ : $\delta=3.71\left(\mathrm{~s}, 3 \mathrm{H}, \mathrm{CH}_{3}\right), 5.25\left(\mathrm{~s}, 2 \mathrm{H}, \mathrm{CH}_{2}\right), 6.06(\mathrm{~d}, J=4.9 \mathrm{~Hz}, 1 \mathrm{H}, 4-\mathrm{H}), 6.95(\mathrm{~d}, J=4.9 \mathrm{~Hz}$, $1 \mathrm{H}, 3-\mathrm{H}) ;{ }^{13} \mathrm{C} \mathrm{NMR}\left(62.9 \mathrm{MHz}, \mathrm{CDCl}_{3}\right): \delta=58.9\left(+, \mathrm{CH}_{3}\right), 104.6\left(-, \mathrm{CH}_{2}\right), 104.7(+, \mathrm{C}-4)$, $116.0(+, \mathrm{C}-3), 117.7\left(\mathrm{C}_{\text {quat }}, \mathrm{C}-2\right), 122.8\left(\mathrm{C}_{\text {quat }}, \mathrm{C}-5\right), 163.6\left(\mathrm{C}_{\text {quat }}, \mathrm{CO}\right)$; IR $(\mathrm{KBr}): \mathrm{nu}($ tilde $)=$ $3451 \mathrm{~cm}^{-1}, 3135,3006,2964,2944,2618,2562,1671,1543,1533,1447,1437,1323,1263$, 1159, 1119; MS (EI, $70 \mathrm{eV}): m / z(\%)=207 / 205(13 / 43)\left[\mathrm{M}^{+}\right], 177 / 175(2 / 5)\left[\mathrm{M}^{+}-\mathrm{CH}_{2} \mathrm{O}\right]$, 145/143 (2/7) $\left[\mathrm{M}^{+}-\mathrm{C}_{2} \mathrm{H}_{6} \mathrm{O}_{2}\right], 129(7), 91 / 89(2 / 6), 75 / 73$ (1/3), 45 (100) $\left[\mathrm{C}_{2} \mathrm{H}_{5} \mathrm{O}^{+}\right]$; HRMS (EI): calcd for $\mathrm{C}_{7} \mathrm{H}_{8} \mathrm{ClN}_{4} \mathrm{O}$ : 205.0142; found 205.0142; elemental analysis calcd (\%) for $\mathrm{C}_{7} \mathrm{H}_{8} \mathrm{ClNO}_{4}$ (205.6): C 40.89, H 3.92, N 6.81; found C 40.93, H 3.94, N 6.68.

Chpca(MOM)-Phe-OFm (112): Boc-Phe-OFm 110 (0.260 g, $0.59 \mathrm{mmol})$ was deprotected with 5 $\mathrm{M} \mathrm{HCl}$ in $\mathrm{Et}_{2} \mathrm{O}(25 \mathrm{~mL})$ for $1.5 \mathrm{~h}$. All volatiles were then removed and the resultant white solid residue was triturated with $\mathrm{Et}_{2} \mathrm{O}$ and filtered off to give $\mathrm{HCl} \cdot \mathrm{H}-\mathrm{Phe}-\mathrm{OFm} 111$ which was directly coupled with acid 109 (0.100 g, $0.48 \mathrm{mmol})$ using EDC (0.100 g, $0.52 \mathrm{mmol})$, HOBt (80 mg, hydrate with $\left.13 \% \mathrm{H}_{2} \mathrm{O}, 0.515 \mathrm{mmol}\right)$ and DIEA $(0.138 \mathrm{~g}, 1.07 \mathrm{mmol})$ in $\mathrm{CH}_{2} \mathrm{Cl}_{2}(7 \mathrm{~mL})$ according to GP 5 for $4 \mathrm{~h}$. After usual aqueous work-up the crude product was purified by column chromatography (EtOAc/hexanes $\left.1: 3, R_{\mathrm{f}}=0.30\right)$ to give $112(0.24 \mathrm{~g}, 93 \%)$ as a turbid glass. ${ }^{1} \mathrm{H}$ NMR $\left(250 \mathrm{MHz}, \mathrm{CDCl}_{3}\right): \delta=3.12(\mathrm{ddt}, J=5.8,14.0,14.0 \mathrm{~Hz}, 2 \mathrm{H}, 3-\mathrm{H}), 3.49(\mathrm{~s}, 3 \mathrm{H}$, 
$\mathrm{OMe}), 4.17\left(\mathrm{t}, J=6.6 \mathrm{~Hz}, 1 \mathrm{H}, 1^{\prime}-\mathrm{H}, F m\right), 4.38-4.59$ (m, $\left.2 \mathrm{H}, 2^{\prime}-\mathrm{H}, F m\right), 5.00\left(\mathrm{~s}, 2 \mathrm{H}, \mathrm{OCH}_{2} \mathrm{O}\right)$, $5.03(\mathrm{dt}, J=5.8,5.8 \mathrm{~Hz}, 1 \mathrm{H}, 2-\mathrm{H}), 5.99$ (d, $J=4.8 \mathrm{~Hz}, 1 \mathrm{H}, 4-\mathrm{H}$, Chpca), 6.63 (d, $J=4.8 \mathrm{~Hz}$, $1 \mathrm{H}, 3-\mathrm{H}$, Chpca), 6.84 (d, J=7.5 Hz, $1 \mathrm{H}, \mathrm{NH}), 7.03-7.11(\mathrm{~m}, 2 \mathrm{H}, \mathrm{Ar}-\mathrm{H}), 7.18-7.33$ (m, $5 \mathrm{H}$, Ar-H), 7.33-7.46 (m, 2 H, Ar-H), 7.52 (d, $J=7.5$ Hz, 2 H, Ar-H), 7.74 (d, $J=7.5$ Hz, 2 H, Ar$\mathrm{H}) ;{ }^{13} \mathrm{C}$ NMR $\left(62.9 \mathrm{MHz}, \mathrm{CDCl}_{3}\right): \delta=37.6(-, \mathrm{C}-3), 46.6\left(+, \mathrm{C}-1^{\prime}, F m\right), 53.0(+, \mathrm{C}-2), 59.1(+$, OMe), $67.0\left(-, \mathrm{C}_{2}{ }^{\prime}, \mathrm{Fm}\right), 104.0(+, \mathrm{C}-4, \mathrm{Chpca}), 104.7$ (-, $\left.\mathrm{OCH}_{2} \mathrm{O}\right), 111.0(+, \mathrm{C}-3$, Chpca), $118.5\left(\mathrm{C}_{\text {quat, }}\right.$ C-2, Chpca), $119.9(+, \mathrm{Ar}-\mathrm{C}), 121.9\left(\mathrm{C}_{\text {quat }}, \mathrm{C}-5\right.$, Chpca $), 124.8(\times 2), 127.1,127.8$, 128.6, $129.2(+, A r-C), 135.7\left(\mathrm{C}_{\text {quat }}, \mathrm{Ar}-\mathrm{C}\right), 141.2\left(\mathrm{C}_{\text {quat }}, \mathrm{Ar}-\mathrm{C}\right), 143.2,143.3\left(\mathrm{C}_{\text {quat }}, \mathrm{Ar}-\mathrm{C}\right), 158.0$ $\left(\mathrm{C}_{\text {quat }}, \mathrm{C}-1\right.$, Chpca $), 171.4\left(\mathrm{C}_{\text {quat }}, \mathrm{C}-1\right)$; MS (EI, $\left.70 \mathrm{eV}\right), \mathrm{m} / z(\%)=532 / 530(1 / 2)\left[\mathrm{M}^{+}\right], 354 / 352$ $(2 / 7)\left[\mathrm{M}^{+}-\mathrm{C}_{14} \mathrm{H}_{10}\right], 178(100)\left[\mathrm{C}_{14} \mathrm{H}_{10}^{+}\right], 128(8), 91(8)\left[\mathrm{C}_{7} \mathrm{H}_{7}^{+}\right], 45(12)\left[\mathrm{C}_{2} \mathrm{H}_{5} \mathrm{O}^{+}\right]$; HRMS (EI): calcd for $\mathrm{C}_{30} \mathrm{H}_{27} \mathrm{ClN}_{2} \mathrm{O}_{5}$ : 530.1608; found 530.1608.

Chpca-Phe-OFm (113): Compound 112 (0.235 g, $0.44 \mathrm{mmol})$ was deprotected using $\mathrm{MgBr}_{2} \cdot \mathrm{Et}_{2} \mathrm{O}(2.50 \mathrm{~g}, 96.81 \mathrm{mmol})$ and $\mathrm{EtSH}(0.12 \mathrm{~mL}, 1.62 \mathrm{mmol})$ in $^{\mathrm{Et}} \mathrm{O}_{2} \mathrm{O}(20 \mathrm{~mL})$ according to GP 10 for $4 \mathrm{~h} .1 \mathrm{M} \mathrm{NaHSO}_{4}(10 \mathrm{~mL})$ and $\mathrm{Et}_{2} \mathrm{O}(40 \mathrm{~mL})$ were then added, the organic layer was separated washed with $1 \mathrm{M} \mathrm{NaHSO}_{4}(3 \times 10 \mathrm{~mL})$, water $(5 \times 10 \mathrm{~mL})$, brine $(2 \times 10 \mathrm{~mL})$, dried, filtered and concentrated under reduced pressure. The residue was purified by column chromatography (twice, EtOAc/hexanes 1:3, $\left.R_{\mathrm{f}}=0.35\right)$ to give $113(0.19 \mathrm{~g}, 88 \%)$ as a white foam. ${ }^{1} \mathrm{H}$ NMR $\left(250 \mathrm{MHz}, \mathrm{CDCl}_{3}\right): \delta=3.11(\mathrm{dd}, J=6.3,6.3 \mathrm{~Hz}, 2 \mathrm{H}, 3-\mathrm{H}), 4.18(\mathrm{t}, J=6.3 \mathrm{~Hz}$, $\left.1 \mathrm{H}, 1^{\prime}-\mathrm{H}, F m\right), 4.47-4.59\left(\mathrm{~m}, 2 \mathrm{H}, 2^{\prime}-\mathrm{H}, F m\right), 5.00\left(\mathrm{~s}, 2 \mathrm{H}, \mathrm{OCH}_{2} \mathrm{O}\right), 5.03(\mathrm{dt}, J=7.5,6.3 \mathrm{~Hz}$, $1 \mathrm{H}, 2-\mathrm{H}), 5.93(\mathrm{~d}, J=5.0 \mathrm{~Hz}, 1 \mathrm{H}, 4-\mathrm{H}, C h p c a), 6.19$ (d, $J=7.5 \mathrm{~Hz}, 1 \mathrm{H}, \mathrm{NH}), 6.35$ (d, $J=$ $5.0 \mathrm{~Hz}, 1 \mathrm{H}, 3-\mathrm{H}$, Chpca), 7.00-7.09 (m, $2 \mathrm{H}, \mathrm{Ar}-\mathrm{H}), 7.21-7.29$ (m, $5 \mathrm{H}, \mathrm{Ar}-\mathrm{H})$, 7.36-7.47 (m, $2 \mathrm{H}, \mathrm{Ar}-\mathrm{H}), 7.52(\mathrm{dd}, J=7.5,3.0 \mathrm{~Hz}, 2 \mathrm{H}, \mathrm{Ar}-\mathrm{H}), 7.76(\mathrm{dd}, J=7.5,4.3 \mathrm{~Hz}, 2 \mathrm{H}, \mathrm{Ar}-\mathrm{H}), 13.2-$ $13.5(\mathrm{br}, 1 \mathrm{H}, \mathrm{OH}) ;{ }^{13} \mathrm{C} \mathrm{NMR}\left(62.9 \mathrm{MHz}, \mathrm{CDCl}_{3}\right): \delta=37.5(-, \mathrm{C}-3), 46.4\left(+, \mathrm{C}-1^{\prime}, F m\right), 52.8(+$, C-2), 67.1 (-, C-2', Fm), 102.5 (+, C-4, Chpca), 106.0 (+, C-3, Chpca), 114.8 (C quat, C-2, Chpca), 115.6 ( $\mathrm{C}_{\text {quat }}, \mathrm{C}-5$, Chpca), 119.9 (+, Ar-C), 124.6, 127.0, 127.2, 127.7, 127.8, 128.5, 
$128.9(+, \mathrm{Ar}-\mathrm{C}), 135.1\left(\mathrm{C}_{\text {quat }}, \mathrm{Ar}-\mathrm{C}\right), 141.1\left(\mathrm{C}_{\text {quat }}, \mathrm{Ar}-\mathrm{C}\right), 142.9,143.0\left(\mathrm{C}_{\text {quat }}, \mathrm{Ar}-\mathrm{C}\right), 161.9\left(\mathrm{C}_{\text {quat }}\right.$, C-1, Chpca), 171.3 (C quat $_{\text {, }}$-1); MS (EI, $\left.70 \mathrm{eV}\right), m / z(\%)=488 / 486(6 / 18)\left[\mathrm{M}^{+}\right], 310 / 308(2 / 8)$ $\left[\mathrm{M}^{+}-\mathrm{C}_{14} \mathrm{H}_{10}\right], 178(100)\left[\mathrm{C}_{14} \mathrm{H}_{10}{ }^{+}\right], 146 / 144(5 / 16)\left[\mathrm{C}_{5} \mathrm{H}_{3} \mathrm{ClNO}_{2}^{+}\right], 91(8)\left[\mathrm{C}_{7} \mathrm{H}_{7}^{+}\right]$; HRMS (EI): calcd for $\mathrm{C}_{28} \mathrm{H}_{23} \mathrm{ClN}_{2} \mathrm{O}_{4}$ : 486.1346; found 486.1346.

Chpca-Phe-OH (114): Compound 113 (70 mg, $0.013 \mathrm{mmol}$ ) was deprotected using 20\% $\mathrm{Et}_{2} \mathrm{NH}$ in $\mathrm{CH}_{2} \mathrm{Cl}_{2}(2 \mathrm{~mL})$ for $40 \mathrm{~min}$. All volatiles were removed, the residue was taken up with EtOAc $(40 \mathrm{~mL})$ and washed with $1 \mathrm{M} \mathrm{NaHSO}_{4}(3 \times 10 \mathrm{~mL})$, water $(3 \times 10 \mathrm{~mL})$, brine $(2 \times 10 \mathrm{~mL})$, dried, filtered and concentrated under reduced pressure to give after drying at 0.02 Torr crude 114 (44 $\mathrm{mg}, 100 \%)$ as a rose oil which was immediately used for the next step.

Chpca-Phe-Phe-Val-OMe (119): Boc-Phe-Val-OMe 117 (60 mg, $0.16 \mathrm{mmol})$ was deprotected with $5 \mathrm{M} \mathrm{HCl}$ in $\mathrm{Et}_{2} \mathrm{O}(5 \mathrm{~mL})$ for $2 \mathrm{~h}$. The mixture was concentrated under reduced pressure and the residual hydrochloride of $\mathrm{H}-\mathrm{Phe}-\mathrm{Val}-\mathrm{OMe} \mathrm{HCl} \cdot 118$ was directly coupled with $\mathrm{N}$-acyl amino acid 114 (44 mg, $0.13 \mathrm{mmol})$ using HATU (97 mg, $0.25 \mathrm{mmol})$ and DIEA $(0.070 \mathrm{~mL}, 0.41$ mmol) in $\mathrm{CH}_{2} \mathrm{Cl}_{2}(4 \mathrm{~mL})$ according to $\mathrm{GP} 7$ for $1.5 \mathrm{~h}$. The mixture was then taken up with $\mathrm{Et}_{2} \mathrm{O}$ $(40 \mathrm{~mL})$ and the crude product obtained after usual aqueous work-up (GP 5) was purified by column chromatography (EtOAc/hexanes $\left.1: 1, R_{\mathrm{f}}=0.27\right)$ and finally recrystallized from EtOAc/hexanes $1: 1$ to give $119(17 \mathrm{mg}, 23 \%)$ as an off-white solid. $[\alpha]_{20}^{D}-32.0(c=1.10$, $\left.\mathrm{CHCl}_{3}\right) ;{ }^{1} \mathrm{H} \mathrm{NMR}\left(300 \mathrm{MHz}, \mathrm{CD}_{3} \mathrm{OD}\right): \delta=0.92(\mathrm{~d}, J=7.0 \mathrm{~Hz}, 6 \mathrm{H}, 4-\mathrm{H}, \mathrm{Val}), 2.08$ (octet, $J=$ 7.0 Hz, $1 \mathrm{H}, 3-\mathrm{H}, V a l), 2.93(\mathrm{dt}, J=13.7,8.0 \mathrm{~Hz}, 2 \mathrm{H}, 3-\mathrm{H}, P h e), 3.11(\mathrm{dt}, J=13.7,6.5 \mathrm{~Hz}, 2 \mathrm{H}$, 3-H, Phe), 3.67 (s, 3 H, OMe), 4.25-4.33 (m, $1 \mathrm{H}, 2-\mathrm{H}, V a l), 4.70$ (dd, J =6.2, 8.0 Hz, 1 H, 2-H, Phe), 4.77 (dd, $J=5.9,8.0 \mathrm{~Hz}, 1 \mathrm{H}, 2-\mathrm{H}, P h e), 5.98$ (d, J = 4.8 Hz, $1 \mathrm{H}, 4-\mathrm{H}$, Chpca), 6.63 (d, $J$ $=4.8 \mathrm{~Hz}, 1 \mathrm{H}, 3-\mathrm{H}$, Chpca), 7.09-7.28 (m, $10 \mathrm{H}, \mathrm{Ar}-\mathrm{H}), 8.25(\mathrm{~d}, J=8.5 \mathrm{~Hz}, 1 \mathrm{H}, \mathrm{NH}) ;{ }^{13} \mathrm{C} \mathrm{NMR}$ (75.5 MHz, CD $\left.{ }_{3} \mathrm{OD}\right): \delta=18.7,19.5(+, \mathrm{C}-4, \mathrm{Val}), 31.9(+, \mathrm{C}-3, \mathrm{Val}), 38.9(-, \mathrm{C}-3$, Phe $), 39.0(-$, C-3, Phe), 52.5 (+, C-2), 55.5 (+, C-2), 55.8 (+, C-2), 59.4 (+, OMe), $103.9(+, \mathrm{C}-4$, Chpca), 


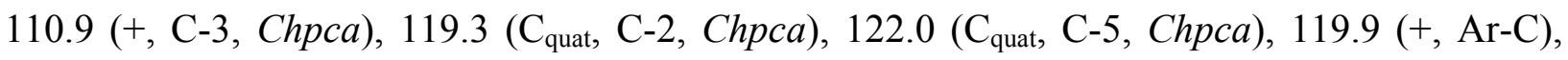
127.7, 127.9, 129.4, 129.5, 130.4, $130.4(+, \mathrm{Ar}-\mathrm{C}), 137.8,138.0\left(\mathrm{C}_{\text {quat }}, \mathrm{Ar}-\mathrm{C}\right), 161.2\left(\mathrm{C}_{\text {quat }}, \mathrm{C}-1\right.$, Chpca), 173.1, 173.1, 173.3 ( $\left.\mathrm{C}_{\text {quat }}, \mathrm{C}-1\right)$; IR (KBr): nu(tilde) $=3063 \mathrm{~cm}^{-1}, 3029,2964,2933$, 1746, 1644, 1630, 1551, 1412, 1259, 1144; MS (EI, $70 \mathrm{eV}), m / z(\%)=570 / 568(9 / 32)\left[\mathrm{M}^{+}\right]$, $439 / 437$ (9/31) $\left[\mathrm{M}^{+}-\mathrm{C}_{6} \mathrm{H}_{13} \mathrm{NO}_{2}\right], 290$ (32), 279 (40), 262 (12), 146/144 (100) $\left[\mathrm{C}_{5} \mathrm{H}_{3} \mathrm{ClNO}_{2}^{+}\right], 91$ (12) $\left[\mathrm{C}_{7} \mathrm{H}_{7}^{+}\right]$; HRMS (EI): calcd for $\mathrm{C}_{29} \mathrm{H}_{33} \mathrm{ClN}_{4} \mathrm{O}_{6}$ : 568.2089; found 568.2089.

2-Acetamido-6-fluoropyridine (123): 2-Acetamido-6-aminopyridine 122 (2.6 g, $17.20 \mathrm{mmol}$ )

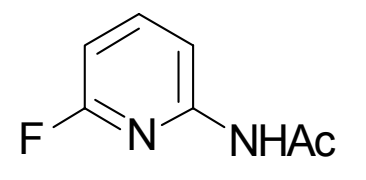
was dissolved in piridinium fluoride $(11 \mathrm{~mL})$, cooled to $-10^{\circ} \mathrm{C}$ (internal temperature) and then $\mathrm{NaNO}_{2}(1.305 \mathrm{~g}, 18.92 \mathrm{mmol})$ was added at the same temperature in small portions and stirring continued for an additional $10 \mathrm{~min}$. The mixture was allowed to warm to $20^{\circ} \mathrm{C}$, poured into ice-cold water (50 $\mathrm{mL})$ and the water fraction was extracted with $\mathrm{CH}_{2} \mathrm{Cl}_{2}(10 \times 20 \mathrm{~mL})$. The combined organic layers were stirred with $\mathrm{K}_{2} \mathrm{CO}_{3}(4 \mathrm{~g})$ and water $(1 \mathrm{~mL})$ for $1 \mathrm{~h}$, magnesium sulfate $(10 \mathrm{~g})$ was then added and stirring continued for an additional $20 \mathrm{~min}$. Salts were filtered off, volatiles were removed under reduced pressure and the residue was recrystallized from $\mathrm{CH}_{2} \mathrm{Cl}_{2} /$ hexanes to give $123(2.09 \mathrm{~g}, 79 \%)$ as an off-white solid. ${ }^{1} \mathrm{H} \mathrm{NMR}\left(250 \mathrm{MHz}, \mathrm{CDCl}_{3}\right): \delta=2.21\left(\mathrm{~s}, 3 \mathrm{H}, \mathrm{CH}_{3}\right)$, $6.65(\mathrm{dd}, J=2.5,8 \mathrm{~Hz}, 1 \mathrm{H}, 5-\mathrm{H}), 7.79$ (ddd, $J=8.0,8.0,8.0 \mathrm{~Hz}, 1 \mathrm{H}, 4-\mathrm{H}), 7.95-8.10$ (br, NH), 8.00-8.07 (m, $1 \mathrm{H}, 3-\mathrm{H})$.

2-Acetamido-6-fluoropyridine- $N$-oxide (124): A solution of 123 (8.89 g, $57.67 \mathrm{mmol})$ and m-<smiles>CC(Cl)(Cl)Nc1cccc(F)[n+]1[O-]</smileschloroperbenzoic acid (33.26 g, 90\% purity, $173.53 \mathrm{mmol})$ in $\mathrm{CH}_{2} \mathrm{Cl}_{2}$ (70 mL) was stirred at $20{ }^{\circ} \mathrm{C}$ for 9 days. The mixture was then stirred with $\mathrm{K}_{2} \mathrm{CO}_{3}(10 \mathrm{~g})$ for $1 \mathrm{~h}$, filtered, intensely shaked with saturated aqueous $\mathrm{K}_{2} \mathrm{CO}_{3}(60 \mathrm{~mL})$ for $15 \mathrm{~min}$, filtered through a pad of magnesium sulfate $(5 \mathrm{~cm})$ and concentrated under reduced pressure to ca. $20 \mathrm{~mL}$. Hexanes $(200 \mathrm{~mL})$ were then added and the 
precipitate formed was filtered off to give $124(3.78 \mathrm{~g}, 38 \%)$ as a white solid. $R_{\mathrm{f}}=0.10$, EtOAc/hexanes $=1: 1 ;$ m.p. $155-157{ }^{\circ} \mathrm{C}$ (decomp.); ${ }^{1} \mathrm{H}$ NMR $\left(250 \mathrm{MHz}, \mathrm{CDCl}_{3}\right): \delta=2.33(\mathrm{~s}$, $\left.3 \mathrm{H}, \mathrm{CH}_{3}\right), 6.86-6.95(\mathrm{~m}, 1 \mathrm{H}, 5-\mathrm{H}), 7.24-7.38(\mathrm{~m}, 1 \mathrm{H}, 4-\mathrm{H}), 8.28(\mathrm{~d}, J=7.5 \mathrm{~Hz}, 1 \mathrm{H}, 3-\mathrm{H})$, 9.80-10.20 (br, $1 \mathrm{H}, \mathrm{NH}) ;{ }^{13} \mathrm{C}$ NMR $\left(62.9 \mathrm{MHz}, \mathrm{D}_{2} \mathrm{O}\right): \delta=24.4\left(+, \mathrm{CH}_{3}\right), 106.0(+, \mathrm{d}, J=$ $19.6 \mathrm{~Hz}, \mathrm{C}-5), 112.2(+, \mathrm{d}, J=4.3 \mathrm{~Hz}, \mathrm{C}-3), 134.1(+, \mathrm{d}, J=8.3 \mathrm{~Hz}, \mathrm{C}-4), 144.7$ (C quat $\mathrm{d}, J=$ $7.0 \mathrm{~Hz}, \mathrm{C}-2), 157.0\left(\mathrm{C}_{\text {quat }}, \mathrm{d}, J=259.9 \mathrm{~Hz}, \mathrm{C}-6\right), 173.3\left(\mathrm{C}_{\text {quat }}, \mathrm{CO}\right)$; IR (KBr): nu(tilde) $=3244$ $\mathrm{cm}^{-1}, 3139,3108,3059,2993,2904,1703,1583,1529,1502,1398,1286,1264,1215$; MS (EI, $70 \mathrm{eV}): m / z(\%)=170(34)\left[\mathrm{M}^{+}\right], 128(100)\left[\mathrm{M}^{+}-\mathrm{C}_{2} \mathrm{H}_{2} \mathrm{O}\right], 112(10), 101(8), 83(8), 70(8), 43$ (72) $\left[\mathrm{C}_{2} \mathrm{H}_{3} \mathrm{O}^{+}\right]$; HRMS (EI): calcd for $\mathrm{C}_{7} \mathrm{H}_{7} \mathrm{FN}_{2} \mathrm{O}_{2}$ : 170.0492; found 170.0492; elemental analysis calcd (\%) for $\mathrm{C}_{7} \mathrm{H}_{7} \mathrm{FN}_{2} \mathrm{O}_{2}$ (170.1): C 49.42, $\mathrm{H} 4.15, \mathrm{~N} 16.46$; found $\mathrm{C} 49.36, \mathrm{H} 4.28, \mathrm{~N}$ 16.27 .

\subsection{Final assembling of hormaomycin}

Preparation and HPLC behavior of (S)-FDVA-derivatives of the amino acids used for synthesis of hormaomycin: The mixture of $\mathrm{H}-(R)-(3-\mathrm{Ncp}) \mathrm{Ala}-\mathrm{OH}, \mathrm{H}-(S)-(3-\mathrm{Ncp}) \mathrm{Ala}-\mathrm{OH}, \mathrm{H}-(2 S, 3 R)-$ ( $\beta \mathrm{Me})$ Phe-OH, $(S)-\mathrm{Ile}, \mathrm{H}-(2 S, 4 R)-(\mathrm{PE})$ Pro-OH, H-(2R)- $a$-Thr (ca. $0.4 \mathrm{mg}$ each) was divided in two equal portions, one of each was directly transformed into mixture of the $(S)$-FDVA derivatives (GP 2) and the second one was dissolved in water $(0.2 \mathrm{~mL})$ and treated with $\mathrm{Et}_{3} \mathrm{~N}$ $(0.08 \mathrm{~mL})$ and $\mathrm{Ac}_{2} \mathrm{O}(0.08 \mathrm{~mL})$. The mixture was then heated at $60^{\circ} \mathrm{C}$ for $1 \mathrm{~h}$, concentrated under reduced pressure to give the residue which was finally hydrolyzed according to GP 1 within $12 \mathrm{~h}$ to give the mixture of pairs of corresponding epimers at $\mathrm{C}-2$ which was further also transformed into the mixture of the appropriate (S)-FDVA derivatives (GP 2). Also $[(S)$ FDVA $_{2}-(2 R)-a-D a b-O H$ was prepared. Detection: UV: $340 \mathrm{~nm}$; ESI-MS, positive, m/z: $454.7-$ 455.7 [(3-Ncp)Ala], 434.7-435.7 [(4-PE)Pro], 459.7-460.7 [( $\beta \mathrm{Me}) \mathrm{Phe}], 411.7-412.7$ (Ile), 399.7-400.7 (a-Thr), 678.7-679.7 (a-Dab); ESI-MS, negative, m/z: 452.6-453.6 [(3-Ncp)Ala], 
433.6-434.6 [(4-PE)Pro], 457.6-458.6 [(ßMe)Phe], 409.6-410.6 (Ile), 397.6-398.6 (a-Thr), 676.7-677.7 (a-Dab); retention times: (3-Ncpa)Ala: $\left(2 S, 1^{\prime} R, 2^{\prime} R\right)$-isomer $-t_{\mathrm{R}}=8.96 \mathrm{~min}$, $\left(2 S, 1^{\prime} R, 2^{\prime} R\right)$-isomer $-t_{\mathrm{R}}=11.44 \mathrm{~min}$; (4-PE)Pro: $(2 S, 4 R)$-isomer $-t_{\mathrm{R}}=13.08 \mathrm{~min}$; the concentration of this amino acid after hydrolysis (GP 1) and derivatization was usually lower then detection limit; $(\beta \mathrm{Me})$ Phe: $(2 S, 3 R)$-isomer $-t_{\mathrm{R}}=13.75 \mathrm{~min} ;(2 R, 3 R)$-isomer $-t_{\mathrm{R}}=18.53$ min; Ile: $(S)$-isomer $-t_{\mathrm{R}}=11.69 \mathrm{~min} ;(R)$ - $a$-isomer $-t_{\mathrm{R}}=17.25 \mathrm{~min}$; $a$-Thr: $(R)$-isomer $-t_{\mathrm{R}}=$ $5.56 \mathrm{~min}$; this amino acid did not epimerize under conditions applied; $a$-Dab: $(R)$-isomer -20.21 $\min$.

Deprotection of N-Fmoc-protected peptides (GP 4): The protected peptides (1 mmol) were taken up with acetonitrile or THF $(2 \mathrm{~mL})$, diethylamine $(2 \mathrm{~mL})$ was added, and the resulting mixture left at ambient temperature for $40 \mathrm{~min}$. All volatiles were evaporated under reduced pressure, the residue was taken up with toluene $(2 \times 5 \mathrm{~mL})$, which was evaporated under reduced pressure to remove the last traces of diethylamine. Obtained crude $N$-deprotected peptides were directly used in the next condensation step.

Peptide condensation step for the preparation of peptides using EDC/HOAt mediated coupling (GP 5): EDC (1.03 mmol) and HOAt $(1.05 \mathrm{mmol})$ were added to a cooled $\left(4^{\circ} \mathrm{C}\right)$ solution of the respective $N$-protected amino acid $(1 \mathrm{mmol})$ in anhydrous $\mathrm{CH}_{2} \mathrm{Cl}_{2}(3 \mathrm{~mL})$. After $20 \mathrm{~min}$, the solution of the appropriate crude $N$-deprotected peptide $(0.97 \mathrm{mmol})$ and TMP $(3 \mathrm{mmol})$ in anhydrous $\mathrm{CH}_{2} \mathrm{Cl}_{2}(1 \mathrm{~mL})$ was added at the same temperature. The temperature was allowed to reach $20{ }^{\circ} \mathrm{C}$ and stirring was continued for $15 \mathrm{~h}$. Then the reaction mixture was diluted with $\mathrm{Et}_{2} \mathrm{O}$ or EtOAc $(30 \mathrm{~mL})$ and washed with water $(2 \times 5 \mathrm{~mL}), 1 \mathrm{M} \mathrm{KHSO}_{4}(3 \times 5 \mathrm{~mL})$, water $(2 \times 5$ $\mathrm{mL}), 3 \%$ aqueous solution of $\mathrm{NaHCO}_{3}(3 \times 5 \mathrm{~mL})$, water $(3 \times 5 \mathrm{~mL})$, brine $(2 \times 5 \mathrm{~mL})$, dried and concentrated under reduced pressure. The residue was purified by column chromatography or recrystallization. 
Preparation of esters using EDC/4-dialkylaminopyridine mediated coupling (GP 6): EDC (1.1$2.2 \mathrm{mmol})$ was added to a cooled $\left(4^{\circ} \mathrm{C}\right)$ solution of the respective $\mathrm{N}$-protected amino acid $(1.03$ mmol), alcohol or $N, C$-protected threonine $(1 \mathrm{mmol})$ and DMAP or 4-pyrrolidinopyridine (0.1-1 mmol) in anhydrous $\mathrm{CH}_{2} \mathrm{Cl}_{2}(3 \mathrm{~mL})$. The temperature was allowed to reach $20{ }^{\circ} \mathrm{C}$ and stirring was continued for $15 \mathrm{~h}$. Then the reaction mixture was diluted with $\mathrm{Et}_{2} \mathrm{O}$ or EtOAc $(30 \mathrm{~mL})$ and washed with $1 \mathrm{M} \mathrm{KHSO}_{4}(3 \times 5 \mathrm{~mL})$, water $(2 \times 5 \mathrm{~mL}), 3 \%$ aqueous solution of $\mathrm{NaHCO}_{3}(3 \times 5$ $\mathrm{mL})$, water $(3 \times 5 \mathrm{~mL})$, brine $(2 \times 5 \mathrm{~mL})$, dried and concentrated under reduced pressure. The residue was purified by column chromatography.

Preparation of depsipeptides using HATU/HOAt mediated coupling (GP 7): Deprotected according to GP 4 tpeptide $(0.1 \mathrm{mmol})$ was dissolved anhydrous $\mathrm{MeCN}(3 \mathrm{~mL})$, depsidipeptide (0.11 mmol), HATU $(0.107 \mathrm{mmol})$ and HOAt $(0.11 \mathrm{mmol})$ were added and the reaction mixture was cooled to $4{ }^{\circ} \mathrm{C}$. DIEA $(0.11 \mathrm{mmol})$ and TMP $(0.3-0.5 \mathrm{mmol})$ were then added, the mixture was allowed to warm to $20^{\circ} \mathrm{C}$ and stirring continued for an additional $15 \mathrm{~h}$. The mixture was then taken up with $\mathrm{Et}_{2} \mathrm{O}$ or EtOAc $(40 \mathrm{~mL})$ and after usual aqueous work-up (GP 5) the organic layer was concentrated to leave crude depsihexapeptide, which was purified by recrystallization and/or column chromatography.

Preparation of cyclohexadepsipeptides (GP 8): Hexadepsipeptide (0.105 mmol) was deprotected and then dissolved with $\mathrm{CH}_{2} \mathrm{Cl}_{2}\left(1.0\right.$ 1). The solution was cooled to $4{ }^{\circ} \mathrm{C}$ (internal temperature), HATU $(0.105 \mathrm{mmol})$ and HOAt $(0.105 \mathrm{mmol})$ were added and then the solution of DIEA $(0.315$ mmol) in $\mathrm{CH}_{2} \mathrm{Cl}_{2}(50 \mathrm{~mL})$ was added over $30 \mathrm{~min}$. The cooling bath was removed and stirring continued for an additional $2 \mathrm{~h}$ at the ambient temperature. Then the reaction mixture was cooled again to $4{ }^{\circ} \mathrm{C}$ and the second portions of HATU $(0.105 \mathrm{mmol})$ and HOAt $(0.105 \mathrm{mmol})$ were added and then the solution of DIEA $(0.315 \mathrm{mmol})$ in $\mathrm{CH}_{2} \mathrm{Cl}_{2}(50 \mathrm{~mL})$ was added over $30 \mathrm{~min}$. 
The temperature was allowed to reach $20^{\circ} \mathrm{C}$ and stirring was continued for $15 \mathrm{~h}$. After this, the solvent was removed under reduced pressure, the residue was taken up with $\mathrm{Et}_{2} \mathrm{O}$, and after usual water work-up (see GP 5) and concentration under reduced pressure, was purified first by column chromatography and then by recrystallization $\left(\mathrm{Et}_{2} \mathrm{O} /\right.$ pentane) to give crude product, which was finally purified by preparative HPLC.

Preparation of OSu-esters from alcohols (GP 9): To a vigorously stirred suspension of dried $\mathrm{K}_{2} \mathrm{CO}_{3}(1.2 \mathrm{~g})$ in a solution of alcohol $(10 \mathrm{mmol})$ in anhydrous $\mathrm{CH}_{2} \mathrm{Cl}_{2}(15 \mathrm{~mL})$ a $20 \%$ phosgene solution in toluene $(7.5 \mathrm{~mL})$ was added at $-10^{\circ} \mathrm{C}$ for $15 \mathrm{~min}$ and stirring continued at the same temperature for $1 \mathrm{~h}$. The cooling bath was then removed and the mixture stirred for a further $1 \mathrm{~h}$. It was then concentrated under reduced pressure at $20^{\circ} \mathrm{C}$, taken up with $\mathrm{Et}_{2} \mathrm{O}(20 \mathrm{~mL})$, filtered through a pad of $\mathrm{MgSO}_{4}$ and concentrated under reduced pressure to give the crude chloroformate which was further used without any purification. To a vigorously stirred ice-cold solution of the crude chloroformate $(10 \mathrm{mmol})$ and $\mathrm{HOSu}(9.5 \mathrm{mmol})$ in $\mathrm{THF}(70 \mathrm{~mL}) \mathrm{Et}_{3} \mathrm{~N}$ (10.1 mmol) was added for $5 \mathrm{~min}$. The reaction mixture was then allowed to warm to $20^{\circ} \mathrm{C}$ and stirred for an additional $15 \mathrm{~h}$. The reaction mixture was filtered, concentrated under reduced pressure, taken up with $\mathrm{Et}_{2} \mathrm{O}$ or EtOAc, filtered again and concentrated. The residue was crystallized from EtOAc/hexanes to give appropriate OSu ester.

Cleavage of MOM-group using $\mathrm{MgBr}_{2} \cdot \mathrm{Et}_{2} \mathrm{O}$ and $\mathrm{EtSH}(\mathrm{GP} 10): \mathrm{MgBr}_{2} \cdot \mathrm{Et}_{2} \mathrm{O}$ (1 mmol) and EtSH $(0.5 \mathrm{mmol})$ were added to a vigorously stirred solution of the $O$-MOM protected derivative $(0.1$ mmol) in $\mathrm{CH}_{2} \mathrm{Cl}_{2}$ or $\mathrm{Et}_{2} \mathrm{O}(15 \mathrm{~mL})$ and stirring continued for a further $3.5 \mathrm{~h}$ (the TLC control was impossible as starting material and product in all cases showed exactly the same $R_{\mathrm{f}}$ in all chromatographic systems). The mixture was then taken up with EtOAc $(40 \mathrm{~mL})$ and washed with $1 \mathrm{M} \mathrm{NaHSO}_{4}(3 \times 10 \mathrm{~mL})$, water $(5 \times 10 \mathrm{~mL})$, brine $(2 \times 5 \mathrm{~mL})$, dried, filtered and concentrated 
under reduced pressure. The residue was purified by column chromatography or preparative TLC, or/and crystallization.

Deprotection of N-MeZ protected cyclohexadepsipeptides (GP 11): The NMe-Z protected cyclodepsipeptide $(10 \mu \mathrm{mol})$ was treated with $10 \%$ anisole in TFA $(1 \mathrm{~mL})$ in dark for $2 \mathrm{~h}$. All volatiles were then removed under reduced pressure at $20^{\circ} \mathrm{C}$. The solid residue was taken up with toluene $(2 \times 10 \mathrm{~mL})$ which was distilled off under reduced pressure and then washed with pentane $(2 \mathrm{~mL} \times 3)$ and hexanes $(2 \mathrm{~mL} \times 3)$. The resultant crude deprotected depsipeptide was directly used for the appropriate coupling reaction.

Boc-Thr-OMTM (132): $\mathrm{MTMCl}(0.918 \mathrm{~g}, 9.51 \mathrm{mmol})$ was added to a vigorously stirred $\mathrm{BOCHN}_{\mathrm{CO}_{2} \mathrm{MTM}}^{\mathrm{OH}}$ suspension of dry $\mathrm{NaI}(1.80 \mathrm{~g}, 12.01 \mathrm{mmol})$ in anhydrous $\mathrm{MeCN}(10$ $\mathrm{mL})$. After $15 \mathrm{~min}$, this mixture was added to a solution of Boc-Thr$\mathrm{OH} 131(2.79 \mathrm{~g}, 10.00 \mathrm{mmol})$ in $\mathrm{MeCN}(20 \mathrm{~mL})$ and the reaction mixture was heated at $80{ }^{\circ} \mathrm{C}$ for $3 \mathrm{~h}$. The mixture was then cooled and concentrated under reduced pressure. The residue was taken up with $\mathrm{Et}_{2} \mathrm{O}(50 \mathrm{~mL})$ and subjected usual aqueous work-up. The organic layer was then dried, filtered and concentrated under reduced pressure. The resultant crude product was purified by column chromatography $\left(\right.$ EtOAc/hexanes 1:3, $R_{\mathrm{f}}=$ $0.15)$ to give $132(2.05 \mathrm{~g}, 77 \%)$ as a colorless oil. ${ }^{1} \mathrm{H}$ NMR $\left(250 \mathrm{MHz}, \mathrm{CDCl}_{3}\right): \delta=1.27(\mathrm{~d}, J=$ $6.3 \mathrm{~Hz}, 3 \mathrm{H}, 4-\mathrm{H}), 1.45$ [s, $\left.9 \mathrm{H}, \mathrm{C}\left(\mathrm{CH}_{3}\right)_{3}\right], 2.04$ (d, $\left.J=5.0 \mathrm{~Hz}, 1 \mathrm{H}, \mathrm{OH}\right), 2.25$ (s, $\left.3 \mathrm{H}, \mathrm{SMe}\right)$, 4.20-4.42 (m, $2 \mathrm{H}, 2-\mathrm{H}, 3-\mathrm{H}), 5.17$ (d, $\left.J=11.8 \mathrm{~Hz}, 1 \mathrm{H}, \mathrm{H}_{\mathrm{a}}, \mathrm{OCH}_{2} \mathrm{SMe}\right), 5.21-5.39$ (br, $1 \mathrm{H}$, $\mathrm{NH}), 5.30\left(\mathrm{~d}, J=11.8 \mathrm{~Hz}, 1 \mathrm{H}, \mathrm{H}_{\mathrm{b}}, \mathrm{OCH}_{2} \mathrm{SMe}\right)$.

Boc-Thr-(Nps-Pro)-OMTM (134): Nps-Pro-OH 133 (0.763 g, $1.70 \mathrm{mmol}$ ) was coupled with alcohol 132 (0.439 g, $1.64 \mathrm{mmol})$ using EDC (0.326 g, $1.18 \mathrm{mmol})$ and DMAP (42 mg, 0.34 mmol) in $\mathrm{CH}_{2} \mathrm{Cl}_{2}(10 \mathrm{~mL})$ according to $\mathrm{GP} 6$ for $15 \mathrm{~h}$. The crude product obtained after usual 
aqueous work-up was finally purified by column chromatography (EtOAc/hexanes 1:4, $R_{\mathrm{f}}=$ $0.13)$ to give the ester $134(0.717 \mathrm{~g}, 85 \%)$ as a bright yellow oil. ${ }^{1} \mathrm{H}$ NMR $\left(250 \mathrm{MHz}, \mathrm{CDCl}_{3}\right): \delta$ =1.11-1.40 (m, 3 H, 4-H, Thr $), 1.46$ [s, 9 H, C( $\left.\left.\mathrm{CH}_{3}\right)_{3}\right], 1.85-2.51$ (m, 4 H, 3-H, 4-H, Pro ), 2.04 (s, 3 H, SMe), 3.01-3.26, 3.27-3.63 (2 × m, 2 H, 5-H, Pro), 3.97 (d, J=8.0 Hz, 1 H, 2-H, Thr), 4.22-4.35, 4.36-4.60 (2 × m, 1 H, 2-H, Pro ), 4.82-5.34 (m, 3 H, $\mathrm{OCH}_{2} \mathrm{SMe}, \mathrm{C}-3$, Thr $), 5.34-$ 5.62 (br, $1 \mathrm{H}, \mathrm{NH}), 7.26$ (dd, $J=8.3,8.3 \mathrm{~Hz}, 1 \mathrm{H}, 4-\mathrm{H}, N p s), 7.46-7.79$ (m, $2 \mathrm{H}, 5-\mathrm{H}, 6-\mathrm{H}, N p s)$, $8.28(\mathrm{~d}, J=8.3 \mathrm{~Hz}, 1 \mathrm{H}, 3-\mathrm{H}, \mathrm{Nps}) ;{ }^{13} \mathrm{C} \mathrm{NMR}\left(62.9 \mathrm{MHz}, \mathrm{CDCl}_{3}\right): \delta=15.0(+, \mathrm{SMe}), 16.2(+, \mathrm{C}-$ 4, Thr), 22.7, 24.2 (-, C-3, Pro), 27.8 [+, C( $\left.\left.\mathrm{CH}_{3}\right)_{3}\right], 31.1$ (-, C-4, Pro), 55.2, 58.9 (-,C-5, Pro), $56.6(+, \mathrm{C}-2, \mathrm{Thr}), 66.1,68.6(+, \mathrm{C}-2$, Pro $), 69.4\left(-, \mathrm{OCH}_{2} \mathrm{SCH}_{3}\right), 70.9(+, \mathrm{C}-3, \mathrm{Thr}), 79.8$ [C quat, $\left.\mathrm{C}\left(\mathrm{CH}_{3}\right)_{3}\right], 123.2(+, \mathrm{C}-3, \mathrm{Nps}), 124.4$ (+, C-6, Nps), 125.1, $125.4(+, \mathrm{C}-4, \mathrm{Nps}), 133.5$ (+, C-5, $N p s), 141.6\left(\mathrm{C}_{\text {quat }}, \mathrm{C}-1, N p s\right), 144.6\left(\mathrm{C}_{\text {quat }}, \mathrm{C}-2, N p s\right), 155.3\left(\mathrm{C}_{\text {quat }}, \mathrm{NCO}_{2}\right), 169.2,171.9\left(\mathrm{C}_{\text {quat }}, \mathrm{C}-\right.$ 1); MS (ESI), positive $m / z(\%)=552(75)\left[\mathrm{M}+\mathrm{Na}^{+}\right]$; negative $m / z(\%)=528(100)\left[\mathrm{M}-\mathrm{H}^{+}\right]$.

Boc-Thr(Nps-Ile-Pro)-MTM (136): The ester 134 (0.109 g, $0.21 \mathrm{mmol})$ was deprotected with 0.2 $\mathrm{M} \mathrm{HCl}$ in $\mathrm{Et}_{2} \mathrm{O}(2.1 \mathrm{~mL}, 0.42 \mathrm{mmol})$ and $\mathrm{EtSH}(0.075 \mathrm{~mL}, 1.0 \mathrm{mmol})$ for $1 \mathrm{~min}$. The mixture was then concentrated under reduced pressure and the solid residue was washed with boiled hexanes to give the crude deprotected amino ester which was immediately coupled with Nps- $N$ protected isoleucine 135 (59 mg, $0.21 \mathrm{mmol}$ ) using EDC (41 mg, $0.21 \mathrm{mmol}$ ), HOAt (29 mg, $0.21 \mathrm{mmol})$ and TMP $(0.125 \mathrm{~g}, 1.03 \mathrm{mmol})$ in $\mathrm{CH}_{2} \mathrm{Cl}_{2}(4 \mathrm{~mL})$ according to GP 5 for $16 \mathrm{~h}$. The crude product obtained after usual aqueous work-up (GP 5) was finally purified by column chromatography (EtOAc/hexanes 1:2.5, $\left.R_{\mathrm{f}}=0.23\right)$ to give $136(0.107 \mathrm{~g}, 80 \%)$ as a bright yellow oil. ${ }^{1} \mathrm{H}$ NMR $\left(250 \mathrm{MHz}, \mathrm{CDCl}_{3}\right): \delta=0.97(\mathrm{t}, J=7.0 \mathrm{~Hz}, 3 \mathrm{H}, 5-\mathrm{H}, I l e), 1.04(\mathrm{~d}, J=6.8 \mathrm{~Hz}, 3 \mathrm{H}$,

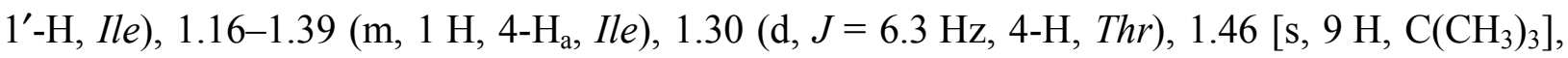
1.71-2.05 (m, 5 H, 4-H , Ile, 3-H, 4-H, Pro), 2.05-2.20 (m, 1 H, 3-H, Ile), 2.23 (s, 3 H, SMe), 3.27-3.43 (m, 2 H, 5- $\mathrm{H}_{\mathrm{a}}$, Pro, NH, Ile), 3.43-3.59 (m, 2 H, 2-H, Ile, 5- $\mathrm{H}_{\mathrm{b}}$, Pro), 4.44-4.56 (m, $2 \mathrm{H}, 2-\mathrm{H}), 5.21\left(\mathrm{~d}, 2 \mathrm{H}, \mathrm{OCH}_{2} \mathrm{SMe}\right), 5.28(\mathrm{~d}, J=9.5 \mathrm{~Hz}, 1 \mathrm{H}, \mathrm{NH}), 5.48(\mathrm{dq}, J=3.0,6.3 \mathrm{~Hz}, 3-$ 
H, Thr), 7.25 (dd, $J=8.0,8.0 \mathrm{~Hz}, 1 \mathrm{H}, 4-\mathrm{H}, N p s), 7.62(\mathrm{dd}, J=8.0 \mathrm{~Hz}, 1 \mathrm{H}, 6-\mathrm{H}, N p s), 8.08(\mathrm{~d}, J$ $=8.0 \mathrm{~Hz}, 1 \mathrm{H}, 5-\mathrm{H}, N p s), 8.25(\mathrm{~d}, J=8.0 \mathrm{~Hz}, 1 \mathrm{H}, 3-\mathrm{H}, N p s) ;{ }^{13} \mathrm{C} \mathrm{NMR}\left(62.9 \mathrm{MHz}, \mathrm{CDCl}_{3}\right): \delta=$ $11.1\left(+, \mathrm{C}-5\right.$, Ile), $15.2(+, \mathrm{SMe}), 15.5\left(+, \mathrm{C}-1^{\prime}\right.$, Ile $), 16.7(+, \mathrm{C}-4$, Thr $), 24.3(-, \mathrm{C}-4$, Pro $), 24.8$ (+, C-4, Ile), $28.1\left[+, \mathrm{C}\left(\mathrm{CH}_{3}\right)_{3}\right], 29.5$ (-, C-3, Pro), 38.6 (+, C-3, Ile), 47.1 (-,C-5, Pro), 57.0 (+, C-2, Thr), 58.9 (+,C-2, Pro), 68.4 (+, C-2, Ile), 69.9 (-, $\left.\mathrm{OCH}_{2} \mathrm{SCH}_{3}\right), 71.3(+, \mathrm{C}-3$, Thr $), 80.1$ $\left[\mathrm{C}_{\text {quat }}, \mathrm{C}\left(\mathrm{CH}_{3}\right)_{3}\right], 124.6(+, \mathrm{C}-3, \mathrm{Nps}), 124.7(+, \mathrm{C}-6, \mathrm{Nps}), 125.5(+, \mathrm{C}-4, \mathrm{Nps}), 133.4(+, \mathrm{C}-5$, $N p s), 142.3\left(\mathrm{C}_{\text {quat }}, \mathrm{C}-1, N p s\right), 145.6\left(\mathrm{C}_{\text {quat }}, \mathrm{C}-2, N p s\right), 155.7\left(\mathrm{C}_{\text {quat }}, \mathrm{NCO}_{2}\right), 169.6,170.7,172.4$ $\left(\mathrm{C}_{\text {quat }}, \mathrm{C}-1\right)$; MS (EI, $\left.70 \mathrm{eV}\right), m / z(\%)=642(5)\left[\mathrm{M}^{+}\right], 586(6)\left[\mathrm{M}^{+}-\mathrm{C}_{4} \mathrm{H}_{8}\right], 521$ (8), 509 (17), 437 (17), 239 (100), 154 (75) $\left[\mathrm{C}_{6} \mathrm{H}_{4} \mathrm{NO}_{2} \mathrm{~S}^{+}\right], 138(36)\left[\mathrm{C}_{6} \mathrm{H}_{4} \mathrm{NOS}^{+}\right], 86(52), 61(38)\left[\mathrm{C}_{2} \mathrm{H}_{5} \mathrm{~S}^{+}\right], 57$ (22) $\left[\mathrm{C}_{4} \mathrm{H}_{9}{ }^{+}\right]$; HRMS (EI): calcd for $\mathrm{C}_{28} \mathrm{H}_{42} \mathrm{~N}_{4} \mathrm{O}_{9} \mathrm{~S}_{2}$ : 642.2393; found 642.2393.

Attempt of the selective cleavage of MTM-group from 134: $\mathrm{MgBr}_{2} \cdot \mathrm{Et}_{2} \mathrm{O}(0.45 \mathrm{~g}, 1.74 \mathrm{mmol})$ was added to a solution of ester $134(0.119 \mathrm{~g}, 0.23 \mathrm{mmol})$ in $\mathrm{CH}_{2} \mathrm{Cl}_{2}(15 \mathrm{~mL})$ and stirring continued for 45 min. TLC analysis of reaction mixture showed full consumption of the starting material and unexpectedly polar, ninhydrin active main product. After usual aqueous work-up (GP 5, excluding washings with an aqueous solution of $\mathrm{NaHCO}_{3}$ ) only several $\mathrm{mg}$ of the mixture containing desired acid (according to ${ }^{1} \mathrm{H}$ NMR) was obtained. An additional experiment with Nps-Ile-OH showed that in this case Nps group completely cleaved within 20 min.

N-Boc-3-(2S,3R)Methylphenylalanine (139): A solution of $\mathrm{Boc}_{2} \mathrm{O}(0.607 \mathrm{~g}, 2.78 \mathrm{mmol})$ in

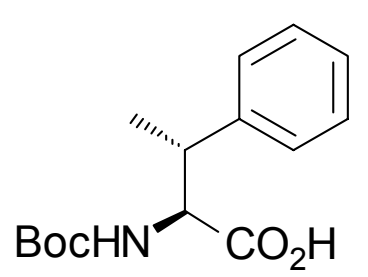
acetone $(5 \mathrm{~mL})$ was added to a solution 3-(2S,3R)-methylphenylalanine hydrochloride $(2 S, 3 R)-78(0.5 \mathrm{~g}, 2.32 \mathrm{mmol})$ in $1 \mathrm{M} \mathrm{NaOH}(4.7 \mathrm{~mL})$ and the resulting mixture was stirred for $15 \mathrm{~h}$. The mixture was then concentrated to ca. $5 \mathrm{~mL}$ under reduced pressure, diluted with water $(20 \mathrm{~mL})$ and extracted with $\mathrm{Et}_{2} \mathrm{O}(3 \times 5 \mathrm{~mL}) \cdot \mathrm{pH}$ of the aqueous layer was adjusted to 2 with solid $\mathrm{NaHSO}_{4}$ and the emulsion obtained was extracted with $\mathrm{Et}_{2} \mathrm{O}(40 \mathrm{~mL})$. The organic layer was washed with $1 \mathrm{M}$ aqueous $\mathrm{NaHSO}_{4}(3 \times 10 \mathrm{~mL})$, water $(3 \times 10 \mathrm{~mL})$, brine $(2 \times 5 \mathrm{~mL})$, dried, filtered and concentrated 
under reduced pressure to give crude product which was recrystallized from hexanes to give $\mathbf{2}$ $(0.53 \mathrm{~g}, 82 \%)$ as a white solid. $[\alpha]_{20}^{D} 18.6\left(c=1.73, \mathrm{CHCl}_{3}\right)\left[\right.$ lit.: $^{\left[{ }^{[76 b}\right]}[\alpha]_{20}^{D} 17.0(c=1.70$, $\left.\mathrm{CHCl}_{3}\right] ;{ }^{1} \mathrm{H} \mathrm{NMR}\left(250 \mathrm{MHz}, \mathrm{CDCl}_{3}\right): \delta=1.19\left(\mathrm{~s}, 3 \mathrm{H}, \mathrm{CH}_{3}\right), 1.39\left(\mathrm{~s}, 9 \mathrm{H}, \mathrm{C}\left(\mathrm{CH}_{3}\right)_{3}\right), 3.33$ (dddd, $J=6.3 \mathrm{~Hz}, 1 \mathrm{H}, 3-\mathrm{H}), 4.36-4.50(\mathrm{~m}, 0.3 \mathrm{H}, 2-\mathrm{H}), 4.45$ (dd, $J=5.0,9.8 \mathrm{~Hz}, 1 \mathrm{H}, 2-\mathrm{H}), 5.04$ (d, $J$ $=8.3 \mathrm{~Hz}, 0.6 \mathrm{H}, \mathrm{NH}), 6.42(\mathrm{~d}, J=8.3 \mathrm{~Hz}, 0.3 \mathrm{H}, \mathrm{NH}), 7.18-7.39(\mathrm{~m}, 5 \mathrm{H}, \mathrm{Ph}-\mathrm{H}), 11.10-12.00$ (br, $\left.1 \mathrm{H}, \mathrm{CO}_{2} \mathrm{H}\right)$.

Boc-( $\beta$ Me)Phe-Ile-Pro-OMe (140): Boc-Ile-Pro-OMe 138 (0.155 g, $0.45 \mathrm{mmol})$ was deprotected with $2 \mathrm{M} \mathrm{HCl}$ in $\mathrm{MeOH}(5 \mathrm{~mL})$ for $2 \mathrm{~h}$. The mixture was then concentrated under reduced pressure and the residue was triturated with $\mathrm{Et}_{2} \mathrm{O} /$ hexanes 1:1. The resultant crude deprotection product was directly coupled with $N$-Boc protected $\beta$-methylphenylalanine $139(0.135 \mathrm{~g}, 0.48$ mmol) using EDC (89 mg, $0.46 \mathrm{mmol})$, HOAt (63 mg, $0.47 \mathrm{mmol})$ and TMP (0.442 g, 3.65 mmol) in $\mathrm{CH}_{2} \mathrm{Cl}_{2}(5 \mathrm{~mL})$ according to $\mathrm{GP} 5$ for $7 \mathrm{~h}$. The solid residue obtained after usual aqueous work-up (GP 5) was taken up with boiling tert-BuOMe (20 mL), the resultant suspension was cooled and hexanes were then added to complete precipitation. The precipitate was filtered off and dried to give $140(0.190 \mathrm{~g}, 83 \%)$ as a colorless solid. $R_{\mathrm{f}}=0.30$ EtOAc/hexanes $1: 1 ;[\alpha]_{20}^{D}-56.9\left(c=0.36, \mathrm{CHCl}_{3}\right) ;{ }^{1} \mathrm{H}$ NMR $\left(250 \mathrm{MHz}, \mathrm{CDCl}_{3}\right): \delta=0.84(\mathrm{t}, J=$ $7.3 \mathrm{~Hz}, 3 \mathrm{H}, 5-\mathrm{H}$, Ile), 0.89 (d, J=7.3 Hz, $3 \mathrm{H}, 1^{\prime}-\mathrm{H}$, Ile), 0.95-1.17 (m, 1 H, 4- $\mathrm{H}_{\mathrm{a}}$, Ile), 1.31 [d, $J=7.0 \mathrm{~Hz}, 3 \mathrm{H}, 4-\mathrm{H},(\beta M e) P h e], 1.39$ [s, $\left.9 \mathrm{H}, \mathrm{C}\left(\mathrm{CH}_{3}\right)_{3}\right], 1.40-1.58\left(\mathrm{~m}, 1 \mathrm{H}, 4-\mathrm{H}_{\mathrm{b}}\right.$, Ile $), 1.59-$ 1.82 (m, 1 H, 3-H, Ile), 1.82-2.10 (m, 3 H, 3-Ha , 4-H, Pro), 2.10-2.31 (m, 1 H, 3-H [dq, $J=7.0,7.0 \mathrm{~Hz}, 1 \mathrm{H}, 3-\mathrm{H},(\beta M e) P h e], 3.42-3.63$ (m, 2 H, 5-H, Pro), 3.69 (s, $3 \mathrm{H}, \mathrm{OMe})$, $4.22(\mathrm{dd}, J=6.5,6.5 \mathrm{~Hz}, 1 \mathrm{H}, 2-\mathrm{H}$, Ile $), 4.29-4.44$ [m, $2 \mathrm{H}, 2-\mathrm{H},(\beta M e)$ Phe, 2-H, Pro], 5.05 (d, J $=6.8 \mathrm{~Hz}, 1 \mathrm{H}, \mathrm{NH}), 6.16(\mathrm{~d}, J=8.8 \mathrm{~Hz}, 1 \mathrm{H}, \mathrm{NH}), 7.08-7.30(\mathrm{~m}, 5 \mathrm{H}, \mathrm{Ar}-\mathrm{H}) ;{ }^{13} \mathrm{C} \mathrm{NMR}(62.9$ $\left.\mathrm{MHz}, \mathrm{CDCl}_{3}\right): \delta=10.9(+, \mathrm{C}-5, I l e), 14.7\left(+, \mathrm{C}-1^{\prime}\right.$, Ile $), 17.0[+, \mathrm{C}-4,(\beta M e) P h e], 24.2(-, \mathrm{C}-4$, Pro), $24.6\left(-, \mathrm{C}-4\right.$, Ile), $28.1\left[+, \mathrm{C}\left(\mathrm{CH}_{3}\right)_{3}\right], 28.9$ (-, C-3, Pro ), 37.7 (+, C-3, Ile), 42.4 [+,C-3, 
(ßMe)Phe], 47.0 (-, C-5, Pro), 51.9 (+, OMe), 54.4 (+, C-2), $58.4(+, \mathrm{C}-2), 60.1(+, \mathrm{C}-2), 79.6$ $\left[\mathrm{C}_{\text {quat }}, \mathrm{C}\left(\mathrm{CH}_{3}\right)_{3}\right], 126.4,127.5,128.1$ (+, $\left.\mathrm{Ar}-\mathrm{C}\right), 142.4\left(\mathrm{C}_{\text {quat }}, \mathrm{Ar}-\mathrm{C}\right), 155.4\left(\mathrm{C}_{\text {quat }}, \mathrm{NCO}_{2}\right), 169.7$, 170.5, $172.1\left(\mathrm{C}_{\text {quat }}, \mathrm{C}-1\right)$; IR $(\mathrm{KBr}): \mathrm{nu}($ tilde $)=3320 \mathrm{~cm}^{-1}, 2970,2878,1754,1706,1642,1516$, 1451, 1367, 1174; MS (EI, $70 \mathrm{eV}), m / z(\%)=503(18)\left[\mathrm{M}^{+}\right], 398(32), 298(18), 269$ (13), 206 (20), 178 (33), 134 (38), 130 (86), 86 (100), 57 (62) $\left[\mathrm{C}_{4} \mathrm{H}_{9}{ }^{+}\right]$; HRMS (EI): calcd for $\mathrm{C}_{27} \mathrm{H}_{41} \mathrm{~N}_{3} \mathrm{O}_{6}$ : 503.2995; found 503.2995.

Boc-( $\beta$ Me)Phe-Ile-Pro-OH (141): A 40\% methanolic solution of Triton X (0.464 g, $1.11 \mathrm{mmol})$ was added to a stirred solution of $140(0.190 \mathrm{~g}, 0.36 \mathrm{mmol})$ in $\mathrm{THF} / \mathrm{H}_{2} \mathrm{O} 5: 1(4 \mathrm{~mL})$ at $4{ }^{\circ} \mathrm{C}$ and stirring continued at the same temperature for a further $4 \mathrm{~h}$. A $1 \mathrm{M} \mathrm{H}_{2} \mathrm{SO}_{4}(2 \mathrm{~mL}) \mathrm{EtOAc}(40$ $\mathrm{mL})$ were then added. Organic layer was separated, washed with $1 \mathrm{M} \mathrm{NaHSO}_{4}(3 \times 10 \mathrm{~mL})$, water $(3 \times 10 \mathrm{~mL})$, brine $(2 \times 5 \mathrm{~mL})$, dried, filtered and concentrated under reduced pressure. The residue was purified by column chromatography $\left(\mathrm{MeOH} / \mathrm{CHCl}_{3} 1: 10, R_{\mathrm{f}}=0.29\right)$ to give 141 $(0.142 \mathrm{~g}, 77 \%)$ as a colorless solid. ${ }^{1} \mathrm{H}$ NMR $\left(250 \mathrm{MHz}, \mathrm{CDCl}_{3}\right): \delta=0.70-0.95(\mathrm{~m}, 3 \mathrm{H}, 5-\mathrm{H}$, Ile), $0.89\left(\mathrm{~d}, J=5.5 \mathrm{~Hz}, 3 \mathrm{H}, 1^{\prime}-\mathrm{H}\right.$, Ile), 0.95-1.18 (m, $2 \mathrm{H}, 4-\mathrm{H}$, Ile), 1.20-1.33 [m, $3 \mathrm{H}, 4-\mathrm{H}$, ( $\beta M e) P h e], 1.40$ [s, $\left.9 \mathrm{H}, \mathrm{C}\left(\mathrm{CH}_{3}\right)_{3}\right], 1.62-1.84(\mathrm{~m}, 1 \mathrm{H}, 3-\mathrm{H}$, Ile $), 1.86-2.32(\mathrm{~m}, 4 \mathrm{H}, 3-\mathrm{H}, 4-\mathrm{H}$, Pro), 3.00-3.23 [m, 1 H, 3-H, ( $\beta M e) P h e], 3.51-3.71$ (m, 2 H, 5-H, Pro), 4.19-4.53 (m, 3 H, 2H), 5.09-5.41 (m, 1 H, NH), 6.48-6.97 (m, $1 \mathrm{H}, \mathrm{NH})$, 7.00-7.29 (m, 5 H, Ar-H); MS (EI, 70 $\mathrm{eV}), m / z(\%)=489(21)\left[\mathrm{M}^{+}\right], 384(50), 347(17), 291$ (13), 284 (30), 206 (22), $178(57), 134$ (63), 116 (80), 86 (100), 57 (76) $\left[\mathrm{C}_{4} \mathrm{H}_{9}{ }^{+}\right]$; HRMS (EI): calcd for $\mathrm{C}_{26} \mathrm{H}_{39} \mathrm{~N}_{3} \mathrm{O}_{6}$ : 489.2839; found 489.2839.

Boc-Thr[Boc-( $\beta M e)$ Phe-Ile-Pro-OMe]-OMTM (142): The acid 141 (0.142 g, $0.28 \mathrm{mmol})$ was coupled with 132 (77 mg, $0.28 \mathrm{mmol})$ using EDC (55 mg, $0.29 \mathrm{mmol})$ and DMAP (5 mg, 0.04 mmol) in $\mathrm{CH}_{2} \mathrm{Cl}_{2}(5 \mathrm{~mL})$ according to $\mathrm{GP} 6$ for $16 \mathrm{~h}$. The crude product obtained after usual aqueous work-up was recrystallized from $\mathrm{Et}_{2} \mathrm{O}$ to give $142(95 \mathrm{mg}, 45 \%)$ as a colorless solid. $R_{\mathrm{f}}$ 
$=0.30$ EtOAc/hexanes $1: 1 ;[\alpha]_{20}^{D}-56.9\left(c=0.36, \mathrm{CHCl}_{3}\right) ;{ }^{1} \mathrm{H}$ NMR $\left(250 \mathrm{MHz}, \mathrm{CDCl}_{3}\right): \delta=$ $0.85\left(\mathrm{t}, J=7.0 \mathrm{~Hz}, 3 \mathrm{H}, 5-\mathrm{H}\right.$, Ile), $0.91\left(\mathrm{~d}, J=6.8 \mathrm{~Hz}, 3 \mathrm{H}, 1^{\prime}-\mathrm{H}\right.$, Ile), $0.94-1.17\left(\mathrm{~m}, 1 \mathrm{H}, 4-\mathrm{H}_{\mathrm{a}}\right.$, Ile), $1.26[\mathrm{~d}, J=6.5 \mathrm{~Hz}, 3 \mathrm{H}, 4-\mathrm{H},(\beta M e) P h e], 1.31(\mathrm{~d}, J=7.0 \mathrm{~Hz}, 3 \mathrm{H}, 4-\mathrm{H}, T h r), 1.40$ [s, $9 \mathrm{H}$, $\left.\mathrm{C}\left(\mathrm{CH}_{3}\right)_{3}\right], 1.43\left[\mathrm{~s}, 9 \mathrm{H}, \mathrm{C}\left(\mathrm{CH}_{3}\right)_{3}\right], 1.62-1.79(\mathrm{~m}, 1 \mathrm{H}, 3-\mathrm{H}$, Ile $), 1.82-2.05\left(\mathrm{~m}, 3 \mathrm{H}, 3-\mathrm{H}_{\mathrm{a}}, 4-\mathrm{H}\right.$, Pro), 2.06-2.31 (m, 1 H, 3-Hb, Pro), 2.21 (s, 3 H, SMe), 3.06-3.27 [m, 1 H, 3-H, ( $\beta M e) P h e]$, 3.39-3.62 (m, 2 H, 5-H, Pro), 4.06-4.30 (m, 2 H, 2-H, Ile, 2-H, Pro), 4.32 [dd, J=8.8, 8.8 Hz, $1 \mathrm{H}, 2-\mathrm{H},(\beta M e) P h e], 4.47(\mathrm{dd}, J=10.0,2.0 \mathrm{~Hz}, 1 \mathrm{H}, 2-\mathrm{H}, T h r), 5.03(\mathrm{~d}, J=9.3 \mathrm{~Hz}, 1 \mathrm{H}, \mathrm{NH})$, $5.18\left(\mathrm{~d}, J=2.0 \mathrm{~Hz}, 2 \mathrm{H}, \mathrm{OCH}_{2} \mathrm{~S}\right), 5.43(\mathrm{dq}, J=7.0,2.0 \mathrm{~Hz}, 1 \mathrm{H}, 3-\mathrm{H}, T h r), 6.10(\mathrm{~d}, J=8.5 \mathrm{~Hz}$, $1 \mathrm{H}, \mathrm{NH}), 7.05-7.31(\mathrm{~m}, 5 \mathrm{H}, \mathrm{Ar}-\mathrm{H})$; The absorption of 4- $\mathrm{H}_{\mathrm{b}}$ of Ile was masked by the signals of $\mathrm{C}\left(\mathrm{CH}_{3}\right)_{3}$ and of $\mathrm{C}-4$ of $\mathrm{Th}$, the signal of one amide proton was masked by the peak of $\mathrm{OCH}_{2} \mathrm{~S}$; ${ }^{13} \mathrm{C}$ NMR $\left(62.9 \mathrm{MHz}, \mathrm{CDCl}_{3}\right): \delta=10.8(+, \mathrm{C}-5$, Ile $), 15.0(+, \mathrm{SMe}), 15.2\left(+, \mathrm{C}-1^{\prime}\right.$, Ile $), 16.6(+$,

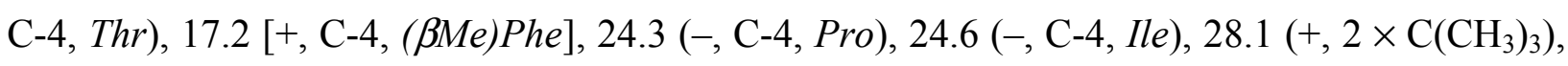

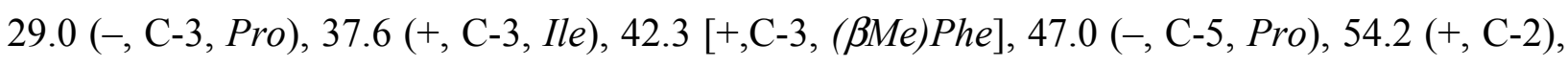
$56.9(+, \mathrm{C}-2), 58.7(+, \mathrm{OMe}), 60.3(+, \mathrm{C}-2), 69.8\left(-, \mathrm{OCH}_{2} \mathrm{~S}\right), 71.3(+, \mathrm{C}-3$, Thr $), 79.5,80.1$ $\left[\mathrm{C}_{\text {quat }}, \mathrm{C}\left(\mathrm{CH}_{3}\right)_{3}\right], 126.5,127.5,128.2(+, \mathrm{Ar}-\mathrm{C}), 142.3\left(\mathrm{C}_{\text {quat }}, \mathrm{Ar}-\mathrm{C}\right), 155.4,155.5\left(\mathrm{C}_{\text {quat }}, \mathrm{NCO}_{2}\right)$, $169.5(\times 2), 170.5(\times 2)\left(\mathrm{C}_{\text {quat }}, \mathrm{C}-1\right)$; MS (EI, $\left.70 \mathrm{eV}\right), m / z(\%)=750(34)\left[\mathrm{M}^{+}\right], 677(15)\left[\mathrm{M}^{+}-\right.$ $\left.\mathrm{C}_{4} \mathrm{H}_{9} \mathrm{O}\right], 645$ (44), 589 (10), 545 (15), 445 (12), 428 (18), 375 (18), 315 (21), 262 (20), 206 (36), 134 (26), 86 (100), 57 (72) $\left[\mathrm{C}_{4} \mathrm{H}_{9}{ }^{+}\right]$; HRMS (EI): calcd for $\mathrm{C}_{37} \mathrm{H}_{58} \mathrm{~N}_{4} \mathrm{O}_{10} \mathrm{~S}$ : 750.3874; found 750.3874 .

N-Boc-3-(2S, 1'S, 2'R)-(trans-2'-Nitrocyclopropyl)alanine $\left(2 S, 1^{\prime} S, 2^{\prime} R\right)-(\mathbf{1 4 3})$ : A solution of $\mathrm{Boc}_{2} \mathrm{O}$

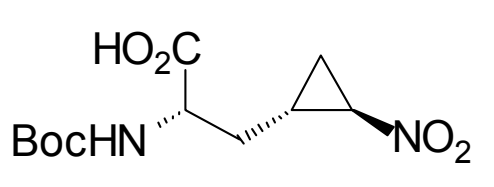
$(0.500 \mathrm{~g}, 2.29 \mathrm{mmol})$ in acetone $(2 \mathrm{~mL})$ was added to a vigorously stirred solution of $3-\quad\left(2 S, 1^{\prime} R, 2^{\prime} R\right)-($ trans-2'nitrocyclopropyl)alanine $\left(2 S, 1^{\prime} R, 2^{\prime} R\right)-83(0.266 \mathrm{~g}, 1.53 \mathrm{mmol})$ in $1 \mathrm{M} \mathrm{NaOH}(1.53 \mathrm{~mL})$ with some amount of $\mathrm{NaHCO}_{3}$ (if precipitate formed acetone and/or water were added to obtain homogeneous solution) and stirring continued for a further $15 \mathrm{~h} . \mathrm{N}, \mathrm{N}$ - 
dimethylaminopropylamine $(0.11 \mathrm{~mL}, 0.88 \mathrm{mmol})$ was then added. After an additional $10 \mathrm{~min}$ acetone was removed under reduced pressure and $\mathrm{pH}$ of the residual water solution was adjusted to 2-3 with $1 \mathrm{M} \mathrm{NaHSO}_{4}$. The resulting emulsion was extracted with $\mathrm{Et}_{2} \mathrm{O}(50 \mathrm{~mL})$ and the ethereal layer was washed with $1 \mathrm{M} \mathrm{NaHSO}_{4}(2 \times 10 \mathrm{~mL})$, water $(3 \times 10 \mathrm{~mL})$, brine $(2 \times 5 \mathrm{~mL})$, dried, filtered and concentrated under reduced pressure. The residue was taken up with hexanes, filtered through Celite pad and concentrated under reduced pressure to give $\left(2 S, 1^{\prime} S, 2^{\prime} R\right)-143$ $(0.353 \mathrm{~g}, 84 \%)$ as an extremely viscous colorless oil. $R_{\mathrm{f}}=0.06$ EtOAc/hexanes 1:3 (2\% AcOH); $[\alpha]_{20}^{D} 20.6\left(c=0.81, \mathrm{CHCl}_{3}\right) ;{ }^{1} \mathrm{H} \mathrm{NMR}\left(250 \mathrm{MHz}, \mathrm{CDCl}_{3}\right): \delta=1.14(\mathrm{ddd}, J=6.5,6,5,6.5 \mathrm{~Hz}$, $\left.1 \mathrm{H}, 3^{\prime}-\mathrm{H}_{\mathrm{a}}\right), 1.45\left[\mathrm{~s}, 9 \mathrm{H}, \mathrm{C}\left(\mathrm{CH}_{3}\right)_{3}\right], 1.59-1.82\left(\mathrm{~m}, 1 \mathrm{H}, 3^{\prime}-\mathrm{H}_{\mathrm{b}}\right), 1.82-1.94\left(\mathrm{~m}, 1 \mathrm{H}, 1^{\prime}-\mathrm{H}\right), 1.94-$ $2.21(\mathrm{~m}, 2 \mathrm{H}, 3-\mathrm{H}), 4.10-4.23\left(\mathrm{~m}, 1 \mathrm{H}, 2^{\prime}-\mathrm{H}\right), 4.25-4.38,4.42-4.53(2 \times \mathrm{m}, 1 \mathrm{H}, 2-\mathrm{H}), 5.22-5.40$, 6.97-7.05 (m, $1 \mathrm{H}, \mathrm{NH}), 6.10-7.30\left(\mathrm{br}, 1 \mathrm{H}, \mathrm{CO}_{2} \mathrm{H}\right) ;{ }^{13} \mathrm{C} \mathrm{NMR}\left(62.9 \mathrm{MHz}, \mathrm{CDCl}_{3}\right): \delta=17.5(-$, C-3'), $22.0\left(+, \mathrm{C}-1^{\prime}\right), 28.1\left[+, \mathrm{C}\left(\mathrm{CH}_{3}\right)_{3}\right], 33.4,33.6(-, \mathrm{C}-3), 52.5,53.7(+, \mathrm{C}-2), 59.2\left(+, \mathrm{C}-2^{\prime}\right)$, 80.6, $82.5\left[\mathrm{C}_{\text {quat }}, \mathrm{C}\left(\mathrm{CH}_{3}\right)_{3}\right], 155.4,156.9\left(\mathrm{C}_{\text {quat }}, \mathrm{NCO}_{2}\right), 175.1,175.4\left(\mathrm{C}_{\text {quat }}, \mathrm{C}-1\right)$; IR (film): $\mathrm{nu}($ tilde $)=3700-2250 \mathrm{~cm}^{-1}, 3101,2979,2935,1715,1545,1437,1394,1369,1253,1163 ; \mathrm{MS}$ (ESI): positive mode, $m / z(\%)=319(32)\left[\mathrm{M}-\mathrm{H}^{+}+2 \mathrm{Na}^{+}\right], 297(86)\left[\mathrm{M}+\mathrm{Na}^{+}\right]$; negative mode, $m / z(\%)=273(30)\left[\mathrm{M}-\mathrm{H}^{+}\right]$.

Boc-(2S, l'SR, 2'SR)-(3-Ncp)Ala-(ßMe)Phe-Ile-Pro-OMe (144): Tripeptide 140 (0.300 g, 0.60 mmol) was deprotected with $3 \mathrm{M} \mathrm{HCl}$ in $\mathrm{MeOH}(7 \mathrm{~mL})$ for $2 \mathrm{~h}$. The mixture was then concentrated under reduced pressure, the residue was triturated with $\mathrm{Et}_{2} \mathrm{O}$ and the resultant precipitate was filtered off and dried to give hydrochloride of deprotected tripeptide which was coupled with Boc-(2S,1'SR,2'SR)-(3-Ncp)Ala-OH (2S,1'SR,2'SR)-143 (0.163 g, 0.59 mmol) (obtained as it was described for $\left(2 S, 1^{\prime} R, 2^{\prime} R\right)$-isomer) using EDC (0.118 g, $\left.0.62 \mathrm{mmol}\right)$, HOAt (83 mg, $0.61 \mathrm{mmol})$, DIEA (77 $\mathrm{mg}, 0.60 \mathrm{mmol})$ and TMP $(0.217 \mathrm{~g}, 1.79 \mathrm{mmol})$ in $\mathrm{CH}_{2} \mathrm{Cl}_{2}(15$ $\mathrm{mL}$ ) according to GP 5 for $15 \mathrm{~h}$. The crude product obtained after usual aqueous work-up (GP 5) was finally purified by column chromatography $\left(\mathrm{MeOH} / \mathrm{CHCl}_{3} 1: 18, R_{\mathrm{f}}=0.42\right)$ to give 144 
$(0.264 \mathrm{~g}, 67 \%)$ as a colorless solid. $[\alpha]_{20}^{D}-87.7\left(c=0.43, \mathrm{CHCl}_{3}\right) ;{ }^{1} \mathrm{H}$ NMR $\left(250 \mathrm{MHz}, \mathrm{CDCl}_{3}\right)$ : $\delta=0.78(\mathrm{dt}, J=2.8,7.3 \mathrm{~Hz}, 3 \mathrm{H}, 5-\mathrm{H}$, Ile $), 0.85\left(\mathrm{~d}, J=6.8 \mathrm{~Hz}, 3 \mathrm{H}, 1^{\prime}-\mathrm{H}\right.$, Ile), $0.91-1.13(\mathrm{~m}$, $1 \mathrm{H}, 4-\mathrm{H}_{\mathrm{a}}$, Ile), 1.27 [ddd, $\left.J=7.0,7.0,7.0 \mathrm{~Hz}, 2^{\prime}-\mathrm{H}_{\mathrm{a}},(3-N c p) A l a\right], 1.36$ [d, J = 7.0 Hz, $3 \mathrm{H}, 4-\mathrm{H}$, ( $\beta M e) P h e], 1.52\left[\mathrm{~s}, 9 \mathrm{H}, \mathrm{C}\left(\mathrm{CH}_{3}\right)_{3}\right], 1.67-2.25\left[\mathrm{~m}, 9 \mathrm{H}, 3-\mathrm{H}\right.$, Ile, 3-H, 1'- $\mathrm{H}, 2^{\prime}-\mathrm{H}_{\mathrm{b}},(3-N c p)$ Ala, $3-$

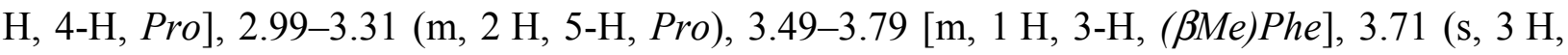
OMe), 3.92-4.03 [m, 0.5 H, 2'-H, (3-Ncp)Ala], 4.24-4.59 [m, 3.5 H, 2-H, 2'-H, (3-Ncp)Ala], 5.19 [ddd, $J=9.6,9.6,9.6 \mathrm{~Hz}, 1 \mathrm{H}, 2-\mathrm{H},(3-N c p)$ Ala $], 6.01-6.19,6.29-6.43(2 \times \mathrm{br}, 1 \mathrm{H}, \mathrm{NH})$, 6.85-6.79 (br, 0.5 H, NH), 7.05-7.17 (m, 1.5 H, Ar-H, NH), 7.18-7.41 (m, 4-H, Ar-H), 7.417.51, 7.54-7.71 $(2 \times \mathrm{m}, 1 \mathrm{H}, \mathrm{NH})$; The absorption of $\mathrm{C}\left(\mathrm{CH}_{3}\right)_{3}$ masked the signal of $4-\mathrm{H}_{\mathrm{b}}$ of Ile; ${ }^{13} \mathrm{C}$ NMR (62.9 MHz, $\left.\mathrm{CDCl}_{3}\right): \delta=11.3(+, \mathrm{C}-5$, Ile $), 14.2,14.3\left(+, \mathrm{C}-1^{\prime}\right.$, Ile $), 16.2,18.1\left[-, \mathrm{C}-3^{\prime}\right.$, (3-Ncp)Ala ], 19.47, 19.55 [+, C-4, (ßMe)Phe], 22.5, 22.9 [+, C-1', (3-Ncp)Ala], 24.4 (-, C-4, Pro), 24.7 (-, C-4, Ile), 28.2, 28.3 [+, C( $\left.\left.\mathrm{CH}_{3}\right)_{3}\right], 28.9$ (-, C-3, Pro), 33.5 [-, 3-H, (3-Ncp)Ala],

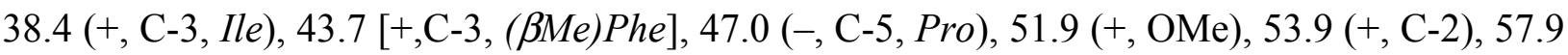
$(+, \mathrm{C}-2), 58.2(+, \mathrm{C}-2), 58.4(+, \mathrm{C}-2), 58.8\left[+, \mathrm{C}-2^{\prime},(3-N c p) A l a\right], 79.2\left[\mathrm{C}_{\text {quat }}, \mathrm{C}\left(\mathrm{CH}_{3}\right)_{3}\right], 126.1$, $128.1(\times 2)(+, A r-C), 142.3\left(\mathrm{C}_{\text {quat }}, \mathrm{Ar}-\mathrm{C}\right), 155.5,155.7\left(\mathrm{C}_{\text {quat }}, \mathrm{NCO}_{2}\right), 169.75,169.78\left(\mathrm{C}_{\text {quat }}, \mathrm{C}-\right.$ 1), 170.7, $170.8\left(\mathrm{C}_{\text {quat }}, \mathrm{C}-1\right), 172.0,172.1\left(\mathrm{C}_{\text {quat }}, \mathrm{C}-1\right), 172.7\left(\mathrm{C}_{\text {quat }}, \mathrm{C}-1\right)$; $\mathrm{IR}(\mathrm{KBr})$ : nu(tilde $)=$ $3085 \mathrm{~cm}^{-1}, 2971,2933,2878,1750,1711,1634,1545,1453,1367,1174 ;$ MS (EI, $\left.70 \mathrm{eV}\right), \mathrm{m} / z$ $(\%)=659(4)\left[\mathrm{M}^{+}\right], 603(7), 531(10), 503$ (27),418 (29), 362 (38), 334 (58), 290 (18), 269 (24), 225 (12), 182 (12), 130 (100), 86 (28), 57 (6) $\left[\mathrm{C}_{4} \mathrm{H}_{9}{ }^{+}\right]$; HRMS (EI): calcd for $\mathrm{C}_{33} \mathrm{H}_{49} \mathrm{~N}_{5} \mathrm{O}_{9}$ : 659.3530; found 659.3530.

Boc-(2S, 1'SR, 2'SR)-(3-Ncp)Ala-( $\beta M e) P h e-I l e-P r o-O H ~(145):$ A 40\% aqueous solution of tetrabutylammonium hydroxide $(0.416 \mathrm{~g}, 0.64 \mathrm{mmol})$ was added to a vigorously stirred solution of tetrapeptide $144(0.13 \mathrm{~g}, 0.20 \mathrm{mmol})$ in THF $(2.0 \mathrm{~mL})$ at $4{ }^{\circ} \mathrm{C}$ and stirring continued at the same temperature for $3.5 \mathrm{~h}$. The reaction was then quenched with $1 \mathrm{M} \mathrm{H}_{2} \mathrm{SO}_{4}(3 \mathrm{~mL})$ and diluted 
with EtOAc $(40 \mathrm{~mL})$, the organic layer was washed with $1 \mathrm{M} \mathrm{NaHSO}_{4}(3 \times 10 \mathrm{~mL})$, water $(3 \times$ $10 \mathrm{~mL})$, brine $(2 \times 10 \mathrm{~mL})$, dried, filtered and concentrated under reduced pressure to give the crude tetrapeptide with free carboxylic group $145\left(0.125 \mathrm{~g}, 100 \%\right.$ crude, $R_{\mathrm{f}}=0.21 \mathrm{MeOH} / \mathrm{CHCl}_{3}$ 1:18) as a white solid which was used for the next step without additional purification.

Fmoc-( $\beta M e)$ Phe-ODmob (147): The $N$-Fmoc protected $\beta$-methylphenylalanine 146 (0.19 g, 0.47 mmol) was esterificated with 2,4-dimethoxybenzyl alcohol (78 mg, $0.46 \mathrm{mmol}$ ) using EDC (93 $\mathrm{mg}, 0.49 \mathrm{mmol})$ and DMAP (12 $\mathrm{mg}, 0.10 \mathrm{mmol})$ in $\mathrm{CH}_{2} \mathrm{Cl}_{2}(5 \mathrm{~mL})$ according to $\mathrm{GP} 7 \mathrm{for} 16 \mathrm{~h}$. The crude product obtained after usual aqueous work-up (GP 7) was finally purified by column chromatography (EtOAc/hexanes 1:3, $\left.R_{\mathrm{f}}=0.33\right)$ to give $147(0.133 \mathrm{~g}, 57 \%)$ as a turbid glass. $[\alpha]_{20}^{D}-2.6\left(c=0.27, \mathrm{CHCl}_{3}\right) ;{ }^{1} \mathrm{H} \operatorname{NMR}\left(250 \mathrm{MHz}, \mathrm{CDCl}_{3}\right): \delta=1.35(\mathrm{~d}, J=7.0 \mathrm{~Hz}, 3 \mathrm{H}, 4-\mathrm{H})$, $3.25(\mathrm{dq}, J=7.0,7.0 \mathrm{~Hz}, 1 \mathrm{H}, 3-\mathrm{H}), 3.80(\mathrm{~s}, 3 \mathrm{H}, \mathrm{OMe}), 3.82(\mathrm{~s}, 3 \mathrm{H}, \mathrm{OMe}), 4.18(\mathrm{t}, J=7.1 \mathrm{~Hz}$, 1 H, 1'-H, Fmoc), 4.23-4.45 (m, 2 H, 2'-H, Fmoc), 4.60 (dd, J = 9.0, 6.0 Hz, 1 H, 2-H), 5.32 (s, 2 H, Bzl-H), 6.41-6.49 (m, 2 H, Ar-H), 7.04-7.18 (m, 3 H, Ar-H), 7.19-7.47 (m, 7 H, Ar-H), 7.57 (d, $J=7.3 \mathrm{~Hz}, 2 \mathrm{H}, \mathrm{Ar}-\mathrm{H}), 7.77$ (d, $J=7.3 \mathrm{~Hz}, 2 \mathrm{H}, \mathrm{Ar}-\mathrm{H}) ;{ }^{13} \mathrm{C} \mathrm{NMR}\left(62.9 \mathrm{MHz}, \mathrm{CDCl}_{3}\right): \delta$ $=16.3(+, \mathrm{C}-4), 42.6(+, \mathrm{C}-3), 47.0\left(+, \mathrm{C}-1^{\prime}\right.$, Fmoc $), 55.1(+, \mathrm{OMe}), 55.2(+, \mathrm{OMe}), 59.2(+, \mathrm{C}-2)$, 62.6 (-, Bzl-C), 66.7 (-, C-2', Fmoc), 98.3 (+, C-3, Dmob), 103.8 (+, C-5, Dmob), 115.7 (C quat, C-1, Dmob), 119.8, 124.9, 126.9, 127.5, $127.6(\times 2), 128.2,131.7(+, A r-C), 141.0,141.1\left(\mathrm{C}_{\text {quat }}\right.$, Ar-C, Fmoc $), 141.1\left(\mathrm{C}_{\text {quat }}, \mathrm{Ar}-\mathrm{C},(\beta M e) P h e\right), 143.7,143.8\left(\mathrm{C}_{\text {quat }}, \mathrm{Ar}-\mathrm{C}, F m o c\right), 155.5\left(\mathrm{C}_{\text {quat }}\right.$, $\left.\mathrm{NCO}_{2}\right), 158.8\left(\mathrm{C}_{\text {quat }}, \mathrm{C}-2, D m o b\right), 161.3\left(\mathrm{C}_{\text {quat }}, \mathrm{C}-4, D m o b\right), 171.1\left(\mathrm{C}_{\text {quat }}, \mathrm{C}-1\right)$; IR (KBr): $\mathrm{nu}($ tilde $)=3063 \mathrm{~cm}^{-1}, 3028,2963,2835,1724,1616,1510,1451,1209,1159,1040$; MS (EI, 70 $\mathrm{eV}), m / z(\%)=551(1)\left[\mathrm{M}^{+}\right], 288(10), 178(100)\left[\mathrm{C}_{14} \mathrm{H}_{10}{ }^{+}\right], 151(32)\left[\mathrm{C}_{9} \mathrm{H}_{11} \mathrm{O}_{2}^{+}\right], 105(27)$ $\left[\mathrm{C}_{8} \mathrm{H}_{9}{ }^{+}\right]$; HRMS (EI): calcd for $\mathrm{C}_{34} \mathrm{H}_{33} \mathrm{NO}_{6}$ : 551.2308; found 551.2308. 
Alloc-a-Thr-OH (148): A solution of AllocOPfp $(0.295 \mathrm{~g}, 1.10 \mathrm{mmol})$ in acetone $(7 \mathrm{~mL})$ was AllocHN " ${ }_{\mathrm{CO}_{2} \mathrm{H}}^{\mathrm{gH}}$ added to a vigorously stirred solution of $(R)$ - $a$-threonine $(R)-a-72(0.203 \mathrm{mmol})$ and $\mathrm{NaHCO}_{3}(0.185 \mathrm{~g}, 2.20 \mathrm{mmol})$ in water $(5 \mathrm{~mL})$ (if emulsion formed acetone or water was added to obtain homogeneous solution) and stirring continued for an additional $1 \mathrm{~h}$. The mixture was then concentrated under reduced pressure, diluted with methanol $(10 \mathrm{~mL})$, treated with $3.9 \mathrm{M} \mathrm{HCl}$ in dioxane $(0.64 \mathrm{~mL})$ and concentrated again. The residue was purified by column chromatography $\left(\mathrm{MeOH} / \mathrm{CHCl}_{3} 1: 8(2.5 \% \mathrm{AcOH}), R_{\mathrm{f}}\right.$ $=0.39)$ to give $148(0.154 \mathrm{~g}, 74 \%)$ as a colorless oil polymerized easily in the presence of the traces of acid. ${ }^{1} \mathrm{H}$ NMR $\left(250 \mathrm{MHz}, \mathrm{CD}_{3} \mathrm{OD}\right): \delta=1.26(\mathrm{~d}, J=6.5 \mathrm{~Hz}, 3 \mathrm{H}, 4-\mathrm{H}), 4.13(\mathrm{dq}, J=$ 6.1, 6.1 Hz, $1 \mathrm{H}, 3-\mathrm{H}), 4.25(\mathrm{~d}, J=5.0 \mathrm{~Hz}, 1 \mathrm{H}, 2-\mathrm{H}), 4.60$ (d, J= 7.8 Hz, $2 \mathrm{H}, 1-\mathrm{H}$, Alloc), 5.16$5.28(\mathrm{~m}, 1 \mathrm{H}$, trans-3-H, Alloc $), 5.30-5.43(\mathrm{~m}, 1 \mathrm{H}$, cis-3-H, Alloc $), 5.87-6.08(\mathrm{~m}, 1 \mathrm{H}, 2-\mathrm{H}$, Alloc); ${ }^{13} \mathrm{C}$ NMR (62.9 MHz, $\left.\mathrm{CD}_{3} \mathrm{OD}\right): \delta=19.5(+, \mathrm{C}-4), 61.3$ (+, C-2), $67.0(-, \mathrm{C}-1$, Alloc $), 69.0$ (+, C-3), $118.0(-, \mathrm{C}-3$, Alloc $), 134.3(+, \mathrm{C}-2$, Alloc $), 158.7\left(\mathrm{C}_{\text {quat }}, \mathrm{NCO}_{2}\right), 174.0\left(\mathrm{C}_{\text {quat }}, \mathrm{C}-1\right)$; IR (film): nu(tilde) $=3750-2500 \mathrm{~cm}^{-1}, 2981,2934,1708,1535,1411,1260,1100,1042$; MS (CI), $m / z(\%): 221(100)\left[\mathrm{M}+\mathrm{NH}_{4}^{+}\right], 204(12)\left[\mathrm{M}+\mathrm{H}^{+}\right]$.

Alloc-a-Thr-( $\beta$ Me)Phe-ODmob (149): Compound 147 (0.128 g, $0.23 \mathrm{mmol})$ was deprotected according to GP 4 and the resultant amino ester was coupled with $N$-Alloc protected allo- $(R)$ threonine 148 (52 mg, $0.26 \mathrm{mmol})$ using EDC (50 mg, $0.26 \mathrm{mmol})$, HOAt (35 mg, $0.26 \mathrm{mmol})$ and TMP (56 mg, $0.46 \mathrm{mmol})$ in $\mathrm{CH}_{2} \mathrm{Cl}_{2} / \mathrm{DMF}$ 1:1 (5 mL) according to GP 5. The crude product obtained after usual aqueous work-up (GP 5) was triturated with hot hexanes, filtered off, washed with cold $\mathrm{Et}_{2} \mathrm{O}$ and dried to give $149(0.129 \mathrm{~g}, 90 \%)$ as a colorless solid. $R_{\mathrm{f}}=0.29$ EtOAc/hexanes $1: 1 ;[\alpha]_{20}^{D} 29.6\left(c=0.27, \mathrm{CHCl}_{3}\right) ;{ }^{1} \mathrm{H} \mathrm{NMR}\left(250 \mathrm{MHz}, \mathrm{CDCl}_{3}\right): \delta=1.15(\mathrm{~d}, J=$ $6.5 \mathrm{~Hz}, 3 \mathrm{H}, 4-\mathrm{H}, a-T h r), 1.32[\mathrm{~d}, J=7.3 \mathrm{~Hz}, 3 \mathrm{H}, 4-\mathrm{H},(\beta M e) P h e], 3.25$ [dq, $J=1.0,7.3 \mathrm{~Hz}$, $1 \mathrm{H}, 3-\mathrm{H},(\beta M e) P h e], 3.30-3.42$ (br, $1 \mathrm{H}, \mathrm{OH}), 3.81$ (s, $3 \mathrm{H}, \mathrm{OMe}), 3.82$ (s, $3 \mathrm{H}, \mathrm{OMe}), 3.87$ (dd, $J=6.5,5.5 \mathrm{~Hz}, 1 \mathrm{H}, 3-\mathrm{H}, a-T h r), 4.01-4.13[\mathrm{~m}, 1 \mathrm{H}, 2-\mathrm{H},(\beta M e) P h e], 4.56(\mathrm{~d}, J=5.5 \mathrm{~Hz}, 2 \mathrm{H}$, 
Alloc), 4.82 (dd, $J=9.0,5.5 \mathrm{~Hz}, 1 \mathrm{H}, 2-\mathrm{H}, a-T h r), 5.08$ (dd, $J=11.8,11.8 \mathrm{~Hz}, 2 \mathrm{H}, 1-H$, Alloc), 5.17-5.26 (m, $1 \mathrm{H}$, trans-3-H, Alloc), 5.26-5.37 (m, $1 \mathrm{H}$, cis-3-H, Alloc), $5.61(\mathrm{~d}, J=7.3 \mathrm{~Hz}$, $1 \mathrm{H}, \mathrm{NH}), 5.78-5.99$ (m, $1 \mathrm{H}, 2-\mathrm{H}$, Alloc $), 6.40-6.49$ (m, $2 \mathrm{H}, \mathrm{Ar}-\mathrm{H}), 6.82(\mathrm{~d}, J=9.0 \mathrm{~Hz}, 1 \mathrm{H}$, $\mathrm{NH}), 7.07-7.19(\mathrm{~m}, 3 \mathrm{H}, \mathrm{Ar}-\mathrm{H}), 7.20-7.30(\mathrm{~m}, 3 \mathrm{H}, \mathrm{Ar}-\mathrm{H}) ;{ }^{13} \mathrm{C} \mathrm{NMR}\left(62.9 \mathrm{MHz}, \mathrm{CDCl}_{3}\right): \delta=$ $16.0[+, \mathrm{C}-4,(\beta M e) P h e], 41.9[+, \mathrm{C}-3,(\beta M e) P h e], 55.29(+, \mathrm{OMe}), 55.34(+, \mathrm{OMe}), 57.4[+, \mathrm{C}-$ 2, (BMe)Phe], 59.1 (+, C-2,a-Thr), 62.8 (-, Bzl-C), 66.1 (-, C-1, Alloc), 69.0 (+, C-3, a-Thr), 98.4 (+, C-3, Dmob), 103.9 (+, C-5, Dmob), 115.8 (C quat C-1, Dmob), 117.9 (-, C-3, Alloc), 127.0, 127.6, 128.3, 131.7 (+, Ar-C), $132.4(+, \mathrm{C}-2$, Alloc $), 141.0\left(\mathrm{C}_{\text {quat }}, \mathrm{Ar}-\mathrm{C}\right), 156.6\left(\mathrm{C}_{\text {quat, }}\right.$, $\left.\mathrm{NCO}_{2}\right), 158.9\left(\mathrm{C}_{\text {quat }}, \mathrm{C}-2, \mathrm{Dmob}\right), 161.4\left(\mathrm{C}_{\text {quat }}, \mathrm{C}-4, \mathrm{Dmob}\right), 170.2,170.9\left(\mathrm{C}_{\text {quat }}, \mathrm{C}-1\right)$; IR $(\mathrm{KBr})$ : $\mathrm{nu}($ tilde $)=3299 \mathrm{~cm}^{-1}, 3065,2962,2934,2838,1743,1694,1539,1510,1259,1239,1045 ; \mathrm{MS}$ $(\mathrm{EI}, 70 \mathrm{eV}), m / z(\%)=514(6)\left[\mathrm{M}^{+}\right], 151(100)\left[\mathrm{C}_{9} \mathrm{H}_{11} \mathrm{O}_{2}{ }^{+}\right], 134(19), 41(3)\left[\mathrm{C}_{3} \mathrm{H}_{5}{ }^{+}\right] ; \mathrm{HRMS}$ (EI): calcd for $\mathrm{C}_{27} \mathrm{H}_{34} \mathrm{~N}_{2} \mathrm{O}_{8}$ : 514.2315; found 514.2315.

Attempts of the preparation of Alloc-a-Thr[Boc-(2S, 1 SR, 2 SR)-(3-Ncp)Ala-( $\beta M e) P h e-I l e-P r o]-$ ( $\beta M e)$ Phe-ODmob (150): Acid $145(0.117 \mathrm{~g}, 0.18 \mathrm{mmol})$ was treated with the alcohol 149 (89 $\mathrm{mg}, 0.17 \mathrm{mmol})$ using DCC (78 $\mathrm{mg}, 0.38 \mathrm{mmol})$ and DMAP (22 mg, $0.18 \mathrm{mmol})$ in THF (5 mL) at $20{ }^{\circ} \mathrm{C}$ according to GP 6 (the carboxylic component was absolute insoluble in $\mathrm{CH}_{2} \mathrm{Cl}_{2}$ or toluene). Solvent was then blown out with the strong flow of nitrogen, dioxane $(10 \mathrm{~mL})$ was then added and stirring continued for a further $24 \mathrm{~h}$. A dilute aqueous acetic acid $(1 \mathrm{~mL})$ was then added and after $10 \mathrm{~min}$ the mixture was filtered and diluted with $\mathrm{Et}_{2} \mathrm{O}(50 \mathrm{~mL})$. The residue (78 mg) obtained after usual aqueous work-up (GP 6) contained according to ${ }^{1} \mathrm{H}$ NMR only the impure alcohol 149.

To a solution of the carboxylic component $(10 \mathrm{mg}, 15.16 \mu \mathrm{mol})$, alcohol $149(7.0 \mathrm{mg}, 13.64$ $\mu \mathrm{mol})$ and HOAt $(2.0 \mathrm{mg}, 15.19 \mu \mathrm{mol})$ in THF $(0.5 \mathrm{~mL})$ was added EDC $(8.7 \mathrm{mg}, 45.47 \mu \mathrm{mol})$ and stirring continued for $0.5 \mathrm{~h}$. Solvent was then blown out with the strong flow of nitrogen, $\mathrm{CH}_{2} \mathrm{Cl}_{2}(0.5 \mathrm{~mL})$ was added and stirring continued for a further $48 \mathrm{~h}$. The separation of the 
mixture obtained after usual aqueous work-up by column chromatography didn't give the desired product.

To a solution of DMAP $(50 \mathrm{mg}, 0.36 \mathrm{mmol})$ in $\mathrm{CH}_{2} \mathrm{Cl}_{2}$ (ca. $\left.3 \mathrm{~mL}\right)$ was added TFA $(20 \mathrm{mg}, 0.18$ mmol) and the resultant suspension was added to a solution of the carboxylic component 145 $(0.120 \mathrm{~g}, 0.18 \mathrm{mmol})$ and alcohol $149(0.112 \mathrm{~g}, 0.22 \mathrm{mmol})$ in THF $(1.5 \mathrm{~mL})$ followed by EDC (0.105 g, $0.55 \mathrm{mmol})$ and stirring continued for $3 \mathrm{~h}$. All volatiles were then removed under reduced pressure under dry conditions, $\mathrm{CH}_{2} \mathrm{Cl}_{2}(5 \mathrm{~mL})$ was added to the residue and stirring continued for a further 48 h. $\mathrm{C}_{2} \mathrm{H}_{4} \mathrm{Cl}_{2}(10 \mathrm{~mL})$ was added and the mixture was heated and stirred at $60{ }^{\circ} \mathrm{C}$ for another $16 \mathrm{~h}$. The crude product obtained after the usual aqueous work-up (GP 6) was then purified by column chromatography (EtOAc/hexanes $1: 1, R_{\mathrm{f}}=0.15$, elongated spot) to give an amorphous solid (0.22 g), which was triturated with $\mathrm{Et}_{2} \mathrm{O}$ to give the residue (54 mg), which was the mixture contained besides the desired depsipeptide $\mathbf{1 5 0}$ at least one side product (ratio 2:1 according to ${ }^{13} \mathrm{C}$ NMR) inseparable by conventional methods.

Alloc-a-(R)-Thr-ONb (152): Boc- $a-(R)-\mathrm{Thr}-\mathrm{ONb} 151$ (0.61 g, $1.72 \mathrm{mmol})$ was deprotected with

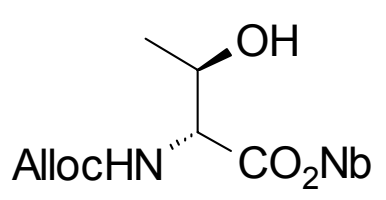
$4 \mathrm{M} \mathrm{HCl}$ in EtOAc (prepared in situ by a careful addition of 1 equivalent of $\mathrm{AcCl}$ to a solution of 1.2 equivalent of $\mathrm{MeOH}$ in EtOAc at $4{ }^{\circ} \mathrm{C}$ ) for $3 \mathrm{~h}$. All volatiles were removed under reduced pressure and the residue was triturated with $\mathrm{Et}_{2} \mathrm{O}$, filtered off and dried to give hydrochloride of the amino ester $(0.48 \mathrm{~g}, 96 \%)$ as a colorless solid which was directly used for the next step. To a solution of this material and DIEA $(0.224 \mathrm{~g}, 1.73 \mathrm{mmol})$ in $\mathrm{CH}_{2} \mathrm{Cl}_{2}(15 \mathrm{~mL})$ was added AllocOBt $(0.38 \mathrm{~g}$, $1.73 \mathrm{mmol})$ and the mixture was stirred for $2 \mathrm{~h} . \mathrm{N}, \mathrm{N}$-dimethylaminopropylamine $(0.1 \mathrm{~g}, 0.98$ mmol) was then added and after 10 min the mixture was diluted with $\mathrm{Et}_{2} \mathrm{O}(60 \mathrm{~mL})$. The crude product obtained after usual aqueous work-up (GP 6) was finally purified by column chromatography (EtOAc/hexanes $\left.1: 1, R_{\mathrm{f}}=0.33\right)$ to give $152(0.44 \mathrm{~g}, 76 \%$ yield on 2 steps $)$ as a colorless solid. $[\alpha]_{20}^{D}-12.3\left(c=0.44, \mathrm{CHCl}_{3}\right) ;{ }^{1} \mathrm{H}$ NMR $\left(250 \mathrm{MHz}, \mathrm{CDCl}_{3}\right): \delta=1.22(\mathrm{~d}, J=$ 
$6.8 \mathrm{~Hz}, 3 \mathrm{H}, 4-\mathrm{H}), 2.85(\mathrm{~d}, J=5.8 \mathrm{~Hz}, 1 \mathrm{H}, \mathrm{OH}), 4.16$ (dq, $J=3.5,6.8 \mathrm{~Hz}, 1 \mathrm{H}, 3-\mathrm{H}), 4.46$ (dd, $J$ $=8.0,3.5 \mathrm{~Hz}, 1 \mathrm{H}, 2-\mathrm{H}), 4.59(\mathrm{dt}, J=5.5,1.3 \mathrm{~Hz}, 2 \mathrm{H}, 1-\mathrm{H}$, Alloc $), 5.17-5.24$ (m, $1 \mathrm{H}$, trans-3H, Alloc), 5.24-5.36 (m, $1 \mathrm{H}$, cis-3-H, Alloc), 5.29 (s, $2 \mathrm{H}, \mathrm{Bzl}-\mathrm{H}), 5.74$ (d, J=8.0 Hz, $1 \mathrm{H}, \mathrm{NH})$, 5.89 (ddt, $J=10.5,10.5,5.5 \mathrm{~Hz}, 1 \mathrm{H}, 2-\mathrm{H}$, Alloc), $7.52(\mathrm{~d}, J=8.8 \mathrm{~Hz}, 2 \mathrm{H}, \mathrm{Ar}-\mathrm{H}), 8.22(\mathrm{~d}, J=$ $8.8 \mathrm{~Hz}, 2 \mathrm{H}, \mathrm{Ar}-\mathrm{H}) ;{ }^{13} \mathrm{C} \mathrm{NMR}\left(62.9 \mathrm{MHz}, \mathrm{CDCl}_{3}\right): \delta=19.1(+, \mathrm{C}-4), 59.4(+, \mathrm{C}-2), 65.5(-$, BzlC), 66.0 (-, C-1, Alloc), 68.4 (+, C-3), 117.9 (-, C-3, Alloc), 123.6, 128.3 (+, Ar-C), $132.1(+, \mathrm{C}-$ 2, Alloc), 142.3, 147.5 ( $\left.\mathrm{C}_{\text {quat }}, \mathrm{Ar}-\mathrm{C}\right), 156.2\left(\mathrm{C}_{\text {quat }}, \mathrm{NCO}_{2}\right), 170.0$ ( $\left.\mathrm{C}_{\text {quat }}, \mathrm{C}-1\right)$; IR (KBr): nu(tilde) $=3315 \mathrm{~cm}^{-1}, 2974,2910,1739,1690,1549,1531,1348,1334,1196,1041 ; \mathrm{MS}(\mathrm{CI}), \mathrm{m} / z(\%)$ : $356(100)\left[\mathrm{M}+\mathrm{NH}_{4}^{+}\right], 339(12)\left[\mathrm{M}+\mathrm{H}^{+}\right]$.

Alloc-a-Thr(Boc-Pro)-ONb (154): Substance 152 (0.43 g, 1.27 mmol) was coupled with BocPro-OH 153 (0.287 g, $1.33 \mathrm{mmol})$ using EDC (0.261 g, $1.36 \mathrm{mmol})$ and DMAP (31 mg, 0.25 mmol) in $\mathrm{CH}_{2} \mathrm{Cl}_{2}(10 \mathrm{~mL})$ according to $\mathrm{GP} 6$ for $15 \mathrm{~h}$. The crude product obtained after usual aqueous work-up (GP 6) was purified by column chromatography (EtOAc/hexanes 1:1, $R_{\mathrm{f}}=$ $0.68)$ to give $154(0.63 \mathrm{~g}, 93 \%)$ as a colorless oil. ${ }^{1} \mathrm{H}$ NMR $\left(250 \mathrm{MHz}, \mathrm{CDCl}_{3}\right): \delta=1.33,1.37(2$ $\times \mathrm{d}, J=6.8 \mathrm{~Hz}, 3 \mathrm{H}, 4-\mathrm{H}, a-T h r), 1.40,1.45\left[2 \times \mathrm{s}, 9 \mathrm{H}, \mathrm{C}\left(\mathrm{CH}_{3}\right)_{3}\right], 1.76-2.26(\mathrm{~m}, 4 \mathrm{H}, 3-\mathrm{H}, 4-\mathrm{H}$, Pro), 3.32-3.55 (m, 2 H, 5-H, Pro), 4.08, 4.22 (dd, J=9.5, 3.5 Hz, 1 H, 2-H, Pro), 4.54, 4.56 (2 $\times \mathrm{d}, J=5.8 \mathrm{~Hz}, 2 \mathrm{H}, 1-\mathrm{H}$, Alloc $), 4.62,4.64(2 \times \mathrm{dd}, J=3.5,6.8 \mathrm{~Hz}, 1 \mathrm{H}, 2-\mathrm{H}, T h r), 5.12-5.42$ (m, $5 \mathrm{H}, 3-\mathrm{H}$, Alloc, 3-H, a-Thr, Bzl-H), 5.50, $6.55(2 \times \mathrm{d}, J=9.5 \mathrm{~Hz}, 1 \mathrm{H}, \mathrm{NH}), 5.89$ (ddt, $J=$ 11.3, 11.3, $5.8 \mathrm{~Hz}, 1 \mathrm{H}, 2-\mathrm{H}$, Alloc $), 7.54,7.58(2 \times \mathrm{d}, J=8.3 \mathrm{~Hz}, 2 \mathrm{H}, \mathrm{Ar}-\mathrm{H}), 8.21,8.25(\mathrm{~d}, J=$ 8.3 Hz, $2 \mathrm{H}, \mathrm{Ar}-\mathrm{H}) ;{ }^{13} \mathrm{C} \mathrm{NMR}\left(62.9 \mathrm{MHz}, \mathrm{CDCl}_{3}\right): \delta=16.0,16.5(+, \mathrm{C}-4, a-T h r), 23.1,24.3(-$, C-4, Pro), $28.0\left[+, \mathrm{C}\left(\mathrm{CH}_{3}\right)_{3}\right], 29.3,30.4$ (-, C-3, Pro), 46.0, 46.3 (-, C-5, Pro), 57.2, 57.6 (+, C2, a-Thr), 58.8, 58.9 (+, C-2, Pro), 65.2, 65.5 (-, Bzl-C), 65.67, 65.72 (-, C-1, Alloc), 70.79, 70.85 (+, C-3, a-Thr), 117.3, 117.6 (-, C-3, Alloc), 123.4, 123.5 (+, Ar-C), 128.0, 128.3 (+, ArC), 132.1, $132.4(+, \mathrm{C}-2$, Alloc $), 141.9,142.4$ ( $\left.\mathrm{C}_{\text {quat }}, \mathrm{Ar}-\mathrm{C}\right), 147.3,147.5$ ( $\left.\mathrm{C}_{\text {quat }}, \mathrm{Ar}-\mathrm{C}\right), 153.2$, 154.0 ( $\left.\mathrm{C}_{\text {quat }}, \mathrm{NCO}_{2}\right), 155.4,155.8\left(\mathrm{C}_{\text {quat }}, \mathrm{NCO}_{2}\right), 168.4\left(\mathrm{C}_{\text {quat }}, \mathrm{C}-1\right), 171.9,172.4\left(\mathrm{C}_{\text {quat }}, \mathrm{C}-1\right)$; IR 
(film): nu(tilde) $=3317 \mathrm{~cm}^{-1}, 3082,2979,2882,1700,1521,1399,1348,1162 ; \mathrm{MS}$ (EI, $\left.70 \mathrm{eV}\right)$, $m / z(\%)=535(1)\left[\mathrm{M}^{+}\right], 479(3)\left[\mathrm{M}^{+}-\mathrm{C}_{4} \mathrm{H}_{8}\right], 434(22)\left[\mathrm{M}^{+}-\mathrm{C}_{5} \mathrm{H}_{9} \mathrm{O}_{2}\right], 321(14), 281(8), 170$ (36), 114 (100), 70 (94) $\left[\mathrm{C}_{4} \mathrm{H}_{8} \mathrm{~N}^{+}\right], 57$ (39) $\left[\mathrm{C}_{4} \mathrm{H}_{9}{ }^{+}\right], 41$ (21) $\left[\mathrm{C}_{3} \mathrm{H}_{5}{ }^{+}\right]$; HRMS (EI): calcd for $\mathrm{C}_{25} \mathrm{H}_{33} \mathrm{~N}_{3} \mathrm{O}_{10}:$ 535.2166; found 535.2166.

Alloc-a-(R)-Thr(Boc-Ile-Pro)-ONb (156): Ester 154 (0.315 g, 0.59 mmol) was deprotected with TFA ( $5 \mathrm{~mL})$ for $40 \mathrm{~min}$. All volatiles were removed, the residue was taken up with toluene $(2 \times$ $20 \mathrm{~mL}$ ) which was distilled off to remove the last traces of TFA. The resultant amino ester was coupled with Boc-Ile-OH 155 (0.15 g, 0.65 mmol) using EDC (0.127 g, 0.66 mmol), HOAt (89 mg, $0.66 \mathrm{mmol})$, DIEA (0.80 g, $0.62 \mathrm{mmol})$ and TMP $(0.214 \mathrm{~g}, 1.77 \mathrm{mmol})$ in $\mathrm{CH}_{2} \mathrm{Cl}_{2}(5 \mathrm{~mL})$ according to GP 5 for $15 \mathrm{~h}$. The crude product, after the usual aqueous work-up (GP 5), was finally purified by column chromatography (EtOAc/hexanes $\left.1: 2, R_{\mathrm{f}}=0.29\right)$ to give $156(0.34 \mathrm{~g}$, $89 \%)$ as white foam. $[\alpha]_{20}^{D}-15.7\left(c=0.42, \mathrm{CHCl}_{3}\right) ;{ }^{1} \mathrm{H}$ NMR $\left(250 \mathrm{MHz}, \mathrm{CDCl}_{3}\right): \delta=0.88(\mathrm{t}, J$ $=7.3 \mathrm{~Hz}, 3 \mathrm{H}, 5-\mathrm{H}$, Ile $), 0.94\left(\mathrm{~d}, J=6.8 \mathrm{~Hz}, 3 \mathrm{H}, 1^{\prime}-\mathrm{H}\right.$, Ile $), 0.99-1.21\left(\mathrm{~m}, 1 \mathrm{H}, 4-\mathrm{H}_{\mathrm{a}}\right.$, Ile $), 1.35$ (d, $J=6.8 \mathrm{~Hz}, 3 \mathrm{H}, 4-\mathrm{H}, a-T h r), 1.42$ [s, $\left.9 \mathrm{H}, \mathrm{C}\left(\mathrm{CH}_{3}\right)_{3}\right], 1.64-1.82\left(\mathrm{~m}, 1 \mathrm{H}, 4-\mathrm{H}_{\mathrm{b}}\right.$, Ile), 1.84-2.27 (m, 5 H, 3-H, Ile, 3-H, 4-H, Pro), 3.54-3.72 (m, 1 H, 5-H, Pro), 3.78-3.92 (m, 1 H, 5-H 4.18-4.33 (m, 2 H, 2-H, Pro, 2-H, Ile), 4.56 (d, J=5.3 Hz, 2 H, 1-H, Alloc), 4.62 (dd, $J=3.0$, $7.0 \mathrm{~Hz}, 1 \mathrm{H}, 2-\mathrm{H}, a-T h r), 5.07-5.45$ (m, $6 \mathrm{H}, 3-\mathrm{H}$, Alloc, 3-H, $a-T h r, \mathrm{Bzl}-\mathrm{H}, \mathrm{NH}), 5.90$ (ddt, $J=$ 11.3, 11.3, $5.3 \mathrm{~Hz}, 1 \mathrm{H}, 2-\mathrm{H}$, Alloc), $6.58(\mathrm{~d}, J=9.0 \mathrm{~Hz}, 1 \mathrm{H}, \mathrm{NH}), 7.57$ (d, $J=9.0 \mathrm{~Hz}, 2 \mathrm{H}, \mathrm{Ar}-$ $\mathrm{H}), 8.21(\mathrm{~d}, J=9.0 \mathrm{~Hz}, 2 \mathrm{H}, \mathrm{Ar}-\mathrm{H}) ;{ }^{13} \mathrm{C} \mathrm{NMR}\left(62.9 \mathrm{MHz}, \mathrm{CDCl}_{3}\right): \delta=11.0(+, \mathrm{C}-5$, Ile $), 15.1$ (+, C-1', Ile $), 16.7(+, \mathrm{C}-4, a-T h r), 24.1(-, \mathrm{C}-4$, Pro $), 25.2(-, \mathrm{C}-4$, Ile $), 28.2\left[+, \mathrm{C}\left(\mathrm{CH}_{3}\right)_{3}\right], 28.8$ (-, C-3, Pro), 37.7 (+, C-3, Ile), 47.4 (-, C-5, Pro), 56.0 (+, C-2), $57.6(+$, C-2), 59.3 (+, C-2), 65.5 (-, Bzl-C), 65.7 (-, C-1, Alloc), 71.2 (+, C-3), 117.5 (-, C-3, Alloc), 123.6, 128.4 (+, Ar-C), 132.6 (+, C-2, Alloc $), 142.6,147.6\left(\mathrm{C}_{\text {quat }}, \mathrm{Ar}-\mathrm{C}\right), 155.5,156.0\left(\mathrm{C}_{\text {quat }}, \mathrm{NCO}_{2}\right), 168.6,171.4,171.6$ $\left(\mathrm{C}_{\text {quat }}, \mathrm{C}-1\right)$; IR $(\mathrm{KBr}): \mathrm{nu}($ tilde $)=3396 \mathrm{~cm}^{-1}, 3323,3083,2974,2936,2878,1749,1715,1638$, 
$1525,1444,1348,1248,1171 ; \mathrm{MS}(\mathrm{ESI})$, positive $m / z(\%)=671(30)\left[\mathrm{M}+\mathrm{Na}^{+}\right], 1319(100)$ $\left[2 \mathrm{M}+\mathrm{Na}^{+}\right]$; negative $m / z(\%)=647(15)\left[\mathrm{M}-\mathrm{H}^{+}\right]$.

Boc-a-Thr(Boc-Ile-Pro)-ONb (157): $\mathrm{Pd}\left(\mathrm{PPh}_{3}\right)_{4}(1.5 \mathrm{mg}, 1.30 \mu \mathrm{mol})$ and then immediately $\mathrm{Bu}_{3} \mathrm{SnH}$ (66 mg, $0.23 \mathrm{mmol}$ ) were added to a vigorously stirred solution of the depsipeptide 156 $(65 \mathrm{mg}, 0.10 \mathrm{mmol})$ and $\mathrm{Boc}_{2} \mathrm{O}(87 \mathrm{mg}, 0.40 \mathrm{mmol})$ in anhydrous degassed DMA $(2 \mathrm{~mL})$ and stirring continued for a further $80 \mathrm{~min}$. All volatiles were removed under reduced pressure and the residue was purified by column chromatography (EtOAc/hexanes $\left.1: 2, R_{\mathrm{f}}=0.19\right)$ to give 157 $(60 \mathrm{mg}, 90 \%)$ as a faint yellow viscous oil. $[\alpha]_{20}^{D}-14.2\left(c=0.60, \mathrm{CHCl}_{3}\right) ;{ }^{1} \mathrm{H} \mathrm{NMR}(250 \mathrm{MHz}$, $\left.\mathrm{CDCl}_{3}\right): \delta=0.89\left(\mathrm{t}, J=7.3 \mathrm{~Hz}, 3 \mathrm{H}, 5-\mathrm{H}\right.$, Ile), $0.97\left(\mathrm{~d}, J=6.8 \mathrm{~Hz}, 3 \mathrm{H}, 1^{\prime}-\mathrm{H}\right.$, Ile), 0.99-1.21 (m, $1 \mathrm{H}, 4-\mathrm{H}_{\mathrm{a}}$, Ile $), 1.32(\mathrm{~d}, J=6.5 \mathrm{~Hz}, 3 \mathrm{H}, 4-\mathrm{H}, a-T h r), 1.42\left[\mathrm{~s}, 9 \mathrm{H}, \mathrm{C}\left(\mathrm{CH}_{3}\right)_{3}\right], 1.44[\mathrm{~s}, 9 \mathrm{H}$, $\left.\mathrm{C}\left(\mathrm{CH}_{3}\right)_{3}\right], 1.49-1.63\left(\mathrm{~m}, 1 \mathrm{H}, 4-\mathrm{H}_{\mathrm{b}}\right.$, Ile $), 1.62-1.85$ (m, $1 \mathrm{H}, 3-\mathrm{H}$, Ile $), 1.87-2.31$ (m, $4 \mathrm{H}, 3-\mathrm{H}, 4-$ H, Pro), 3.51-3.70 (m, $1 \mathrm{H}, 5-\mathrm{H}_{\mathrm{a}}$, Pro), 3.75-3.88 (m, 1 H, 5-H $\mathrm{H}_{\mathrm{b}}$ Pro), 4.21-4.37 (m, 2 H, 2-H, Pro, 2-H, Ile), 4.53 (dd, J=3.0, 7.0 Hz, $1 \mathrm{H}, 2-\mathrm{H}, a-T h r), 5.16$ (d, $J=9.3 \mathrm{~Hz}, 1 \mathrm{H}, \mathrm{NH}), 5.32$ (d, $J=3.5 \mathrm{~Hz}, 2 \mathrm{H}, \mathrm{Bzl}-\mathrm{H}), 6.17$ (d, $J=7.0 \mathrm{~Hz}, 1 \mathrm{H}, \mathrm{NH}), 7.56(\mathrm{~d}, J=8.8 \mathrm{~Hz}, 2 \mathrm{H}, \mathrm{Ar}-\mathrm{H}), 8.21(\mathrm{~d}, J$ $=8.8 \mathrm{~Hz}, 2 \mathrm{H}, \mathrm{Ar}-\mathrm{H})$; The peak of Bzl-H masked absorption for $3-\mathrm{H}$ of $a-T h r ;{ }^{13} \mathrm{C} \mathrm{NMR}(62.9$ $\left.\mathrm{MHz}, \mathrm{CDCl}_{3}\right): \delta=11.1(+, \mathrm{C}-5$, Ile $), 15.3\left(+, \mathrm{C}-1^{\prime}\right.$, Ile $), 16.8(+, \mathrm{C}-4, a-T h r), 24.0$ (-, C-4, Pro), $25.2(-, \mathrm{C}-4$, Ile $), 28.2\left[+, 2 \times \mathrm{C}_{\left.\left(\mathrm{CH}_{3}\right)_{3}\right],} 28.8\right.$ (-, C-3, Pro), 37.8 (+, C-3, Ile), 47.3 (-, C-5, Pro),

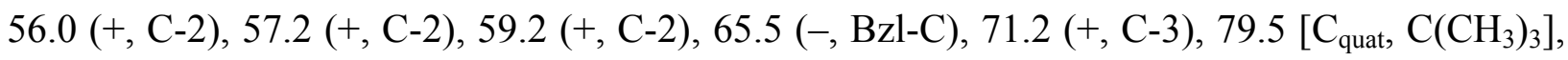
$79.9\left[\mathrm{C}_{\text {quat }}, \mathrm{C}\left(\mathrm{CH}_{3}\right)_{3}\right], 123.7,128.4(+, \mathrm{Ar}-\mathrm{C}), 142.6,147.6\left(\mathrm{C}_{\text {quat }}, \mathrm{Ar}-\mathrm{C}\right), 155.6\left(\mathrm{C}_{\text {quat }}, 2 \times \mathrm{NCO}_{2}\right)$, 169.0, 171.3, 171.6 (C $\left.\mathrm{C}_{\text {quat }}, \mathrm{C}-1\right)$; IR $(\mathrm{KBr}): \mathrm{nu}(\mathrm{tilde})=2979 \mathrm{~cm}^{-1}, 2932,1751,1717,1646,1526$, 1456, 1368, 1348, 1251, 1165; MS (EI, $70 \mathrm{eV}), m / z(\%)=664(1)\left[\mathrm{M}^{+}\right], 608(3)\left[\mathrm{M}^{+}-\mathrm{C}_{4} \mathrm{H}_{8}\right]$, 491 (8), 186 (45), 130 (100), 86 (69), 57 (45) $\left[\mathrm{C}_{4} \mathrm{H}_{9}{ }^{+}\right]$; HRMS (EI): calcd for $\mathrm{C}_{32} \mathrm{H}_{48} \mathrm{~N}_{4} \mathrm{O}_{11}$ : 664.3320; found 664.3320. 
Boc-(3-Ncp)Ala-a-Thr(Boc-Ile-Pro)-ONb (158): DCC (29 mg, $0.14 \mathrm{mmol})$ was added to an icecold solution of $N$-Boc protected (3-nitrocyclopropyl)alanine (2S)-143 (39 $\mathrm{mg}, 0.14 \mathrm{mmol}$ ) (the mixture of diastereomers partially enriched with $\left(1^{\prime} S, 2^{\prime} R\right)$-isomer was used for this synthesis) depsipeptide 156 (30 mg, $46.24 \mu \mathrm{mol}$ ) and HOBt (19 mg, hydrate, 87\% purity, $0.14 \mathrm{mmol}$ ) in $\mathrm{CH}_{2} \mathrm{Cl}_{2}(5 \mathrm{~mL})$ Cooling bath was then removed and stirring continued for a further $90 \mathrm{~min}$. The mixture was then degassed with three freeze-pump-thaw cycles at $-70{ }^{\circ} \mathrm{C} . \mathrm{Pd}\left(\mathrm{PPh}_{3}\right)_{4}(1.1 \mathrm{mg}$, $1.0 \mu \mathrm{mol}$ ) and $\mathrm{Bu}_{3} \mathrm{SnH}(28 \mathrm{mg}, 96.20 \mu \mathrm{mol}$ ) was then added and the mixture was stirred for a further $3 \mathrm{~h}$. AcOH ( 1 drop) and then 5 min later $N, N$-dimethylaminopropylamine (1 drop) were added, and after an additional $10 \mathrm{~min}$, the mixture was diluted with $\mathrm{Et}_{2} \mathrm{O}(50 \mathrm{~mL})$ and filtered. The residue obtained after usual aqueous work-up (GP 5) was dissolved in $\mathrm{Et}_{2} \mathrm{O}(4 \mathrm{~mL})$, the resultant solution was filtered and concentrated again under reduced pressure. The crude product was purified by column chromatography (twice, EtOAc/hexanes $1: 2, R_{\mathrm{f}}=0.11$ ) and finally by preparative TLC (EtOAc/hexanes 1:2.5, 3 runs) to give 158 (36 mg, 95\%) as an extremely viscous colorless oil or white foam. $[\alpha]_{20}^{D}-4.3\left(c=0.40, \mathrm{CHCl}_{3}\right) ;{ }^{1} \mathrm{H} \mathrm{NMR}\left(500 \mathrm{MHz}, \mathrm{CDCl}_{3}\right)$ : $\delta=0.87(\mathrm{t}, J=7.0 \mathrm{~Hz}, 3 \mathrm{H}, 5-\mathrm{H}$, Ile $), 0.94\left(\mathrm{~d}, J=6.8 \mathrm{~Hz}, 3 \mathrm{H}, 1^{\prime}-\mathrm{H}\right.$, Ile $), 0.99$ [ddd, $J=6.5,6.5$, $6.5 \mathrm{~Hz}, 1 \mathrm{H}, 3^{\prime}-\mathrm{H}_{\mathrm{a}},(3-N c p)$ Ala $], 1.14-1.24\left[\mathrm{~m}, 2 \mathrm{H}, 4-\mathrm{H}_{\mathrm{a}}\right.$, Ile, 3'- $\mathrm{H}_{\mathrm{b}},(3-N c p)$ Ala $], 1.37$ (d, J= $6.8 \mathrm{~Hz}, 3 \mathrm{H}, 4-\mathrm{H}, a-T h r), 1.40\left[\mathrm{~s}, 9 \mathrm{H}, \mathrm{C}\left(\mathrm{CH}_{3}\right)_{3}\right], 1.41\left[\mathrm{~s}, 9 \mathrm{H}, \mathrm{C}\left(\mathrm{CH}_{3}\right)_{3}\right], 1.56-1.67$ [m, $2 \mathrm{H}, 4-$ $\mathrm{H}_{\mathrm{b}}$, Ile, 1'-H, (3-Ncp)Ala ], 1.70-1.80 (m, 1 H, 3-H, Ile), 1.85-2.01 [m, 4 H, 4-H, Pro, 3-H, (3Ncp)Ala], 2.06-2.15 (m, $1 \mathrm{H}, 3-\mathrm{H}_{\mathrm{a}}$, Pro), 2.17-2.25 (m, $1 \mathrm{H}, 3-\mathrm{H}_{\mathrm{b}}$, Pro) 3.64 (ddd, J=8.0, 8.0, 8.0 Hz, $1 \mathrm{H}, 5-\mathrm{H}_{\mathrm{a}}$, Pro ), 3.94-4.02 (m, $1 \mathrm{H}, 5-\mathrm{H}_{\mathrm{b}}$, Pro), 4.04-4.16 (m, $\left.2 \mathrm{H}, 2-\mathrm{H}\right), 4.27$ (dd, J= 9.5, 9.5 Hz, $1 \mathrm{H}, 2-\mathrm{H}), 4.63-4.72\left[\mathrm{~m}, 1.7 \mathrm{H}, 2-\mathrm{H}, 0.72^{\prime}-\mathrm{H},(3-N c p) A l a\right], 5.27$ (d, J = $14.5 \mathrm{~Hz}$, $\left.1 \mathrm{H}, \mathrm{H}_{\mathrm{a}}, \mathrm{Bzl}-\mathrm{H}\right), 5.34\left(\mathrm{~d}, J=14.5 \mathrm{~Hz}, 2 \mathrm{H}, \mathrm{H}_{\mathrm{b}}, \mathrm{Bzl}-\mathrm{H}\right), 5.38-5.45(\mathrm{~m}, 1 \mathrm{H}, a-T h r), 5.49$ (d, $J=$ $8.5 \mathrm{~Hz}, 1 \mathrm{H}, \mathrm{NH}), 5.66(\mathrm{~d}, J=9.0 \mathrm{~Hz}, 1 \mathrm{H}, \mathrm{NH}), 7.52(\mathrm{~d}, J=8.5 \mathrm{~Hz}, 2 \mathrm{H}, \mathrm{Ar}-\mathrm{H}), 8.12-8.20$ (br, $1 \mathrm{H}, \mathrm{NH}), 8.20(\mathrm{~d}, J=8.5 \mathrm{~Hz}, 2 \mathrm{H}, \mathrm{Ar}-\mathrm{H})$; The peak of $\mathrm{H}_{\mathrm{b}}$ of Bzl-H masked absorption for $0.7 \mathrm{H}$, $2^{\prime}-\mathrm{H}$ of $(3-N c p) A l a ;{ }^{13} \mathrm{C} \mathrm{NMR}\left(125.7 \mathrm{MHz}, \mathrm{CDCl}_{3}\right): \delta=10.6(+, \mathrm{C}-5$, Ile $), 14.8\left(+, \mathrm{C}-1^{\prime}\right.$, Ile $)$, $17.1(+, \mathrm{C}-4, a-T h r), 17.26\left[-, \mathrm{C}-3^{\prime},(3-N c p) A l a\right], 21.9$ [+, C-1', (3-Ncp)Ala], 24.7 (-, C-4, Pro), 
25.3 (-, C-4, Ile), $28.2\left[+, \mathrm{C}\left(\mathrm{CH}_{3}\right)_{3}\right], 28.3$ [+, C( $\left.\left(\mathrm{CH}_{3}\right)_{3}\right], 29.0$ (-, C-3, Pro), 34.4 [-, C-3, (3Ncp)Ala], 37.7 (+, C-3, Ile), 47.8 (-, C-5, Pro), 52.9 (+, C-2), 56.1 (+, C-2), 59.4 (+, C-2), 59.6 [+, C-2 and C-2', (3-Ncp)Ala $], 65.4(-, \mathrm{Bzl}-\mathrm{C}), 70.6(+, \mathrm{C}-3), 79.6\left[\mathrm{C}_{\text {quat }}, \mathrm{C}\left(\mathrm{CH}_{3}\right)_{3}\right], 80.0\left[\mathrm{C}_{\text {quat, }}\right.$, $\left.\mathrm{C}\left(\mathrm{CH}_{3}\right)_{3}\right], 123.7,128.3(+, \mathrm{Ar}-\mathrm{C}), 142.5,147.6\left(\mathrm{C}_{\text {quat }}, \mathrm{Ar}-\mathrm{C}\right), 154.9,155.8\left(\mathrm{C}_{\text {quat }}, 2 \times \mathrm{NCO}_{2}\right)$, 167.9, 171.3, 171.5, $172.2\left(\mathrm{C}_{\text {quat }}, \mathrm{C}-1\right)$; IR $(\mathrm{KBr})$ : nu(tilde $)=2977 \mathrm{~cm}^{-1}, 2935,2879,1751,1700$, $1630,1543,1525,1448,1368,1348,1252,1170 ;$ MS (ESI), positive $m / z(\%)=843(100)[\mathrm{M}+$ $\left.\mathrm{Na}^{+}\right]$; negative $m / z(\%)=820(100)\left[\mathrm{M}-\mathrm{H}^{+}\right]$.

Alloc-a-Thr-OSEM (159): To a vigorously stirred suspension of $\mathrm{Li}_{2} \mathrm{CO}_{3}(0.122 \mathrm{~g}, 1.65 \mathrm{mmol})$ in

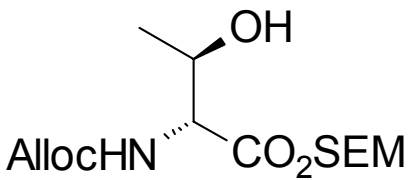
a solution of $148(0.305 \mathrm{~g}, 1.50 \mathrm{mmol})$ in DMF (6 mL), SEMCl $(0.2$ g, $1.2 \mathrm{mmol}$ ) was added for $5 \mathrm{~min}$ and stirring continued for $23 \mathrm{~h}$. The second portion of SEMCl $(0.1 \mathrm{~g}, 0.6 \mathrm{mmol})$ was then added and after stirring for an additional 3 $\mathrm{h}$ the mixture was diluted with $\mathrm{Et}_{2} \mathrm{O}(50 \mathrm{~mL})$, washed with water $(8 \times 10 \mathrm{~mL})$, brine $(2 \times 5 \mathrm{~mL})$, dried, filtered and concentrated under reduced pressure. The residue was purified by column chromatography ( EtOAc/hexanes $\left.1: 3, R_{\mathrm{f}}=0.22\right)$ to give $159(0.288 \mathrm{~g}, 58 \%)$ as a colorless oil. $[\alpha]_{20}^{D}-7.3\left(c=0.33, \mathrm{CHCl}_{3}\right) ;{ }^{1} \mathrm{H} \mathrm{NMR}\left(250 \mathrm{MHz}, \mathrm{CDCl}_{3}\right): \delta=0.02\left[\mathrm{~s}, 9 \mathrm{H}, \mathrm{Si}\left(\mathrm{CH}_{3}\right)_{3}\right], 0.96(\mathrm{t}$, $\left.J=8.4 \mathrm{~Hz}, 2 \mathrm{H}, 2^{\prime}-\mathrm{H}, S E M\right), 1.23(\mathrm{~d}, J=6.5 \mathrm{~Hz}, 3 \mathrm{H}, 4-\mathrm{H}), 2.73-2.82(\mathrm{br}, 1 \mathrm{H}, \mathrm{OH}), 3.72$ (t, $J=$ $\left.8.4 \mathrm{~Hz}, 2 \mathrm{H}, 1^{\prime}-\mathrm{H}, S E M\right), 4.11-4.28$ (m, $\left.1 \mathrm{H}, 3-\mathrm{H}\right), 4.47$ (dd, $\left.J=7.8,4.0 \mathrm{~Hz}, 1 \mathrm{H}, 2-\mathrm{H}\right), 4.59$ (d, $J$ $=5.5 \mathrm{~Hz}, 2 \mathrm{H}, 1-\mathrm{H}$, Alloc $), 5.22-5.29(\mathrm{~m}, 1 \mathrm{H}$, trans $-3-\mathrm{H}$, Alloc $), 5.30-5.39(\mathrm{~m}, 1 \mathrm{H}$, cis $-3-\mathrm{H}$, Alloc), 5.33 (d, $J=6.0 \mathrm{~Hz}, 1 \mathrm{H}, 1-\mathrm{H}, S E M), 5.66$ (d, $J=7.8 \mathrm{~Hz}, 1 \mathrm{H}, \mathrm{NH}), 5.89$ (ddt, $J=10.4$, 10.4, $5.6 \mathrm{~Hz}, 1 \mathrm{H}, 2-\mathrm{H}$, Alloc $) ;{ }^{13} \mathrm{C} \mathrm{NMR}\left(62.9 \mathrm{MHz}, \mathrm{CDCl}_{3}\right): \delta=-1.7\left[+, \mathrm{Si}\left(\mathrm{CH}_{3}\right)_{3}\right], 17.7(-, \mathrm{C}-$ 2', SEM), 18.6 (+,C-4), 59.3 (+, C-2), 65.8 (-, C-1', SEM), 67.9 (-, C-1, Alloc), 68.2 (+, C-3), 89.6 (-, C-1, SEM), 117.7 (-, C-3, Alloc), 132.2 (+, C-2, Alloc), $156.2\left(\mathrm{C}_{\text {quat }}, \mathrm{NCO}_{2}\right), 169.9$ $\left(\mathrm{C}_{\text {quat}}, \mathrm{C}-1\right)$; IR (film): nu(tilde) $=3390 \mathrm{~cm}^{-1}, 3086,2954,2898,1728,1521,1457,1412,1382$, 1250, 1115; MS (ESI), positive $m / z(\%)=356(100)\left[\mathrm{M}+\mathrm{Na}^{+}\right]$. 
N-Fmoc-3-(2R,1 R,2 R)-(trans-2'-Nitrocyclopropyl)alanine $\left(2 R, 1^{\prime} R, 2^{\prime} R\right)-(\mathbf{1 6 0}):$ A solution of

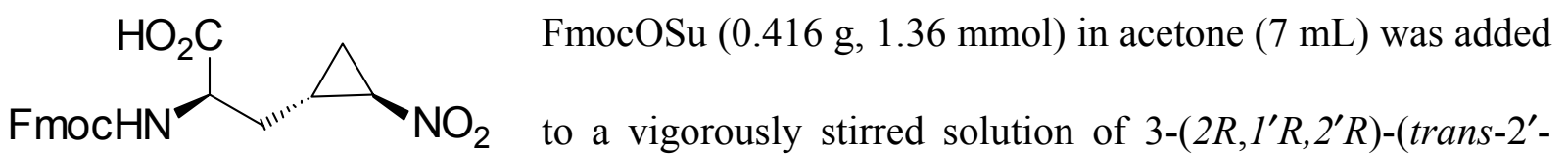
nitrocyclopropyl)alanine $\left(2 R, 1^{\prime} R, 2^{\prime} R\right)-83(0.2 \mathrm{~g}, 1.15 \mathrm{mmol})$ and $\mathrm{NaHCO}_{3}(0.202 \mathrm{~g}, 2.40 \mathrm{mmol})$ in water $(5 \mathrm{~mL})$ (if precipitate formed acetone and/or water were added to obtain homogeneous solution) and stirring continued for an additional $3 \mathrm{~h}$. Acetone was then removed under reduced pressure and $\mathrm{pH}$ of the residual water solution was adjusted to 1 with $3 \mathrm{M} \mathrm{HCl}$. The resulting emulsion was extracted with $\mathrm{Et}_{2} \mathrm{O}(30 \mathrm{~mL})$ and the ethereal layer was then back extracted with $3 \%$ aqueous $\mathrm{NaHCO}_{3}(5 \times 10 \mathrm{~mL}$, TLC control of the completeness of extraction was necessary). The aqueous fractions were pooled, washed with $\mathrm{Et}_{2} \mathrm{O}(2 \times 10 \mathrm{~mL})$, acidified to $\mathrm{pH} 2$ with $3 \mathrm{M} \mathrm{HCl}$ and the resulting emulsion was extracted with $\mathrm{Et}_{2} \mathrm{O}(40 \mathrm{~mL})$. The organic phase was washed with $3 \mathrm{M} \mathrm{HCl}(2 \times 10 \mathrm{~mL})$, water $(3 \times 10 \mathrm{~mL})$, brine $(2 \times 5 \mathrm{~mL})$, dried, filtered and concentrated under reduced pressure. The residue was triturated with cold pentane and filtered. The resulting semisolid was dried at 0.02 Torr for prolonged time to give $\left(2 R, 1^{\prime} R, 2^{\prime} R\right)-\mathbf{1 6 0}$ $(0.423 \mathrm{~g}, 93 \%)$ as a white foam. $R_{\mathrm{f}}=0.08$ EtOAc/hexanes $1: 1 ;$ m.p. (softening) $50-57^{\circ} \mathrm{C} ;[\alpha]_{20}^{D}$ $-56.7\left(c=0.36, \mathrm{CHCl}_{3}\right) ;{ }^{1} \mathrm{H}$ NMR $\left(250 \mathrm{MHz}, \mathrm{CDCl}_{3}\right): \delta=0.71-0.82\left(\mathrm{~m}, 0.4 \mathrm{H}, 3^{\prime}-\mathrm{H}_{\mathrm{a}}\right), 1.11$ (ddd $\left.J=6.2,6.2,6.2 \mathrm{~Hz}, 0.6 \mathrm{H}, 3^{\prime}-\mathrm{H}_{\mathrm{a}}\right), 1.17-1.51\left(\mathrm{~m}, 1 \mathrm{H}, 3^{\prime}-\mathrm{H}_{\mathrm{b}}\right), 1.75-2.13(\mathrm{~m}, 2 \mathrm{H}, 3-\mathrm{H})$, 3.61-3.76, 3.76-3.89 (2 × m, 1 H, 1'-H), 3.99-4.12 (m, 0.5 H, 2-H), 4.12-4.27 (m, 1.5 H, 2"-H, 2-H), 4.27-4.56 (m, 2 H, 1'-H), 4.56-4.69, 4.71-4.87 (2 × m, $\left.1 \mathrm{H}, 2^{\prime}-\mathrm{H}\right), 5.48$ (d, J = 7.0 Hz, 0.6 H, NH), 7.01-7.13 (m, 0.4 H, NH), 7.23-7.42 (m, $5 \mathrm{H}, \mathrm{Ar}-\mathrm{H}), 7.42-7.61(\mathrm{~m}, 2 \mathrm{H}, \mathrm{Ar}-\mathrm{H})$, $7.75(\mathrm{~d}, J=7.5 \mathrm{~Hz}, 2 \mathrm{H}, \mathrm{Ar}-\mathrm{H}) ;{ }^{13} \mathrm{C} \mathrm{NMR}\left(62.9 \mathrm{MHz}, \mathrm{CDCl}_{3}\right): \delta=17.3,17.6\left(-, \mathrm{C}-3^{\prime}\right), 21.4$, $22.0\left(+, \mathrm{C}-1^{\prime}\right), 32.8,33.2(-, \mathrm{C}-3), 46.8\left(+, \mathrm{C}-2^{\prime \prime}\right), 53.0(+, \mathrm{C}-2), 59.0\left(+, \mathrm{C}-2^{\prime}\right), 66.7,67.0(-, \mathrm{C}-$ $\left.1^{\prime \prime}\right), 119.8(+, \mathrm{Ar}-\mathrm{C}), 124.2,124.4(+, \mathrm{Ar}-\mathrm{C}), 124.8(+, \mathrm{Ar}-\mathrm{C}), 126.9,127.6(+, \mathrm{Ar}-\mathrm{C}), 141.1$ $\left(\mathrm{C}_{\text {quat }}, \mathrm{Ar}-\mathrm{C}\right), 143.0,143.3\left(\mathrm{C}_{\text {quat }}, \mathrm{Ar}-\mathrm{C}\right), 143.3,143.5\left(\mathrm{C}_{\text {quat }}, \mathrm{Ar}-\mathrm{C}\right), 155.9,156.8\left(\mathrm{C}_{\text {quat }}, \mathrm{NCO}_{2}\right)$, 173.8, $174.6\left(\mathrm{C}_{\text {quat }}, \mathrm{C}-1\right)$; IR $(\mathrm{KBr}): \mathrm{nu}($ tilde $)=3331 \mathrm{~cm}^{-1}, 2965,2934,1736,1689,1653,1525$, 
1455, 1391, 1367, 1251, 1176; MS (EI, $70 \mathrm{eV}), \mathrm{m} / z(\%)=396(1)\left[\mathrm{M}^{+}\right], 196(36)\left[\mathrm{C}_{14} \mathrm{H}_{12} \mathrm{O}^{+}\right]$, 178 (51) $\left[\mathrm{C}_{14} \mathrm{H}_{10}{ }^{+}\right], 165$ (100), 139 (6); HRMS (EI): calcd for $\mathrm{C}_{21} \mathrm{H}_{20} \mathrm{~N}_{2} \mathrm{O}_{6}$ : 396.1321; found 396.1321; elemental analysis calcd (\%) for $\mathrm{C}_{21} \mathrm{H}_{20} \mathrm{~N}_{2} \mathrm{O}_{6}$ (396.4): C 63.63, H 5.09, N 7.07; found C 63.56, H 5.21, N 7.00.

Boc-( $\beta M e)$ Phe-Ile-OTMSE (162): To a solution of Boc-Ile-OH 155 (0.5 g, 2.16 mmol), 2trimethylsilylethanol $(0.256 \mathrm{~g}, 2.16 \mathrm{mmol})$ and DMAP $(0.053 \mathrm{~g}, 0.43 \mathrm{mmol})$ in $\mathrm{CH}_{2} \mathrm{Cl}_{2}(5 \mathrm{~mL})$ EDC $(0.427 \mathrm{~g}, 2.23 \mathrm{mmol})$ was added at $-10^{\circ} \mathrm{C}$ and the mixture was then allowed to warm to $20{ }^{\circ} \mathrm{C}$ and stirred for $3 \mathrm{~h}$. The mixture was then diluted with $\mathrm{Et}_{2} \mathrm{O}(50 \mathrm{~mL})$ and washed with water $(2 \times 10 \mathrm{~mL}), 1 \mathrm{M} \mathrm{NaHSO}_{4}(3 \times 10 \mathrm{~mL})$, water $(2 \times 10 \mathrm{~mL})$, brine $(2 \times 5 \mathrm{~mL})$, dried, filtered and concentrated to give after prolonged drying at 5 Torr crude Boc-Ile-OTMSE $(0.47 \mathrm{~g}, 66 \%$ crude yield) as a colorless oil which was used without purification for the next step. This material (0.47 $\mathrm{g}, 1.42 \mathrm{mmol})$ and $p \mathrm{TsOH} \cdot \mathrm{H}_{2} \mathrm{O}(0.297 \mathrm{~g}, 1.56 \mathrm{mmol})$ were dissolved in dry benzene $(15 \mathrm{~mL})$ and heated under reflux for $1.5 \mathrm{~h}$. The mixture was concentrated under reduced pressure and the residue was recrystallized from EtOAc/hexanes to give TsOH $\cdot \mathrm{H}_{2} \mathrm{~N}-\mathrm{Ile}-\mathrm{OTMSE}(0.505 \mathrm{~g}, 88 \%$ crude yield) as a white solid which was used without further purification for the next step. This tosylate $(0.283 \mathrm{~g}, 0.7 \mathrm{mmol})$ was dissolved $\mathrm{Et}_{2} \mathrm{O}(40 \mathrm{~mL})$ and was washed with saturated aqueous $\mathrm{NaHCO}_{3}(3 \times 5 \mathrm{~mL})$, brine $(2 \times 5 \mathrm{~mL})$, dried, filtered and concentrated under reduced pressure to leave $\mathrm{H}_{2} \mathrm{~N}$-Ile-OTMSE $161(0.150 \mathrm{~g}, 93 \%$ crude) as a colorless oil which was directly used for the peptide condensation in $\mathrm{CH}_{2} \mathrm{Cl}_{2}(5 \mathrm{~mL})$ according to GP 5 with $\mathrm{N}$-Boc protected $\beta$-methylphenylalanine $139(0.193 \mathrm{~g}, 0.65 \mathrm{mmol})$ using $\mathrm{EDC}(0.127 \mathrm{~g}, 0.66 \mathrm{mmol})$, HOAt $(0.089 \mathrm{~g}, 0.66 \mathrm{mmol})$ and TMP $(0.089 \mathrm{~g}, 0.73 \mathrm{mmol})$. The organic layer, after aqueous work-up, was concentrated under reduced pressure to leave pure $162(0.275 \mathrm{~g}, 86 \%)$ as a colorless glass. $R_{\mathrm{f}}=0.38 \mathrm{EtOAc} /$ hexanes $1: 4 ;[\alpha]_{20}^{D}-3.3\left(c=0.4, \mathrm{CHCl}_{3}\right) ;{ }^{1} \mathrm{H} \mathrm{NMR}(250 \mathrm{MHz}$, $\left.\mathrm{CDCl}_{3}\right): \delta=0.04\left[\mathrm{~s}, 9 \mathrm{H}, \mathrm{Si}\left(\mathrm{CH}_{3}\right)_{3}\right], 0.79\left(\mathrm{~d}, J=6.8 \mathrm{~Hz}, 3 \mathrm{H}, 1^{\prime}-\mathrm{H}, I l e\right), 0.86(\mathrm{~d}, J=7.4 \mathrm{~Hz}, 3 \mathrm{H}$, 5-H, Ile), 0.82-0.95 (m, $2 \mathrm{H}, 2-\mathrm{H}, T M S E), 0.95-1.15\left(\mathrm{~m}, 1 \mathrm{H}, 4-\mathrm{H}_{\mathrm{a}}\right.$, Ile $), 1.31[\mathrm{~d}, J=7.3 \mathrm{~Hz}, 3 \mathrm{H}$, 
4-H, ( $\beta M e) P h e], 1.40$ [s, $\left.9 \mathrm{H}, \mathrm{C}\left(\mathrm{CH}_{3}\right)_{3}\right], 1.73-1.82(\mathrm{~m}, 1 \mathrm{H}, 3-\mathrm{H}$, Ile $), 3.16$ [quint, $J=6.9 \mathrm{~Hz}$, $1 \mathrm{H}, 3-\mathrm{H},(\beta M e) P h e], 3.95-4.19(\mathrm{~m}, 2 \mathrm{H}, 1-\mathrm{H}, T M S E), 4.20-4.36$ [m, $2 \mathrm{H}, 2-\mathrm{H}$, Ile and 2-H, (ßMe)Phe], $5.15(\mathrm{~d}, J=8.8 \mathrm{~Hz}, 1 \mathrm{H}, \mathrm{NH}), 6.17(\mathrm{~d}, J=8.3 \mathrm{~Hz}, 1 \mathrm{H}, \mathrm{NH}), 7.01-7.33(\mathrm{~m}, 5 \mathrm{H}, \mathrm{Ar}-$ $\mathrm{H})$; the peaks of $\mathrm{C}\left(\mathrm{CH}_{3}\right)_{3}$ and $\mathrm{CH}_{3},(\beta M e)$ Phe masked absorption for $4-\mathrm{H}_{\mathrm{b}}$, Ile; ${ }^{13} \mathrm{C} \mathrm{NMR}(62.9$ $\left.\mathrm{MHz}, \mathrm{CDCl}_{3}\right): \delta=-1.79\left[+, \mathrm{Si}\left(\mathrm{CH}_{3}\right)_{3}\right], 11.3(+, \mathrm{C}-5$, Ile $), 15.0\left(+, \mathrm{C}-1^{\prime}\right.$, Ile $), 16.9[+, \mathrm{C}-4$, (ßMe)Phe], 17.1 (-, C-2, TMSE), 24.9 (-,C-4, Ile), $28.0\left[+, \mathrm{C}\left(\mathrm{CH}_{3}\right)_{3}\right], 37.7(+, \mathrm{C}-3$, Ile $), 42.1$ [+,C-3, $(\beta M e) P h e], 56.2(+, \mathrm{C}-2$, Ile $), 60.0$ [+,C-2, $(\beta M e) P h e], 63.0(-, \mathrm{C}-1, T M S E), 79.4$ [C $\mathrm{C}_{\text {quat }}$, $\left.\mathrm{C}\left(\mathrm{CH}_{3}\right)_{3}\right], 126.5,127.4,128.2(+, \mathrm{Ar}-\mathrm{C}), 142.3\left(\mathrm{C}_{\text {quat }}, \mathrm{Ar}-\mathrm{C}\right), 155.5\left(\mathrm{C}_{\text {quat }}, \mathrm{NCO}_{2}\right), 170.6\left(\mathrm{C}_{\text {quat }}\right.$, $\mathrm{C}-1$, Ile $), 170.8\left[\mathrm{C}_{\text {quat }}, \mathrm{C}-1,(\beta M e) P h e\right]$; IR (film): nu(tilde) $=3500-2500 \mathrm{~cm}^{-1}, 3066,2953,1720$, 1542, 1478, 1369, 1234, 1106, 1078; MS (EI, $70 \mathrm{eV}), m / z(\%)=492(7)\left[\mathrm{M}^{+}\right], 436(4)\left[\mathrm{M}^{+}-\right.$ $\left.\mathrm{C}_{4} \mathrm{H}_{8}\right], 375(8)\left[\mathrm{M}^{+}-\mathrm{C}_{5} \mathrm{H}_{13} \mathrm{OSi}\right], 259$ (38), 178 (52), 134 (58), 73 (40), 57 (100) $\left[\mathrm{C}_{4} \mathrm{H}_{9}^{+}\right]$; HRMS (EI): calcd for $\mathrm{C}_{26} \mathrm{H}_{44} \mathrm{~N}_{2} \mathrm{O}_{5} \mathrm{Si}$ : 492.3020; found 492.3020 .

Fmoc-(2S)-(3-Ncp)Ala-( $\beta M e)$ Phe-Ile-OTMSE (164): The dipeptide 162 (0.268 g, $0.544 \mathrm{mmol})$ was deprotected with $3.9 \mathrm{M} \mathrm{HCl}$ in dioxane $(4 \mathrm{~mL})$ for $1 \mathrm{~h}$. The mixture was then concentrated under reduced pressure and the residue was taken up with $\mathrm{Et}_{2} \mathrm{O}(40 \mathrm{~mL})$ and washed with saturated aqueous $\mathrm{NaHCO}_{3}(3 \times 10 \mathrm{~mL})$, brine $(2 \times 5 \mathrm{~mL})$, dried, filtered and concentrated under reduced pressure to give $\mathrm{H}-(\beta \mathrm{Me})$ Phe-Ile-OTMSE $(0.167 \mathrm{~g}, 78 \%)$ as a colorless oil which was directly coupled with $160(0.178 \mathrm{~g}, 0.449 \mathrm{mmol})$ in $\mathrm{CH}_{2} \mathrm{Cl}_{2}(4 \mathrm{~mL})$ according to $\mathrm{GP} 5$ employing EDC (89 mg, $0.463 \mathrm{mmol})$, HOAt (61 mg, $0.450 \mathrm{mmol})$ and TMP (54 mg, $0.449 \mathrm{mmol})$ to give after usual water work-up crude product which was crystallized twice from $\mathrm{CH}_{2} \mathrm{Cl}_{2} /$ hexanes and finally purified by column chromatography $\left(\mathrm{CHCl}_{3} / \mathrm{EtOAc} 7: 1\right)$ to give $164(0.240 \mathrm{~g}, 73 \%)$ as a white solid. $R_{\mathrm{f}}=0.28 \mathrm{EtOAc} /$ hexanes $1: 2 ;[\alpha]_{20}^{D}-7.5\left(c=0.12, \mathrm{CHCl}_{3}\right) ;{ }^{1} \mathrm{H} \mathrm{NMR}(250 \mathrm{MHz}$, $\left.\mathrm{CDCl}_{3}\right): \delta=0.04\left[\mathrm{~s}, 9 \mathrm{H}, \mathrm{Si}\left(\mathrm{CH}_{3}\right)_{3}\right], 0.79\left(\mathrm{~d}, J=7.0 \mathrm{~Hz}, 3 \mathrm{H}, 1^{\prime}-\mathrm{H}\right.$, Ile $), 0.86(\mathrm{t}, J=7.6 \mathrm{~Hz}, 3 \mathrm{H}$, 5-H, Ile), 0.87-1.14 [m, 4 H, 2-H, TMSE, 2'- $\mathrm{H}_{\mathrm{a}},(3-N c p) A l a, 4-\mathrm{H}_{\mathrm{a}}$, Ile ], 1.24-1.41 (m, $1 \mathrm{H}, 4-\mathrm{H}_{\mathrm{b}}$, 
Ile), $1.31[\mathrm{~d}, \mathrm{~J}=7.0 \mathrm{~Hz}, 3 \mathrm{H}, 4-\mathrm{H},(\beta M e) P h e], 1.49-1.67$ [m, $1 \mathrm{H}, 2^{\prime}-\mathrm{H}_{\mathrm{b}},(3-N c p)$ Ala $], 1.67-1.82$ $[\mathrm{m}, 2 \mathrm{H}, 3-\mathrm{H}$, Ile, 1'-H, (3-Ncp)Ala], 1.82-2.03 [m, $2 \mathrm{H}, 3-\mathrm{H},(3-N c p)$ Ala], 3.21 [quint, $J=$ $7.5 \mathrm{~Hz}, 1 \mathrm{H}, 3-\mathrm{H},(\beta M e) P h e], 3.97-4.15$ (m, $3 \mathrm{H}, 1-\mathrm{H}$, TMSE, 2-H), 4.16-4.54 (m, $5 \mathrm{H}, 2 \times 2-\mathrm{H}$ and $1^{\prime}-\mathrm{H}, 2^{\prime}-\mathrm{H}$, Fmoc), 4.62 [ddd, $\left.J=8.5 \mathrm{~Hz}, 1 \mathrm{H}, 2^{\prime}-\mathrm{H},(3-N c p) A l a\right], 5.49-5.83$ (br, $1 \mathrm{H}, \mathrm{NH}$ ), 6.00-6.34 (br, 1 H, NH), 7.08-7.46 (m, 9 H, Ar-H), 7.52-7.66 (m, 2 H, Ar-H), 7.77 (d, $J=$ 7.3 Hz, $2 \mathrm{H}, \mathrm{Ar}-\mathrm{H}) ;{ }^{13} \mathrm{C} \mathrm{NMR}\left(62.9 \mathrm{MHz}, \mathrm{CDCl}_{3}\right): \delta=-1.79\left[+, \mathrm{Si}\left(\mathrm{CH}_{3}\right)_{3}\right], 11.3(+, \mathrm{C}-5$, Ile $)$, $15.0\left(+, \mathrm{C}-1^{\prime}\right.$, Ile $), 17.1[+, \mathrm{C}-4,(\beta M e) P h e], 17.2$ (-, C-2, TMSE), 17.9 [-, C-3', (3-Ncp)Ala], $22.2\left[+, \mathrm{C}-1^{\prime},(3-N c p) A l a\right], 25.0(-, \mathrm{C}-4$, Ile $), 34.0[-, \mathrm{C}-3,(3-N c p) A l a], 37.9(+, \mathrm{C}-3$, Ile $), 41.9$ [+,C-3, (BMe)Phe], $46.9\left(+, \mathrm{C}-1^{\prime}\right.$, Fmoc $), 54.0$ [+, C-2, (3-Ncp)Ala], $56.3(+, \mathrm{C}-2$, Ile $), 58.7[+$, C-2, (BMe)Phe], 58.9 [+,C-2', (3-Ncp)Ala], 63.3(-, C-1, TMSE), $67.0\left(-, \mathrm{C}-2^{\prime}\right.$, Fmoc), $112.3(+$, Ar-C), 124.9 (+, Ar-C), 126.8 (+, Ar-C), 126.9 (+, Ar-C), 127.0 (+, Ar-C), 127.6 (+, Ar-C), $128.3(+, \mathrm{Ar}-\mathrm{C}), 141.1,141.1\left(\mathrm{C}_{\text {quat }}, \mathrm{Ar}-\mathrm{C}\right), 141.7\left(\mathrm{C}_{\text {quat }}, \mathrm{Ar}-\mathrm{C}\right), 143.5,143.7\left(\mathrm{C}_{\text {quat }}, \mathrm{Ar}-\mathrm{C}\right), 156.0$ $\left(\mathrm{C}_{\text {quat }}, \mathrm{NCO}_{2}\right), 170.2,171.2,171.4\left(\mathrm{C}_{\text {quat }}, \mathrm{C}-1\right)$; IR $(\mathrm{KBr}): \mathrm{nu}($ tilde $)=3341 \mathrm{~cm}^{-1}, 3261,3068$, 2964, 2877, 1732, 1712 1642, 1544, 1368, 1251, 1228; MS (ESI), positive $m / z(\%)=794(30)$ $\left[\mathrm{M}+\mathrm{Na}^{+}\right], 1564(100)\left[2 \mathrm{M}+\mathrm{Na}^{+}\right]$; negative $m / z(\%)=805(20)\left[\mathrm{M}+\mathrm{Cl}^{-}\right]$.

Fmoc-( $\beta M e)$ Phe-(2S)-(3-Ncp)Ala-( $\beta M e)$ Phe-Ile-OTMSE (167): The $N$-Fmoc protected amino acid $146(0.132 \mathrm{~g}, 0.328 \mathrm{mmol})$ was coupled according to GP2 with the tripeptide obtained after deprotection of $164(0.230 \mathrm{~g}, 0.298 \mathrm{mmol})$ according to $\mathrm{GP} 4$ in $\mathrm{CH}_{2} \mathrm{Cl}_{2} / \mathrm{DMF}$ 3:1 (3 mL) employing EDC (65 mg, $0.339 \mathrm{mmol})$, HOAt (46 mg, $0.340 \mathrm{mmol})$ and TMP (41 mg, 0.338 mmol). The mixture was then taken up with EtOAc $(30 \mathrm{~mL})$ and concentrated after usual aqueous work-up to give crude product which was triturated with $\mathrm{Et}_{2} \mathrm{O} /$ hexanes $1: 1$ and then recrystallized from THF/hexanes to give $167(0.248 \mathrm{~g}, 89 \%)$ as an off-white solid. $R_{\mathrm{f}}=0.21$, EtOAc/ $\mathrm{CHCl}_{3} 2: 9 ;[\alpha]_{20}^{D}-7.5\left(c=0.12, \mathrm{CHCl}_{3}\right) ;{ }^{1} \mathrm{H}$ NMR $\left(250 \mathrm{MHz},\left[\mathrm{D}_{8}\right] \mathrm{THF}\right): \delta=0.03[\mathrm{~s}$, $\left.9 \mathrm{H}, \mathrm{Si}\left(\mathrm{CH}_{3}\right)_{3}\right], 0.78\left(\mathrm{q}, J=7.1 \mathrm{~Hz}, 6 \mathrm{H}, 5-\mathrm{H}, 1^{\prime}-\mathrm{H}\right.$, Ile), $0.91(\mathrm{dd}, J=8.6,8.6 \mathrm{~Hz}, 2 \mathrm{H}, 2-\mathrm{H}$, 
TMSE), 0.98-1.17 [m, $\left.2 \mathrm{H}, 2^{\prime}-\mathrm{H}_{\mathrm{a}},(3-N c p) A l a, 4-\mathrm{H}_{\mathrm{a}}, I l e\right], 1.20$ [d, J= $\left.7 \mathrm{~Hz}, 3 \mathrm{H}, 4-\mathrm{H},(\beta M e) P h e\right]$, 1.30-1.51 [m, $4 \mathrm{H}, 1^{\prime}-\mathrm{H}, 2^{\prime}-\mathrm{H}_{\mathrm{b}}$, (3-Ncp)Ala and 3-H, 4- $\left.\mathrm{H}_{\mathrm{b}}, I l e\right], 1.33\left[\mathrm{~d}, J=7.0 \mathrm{~Hz}, 4-\mathrm{H}, \mathrm{CH}_{3}\right.$, (BMe)Phe], 1.54-1.79 [m, $2 \mathrm{H}, 3-\mathrm{H},(3-N c p) A l a], 3.10[\mathrm{~m}, 1 \mathrm{H}, 3-\mathrm{H},(\beta M e) P h e], 3.24[\mathrm{t}, J=$ $7.3 \mathrm{~Hz}, 1 \mathrm{H}, 3-\mathrm{H},(\beta M e) P h e], 3.91-4.08$ (m, $3 \mathrm{H}, 1-\mathrm{H}, T M S E, 2-\mathrm{H}), 4.19-4.48$ [m, $6 \mathrm{H}, 2 \times 2-\mathrm{H}$ and $1^{\prime}-\mathrm{H}, 2^{\prime}-\mathrm{H}$, Fmoc and $\left.2^{\prime}-\mathrm{H},(3-N c p) A l a\right], 4.55(\mathrm{dd}, J=8.5,8.5 \mathrm{~Hz}, 1 \mathrm{H}, 2-\mathrm{H}), 7.02-7.38(\mathrm{~m}$, $16 \mathrm{H}, \mathrm{Ar}-\mathrm{H}), 7.52$ (d, $J=9.0 \mathrm{~Hz}, 1 \mathrm{H}, \mathrm{NH}), 7.57$ (d, $J=8.3 \mathrm{~Hz}, 1 \mathrm{H}, \mathrm{NH}), 7.64(\mathrm{~d}, J=7.5 \mathrm{~Hz}$, $1 \mathrm{H}, \mathrm{NH}), 7.68(\mathrm{~d}, J=7.3 \mathrm{~Hz}, 1 \mathrm{H}, \mathrm{NH}), 7.77$ (d, J = 7.3 Hz, $2 \mathrm{H}, \mathrm{Ar}-\mathrm{H}) ;{ }^{13} \mathrm{C}$ NMR $(62.9 \mathrm{MHz}$, $\left.\left[\mathrm{D}_{8}\right] \mathrm{THF}\right): \delta=-1.3\left[+, \mathrm{Si}\left(\mathrm{CH}_{3}\right)_{3}\right], 11.9(+, \mathrm{C}-5$, Ile $), 15.9\left(+, \mathrm{C}-1^{\prime}\right.$, Ile $), 18.0[+, \mathrm{C}-4,(\beta M e) P h e]$, 18.2 (-, C-2, TMSE), 18.7 [+, C-4, (BMe)Phe], 18.7 [-, C-3', (3-Ncp)Ala], $23.1\left[+, \mathrm{C}^{\prime} 1^{\prime},(3-\right.$ Ncp)Ala], 26.0 (-,C-4, Ile), 34.8 [-, C-3, (3-Ncp)Ala], 38.8 (+, C-3, Ile), 42.8 [+,C-3, (BMe)Phe], $44.1[+, \mathrm{C}-3,(\beta M e) P h e], 48.4\left(+, \mathrm{C}-1^{\prime}\right.$, Fmoc $), 52.7(+, \mathrm{C}-2), 57.1(+, \mathrm{C}-2), 59.5(+, \mathrm{C}-2), 60.1$ (+, C-2), $62.0\left[+, \mathrm{C}-2^{\prime},(3-N c p)\right.$ Ala $], 63.5(-, \mathrm{C}-1, T M S E), 67.8$ (-, C-2', Fmoc), 120.7 (+, Ar-C), $126.3(+, \mathrm{Ar}-\mathrm{C}), 127.4$ (+, Ar-C), 127.6 (+, Ar-C), $128.0(+, \mathrm{Ar}-\mathrm{C}), 128.5$ (+, Ar-C), $129.0(+$, Ar-C), 129.1 (+, Ar-C), 129.2 (+, Ar-C), 129.3 (+, Ar-C), $142.4\left(2 \times \mathrm{C}_{\text {quat }}\right.$, Ar-C), 143.9, 144.3 $\left(\mathrm{C}_{\text {quat }}, \mathrm{Ar}-\mathrm{C}\right), 145.3,145.5\left(\mathrm{C}_{\text {quat }}, \mathrm{Ar}-\mathrm{C}\right), 157.7\left(\mathrm{C}_{\text {quat }}, \mathrm{NCO}_{2}\right), 171.2,171.4,172.0,172.1\left(\mathrm{C}_{\text {quat }}\right.$, $\mathrm{C}-1)$; IR (KBr): nu(tilde) $=3276 \mathrm{~cm}^{-1}, 3064,2965,1710,1668,1637,1543,1452,1369,1249$; MS (ESI), positive $m / z(\%)=955(90)\left[\mathrm{M}+\mathrm{Na}^{+}\right], 1886(80)\left[2 \mathrm{M}+\mathrm{Na}^{+}\right]$; negative $m / z(\%)=$ $966(22)\left[\mathrm{M}+\mathrm{Cl}^{-}\right]$.

Alloc-a-Thr[Boc-(4-PE)Pro]-OSEM (166): The ester 166 (0.203 g, 64\%) was prepared from the protected threonine $159(0.185 \mathrm{~g}, 0.555 \mathrm{mmol}), N$-Boc protected propenylproline $16(0.146 \mathrm{~g}$, $0.572 \mathrm{mmol}), 4-p y r r o l i d i n o p y r i d i n e ~(0.01 \mathrm{~g}, 0.067 \mathrm{mmol})$ and EDC $(0.113 \mathrm{~g}, 0.589 \mathrm{mmol})$ in $\mathrm{CH}_{2} \mathrm{Cl}_{2}(5 \mathrm{~mL})$ according to GP 6 after column chromatography (EtOAc/hexanes 1:6, $\left.R_{\mathrm{f}}=0.19\right)$ as a turbid oil. $[\alpha]_{20}^{D}-21.0\left(c=0.40, \mathrm{CHCl}_{3}\right) ;{ }^{1} \mathrm{H} \mathrm{NMR}\left(250 \mathrm{MHz}, \mathrm{CDCl}_{3}\right): \delta=0.01[\mathrm{~s}, 9 \mathrm{H}$, $\left.\mathrm{Si}\left(\mathrm{CH}_{3}\right)_{3}\right], 0.96\left(\mathrm{t}, J=8.6 \mathrm{~Hz}, 2 \mathrm{H}, 2^{\prime}-\mathrm{H}, S E M\right), 1.32,1.36(2 \times \mathrm{d}, J=6.7 \mathrm{~Hz}, 3 \mathrm{H}, 4-\mathrm{H}, a-T h r)$, 
1.41, $1.43\left[2 \times \mathrm{s}, 9 \mathrm{H}, \mathrm{C}\left(\mathrm{CH}_{3}\right)_{3}\right], 1.63,1.64\left[2 \times \mathrm{d}, J=7.0 \mathrm{~Hz}, 3 \mathrm{H}, 3^{\prime}-\mathrm{H},(4-P E)\right.$ Pro $], 1.67-1.86$ [m, $\left.1 \mathrm{H}, 3-\mathrm{H}_{\mathrm{a}},(4-P E) P r o\right], 2.17-2.49$ [m, $1 \mathrm{H}, 3-\mathrm{H}_{\mathrm{b}},(4-P E)$ Pro], 2.94-3.19 [m, $2 \mathrm{H}, 4-\mathrm{H}, 5-\mathrm{H}_{\mathrm{a}}$, (4-PE)Pro], 3.51-3.64 [m, 0.5 H, 5-Hb, (4-PE)Pro], 3.72 (t, J = 8.3 Hz, $\left.2 \mathrm{H}, 1^{\prime}-\mathrm{H}, S E M\right), 4.15$ [dd, $J=9.6,6.9 \mathrm{~Hz}, 0.5 \mathrm{H}, 2-\mathrm{H},(4-P E)$ Pro], 4.22 [dd, $J=8.2,8.2 \mathrm{~Hz}, 0.5 \mathrm{H}, 2-\mathrm{H},(4-P E)$ Pro], 4.45-4.67 (m, 3 H, 1-H, Alloc and 2-H, a-Thr), 5.09-5.44 [m, 6 H, 1-H, SEM and 2-H, 3-H, Alloc and 3-H, a-Thr and 1'-H, (4-PE)Pro], 5.53 [dq, $J=9.2,7.0 \mathrm{~Hz}, 1 \mathrm{H}, 2^{\prime}-\mathrm{H},(4-P E)$ Pro], $6.51(\mathrm{~d}, J=9.2 \mathrm{~Hz}, 1 \mathrm{H}, \mathrm{NH})$; the peak of 1'- $\mathrm{H}, S E M$ masked absorption for $0.5 \mathrm{H}, 5-\mathrm{H}_{\mathrm{b}}$ of $(4-$ PE)Pro; ${ }^{13} \mathrm{C}$ NMR $\left(62.9 \mathrm{MHz}, \mathrm{CDCl}_{3}\right): \delta=-1.6\left[+, \mathrm{Si}\left(\mathrm{CH}_{3}\right)_{3}\right], 13.0\left[+, \mathrm{C}-3^{\prime},(4-\right.$ PE)Pro $], 15.8$, $16.6(+, \mathrm{C}-4, a-T h r), 17.8\left(-, \mathrm{C}-2^{\prime}, \mathrm{SEM}\right), 28.2,28.3\left[+, \mathrm{C}\left(\mathrm{CH}_{3}\right)_{3}\right], 35.5,36.3[+, \mathrm{C}-4$, (4PE)Pro], 36.1, 37.1 [-, C-3, (4-PE)Pro], 51.3, 51.6 [-, C-5, (4-PE)Pro], 57.157 .6 [+, C-2, (4PE)Pro], 59.2, 59.3 (+, C-2, a-Thr), 65.6, 65.9 (-, C-1', SEM), 68.0, 68.3 (-, C-1, Alloc), 70.9, $71.1(+, \mathrm{C}-3, a-T h r), 80.0,80.2\left(\mathrm{C}_{\text {quat }}, \mathrm{C}\left(\mathrm{CH}_{3}\right)_{3}\right), 89.7,90.1$ (-, C-1, SEM), 117.4, 117.9 (-, C-3, Alloc), 126.6, 126.7 [+, C-2', (4-PE)Pro], 129.1, 129.2 [+, C-1', (4-PE)Pro], 132.3, 132.7 (+, C2, Alloc $), 153.2,153.8\left[\mathrm{C}_{\text {quat }}, \mathrm{NCO}_{2},(4-P E)\right.$ Pro $], 155.4,156.0\left(\mathrm{C}_{\text {quat }}, \mathrm{NCO}_{2}, a-T h r\right), 168.7\left(\mathrm{C}_{\text {quat, }}\right.$, C-1), 171.9, 172.3 (C quat, C-1); IR (film): nu(tilde) $=3333 \mathrm{~cm}^{-1}, 3082,2977,2953,2930,1746$, 1703, 1367, 1250, 1113; MS (EI, $70 \mathrm{eV}), m / z(\%)=570(1)\left[\mathrm{M}^{+}\right], 469(16)\left[\mathrm{M}^{+}-\mathrm{C}_{5} \mathrm{H}_{9} \mathrm{O}_{2}\right], 339$ (15), 168 (9), 210 (17), 154 (100) $\left[\mathrm{C}_{8} \mathrm{H}_{12} \mathrm{NO}_{2}^{+}\right], 110$ (98) $\left[\mathrm{C}_{7} \mathrm{H}_{12} \mathrm{~N}^{+}\right], 57$ (92) $\left[\mathrm{C}_{4} \mathrm{H}_{9}^{+}\right], 41$ (12) $\left[\mathrm{C}_{3} \mathrm{H}_{5}{ }^{+}\right]$; HRMS (EI): calcd for $\mathrm{C}_{27} \mathrm{H}_{46} \mathrm{~N}_{2} \mathrm{O}_{9} \mathrm{Si}$ : 570.2972; found 570.2972.

Alloc-a-Thr[Boc-(4-PE)Pro]-OH (168): $\mathrm{MgBr}_{2} \cdot \mathrm{Et}_{2} \mathrm{O}(0.335 \mathrm{~g}, 1.297 \mathrm{mmol})$ was added to a solution of ester $166(0.200 \mathrm{~g}, 0.350 \mathrm{mmol})$ in $\mathrm{CH}_{2} \mathrm{Cl}_{2}(15 \mathrm{~mL})$ at $-30{ }^{\circ} \mathrm{C}$, the mixture was allowed to warm to $4{ }^{\circ} \mathrm{C}$ and stirring continued for $2 \mathrm{~h}$. The reaction mixture was then diluted with $\mathrm{Et}_{2} \mathrm{O}(50 \mathrm{~mL})$, washed with $1 \mathrm{M} \mathrm{NaHSO}_{4}(3 \times 10 \mathrm{~mL})$, water $(3 \times 10 \mathrm{~mL})$, brine $(2 \times 5 \mathrm{~mL})$, dried, filtered and concentrated under reduced pressure to give crude $168(0.103 \mathrm{~g}, 65 \%)$ as a turbid oil which was used for next step without purification. $R_{\mathrm{f}}=0.17 \mathrm{EtOAc} / \mathrm{hexanes} 1: 3(2 \%$ $\mathrm{AcOH})$. 
Alloc-a-Thr[Boc-(4-PE)Pro]-( $\beta M e) P h e-(2 S)-(3-N c p)$ Ala-( $\beta M e) P h e-I l e-O T M S E$ (170):

Tetrapeptide 167 ( $0.165 \mathrm{~g}, 0.174 \mathrm{mmol})$ was deprotected according to GP 4 and the condensation between the resultant $N$-deprotected peptide 169 and peptide acid 168 (88 $\mathrm{mg}, 0.193 \mathrm{mmol})$ was then carried out in MeCN (3 mL) employing HATU (82 mg, $0.216 \mathrm{mmol}$ ), HOAt (27 mg, 0.200 mmol), DIEA (25 mg, $0.193 \mathrm{mmol}$ ) and TMP (70 $\mathrm{mg}, 0.578 \mathrm{mmol})$ according to GP 7 to give after usual aqueous work-up and evaporation of solvents the red solid which was triturated with hexanes, filtered and finally purified by column chromatography $\left(\mathrm{EtOAc} /\right.$ hexanes $1: 1.5, R_{\mathrm{f}}=$ $0.28)$ to give $170(0.156 \mathrm{~g}, 80 \%)$ as white solid. $[\alpha]_{20}^{D} 16.9\left(c=0.16, \mathrm{CHCl}_{3}\right) ;{ }^{1} \mathrm{H}$ NMR (500 $\left.\mathrm{MHz}, \mathrm{CDCl}_{3}\right): \delta=0.07\left[\mathrm{~s}, 9 \mathrm{H}, \mathrm{Si}\left(\mathrm{CH}_{3}\right)_{3}\right], 0.72\left(\mathrm{~d} J=7.0 \mathrm{~Hz}, 3 \mathrm{H}, 1^{\prime}-\mathrm{H}\right.$, Ile $), 0.86(\mathrm{t}, J=7.0 \mathrm{~Hz}$, $3 \mathrm{H}, 5-\mathrm{H}$, Ile), 0.97 [ddd, $J=6.5 \mathrm{~Hz}, 1 \mathrm{H}, 2^{\prime}-\mathrm{H}_{\mathrm{a}},(3-N c p)$ Ala], 1.02 (dd, $J=8.8 \mathrm{~Hz}, 2 \mathrm{H}, 2-\mathrm{H}$, TMSE), 1.06-1.21 [m, $\left.2 \mathrm{H}, 1^{\prime}-\mathrm{H},(3-N c p) A l a, 4-\mathrm{H}_{\mathrm{a}}, I l e\right], 1.21-1.50$ [m, $4 \mathrm{H}, 2^{\prime}-\mathrm{H}_{\mathrm{b}}, 3-\mathrm{H},(3-$ Ncp)Ala, 4- $\left.\mathrm{H}_{\mathrm{b}}, I l e\right], 1.25$ [d, $\left.J=6.5 \mathrm{~Hz}, 3 \mathrm{H}, 4-\mathrm{H},(\beta M e) P h e\right], 1.35$ [d, $J=7.0 \mathrm{~Hz}, 3 \mathrm{H}, 4-\mathrm{H}$, ( $\beta M e) P h e], 1.42(\mathrm{~d}, J=6.5 \mathrm{~Hz}, 3 \mathrm{H}, 4-\mathrm{H}, a-T h r), 1.49$ [s, $\left.9 \mathrm{H}, \mathrm{C}\left(\mathrm{CH}_{3}\right)_{3}\right], 1.69$ [dd, $J=1.5$, $6.5 \mathrm{~Hz}, 3 \mathrm{H}, 3^{\prime}-\mathrm{H},(4-P E)$ Pro], 1.75 [ddd, $J=11.8,10.3,12.0 \mathrm{~Hz}, 1 \mathrm{H}, 3-\mathrm{H}_{\mathrm{a}}$, (4-PE)Pro], $1.79-$ 1.89 (m, $1 \mathrm{H}, 3-\mathrm{H}$, Ile), 2.43 [ddd, $J=6.5,6.5,6.5 \mathrm{~Hz}, 1 \mathrm{H}, 3-\mathrm{H}_{\mathrm{b}},(4-P E)$ Pro], 3.17 [dd, $J=10.5$,

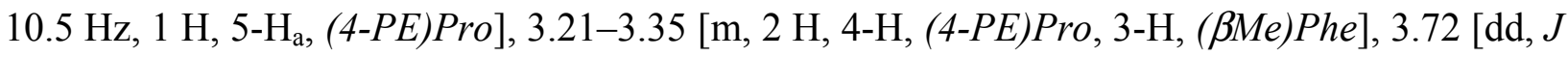
= 7.8, $10.5 \mathrm{~Hz}, 5-\mathrm{H},(4-P E)$ Pro], $3.86[\mathrm{dq}, J=3.0,7.0 \mathrm{~Hz}, 1 \mathrm{H}, 3-\mathrm{H},(\beta M e) P h e], 4.17-4.32(\mathrm{~m}$, $3 \mathrm{H}, 1-\mathrm{H}, T M S E, 2-\mathrm{H}), 4.35(\mathrm{dd}, J=4.5,9 \mathrm{~Hz}, 1 \mathrm{H}, 2-\mathrm{H}), 4.40(\mathrm{dd}, J=1.5,8.5 \mathrm{~Hz}, 1 \mathrm{H}, 2-\mathrm{H})$, $4.49(\mathrm{dd}, J=7.0,10.0 \mathrm{~Hz}, 1 \mathrm{H}, 2-\mathrm{H}), 4.51-4.62$ [m, $4 \mathrm{H}, 1-\mathrm{H}$, Alloc and 2'-H, (3-Ncp)Ala and 2$\mathrm{H}], 4.63(\mathrm{dd}, J=10.3,10.3 \mathrm{~Hz}, 1 \mathrm{H}, 2-\mathrm{H}), 5.16-5.21$ (m, $1 \mathrm{H}$, trans-3-H, Alloc), 5.24-5.33 [m, $2 \mathrm{H}, 1^{\prime}-\mathrm{H},(4-P E)$ Pro, cis-3-H, Alloc], 5.50 (dq, $\left.J=1.5,6.5 \mathrm{~Hz}, 1 \mathrm{H}, 3-\mathrm{H}, a-T h r\right), 5.58$ [dq, $J=$ 10.5, 6.5 Hz, $1 \mathrm{H}, 2^{\prime}-\mathrm{H},(4-P E)$ Pro], 5.86 (ddt, $J=17.0,10.5,6.0 \mathrm{~Hz}, 1 \mathrm{H}, 2-\mathrm{H}$, Alloc), 6.59 (d, $J$ $=8 \mathrm{~Hz}, \mathrm{NH}), 6.85(\mathrm{~d}, J=9.5 \mathrm{~Hz}, \mathrm{NH}), 7.05(\mathrm{~d}, J=10 \mathrm{~Hz}, 1 \mathrm{H}, \mathrm{NH}), 7.14-7.37(\mathrm{~m}, 10 \mathrm{H}, \mathrm{Ar}-\mathrm{H})$, $7.50(\mathrm{~d}, J=9.5 \mathrm{~Hz}, 1 \mathrm{H}, \mathrm{NH}), 7.91(\mathrm{~d}, J=6.0 \mathrm{~Hz}, 1 \mathrm{H}, \mathrm{NH}) ;{ }^{13} \mathrm{C} \mathrm{NMR}\left(125.7 \mathrm{MHz}, \mathrm{CDCl}_{3}\right): \delta=$ -1.5 [+, $\left.\mathrm{Si}\left(\mathrm{CH}_{3}\right)_{3}\right], 11.7$ (+, C-5, Ile $), 13.3$, [+, C-3', (4-PE)Pro $], 15.9$ (+, C-1', Ile), 17.3 [-, C-3', 
(3-Ncp)Ala $], 17.9$ (+, C-4, a-Thr), 18.3 (-, C-2, TMSE), 19.0 [+, C-4, (ßMe)Phe], 19.5 [+, C-4, (BMe)Phe], 21.9 [+, C-1', (3-Ncp)Ala], $25.2\left(-, \mathrm{C}-4\right.$, Ile), $28.5\left[+, \mathrm{C}\left(\mathrm{CH}_{3}\right)_{3}\right], 31.6[-, \mathrm{C}-3,(3-$ Ncp)Ala], 36.4 [-, C-3, (4-PE)Pro], 36.4 [+, C-4, (4-PE)Pro], 37.4 (+, C-3, Ile), 40.6 [+,C-3,

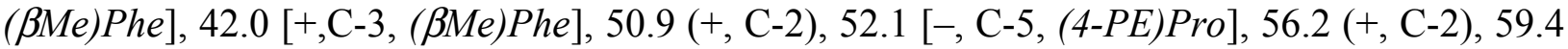
(+, C-2), $59.5(+, \mathrm{C}-2), 61.1(+, \mathrm{C}-2), 61.6(+, \mathrm{C}-2), 62.0\left[+, \mathrm{C}-2^{\prime},(3-N c p) A l a\right], 63.2(-, \mathrm{C}-1$, TMSE), 65.6 (-, C-1, Alloc), 70.7 (+, C-3, a-Thr), 118.1 (-, C-3, Alloc), 127.0, 127.2, 127.7, 127.8, 128.5 (x2), 128.7, 128.9 [+, Ar-C and C-1', C-2', (4-PE)Pro], 132.7 (+, C-2, Alloc), 141.8, $142.0\left(\mathrm{C}_{\text {quat }}, \mathrm{Ar}-\mathrm{C}\right), 154.9,155.6\left(\mathrm{C}_{\text {quat }}, \mathrm{NCO}_{2}\right), 170.5(\mathrm{x} 2), 170.8,171.3,173.8,174.2\left(\mathrm{C}_{\text {quat }}, \mathrm{C}-\right.$ 1); IR $(\mathrm{KBr}): \mathrm{nu}($ tilde $)=3390 \mathrm{~cm}^{-1}, 3086,2954,2898,1728,1521,1412,1382,1250,1150 ; \mathrm{MS}$ $($ ESI $)$, positive $m / z(\%)=1155(100)\left[\mathrm{M}+\mathrm{Na}^{+}\right]$; negative $m / z(\%)=1131(95)\left[\mathrm{M}-\mathrm{H}^{+}\right]$.

$N$-Alloc protected cyclodepsipeptide (171a): Depsipeptide 170 (0.136 g, 0.120 mmol) was deprotected with TFA $(2 \mathrm{~mL})$ at $20^{\circ} \mathrm{C}$ for $2 \mathrm{~h}$. The mixture was then concentrated under reduced pressure to give residue which was triturated with $\mathrm{Et}_{2} \mathrm{O}$, filtered and quickly washed with cold $\mathrm{CH}_{2} \mathrm{Cl}_{2}$ to give trifluoroacetate of deprotected depsipeptide $(0.126 \mathrm{~g}, 100 \%)$ as a white solid which was used without further purification for the cyclisation step. $R_{\mathrm{f}}=0.10 \mathrm{CH}_{2} \mathrm{Cl}_{2} / \mathrm{MeOH}$ 10:1 (1\% of $28 \%$ aqueous $\left.\mathrm{NH}_{3}\right)$. It was dissolved in anhydrous $\mathrm{CH}_{2} \mathrm{Cl}_{2}(1.201)$ and cyclizated according to GP 9 employing HATU $(2 \times 0.059 \mathrm{~g}, 0.155 \mathrm{mmol})$ and DIEA $(2 \times 0.062 \mathrm{~g}, 0.480$ $\mathrm{mmol})$ to give after aqueous work-up, column chromatography $\left(\right.$ EtOAc/hexanes 1:1.5, $\left.\mathrm{R}_{\mathrm{f}}=0.24\right)$ and crystallization from $\mathrm{Et}_{2} \mathrm{O}$ /pentane the crude product which was finally purified by preparative HPLC to give 171a (29 mg, 26\%) as a colorless solid. Preparative HPLC: isocratic, $70 \% \mathrm{MeCN}$ in $\mathrm{H}_{2} \mathrm{O}\left(0.1 \%\right.$ TFA); analytical HPLC 1: column A, isocratic $70 \% \mathrm{MeCN}$ in $\mathrm{H}_{2} \mathrm{O}$ $\left(0.1 \%\right.$ TFA), flow rate $1 \mathrm{~mL} / \mathrm{min}, t_{\mathrm{R}}=12.96 \mathrm{~min}$, purity $>98 \%$; analytical HPLC 2: column $\mathrm{B}$, gradient $0 \rightarrow 99 \% \mathrm{MeCN}$ in $\mathrm{H}_{2} \mathrm{O}(0.1 \% \mathrm{TFA})$ for $25 \mathrm{~min}$, then isocratic $99 \% \mathrm{MeCN}(0.1 \%$ TFA), flow rate $=0.5 \mathrm{~mL} / \mathrm{min}, t_{\mathrm{R}}=28.61 \mathrm{~min}$, purity $>98 \% ;[\alpha]_{20}^{D}-8.5\left(c=0.13, \mathrm{CHCl}_{3}\right) ;{ }^{1} \mathrm{H}$ $\operatorname{NMR}\left(500 \mathrm{MHz}, \mathrm{CDCl}_{3}\right): \delta=0.61\left[\mathrm{ddd}, J=14.3,7.5,7.5 \mathrm{~Hz}, 1 \mathrm{H}, 3-\mathrm{H}_{\mathrm{a}},(3-N c p)\right.$ Ala $], 0.76$ 
[ddd, $\left.J=6.5,6.5,6.5 \mathrm{~Hz}, 1 \mathrm{H}, 2^{\prime}-\mathrm{H}_{\mathrm{a}},(3-N c p) A l a\right], 0.88\left(\mathrm{~d} J=6.5 \mathrm{~Hz}, 3 \mathrm{H}, 1^{\prime}-\mathrm{H}\right.$, Ile $), 0.91(\mathrm{t}, J=$ $7.5 \mathrm{~Hz}, 3 \mathrm{H}, 5-\mathrm{H}$, Ile), 1.10-1.20 (m, $\left.1 \mathrm{H}, 4-\mathrm{H}_{\mathrm{a}}, I l e\right), 1.27$ [d, J = 7.0 Hz, $\left.3 \mathrm{H}, 4-\mathrm{H},(\beta M e) P h e\right]$, 1.28-1.34 [m, $\left.1 \mathrm{H}, 1^{\prime}-\mathrm{H},(3-N c p) A l a\right], 1.38$ [d, $\left.J=7.0 \mathrm{~Hz}, 4-\mathrm{H},(\beta M e) P h e\right], 1.42-1.53$ [m, $1 \mathrm{H}$, 3-H $\left.\mathrm{H}_{\mathrm{b}},(3-N c p) A l a\right], 1.53-1.61$ [m, $2 \mathrm{H}, 2^{\prime}-\mathrm{H}_{\mathrm{b}},(3-N c p)$ Ala $\left., 4-\mathrm{H}_{\mathrm{b}}, I l e\right], 1.59$ (d, J = 6.5 Hz, $3 \mathrm{H}, 4-$ $\mathrm{H}, a-T h r), 1.65\left[\mathrm{dd}, J=1.5,6.8 \mathrm{~Hz}, 3 \mathrm{H}, 3^{\prime}-\mathrm{H},(4-P E)\right.$ Pro], 1.74 [ddd, $J=11.5,11.5,11.5 \mathrm{~Hz}$, $1 \mathrm{H}, 3-\mathrm{H}_{\mathrm{a}},(4-P E)$ Pro], 1.77-1.87 (m, $1 \mathrm{H}, 3-\mathrm{H}$, Ile), 2.23 [ddd, $J=5.5,5.5,12.5 \mathrm{~Hz}, 1 \mathrm{H}, 3-\mathrm{H}_{\mathrm{b}}$, (4-PE)Pro], 3.07 [dq, $J=7.0,7.0 \mathrm{~Hz}, 1 \mathrm{H}, 3-\mathrm{H},(\beta M e) P h e], 3.15-3.30$ [m, $1 \mathrm{H}, 4-\mathrm{H},(4-P E) P r o]$, $3.25\left[\mathrm{dd}, J=11.0,11.0 \mathrm{~Hz}, 1 \mathrm{H}, 5-\mathrm{H}_{\mathrm{a}},(4-P E)\right.$ Pro $], 3.55[\mathrm{dq}, J=5.0,7.0 \mathrm{~Hz}, 1 \mathrm{H}, 3-\mathrm{H}$, ( $\beta M e) P h e], 3.71(\mathrm{dd}, J=6.5,6.5 \mathrm{~Hz}, 1 \mathrm{H}, 2-\mathrm{H}), 3.78(\mathrm{ddd}, J=3.0,3.0,7.0 \mathrm{~Hz}, 1 \mathrm{H}, 2-\mathrm{H}), 3.98$ [dd, $J=6.3,11.0 \mathrm{~Hz}, 5-\mathrm{H},(4-P E) P r o], 4.07$ [dd, $J=7.3,7.3 \mathrm{~Hz}, 1 \mathrm{H}, 2-\mathrm{H},(4-P E) P r o], 4.49$ (dd, $J=1.5,9.5 \mathrm{~Hz}, 2-\mathrm{H}), 4.53[\mathrm{dd}, J=4.5,7.0 \mathrm{~Hz}, 1 \mathrm{H}, 2-\mathrm{H},(\beta M e) P h e], 4.54-4.60$ [m, $2 \mathrm{H}, 2^{\prime}-\mathrm{H}$, (3-Ncp)Ala, 1- $\mathrm{H}_{\mathrm{a}}$, Alloc], 4.60-4.64 (m, $1 \mathrm{H}, 1-\mathrm{H}$, Alloc), 4.66 [dd, J =8.0, 8.0 Hz, $1 \mathrm{H}, 2-\mathrm{H}$, (ßMe)Phe], 5.19-5.26 [m, $\left.1 \mathrm{H}, 1^{\prime}-\mathrm{H},(4-P E) P r o\right], 5.22$ (ddt, $J=1.5,1.5,10.3 \mathrm{~Hz}, 1 \mathrm{H}$, trans-3H, Alloc), 5.32 (ddt, $J=1.5,1.5,17.3 \mathrm{~Hz}, 1 \mathrm{H}$, cis-3-H, Alloc), 5.42 (dq, $J=10.5,7.0 \mathrm{~Hz}, 1 \mathrm{H}, 3-$ H, a-Thr), 5.58 [dq, $\left.J=10.5,6.8 \mathrm{~Hz}, 1 \mathrm{H}, 2^{\prime}-\mathrm{H},(4-P E) P r o\right], 5.91$ (ddt, $J=17.3,10.5,5.5 \mathrm{~Hz}$, $1 \mathrm{H}, 2-\mathrm{H}$, Alloc), 6.12-6.21 (br, $1 \mathrm{H}, \mathrm{NH}), 6.36$ (d, $J=7.0 \mathrm{~Hz}, 1 \mathrm{H}, \mathrm{NH}), 6.46$ (d, $J=9.5 \mathrm{~Hz}$, $\mathrm{NH}), 6.54(\mathrm{~d}, J=9.5 \mathrm{~Hz}, \mathrm{NH}), 7.11-7.34(\mathrm{~m}, 10 \mathrm{H}, \mathrm{Ar}-\mathrm{H}), 7.45(\mathrm{~d}, J=8.0 \mathrm{~Hz}, 1 \mathrm{H}, \mathrm{NH}) ;{ }^{13} \mathrm{C}$ NMR (125.7 MHz, $\left.\mathrm{CDCl}_{3}\right): \delta=10.5(+, \mathrm{C}-5$, Ile), 13.3, [+, C-3', (4-PE)Pro], 14.6 [+, C-4, (ßMe)Phe $], 14.8\left(+, \mathrm{C}-1^{\prime}\right.$, Ile $), 17.3$ [-, C-3', (3-Ncp)Ala $], 17.8$ [+, C-4, $\left.(\beta M e) P h e\right], 18.4(+, \mathrm{C}-4$, a-Thr), 21.4 [+, C-1', (3-Ncp)Ala $], 24.8$ (-,C-4, Ile), 32.0 [-, C-3, (3-Ncp)Ala], 35.4 [-, C-3, (4PE)Pro], 36.6 [+, C-4, (4-PE)Pro], $37.0(+, \mathrm{C}-3$, Ile $), 39.6[+, \mathrm{C}-3,(\beta M e) P h e], 44.7[+, \mathrm{C}-3$, (BMe)Phe], 52.5 [-, C-5, (4-PE)Pro], 53.3 [+, C-2, (3-Ncp)Ala], $54.6(+, \mathrm{C}-2$, Ile $), 58.5$ [+, C-2, ( $\beta M e) P h e], 59.1(+, \mathrm{C}-2, \mathrm{a}-T h r), 59.2$ [+, C-2, $(\beta M e) P h e], 59.9$ [+, C-2, (4-PE)Pro], $60.6[+, \mathrm{C}-$ 2', (3-Ncp)Ala], 65.9 (-, C-1, Alloc), 72.6 (+, C-3, a-Thr), 117.7 (-, C-3, Alloc), 126.9, 127.2, 127.4, 127.7, 127.8, 127.9, 128.5, 128.7 [+, Ar-C and C-1', C-2', (4-PE)Pro], 132.7 (+, C-2, 
Alloc $), 141.1,142.6\left(\mathrm{C}_{\text {quat }}, \mathrm{Ar}-\mathrm{C}\right), 156.0\left(\mathrm{C}_{\text {quat }}, \mathrm{NCO}_{2}\right), 168.7,170.0,170.5,171.2,171.3,173.0$ $\left(\mathrm{C}_{\text {quat }}, \mathrm{C}-1\right)$; MS (ESI), positive $m / z(\%)=937(100)\left[\mathrm{M}+\mathrm{Na}^{+}\right]$; negative $m / z(\%)=913(100)$ $\left[\mathrm{M}-\mathrm{H}^{+}\right]$.

Attempt of the transprotection of cyclodepsipeptide 171a: To a vigorously stirred suspension of $\operatorname{Pd}\left(\mathrm{P}(\mathrm{Ph})_{3}\right)_{4}(1 \mathrm{mg}, 0.87 \mu \mathrm{mol})$ in a solution of the $N$-Alloc protected cyclohexadepsipeptide 171a $(25 \mathrm{mg}, 27.38 \mu \mathrm{mol})$ and $\mathrm{Boc}_{2} \mathrm{O}(2 \mathrm{mg}, 91.64 \mu \mathrm{mol})$ in $\mathrm{DME}(4 \mathrm{~mL}) \mathrm{Bu}_{3} \mathrm{SnH}(24 \mathrm{mg}$, $82.45 \mu \mathrm{mol})$ was added and stirring continued for $2.5 \mathrm{~h}$. All volatiles were removed under reduced pressure and the residue was purified by preparative TLC $(20 \times 20$, EtOAc/hexanes 1:2, $R_{\mathrm{f}}=0.18,3$ runs) to give $10 \mathrm{mg}$ of the mixture of unreacted and transprotected peptide, 171a and 171b, in ratio 40:60 (according to ESI-MS spectra).

Study of the acidolytic cleavage of $Z, m-M e Z, M e Z$ and $D m Z$ : To test a cleavage of Z-group by “push-pool” mechanism ester Z-Thr(FmocPro)-OMe 172 (prepared according to GP 6) (0.005 g) was treated with mixture of TFA $(1.5 \mathrm{~mL})$, dimethylsulfide $(0.2 \mathrm{~mL})$ and thioanisole $(0.4 \mathrm{~mL})$ at $20{ }^{\circ} \mathrm{C}$ for $7.5 \mathrm{~h}$ and then at $4{ }^{\circ} \mathrm{C}$ for $12 \mathrm{~h}$. According to TLC the deprotection was incomplete. Dipeptide Boc-Phe-Val-OMe 117 was deprotected by TFA for $20 \mathrm{~min}$. The aliquots of the resultant trifluoroacetate of dipeptide $(3 \times 0.25 \mathrm{mmol})$ were dissolved in $\mathrm{CH}_{2} \mathrm{Cl}_{2}(5 \mathrm{~mL})$, DIEA $(0.510 \mathrm{mmol})$ and then appropriate OSu-ester: MeZOSu or $m$-MeZOSu, or DmZ-OSu (0.275 mmol) (prepared according to GP 9) was added to each portion of the deprotected dipeptide. After $3 \mathrm{~h}$, the reaction mixtures were diluted with $\mathrm{Et}_{2} \mathrm{O}(50 \mathrm{~mL})$ and subjected usual aqueous work-up (GP 5). The ethereal solutions were dried, filtered and concentrated under reduced pressure. The residues were purified by crystallization from EtOAc/hexanes (MeZ-Phe-Val-OMe 173a) or by column chromatography ( $m$-MeZ-Phe-Val-OMe 173b and DmZ-Phe-Val-OMe 173c) to give the desired dipeptides in $75-90 \%$ yield. These dipeptides and hexadepsipeptide 170 (6 mg each) were placed in four $10-\mathrm{mL}$ flasks and then freshly prepared $2 \mathrm{M} \mathrm{HCl}$ in dry 
EtOAc ( $1 \mathrm{~mL}$ to each sample) was added. TLC control (EtOAc/hexanes 1:1, $\mathrm{MeOH} / \mathrm{CH}_{2} \mathrm{Cl}_{2} 1: 10$ ( $1 \%$ of $28 \%$ aqueous $\mathrm{NH}_{3}$ ); visualization with phosphomolibdic acid or with ninhydrin) showed full $\mathrm{N}$-deprotection of $\mathbf{1 7 0}$ for $2 \mathrm{~min}$, MeZ-group and 3.5-DeZ-groups were stable to these conditions at least for $2 \mathrm{~h}$ and showed only traces of the deprotected products after $5 \mathrm{~h} . \mathrm{m}$-MeZgroup was stable to these conditions. TFA or $20 \% N$-methylindole, or $10 \%$ anisole, or $20 \%$ thioanisole in TFA $(1 \mathrm{~mL})$ were added to aliquots of $N$-protected dipeptides $(4 \times 5 \mathrm{mg}$ each). TLC control (see above) showed smooth cleavage (full consumption of the starting material and one ninhidrin active spot on chromatogram) of MeZ and DmZ groups with $10 \%$ anisole and $20 \%$ thioanisole in TFA for $1 \mathrm{~h}$ and $m$-MeZ with $20 \%$ thioanisole in TFA for $3.5 \mathrm{~h}$.

MeZ-a-Thr-OH (175): $\mathrm{NaHCO}_{3}(0.180 \mathrm{~g}, 2.14 \mathrm{mmol})$ and then a solution of MeZOSu $(0.608 \mathrm{~g}$, $2.31 \mathrm{mmol})$ in dioxane $(7 \mathrm{~mL})$ were added to a vigorously stirred solution of allo-D-threonine $(R)$-a-72 $(0.25 \mathrm{~g}, 2.10 \mathrm{mmol})$ in water $(7 \mathrm{~mL})$ and stirring continued for $3 \mathrm{~h}$ (if precipitate formed dioxane and/or water were added to obtain homogeneous solution). The mixture was then concentrated under reduced pressure, diluted with water $(40 \mathrm{~mL})$ and washed with $\mathrm{CH}_{2} \mathrm{Cl}_{2}$ $(4 \times 10 \mathrm{~mL}) \cdot \mathrm{pH}$ of the water fraction was adjusted to ca. $1-2$ with solid $\mathrm{NaHSO}_{4}$ and the resulting emulsion was extracted with EtOAc $(2 \times 40 \mathrm{~mL})$. The organic layer was washed with water $(4 \times 20 \mathrm{~mL})$, brine $2 \times 10 \mathrm{~mL})$, dried and concentrated under reduced pressure. The residue was recrystallized from $\mathrm{Et}_{2} \mathrm{O} /$ hexanes and then from $\mathrm{CH}_{2} \mathrm{Cl}_{2} /$ hexanes to give $\mathbf{1 7 5}(0.175 \mathrm{~g}, 39 \%)$ as a white solid. The mother liquor from the second crystallization was concentrated and recrystallized again from $\mathrm{Et}_{2} \mathrm{O} /$ hexanes to give a second crop of $\mathbf{1 7 5}(0.23 \mathrm{~g}, 90 \%$ overall yield). $R_{\mathrm{f}}=0.13 \mathrm{EtOAc} / \mathrm{hexanes}(2 \% \mathrm{AcOH}), 3$ runs; m.p. $78-80{ }^{\circ} \mathrm{C} ;[\alpha]_{20}^{D}-24.6\left(c=0.32, \mathrm{CHCl}_{3}\right)$; ${ }^{1} \mathrm{H}$ NMR $\left(250 \mathrm{MHz}, \mathrm{CDCl}_{3}\right): \delta=1.27(\mathrm{~d}, J=6.5 \mathrm{~Hz}, 3 \mathrm{H}, 4-\mathrm{H}), 2.34\left(\mathrm{~s}, 3 \mathrm{H}, 1^{\prime}-\mathrm{H}\right), 3.70-4.40$ (br, $\left.1 \mathrm{H}, \mathrm{CO}_{2} \mathrm{H}\right), 4.05-4.27(\mathrm{~m}, 1 \mathrm{H}, 3-\mathrm{H}), 4.33-4.44$ (m, $\left.1 \mathrm{H}, 2-\mathrm{H}\right), 5.07$ (s, $\left.2 \mathrm{H}, \mathrm{Bzl}-\mathrm{H}\right), 5.77$ (d, $J=7.5 \mathrm{~Hz}, 1 \mathrm{H}, \mathrm{NH}), 7.15(\mathrm{~d}, J=8.0 \mathrm{~Hz}, 2 \mathrm{H}, \mathrm{Ar}-\mathrm{H}), 7.24(\mathrm{~d}, J=8.0 \mathrm{~Hz}, 2 \mathrm{H}, \mathrm{Ar}-\mathrm{H}) ;{ }^{13} \mathrm{C} \mathrm{NMR}$ $\left(62.9 \mathrm{MHz}, \mathrm{CDCl}_{3}\right): \delta=18.7(+, \mathrm{C}-4), 21.1\left(+, \mathrm{C}-1^{\prime}\right), 59.3(+, \mathrm{C}-2), 67.4(-, \mathrm{Bzl}-\mathrm{C}), 69.0(+, \mathrm{C}-$ 
3), 128.3, $129.2(+, \mathrm{Ar}-\mathrm{C}), 132.7,138.1\left(\mathrm{C}_{\text {quat }}, \mathrm{Ar}-\mathrm{C}\right), 156.9\left(\mathrm{C}_{\text {quat }}, \mathrm{NCO}_{2}\right), 173.3\left(\mathrm{C}_{\text {quat }}, \mathrm{C}-1\right)$; IR $(\mathrm{KBr}): \mathrm{nu}(\mathrm{tilde})=3400 \mathrm{~cm}^{-1}, 3213,2988,2931,2889,1723,1678,1537,1422,1275,1225$, 1210; MS (EI, $70 \mathrm{eV}), m / z(\%)=267(3)\left[\mathrm{M}^{+}\right], 223(5)\left[\mathrm{M}^{+}-\mathrm{CO}_{2}\right], 162(8)\left[\mathrm{M}^{+}-\mathrm{C}_{8} \mathrm{H}_{9}\right], 105$ (100) $\left[\mathrm{C}_{8} \mathrm{H}_{9}{ }^{+}\right], 77$ (6) $\left[\mathrm{C}_{6} \mathrm{H}_{5}{ }^{+}\right], 45$ (5) $\left[\mathrm{CHO}_{2}{ }^{+}\right]$; HRMS (EI): calcd for $\mathrm{C}_{13} \mathrm{H}_{17} \mathrm{NO}_{5}$ : 267.1107; found 267.1107; elemental analysis calcd (\%) for $\mathrm{C}_{13} \mathrm{H}_{17} \mathrm{NO}_{5}$ (267.3): calcd. C 58.42, H 6.41, N 5.24; found C 58.59, H 6.13, N 5.08.

MeZ-a-Thr-OAll (176): A suspension of dried $\mathrm{K}_{2} \mathrm{CO}_{3}(0.034 \mathrm{~g}, 0.247 \mathrm{mmol})$ in a solution of the $N$-protected acid $175(0.12 \mathrm{~g}, 0.449 \mathrm{mmol})$ and allyl bromide $(0.08 \mathrm{~mL}, 0.946 \mathrm{mmol})$ in anhydrous $\mathrm{MeCN}(4 \mathrm{~mL})$ was vigorously stirred in a sealed tube at $85^{\circ} \mathrm{C}$ for $2 \mathrm{~h}$. The mixture was then allowed to cool to $60^{\circ} \mathrm{C}$ and stirring continued for an additional $16 \mathrm{~h}$. The reaction mixture was cooled to $20^{\circ} \mathrm{C}$, and $\mathrm{Et}_{2} \mathrm{O}(50 \mathrm{~mL})$ and water $(20 \mathrm{~mL})$ were then added. The organic layer was washed with water $(4 \times 10 \mathrm{~mL})$, saturated aqueous $\mathrm{NaHCO}_{3}(2 \times 10 \mathrm{~mL})$, brine $(2 \times 5$ $\mathrm{mL}$ ), dried, filtered and concentrated under reduced pressure. The residual oil was triturated with $\mathrm{Et}_{2} \mathrm{O}$ /pentane 1:2 to give a white solid. Then more pentane was added to complete precipitation and 176 was filtered off and dried under reduced pressure $(0.116 \mathrm{~g}, 84 \%) . R_{\mathrm{f}}=0.16$ EtOAc/hexanes 1:3; m.p. $47-48{ }^{\circ} \mathrm{C} ;[\alpha]_{20}^{D}-20.4\left(c=0.30, \mathrm{CHCl}_{3}\right) ;{ }^{1} \mathrm{H}$ NMR $(250 \mathrm{MHz}$, $\left.\mathrm{CDCl}_{3}\right): \delta=1.20(\mathrm{~d}, J=6.5 \mathrm{~Hz}, 3 \mathrm{H}, 4-\mathrm{H}), 2.35\left(\mathrm{~s}, 3 \mathrm{H}, 1^{\prime}-\mathrm{H}\right), 2.74(\mathrm{~d}, J=6.3 \mathrm{~Hz}, 1 \mathrm{H}, \mathrm{OH})$, 4.09-4.26 (m, $1 \mathrm{H}, 3-\mathrm{H}), 4.47(\mathrm{dd}, J=7.5,3.8 \mathrm{~Hz}, 1 \mathrm{H}, 2-\mathrm{H}), 4.67$ (d, $J=5.3 \mathrm{~Hz}, 2 \mathrm{H}, 1-\mathrm{H}, A l l)$, 5.08 (s, $2 \mathrm{H}, \mathrm{Bzl}-\mathrm{H}), 5.27$ (ddt, $J=10.3,1.0,1.0 \mathrm{~Hz}, 1 \mathrm{H}$, trans-3-H, All), 5.34 (ddt, $J=17.3$, 1.0, $1.0 \mathrm{~Hz}, 1 \mathrm{H}$, cis-3-H, All), 5.66 (d, $J=7.3 \mathrm{~Hz}, 1 \mathrm{H}, \mathrm{NH}), 5.90$ (ddt, $J=17.3,10.3,5.8 \mathrm{~Hz}$, $1 \mathrm{H}, 2-\mathrm{H}, A l l), 7.17(\mathrm{~d}, J=8.0 \mathrm{~Hz}, 2 \mathrm{H}, \mathrm{Ar}-\mathrm{H}), 7.26(\mathrm{~d}, J=8.0 \mathrm{~Hz}, 2 \mathrm{H}, \mathrm{Ar}-\mathrm{H}) ;{ }^{13} \mathrm{C}$ NMR $(62.9$ $\left.\mathrm{MHz}, \mathrm{CDCl}_{3}\right): \delta=18.7(+, \mathrm{C}-4), 20.9\left(+, \mathrm{C}-1^{\prime}\right), 59.3(+, \mathrm{C}-2), 65.9(-, \mathrm{C}-1, \mathrm{All}), 67.4$ (-, Bzl-C), $68.4(+, \mathrm{C}-3), 118.7(-, \mathrm{C}-3, \mathrm{All}), 128.0,128.9$ (+, Ar-C), $132.8(+, \mathrm{C}-2, \mathrm{All}), 132.8,137.7\left(\mathrm{C}_{\text {quat }}\right.$, Ar-C), $156.4\left(\mathrm{C}_{\text {quat }}, \mathrm{NCO}_{2}\right), 169.9\left(\mathrm{C}_{\text {quat }}, \mathrm{C}-1\right)$; IR $(\mathrm{KBr}): \mathrm{nu}($ tilde $)=3409 \mathrm{~cm}^{-1}, 3327,3056$, 
2972, 2941, 2889, 1744, 1690, 1536, 1463, 1382, 1338, 1284, 1233, 1183; MS (EI, 70 eV), m/z

$(\%)=307(2)\left[\mathrm{M}^{+}\right], 263(4)\left[\mathrm{M}^{+}-\mathrm{C}_{2} \mathrm{H}_{4} \mathrm{O}\right], 202(8)\left[\mathrm{M}^{+}-\mathrm{C}_{8} \mathrm{H}_{9}\right], 105(100)\left[\mathrm{C}_{8} \mathrm{H}_{9}^{+}\right], 77(5)$

$\left[\mathrm{C}_{6} \mathrm{H}_{5}^{+}\right], 41(5)\left[\mathrm{C}_{3} \mathrm{H}_{5}^{+}\right]$; HRMS (EI): calcd for $\mathrm{C}_{16} \mathrm{H}_{21} \mathrm{NO}_{5}$ : 307.1420; found 307.1420; elemental analysis calcd (\%) for $\mathrm{C}_{16} \mathrm{H}_{21} \mathrm{NO}_{5}$ (307.5): C 62.53, H 6.89, $\mathrm{N} 4.56$; found $\mathrm{C} 62.80, \mathrm{H} 6.84, \mathrm{~N}$ 4.41 .

$N-Z-3-(2 S, 3 R)-\beta$-Methylphenylalanine (177): A solution of ZOSu $(0.396 \mathrm{~g}, 1.59 \mathrm{mmol})$ in acetone $(5 \mathrm{~mL})$ was added to a vigorously stirred solution of $3-(2 S, 3 R)$-methylphenylalanine hydrochloride $\mathrm{HCl} .78(0.35 \mathrm{~g}, 1.62 \mathrm{mmol})$ and $\mathrm{NaHCO}_{3}(0.409 \mathrm{~g}, 4.87 \mathrm{mmol})$ in water $(5 \mathrm{~mL})$ and stirring continued for $2 \mathrm{~h}$ (if emulsion formed acetone and/or water were added to obtain homogeneous solution). Acetone was then removed under reduced pressure, the residual fraction was diluted with water $(25 \mathrm{~mL})$ and washed with $\mathrm{Et}_{2} \mathrm{O}(3 \times 10 \mathrm{~mL})$. The organic fraction was back extracted with $5 \%$ aqueous $\mathrm{NaHCO}_{3}(3 \times 10 \mathrm{~mL})$, $\mathrm{pH}$ of the combined water fractions were adjusted to 1 with $1 \mathrm{M} \mathrm{HCl}$ and the resulting emulsion was extracted with $\mathrm{Et}_{2} \mathrm{O}(2 \times 50 \mathrm{~mL})$. The organic layer was washed with $1 \mathrm{M} \mathrm{HCl}(2 \times 10 \mathrm{~mL})$, water $(10 \times 10 \mathrm{~mL})$, brine $(2 \times 10 \mathrm{~mL})$, dried, filtered and concentrated under reduced pressure. The residual oil was dissolved in $\mathrm{Et}_{2} \mathrm{O}(3$ $\mathrm{mL})$ and dicyclohexylamine $(0.277 \mathrm{~g}, 1.53 \mathrm{mmol})$ was added followed by hexanes $(20 \mathrm{~mL})$ and the resulting precipitate was filtered and crystallized twice from EtOAc/hexanes to give dicyclohexylammomium salt of $177(0.67 \mathrm{~g}, 85 \%)$ as a white solid. To obtain an analytical sample of 177 a small quantity of the dicyclohexylammionium salt dissolved in EtOAc and washed twice with $1 \mathrm{M} \mathrm{HCl}$, thrice with water, twice with brine to give after prolonged drying at 0.02 Torr and at $60{ }^{\circ} \mathrm{C} 177$ as a colorless solid. M.p. $76.5-79{ }^{\circ} \mathrm{C} ;[\alpha]_{20}^{D} 17.3\left(c=0.76, \mathrm{CHCl}_{3}\right)$ ${ }^{1} \mathrm{H}$ NMR $\left(250 \mathrm{MHz}, \mathrm{CDCl}_{3}\right): \delta=1.31,1.36(2 \times \mathrm{d}, J=7.0 \mathrm{~Hz}, 3 \mathrm{H}, 4-\mathrm{H}), 3.33(\mathrm{dq}, J=7.0$, $7.0 \mathrm{~Hz}, 1 \mathrm{H}, 3-\mathrm{H}), 4.43-4.55(\mathrm{~m}, 0.25 \mathrm{H}, 2-\mathrm{H}), 4.60$ (dd, $J=5.5 \mathrm{~Hz}, 9 \mathrm{~Hz}, 0.75 \mathrm{H}, 3-\mathrm{H}), 4.78$ (d, $J=12.5 \mathrm{~Hz}, 0.25 \mathrm{H}, \mathrm{Bzl}-\mathrm{H}), 4.96-5.20(\mathrm{~m}, 1.75 \mathrm{H}, \mathrm{Bzl}-\mathrm{H}), 5.30$ (d, $J=9 \mathrm{~Hz}, 0.75 \mathrm{H}, \mathrm{NH}), 6.25$ 
$(\mathrm{d}, J=8.8 \mathrm{~Hz}, 0.20 \mathrm{H}, \mathrm{NH}), 7.03-7.52\left(\mathrm{~m}, 10 \mathrm{H}, 1^{\prime \prime}-\mathrm{H}\right), 4.56-4.69,4.71-4.87\left(2 \times \mathrm{m}, 1 \mathrm{H}, 2^{\prime}-\right.$ H), $5.48(\mathrm{~d}, J=7.0 \mathrm{~Hz}, 0.6 \mathrm{H}, \mathrm{NH}), 7.01-7.13(\mathrm{~m}, 10 \mathrm{H}, \mathrm{Ar}-\mathrm{H}), 7.30-7.90\left(\mathrm{br}, 1 \mathrm{H}, \mathrm{CO}_{2} \mathrm{H}\right) ;{ }^{13} \mathrm{C}$ NMR (62.9 MHz, $\left.\mathrm{CDCl}_{3}\right): \delta=14.3,15.8(+, \mathrm{C}-4), 41.5,41.8(+, \mathrm{C}-3), 59.1,59.8(+, \mathrm{C}-2), 67.0$, 67.4 (-, Bzl-C), 127.0 (+, Ar-C), 127.5 (+, Ar-C), 127.6 (+, Ar-C), 127.9 (+, Ar-C), 128.0 (+, Ar-C), 128.3, 128.3 (+, Ar-C), 135.2, 135.9 ( $\left.\mathrm{C}_{\text {quat }}, \mathrm{Ar}-\mathrm{C}\right), 140.9,141.4\left(\mathrm{C}_{\text {quat }}, \mathrm{Ar}-\mathrm{C}\right), 156.0$, $157.1\left(\mathrm{C}_{\text {quat }}, \mathrm{NCO}_{2}\right), 175.1,175.4\left(\mathrm{C}_{\text {quat }}, \mathrm{C}-1\right)$; IR $(\mathrm{KBr}): \mathrm{nu}($ tilde $)=3750-2250 \mathrm{~cm}^{-1}, 1718$, $1518,1497,1454,1416,1347,1217$; MS (EI, $70 \mathrm{eV}), m / z(\%)=313(1)\left[\mathrm{M}^{+}\right], 209(4)\left[\mathrm{M}^{+}-\right.$ $\left.\mathrm{C}_{8} \mathrm{H}_{8}\right], 162$ (10), $105(100)\left[\mathrm{C}_{8} \mathrm{H}_{9}^{+}\right], 91(48)\left[\mathrm{C}_{7} \mathrm{H}_{7}^{+}\right], 65$ (7) $\left[\mathrm{C}_{5} \mathrm{H}_{5}{ }^{+}\right]$; HRMS (EI): calcd for $\mathrm{C}_{18} \mathrm{H}_{19} \mathrm{NO}_{4}$ : 313.1314; found 313.1314; elemental analysis calcd (\%) for $\mathrm{C}_{18} \mathrm{H}_{19} \mathrm{NO}_{4}$ (313.4): C 69.00, H 6.11, N 4.47; found C 69.16, H 6.00, N 4.45.

N-Z Isoleucine methoxymethyl ester (179): To an ice-cold solution of MOM-Cl (0.43 mL, 5.65 mmol) in acetonitrile $(7 \mathrm{~mL}) \mathrm{LiI}(0.783 \mathrm{~g}, 5.65 \mathrm{mmol})$ was added. The mixture was stirred at the same temperature for $20 \mathrm{~min}$ and a solution of Z-Ile-OH $178(0.75 \mathrm{~g}, 2.83 \mathrm{mmol})$, DIEA (0.731 $\mathrm{g}, 5.65 \mathrm{mmol})$ in acetonitrile $(5 \mathrm{~mL})$ was then added dropwise for $5 \mathrm{~min}$. The reaction mixture was allowed to warm to $20^{\circ} \mathrm{C}$, stirred for an additional $2 \mathrm{~h}$ and then diluted with $\mathrm{Et}_{2} \mathrm{O}(50 \mathrm{~mL})$. After usual aqueous work-up (GP 5), the organic layer was dried, and concentrated under reduced pressure to give the oily residue which was purified by column chromatography (twice, EtOAc/hexanes 1:6, $\left.R_{\mathrm{f}}=0.21\right)$ to give $179(0.665 \mathrm{~g}, 76 \%)$ as a colorless oil. $[\alpha]_{20}^{D} 3.9(c=1.90$, $\left.\mathrm{CHCl}_{3}\right) ;{ }^{1} \mathrm{H} \mathrm{NMR}\left(250 \mathrm{MHz}, \mathrm{CDCl}_{3}\right): \delta=0.93(\mathrm{t}, J=6.9 \mathrm{~Hz}, 3 \mathrm{H}, 5-\mathrm{H}), 0.97(\mathrm{~d}, J=6.8 \mathrm{~Hz}$, $\left.3 \mathrm{H}, 1^{\prime}-\mathrm{H}\right), 1.06-1.32\left(\mathrm{~m}, 1 \mathrm{H}, 4-\mathrm{H}_{\mathrm{a}}\right), 1.35-1.54\left(\mathrm{~m}, 1 \mathrm{H}, 4-\mathrm{H}_{\mathrm{b}}\right), 1.81-2.03$ (m, $\left.1 \mathrm{H}, 3-\mathrm{H}\right), 3.47$ (s, $3 \mathrm{H}, \mathrm{OMe}), 4.38$ (dd, $J=9,4.5 \mathrm{~Hz}, 1 \mathrm{H}, 2-\mathrm{H}), 5.11(\mathrm{~s}, 2 \mathrm{H}, \mathrm{Bzl}-\mathrm{H}), 5.22\left(\mathrm{~d}, J=5.8 \mathrm{~Hz}, 1 \mathrm{H}, \mathrm{H}_{\mathrm{a}}\right.$, $\left.\mathrm{OCH}_{2} \mathrm{O}\right), 5.28(\mathrm{~d}, J=6.0 \mathrm{~Hz}, 1 \mathrm{H}, \mathrm{NH}), 5.35\left(\mathrm{~d}, J=5.8 \mathrm{~Hz}, 1 \mathrm{H}, \mathrm{H}_{\mathrm{b}}, \mathrm{OCH}_{2} \mathrm{O}\right), 7.28-7.42(\mathrm{~m}$, $5 \mathrm{H}, \mathrm{Ar}-\mathrm{H}) ;{ }^{13} \mathrm{C}$ NMR $\left(62.9 \mathrm{MHz}, \mathrm{CDCl}_{3}\right): \delta=11.2(+, \mathrm{C}-5), 15.1\left(+, \mathrm{C}-1^{\prime}\right), 24.5(-, \mathrm{C}-4), 57.3$ (+, OMe), $37.4(+, \mathrm{C}-3), 58.1(+, \mathrm{C}-2), 66.5$ (-, Bzl-C), $90.6\left(-, \mathrm{OCH}_{2} \mathrm{O}\right), 127.7,127.7,128.1(+$, 
Ar-C), $136.0\left(\mathrm{C}_{\text {quat }}, \mathrm{Ar}-\mathrm{C}\right), 155.9\left(\mathrm{C}_{\text {quat }}, \mathrm{NCO}_{2}\right), 171.4\left(\mathrm{C}_{\text {quat }}, \mathrm{C}-1\right)$; IR (film): nu(tilde) $=3390$ $\mathrm{cm}^{-1}, 3066,3034,2965,2937,2878,2832,1724,1525,1455,1412,1337,1220,1087,1041$.

N-Fmoc Isoleucine dicyclopropylmethyl ester (182): To a stirred ice-cold solution of $N$-Fmoc protected isoleucine $\mathbf{1 8 1}(1 \mathrm{~g}, 28.29 \mathrm{mmol})$ in anhydrous $\mathrm{CH}_{2} \mathrm{Cl}_{2}(10 \mathrm{~mL})$ oxalyl chloride $(0.898$ g, $7.07 \mathrm{mmol}$ ) and then DMF (5 drops) were added and stirring continued at the same temperature for $2 \mathrm{~h}$. The mixture was then allowed to warm to $20^{\circ} \mathrm{C}$ and stirred for an additional $1 \mathrm{~h}$. The solvent was then flow out with nitrogen stream and the crude chloranhydride was dried at 0.01 Torr for $2 \mathrm{~h}$ and used further without purification. The chloranhydride was dissolved in anhydrous $\mathrm{CH}_{2} \mathrm{Cl}_{2}(10 \mathrm{~mL})$ and the mixture of pyridine/dicyclopropylmethanol 1:1 $(1.5 \mathrm{~mL})$ was then added. After 40 min DMAP (0.02 g) was added to the mixture and stirring continued for an additional $3 \mathrm{~h}$. The reaction mixture was then diluted with $\mathrm{Et}_{2} \mathrm{O}(50 \mathrm{~mL})$, washed (according to GP 5), dried, filtered and concentrated under reduced pressure. The residue was purified by column chromatography $\left(\right.$ EtOAc/hexanes 1:10 $\left.\left(0.5 \% \mathrm{Et}_{3} \mathrm{~N}\right), R_{\mathrm{f}}=0.24\right)$. The appropriate fractions were pooled, concentrated under reduced pressure, taken up with $\mathrm{Et}_{2} \mathrm{O} /$ hexanes 1:1 (100 mL), washed with water $(3 \times 20 \mathrm{~mL}), 3 \%$ aqueous $\mathrm{NaHCO}_{3}(3 \times 20 \mathrm{~mL})$, water $(3 \times 20 \mathrm{~mL})$, brine $(2$ $\times 10 \mathrm{~mL})$, dried, filtered and concentrated under reduced pressure to give $\mathbf{1 8 2}(0.72 \mathrm{~g}, 57 \%)$ as a turbid oil. . $[\alpha]_{20}^{D}-3.8\left(c=0.26, \mathrm{CHCl}_{3}\right) ;{ }^{1} \mathrm{H} \mathrm{NMR}\left(250 \mathrm{MHz}, \mathrm{CDCl}_{3}\right): \delta=0.16-0.38(\mathrm{~m}, 4 \mathrm{H}$, $\left.2^{\prime}-\mathrm{H}_{\mathrm{a}}, D C P M\right), 0.38-0.51\left(\mathrm{~m}, 2 \mathrm{H}, 2^{\prime}-\mathrm{H}_{\mathrm{b}}, D C P M\right), 0.51-0.64\left(\mathrm{~m}, 2 \mathrm{H}, 2^{\prime}-\mathrm{H}_{\mathrm{c}}, D C P M\right), 0.94(\mathrm{t}, J=$ $7.5 \mathrm{~Hz}, 3 \mathrm{H}, 5-\mathrm{H}$, Ile), 0.96 (d, $J=7.5 \mathrm{~Hz}, 3 \mathrm{H}, 1^{\prime}-\mathrm{H}$, Ile), 1.02-1.16 (m, $\left.2 \mathrm{H}, 1^{\prime}-\mathrm{H}, D C P M\right)$, 1.17-1.34 (m, $1 \mathrm{H}, 4-\mathrm{H}_{\mathrm{a}}$, Ile), $1.39-1.47$ (m, $1 \mathrm{H}, 4-\mathrm{H}_{\mathrm{b}}$, Ile), 1.86-2.09 (m, $1 \mathrm{H}, 3-\mathrm{H}$, Ile), 3.90 (t, $J=8.8 \mathrm{~Hz}, 1 \mathrm{H}, 1-\mathrm{H}, D C P M), 4.23$ (dd, $J=7.3,7.3 \mathrm{~Hz}, 1 \mathrm{H}, 2-\mathrm{H}$, Ile), $4.34-4.44$ (m, $3 \mathrm{H}, 1^{\prime}-\mathrm{H}$, 2'-H, Fmoc), 5.36 (d, $J=9.8 \mathrm{~Hz}, 1 \mathrm{H}, \mathrm{NH}), 5.16(\mathrm{~d}, J=6.0 \mathrm{~Hz}, 1 \mathrm{H}, \mathrm{NH}), 7.23-7.46$ (m, $4 \mathrm{H}$, Ar-H), $7.6(\mathrm{~d}, J=7.5 \mathrm{~Hz}, 2 \mathrm{H}, \mathrm{Ar}-\mathrm{H}), 7.76(\mathrm{~d}, J=8.3 \mathrm{~Hz}, 2 \mathrm{H}, \mathrm{Ar}-\mathrm{H}) ;{ }^{13} \mathrm{C} \mathrm{NMR}(62.9 \mathrm{MHz}$, $\left.\mathrm{CDCl}_{3}\right): \delta=2.4,2.6,2.9\left(-, \mathrm{C}-2^{\prime}, D C P M\right), 11.6(+, \mathrm{C}-5$, Ile $), 14.5,15.3\left(+, \mathrm{C}-1^{\prime}\right.$, DCPM $), 14.6$ 
(+, C-1', Ile), 24.9 (-, C-4, Ile), 38.1 (+, C-3, Ile), 47.1 (+, C-2', Fmoc), 58.3 (+, C-2, Ile), $66.8(-$ , C-1', Fmoc), 83.4 (+, C-1, DCPM), 119.8, 125.0, 126.9, 127.5 (+, Ar-C), 141.1 (C quat, Ar-C), 143.7, $143.8\left(\mathrm{C}_{\text {quat }}, \mathrm{Ar}-\mathrm{C}\right), 156.0\left(\mathrm{C}_{\text {quat }}, \mathrm{NCO}_{2}\right), 171.5,\left(\mathrm{C}_{\text {quat }}, \mathrm{C}-1\right.$, Ile $)$; IR (film): nu(tilde) $=$ $3400 \mathrm{~cm}^{-1}, 3068,3009,2964,2935,2876,2832,1805,1717,1508,1451,1331,1209,1220$, $1091,1030$.

Z-( $\beta M e)$ Phe-Ile-OMOM (183a): The compound 179 (0.415 g, $1.34 \mathrm{mmol})$ was hydrogenated on $10 \% \mathrm{Pd}$ on charcoal $(0.150 \mathrm{~g})$ under ambient pressure of hydrogen in EtOAc $(10 \mathrm{~mL})$ with AcOH (0.080 g, $1.34 \mathrm{mmol})$ for $1 \mathrm{~h}$. The mixture was then filtered, concentrated under reduced pressure, taken up with $\mathrm{Et}_{2} \mathrm{O}(40 \mathrm{~mL})$, washed with saturated aqueous $\mathrm{NaHCO}_{3}(3 \times 10 \mathrm{~mL})$, brine $(2 \times 10 \mathrm{~mL})$, dried, filtered and concentrated under reduced pressure to give deprotected ester of isoleucine 180a as a free base which was directly used for the condensation with $N-Z$ protected $\beta$-methylphenylalanine $177(0.400 \mathrm{~g}, 1.28 \mathrm{mmol})$ employing EDC (0.257 g, 1.34 mmol), HOAt $(0.173 \mathrm{~g}, 1.28 \mathrm{mmol})$ and TMP $(0.500 \mathrm{~mL}, 3.78 \mathrm{mmol})$ in $\mathrm{CH}_{2} \mathrm{Cl}_{2}(5 \mathrm{~mL})$ according to GP 5. After $2 \mathrm{~h}$, the reaction mixture was subjected usual aqueous work-up and the resulting crude product was purified by crystallization from EtOAc/hexanes to give dipeptide 183a $(0.400 \mathrm{~g}, 66 \%)$ as a white solid. $R_{\mathrm{f}}=0.17$, EtOAc/hexanes $1: 6 ; R_{\mathrm{f}}=0.35$, EtOAc/hexanes 1:4; m.p. $111-112{ }^{\circ} \mathrm{C} ;[\alpha]_{20}^{D}-8.5\left(c=0.30, \mathrm{CHCl}_{3}\right) ;{ }^{1} \mathrm{H}$ NMR $\left(250 \mathrm{MHz}, \mathrm{CDCl}_{3}\right): \delta=0.81(\mathrm{~d}$, $J=6.8 \mathrm{~Hz}, 3 \mathrm{H}, 1^{\prime}-\mathrm{H}$, Ile), 0.87 (t, $J=6.8 \mathrm{~Hz}, 3 \mathrm{H}, 5-\mathrm{H}$, Ile), 0.94-1.17 (m, $1 \mathrm{H}, 4-\mathrm{H}_{\mathrm{a}}$, Ile), $1.24-$ $1.45\left(\mathrm{~m}, 1 \mathrm{H}, 4-\mathrm{H}_{\mathrm{b}}, I l e\right), 1.35$ [d, $\left.J=7.3 \mathrm{~Hz}, 3 \mathrm{H}, 4-\mathrm{H},(\beta M e) P h e\right], 1.68-1.87$ (m, $1 \mathrm{H}, 3-\mathrm{H}$, Ile), $3.17[\mathrm{dq}, J=7.3 \mathrm{~Hz}, 1 \mathrm{H}, 3-\mathrm{H},(\beta M e) P h e], 3.42(\mathrm{~s}, 3 \mathrm{H}, \mathrm{OMe}), 4.27-4.41(\mathrm{~m}, 2 \mathrm{H}, 2 \times 2-\mathrm{H})$, $5.09(\mathrm{~s}, 2 \mathrm{H}, \mathrm{Bzl}-\mathrm{H}), 5.13\left(\mathrm{~d}, J=6.0 \mathrm{~Hz}, 1 \mathrm{H}, \mathrm{H}_{\mathrm{a}}, \mathrm{OCH}_{2} \mathrm{O}\right), 5.21\left(\mathrm{~d}, J=5.8 \mathrm{~Hz}, 1 \mathrm{H}, \mathrm{H}_{\mathrm{b}}\right.$, $\left.\mathrm{OCH}_{2} \mathrm{O}\right), 5.43(\mathrm{~d}, J=9 \mathrm{~Hz}, 1 \mathrm{H}, \mathrm{NH}), 5.98(\mathrm{~d}, J=7.8 \mathrm{~Hz}, \mathrm{NH}), 7.15-7.30(\mathrm{~m}, 6 \mathrm{H}, \mathrm{Ar}-\mathrm{H}), 7.30-$ $7.42(\mathrm{~m}, 4 \mathrm{H}, \mathrm{Ar}-\mathrm{H}) ;{ }^{13} \mathrm{C} \mathrm{NMR}\left(62.9 \mathrm{MHz}, \mathrm{CDCl}_{3}\right): \delta=11.2(+, \mathrm{C}-5$, Ile $), 14.9\left(+, \mathrm{C}-1^{\prime}\right.$, Ile $), 16.9$ [+, C-4, (ßMe)Phe], $24.8(-, \mathrm{C}-4$, Ile $), 37.5(+, \mathrm{C}-3$, Ile $), 42.2[+, \mathrm{C}-3,(\beta M e) P h e], 56.1(+, \mathrm{C}-2$, 
Ile), $57.5(+, \mathrm{OMe}), 60.2$ [+, C-2, ( $\beta M e) P h e], 66.5$ (-, Bzl-C), $90.6\left(-, \mathrm{OCH}_{2} \mathrm{O}\right), 126.6,127.5$, 127.5, 127.7, $128.2(\times 2)(+, A r-C), 136.2,141.9\left(\mathrm{C}_{\text {quat }}, \mathrm{Ar}-\mathrm{C}\right), 156.2,156.8\left(\mathrm{C}_{\text {quat }}, \mathrm{NCO}_{2}\right), 170.4$, $170.6\left(\mathrm{C}_{\text {quat }}, \mathrm{C}-1\right)$; IR $(\mathrm{KBr}): \mathrm{nu}($ tilde $)=3330 \mathrm{~cm}^{-1}, 3275,3065,3033,2965,2928,2874,2832$, $1741,1689,1654,1536,1293,1275,1253,1242,1155,1071,1012 ;$ MS (EI, $70 \mathrm{eV}), \mathrm{m} / z(\%)=$ 470 (4) $\left[\mathrm{M}^{+}\right], 366(11)\left[\mathrm{M}^{+}-\mathrm{C}_{8} \mathrm{H}_{8}\right], 319$ (18) $\left[\mathrm{M}^{+}-\mathrm{C}_{8} \mathrm{H}_{8} \mathrm{NO}_{2}\right], 289$ (15), 268 (8), 224 (20), 199 (6), 105 (10) $\left[\mathrm{C}_{8} \mathrm{H}_{9}^{+}\right], 91(100)\left[\mathrm{C}_{7} \mathrm{H}_{7}^{+}\right], 86$ (10), 45 (12) $\left[\mathrm{CHO}_{2}^{+}\right]$; HRMS (EI): calcd for $\mathrm{C}_{26} \mathrm{H}_{34} \mathrm{~N}_{2} \mathrm{O}_{6}$ : 470.2417; found 470.2417; elemental analysis calcd (\%) for $\mathrm{C}_{26} \mathrm{H}_{34} \mathrm{~N}_{2} \mathrm{O}_{6}$ (470.6): C 66.36, H 7.28, N 5.95; found C 66.58, H 7.19, N 6.10.

Z-( $\beta M e) P h e-I l e-O D C P M$ (183b): The substance $182(0.540 \mathrm{~g}, 1.21 \mathrm{mmol})$ was $N$-deprotected according to GP 4 and the resultant amino ester was coupled with $N-Z$ protected ( $\beta$ methylphenylalanine) 177 (0.36 g, $1.15 \mathrm{mmol})$ employing EDC (0.277 g, $1.18 \mathrm{mmol})$, HOAt $(0.160 \mathrm{~g}, 1.18 \mathrm{mmol})$ and TMP $(0.418 \mathrm{~g}, 3.45 \mathrm{mmol})$ in $\mathrm{CH}_{2} \mathrm{Cl}_{2}(5 \mathrm{~mL})$ according to GP 5. After $6 \mathrm{~h}$, the reaction mixture was subjected usual aqueous work-up and the resulting crude product was triturated with pentane and then purified by crystallization from hexanes to give dipeptide 183b $(0.500 \mathrm{~g}, 84 \%)$ as a white solid. $R_{\mathrm{f}}=0.17$, EtOAc/hexanes 1:6 $\left(0.5 \% \mathrm{Et}_{3} \mathrm{~N}\right)$; m.p 105$106{ }^{\circ} \mathrm{C} ;[\alpha]_{20}^{D} 9.0\left(c=0.31, \mathrm{CHCl}_{3}\right) ;{ }^{1} \mathrm{H} \mathrm{NMR}\left(250 \mathrm{MHz}, \mathrm{CDCl}_{3}\right): \delta=0.23-0.41\left(\mathrm{~m}, 4 \mathrm{H}, 2^{\prime}-\mathrm{H}_{\mathrm{a}}\right.$, $D C P M), 0.41-0.53\left(\mathrm{~m}, 2 \mathrm{H}, 2^{\prime}-\mathrm{H}_{\mathrm{b}}, D C P M\right), 0.53-0.64\left(\mathrm{~m}, 2 \mathrm{H}, 2^{\prime}-\mathrm{H}_{\mathrm{c}}, D C P M\right), 0.81(\mathrm{~d}, J=$ $6.8 \mathrm{~Hz}, 3 \mathrm{H}, 1^{\prime}-\mathrm{H}$, Ile), 0.89 (t, $J=7.3 \mathrm{~Hz}, 3 \mathrm{H}, 5-\mathrm{H}$, Ile), 0.95-1.21 (m, $3 \mathrm{H}, 4-\mathrm{H}_{\mathrm{a}}$, Ile, 1'-H, $D C P M), 1.35[\mathrm{~d}, J=7.3 \mathrm{~Hz}, 3 \mathrm{H},(\beta M e) P h e], 1.37-1.48\left(\mathrm{~m}, 1 \mathrm{H}, 4-\mathrm{H}_{\mathrm{b}}\right.$, Ile $), 1.71-1.90(\mathrm{~m}, 1 \mathrm{H}$, 3-H, Ile), 3.19 [dq, $J=7.3 \mathrm{~Hz}, 1 \mathrm{H},(\beta M e) P h e], 3.90$ (t, $J=8.5 \mathrm{~Hz}, 1 \mathrm{H}, 1-\mathrm{H}, D C P M), 4.26-4.42$ (m, $2 \mathrm{H}, 2 \times 2-\mathrm{H}), 5.09(\mathrm{~s}, 2 \mathrm{H}, \mathrm{Bzl}-\mathrm{H}), 5.42(\mathrm{~d}, J=9.0 \mathrm{~Hz}, 1 \mathrm{H}, \mathrm{NH}), 6.06(\mathrm{~d}, J=7.5 \mathrm{~Hz}, 1 \mathrm{H}$, $\mathrm{NH}), 7.15-7.30(\mathrm{~m}, 6 \mathrm{H}, \mathrm{Ar}-\mathrm{H}), 7.30-7.40(\mathrm{~m}, 4 \mathrm{H}, \mathrm{Ar}-\mathrm{H}) ;{ }^{13} \mathrm{C} \mathrm{NMR}\left(62.9 \mathrm{MHz}, \mathrm{CDCl}_{3}\right): \delta=$ 2.4, $2.7\left(-, \mathrm{C}-2^{\prime}\right.$, DCPM), $11.4(+, \mathrm{C}-5$, Ile $), 14.3,14.5\left(+, \mathrm{C}-1^{\prime}\right.$, DCPM $), 14.8\left(+, \mathrm{C}-1^{\prime}\right.$, Ile $), 16.7$ [+, C-4, ( $\beta M e) P h e], 24.9(-, \mathrm{C}-4$, Ile $), 37.9(+, \mathrm{C}-3$, Ile $), 42.2[+, \mathrm{C}-3,(\beta M e) P h e], 56.1(+, \mathrm{C}-2$, 
Ile), 60.1 [+, C-2, (ßMe)Phe], 66.5 (-, Bzl-C), 82.9 (+, C-1, DCPM), 126.5, 127.4, 127.5, 127.6, $128.1(\times 2)(+, \mathrm{Ar}-\mathrm{C}), 136.2,141.9\left(\mathrm{C}_{\text {quat }}, \mathrm{Ar}-\mathrm{C}\right), 156.1\left(\mathrm{C}_{\text {quat }}, \mathrm{NCO}_{2}\right), 170.2,170.5\left(\mathrm{C}_{\text {quat }}, \mathrm{C}-1\right.$, Ile); IR (KBr): nu(tilde) $=3300 \mathrm{~cm}^{-1}, 3270,3010,2965,2875,1732,1690,1655,1535,1454$, 1379, 1334, 1292, 1275, 1255, 1240, 1196, 1137, 1013; MS (EI, $70 \mathrm{eV}), \mathrm{m} / z(\%)=520(8)\left[\mathrm{M}^{+}\right]$, 416 (7) $\left[\mathrm{M}^{+}-\mathrm{C}_{8} \mathrm{H}_{8}\right], 381(8)\left[\mathrm{M}^{+}-\mathrm{C}_{7} \mathrm{H}_{9} \mathrm{NO}_{2}\right], 296$ (20) $\left[\mathrm{C}_{18} \mathrm{H}_{18} \mathrm{NO}_{2}\right], 268$ (11), 224 (20), 176 (6), 105 (7) $\left[\mathrm{C}_{8} \mathrm{H}_{9}^{+}\right], 95$ (100), 86 (17), 67 (10); HRMS (EI): calcd for $\mathrm{C}_{31} \mathrm{H}_{40} \mathrm{~N}_{2} \mathrm{O}_{5}$ : 520.2937; found 520.2937; elemental analysis calcd (\%) for $\mathrm{C}_{26} \mathrm{H}_{34} \mathrm{~N}_{2} \mathrm{O}_{6}$ (470.6): C 71.51, H 7.74, N 5.38; found C 71.51, H 7.42, N 5.40.

Fmoc-(2S)-(3-Ncp)Ala-( $\beta M e)$ Phe-Ile-OMOM (185a): The compound 183a $(0.370 \mathrm{~g}, 0.786$ mmol) was hydrogenated on $10 \% \mathrm{Pd}$ on charcoal $(0.100 \mathrm{~g})$ under ambient pressure of hydrogen in EtOAc $(10 \mathrm{~mL})$ with $\mathrm{AcOH}(0.048 \mathrm{~g}, 0.800 \mathrm{mmol})$ for $1 \mathrm{~h}$. The mixture was then filtered, concentrated under reduced pressure, taken up with $\mathrm{Et}_{2} \mathrm{O}(40 \mathrm{~mL})$, washed with saturated aqueous $\mathrm{NaHCO}_{3}(3 \times 10 \mathrm{~mL})$, brine $(2 \times 10 \mathrm{~mL})$, dried, filtered and concentrated under reduced pressure to give deprotected dipeptide 184a as a free base together with cyclo-[( $\beta \mathrm{Me}) \mathrm{Phe}-\mathrm{Ile}]$. This mixture was directly used for the condensation with $N$-Fmoc protected amino acid $\mathbf{1 6 0}$ (0.320 g, $0.807 \mathrm{mmol})$ employing EDC (0.160 g, $0.835 \mathrm{mmol})$, HOAt (0.111 g, $0.821 \mathrm{mmol})$ and TMP (0.430 mL, $3.254 \mathrm{mmol})$ in $\mathrm{CH}_{2} \mathrm{Cl}_{2}(5 \mathrm{~mL})$ according to GP 5. After $15 \mathrm{~h}$, the reaction mixture was subjected usual aqueous work-up and the resulting crude product was first crystallized from $\mathrm{CH}_{2} \mathrm{Cl}_{2} /$ hexanes and then purified by column chromatography $\left(\mathrm{CHCl}_{3} / \mathrm{MeOH}\right.$ $\left.=60: 1(1 \% \mathrm{EtOH}), R_{\mathrm{f}}=0.28\right)$ to give tripeptide $\mathbf{1 8 5 a}(0.220 \mathrm{~g}, 39 \%)$ as a white solid. $[\alpha]_{20}^{D}-$ $15.3\left(c=0.40, \mathrm{CHCl}_{3}\right) ;{ }^{1} \mathrm{H} \mathrm{NMR}\left(250 \mathrm{MHz}, \mathrm{CDCl}_{3}\right): \delta=0.82\left(\mathrm{q}, J=7.3 \mathrm{~Hz}, 6 \mathrm{H}, 5-\mathrm{H}, 1^{\prime}-\mathrm{H}\right.$, Ile), 0.93-1.17 [m, $2 \mathrm{H}, 4-\mathrm{H}_{\mathrm{a}}$, Ile, 3'- $\left.\mathrm{H}_{\mathrm{a}},(3-N c p) A l a\right], 1.26-1.43\left(\mathrm{~m}, 1 \mathrm{H}, 4-\mathrm{H}_{\mathrm{b}}\right.$, Ile $), 1.32$ [d, J= $6.5 \mathrm{~Hz}, 3 \mathrm{H}, 4-\mathrm{H},(\beta M e) P h e], 1.48-1.70\left[\mathrm{~m}, 1 \mathrm{H}, 3^{\prime}-\mathrm{H}_{\mathrm{b}},(3-N c p)\right.$ Ala $], 1.70-1.84[\mathrm{~m}, 2 \mathrm{H}, 3-\mathrm{H}$, Ile, 1'-H, (3-Ncp)Ala], 1.84-1.99 [m, 2 H, 3-H, (3-Ncp)Ala], 3.22 [dq, J = 7.4 Hz, $1 \mathrm{H}, 3-\mathrm{H}$, 
(ßMe)Phe], 3.40 (s, $3 \mathrm{H}, \mathrm{OMe}), 4.00-4.10(\mathrm{~m}, 1 \mathrm{H}, 2-\mathrm{H}), 4.20(\mathrm{dd}, J=6.5,6.5 \mathrm{~Hz}, \mathrm{C}-2), 4.26-$ $4.44\left[\mathrm{~m}, 3 \mathrm{H}, 1^{\prime}-\mathrm{H}, 2^{\prime}-\mathrm{H}_{\mathrm{a}}, F m o c, 2^{\prime}-\mathrm{H},(3-N c p) A l a\right], 4.49$ (dd, J=10.6, 6.9 Hz, $1 \mathrm{H}, 2^{\prime}-\mathrm{H}$, Fmoc), $4.62(\mathrm{dd}, J=8.5,8.5 \mathrm{~Hz}, 1 \mathrm{H}, \mathrm{C}-2), 5.09\left(\mathrm{~d}, J=5.9 \mathrm{~Hz}, 1 \mathrm{H}, \mathrm{H}_{\mathrm{a}}, \mathrm{OCH}_{2} \mathrm{O}\right), 5.20(\mathrm{~d}, J=5.9 \mathrm{~Hz}$, $\left.1 \mathrm{H}, \mathrm{H}_{\mathrm{b}}, \mathrm{OCH}_{2} \mathrm{O}\right), 5.63(\mathrm{~d}, J=8.0 \mathrm{~Hz}, 1 \mathrm{H}, \mathrm{NH}), 6.17(\mathrm{~d}, J=8.0 \mathrm{~Hz}, \mathrm{NH}), 6.95(\mathrm{~d}, J=8.5 \mathrm{~Hz}$, $1 \mathrm{H}, \mathrm{NH}), 7.14-7.31(\mathrm{~m}, 7 \mathrm{H}, \mathrm{Ar}-\mathrm{H}), 7.40(\mathrm{dd}, J=7.5,7.5 \mathrm{~Hz}, 2 \mathrm{H}, \mathrm{Ar}-\mathrm{H}), 7.58(\mathrm{dd}, J=7.3$, $5.0 \mathrm{~Hz}, 2 \mathrm{H}, \mathrm{Ar}-\mathrm{H}), 7.77(\mathrm{~d}, J=7 \mathrm{~Hz}, 2 \mathrm{H}, \mathrm{Ar}-\mathrm{H}) ;{ }^{13} \mathrm{C} \mathrm{NMR}\left(62.9 \mathrm{MHz}, \mathrm{CDCl}_{3}\right): \delta=11.4(+, \mathrm{C}-$ 5, Ile $), 15.0\left(+, \mathrm{C}-1^{\prime}\right.$, Ile $), 17.1[+, \mathrm{C}-4,(\beta M e) P h e], 17.9\left[-, \mathrm{C}-3^{\prime},(3-N c p) A l a\right], 22.1\left[+, \mathrm{C}-1^{\prime},(3-\right.$ Ncp)Ala], 24.9 (-, C-4, Ile), 34.0 [-, C-3, (3-Ncp)Ala], 37.7 (+, C-3, Ile), 41.9 [+, C-3,

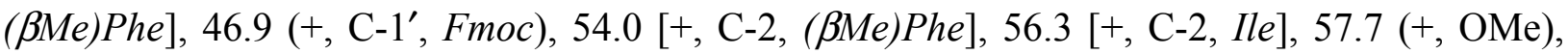
$58.7[+, \mathrm{C}-2,(3-N c p) A l a], 59.0\left[+, \mathrm{C}-2^{\prime},(3-N c p) A l a\right], 67.1\left(+, \mathrm{C}-2^{\prime}\right.$, Fmoc $), 90.9\left(-, \mathrm{OCH}_{2} \mathrm{O}\right)$, $119.9,124.9,126.9,127.0,127.6,127.7,128.4$ (+, Ar-C), 141.1, $141.2\left(\mathrm{C}_{\text {quat }}\right.$, Ar-C, Fmoc), 141.6 [ $\left.\mathrm{C}_{\text {quat }}, \mathrm{Ar}-\mathrm{C},(\beta M e) P h e\right], 143.5,143.7\left(\mathrm{C}_{\text {quat }}, \mathrm{Ar}-\mathrm{C}, F m o c\right), 156.0\left(\mathrm{C}_{\text {quat }}, \mathrm{NCO}_{2}\right), 170.2$, 170.6, $171.2\left(\mathrm{C}_{\text {quat }}, \mathrm{C}-1\right)$; IR $(\mathrm{KBr}): \mathrm{nu}($ tilde $)=3330 \mathrm{~cm}^{-1}, 3275,3067,2966,1718,1643,1542$, 1451, 1368, 1227, 1163, 1139, 1091; MS (EI, $70 \mathrm{eV}), \mathrm{m} / z(\%)=715(0.04)\left[\mathrm{M}^{+}\right], 518(3), 429$ (13), 321 (5), 196 (20), 178 (100), 165 (54), 199 (6), 134 (16), 86 (23), 45 (20) $\left[\mathrm{CHO}_{2}^{+}\right]$; MS (ESI): positive mode, $m / z(\%)=737(30)\left[\mathrm{M}+\mathrm{Na}^{+}\right]$; negative mode, $m / z(\%)=749(40)[\mathrm{M}+$ $\left.\mathrm{Cl}^{-}\right]$.

Fmoc-(3-Ncp)Ala-(ßMe)Phe-Ile-ODCPM (185b): Dipeptide 183b (0.35 g, $0.67 \mathrm{mmol})$ was taken up with EtOAc $(10 \mathrm{~mL})$ and hydrogenated over $10 \% \mathrm{Pd} / \mathrm{C}(0.15 \mathrm{~g})$ under ambient pressure of hydrogen for $2 \mathrm{~h}$. The reaction mixture was filtered through a pad of Celite and concentrated under reduced pressure to give deprotected dipeptide $\mathbf{1 8 4 b}$, which was directly used for the coupling with 160 (274 mg, $0.69 \mathrm{mmol})$, using EDC (137 mg, $0.72 \mathrm{mmol})$, HOAt (96 mg, 0.71 mmol) and TMP $(0.25 \mathrm{~mL}, 2.02 \mathrm{mmol})$ according to GP 5 to give compound $\mathbf{1 8 5} \mathbf{b}$ (405 $\mathrm{mg}$, $79 \%$ ) as a colorless solid after 2 recrystallizations from THF/hexanes 1:1. $R_{\mathrm{f}}=0.52$, 
EtOAc/hexanes 2:3; m.p $151-155{ }^{\circ} \mathrm{C},[\alpha]_{20}^{D}-3.8\left(c=0.26, \mathrm{CHCl}_{3}\right) ;{ }^{1} \mathrm{H}$ NMR $(250 \mathrm{MHz}$, $\left.\mathrm{CDCl}_{3}\right): \delta=0.20-0.38\left(\mathrm{~m}, 4 \mathrm{H}, 2^{\prime}-\mathrm{H}, D C P M\right), 0.37-0.49\left(\mathrm{~m}, 2 \mathrm{H}, 2^{\prime}-\mathrm{H}, D C P M\right), 0.49-0.62(\mathrm{~m}$, 2 H, 2'-H, DCPM), 0.80 (d, J = 7.0 Hz, 3 H, 1'-H, Ile), 0.86 (t, J=7.5 Hz, 3 H, 5-H, Ile), 0.94$1.20\left[\mathrm{~m}, 4 \mathrm{H}, 4-\mathrm{H}_{\mathrm{a}}\right.$, Ile, $3^{\prime}-\mathrm{H}_{\mathrm{a}}, 3-(N c p)$ Ala $\left., 1^{\prime}-\mathrm{H}, D C P M\right], 1.33-1.49$ [m, $1 \mathrm{H}, 3^{\prime}-\mathrm{H}_{\mathrm{b}}, 3-(N c p)$ Ala $]$, $1.34[\mathrm{~d}, J=7.0 \mathrm{~Hz}, 3 \mathrm{H}, 4-\mathrm{H},(\beta-M e) P h e], 1.50-1.68\left(\mathrm{~m}, 1 \mathrm{H}, 4-\mathrm{H}_{\mathrm{b}}, I l e\right), 1.71-1.85$ [m, $2 \mathrm{H}, 3-$ H, Ile, 1'-H, 3-(Ncp)Ala], 1.85-2.05 [m, 2 H, 3-H, 3-(Ncp)Ala], 3.22 [dq, J= 7.0 Hz, $1 \mathrm{H}, 3-\mathrm{H}$, ( $\beta$-Me)Phe], 3.79 (t, $J=8.6 \mathrm{~Hz}, 1 \mathrm{H}, 1-\mathrm{H}, D C P M), 4.01-4.1(\mathrm{~m}, 1 \mathrm{H}, 2-\mathrm{H}), 4.21(\mathrm{dd}, J=6.5 \mathrm{~Hz}$, $1 \mathrm{H}, 2-\mathrm{H}), 4.26-4.43$ [m, $\left.3 \mathrm{H}, 2^{\prime}-\mathrm{H}, 3-(N c p) A l a, 1^{\prime}-\mathrm{H}, 2^{\prime}-\mathrm{H}_{\mathrm{a}}, F m o c\right], 4.49$ (dd, J = 10.3, $7.0 \mathrm{~Hz}$, $\left.1 \mathrm{H}, 2^{\prime}-\mathrm{H}_{\mathrm{b}}, F m o c\right), 4.61(\mathrm{dd}, J=5.5 \mathrm{~Hz}, 1 \mathrm{H}, 2-\mathrm{H}), 5.58(\mathrm{~d}, J=8.3 \mathrm{~Hz}, 1 \mathrm{H}, \mathrm{NH}), 6.14(\mathrm{~d}, J=$ $7.8 \mathrm{~Hz}, 1 \mathrm{H}, \mathrm{NH}), 6.84(\mathrm{~d}, J=8.0 \mathrm{~Hz}, 1 \mathrm{H}, \mathrm{NH}), 7.12-7.31(\mathrm{~m}, 5 \mathrm{H}, \mathrm{Ar}-\mathrm{H}), 7.33(\mathrm{~d}, J=7.8 \mathrm{~Hz}$, $2 \mathrm{H}, \mathrm{Ar}-\mathrm{H}), 7.40(\mathrm{dd}, J=7.3,7.3 \mathrm{~Hz}, 2 \mathrm{H}, \mathrm{Ar}-\mathrm{H}), 7.58(\mathrm{~d}, J=7.0 \mathrm{~Hz}, 2 \mathrm{H}, \mathrm{Ar}-\mathrm{H}), 7.77$ (d, $J=$ $7.3 \mathrm{~Hz}, 2 \mathrm{H}, \mathrm{Ar}-\mathrm{H}) ;{ }^{13} \mathrm{C} \mathrm{NMR}\left(62.9 \mathrm{MHz}, \mathrm{CDCl}_{3}\right): \delta=2.4,2.7\left(-, \mathrm{C}-2^{\prime}, D C P M\right), 11.4(+, \mathrm{C}-5$, Ile), 14.1, $14.4\left(+, \mathrm{C}-1^{\prime}\right.$, DCPM), $14.8\left(+, \mathrm{C}-1^{\prime}\right.$, Ile $), 16.7[+, \mathrm{C}-4,(\beta-M e) P h e], 17.7$ [-, C-3', $3-$ (Ncp)Ala ], 21.97 [+, C-1', 3-(Ncp)Ala], 24.9 (-, C-4, Ile), 33.9 [-, C-3, 3-(Ncp)Ala], 38.1 (+, C3, Ile), 41.7 [+, C-3, $(\beta-M e) P h e], 46.8\left(+, \mathrm{C}-1^{\prime}, F m o c\right), 53.8(+, \mathrm{C}-2), 56.2(+, \mathrm{C}-2), 58.5(+, \mathrm{C}-$ 2), 58.8 [+, C-2', 3-(Ncp)Ala], 67.0 (-, C-2', Fmoc), 83.1 (+, C-1, DCPM), 119.7, 124.8, 126.6, $127.4(\times 2), 128.2(\times 2), 140.96,141.49\left(\mathrm{C}_{\text {quat }}, \mathrm{Ar}-\mathrm{C}, F m o c\right), 141.5$ [C $\left.\mathrm{C}_{\text {quat }}, \mathrm{Ar}-\mathrm{C},(\beta-M e) P h e\right]$, 143.5, $143.6\left(\mathrm{C}_{\text {quat }}, \mathrm{Ar}-\mathrm{C}, F m o c\right), 155.9\left(\mathrm{C}_{\text {quat }}, \mathrm{NCO}_{2}\right), 169.8,170.5,171.0\left(\mathrm{C}_{\text {quat }}, \mathrm{C}-1\right)$; IR (KBr): $v=2966 \mathrm{~cm}^{-1}, 1717,1645,1543,1451,1368,1147$; MS (ESI): positive mode, $\mathrm{m} / z(\%)=787$ (70) $\left[\mathrm{M}+\mathrm{Na}^{+}\right]$; negative mode, $m / z(\%)=799(38)\left[\mathrm{M}+\mathrm{Cl}^{-}\right]$; elemental analysis calcd $(\%)$ for $\mathrm{C}_{44} \mathrm{H}_{52} \mathrm{~N}_{4} \mathrm{O}_{8}$ (764.9): C 69.09, H 6.85, N 7.32; found C 69.06, H 6.79, N 7.19.

Fmoc-( $\beta M e)$ Phe-(3-Ncp)Ala-( $\beta M e)$ Phe-Ile-OMOM (188a): Tripeptide 185a (126 mg, 0.29 mmol) was deprotected according to GP 4 and then directly coupled with 146 (126 mg, 0.31 mmol) according to GP 5 using EDC (6 mg, $0.31 \mathrm{mmol})$, HOAt (42 mg, $0.31 \mathrm{mmol})$ and TMP 
(106 mg, $0.88 \mathrm{mmol})$ in $\mathrm{CH}_{2} \mathrm{Cl}_{2} / \mathrm{MeCN} 2: 1(3 \mathrm{~mL})$ to give 188a (195 mg, 78\%) as a colorless solid after recrystallization from $\mathrm{CHCl}_{3} /$ hexanes. $R_{\mathrm{f}}=0.27, \mathrm{CHCl}_{3} / \mathrm{MeOH} 70: 1(1 \% \mathrm{EtOH}) ;$ m.p. 209-212 ${ }^{\circ} \mathrm{C}$ (decomp.), $[\alpha]_{20}^{D}-40.2(c=0.3, \mathrm{THF}) ;{ }^{1} \mathrm{H}$ NMR $\left(250 \mathrm{MHz},\left[\mathrm{D}_{8}\right] \mathrm{THF}\right): \delta=0.78(\mathrm{~d}$, $J=7.0 \mathrm{~Hz}, 3 \mathrm{H}, 1^{\prime}-\mathrm{H}$, Ile), $0.80(\mathrm{t}, J=7.0 \mathrm{~Hz}, 3 \mathrm{H}, 5-\mathrm{H}$, Ile $), 0.94-1.17$ [m, $2 \mathrm{H}, 4-\mathrm{H}_{\mathrm{a}}$, Ile, $3^{\prime}-\mathrm{H}_{\mathrm{a}}$, (3-Ncp)Ala], $1.20[\mathrm{~d}, J=6.8 \mathrm{~Hz}, 3 \mathrm{H}, 4-\mathrm{H},(\beta-M e) P h e], 1.33[\mathrm{~d}, J=7.0 \mathrm{~Hz}, 3 \mathrm{H}, 4-\mathrm{H},(\beta-$ Me)Phe], 1.34-1.54 [m, 4 H, 3-H, 4- $\mathrm{H}_{\mathrm{a}}$, Ile, 1'- $\left.\mathrm{H}, 3^{\prime}-\mathrm{H}_{\mathrm{b}},(3-N c p) A l a\right], 1.59-1.87$ [m, $2 \mathrm{H}, 3-\mathrm{H}$, (3-Ncp)Ala], 3.10 [dq, $J=10.3,7.1 \mathrm{~Hz}, 1 \mathrm{H}, 3-\mathrm{H},(\beta-M e) P h e], 3.21$ [dq, $J=7.5,7.5 \mathrm{~Hz}, 1 \mathrm{H}, 3-$ $\mathrm{H},(\beta-M e) P h e], 3.31$ (s, $3 \mathrm{H}, \mathrm{OMe}), 3.95\left[\mathrm{ddd}, J=6.8,3.3,3.3 \mathrm{~Hz}, 1 \mathrm{H}, 2^{\prime}-\mathrm{H},(3-N c p)\right.$ Ala $], 4.13-$ $4.40\left(\mathrm{~m}, 5 \mathrm{H}, 2 \times 2-\mathrm{H}, 1^{\prime}-\mathrm{H}, 2^{\prime}-\mathrm{H}, F m o c\right), 4.46$ [ddd, $J=3.3,3.3,3.3 \mathrm{~Hz}, 1 \mathrm{H}, 2-\mathrm{H},(3-N c p)$ Ala $]$, $4.55(\mathrm{dd}, J=8.6,8.6 \mathrm{~Hz}, 1 \mathrm{H}, 2-\mathrm{H}), 5.03\left(\mathrm{~d}, J=5.6 \mathrm{~Hz}, 1 \mathrm{H}, \mathrm{H}_{\mathrm{a}}, \mathrm{OCH}_{2} \mathrm{O}\right), 5.11(\mathrm{~d}, J=5.6 \mathrm{~Hz}$, $\left.1 \mathrm{H}, \mathrm{H}_{\mathrm{b}}, \mathrm{OCH}_{2} \mathrm{O}\right), 6.97-7.46(\mathrm{~m}, 16 \mathrm{H}, 4 \times \mathrm{NH}, 12 \times \mathrm{Ar}-\mathrm{H}), 7.54(\mathrm{dd}, J=10.9,8.9 \mathrm{~Hz}, 2 \mathrm{H}, \mathrm{Ar}-$ H), 7.66 (dd, $J=9.9,7.6 \mathrm{~Hz}, 2 \mathrm{H}, \mathrm{Ar}-\mathrm{H}), 7.77(\mathrm{~d}, J=7.5 \mathrm{~Hz}, 2 \mathrm{H}, \mathrm{Ar}-\mathrm{H}) ;{ }^{13} \mathrm{C}$ NMR $(62.9 \mathrm{MHz}$, $\left.\left[\mathrm{D}_{8}\right] \mathrm{THF}\right): \delta=11.9(+, \mathrm{C}-5$, Ile $), 15.9\left(+, \mathrm{C}-1^{\prime}\right.$, Ile $), 17.9[+, \mathrm{C}-4,(\beta-M e) P h e], 18.6[+, \mathrm{C}-4,(\beta-$ Me)Phe ], 18.8 [-, C-3', (3-Ncp)Ala], 23.1 [+, C-1', (3-Ncp)Ala], 26.0 (-, C-4, Ile), 34.7 [-, C-3, (3-Ncp)Ala], $38.6(+, \mathrm{C}-3$, Ile $), 42.7$ [+, C-3, $(\beta-M e) P h e], 44.0[+, \mathrm{C}-3,(\beta-M e) P h e], 48.4(+, \mathrm{C}-$ 1', Fmoc), 52.7 (+, C-2), $57.3(+, \mathrm{C}-2), 57.9(+, \mathrm{OMe}), 59.6(+, \mathrm{C}-2), 60.1$ [+, C-2', (3-Ncp)Ala $]$, 62.1 (+, C-2), $67.6\left(-, \mathrm{C}-2^{\prime}\right.$, Fmoc $), 91.4\left(-, \mathrm{OCH}_{2} \mathrm{O}\right), 126.3,126.3,127.4,127.7,128.0,128.5$, $128.9,129.1,129.2,129.3(+, A r-C), 142.4,142.4\left(\mathrm{C}_{\text {quat }}, \mathrm{Ar}-\mathrm{C}, F m o c\right), 143.9$ [C $\mathrm{C}_{\text {quat }}, \mathrm{Ar}-\mathrm{C},(\beta-$ Me)Phe], 144.2 [C $\left.\mathrm{C}_{\text {quat }}, \mathrm{Ar}-\mathrm{C},(\beta-M e) P h e\right], 145.3,145.5\left(\mathrm{C}_{\text {quat }}, \mathrm{Ar}-\mathrm{C}, F m o c\right), 157.7\left(\mathrm{C}_{\text {quat }}, \mathrm{NCO}_{2}\right)$, 171.3, 171.4, 171.6, 172.1 ( $\left.\mathrm{C}_{\text {quat }}, \mathrm{C}-1\right)$; IR $(\mathrm{KBr}): \mathrm{v}=3300 \mathrm{~cm}^{-1}, 2967,1708,1668,1637,1543$, 1370, 1247; MS (ESI): positive mode, $m / z(\%)=898(100)\left[\mathrm{M}+\mathrm{Na}^{+}\right]$; elemental analysis calcd (\%) for $\mathrm{C}_{49} \mathrm{H}_{57} \mathrm{~N}_{5} \mathrm{O}_{10}$ (876.0): C 67.18, H 6.56, N 7.99; found C 67.15, H 6.46, N 8.06.

Fmoc-( $\beta M e)$ Phe-(2S)-(3-Ncp)Ala-( $\beta M e) P h e-I l e-O D C P M(\mathbf{1 8 8 b})$ : The tripeptide 185b $(0.420 \mathrm{~g}$, $0.549 \mathrm{mmol}$ ) was deprotected according to GP 4 and the resultant product $\mathbf{1 8 6} \mathbf{b}$ was then directly 
coupled with 146 (0.242 g, $0.603 \mathrm{mmol})$ according to GP 5 using EDC (0.114 g, $0.595 \mathrm{mmol})$, HOAt $(0.080 \mathrm{~g}, 0.592 \mathrm{mmol})$ and TMP $(0.200 \mathrm{~g}, 1.650 \mathrm{mmol})$ in $\mathrm{CH}_{2} \mathrm{Cl}_{2}(3 \mathrm{~mL})$. After $1 \mathrm{~h}$, a precipitate fell down from the reaction mixture and anhydrous DMF (2 mL) was added to obtain homogeneous solution. After $15 \mathrm{~h}$ the reaction mixture was concentrated under reduced pressure. The resulting solid was washed with water $(100 \mathrm{~mL}), 3 \%$ aqueous $\mathrm{NaHCO}_{3}(100 \mathrm{~mL})$, water $(100 \mathrm{~mL}), \mathrm{Et}_{2} \mathrm{O}(100 \mathrm{~mL})$, pentane $(50 \mathrm{~mL})$, dried at 0.5 Torr and then crystallized twice from THF/hexanes to give $\mathbf{1 8 8 b}(0.430 \mathrm{~g}, 85 \%)$ as an off-white solid. $R_{\mathrm{f}}=0.29, \mathrm{CHCl}_{3} / \mathrm{MeOH} 70: 1$; m.p $210-215{ }^{\circ} \mathrm{C}$ (decomp.), $[\alpha]_{20}^{D}-26.3\left(c=0.32\right.$, THF); ${ }^{1} \mathrm{H}$ NMR $\left(250 \mathrm{MHz},\left[\mathrm{D}_{8}\right] \mathrm{THF}\right): \delta=$ $0.22-0.57\left(\mathrm{~m}, 7 \mathrm{H}, 2^{\prime}-\mathrm{H}, D C P M\right), 0.67-0.90$ (m, 2 H, 1'-H, 2'-H, DCPM), 0.78-0.86 (m, $1 \mathrm{H}, 1^{\prime}-$ H, DCPM), $0.81\left(\mathrm{~d}, J=6.5 \mathrm{~Hz}, 3 \mathrm{H}, 1^{\prime}-\mathrm{H}\right.$, Ile), 0.83 (t, $J=7.3 \mathrm{~Hz}, 3 \mathrm{H}, 5-\mathrm{H}$, Ile), 0.92-1.19 [m, $4 \mathrm{H}, 4-\mathrm{H}$, Ile, 3'-H, (3-Ncp)Ala], 1.20 [d, $J=7.3 \mathrm{~Hz}, 3 \mathrm{H}, 4-\mathrm{H},(\beta M e) P h e], 1.32$ [d, J= 7.0 Hz, $3 \mathrm{H}, 4-\mathrm{H},(\beta M e) P h e], 1.30-1.50$ [m, 4 H, 3-H, Ile, 1'-H, 3-H, (3-Ncp)Ala], 3.11 [dq, J = 9.6, $7.2 \mathrm{~Hz}, 1 \mathrm{H}, 3-\mathrm{H},(\beta M e) P h e], 3.24$ [dq, $J=7.4,7.4 \mathrm{~Hz}, 1 \mathrm{H}, 3-\mathrm{H},(\beta M e) P h e], 3.81$ (t, $J=8.5 \mathrm{~Hz}$, $1 \mathrm{H}, 1-\mathrm{H}, D C P M), 3.94\left[\mathrm{ddd}, J=6.8,3.3,3.3 \mathrm{~Hz}, 1 \mathrm{H}, 2^{\prime}-\mathrm{H},(3-N c p) A l a\right], 4.13-4.41(\mathrm{~m}, 5 \mathrm{H}, 2 \times$ 2-H, 1'-H, 2'-H, Fmoc), 4.41-4.51 (m, 1 H, 2-H), 4.61 (dd, J = 8.5, 7.8 Hz, 1 H, 2-H), 6.97-7.40 $(\mathrm{m}, 16 \mathrm{H}, 2 \times \mathrm{NH}, 14 \times \mathrm{Ar}-\mathrm{H}), 7.48(\mathrm{~d}, J=8.5 \mathrm{~Hz}, 1 \mathrm{H}, \mathrm{NH}), 7.58(\mathrm{~d}, J=8.3 \mathrm{~Hz}, 1 \mathrm{H}, \mathrm{NH}), 7.66$ $(\mathrm{dd}, J=8.1 \mathrm{~Hz}, 2 \mathrm{H}, \mathrm{Ar}-\mathrm{H}), 7.77(\mathrm{~d}, J=7.8 \mathrm{~Hz}, 2 \mathrm{H}, \mathrm{Ar}-\mathrm{H}) ;{ }^{13} \mathrm{C}$ NMR $\left(62.9 \mathrm{MHz},\left[\mathrm{D}_{8}\right] \mathrm{THF}\right): \delta$ $=3.3,3.5,3.6,3.7\left(-, \mathrm{C}-2^{\prime}, D C P M\right), 12.2(+, \mathrm{C}-5$, Ile $), 15.6,15.9\left(+, \mathrm{C}-1^{\prime}, D C P M\right), 16.1(+, \mathrm{C}-$

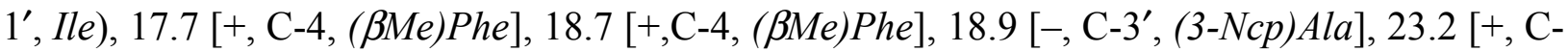
1', (3-Ncp)Ala], 26.2 (-, C-4, Ile), 35.0 [-,C-3, (3-Ncp)Ala], 39.1 (+, C-3, Ile), 42.8 [+, C-3,

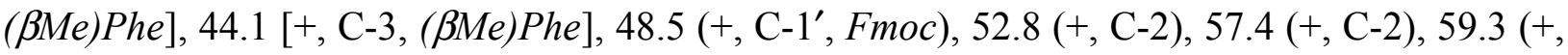
C-2), $60.2\left[+, \mathrm{C}-2^{\prime},(3-N c p) A l a\right], 62.1$ (+, C-2), 67.8 (-, C-2', Fmoc), 83.4 (+, C-1, DCPM), 126.4 $(\times 2), 127.5,127.7,128.1,128.6,129.1,129.1,129.3,129.4(+, A r-C), 142.5\left(C_{\text {quat }}\right.$, Ar-C, Fmoc $), 144.1\left[\mathrm{C}_{\text {quat }}, \mathrm{Ar}-\mathrm{C},(\beta M e) P h e\right], 144.4\left[\mathrm{C}_{\text {quat }}, \mathrm{Ar}-\mathrm{C},(\beta M e) P h e\right], 145.4,145.6\left(\mathrm{C}_{\text {quat }}, \mathrm{Ar}-\mathrm{C}\right.$, Fmoc), $157.8\left(\mathrm{C}_{\text {quat }}, \mathrm{NCO}_{2}\right), 171.1,171.5,171.9,172.1\left(\mathrm{C}_{\text {quat }}, \mathrm{C}-1\right)$; IR $(\mathrm{KBr}): \mathrm{v}=3250 \mathrm{~cm}^{-1}$, 
3065, 2967, 2933, 1709, 1665, 1636, 1542, 1451, 1369, 1246; MS (ESI): positive mode, $\mathrm{m} / z$ (\%) $=949(100)\left[\mathrm{M}+\mathrm{Na}^{+}\right]$; elemental analysis calcd $(\%)$ for $\mathrm{C}_{54} \mathrm{H}_{63} \mathrm{~N}_{5} \mathrm{O}_{9}(926.1): \mathrm{C} 70.03, \mathrm{H} 6.86, \mathrm{~N}$ 7.56; found C 69.94, H 6.68, N 7.32.

MeZ-a-Thr[(4-PE)Pro]-OAll (187): Ester $187(0.588 \mathrm{~g}, 83 \%)$ was prepared from protected threonine 176 (0.400 g, $1.30 \mathrm{mmol})$ and $N$-Boc protected 4-(Z)-propenylproline 16 (0.340 g, 1.33 mmol), 4-pyrrolidinopyridine $(0.250 \mathrm{~g}, 1.69 \mathrm{mmol})$ and $\mathrm{EDC}(0.324 \mathrm{~g}, 1.69 \mathrm{mmol})$ in $\mathrm{CH}_{2} \mathrm{Cl}_{2}(7$ $\mathrm{mL}$ ) according to GP 6 after column chromatography (EtOAc/hexanes 1:6) as a turbid oil. $R_{\mathrm{f}}=$ $0.43 \mathrm{EtOAc} / \mathrm{hexanes} 1: 3 ;[\alpha]_{20}^{D}-35.4\left(c=0.28, \mathrm{CHCl}_{3}\right) ;{ }^{1} \mathrm{H} \mathrm{NMR}\left(250 \mathrm{MHz}, \mathrm{CDCl}_{3}\right): \delta=1.32$, $1.34(2 \times \mathrm{d}, J=6.5 \mathrm{~Hz}, 3 \mathrm{H}, 4-\mathrm{H}, a-T h r), 1.36,1.39\left[2 \times \mathrm{s}, 9 \mathrm{H}, \mathrm{C}\left(\mathrm{CH}_{3}\right)_{3}\right], 1.64,1.64[2 \times \mathrm{dd}, J=$ 1.5, 7.0 Hz, 3 H, 3'-H, (4-PE)Pro], 1.63-1.81 [m, 1 H, 3-H $\mathrm{H}_{\mathrm{a}}$ (4-PE)Pro], 2.21-2.46 [m, $1 \mathrm{H}, 3-$ $\mathrm{H}_{\mathrm{b}},(4-P E)$ Pro], 2.33, 2.35 (2 × s, $\left.3 \mathrm{H}, 1^{\prime}-\mathrm{H}, M e Z\right), 2.93-3.19$ [m, $2 \mathrm{H}, 4-\mathrm{H}, 5-\mathrm{H}_{\mathrm{a}},(4-P E)$ Pro], 3.51-3.67, 3.69-3.83 [2 × m, 1 H, 5-Hb, (4-PE)Pro], 4.10-4.27 [m, 1 H, 2-H, (4-PE)Pro], 4.484.65 (m, 1 H, 2-H, a-Thr), 4.68 (d, J = 5.2 Hz, 2 H, 1-H, All), 4.97-5.13 (m, 2 H, Bzl-H, MeZ), 5.17-5.43 [m, 4 H, 3-H, All, 3-H, a-Thr, 1'-H, (4-PE)Pro], 5.54 [dq, J = 10.2, $7.0 \mathrm{~Hz}, 1 \mathrm{H}, 2^{\prime}-\mathrm{H}$, (4-PE)Pro], 5.80-6.01 (m, $1 \mathrm{H}, 2-\mathrm{H}, A l l), 6.42(\mathrm{~d}, J=9.2 \mathrm{~Hz}, 1 \mathrm{H}, \mathrm{NH}), 7.13,7.17(2 \times \mathrm{d}, J=$ $7.9 \mathrm{~Hz}, 2 \mathrm{H}, \mathrm{Ar}-\mathrm{H}), 7.23,7.25(\mathrm{~d}, J=7.9 \mathrm{~Hz}, 2 \mathrm{H}, \mathrm{Ar}-\mathrm{H}) ;{ }^{13} \mathrm{C} \mathrm{NMR}\left(62.9 \mathrm{MHz}, \mathrm{CDCl}_{3}\right): \delta=$

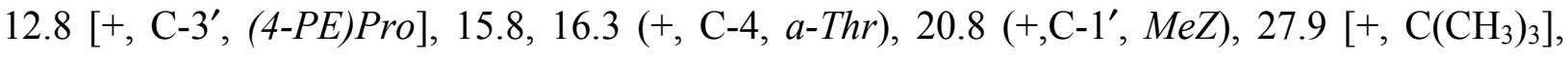
35.3, $36.1[+, \mathrm{C}-4,(4-P E) P r o], 35.8,36.9$ [-, C-3, (4-PE)Pro], 51.1, 51.3 [-, C-5, (4-PE)Pro], 57.0, 57.4 [+, C-2, (4-PE)Pro], 59.0, 59.1 (+, C-2, a-Thr), 65.5, 66.0 (-, C-1, All), 66.4, 67.0 (-, Bzl-C), 70.8, $70.8(+, \mathrm{C}-3), 79.6,79.7\left[\mathrm{C}_{\text {quat }}, \mathrm{C}\left(\mathrm{CH}_{3}\right)_{3}\right] 118.2,118.9(-, \mathrm{C}-3, \mathrm{All}), 126.3,126.3$ (+, Ar-C), 127.9 (+, Ar-C), 128.7, 128.8 [+, C-2', (4-PE)Pro], 129.0, 129.1 [+, C-1', (4-PE)Pro], 130.9, $131.2(+, \mathrm{C}-2, A l l), 132.7,133.1$ ( $\left.\mathrm{C}_{\text {quat, }}, \mathrm{Ar}-\mathrm{C}\right), 137.2,137.6\left(\mathrm{C}_{\text {quat }}, \mathrm{Ar}-\mathrm{C}\right), 153.0,153.5$ $\left(\mathrm{C}_{\text {quat }}, \mathrm{NCO}_{2}, B o c\right), 155.4,155.9\left(\mathrm{C}_{\text {quat }}, \mathrm{NCO}_{2}, M e Z\right), 168.4,168.4\left(\mathrm{C}_{\text {quat }}, \mathrm{C}-1, a-T h r\right), 171.6$, 171.9 [ $\mathrm{C}_{\text {quat }}, \mathrm{C}-1,(4-P E)$ Pro]; IR (KBr): nu(tilde) $=3009 \mathrm{~cm}^{-1}, 2977,2932,2871,1747,1703$, $1520,1478,1453,1399,1367,1253,1161 ; \mathrm{MS}(\mathrm{EI}, 70 \mathrm{eV}), m / z(\%)=544(2)\left[\mathrm{M}^{+}\right], 488(6)\left[\mathrm{M}^{+}\right.$ 
$\left.-\mathrm{C}_{4} \mathrm{H}_{8}\right], 444(5)\left[\mathrm{M}^{+}-\mathrm{C}_{5} \mathrm{H}_{8} \mathrm{O}_{2}\right], 339$ (26), 210 (11), 154 (100) $\left[\mathrm{C}_{8} \mathrm{H}_{12} \mathrm{NO}_{2}^{+}\right], 110$ (83) $\left[\mathrm{C}_{7} \mathrm{H}_{12} \mathrm{~N}^{+}\right], 105$ (100) $\left[\mathrm{C}_{8} \mathrm{H}_{9}{ }^{+}\right], 57$ (49) $\left[\mathrm{C}_{4} \mathrm{H}_{9}{ }^{+}\right], 41$ (15) $\left[\mathrm{C}_{3} \mathrm{H}_{5}{ }^{+}\right]$; HRMS (EI): calcd for $\mathrm{C}_{29} \mathrm{H}_{40} \mathrm{~N}_{2} \mathrm{O}_{8}$ : 544.2785; found 544.2785; elemental analysis calcd (\%) for $\mathrm{C}_{29} \mathrm{H}_{40} \mathrm{~N}_{2} \mathrm{O}_{8}$ (544.7): C 63.95, H 7.40, N 5.14; found C 63.99, H 7.32, N 4.99.

MeZ-a-Thr[(4-PE)Pro]-OH (189): $\mathrm{Pd}\left(\mathrm{P}(\mathrm{Ph})_{3}\right)_{4}(0.034 \mathrm{~g}, 2.94 \mu \mathrm{mol})$ was added to a vigorously stirred solution of ester $187(0.108 \mathrm{~g}, 0.198 \mathrm{mmol})$ and $N$-methyl aniline $(0.08 \mathrm{~mL}, 0.738 \mathrm{mmol})$ in DME (3.5 mL) and the resulting suspension was carefully heated with heat-gun to obtain a homogeneous solution. The mixture was left to stir at $20^{\circ} \mathrm{C}$ for $45 \mathrm{~min}$, was then again carefully heated with heat-gun till catalyst dissolved and stirred for a further $15 \mathrm{~min}$. The reaction mixture was then diluted with $\mathrm{Et}_{2} \mathrm{O}(40 \mathrm{~mL})$, washed with $1 \mathrm{M} \mathrm{NaHSO}_{4}(3 \times 10 \mathrm{~mL})$, water $(10 \times 10$ $\mathrm{mL})$, brine $(2 \times 10 \mathrm{~mL})$, dried, filtered and concentrated under reduced pressure. The residue was taken up with $\mathrm{Et}_{2} \mathrm{O} /$ hexanes 1:2, filtered, concentrated and purified by column chromatography $\left(\right.$ EtOAc/hexanes 1:2 $\left.(1.5 \% \mathrm{AcOH}), R_{\mathrm{f}}=0.34\right)$ to give $189(0.121 \mathrm{~g}, 90 \%)$ as an yellow oil. $[\alpha]_{20}^{D}$

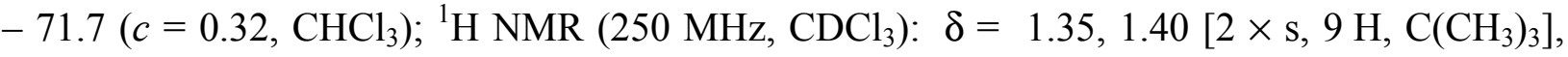
$1.41,1.43(2 \times \mathrm{d}, J=5.7 \mathrm{~Hz}, 3 \mathrm{H}, 4-\mathrm{H}, a-T h r), 1.63,1.65\left[2 \times \mathrm{dd}, J=1.5,6.7 \mathrm{~Hz}, 3 \mathrm{H}, 3^{\prime}-\mathrm{H},(4-\right.$ PE)Pro], 1.67-1.86 [m, $\left.1 \mathrm{H}, 3-\mathrm{H}_{\mathrm{a}},(4-P E) P r o\right], 2.24-2.49$ [m, $1 \mathrm{H}, 3-\mathrm{H}_{\mathrm{b}},(4-P E)$ Pro $], 2.33,2.34$ $\left(2 \times \mathrm{s}, 3 \mathrm{H}, 1^{\prime}-\mathrm{H}, M e Z\right), 2.91-3.20$ [m, $2 \mathrm{H}, 4-\mathrm{H}, 5-\mathrm{H}_{\mathrm{a}},(4-P E)$ Pro], 3.40-4.30 (br, $\left.1 \mathrm{H}, \mathrm{CO}_{2} \mathrm{H}\right)$, 3.61, 3.73 [2 $\times$ ddd, $J=2.7 \mathrm{~Hz}, 1 \mathrm{H}, 5-\mathrm{H}_{\mathrm{b}},(4-P E)$ Pro], 4.11-4.29 [m, $1 \mathrm{H}, 2-\mathrm{H},(4-P E)$ Pro], 4.51 $(\mathrm{dd}, J=8.6,3.5 \mathrm{~Hz}, 1 \mathrm{H}, 2-\mathrm{H}, a-T h r), 5.05$ (s, $2 \mathrm{H}, \mathrm{Bzl}-\mathrm{H}, M e Z), 5.15-5.31$ [m, $1 \mathrm{H}, 1^{\prime}-\mathrm{H},(4-$ PE)Pro], 5.31-5.44 (m, 1 H, 3-H, a-Thr), 5.45-5.60 [m, $1 \mathrm{H}, 2^{\prime}-\mathrm{H},(4-P E)$ Pro], 5.63 (d, $J=$ 7.6 Hz, 0.4 H, NH), 6.46 (d, $J=9.2 \mathrm{~Hz}, 0.6 \mathrm{H}, \mathrm{NH}), 7.13,7.17(2 \times \mathrm{d}, J=7.9 \mathrm{~Hz}, 2 \mathrm{H}, \mathrm{Ar}-\mathrm{H})$, 7.21, $7.22(\mathrm{~d}, J=7.9 \mathrm{~Hz}, 2 \mathrm{H}, \mathrm{Ar}-\mathrm{H}) ;{ }^{13} \mathrm{C} \mathrm{NMR}\left(62.9 \mathrm{MHz}, \mathrm{CDCl}_{3}\right): \delta=13.0\left[+, \mathrm{C}-3^{\prime},(4-\right.$ PE)Pro], 15.8, 16.3 (+, C-4, a-Thr), $21.0\left(+, \mathrm{C}-1^{\prime}, \mathrm{MeZ}\right), 28.1\left[+, \mathrm{C}\left(\mathrm{CH}_{3}\right)_{3}\right], 35.5,36.2[+, \mathrm{C}-4$, (4-PE)Pro $], 36.0,37.0$ [-, C-3, (4-PE)Pro], 51.3, 51.6 [-, C-5, (4-PE)Pro], 57.0, 57.5 [+, C-2, 
(4-PE)Pro], 59.1, 59.3 (+, C-2, a-Thr), 66.8, 67.0 (-, Bzl-C), 71.2, 71.4 (+, C-3), 80.5, 80.6 $\left[\mathrm{C}_{\text {quat }}, \mathrm{C}\left(\mathrm{CH}_{3}\right)_{3}\right] 126.5,126.5$ (+, Ar-C), 128.1 (+, Ar-C), $128.1\left[+, \mathrm{C}-2^{\prime}\right.$, (4-PE)Pro], 128.9, 129.0 [+, C-1', (4-PE)Pro], 132.8, 133.0 ( $\left.\mathrm{C}_{\text {quat }}, \mathrm{Ar}-\mathrm{C}\right), 137.6,137.9\left(\mathrm{C}_{\text {quat }}, \mathrm{Ar}-\mathrm{C}\right), 153.7,154.2$ $\left(\mathrm{C}_{\text {quat }}, \mathrm{NCO}_{2}, B o c\right), 155.8,156.4\left(\mathrm{C}_{\text {quat }}, \mathrm{NCO}_{2}, \mathrm{MeZ}\right), 171.2,171.7\left(\mathrm{C}_{\text {quat }}, \mathrm{C}-1\right), 172.0,172.2$ $\left(\mathrm{C}_{\text {quat }}, \mathrm{C}-1\right)$; IR $(\mathrm{KBr}): \mathrm{nu}($ tilde $)=3700-2750 \mathrm{~cm}^{-1}, 2979,1729,1520,1404,1369,1257,1162$, 1061; MS (EI, $70 \mathrm{eV}), m / z(\%)=504(2)\left[\mathrm{M}^{+}\right], 448(7)\left[\mathrm{M}^{+}-\mathrm{C}_{4} \mathrm{H}_{8}\right], 404$ (27) $\left[\mathrm{M}^{+}-\mathrm{C}_{5} \mathrm{H}_{8} \mathrm{O}_{2}\right]$, 299 (21), 210 (14), 154 (100) $\left[\mathrm{C}_{8} \mathrm{H}_{12} \mathrm{NO}_{2}{ }^{+}\right], 110$ (92) $\left[\mathrm{C}_{7} \mathrm{H}_{12} \mathrm{~N}^{+}\right], 105$ (100) $\left[\mathrm{C}_{8} \mathrm{H}_{9}^{+}\right], 57$ (50) $\left[\mathrm{C}_{4} \mathrm{H}_{9}{ }^{+}\right], 41$ (16) $\left[\mathrm{C}_{3} \mathrm{H}_{5}{ }^{+}\right]$; HRMS (EI): calcd for $\mathrm{C}_{26} \mathrm{H}_{36} \mathrm{~N}_{2} \mathrm{O}_{8}$ : 504.2472; found 504.2472.

MeZ-a-Thr[Boc-(4-PE)Pro]-( $\beta M e) P h e-(3-N c p) A l a-(\beta M e) P h e-I l e-O M O M$ (191a): Tetrapeptide 188a (180 mg, $0.21 \mathrm{mmol})$ was deprotected according to GP 4 in MeCN (2 mL), taken up with anhydrous $\mathrm{CH}_{2} \mathrm{Cl}_{2}(5 \mathrm{~mL})$, dipeptide acid 189 (0.114 g, $\left.0.23 \mathrm{mmol}\right)$, HATU (96 mg, $\left.0.25 \mathrm{mmol}\right)$ and HOAt $(31 \mathrm{mg}, 0.23 \mathrm{mmol})$ were added and the reaction mixture was cooled to $4{ }^{\circ} \mathrm{C}$. After this, a solution of DIEA (29 mg, 0,22 mmol) and TMP (75 mg, $0.62 \mathrm{mmol})$ in $\mathrm{CH}_{2} \mathrm{Cl}_{2}(2 \mathrm{~mL})$ were added at the same temperature within $5 \mathrm{~min}$. The temperature was allowed to reach $20^{\circ} \mathrm{C}$, and stirring continued for an additional $15 \mathrm{~h}$. After aqueous work-up according to GP 5 and two recrystallizations from EtOAc/hexanes 1:2, depsipeptide 191a (185 mg, 79\%) was obtained as a colorless powder. $R_{\mathrm{f}}=0.46$, EtOAc/hexanes 1:1; m.p. $125-127^{\circ} \mathrm{C},[\alpha]_{20}^{D} 0\left(c=0.21, \mathrm{CHCl}_{3}\right)$, $[\alpha]_{20}^{D}-29.0(c=0.2, \mathrm{THF}) ;{ }^{1} \mathrm{H} \mathrm{NMR}\left(250 \mathrm{MHz}, \mathrm{CDCl}_{3}\right): \delta=0.76\left(\mathrm{~d}, J=6.8 \mathrm{~Hz}, 3 \mathrm{H}, 1^{\prime}-\mathrm{H}\right.$, Ile $)$, $0.89\left(\mathrm{t}, J=7.3 \mathrm{~Hz}, 3 \mathrm{H}, 5-\mathrm{H}\right.$, Ile), 0.95-1.11 [m, $2 \mathrm{H}, 4-\mathrm{H}_{\mathrm{a}}$, Ile, 3'- $\mathrm{H}_{\mathrm{a}},(3-N c p)$ Ala ], 1.11-1.60 [m, $5 \mathrm{H}, 3-\mathrm{H}, 4-\mathrm{H}_{\mathrm{b}}$, Ile, 3-H $\left.\mathrm{H}_{\mathrm{a}} 1^{\prime}-\mathrm{H}, 3^{\prime}-\mathrm{H}_{\mathrm{a}},(3-N c p) A l a\right], 1.24[\mathrm{~d}, J=7.5 \mathrm{~Hz}, 3 \mathrm{H},(\beta-M e) P h e], 1.27$ [d, $J=7.5 \mathrm{~Hz}, 3 \mathrm{H}, 4-\mathrm{H},(\beta-M e) P h e], 1.36\left(\mathrm{~s}, 9 \mathrm{H}, \mathrm{C}\left(\mathrm{CH}_{3}\right)_{3}\right), 1.43(\mathrm{~d}, J=6.5 \mathrm{~Hz}, 3 \mathrm{H}, 4-\mathrm{H}, a-T h r)$, $1.68\left[\mathrm{dd}, J=6.6,1.6 \mathrm{~Hz}, 3 \mathrm{H}, 3^{\prime}-\mathrm{H},(4-P E)\right.$ Pro], 1.76 [dd, $J=11.6,11.6 \mathrm{~Hz}, 1 \mathrm{H}, 3-\mathrm{H}_{\mathrm{a}}$, (4PE)Pro], 1.87-2.02 [m, $\left.1 \mathrm{H}, 3-\mathrm{H}_{\mathrm{b}},(3-N c p) A l a\right], 2.32$ (s, $\left.3 \mathrm{H}, 1^{\prime}-\mathrm{H}, \mathrm{MeZ}\right), 2.43$ [ddd, J = 12.3, 6.5, $6.5 \mathrm{~Hz}, 1 \mathrm{H}, 3-\mathrm{H}_{\mathrm{b}}$, (4-PE)Pro], 2.99-3.20 [m, $1 \mathrm{H}, 4-\mathrm{H},(4-P E)$ Pro], 3.11 [dd, $J=9.8$, 
$9.8 \mathrm{~Hz}, 1 \mathrm{H}, 5-\mathrm{H}_{\mathrm{a}},(4-P E)$ Pro], 3.21-3.38 [m, $2 \mathrm{H}, 3-\mathrm{H},(\beta-M e)$ Phe, 5- $\mathrm{H}_{\mathrm{b}},(4-P E)$ Pro], 3.49 (s, $3 \mathrm{H}, \mathrm{OMe}), 3.70(\mathrm{dd}, J=9.8,7.0 \mathrm{~Hz}, 1 \mathrm{H}, 2-\mathrm{H}), 3.86$ [dq, $J=3.9,6.5 \mathrm{~Hz}, 1 \mathrm{H}, 3-\mathrm{H},(\beta-M e) P h e]$, $4.14(\mathrm{dd}, J=11.1,6.3 \mathrm{~Hz}, 1 \mathrm{H}, 2-\mathrm{H}), 4.34-4.55$ (m, $\left.3 \mathrm{H}, 2-\mathrm{H}, 2^{\prime}-\mathrm{H},(3-N c p) A l a\right), 4.59$ (dd, $J=$ 9.9, $5.1 \mathrm{~Hz}, 1 \mathrm{H}, 2-\mathrm{H}), 4.67$ (dd, $J=10.4,10.4 \mathrm{~Hz}, 1 \mathrm{H}, 2-\mathrm{H}), 5.03$ (dd, $J=2.9,2.9 \mathrm{~Hz}, 2 \mathrm{H}$, BzlH), 5.27 [ddq, $\left.J=10.5,10.5,1.6 \mathrm{~Hz}, 1 \mathrm{H}, 1^{\prime}-\mathrm{H},(4-P E) P r o\right], 5.46-5.60$ [m, $2 \mathrm{H}, 3-\mathrm{H}, a-T h r, 2^{\prime}-$ $\mathrm{H},(4-P E) P r o], 5.61\left(\mathrm{~d}, J=6.3 \mathrm{~Hz}, 1 \mathrm{H}, \mathrm{H}_{\mathrm{b}}, \mathrm{OCH}_{2} \mathrm{O}\right), 6.57$ (d, $\left.J=8.3 \mathrm{~Hz}, 1 \mathrm{H}, \mathrm{NH}\right), 6.99(\mathrm{~d}, J=$ $6.0 \mathrm{~Hz}, 1 \mathrm{H}, \mathrm{NH}), 7.02(\mathrm{~d}, J=6.0 \mathrm{~Hz}, 1 \mathrm{H}, \mathrm{NH}), 7.07-7.38(\mathrm{~m}, 14 \mathrm{H}, \operatorname{Ar}-\mathrm{H}), 7.57(\mathrm{~d}, J=$ $10.0 \mathrm{~Hz}, 1 \mathrm{H}, \mathrm{NH}), 7.95(\mathrm{~d}, J=5.8 \mathrm{~Hz}, 1 \mathrm{H}, \mathrm{NH})$; The peak of Bzl-H masked absorption for $\mathrm{H}_{\mathrm{a}}$ of $\mathrm{OCH}_{2} \mathrm{O} ;{ }^{13} \mathrm{C}$ NMR $\left(62.9 \mathrm{MHz}, \mathrm{CDCl}_{3}\right): \delta=11.5(+, \mathrm{C}-5$, Ile $), 13.0\left[+, \mathrm{C}-3^{\prime},(4-P E)\right.$ Pro $], 15.6$ $\left(+, \mathrm{C}-1^{\prime}\right.$, Ile $), 17.5[+, \mathrm{C}-4,(\beta-M e) P h e], 18.2\left[-, \mathrm{C}-3^{\prime},(3-N c p) A l a\right], 18.4[+, \mathrm{C}-4,(\beta-M e) P h e]$, $19.2(+, \mathrm{C}-4, a-T h r), 20.9\left(+, \mathrm{C}-1^{\prime}, \mathrm{MeZ}\right), 21.7\left[+, \mathrm{C}^{\prime} 1^{\prime},(3-N c p) A l a\right], 24.9(-, \mathrm{C}-4$, Ile $), 28.1[+$, $\left.\mathrm{C}\left(\mathrm{CH}_{3}\right)_{3}\right], 31.5$ [-, C-3, (3-Ncp)Ala], 36.1 [-, C-3, (4-PE)Pro], 36.1 [+, C-4, (4-PE)Pro], $36.9(+$, C-3, Ile $), 40.4$ [+, C-3, $(\beta-M e) P h e], 41.8$ [+, C-3, $(\beta-M e) P h e], 50.8(+, \mathrm{C}-2), 51.9$ [-, C-5, (4PE)Pro], 56.1 (+, C-2), 57.5 (+, OMe), 59.1 (+, C-2), 59.3 (+, C-2), $60.7(+, \mathrm{C}-2), 61.2(+, \mathrm{C}-2)$, $61.6\left[+, \mathrm{C}-2^{\prime},(3-N c p) A l a\right], 66.7$ (-, Bzl-C), $70.4(+, \mathrm{C}-3, a-T h r), 80.7\left[\mathrm{C}_{\text {quat }}, \mathrm{C}\left(\mathrm{CH}_{3}\right)_{3}\right], 90.9(-$, $\left.\mathrm{OCH}_{2} \mathrm{O}\right), 126.3,126.7,127.5,127.7,128.2,128.3,128.4,128.5,128.7,128.8\left[+, \mathrm{Ar}-\mathrm{C}\right.$ and $\mathrm{C}-1^{\prime}$, C-2', (4-PE)Pro], 133.0, 137.6, 141.6, 141.8 ( $\left.\mathrm{C}_{\text {quat }}, \mathrm{Ar}-\mathrm{C}\right), 154.5,154.6\left(\mathrm{C}_{\text {quat }}, \mathrm{NCO}_{2}\right), 170.31$, 170.33, 170.8, 171.1, 173.0, $173.8\left(\mathrm{C}_{\text {quat }}, \mathrm{C}-1\right)$; IR $(\mathrm{KBr}): v=3062 \mathrm{~cm}^{-1}, 2972,2934,1729$, $1648,1543,1454,1368,1254,1162,1091$; MS (ESI): positive mode, $m / z(\%)=1163(100)[\mathrm{M}+$ $\left.\mathrm{Na}^{+}\right]$; negative mode, $m / z(\%)=1138(50)\left[\mathrm{M}-\mathrm{H}^{+}\right], 1175(50)\left[\mathrm{M}+\mathrm{Cl}^{-}\right]$; elemental analysis calcd (\%) for $\mathrm{C}_{60} \mathrm{H}_{81} \mathrm{~N}_{7} \mathrm{O}_{15}$ (1140.4): C 63.20, H 7.16, N 8.60; found C 63.09, H 7.27, N 8.46.

N-MeZ protected cyclohexadepsipeptide (171c): To the hexadepsipeptide 191a (0.188 g, 0.165 mmol) $2 \mathrm{M} \mathrm{HCl}$ in EtOAc $(2 \mathrm{~mL})$ was added, the reaction mixture was stirred for $45 \mathrm{~min}$ at 20 ${ }^{\circ} \mathrm{C}$ in the dark place and then was concentrated under reduced pressure at $20{ }^{\circ} \mathrm{C}$. The residue was triturated with dry $\mathrm{Et}_{2} \mathrm{O}$ to give deprotected material as a white solid (0.160 g; MS (ESI): 
positive mode, $m / z(\%)=997(100)\left[\mathrm{M}+\mathrm{H}^{+}\right], 1019(5)\left[\mathrm{M}+\mathrm{Na}^{+}\right]$; negative mode, $m / z(\%)=$ $\left.995(50)\left[\mathrm{M}-\mathrm{H}^{+}\right]\right)$, which was taken up with anhydrous $\mathrm{CH}_{2} \mathrm{Cl}_{2}(1.51)$ and cyclizated employing HATU $(2 \times 0.073 \mathrm{~g}, 2 \times 0.192 \mathrm{mmol})$ and HOAt $(2 \times 0.022 \mathrm{~g}, 2 \times 0.163 \mathrm{mmol})$ and solution of DIEA $(2 \times 0.062 \mathrm{~g}, 2 \times 0.480 \mathrm{mmol})$ in $\mathrm{CH}_{2} \mathrm{Cl}_{2}(2 \times 50 \mathrm{~mL})$ according to GP 8 for $16 \mathrm{~h}$. After this, the solvent was removed under reduced pressure, the residue was taken up with $\mathrm{Et}_{2} \mathrm{O}(50$ $\mathrm{mL}$ ), and after usual aqueous work-up (GP 5), drying and filtration, the organic layer was concentrated under reduced pressure. The residue was purified first by column chromatography (acetone/hexanes 1:3, $\left.R_{\mathrm{f}}=0.17\right)$ and then by recrystallization $\left(\mathrm{Et}_{2} \mathrm{O} /\right.$ pentane) to give crude product $(0.12 \mathrm{~g})$, which was finally purified by preparative HPLC to give cyclodepsipeptide 171c ( $86 \mathrm{mg}, 53 \%$ on 2 steps) as a white solid. Preparative HPLC: isocratic, $75 \% \mathrm{MeCN}_{\text {in }} \mathrm{H}_{2} \mathrm{O}$ $\left(0.1 \%\right.$ TFA); analytical HPLC 1: column $\mathrm{B}$, isocratic, $50 \% \mathrm{MeCN}$ in $\mathrm{H}_{2} \mathrm{O}(0.1 \%$ TFA) for 20 min, then gradient $50 \% \rightarrow 99 \% \mathrm{MeCN}(0.1 \%$ TFA) for $15 \mathrm{~min}$, then isocratic, $99 \% \mathrm{MeCN}$ in $\mathrm{H}_{2} \mathrm{O}(0.1 \% \mathrm{TFA})$ for $10 \mathrm{~min}$, flow rate $=0.5 \mathrm{~mL} / \mathrm{min}, t_{\mathrm{R}}=34.93 \mathrm{~min}$, purity $>98 \%$; analytical HPLC 2: column B, gradient $40 \% \rightarrow 70 \% \mathrm{MeCN}(0.1 \%$ TFA) for $20 \mathrm{~min}$, then gradient $70 \% \rightarrow$ $99 \% \mathrm{MeCN}(0.1 \% \mathrm{TFA})$ for $15 \mathrm{~min}$, flow rate $=0.5 \mathrm{~mL} / \mathrm{min}, t_{\mathrm{R}}=29.00 \mathrm{~min}$, purity $>98 \%$; $[\alpha]_{20}^{D}-15.5\left(c=0.20, \mathrm{CHCl}_{3}\right) ;{ }^{1} \mathrm{H}$ NMR $\left(600 \mathrm{MHz}, \mathrm{CDCl}_{3}\right): \delta=0.64[\mathrm{ddd}, J=14.4,7.2$, $\left.7.2 \mathrm{~Hz}, 1 \mathrm{H}, 3-\mathrm{H}_{\mathrm{a}},(3-N c p) A l a\right], 0.72$ (d, $J=6.6 \mathrm{~Hz}, 3 \mathrm{H}, 1^{\prime}-\mathrm{H}$, Ile), 0.74 [ddd, J = 6.6, 6.6, $6.6 \mathrm{~Hz}, 1 \mathrm{H}, 3^{\prime}-\mathrm{H}_{\mathrm{a}},(3-N c p)$ Ala], 0.79 (t, J = 7.2 Hz, $3 \mathrm{H}, 5-\mathrm{H}$, Ile), 1.04-1.12 (m, $1 \mathrm{H}, 4-\mathrm{H}_{\mathrm{a}}$, Ile), $1.23[\mathrm{~d}, J=6.6 \mathrm{~Hz}, 3 \mathrm{H}, 4-\mathrm{H},(\beta-M e) P h e], 1.27-1.34$ [m, $\left.1 \mathrm{H}, 1^{\prime}-\mathrm{H},(3-N c p) A l a\right], 1.37$ [d, $J=$ $6.6 \mathrm{~Hz}, 3 \mathrm{H}, 4-\mathrm{H},(\beta-M e) P h e], 1.37-1.43\left[\mathrm{~m}, 1 \mathrm{H}, 3-\mathrm{H}_{\mathrm{b}},(3-N c p) A l a\right], 1.45-1.54\left(\mathrm{~m}, 1 \mathrm{H}, 4-\mathrm{H}_{\mathrm{b}}\right.$, Ile), 1.54-1.57 [m, $\left.1 \mathrm{H}, 3^{\prime}-\mathrm{H}_{\mathrm{b}},(3-N c p) A l a\right], 1.57(\mathrm{~d}, J=6.6 \mathrm{~Hz}, 3 \mathrm{H}, 4-\mathrm{H}, a-T h r), 1.65$ [dd, $J=$ 6.6, 1.5 Hz, 3 H, 3'-H, (4-PE)-Pro], 1.66-1.76 [m, 2 H, 3-H, Ile, 3-Ha, (4-PE)Pro], 2.23 [ddd, J $\left.=12.0,5.4,5.4 \mathrm{~Hz}, 1 \mathrm{H}, 3-\mathrm{H}_{\mathrm{b}},(4-P E)-P r o\right], 2.35$ (s, $\left.3 \mathrm{H}, 1^{\prime}-\mathrm{H}, M e Z\right), 3.05[\mathrm{dq}, J=6.6,6.6 \mathrm{~Hz}$, $1 \mathrm{H}, 3-\mathrm{H},(\beta-M e) P h e], 3.15-3.28\left[\mathrm{~m}, 2 \mathrm{H}, 4-\mathrm{H}, 5-\mathrm{H}_{\mathrm{a}},(4-P E)-P r o\right], 3.54[\mathrm{dq}, J=7.2,6.6 \mathrm{~Hz}, 1 \mathrm{H}$, 3-H, $(\beta-M e) P h e], 3.71(\mathrm{dd}, J=6.0,5.4 \mathrm{~Hz}, 1 \mathrm{H}, 2-\mathrm{H}), 3.76$ [ddd, $J=6.6,3.3,3.3 \mathrm{~Hz}, 1 \mathrm{H}, 2^{\prime}-\mathrm{H}$, 
(3-Ncp)Ala], 3.98 (dd, $J=10.5,6.3 \mathrm{~Hz}, 1 \mathrm{H}, 2-\mathrm{H}), 4.01-4.08$ [m, $1 \mathrm{H}, 5-\mathrm{H}_{\mathrm{b}},(4-P E)-$ Pro], 4.464.54 (m, 2 H, 2-H), 4.54 (dd, $J=9.6,9.6 \mathrm{~Hz}, 1 \mathrm{H}, 2-\mathrm{H}), 4.69$ (dd, $J=8.4 \mathrm{~Hz}, 1 \mathrm{H}, 2-\mathrm{H}), 5.03$ (d, $\left.J=12.0 \mathrm{~Hz}, 1 \mathrm{H}, \mathrm{Bzl}-\mathrm{H}_{\mathrm{a}}\right), 5.15\left(\mathrm{~d}, J=12.0 \mathrm{~Hz}, 1 \mathrm{H}, \mathrm{Bzl}-\mathrm{H}_{\mathrm{b}}\right), 5.19-5.25$ [m, $1 \mathrm{H}, 1^{\prime}-\mathrm{H},(4-P E)-$ Pro], 5.39 (dq, $J=1.8,6.6 \mathrm{~Hz}, 1 \mathrm{H}, 3-\mathrm{H}, a-T h r), 5.56$ [dq, $J=10.8,6.6 \mathrm{~Hz}, 1 \mathrm{H}, 2^{\prime}-\mathrm{H},(4-P E)-$ Pro], 6.21-6.37 (br, $2 \mathrm{H}, \mathrm{NH}), 6.44(\mathrm{~d}, J=8.4 \mathrm{~Hz}, 1 \mathrm{H}, \mathrm{NH}), 6.48(\mathrm{~d}, J=9.6 \mathrm{~Hz}, 1 \mathrm{H}, \mathrm{NH})$, 7.06-7.18 (m, 4 H, Ar-H), 7.19-7.27 (m, 9 H, Ar-H, NH), 7.26-7.34 (m, 2 H, Ar-H), 7.48 (d, J= 8.4 Hz, $1 \mathrm{H}, \mathrm{NH}) ;{ }^{13} \mathrm{C} \mathrm{NMR}\left(150.8 \mathrm{MHz}, \mathrm{CDCl}_{3}\right): \delta=10.3(+, \mathrm{C}-5$, Ile $), 13.3\left[+, \mathrm{C}-3{ }^{\prime},(4-P E)-\right.$ Pro], 14.6 [+, C-1', Ile, C-4, $(\beta-M e) P h e], 17.3$ [-, C-3', (3-Ncp)Ala], 17.7 [+, C-4, $(\beta-M e) P h e]$, $18.4(+, \mathrm{C}-4, a-T h r), 21.2\left(+, \mathrm{C}-1^{\prime}, \mathrm{MeZ}\right), 21.3\left[+, \mathrm{C}-1^{\prime},(3-N c p) A l a\right], 24.7(-, \mathrm{C}-4$, Ile $), 32.0[-$, C-3, (3-Ncp)Ala], 35.4 [-, C-3, (4-PE)-Pro], 36.6 [+, C-3, Ile, C-4, (4-PE)-Pro], 39.4 [+, C-3, $(\beta-M e) P h e], 44.5$ [+, C-3, ( $\beta-M e) P h e], 52.5$ [-, C-5, (4-PE)-Pro], $53.3(+, \mathrm{C}-2), 54.6(+, \mathrm{C}-2)$, $58.6(+, \mathrm{C}-2), 59.0\left[+, \mathrm{C}-2^{\prime},(3-N c p)\right.$ Ala $], 59.4(+, \mathrm{C}-2), 60.1(+, \mathrm{C}-2), 60.7$ (+, C-2), 67.2 (-, BzlC), $72.6(+, \mathrm{C}-3, a-T h r), 127.1,127.2,127.5,127.6,128.3,128.6,128.8,129.2(+$, Ar-C), 127.8 [+, C-1', (4-PE)-Pro], 128.0 [+, C-2', (4-PE)-Pro], 133.2, 137.9, 140.9, 142.6 (C $\left.\mathrm{C}_{\text {quat }}, \mathrm{Ar}-\mathrm{C}\right)$, $156.3\left(\mathrm{C}_{\text {quat }}, \mathrm{NCO}_{2}\right), 169.0,170.3,170.6,171.1,171.4,173.1\left(\mathrm{C}_{\text {quat }}, \mathrm{C}-1\right)$; IR $(\mathrm{KBr}): \mathrm{v}=2969$ $\mathrm{cm}^{-1}, 2936,2878,1730,1637,1541,1515,1452,1370,1179$; MS (ESI): positive mode, $m / z(\%)$ $=1001(100)\left[\mathrm{M}+\mathrm{Na}^{+}\right]$; negative mode, $m / z(\%)=977(100)\left[\mathrm{M}-\mathrm{H}^{+}\right]$; HRMS (ESI): calcd for $\left[\mathrm{C}_{53} \mathrm{H}_{67} \mathrm{~N}_{7} \mathrm{O}_{11} \mathrm{Na}^{+}\right]:$1000.4792; found 1000.4792.

Boc-(S)-(3-Ncp)Ala-OFm (192): The ester 192 was obtained from $N$-protected aminoacid $\left(2 S, 1^{\prime} R, 2^{\prime} R\right)-143 \quad(0.213 \mathrm{~g}, \quad 0.777 \mathrm{mmol})$ employing $\operatorname{EDC}(0.223 \mathrm{~g}, 1.163 \mathrm{mmol}), 4-$ pyrrolidinopyridine $(0.036 \mathrm{~g}, 0.242 \mathrm{mmol})$ and 9-fluorenylmethanol $(0.152 \mathrm{~g}, 0.775 \mathrm{mmol})$ in $\mathrm{CH}_{2} \mathrm{Cl}_{2}(6 \mathrm{~mL})$ according to GP 6 for $5 \mathrm{~h}$. The mixture was then diluted with $\mathrm{Et}_{2} \mathrm{O}(50 \mathrm{~mL})$ and subjected to usual aqueous work-up. The organic layer was dried, filtered and concentrated under reduced pressure. The residue was crystallized from hexanes, taken up with $\mathrm{Et}_{2} \mathrm{O}(4 \mathrm{~mL})$ and filtered through a silica gel pad $(1.5 \mathrm{~cm})$ to give after concentration of the filtrate under reduced 
pressure the desired product $192(0.26 \mathrm{~g}, 74 \%)$ as a colorless solid. $R_{\mathrm{f}}=0.30 \mathrm{EtOAc} / \mathrm{hexanes}$ 1:4; m.p 79-81 ${ }^{\circ} \mathrm{C} ;[\alpha]_{20}^{D}-27.0\left(c=0.3, \mathrm{CHCl}_{3}\right) ;{ }^{1} \mathrm{H}$ NMR $\left(250 \mathrm{MHz}, \mathrm{CDCl}_{3}\right): \delta=1.14(\mathrm{ddd}, J$ $\left.=6.8,6,8,5.3 \mathrm{~Hz}, 1 \mathrm{H}, 3^{\prime}-\mathrm{H}_{\mathrm{a}}\right), 1.43\left[\mathrm{~s}, 9 \mathrm{H}, \mathrm{C}\left(\mathrm{CH}_{3}\right)_{3}\right], 1.43-1.60\left(\mathrm{~m}, 1 \mathrm{H}, 3^{\prime}-\mathrm{H}_{\mathrm{b}}\right), 1.58-1.71(\mathrm{~m}$, $\left.1 \mathrm{H}, 1^{\prime}-\mathrm{H}\right), 1.72-1.92(\mathrm{~m}, 2 \mathrm{H}, 3-\mathrm{H}), 3.90(\mathrm{ddd}, J=6.9,3.5,3.5 \mathrm{~Hz}, 1 \mathrm{H}, 2-\mathrm{H}), 4.23(\mathrm{t}, J=$ $\left.6.0 \mathrm{~Hz}, 1 \mathrm{H}, 1^{\prime \prime}-\mathrm{H}\right), 4.35\left(\mathrm{ddd}, J=6.8,6.8,6.8 \mathrm{~Hz}, 1 \mathrm{H}, 1^{\prime}-\mathrm{H}\right), 4.55(\mathrm{dd}, J=10.8,6.0 \mathrm{~Hz}, 1 \mathrm{H}$, $\left.2^{\prime \prime}-\mathrm{H}_{\mathrm{a}}\right), 4.66\left(\mathrm{dd}, J=10.8,6.0 \mathrm{~Hz}, 1 \mathrm{H}, 2^{\prime \prime}-\mathrm{H}_{\mathrm{b}}\right), 5.13(\mathrm{~d}, J=8 \mathrm{~Hz}, 1 \mathrm{H}, \mathrm{NH}), 7.26-7.48(\mathrm{~m}, 4 \mathrm{H}$, Ar-H), 7.57 (d, $J=7.4 \mathrm{~Hz}, 2 \mathrm{H}, \mathrm{Ar}-\mathrm{H}), 7.77$ (d, $J=7.5 \mathrm{~Hz}, 2 \mathrm{H}, \mathrm{Ar}-\mathrm{H}) ;{ }^{13} \mathrm{C} \mathrm{NMR}(62.9 \mathrm{MHz}$, $\left.\mathrm{CDCl}_{3}\right): \delta=17.5\left(-, \mathrm{C}-3^{\prime}\right), 21.8\left(+, \mathrm{C}-1^{\prime}\right), 28.1\left[+, \mathrm{C}\left(\mathrm{CH}_{3}\right)_{3}\right], 33.6(-, \mathrm{C}-3), 46.6\left(+, \mathrm{C}-1^{\prime \prime}\right), 52.6$ (+, C-2), $59.0\left(+, \mathrm{C}-2^{\prime}\right), 66.9\left(-, \mathrm{C}-2^{\prime \prime}\right), 80.2\left[\mathrm{C}_{\text {quat }}, \mathrm{C}\left(\mathrm{CH}_{3}\right)_{3}\right], 119.9,119.9(+, \mathrm{Ar}-\mathrm{C}), 124.6$, $124.7(+, \mathrm{Ar}-\mathrm{C}), 127.1(+, \mathrm{Ar}-\mathrm{C}), 127.9$ (+, Ar-C), 141.2, $141.3\left(\mathrm{C}_{\text {quat }}, \mathrm{Ar}-\mathrm{C}\right), 143.0,143.2\left(\mathrm{C}_{\text {quat, }}\right.$ Ar-C), $155.0\left(\mathrm{C}_{\text {quat }}, \mathrm{NCO}_{2}\right), 175.4\left(\mathrm{C}_{\text {quat }}, \mathrm{C}-1\right)$; IR (KBr): nu(tilde) $=3420 \mathrm{~cm}^{-1}, 3067,2980$, $2934,1738,1715,1684,1545,1521,1451,1369,1208,1163$; MS (EI, $70 \mathrm{eV}), \mathrm{m} / z(\%)=452(3)$ $\left[\mathrm{M}^{+}\right], 178(100)\left[\mathrm{C}_{14} \mathrm{H}_{10}{ }^{+}\right], 129(2)\left[\mathrm{C}_{5} \mathrm{H}_{9} \mathrm{~N}_{2} \mathrm{O}_{2}{ }^{+}\right], 93(3)\left[\mathrm{C}_{7} \mathrm{H}_{7}{ }^{+}\right], 57(16)\left[\mathrm{C}_{4} \mathrm{H}_{9}{ }^{+}\right], 41(5)\left[\mathrm{C}_{3} \mathrm{H}_{5}{ }^{+}\right]$; HRMS (EI): calcd for $\mathrm{C}_{25} \mathrm{H}_{28} \mathrm{~N}_{2} \mathrm{O}_{6}$ : 452.1947; found 452.1947; elemental analysis calcd (\%) for $\mathrm{C}_{25} \mathrm{H}_{28} \mathrm{~N}_{2} \mathrm{O}_{6}$ (452.5): C 66.36, H 6.24, N 6.19; found C 66.11, H 5.99, N 6.02.

Chpca(MOM)-(2S)-(3-Ncp)Ala-OFm (193): Ester 192 (0.223 g, $0.493 \mathrm{mmol})$ was deprotected with $4 \mathrm{M} \mathrm{HCl}$ in EtOAc $(5 \mathrm{~mL})$ for $90 \mathrm{~min}$ to give $\mathrm{HCl} \cdot \mathrm{H}-(3-\mathrm{Ncp})$ Ala-OFm $(0.182 \mathrm{~g}, 95 \%)$ as a white solid, which was coupled with acid Chpca(MOM)-OH 109 (96 mg, 0.467 mmol) using EDC (93 mg, $0.485 \mathrm{mmol})$, HOAt (64 mg, $0.474 \mathrm{mmol})$, DIEA (61 mg, $0.472 \mathrm{mmol})$ and TMP (0.114 g, $0.941 \mathrm{mmol})$ in $\mathrm{CH}_{2} \mathrm{Cl}_{2}(5 \mathrm{~mL})$ according to GP 5 . After $3 \mathrm{~h}$, the reaction mixture was diluted with $\mathrm{Et}_{2} \mathrm{O}(50 \mathrm{~mL})$ and subjected to usual aqueous work-up (GP 5). The organic layer was dried, filtered and concentrated under reduced pressure. The residue was crystallized from $\mathrm{Et}_{2} \mathrm{O} /$ pentane to give $193(0.202 \mathrm{~g}, 80 \%$ on two steps $)$ as a white solid. $R_{\mathrm{f}}=0.20 \mathrm{EtOAc} /$ hexanes 1:3; m.p 94-95 ${ }^{\circ} \mathrm{C} ;[\alpha]_{20}^{D}-33.3\left(c=0.3, \mathrm{CHCl}_{3}\right) ;{ }^{1} \mathrm{H}$ NMR $\left(250 \mathrm{MHz}, \mathrm{CDCl}_{3}\right): \delta=1.14[\mathrm{ddd}, J$ 
$\left.=6.8,6,8,5.5 \mathrm{~Hz}, 1 \mathrm{H}, 3^{\prime}-\mathrm{H}_{\mathrm{a}},(3-N c p) A l a\right], 1.47-1.60\left[\mathrm{~m}, 1 \mathrm{H}, 3^{\prime}-\mathrm{H}_{\mathrm{b}},(3-N c p) A l a\right], 1.69-1.90[\mathrm{~m}$,

$\left.3 \mathrm{H}, 1^{\prime}-\mathrm{H}, 3-\mathrm{H},(3-N c p) A l a\right], 3.57$ (s, $\left.3 \mathrm{H}, \mathrm{OMe}\right), 3.89$ [ddd, $J=7.0,3.3,3.3 \mathrm{~Hz}, 1 \mathrm{H}, 2-\mathrm{H},(3-$ Ncp)Ala], 4.25 (t, $\left.J=5.8 \mathrm{~Hz}, 1 \mathrm{H}, 1^{\prime}-\mathrm{H}, F m\right), 4.63\left(\mathrm{dd}, J=10.6,5.8 \mathrm{~Hz}, 1 \mathrm{H}, 2^{\prime}-\mathrm{H}_{\mathrm{a}}, F m\right), 4.73$ $\left(\mathrm{dd}, J=10.6,5.8 \mathrm{~Hz}, 1 \mathrm{H}, 2^{\prime}-\mathrm{H}_{\mathrm{b}}, F m\right), 5.17\left(\mathrm{~d}, J=6.8 \mathrm{~Hz}, 1 \mathrm{H}, \mathrm{H}_{\mathrm{a}}, \mathrm{OCH}_{2} \mathrm{O}\right), 5.25(\mathrm{~d}, \mathrm{~J}=6.8 \mathrm{~Hz}$, $\left.1 \mathrm{H}, \mathrm{H}_{\mathrm{b}}, \mathrm{OCH}_{2} \mathrm{O}\right), 6.04$ (d, J=4.7 Hz, $1 \mathrm{H}, 4-\mathrm{H}$, Chpca), 6.68 (d, J=4.7 Hz, $1 \mathrm{H}, 3-\mathrm{H}$, Chpca), $7.17(\mathrm{~d}, J=7.0 \mathrm{~Hz}, 1 \mathrm{H}, \mathrm{NH}), 7.26-7.37$ (m, $2 \mathrm{H}, \mathrm{Ar}-\mathrm{H}, F m), 7.42(\mathrm{dd}, J=7.0,7.0 \mathrm{~Hz}, 2 \mathrm{H}, \mathrm{Ar}-$ H, Fm), 7.57 (d, $J=7.3 \mathrm{~Hz}, 2 \mathrm{H}, \mathrm{Ar}-\mathrm{H}, F m), 7.77$ (dd, $J=7.5,3.6 \mathrm{~Hz}, 2 \mathrm{H}, \mathrm{Ar}-\mathrm{H}, F m)$; The adsorption of $2^{\prime}-\mathrm{H}_{\mathrm{b}}, F m$ masked signal of $1^{\prime}-\mathrm{H},(3-N c p) A l a ;{ }^{13} \mathrm{C} \mathrm{NMR}\left(62.9 \mathrm{MHz}, \mathrm{CDCl}_{3}\right): \delta=$ $17.5\left[-, \mathrm{C}-3^{\prime},(3-N c p) A l a\right], 21.7$ [+,C-1', (3-Ncp)Ala $], 33.3[-, \mathrm{C}-3,(3-N c p) A l a], 46.6\left(+, \mathrm{C}-1^{\prime}\right.$, Fm), $51.3[+, \mathrm{C}-2,(3-N c p) A l a], 58.9\left[+, \mathrm{C}-2^{\prime},(3-N c p) A l a\right], 59.4(+, \mathrm{OMe}), 66.9\left(-, \mathrm{C}-2^{\prime}, \mathrm{Fm}\right)$, 104.2 (+, C-4, Chpca), $104.8\left(-, \mathrm{OCH}_{2} \mathrm{O}\right), 111.1$ (+, C-3, Chpca), $118.7\left(\mathrm{C}_{\mathrm{quat}}, \mathrm{C}-2\right.$, Chpca),

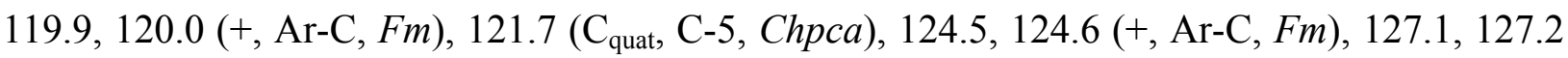
$(+, \operatorname{Ar}-\mathrm{C}, F m), 127.9(+, \mathrm{Ar}-\mathrm{C}, F m), 141.2,141.3\left(\mathrm{C}_{\text {quat }}, \mathrm{Ar}-\mathrm{C}, F m\right), 143.0,143.1\left(\mathrm{C}_{\text {quat }}, \mathrm{Ar}-\mathrm{C}\right.$, Fm), 158.0 ( $\mathrm{C}_{\text {quat }}, \mathrm{C}-1$, Chpca $), 171.1\left[\mathrm{C}_{\text {quat }}, \mathrm{C}-1,(3-N c p) A l a\right]$; IR $(\mathrm{KBr}):$ nu(tilde $)=3370 \mathrm{~cm}^{-1}$, $3038,2962,2935,2836,1729,1638,1538,1428,1374,1339,1239,1164 ; \mathrm{MS}(\mathrm{EI}, 70 \mathrm{eV}), \mathrm{m} / z$ $(\%)=542 / 540(1 / 2)\left[\mathrm{M}^{+}\right], 510 / 508(1 / 3)\left[\mathrm{M}^{+}-\mathrm{CH}_{2} \mathrm{O}\right], 191(10), 178(100)\left[\mathrm{C}_{14} \mathrm{H}_{10}{ }^{+}\right], 165(12)$, $130 / 128(3 / 10) \quad\left[\mathrm{C}_{5} \mathrm{H}_{3} \mathrm{ClNO}^{+}\right], 45$ (36) $\left[\mathrm{C}_{2} \mathrm{H}_{5} \mathrm{O}^{+}\right]$; HRMS (EI): calcd for $\mathrm{C}_{27} \mathrm{H}_{26} \mathrm{ClN}_{3} \mathrm{O}_{7}$ : 539.1459; found 539.1459; elemental analysis (\%) for $\mathrm{C}_{27} \mathrm{H}_{26} \mathrm{ClN}_{3} \mathrm{O}_{7}(540)$ : calcd. $\mathrm{C} 60.06, \mathrm{H}$ 4.85, N 7.78; found C 60.10, H 5.00, N 7.71.

Chpca-(2S)-(3-Ncp)Ala-OFm (194): The acyl amino ester 193 (50 mg, $92.60 \mu \mathrm{mol})$ was deprotected with $\mathrm{MgBr}_{2} \cdot \mathrm{Et}_{2} \mathrm{O}(0.239 \mathrm{~g}, 0.925 \mathrm{mmol})$ and $\mathrm{EtSH}(0.07 \mathrm{~mL}, 0.945 \mathrm{mmol})$ in $\mathrm{CH}_{2} \mathrm{Cl}_{2}(15 \mathrm{~mL})$ according to GP 10 for $3 \mathrm{~h}$. The usual aqueous work-up (GP 10) followed by preparative TLC $(200 \times 200 \mathrm{~mm}$, EtOAc/hexanes 1:4, 2 runs $)$ gave $194(0.036 \mathrm{~g}, 78 \%)$ as an extremely viscous oil which was unlimitedly stable by storage under argon at $-28^{\circ} \mathrm{C} . R_{\mathrm{f}}=0.20$ EtOAc/hexanes 1:3; ${ }^{1} \mathrm{H}$ NMR $\left(250 \mathrm{MHz}, \mathrm{CDCl}_{3}\right): \delta=0.83-0.96\left[\mathrm{~m}, 1 \mathrm{H}, 3^{\prime}-\mathrm{H}_{\mathrm{a}},(3-N c p)\right.$ Ala $]$, 
1.42-1.56 [m, 1 H, 3'- $\left.\mathrm{H}_{\mathrm{b}},(3-N c p) A l a\right], 1.67-1.82$ [m, $\left.3 \mathrm{H}, 1^{\prime}-\mathrm{H}, 3-\mathrm{H},(3-N c p) A l a\right], 3.89$ [ddd, $J=$ 7.0, 3.1, 3.1 Hz, $1 \mathrm{H}, 2-\mathrm{H},(3-N c p) A l a], 4.25$ (t, $\left.J=5.1 \mathrm{~Hz}, 1 \mathrm{H}, 1^{\prime}-\mathrm{H}, F m\right), 4.67$ (dd, $J=10.7$, $\left.5.1 \mathrm{~Hz}, 1 \mathrm{H}, 2^{\prime}-\mathrm{H}_{\mathrm{a}}, F m\right), 4.82\left(\mathrm{dd}, J=10.7,5.1 \mathrm{~Hz}, 1 \mathrm{H}, 2^{\prime}-\mathrm{H}_{\mathrm{b}}, F m\right), 5.96(\mathrm{~d}, J=5.0 \mathrm{~Hz}, 1 \mathrm{H}, 4-$ H, Chpca), 6.37 (d, J=7.0 Hz, 1 H, NH), 6.40 (d, J=5.0 Hz, $1 \mathrm{H}, 3-\mathrm{H}$, Chpca), 7.26-7.37 (m, 2 H, Ar-H, Fm), 7.42 (dd, J= 7.3, 7.3 Hz, 2 H, Ar-H, Fm), 7.56 (d, J=7.3 Hz, 2 H, Ar-H, Fm), $7.76(\mathrm{dd}, J=7.1,5.3 \mathrm{~Hz}, 2 \mathrm{H}, \mathrm{Ar}-\mathrm{H}, F m), 13.0-13.3$ (br, $1 \mathrm{H}, \mathrm{OH})$; The adsorption of $2^{\prime}-\mathrm{H}_{\mathrm{a}}, F m$ masked signal of $1^{\prime}-\mathrm{H},(3-N c p)$ Ala; ${ }^{13} \mathrm{C}$ NMR $\left(62.9 \mathrm{MHz}, \mathrm{CDCl}_{3}\right): \delta=17.4\left[-, \mathrm{C}-3^{\prime},(3-\right.$ Ncp)Ala $], 21.6\left[+, \mathrm{C}-1^{\prime},(3-N c p) A l a\right], 33.4[-, \mathrm{C}-3,(3-N c p) A l a], 46.7\left(+, \mathrm{C}-1^{\prime}, F m\right), 51.2[+, \mathrm{C}-2$, (3-Ncp)Ala ], 58.9 [+, C-2', (3-Ncp)Ala], 66.9 (-,C-2', Fm), 102.8 (+, C-4, Chpca), 106.1 (+, C-3, Chpca), 114.4 ( $\mathrm{C}_{\text {quat }}, \mathrm{C}-2$, Chpca $), 116.0$ ( $\mathrm{C}_{\text {quat }}, \mathrm{C}-5$, Chpca), 120.0, 120.1 (+, Ar-C, Fm), 124.4, $124.5(+, \mathrm{Ar}-\mathrm{C}, F m), 127.2,127.3(+, \mathrm{Ar}-\mathrm{C}, F m), 128.0(+, \mathrm{Ar}-\mathrm{C}, F m), 141.3,141.4\left(\mathrm{C}_{\text {quat }}, \mathrm{Ar}-\mathrm{C}\right.$, $F m), 142.9,143.0\left(\mathrm{C}_{\text {quat }}, \mathrm{Ar}-\mathrm{C}, F m\right), 162.2\left(\mathrm{C}_{\text {quat }}, \mathrm{C}-1\right.$, Chpca $), 170.9$ [C $\mathrm{C}_{\text {quat }}, \mathrm{C}-1,(3-N c p)$ Ala $]$; IR $(\mathrm{KBr}): \mathrm{nu}($ tilde $)=3750-1800 \mathrm{~cm}^{-1}, 3067,1740,1542,1451,1426,1368,1198$; MS (EI, $\left.70 \mathrm{eV}\right)$, $m / z(\%)=497 / 495(2 / 7)\left[\mathrm{M}^{+}\right], 319 / 317(1 / 4)\left[\mathrm{M}^{+}-\mathrm{C}_{14} \mathrm{H}_{10}\right], 178(100)\left[\mathrm{C}_{14} \mathrm{H}_{10}{ }^{+}\right], 146 / 144(3 / 10)$ $\left[\mathrm{C}_{5} \mathrm{H}_{2} \mathrm{ClNO}_{2}{ }^{+}\right]$; HRMS (EI): calcd for $\mathrm{C}_{25} \mathrm{H}_{22} \mathrm{ClN}_{3} \mathrm{O}_{6}$ : 495.1197; found 495.1197.

Chpca-(2S)-(3-Ncp)Ala-OH (195): Ester $194(35 \mathrm{mg}, 70.58 \mu \mathrm{mol})$ was deprotected with 10\% tris(2-aminoethyl)amine (TAEA) in $\mathrm{CH}_{2} \mathrm{Cl}_{2}(1 \mathrm{~mL})$ for 20 min and the mixture was then taken up with EtOAc $(30 \mathrm{~mL})$. The organic layer briefly washed $1 \mathrm{M} \mathrm{NaHSO}_{4}(3 \times 10 \mathrm{~mL})$, water $(3 \times$ $5 \mathrm{~mL})$, brine $(2 \times 5 \mathrm{~mL})$, dried, filtered and concentrated to give 195 as a turbid oil which completely polymerized into white insoluble solid at $20^{\circ} \mathrm{C}$ for ca. $2 \mathrm{~h}$. In solution 195 was even less stable. ${ }^{1} \mathrm{H}$ NMR $\left(250 \mathrm{MHz},\left[\mathrm{D}_{6}\right]\right.$-acetone): $\delta=1.30$ [ddd, $J=7.0,7.0,7.0 \mathrm{~Hz}, 1 \mathrm{H}, 3^{\prime}-\mathrm{H}_{\mathrm{a}}$, (3-Ncp)Ala ], 1.71-1.87 [m, $\left.1 \mathrm{H}, 3^{\prime}-\mathrm{H}_{\mathrm{b}},(3-N c p) A l a\right], 1.90-2.21$ [m, $3 \mathrm{H}, 1^{\prime}-\mathrm{H}, 3-\mathrm{H},(3-N c p)$ Ala $]$, $4.42[\mathrm{ddd}, J=6.3,3.5,3.5 \mathrm{~Hz}, 1 \mathrm{H}, 2-\mathrm{H},(3-N c p) A l a], 4.71-4.84$ [m, 2'-H, (3-Ncp)Ala], 6.03 (d, $J=5.0 \mathrm{~Hz}, 1 \mathrm{H}, 4-\mathrm{H}$, Chpca), $6.82\left(\mathrm{~d}, J=5.0 \mathrm{~Hz}, 1 \mathrm{H}\right.$, Chpca), 7.0-8.0 (br, $\left.2 \mathrm{H}, \mathrm{OH}, \mathrm{CO}_{2} \mathrm{H}\right)$, 
$8.17(\mathrm{~d}, J=7.5 \mathrm{~Hz}, 1 \mathrm{H}, \mathrm{NH})$; It was impossible to measure ${ }^{13} \mathrm{C}$ NMR because of complete decomposition of 195 during measurement; MS (EI, $70 \mathrm{eV}), m / z(\%)=319 / 317(12 / 40)\left[\mathrm{M}^{+}\right]$, 283 (6), 144 (100), 130/128 (15/46) [ $\left.\mathrm{C}_{5} \mathrm{H}_{2} \mathrm{ClNO}^{+}\right], 112$ (6), 91/89 (4/12), $80(18)\left[\mathrm{C}_{5} \mathrm{H}_{6} \mathrm{~N}^{+}\right], 64$ (12), 45 (10) $\left[\mathrm{CO}_{2} \mathrm{H}^{+}\right]$; HRMS (EI): calcd for $\mathrm{C}_{11} \mathrm{H}_{12} \mathrm{ClN}_{3} \mathrm{O}_{6}$ : 317.0414; found 317.0414.

O-MOM protected hormaomycin O-MOM-3 and its epimer epi-O-MOM-3: The ester 193 (5.4 $\mathrm{mg}, 10.00 \mu \mathrm{mol})$ was deprotected with $20 \% \mathrm{Et}_{2} \mathrm{~N}$ in $\mathrm{MeCN}(1 \mathrm{~mL})$ for $40 \mathrm{~min}$. The mixture was then concentrated under reduced pressure, the residue was washed with hexanes, taken up with $\mathrm{Et}_{2} \mathrm{O}(30 \mathrm{~mL})$, washed with $1 \mathrm{M} \mathrm{NaHSO}_{4}(3 \times 5 \mathrm{~mL})$, water $(3 \times 5 \mathrm{~mL})$, brine $(2 \times 5 \mathrm{~mL})$. The organic layer was dried, filtered and concentrated under reduced pressure. The resultant crude $N$ acyl amino acid $196\left(R_{\mathrm{f}}=0.42 \mathrm{EtOAc} / \mathrm{hexanes} 1: 2(2 \% \mathrm{AcOH})\right)$ was dried at 0.01 Torr for $1 \mathrm{~h}$ and then introduced in the coupling reaction with the deprotected depsipeptide obtained after treatment of the $N \mathrm{MeZ}$ protected cyclodepsipeptide 171c $(8.0 \mathrm{mg}, 8.179 \mu \mathrm{mol})$ with $10 \%$ anisole in TFA $(1 \mathrm{~mL})$ according to GP $11 \mathrm{using}$ PyAOP $(8.4 \mathrm{mg}, 16.11 \mu \mathrm{mol})$, HOAt $(2.2 \mathrm{mg}, 16.28$ $\mu \mathrm{mol})$, DIEA $(1.3 \mathrm{mg}, 10,06 \mu \mathrm{mol})$ and TMP $(3.8 \mathrm{mg}, 31.36 \mu \mathrm{mol})$ in $\mathrm{CH}_{2} \mathrm{Cl}_{2}(1 \mathrm{~mL})$ according to GP 7 for $16 \mathrm{~h}$. The mixture was then diluted with $\mathrm{Et}_{2} \mathrm{O}$ and subjected to usual aqueous workup (GP 5). TLC analysis showed the presence of the two main reaction products which were separated by preparative TLC $(100 \times 200 \mathrm{~mm}$, acetone/hexanes $1: 3,3$ runs $)$. Each fraction was additionally purified by preparative TLC $(40 \times 80 \mathrm{~mm}$, acetone/hexanes $1: 3,3$ runs $)$ to give $O$ MOM-3 (2.5 mg, 26\%) as a colorless glass and epi-O-MOM-3 (1.5 mg, 16\%) as colorless glass. $O$-MOM-3: $\mathrm{R}_{\mathrm{f}}=0.11$ acetone/hexanes $1: 3 ;{ }^{1} \mathrm{H}$ NMR $\left(600 \mathrm{MHz}, \mathrm{CDCl}_{3}\right): \delta=-0.26[\mathrm{ddd}, J=$ 6.0, 6.0, $\left.6.0 \mathrm{~Hz}, 1 \mathrm{H}, 3^{\prime}-\mathrm{H}_{\mathrm{a}},(3-N c p) A l a\right], 0.07-0.17\left[\mathrm{~m}, 1 \mathrm{H}, 3-\mathrm{H}_{\mathrm{a}},(3-N c p) A l a\right], 0.52-0.61[\mathrm{~m}$, $\left.1 \mathrm{H}, 1^{\prime}-\mathrm{H},(3-N c p) A l a\right], 0.68-0.79$ [m, $\left.1 \mathrm{H}, 3-\mathrm{H}_{\mathrm{b}},(3-N c p) A l a\right], 0.88$ (t, J= $7.2 \mathrm{~Hz}, 3 \mathrm{H}, 5-\mathrm{H}$, Ile), $1.02\left(\mathrm{~d}, J=6.6 \mathrm{~Hz}, 3 \mathrm{H}, 1^{\prime}-\mathrm{H}, I l e\right), 1.05\left[\mathrm{ddd}, \mathrm{J}=6.0,6.0,6.0 \mathrm{~Hz}, 1 \mathrm{H}, 3^{\prime}-\mathrm{H}_{\mathrm{b}},(3-N c p)\right.$ Ala $], 1.10$ $1.14\left[\mathrm{~m}, 1 \mathrm{H}, 3^{\prime}-\mathrm{H}_{\mathrm{a}},(3-N c p)\right.$ Ala $], 1.19-1.27\left[\mathrm{~m}, 2 \mathrm{H}, 4-\mathrm{H}_{\mathrm{a}}\right.$, Ile, 3'- $\left.\mathrm{H}_{\mathrm{b}},(3-N c p) A l a\right], 1.29[\mathrm{~d}, J=$ $6.6 \mathrm{~Hz}, 3 \mathrm{H}, 4-\mathrm{H},(\beta M e) P h e], 1.39$ [d, J = 6.6 Hz, $3 \mathrm{H}, 4-\mathrm{H},(\beta M e) P h e], 1.50-1.56\left(\mathrm{~m}, 1 \mathrm{H}, 4-\mathrm{H}_{\mathrm{b}}\right.$, 
Ile), 1.59 (d, $J=7.2 \mathrm{~Hz}, 3 \mathrm{H}, 4-\mathrm{H}, a-T h r), 1.64-1.70$ [m, $\left.1 \mathrm{H}, 3-\mathrm{H}_{\mathrm{a}},(3-N c p) A l a\right], 1.69$ [dd, $J=$ 7.2, $\left.1.8 \mathrm{~Hz}, 3 \mathrm{H}, 3^{\prime}-\mathrm{H},(4-P E)-P r o\right], 1.75-1.85$ [m, $2 \mathrm{H}, 3-\mathrm{H}$, Ile, 3-H $\mathrm{H}_{\mathrm{a}}$ (4-PE)Pro], 1.90-1.95 $\left[\mathrm{m}, 1 \mathrm{H}, 3-\mathrm{H}_{\mathrm{b}},(3-N c p) A l a\right], 1.95-2.00\left[\mathrm{~m}, 1 \mathrm{H}, 1^{\prime}-\mathrm{H},(3-N c p) A l a\right], 2.40$ [ddd, $J=12.0,6.0$, $\left.6.0 \mathrm{~Hz}, 1 \mathrm{H}, 3-\mathrm{H}_{\mathrm{b}},(4-P E)-P r o\right], 3.05$ [dq, $\left.J=10.8,6.6 \mathrm{~Hz}, 1 \mathrm{H}, 3-\mathrm{H},(\beta M e) P h e\right], 3.11-3.17$ [m, $\left.1 \mathrm{H}, 2^{\prime}-\mathrm{H},(3-N c p) A l a\right], 3.24-3.32\left[\mathrm{~m}, 2 \mathrm{H}, 4-\mathrm{H}, 5-\mathrm{H}_{\mathrm{a}},(4-P E)-P r o\right], 3.71[\mathrm{dq}, J=4.2,7.2 \mathrm{~Hz}$, $1 \mathrm{H}, 3-\mathrm{H},(\beta M e) P h e], 3.75$ (s, $3 \mathrm{H}, \mathrm{OMe}), 3.90(\mathrm{dd}, J=6.6,5.4 \mathrm{~Hz}, 1 \mathrm{H}, 2-\mathrm{H}), 3.93-3.98$ [m, $\left.1 \mathrm{H}, 5-\mathrm{H}_{\mathrm{b}},(4-P E)-P r o\right], 4.09$ [ddd, $\left.J=6.6,3.0,3.0 \mathrm{~Hz}, 1 \mathrm{H}, 2^{\prime}-\mathrm{H},(3-N c p) A l a\right], 4.26(\mathrm{dd}, J=$ $13.2,13.2 \mathrm{~Hz}, 1 \mathrm{H}, 2-\mathrm{H}), 4.30(\mathrm{dd}, J=11.4,6.0 \mathrm{~Hz}, 1 \mathrm{H}, 2-\mathrm{H}), 4.61(\mathrm{dd}, J=8.4,4.8 \mathrm{~Hz}, 1 \mathrm{H}, 2-$ H), 4.64-4.70 (m, 2 H, 2-H), 5.10-5.17 [m, $1 \mathrm{H}, 2-\mathrm{H},(3-N c p) A l a], 5.24-5.29$ [m, $1 \mathrm{H}, 1^{\prime}-\mathrm{H},(4-$ PE)-Pro], 5.39 (dq, $J=1.8,7.2 \mathrm{~Hz}, 1 \mathrm{H}, 3-\mathrm{H}, a-T h r), 5.47$ (d, $\left.J=6.0 \mathrm{~Hz}, 1 \mathrm{H}, \mathrm{H}_{\mathrm{a}}, \mathrm{OCH}_{2} \mathrm{O}\right), 5.50$ $\left(\mathrm{d}, J=6.0 \mathrm{~Hz}, 1 \mathrm{H}, \mathrm{H}_{\mathrm{b}}, \mathrm{OCH}_{2} \mathrm{O}\right), 5.64\left[\mathrm{dq}, J=10.5,7.2 \mathrm{~Hz}, 1 \mathrm{H}, 2^{\prime}-\mathrm{H},(4-P E)-P r o\right], 5.75-5.81$ (br, $2 \mathrm{H}, \mathrm{NH}), 6.17$ (d, $J=4.5 \mathrm{~Hz}, 1 \mathrm{H}, 4-\mathrm{H}, \mathrm{Chpca}), 6.70(\mathrm{~d}, J=9.0 \mathrm{~Hz}, 1 \mathrm{H}, \mathrm{NH}), 6.84(\mathrm{~d}, J=$ 4.5 Hz, $1 \mathrm{H}, 3-\mathrm{H}$, Chpca), 7.06 (dd, $J=7.5,7.5 \mathrm{~Hz}, 1 \mathrm{H}, \mathrm{Ar}-\mathrm{H}), 7.09$ (d, J=7.2 Hz, $2 \mathrm{H}, \mathrm{Ar}-\mathrm{H}$ ), $7.15(\mathrm{dd}, J=7.5,7.5 \mathrm{~Hz}, 3 \mathrm{H}, \mathrm{Ar}-\mathrm{H}), 7.16(\mathrm{~d}, J=7.2 \mathrm{H}, 1 \mathrm{H}, \mathrm{NH}), 7.22-7.31(\mathrm{~m}, 5 \mathrm{H}$, Ar-H, $\mathrm{NH}), 7.38(\mathrm{~d}, J=9.6 \mathrm{~Hz}, 1 \mathrm{H}, \mathrm{NH}), 7.75(\mathrm{~d}, J=9.0 \mathrm{~Hz}, 1 \mathrm{H}, \mathrm{NH}), 8.79(\mathrm{~d}, J=9.0 \mathrm{~Hz}, 1 \mathrm{H}, \mathrm{NH})$; ${ }^{13} \mathrm{C}$ NMR $\left(150.8 \mathrm{MHz}, \mathrm{CDCl}_{3}\right): \delta=10.8(+, \mathrm{C}-5$, Ile $), 13.2[+, \mathrm{C}-4,(\beta M e) P h e], 13.3\left[+, \mathrm{C}-3^{\prime},(4-\right.$ PE)-Pro], $15.5\left(+, \mathrm{C}-1^{\prime}\right.$, Ile $), 17.2$ [-, C-3', (3-Ncp)Ala $], 17.3$ [-, C-3', (3-Ncp)Ala $], 17.5(+, \mathrm{C}-4$, a-Thr), 18.3 [+, C-4, (BMe)Phe], 20.7 [+, C-1', (3-Ncp)Ala ], 21.3 [+, C-1', (3-Ncp)Ala], 24.9 (-, C-4, Ile), 29.7 [-, C-3, (3-Ncp)Ala], 34.0 [-, C-3, (3-Ncp)Ala], 35.4 [-, C-3, (4-PE)-Pro], 36.7 [+, C-4, (4-PE)-Pro], $38.6(+, \mathrm{C}-3, \mathrm{Ile}), 39.4[+, \mathrm{C}-3,(\beta M e) P h e], 42.7$ [+, C-3, (ßMe)Phe], 50.2 [+, C-2', (3-Ncp)Ala], $51.5(+, \mathrm{C}-2), 52.9$ [-, C-5, (4-PE)-Pro], 54.7 (+, C-2), 54.9 (+, C-2), 58.5 $\left[+, \mathrm{C}-2^{\prime},(3-N c p)\right.$ Ala $], 59.4(+, \mathrm{OMe}, \mathrm{C}-2), 59.6(+, \mathrm{C}-2), 60.2(+, \mathrm{C}-2), 61.5(+, \mathrm{C}-2), 69.9(+, \mathrm{C}-$ 3, a-Thr), $104.5\left(-, \mathrm{OCH}_{2} \mathrm{O}\right), 106.1\left(+, \mathrm{C}-4\right.$, Chpca), $111.5(+, \mathrm{C}-3$, Chpca $), 119.8\left(\mathrm{C}_{\text {quat }}, \mathrm{C}-2\right.$, Chpca), $121.5\left(\mathrm{C}_{\text {quat }}, \mathrm{C}-2\right.$, Chpca $), 126.9,127.1,127.5,127.6,128.5,128.6(+$, Ar-C), $127.4[+$, C-1', (4-PE)-Pro], 128.5 [+, C-2', (4-PE)-Pro], 142.3 (2 × $\left.\mathrm{C}_{\text {quat }}, \mathrm{Ar}-\mathrm{C}\right), 158.6\left(\mathrm{C}_{\text {quat }}, \mathrm{C}-1\right.$, 
Chpca), 168.8, 169.9, 170.1, 170.2, 171.1, 171.3, $171.4\left(\mathrm{C}_{\text {quat }}, \mathrm{C}-1\right)$; UV (MeOH): neutral: $\lambda_{\max }(\varepsilon)=268\left(1.8 \times 10^{4}\right), 201\left(7.5 \times 10^{5}\right) \mathrm{nm} ;$ basic: $341\left(1.4 \times 10^{3}\right), 268\left(1.5 \times 10^{4}\right), 205(9.1 \times$ $\left.10^{4}\right)$; acidic: $341\left(2.7 \times 10^{3}\right), 268\left(1.4 \times 10^{4}\right) \mathrm{nm}$; $\mathrm{CD}(\mathrm{MeOH}): \lambda_{\max }[\Theta]=268.2\left(2.71 \times 10^{4}\right)$, $259.3\left(2.40 \times 10^{4}\right), 222.1\left(-41.7 \times 10^{4}\right) \mathrm{nm}\left(c=8.18 \times 10^{-6} \mathrm{M}\right) ;$ MS (ESI): positive mode, $m / z$ $(\%)=1196(100)\left[\mathrm{M}+\mathrm{Na}^{+}\right]$; negative mode, $m / z(\%)=1171(100)\left[\mathrm{M}-\mathrm{H}^{+}\right]$.

epi-O-MOM-3: $\mathrm{R}_{\mathrm{f}}=0.09$ acetone/hexanes $1: 3 ;{ }^{1} \mathrm{H}$ NMR $\left(600 \mathrm{MHz}, \mathrm{CDCl}_{3}\right): \delta=0.68[\mathrm{ddd}, J=$ 6.6, 6.6, 6.6 Hz, $\left.1 \mathrm{H}, 3^{\prime}-\mathrm{H}_{\mathrm{a}},(3-N c p) A l a\right], 0.79-0.92\left[\mathrm{~m}, 2 \mathrm{H}, 1^{\prime}-\mathrm{H}, 3-\mathrm{H}_{\mathrm{a}},(3-N c p) A l a\right], 0.98(\mathrm{t}, J=$ 7.2 Hz, $3 \mathrm{H}, 5-\mathrm{H}$, Ile), $1.01\left(\mathrm{~d}, J=6.6 \mathrm{~Hz}, 3 \mathrm{H}, 1^{\prime}-\mathrm{H}\right.$, Ile), 1.15 [ddd, J = 6.6, 6.6, 6.6 Hz, 1 H, 3'$\left.\mathrm{H}_{\mathrm{b}},(3-N c p) A l a\right], 1.19-1.32$ [m, $6 \mathrm{H}, 3^{\prime}-\mathrm{H},(3-N c p)$ Ala $\left., 4-\mathrm{H},(\beta M e) P h e, 4-\mathrm{H}_{\mathrm{a}}, I l e\right], 1.34$ [d, J= 7.2 Hz, 3 H, 4-H, (ßMe)Phe], 1.46-1.62 [m, 3 H, 4-Hb, Ile, 3-H, (3-Ncp)Ala], 1.64-1.70 [m, 1 H, 3- $\left.\mathrm{H}_{\mathrm{a}},(3-N c p) A l a\right], 1.69$ [dd, $\left.J=7.2,1.8 \mathrm{~Hz}, 3 \mathrm{H}, 3{ }^{\prime}-\mathrm{H},(4-P E)-P r o\right], 1.76$ [ddd, $J=11.4,11.4$, $11.4 \mathrm{~Hz}, 3-\mathrm{H}_{\mathrm{a}}$, (4-PE)Pro], 1.90-1.99 (m, 1 H, 3-H, Ile), 2.05-2.13 [m, $\left.1 \mathrm{H}, 1^{\prime}-\mathrm{H},(3-N c p) A l a\right]$, 2.40 [ddd, $\left.J=11.4,6.0,6.0 \mathrm{~Hz}, 1 \mathrm{H}, 3-\mathrm{H}_{\mathrm{b}},(4-P E)-P r o\right], 3.13-3.23$ [m, $\left.1 \mathrm{H}, 3-\mathrm{H},(\beta M e) P h e\right]$, 3.23-3.32 [m, $2 \mathrm{H}, 4-\mathrm{H}, 5-\mathrm{H}_{\mathrm{a}}$, (4-PE)-Pro], $3.62(\mathrm{~s}, 3 \mathrm{H}, \mathrm{OMe}), 3.67-3.75[\mathrm{~m}, 1 \mathrm{H}, 3-\mathrm{H}$, (BMe)Phe], 3.85 [ddd, $\left.J=6.6,3.0,3.0 \mathrm{~Hz}, 2^{\prime}-\mathrm{H},(3-N c p) A l a\right], 3.96-4.01$ (m, $\left.1 \mathrm{H}, 2-\mathrm{H}\right), 4.05-$ $4.09\left[\mathrm{~m}, 1 \mathrm{H}, 5-\mathrm{H}_{\mathrm{b}},(4-P E)-P r o\right], 4.13$ [ddd, $J=6.6,3.0,3.0 \mathrm{~Hz}, 1 \mathrm{H}, 2^{\prime}-\mathrm{H},(3-N c p)$ Ala $]$, 4.13$4.21(\mathrm{~m}, 1 \mathrm{H}, 2-\mathrm{H}), 4.56-4.72(\mathrm{~m}, 3 \mathrm{H}, 2-\mathrm{H}), 4.61(\mathrm{dd}, J=9.0,4.5 \mathrm{~Hz}, 1 \mathrm{H}, 2-\mathrm{H}), 4.84(\mathrm{dd}, J=$ 9.0, $2.4 \mathrm{~Hz}, 1 \mathrm{H}, 2-\mathrm{H}), 5.20-5.35\left[\mathrm{~m}, 2 \mathrm{H}, 1^{\prime}-\mathrm{H},(4-P E)\right.$ Pro, 3-H, $\left.a-T h r\right], 5.22$ (d, J=6.0 Hz, $\left.1 \mathrm{H}, \mathrm{H}_{\mathrm{a}}, \mathrm{OCH}_{2} \mathrm{O}\right), 5.28\left(\mathrm{~d}, J=6.0 \mathrm{~Hz}, 1 \mathrm{H}, \mathrm{H}_{\mathrm{b}}, \mathrm{OCH}_{2} \mathrm{O}\right), 5.61$ [dq, $J=10.5,7.2 \mathrm{~Hz}, 1 \mathrm{H}, 2^{\prime}-\mathrm{H}$, (4-PE)-Pro], 6.03 (d, $J=4.8$ Hz, $1 \mathrm{H}, 4-\mathrm{H}$, Chpca), 6.33-6.46 (br, $1 \mathrm{H}, \mathrm{NH}), 6.57-6.69$ (br, $1 \mathrm{H}$, NH), 6.70 (d, $J=4.8 \mathrm{~Hz}, 1 \mathrm{H}, 3-\mathrm{H}$, Chpca), 7.13-7.34 (m, $14 \mathrm{H}, \mathrm{Ar}-\mathrm{H}, \mathrm{NH}), 7.57-7.71$ (br, $1 \mathrm{H}$, $\mathrm{NH})$; Absorption of water masked signal of $4-\mathrm{H}, a-T h r ;{ }^{13} \mathrm{C} \mathrm{NMR}\left(150.8 \mathrm{MHz}, \mathrm{CDCl}_{3}\right): \delta=$ $10.2(+, \mathrm{C}-5$, Ile), 13.4 [+, C-4, (ßMe)Phe, C-3', (4-PE)-Pro], 14.7 (+, C-1', Ile), 17.4 [-, C-3', (3-Ncp)Ala], 17.9 [-, C-3', (3-Ncp)Ala $], 18.6(+, \mathrm{C}-4, a-T h r), 21.5[+, \mathrm{C}-4,(\beta M e) P h e], 24.4[+$, C-1', (3-Ncp)Ala], 24.9 (-, C-4, Ile), 29.3 [+, C-1', (3-Ncp)Ala], 29.7 [-, C-3, (3-Ncp)Ala ], 30.9 
[-, C-3, (3-Ncp)Ala], 35.6 [-, C-3, (4-PE)-Pro], 36.6 [+, C-4, (4-PE)-Pro], 37.5 (+,C-3, Ile), 39.0 [+, C-3, ( $\beta M e) P h e], 40.2[+, \mathrm{C}-3,(\beta M e) P h e], 52.3(+, \mathrm{C}-2), 52.9$ [-, C-5, (4-PE)-Pro], $53.2(+$, C-2), $54.4(+, \mathrm{C}-2), 55.8(+, \mathrm{C}-2), 59.0\left[+, \mathrm{C}-2^{\prime},(3-N c p) A l a\right], 59.3\left[+, \mathrm{C}-2^{\prime},(3-N c p)\right.$ Ala $], 59.5(+$, OMe, C-2), 59.5 (+, C-2), $60.7(+, \mathrm{C}-2), 72.2(+, \mathrm{C}-3, a-T h r), 104.2\left(-, \mathrm{OCH}_{2} \mathrm{O}\right), 104.9(+, \mathrm{C}-4$, Chpca), 110.9 (+, C-3, Chpca), 118.7 (C quat $_{1}$ C-2, Chpca), 122.1 ( $\mathrm{C}_{\text {quat }}, \mathrm{C}-2$, Chpca), 126.8, 127.1, 127.3, 128.6, 128.7, 128.8 (+, Ar-C), 127.7 [+, C-1', (4-PE)-Pro], 128.1 [+, C-2', (4-PE)Pro], $141.6\left(2 \times \mathrm{C}_{\text {quat }}, \mathrm{Ar}-\mathrm{C}\right), 157.9\left(\mathrm{C}_{\text {quat }}, \mathrm{C}-1\right.$, Chpca $), 169.8,170.0,170.2,170.9(\times 2), 171.2$, $171.3\left(\mathrm{C}_{\text {quat }}, \mathrm{C}-1\right)$; UV $(\mathrm{MeOH})$ : neutral: $\lambda_{\max }(\varepsilon)=261\left(1.7 \times 10^{4}\right), 201\left(6.6 \times 10^{5}\right) \mathrm{nm}$; basic: $341\left(1.4 \times 10^{3}\right), 267\left(1.4 \times 10^{4}\right), 226\left(2.1 \times 10^{4}\right), 204\left(7.6 \times 10^{4}\right)$; acidic: $341\left(2.7 \times 10^{3}\right), 269$ $\left(2.0 \times 10^{4}\right) \mathrm{nm} ; \mathrm{CD}(\mathrm{MeOH}): \lambda_{\max }[\Theta]=274\left(2.08 \times 10^{4}\right), 221.6\left(-4.99 \times 10^{4}\right) \mathrm{nm}\left(c=6.48 \times 10^{-}\right.$ $\left.{ }^{6} \mathrm{M}\right)$; MS (ESI): positive mode, $m / z(\%)=1196(100)\left[\mathrm{M}+\mathrm{Na}^{+}\right]$; negative mode, $m / z(\%)=1207$ (40) $\left[\mathrm{M}+\mathrm{Cl}^{\top}\right], 1171(100)\left[\mathrm{M}-\mathrm{H}^{+}\right]$.

N-Teoc-3-(2S,1 S, 2 R)-(trans-2'-Nitrocyclopropyl)alanine (S)-(197): A solution of TeocOSu TeocHN “.' nitrocyclopropyl)alanine $\left(2 S, 1^{\prime} R, 2^{\prime} R\right)-83(0.200 \mathrm{~g}, 1.15 \mathrm{mmol})$ and $\mathrm{NaHCO}_{3}(0.202 \mathrm{~g}, 2.40$ $\mathrm{mmol}$ ) in water $(7 \mathrm{~mL})$ (if emulsion formed acetone and/or water were added to obtain homogeneous solution) and stirring continued for a further 2 h. $N, N$-dimethylaminopropylamine $(0.055 \mathrm{~mL}, 0.44 \mathrm{mmol})$ was then added. After an additional $10 \mathrm{~min}$ acetone was removed under reduced pressure and $\mathrm{pH}$ of the residual water solution was adjusted to 2-3 with $1 \mathrm{M} \mathrm{NaHSO}_{4}$. The resulting emulsion was extracted with $\mathrm{Et}_{2} \mathrm{O}(50 \mathrm{~mL})$ and the ethereal layer was washed with $1 \mathrm{M} \mathrm{NaHSO}_{4}(2 \times 10 \mathrm{~mL})$, water $(10 \times 10 \mathrm{~mL})$, brine $(2 \times 5 \mathrm{~mL})$, dried, filtered and concentrated under reduced pressure. The residual oil $(0.300 \mathrm{~g})$ was dissolved in $\mathrm{Et}_{2} \mathrm{O}(5 \mathrm{~mL})$ and cyclohexylamine $(0.094 \mathrm{~g}, 0.95 \mathrm{mmol})$ was added. The mixture was concentrated under reduced pressure and treated with boiling hexanes. The resultant precipitate was filtered off and washed 
with $\mathrm{Et}_{2} \mathrm{O}$ /pentane 1:4 to give cyclohexylammonium salt of $(S)-197(0.386 \mathrm{~g}, 81 \%)$ as a white solid. $R_{\mathrm{f}}=0.24 \mathrm{EtOAc} /$ hexanes $1: 3(2 \% \mathrm{AcOH}) ;[\alpha]_{20}^{D} 22.80\left(c=0.46, \mathrm{CHCl}_{3}\right)$ for CHA salt; ${ }^{1} \mathrm{H}$ $\operatorname{NMR}\left(250 \mathrm{MHz}, \mathrm{CDCl}_{3}\right): \delta=0.04\left[\mathrm{~s}, 9 \mathrm{H}, \mathrm{Si}\left(\mathrm{CH}_{3}\right)_{3}\right], 1.00(\mathrm{dd}, J=9.5,7.3 \mathrm{~Hz}, 2 \mathrm{H}, 2-\mathrm{H}$, Teoc $)$, $1.14\left(\mathrm{ddd}, J=6.0,6.0,6.0 \mathrm{~Hz}, 1 \mathrm{H}, 3^{\prime}-\mathrm{H}_{\mathrm{a}}\right), 1.60-1.95\left(\mathrm{~m}, 1 \mathrm{H}, 1^{\prime}-\mathrm{H}, 3^{\prime}-\mathrm{H}_{\mathrm{b}}\right), 1.98-2.19$ (m, $2 \mathrm{H}$, 3-H), 4.18 (dd, $J=8.4,8.4$ Hz, $2 \mathrm{H}, 1-\mathrm{H}$, Teoc), 4.33-4.46, 4.46-4.59 (2 × m, $1 \mathrm{H}, 2-\mathrm{H}), 5.33-$ $5.46(\mathrm{~m}, 1 \mathrm{H}, \mathrm{NH}), 7.08-7.25\left(\mathrm{br}, 1 \mathrm{H}, \mathrm{CO}_{2} \mathrm{H}\right) ;{ }^{13} \mathrm{C} \mathrm{NMR}\left(62.9 \mathrm{MHz}, \mathrm{CDCl}_{3}\right): \delta=-1.9[+$, $\left.\mathrm{Si}\left(\mathrm{CH}_{3}\right)_{3}\right], 17.3\left(-, \mathrm{C}^{\prime} 3^{\prime}\right.$ and $\mathrm{C}-2$, Teoc $), 22.0\left(+, \mathrm{C}^{\prime} 1^{\prime}\right), 33.1,33.3(-, \mathrm{C}-3), 52.7,53.2(+, \mathrm{C}-2)$, $59.0\left(+, \mathrm{C}-2^{\prime}\right), 63.7,64.8(-, \mathrm{C}-1$, Teoc $), 156.4,157.4\left(\mathrm{C}_{\text {quat }}, \mathrm{NCO}_{2}\right), 174.5,174.8\left(\mathrm{C}_{\text {quat }}, \mathrm{C}-1\right)$; IR $\left(\mathrm{KBr}\right.$; for CHA salt): nu(tilde) $=3419 \mathrm{~cm}^{-1}, 3250-2600,2952,2863,1722,1632,1541,1492$, 1406, 1368, 1248, 1206, 1163, 1053; MS (ESI): positive, $m / z=363(28)\left[\mathrm{M}-\mathrm{H}^{+}+2 \mathrm{Na}^{+}\right], 341$ (26) $\left[\mathrm{M}+\mathrm{Na}^{+}\right]$; negative, $m / z=657(100)\left[2 \mathrm{M}-2 \mathrm{H}^{+}+\mathrm{Na}^{+}\right], 317(28)\left[\mathrm{M}-\mathrm{H}^{+}\right]$.

Hormaomycin (3): An ethereal solution $(50 \mathrm{~mL})$ of the CHA salt of $N$-Teoc protected $\left(2 S, 1^{\prime} R, 2^{\prime} R\right)-\left(3-\right.$ nitrocyclopropyl)alanine $(8.1 \mathrm{mg}, 19.41 \mu \mathrm{mol})$ was washed with $1 \mathrm{M} \mathrm{H}_{2} \mathrm{SO}_{4}(3 \times$ $5 \mathrm{~mL}), 1 \mathrm{M} \mathrm{KHSO}_{4}(2 \times 5 \mathrm{~mL})$, water $(3 \times 5 \mathrm{~mL})$, brine $(2 \times 5 \mathrm{~mL})$, dried, filtered and concentrated under reduced pressure. The resultant $N$-protected amino acid (S)-197 was dried at 0.02 Torr for $2 \mathrm{~h}$ and then coupled with the depsipeptide [obtained after deprotection of $N$-MeZ protected cyclodepsipeptide 171c $(9.5 \mathrm{mg}, 9.71 \mu \mathrm{mol})$ with $10 \%$ anisole in TFA $(1.1 \mathrm{~mL})$ for $2 \mathrm{~h}$ according to GP 11] using HATU (7.4 mg, $19.46 \mu \mathrm{mol})$, HOAt (2.6 mg, $19.24 \mu \mathrm{mol})$, DIEA (1.25 mg, $9.67 \mu \mathrm{mol})$ and TMP $(7.04 \mathrm{mg}, 58.10 \mu \mathrm{mol})$ in $\mathrm{CH}_{2} \mathrm{Cl}_{2}(0.7 \mathrm{~mL})$ according to $\mathrm{GP} 7$ for $15 \mathrm{~h}$. The mixture was then diluted with EtOAc/Et $2 \mathrm{O}$ 1:1 $(20 \mathrm{~mL})$ and the crude product obtained after usual aqueous work-up (GP 2) was purified by preparative TLC $(100 \times 200 \mathrm{~mm}$, acetone/hexanes 1:3, 3 runs) to give Teoc- $(S)$-(3-Ncp)Ala-cyclo-hexadepsipeptide (8.0 mg, 73\%, $R_{\mathrm{f}}=0.43$ acetone/hexanes $\left.1: 2\right)$ as a colorless glass which was used for the next step without any characterization. Teoc group was cleaved from this substance $(8.0 \mathrm{mg}, 7.08 \mu \mathrm{mol})$ with TFA $(0.6$ 
$\mathrm{mL}$ ) for $1 \mathrm{~h}$. The mixture was concentrated under reduced pressure at $20{ }^{\circ} \mathrm{C}$ and then taken up with toluene $(3 \times 15 \mathrm{~mL})$ which was distilled off to remove the last traces of TFA. The resultant deprotected depsipeptide was coupled with $109(2.9 \mathrm{mg}, 14.10 \mu \mathrm{mol})$ using HATU (5.4 mg, 14.20 $\mu \mathrm{mol})$, DIEA $(0.92 \mathrm{mg}, 7.12 \mu \mathrm{mol})$ and TMP $(5.14 \mathrm{mg}, 42.42 \mu \mathrm{mol})$ in $\mathrm{CH}_{2} \mathrm{Cl}_{2}(1 \mathrm{~mL})$ according to GP 7 for $2.5 \mathrm{~h}$. The mixture was then taken up with $\mathrm{Et}_{2} \mathrm{O}(20 \mathrm{~mL})$ and the crude product obtained after usual aqueous work-up (GP 7) was purified by preparative TLC $(200 \times$ $200 \mathrm{~mm}$, acetone/hexanes $1: 2.5)$ to give $O-M O M-3\left(8.0 \mathrm{mg}, 96 \%, R_{\mathrm{f}}=0.36\right.$ acetone/hexanes 1:2) as a colorless glass which was used for the next step without any characterization. MOM-3 (8.0 mg, $6.82 \mu \mathrm{mol})$ was deprotected using $\mathrm{MgBr}_{2} \cdot \mathrm{Et}_{2} \mathrm{O}(52 \mathrm{mg}, 201.36 \mu \mathrm{mol})$ and $\mathrm{EtSH}(0.10$ $\mathrm{mL}, 1.918 \mathrm{mmol})$ in $\mathrm{CH}_{2} \mathrm{Cl}_{2}(10 \mathrm{~mL})$ according to 10 for $3 \mathrm{~h}$. The mixture was taken up with EtOAc and the crude product obtained after usual aqueous work-up was purified by preparative TLC $(200 \times 200 \mathrm{~mm}$, acetone/hexanes $1: 3,2$ runs $)$ and finally by trituration with pentane to give $3\left(5.5 \mathrm{mg}, 72 \%, 50 \%\right.$ on 5 steps from 171c) as a white solid. $R_{\mathrm{f}}=0.24$ acetone/hexanes $3: 7$; analytical HPLC: column B, isocratic, $65 \% \mathrm{MeCN}$ in $\mathrm{H}_{2} \mathrm{O}$ for $15 \mathrm{~min}$, then gradient $65 \rightarrow 99 \%$ $\mathrm{MeCN}$ in $\mathrm{H}_{2} \mathrm{O}$ for $5 \mathrm{~min}$, then isocratic, $99 \% \mathrm{MeCN}$, flow rate $=0.5 \mathrm{~mL} / \mathrm{min}, t_{\mathrm{R}}=14.54 \mathrm{~min}$, purity $>92 \% ;[\alpha]_{20}^{D} 20.0(c=0.1, \mathrm{MeOH}) ;{ }^{1} \mathrm{H} \mathrm{NMR}\left(600 \mathrm{MHz}, \mathrm{CDCl}_{3}\right): \delta=-0.65--0.36[\mathrm{~m}$, $\left.1 \mathrm{H}, 3^{\prime}-\mathrm{H}_{\mathrm{a}},(3-N c p) A l a\right],-0.11-0.16\left[\mathrm{~m}, 1 \mathrm{H}, 3-\mathrm{H}_{\mathrm{a}},(3-N c p) A l a\right], 0.27-0.36\left[\mathrm{~m}, 1 \mathrm{H}, 1^{\prime}-\mathrm{H},(3-\right.$ Ncp)Ala], 0.38-0.51 [m, $\left.1 \mathrm{H}, 3-\mathrm{H}_{\mathrm{b}},(3-N c p) A l a\right], 0.90$ (t, J = 7.2 Hz, 3 H, 5-H, Ile), 0.98-1.07 [m, $\left.1 \mathrm{H}, 3^{\prime}-\mathrm{H}_{\mathrm{b}},(3-N c p) A l a\right], 1.04\left(\mathrm{~d}, J=6.6 \mathrm{~Hz}, 3 \mathrm{H}, 1^{\prime}-\mathrm{H}\right.$, Ile $), 1.08$ [(ddd, $J=7.2,7.2,7.2 \mathrm{~Hz}$, $\left.1 \mathrm{H}, 3^{\prime}-\mathrm{H}_{\mathrm{a}},(3-N c p) A l a\right], 1.27-1.35\left(\mathrm{~m}, 1 \mathrm{H}, 4-\mathrm{H}_{\mathrm{a}}, I l e\right), 1.30$ [d, J=7.2 Hz, $\left.3 \mathrm{H}, 4-\mathrm{H},(\beta-M e) P h e\right]$, $1.42[\mathrm{~d}, J=7.2 \mathrm{~Hz}, 3 \mathrm{H}, 4-\mathrm{H},(\beta-M e) P h e], 1.53-1.59\left(\mathrm{~m}, 1 \mathrm{H}, 4-\mathrm{H}_{\mathrm{b}}, I l e\right), 1.54(\mathrm{~d}, J=6.6 \mathrm{~Hz}$, $3 \mathrm{H}, a-T h r), 1.59-1.67\left[\mathrm{~m}, 1 \mathrm{H}, 3-\mathrm{H}_{\mathrm{a}},(3-N c p) A l a\right], 1.70$ [dd, $J=6.6,1.8 \mathrm{~Hz}, 3 \mathrm{H}, 3^{\prime}-\mathrm{H},(4-$ PE)Pro], 1.73-1.99 [m, 4 H, 3-H $, 1^{\prime}-\mathrm{H}, 3^{\prime}-\mathrm{H}_{\mathrm{b}},(3-N c p)$ Ala, 3-H, Ile], 1.82 [ddd, $J=11.4,11.4$, 11,4 Hz, $1 \mathrm{H}, 3-\mathrm{H}_{\mathrm{a}},(4-P E)$ Pro], 2.40 [ddd, $J=11.4,6.0,5.4 \mathrm{~Hz}, 1 \mathrm{H}, 4-\mathrm{H}_{\mathrm{b}}$, (4-PE)Pro], 2.912.98 [m, 1 H, 2'-H, (3-Ncp)Ala], 3.02 [dq, $J=11.1,7.2 \mathrm{~Hz}, 1 \mathrm{H}, 3-\mathrm{H},(\beta-M e) P h e], 3.24-3.34$ [m, 
$\left.2 \mathrm{H}, 4-\mathrm{H}, 5-\mathrm{H}_{\mathrm{a}},(4-P E) P r o\right], 3.53-3.63$ [m, $\left.1 \mathrm{H}, 2-\mathrm{H},(3-N c p) A l a\right], 3.68$ [dq, $J=4.5,7.2 \mathrm{~Hz}, 1 \mathrm{H}$, 3-H, $(\beta-M e) P h e], 3.94-4.00\left[\mathrm{~m}, 1 \mathrm{H}, 5-\mathrm{H}_{\mathrm{b}},(4-P E)\right.$ Pro], 4.05 [ddd, $J=6.6,3.6,3.6 \mathrm{~Hz}, 1 \mathrm{H}, 2^{\prime}-$ H, (3-Ncp)Ala], 4.30 [dd, $J=12.0,6.6 \mathrm{~Hz}, 1 \mathrm{H}, 2-\mathrm{H},(4-P E) P r o], 4.33$ [dd, $J=10.8,10.8 \mathrm{~Hz}$, $1 \mathrm{H}, 2-\mathrm{H},(\beta-M e) P h e], 4.50-4.58[\mathrm{~m}, 1 \mathrm{H}, 2-\mathrm{H},(\beta-M e) P h e], 4.54(\mathrm{dd}, J=9.0,1.8 \mathrm{~Hz}, 1 \mathrm{H}, 2-\mathrm{H}$, $a-T h r), 4.69(\mathrm{dd}, J=9.0,9.0 \mathrm{~Hz}, 1 \mathrm{H}, 2-\mathrm{H}$, Ile), 5.19 [ddd, $J=7.8,7.8,7.8 \mathrm{~Hz}, 1 \mathrm{H}, 2-\mathrm{H},(3-$ Ncp)Ala], 5.24-5.31 [m, $\left.1 \mathrm{H}, 1^{\prime}-\mathrm{H},(4-P E) P r o\right], 5.43$ (dq, $\left.J=6.6,2.4 \mathrm{~Hz}, 1 \mathrm{H}, 3-\mathrm{H}, a-T h r\right), 5.65$ [dq, $J=10.2,6.6 \mathrm{~Hz}, 1 \mathrm{H}, 2^{\prime}-\mathrm{H},(4-P E)$ Pro $], 6.13$ (d, $J=4.8 \mathrm{~Hz}, 1 \mathrm{H}, 4-\mathrm{H}$, Chpca), 6.26-6.36 (br, $1 \mathrm{H}, \mathrm{NH}), 6.83$ (d, $J=4.8 \mathrm{~Hz}, 1 \mathrm{H}, 3-\mathrm{H}, C h p c a), 6.84$ (d, $J=9.6 \mathrm{~Hz}, 1 \mathrm{H}, \mathrm{NH}), 7.02-7.07$ (m, 1 H, Ar-H), 7.08-7.13 (m, 2 H, Ar-H), 7.14-7.19 (m, 3 H, Ar-H), 7.22-7.28 (m, 7 H, Ar-H, $\mathrm{NH}), 7.31(\mathrm{~d}, J=8.4 \mathrm{~Hz}, 1 \mathrm{H}, \mathrm{NH}), 9.03(\mathrm{~d}, J=7.8 \mathrm{~Hz}, 1 \mathrm{H}, \mathrm{NH}) ;{ }^{13} \mathrm{C} \mathrm{NMR}(150.8 \mathrm{MHz}$, $\left.\mathrm{CDCl}_{3}\right): \delta=10.6(+, \mathrm{C}-5$, Ile $), 13.3\left[+, \mathrm{C}-3^{\prime},(4-P E)\right.$ Pro $], 13.3[+, \mathrm{C}-4,(\beta-M e)$ Phe $], 15.0(+, \mathrm{C}-$ 1', Ile), $17.0(+, \mathrm{C}-4, a-T h r), 17.4\left[-, 2 \times \mathrm{C}-3^{\prime},(3-N c p) A l a\right], 18.0[+, \mathrm{C}-4,(\beta-M e) P h e], 20.5[+$, C-1', (3-Ncp)Ala], 21.8 [+, C-1', (3-Ncp)Ala], 24.9 (-, C-4, Ile), 33.4 [-, C-3, (3-Ncp)Ala $], 35.3$ [-, C-3, (3-Ncp)Ala], 35.6 [-, C-3, (4-PE)Pro], 36.7 [+, C-4, (4-PE)Pro], 38.1 (+, C-3, Ile), 39.3 $[+, \mathrm{C}-3,(\beta-M e) P h e], 41.9[+, \mathrm{C}-3,(\beta-M e) P h e], 50.7[+, \mathrm{C}-2,(3-N c p) A l a], 52.0[+, \mathrm{C}-2,(3-$ Ncp)Ala], 52.8 [-, C-5, (4-PE)Pro], 54.7 (+, C-2, Ile), $55.2(+, \mathrm{C}-2, a-T h r), 58.2$ [+, C-2', (3Ncp)Ala], 59.3 [+, C-2', (3-Ncp)Ala], 59.9 [+, C-2, ( $\beta$-Me)Phe], 60.2 [+, C-2, $(\beta-M e) P h e], 61.4$ [+, C-2, (4-PE)Pro], 69.2 (+, C-3, a-Thr), $103.2(+, \mathrm{C}-4$, Chpca), 109.3 (+, C-3, Chpca), 119.5 $\left(\mathrm{C}_{\text {quat }}, \mathrm{C}-2\right.$, Chpca $), 121.4\left(\mathrm{C}_{\text {quat }}, \mathrm{C}-5\right.$, Chpca $), 126.9,127.2,127.5,127.6,128.5,128.7(+, \mathrm{Ar}-\mathrm{C})$, 127.5 [+, C-1', (4-PE)Pro], 128.4 [+, C-2', (4-PE)Pro $], 141.6,142.2\left(\mathrm{C}_{\text {quat }}, \mathrm{Ar}-\mathrm{C}\right), 159.6\left(\mathrm{C}_{\text {quat, }}\right.$, C-1, Chpca), 168.7, 168.9, 169.8, 170.9, 171.3, 171.5, $172.1\left(\mathrm{C}_{\text {quat }}, \mathrm{C}-1\right)$; UV (MeOH): $\lambda_{\max }(\varepsilon)$ $=204\left(5 \times 10^{4}\right), 275\left(9.6 \times 10^{3}\right) \mathrm{nm} ; \mathrm{CD}(\mathrm{MeOH}): \lambda_{\max }[\Theta]=279\left(6.01 \times 10^{4}\right) ; 224.2(-6.500 \times$ $\left.10^{3}\right) \mathrm{nm}\left(c=1.754 \times 10^{-4} \mathrm{M}\right) ; \mathrm{MS}(\mathrm{ESI}):$ positive, $m / z=1174(100)\left[\mathrm{M}-\mathrm{H}^{+}+2 \mathrm{Na}^{+}\right], 1151(80)$ $\left[\mathrm{M}+\mathrm{Na}^{+}\right]$; negative, $m / z=1127(100)\left[\mathrm{M}-\mathrm{H}^{+}\right]$. 
Comparison of amino acidic content and their configuration of synthetic and natural hormaomycins 3 by HPLC/MS experiments: The natural and synthetic hormaomycins 3 (0.6 mg each) were hydrolyzed according to GP 1 and concentrated under reduced pressure. A half portion of each probe was directly treated with $(S)$-FDVA and other half was first epimerized and then treated with $(S)$-FDVA according GP 2. The HPLC/MS experiments with this material using the mixtures of $(S)$-FDVA derivatives of synthetic amino acids as standards failed to find any difference between synthetic and natural products.

\subsection{Synthesis of hormaomycin analogs. Evaluation of biological activity}

$N_{\omega^{-}}$Z protected (2S, 1'S, 2'R)-3-(2'-Aminocyclopropyl)alanine (S)-(199): (2S, 1'S,2'R)-3-(2'-Amino-<smiles>[Z]NC1C[C@H]1C[C@H](N)C(=O)O</smiles$(20 \mathrm{~mL})$ under ambient pressure of hydrogen for $15 \mathrm{~h}$. The mixture was then filtered though Celite pad and concentrated under reduced pressure to give a crude diamino acid as oil which was directly used without purification for the next step. This material was dissolved in water (4 $\mathrm{mL})$ and $\mathrm{CuSO}_{4}(72 \mathrm{mg}, 0.451 \mathrm{mmol})$ was added. $\mathrm{NaHCO}_{3}(0.145 \mathrm{~g}, 1.726 \mathrm{mmol})$ and then a solution of ZOSu $(0.28 \mathrm{~g}, 1.123 \mathrm{mmol})$ in acetone $(2 \mathrm{~mL})$ were added to the resultant deep violet solution after $1 \mathrm{~h}$ and stirring continued for a further $1.5 \mathrm{~h}$. The precipitate formed was then filtered off and washed with water $(100 \mathrm{~mL}), \mathrm{Et}_{2} \mathrm{O}(100 \mathrm{~mL})$ and dried. The resulting copper complex $(0.265 \mathrm{~g})$ was dissolved in boiling water $(5 \mathrm{~mL})$ and Trilon B $(0.509 \mathrm{~g}, 1.367 \mathrm{mmol})$ was then added. The mixture was vigorously stirred for $10 \mathrm{~min}$ and then cooled in ice bath for 30 min. The precipitate formed was filtered off, washed with ice-cold water $(50 \mathrm{~mL}), \mathrm{Et}_{2} \mathrm{O}(100$ $\mathrm{mL})$ and dried to give the first crop of $(S)-199(0.112 \mathrm{~g})$ as a white solid. The mother liquor was concentrated under reduced pressure to ca. $5 \mathrm{~mL}$ and cooled in ice bath. The precipitate formed was filtered off and crystallized from methanol to give the second crop of $(S)$-199 (12 mg, 52\% 
overall yield over 2 steps). $R_{\mathrm{f}}=0.19 \mathrm{MeCN} / \mathrm{H}_{2} \mathrm{O} / \mathrm{AcOH} 1: 1: 1 ;$ m.p $212-216^{\circ} \mathrm{C} ;[\alpha]_{20}^{D}-14.47$ $(c=0.32,0.1 \mathrm{~N} \mathrm{HCl}) ;{ }^{1} \mathrm{H} \mathrm{NMR}\left(250 \mathrm{MHz}, \mathrm{CD}_{3} \mathrm{OD}\right): \delta=0.56-0.68\left(\mathrm{~m}, 1 \mathrm{H}, 3^{\prime}-\mathrm{H}_{\mathrm{a}}\right), 0.78-0.98$ $\left(\mathrm{m}, 2 \mathrm{H}, 3^{\prime}-\mathrm{H}_{\mathrm{b}}, 3-\mathrm{H}_{\mathrm{a}}\right), 1.06\left(\mathrm{ddd}, J=10.5,10.5,10.5 \mathrm{~Hz}, 1 \mathrm{H}, 3-\mathrm{H}_{\mathrm{b}}\right), 2.34(\mathrm{ddd}, J=7.0,3.5$, $\left.3.5 \mathrm{~Hz}, 1 \mathrm{H}, 1^{\prime}-\mathrm{H}\right), 2.60$ (d, $\left.J=14.3 \mathrm{~Hz}, 1 \mathrm{H}, 2^{\prime}-\mathrm{H}\right), 3.74(\mathrm{~d}, J=10.8 \mathrm{~Hz}, 1 \mathrm{H}, 2-\mathrm{H}), 5.20$ (s, $2 \mathrm{H}$, Bzl-H), 7.28-7.44 (m, $5 \mathrm{H}, \mathrm{Ar}-\mathrm{H}) ;{ }^{13} \mathrm{C}$ NMR (62.9 MHz, DCl in $\left.\mathrm{D}_{2} \mathrm{O}\right): \delta=11.5\left(-, \mathrm{C}-3^{\prime}\right), 17.3$ $\left(+, \mathrm{C}-1^{\prime}\right), 29.1\left(+, \mathrm{C}-2^{\prime}\right), 32.4(-, \mathrm{C}-3), 54.2$ (+, C-2), 68.4 (-, Bzl-C), 128.3, 129.1, 129.4 (+, ArC), $136.8\left(\mathrm{C}_{\text {quat }}, \mathrm{Ar}-\mathrm{C}\right), 160.6\left(\mathrm{C}_{\text {quat }}, \mathrm{NCO}_{2}\right), 172.2\left(\mathrm{C}_{\text {quat }}, \mathrm{C}-1\right)$; IR $(\mathrm{KBr})$ : nu(tilde $)=3730-2700$ $\mathrm{cm}^{-1}, 3341,3035,2949,1696,1599,1529,1276$; MS (ESI): positive, $m / z=323(8)\left[\mathrm{M}-\mathrm{H}^{+}+\right.$ $\left.2 \mathrm{Na}^{+}\right], 301(80)\left[\mathrm{M}+\mathrm{Na}^{+}\right], 279(3)\left[\mathrm{M}+\mathrm{H}^{+}\right]$; negative, $m / z=277(100)\left[\mathrm{M}-\mathrm{H}^{+}\right]$; elemental analysis calcd (\%) for $\mathrm{C}_{14} \mathrm{H}_{18} \mathrm{~N}_{2} \mathrm{O}_{4}$ (278.3): calcd. C 60.42, $\mathrm{H} \mathrm{6.52,} \mathrm{N} \mathrm{10.07;} \mathrm{found} \mathrm{C} 60.26, \mathrm{H}$ 6.32, N 9.89 .

Teoc-(2S)-(3-Acp)Ala(Z)-OH (200): The $N_{\omega}$-monoprotected diamino acid 199 (0.112 g, 0.402 TeocHN TeocOSu $(0.131 \mathrm{~g}, 0.505 \mathrm{mmol})$ in acetone $(7 \mathrm{~mL})$ was added, the cooling bath was then removed and stirring continued for an additional $15 \mathrm{~h}$. Acetone was then removed from the mixture under reduced pressure and the resulting water fraction was filtered through Celite pad and treated with 3-N,N-dimethylaminopropylamine (3 drops). $\mathrm{pH}$ was adjusted to 2 with $1 \mathrm{M}$ $\mathrm{KHSO}_{4}$ and the resulting emulsion was extracted with $\mathrm{Et}_{2} \mathrm{O}(50 \mathrm{~mL})$. The organic layer was washed with $1 \mathrm{M} \mathrm{KHSO}_{4}(3 \times 10 \mathrm{~mL})$, water $(10 \times 10 \mathrm{~mL})$, brine $(2 \times 5 \mathrm{~mL})$, dried, filtered and concentrated under reduced pressure to give $N, N^{\prime}$-diprotected amino acid $200(0.112 \mathrm{~g}, 66 \%)$ as an extremely viscous colorless oil. $R_{\mathrm{f}}=0.20 \mathrm{EtOAc} / \mathrm{h}$ exane $1: 3(2 \% \mathrm{AcOH}) ;[\alpha]_{20}^{D} 37.1(c=1.1$, $\left.\mathrm{CHCl}_{3}\right) ;{ }^{1} \mathrm{H} \mathrm{NMR}\left(250 \mathrm{MHz}, \mathrm{CDCl}_{3}\right): \delta=0.03\left[\mathrm{~s}, 9 \mathrm{H}, \mathrm{Si}\left(\mathrm{CH}_{3}\right)_{3}\right], 0.56(\mathrm{ddd}, J=6.0,6.0$, $\left.6.0 \mathrm{~Hz}, 1 \mathrm{H}, 3^{\prime}-\mathrm{H}_{\mathrm{a}}\right), 0.71-0.84\left(\mathrm{~m}, 1 \mathrm{H}, 3^{\prime}-\mathrm{H}_{\mathrm{b}}\right), 0.98$ (t, $J=8.5 \mathrm{~Hz}, 2 \mathrm{H}, 2-\mathrm{H}$, Teoc), 1.22-1.43 
$\left(\mathrm{m}, 1 \mathrm{H}, 3-\mathrm{H}_{\mathrm{b}}\right), 2.02-2.22,2.25-2.36\left(2 \times \mathrm{m}, 1 \mathrm{H}, 1^{\prime}-\mathrm{H}\right), 2.36-2.51\left(\mathrm{~m}, 1 \mathrm{H}, 2^{\prime}-\mathrm{H}\right), 4.14(\mathrm{t}, J=$ 8.5 Hz, 2 H, 1-H, Teoc), 4.39-4.65 (m, 1 H, 2-H), 5.10 (s, 2 H, Bzl-H), 5.22-5.28, 5.60-5.71 (2 $\times$ br, $1 \mathrm{H}, \mathrm{NH})$, 5.76-5.88, 6.81-6.90 (m, $1 \mathrm{H}, \mathrm{NH}), 7.27-7.39$ (m, $5 \mathrm{H}, \mathrm{Ar}-\mathrm{H}), 10.50-11.40$ (br, $\left.1 \mathrm{H}, \mathrm{CO}_{2} \mathrm{H}\right)$; The peak of 2-H, Teoc masked absorption of $3-\mathrm{H}_{\mathrm{a}} ;{ }^{13} \mathrm{C} \mathrm{NMR}\left(62.9 \mathrm{MHz}, \mathrm{CDCl}_{3}\right): \delta$ $=-1.7\left[+, \mathrm{Si}\left(\mathrm{CH}_{3}\right)_{3}\right], 13.6,13.8\left(-, \mathrm{C}-3^{\prime}\right), 15.4,17.0\left(+, \mathrm{C}-1^{\prime}\right), 17.6(-, \mathrm{C}-2$, Teoc $), 28.9,29.4(+$, C-2'), 34.7, 35.1 (-, C-3), 53.0, 53.7 (+, C-2), 63.4, 64.3 (-, C-1, Teoc), 67.1 (-, Bzl-C), 127.5, 128.2, $128.5(+, A r-C), 135.9\left(\mathrm{C}_{\text {quat }}, \mathrm{Ar}-\mathrm{C}\right), 155.9,156.3\left(\mathrm{C}_{\text {quat }}, \mathrm{NCO}_{2}\right), 157.8,159.2\left(\mathrm{C}_{\text {quat }}\right.$, $\left.\mathrm{NCO}_{2}\right), 174.8,175.4\left(\mathrm{C}_{\text {quat }}, \mathrm{C}-1\right)$; IR $(\mathrm{KBr}): \mathrm{nu}(\mathrm{tilde})=3730-2500 \mathrm{~cm}^{-1}, 3035,2954,2898$, $1724,1690,1531,1349,1269,1250,1065 ; \mathrm{MS}$ (ESI): positive, $m / z=867(100)\left[2 \mathrm{M}+\mathrm{Na}^{+}\right], 445$ (26) $\left[\mathrm{M}+\mathrm{Na}^{+}\right]$; negative, $m / z=421(78)\left[\mathrm{M}-\mathrm{H}^{+}\right]$.

Teoc-(2S)-(3-Acp)Ala(Npys)-OH (201): The N,N'-Diprotected diamino acid 200 (60 mg, 0.142

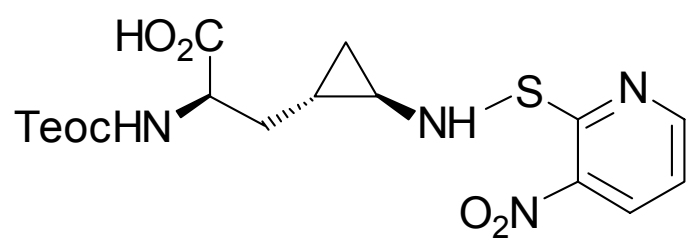
mmol) was hydrogenated on $10 \%$ Pd on charcoal $(30$ $\mathrm{mg})$ in methanol $(7 \mathrm{~mL})$ under ambient pressure of hydrogen for $2.5 \mathrm{~h}$. The mixture was then filtered and concentrated under reduced pressure to give Teoc-(3-Acp)Ala-OH as a white solid which was directly used for the next step. This material was taken up with anhydrous DMF (1 mL), triethylamine $(60 \mathrm{mg}, 0.593 \mathrm{mmol})$ was added, and the mixture was heated to $80{ }^{\circ} \mathrm{C}$ with heatgun and cooled then in water/ice bath. A solution of $\mathrm{NpysCl}(25 \mathrm{mg}, 0.131 \mathrm{mmol})$ in $\mathrm{DMF}(0.4$ $\mathrm{mL}$ ) was then added dropwise for $5 \mathrm{~min}$ and the mixture was stirred at $4{ }^{\circ} \mathrm{C}$ for $1 \mathrm{~h}$. After this, triethylamine (14 mg, $0.138 \mathrm{mmol})$, then (dropwise) solution of $\mathrm{NpysCl}(25 \mathrm{mg}, 0.131 \mathrm{mmol})$ in DMF (0.4 mL) and finally a third portion of triethylamine $(28 \mathrm{mg}, 0.277 \mathrm{mmol})$ were added and the mixture was allowed to reach $20^{\circ} \mathrm{C}$. Stirring continued for a further $15 \mathrm{~h}$, the reaction mixture was then diluted with water $(30 \mathrm{~mL})$, filtered and extracted with $\mathrm{Et}_{2} \mathrm{O}(3 \times 10 \mathrm{~mL}) \cdot \mathrm{pH}$ of the water fraction was adjusted to 2 with solid $\mathrm{KHSO}_{4}$ and the resulting emulsion was extracted with $\mathrm{Et}_{2} \mathrm{O}(50 \mathrm{~mL})$. The organic layer was washed with $1 \mathrm{M} \mathrm{KHSO}_{4}(2 \times 10 \mathrm{~mL})$, 
water $(10 \times 10 \mathrm{~mL})$, brine $(2 \times 5 \mathrm{~mL})$, dried, filtered and concentrated under reduced pressure. The resultant bright orange oil $(0.049 \mathrm{~g})$ was purified by preparative TLC $(200 \times 200 \mathrm{~mm}$, EtOAc/hexane 1:2 (1.5\% AcOH), $1 \mathrm{run})$ to give $201(0.028 \mathrm{~g}, 44 \%)$ as an orange foam. To prevent decomposition this acid was transformed in its tert-butyl ammonium salt. $R_{\mathrm{f}}=0.26$ EtOAc/hexane 1:2 (1.5\% AcOH); $[\alpha]_{20}^{D}=32.0\left(c=0.8, \mathrm{CHCl}_{3}\right)$ (for tert-butyl ammonium salt); ${ }^{1} \mathrm{H}$ NMR $\left(250 \mathrm{MHz}, \mathrm{CDCl}_{3}\right): \delta=0.03\left[\mathrm{~s}, 9 \mathrm{H}, \mathrm{Si}\left(\mathrm{CH}_{3}\right)_{3}\right], 0.44\left(\mathrm{ddd}, J=6.0,6.0,6.0 \mathrm{~Hz}, 1 \mathrm{H}, 3^{\prime}-\right.$ $\left.\mathrm{H}_{\mathrm{a}}\right), 0.80-0.94\left(\mathrm{~m}, 1 \mathrm{H}, 3^{\prime}-\mathrm{H}_{\mathrm{b}}\right), 0.94-1.15\left(\mathrm{~m}, 1 \mathrm{H}, 1^{\prime}-\mathrm{H}\right), 0.99(\mathrm{t}, J=8.5 \mathrm{~Hz}, 2 \mathrm{H}, 2-\mathrm{H}$, Teoc), 1.54-1.71 (m, $\left.1 \mathrm{H}, 3-\mathrm{H}_{\mathrm{a}}\right), 1.71-1.86\left(\mathrm{~m}, 1 \mathrm{H}, 3-\mathrm{H}_{\mathrm{b}}\right), 2.62\left(\mathrm{ddd}, J=6.8,3.5,3.5 \mathrm{~Hz}, 1 \mathrm{H}, 2^{\prime}-\mathrm{H}\right)$, $4.16(\mathrm{t}, J=8.5 \mathrm{~Hz}, 2 \mathrm{H}, 1-\mathrm{H}$, Teoc), 4.40 (ddd, $J=6.4,6.4,6.4 \mathrm{~Hz}, 1 \mathrm{H}, 2-\mathrm{H}), 4.60-6.30$ (br, $\left.2 \mathrm{H}, \mathrm{CO}_{2} \mathrm{H}, \mathrm{NH}\right), 5.34(\mathrm{~d}, J=7.8 \mathrm{~Hz}, 1 \mathrm{H}, \mathrm{CONH}), 8.52(\mathrm{dd}, \mathrm{J}=8.3,1.3 \mathrm{~Hz}, 1 \mathrm{H}, 4-\mathrm{H}$, Npys), 8.83 (dd, $J=4.5,1.3 \mathrm{~Hz}, 1 \mathrm{H}, 6-\mathrm{H}$, Npys); Absorption of 5-H, Npys was masked by residual signal of $\mathrm{CDCl}_{3} ;{ }^{13} \mathrm{C} \mathrm{NMR}\left(62.9 \mathrm{MHz}, \mathrm{CDCl}_{3}\right): \delta=-1.5\left[+, \mathrm{Si}\left(\mathrm{CH}_{3}\right)_{3}\right], 14.5\left(-, \mathrm{C}-3^{\prime}\right), 15.2,18.0$ (+,C-1') 17.7 (-, C-2, Teoc), 34.5 (-, C-3), 38.5 (+, C-2'), 53.4, $53.8(+, \mathrm{C}-2), 63.7,65.8$ (-, C-1,

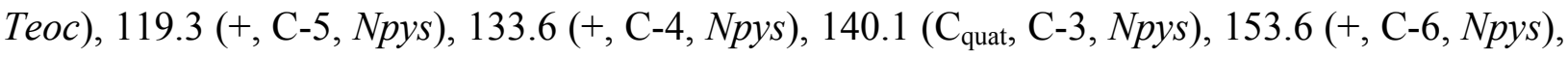
$156.4\left(\mathrm{C}_{\text {quat }}, \mathrm{NCO}_{2}\right), 164.0\left(\mathrm{C}_{\text {quat }}, \mathrm{C}-2, \mathrm{Npys}\right), 176.7\left(\mathrm{C}_{\text {quat }}, \mathrm{C}-1\right)$; IR $(\mathrm{KBr})$ : nu(tilde $)=3730$ $2500 \mathrm{~cm}^{-1}, 3077,2954,2896,1718,1587,1557,1511,1397,1336,1254,1230,1069$; MS (ESI): positive, $m / z=487(50)\left[\mathrm{M}-\mathrm{H}^{+}+2 \mathrm{Na}^{+}\right], 466(100)\left[\mathrm{M}+\mathrm{Na}^{+}\right]$; negative, $m / z=441(100)[\mathrm{M}-$ $\left.\mathrm{H}^{+}\right]$.

$N_{\alpha} Z, N_{\delta}$-Npys-(S)-Ornithine $\quad 1-(S)$-phenethylamide (204): $\quad N_{\alpha}-Z, N_{\delta}-$ Boc- $(S)$-Ornithine $\quad 1-(S)$ -

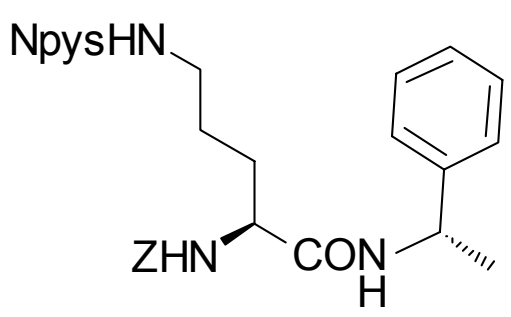
phenethylamide 203 (0.145 g, 76\%) was obtained from ZOrn(Boc)-OH $202 \quad(0.150$ g, 0.409 mmol $)$ and $1-(S)-$ phenethylamine (99 mg, $0.919 \mathrm{mmol})$ employing EDC (81 mg, $0.423 \mathrm{mmol}$ ) and HOBt (65 mg, crystallohydrate with $13 \%$ $\left.\mathrm{H}_{2} \mathrm{O}, 0.422 \mathrm{mmol}\right)$ in $\mathrm{CH}_{2} \mathrm{Cl}_{2}(5 \mathrm{~mL})$ according to $\mathrm{GP} 5$ as a white solid. This amide was treated with TFA (2 mL) within 20 min. All volatiles were then removed under reduced pressure and the 
residue was taken up with toluene $(2 \times 15 \mathrm{~mL})$ which was then distilled off to remove the last traces of TFA. A solution of NpysCl $(77 \mathrm{mg}, 0.404 \mathrm{mmol})$ was added dropwise for $5 \mathrm{~min}$ to an ice-cold solution of the amino amide trifluoroacetate and TEA (90 mg, $0.889 \mathrm{mmol})$ in THF (5 $\mathrm{mL}$ ), the mixture was allowed to warm to $20^{\circ} \mathrm{C}$ and stirring continued for a further $15 \mathrm{~h}$. The reaction mixture was then taken up with $\mathrm{Et}_{2} \mathrm{O}(50 \mathrm{~mL})$ and subjected to usual aqueous work-up (GP 5). The organic layer was dried, filtered and concentrated under reduced pressure. The residue was purified by two preparative TLC $(200 \times 200 \mathrm{~mm}$, EtOAc/hexane 1:2, 2 runs and then $200 \times 200 \mathrm{~mm}, \mathrm{CHCl}_{3} / \mathrm{MeOH} 110: 1,3$ runs) and finally by crystallization from $\mathrm{Et}_{2} \mathrm{O} /$ hexane to give $204(95 \mathrm{mg}, 59 \%)$ as a bright yellow solid. $R_{\mathrm{f}}=0.24 \mathrm{CHCl}_{3} / \mathrm{MeOH}^{110: 1,2}$ runs; $[\alpha]_{20}^{D}=-27.3\left(c=0.34, \mathrm{CHCl}_{3}\right) ;{ }^{1} \mathrm{H} \mathrm{NMR}\left(250 \mathrm{MHz}, \mathrm{CDCl}_{3}\right): \delta=1.47(\mathrm{~d}, J=7.0 \mathrm{~Hz}, 3 \mathrm{H}$, 2'-H, Phet), 1.50-1.65 (m, $2 \mathrm{H}, 4-\mathrm{H}), 1.65-1.82\left(\mathrm{~m}, 1 \mathrm{H}, 3-\mathrm{H}_{\mathrm{a}}\right), 1.83-2.00\left(\mathrm{~m}, 1 \mathrm{H}, 3-\mathrm{H}_{\mathrm{b}}\right), 1.54-$ $1.71\left(\mathrm{~m}, 1 \mathrm{H}, 3-\mathrm{H}_{\mathrm{a}}\right), 1.71-1.86\left(\mathrm{~m}, 1 \mathrm{H}, 3-\mathrm{H}_{\mathrm{b}}\right), 2.82-2.98(\mathrm{~m}, 2 \mathrm{H}, 5-\mathrm{H}), 3.53-3.64\left(\mathrm{~m}, 1 \mathrm{H}, 1^{\prime}-\mathrm{H}\right.$, Phet), 4.20 (ddd, $J=8.0,6.8,6.8 \mathrm{~Hz}, 1 \mathrm{H}, 2-\mathrm{H}), 4.99-5.14$ (br, NH), 5.10 (s, 2 H, Bzl-H), 5.42 (d, $J=8.0 \mathrm{~Hz}, 1 \mathrm{H}, \mathrm{CONH}), 6.20-6.43$ (br, $1 \mathrm{H}, \mathrm{CONH}), 7.14-7.38(\mathrm{~m}, 10 \mathrm{H}, \mathrm{Ar}-\mathrm{H}), 7.22$ (dd, $J$ $=8.4,4.5 \mathrm{~Hz}, 1 \mathrm{H}, 5-\mathrm{H}$, Npys), $8.49(\mathrm{dd}, \mathrm{J}=8.4,1.5 \mathrm{~Hz}, 1 \mathrm{H}, 4-\mathrm{H}$, Npys), $8.72(\mathrm{dd}, J=4.5$, $1.5 \mathrm{~Hz}, 1 \mathrm{H}, 6-\mathrm{H}$, Npys; ${ }^{13} \mathrm{C} \mathrm{NMR}\left(62.9 \mathrm{MHz}, \mathrm{CDCl}_{3}\right): \delta=21.7$ (+, C-2', Phet), 25.8 (-, C-4), 30.2 (-, C-3), 45.4 (+, C-1', Phet), 48.7 (-, C-5), 54.5 (+, C-2), 66.8 (+, Bzl-C), 119.3 (+, C-5, Npys), 125.9, 127.1, 127.7, 128.0, 128.4, 128.5 (+, Ar-C), 133.6 (+, C-4, Npys), 136.0, 140.0 $\left(\mathrm{C}_{\text {quat }}, \mathrm{Ar}-\mathrm{C}\right), 143.0\left(\mathrm{C}_{\text {quat }}, \mathrm{C}-5, \mathrm{Npys}\right), 153.4(+, \mathrm{C}-6$, Npys $), 156.2\left(\mathrm{C}_{\text {quat }}, \mathrm{NCO}_{2}\right), 163.9\left(\mathrm{C}_{\text {quat }}, \mathrm{C}-\right.$ 2, Npys), 170.8 ( $\left.\mathrm{C}_{\text {quat }}, \mathrm{C}-1\right)$; IR (KBr): nu(tilde) $=3066 \mathrm{~cm}^{-1}, 3030,2954,2868,1710,1650$, $1586,1556,1510,1451,1394,1334,1233,1068$; MS (ESI): positive, $m / z=1069$ (100) [2M + $\left.\mathrm{Na}^{+}\right], 546(16)\left[\mathrm{M}+\mathrm{Na}^{+}\right]$.

Attempts of the cleavage of Npys-group from 204: An aliquot of the amide 204 (10 mg, 19.10 $\mu \mathrm{mol})$ was dissolved in $\mathrm{CH}_{2} \mathrm{Cl}_{2}(15 \mathrm{~mL})$ and treated with $\mathrm{MgBr}_{2} \cdot \mathrm{Et}_{2} \mathrm{O}(99 \mathrm{mg}, 0.383 \mathrm{mmol})$ and EtSH (12 $\mathrm{mg}, 0.193 \mathrm{mmol})$ according to GP 10 . After $4.5 \mathrm{~h}$ only the partial deprotection took 
place (ca. 30\% according to TLC). Another aliquot of 204 (10 mg, $19.10 \mu \mathrm{mol})$ was dissolved in $\mathrm{CH}_{2} \mathrm{Cl}_{2}(4 \mathrm{~mL})$ and 2-mercaptopyridine-N-oxide $(0.150 \mathrm{~g}, 1.175 \mathrm{mmol})$ was added. After $5 \mathrm{~h}$, the mixture contained (according to TLC) only traces of the deprotected material. The third aliquot of $34(10 \mathrm{mg}, 19.10 \mu \mathrm{mol})$ was dissolved in $\mathrm{CH}_{2} \mathrm{Cl}_{2}(0.5 \mathrm{~mL})$ and treated with $0.1 \mathrm{M}$ $\mathrm{PPh}_{3}$ in $\mathrm{CH}_{2} \mathrm{Cl}_{2}(0.2 \mathrm{~mL})$ and $0.1 \mathrm{M} \mathrm{Py} \cdot \mathrm{HCl}$ in $\mathrm{CH}_{2} \mathrm{Cl}_{2}(0.2 \mathrm{~mL})$. The starting material was completely consumed in $2 \mathrm{~min}$ (according to TLC). The mixture was concentrated under reduced pressure and the residue was triturated with EtOAc and filtered to give the mixture of the product of deprotection and $\mathrm{Py} \cdot \mathrm{HCl}$ (4 mg, 2:1 molar ratio according to $\left.{ }^{1} \mathrm{H} \mathrm{NMR}\right)$.

$\left[(S)-(3-A c p) A l a(N p y s)^{7}\right]-M O M-3:$ An ethereal solution $(50 \mathrm{~mL})$ of the tert-butylammonium salt of $201(10.0 \mathrm{mg}, 19.39 \mu \mathrm{mol})$ was washed with $1 \mathrm{M} \mathrm{KHSO}_{4}(3 \times 5 \mathrm{~mL})$, water $(3 \times 5 \mathrm{~mL})$, brine $(2 \times 5 \mathrm{~mL})$, dried, filtered and concentrated under reduced pressure. The resultant $N$-protected amino acid was dried at 0.02 Torr for $2 \mathrm{~h}$ and then coupled with the depsipeptide, obtained after deprotection of $171 \mathrm{c}(10.0 \mathrm{mg}, 10.22 \mu \mathrm{mol})$ with $10 \%$ anisole in TFA $(1.1 \mathrm{~mL})$ according to GP 11 for $2 \mathrm{~h}$, using HATU (7.4 mg, $19.46 \mu \mathrm{mol})$, HOAt (2.6 mg, $19.24 \mu \mathrm{mol})$, DIEA (1.25 mg, $9.67 \mu \mathrm{mol})$ and TMP $(7.04 \mathrm{mg}, 58.10 \mu \mathrm{mol})$ in $\mathrm{CH}_{2} \mathrm{Cl}_{2}(0.7 \mathrm{~mL})$ according to $\mathrm{GP} 7$ for $15 \mathrm{~h}$. The mixture was then taken up with $\mathrm{Et}_{2} \mathrm{O}(20 \mathrm{~mL})$ and the crude product obtained after usual aqueous work-up (GP 5) was purified by preparative TLC $(100 \times 200 \mathrm{~mm}$, acetone/hexane 1:3, 2 runs $)$ to give Teoc-(S)-(3-Acp)Ala(Npys)-cyclo-hexadepsipeptide $\quad\left(10.9 \quad \mathrm{mg}, \quad 83 \%, \quad R_{\mathrm{f}}=0.22\right.$ acetone/hexane 1:2) as a yellow foam which was used for the next step without any characterization. This substance $(10.9 \mathrm{mg}, 8.50 \mu \mathrm{mol})$ was deprotected with TFA $(1 \mathrm{~mL})$ for $1 \mathrm{~h}$. The mixture was concentrated under reduced pressure at $20^{\circ} \mathrm{C}$ and then taken up with toluene (3 $\times 15 \mathrm{~mL}$ ) which was distilled off to remove the last traces of TFA. The resultant deprotected depsipeptide was coupled with Chpca(MOM)-OH 109 (3.5 mg, $17.02 \mu \mathrm{mol})$ using HATU (6.5 $\mathrm{mg}, 17.09 \mu \mathrm{mol})$, DIEA $(1.10 \mathrm{mg}, 8.51 \mu \mathrm{mol})$ and TMP $(6.2 \mathrm{mg}, 51.16 \mu \mathrm{mol})$ in $\mathrm{CH}_{2} \mathrm{Cl}_{2}(1 \mathrm{~mL})$ 
according to GP 7 for $3.5 \mathrm{~h}$. The mixture was then taken up with $\mathrm{Et}_{2} \mathrm{O}(20 \mathrm{~mL})$ and the crude product obtained after usual aqueous work-up (GP 5) was purified by preparative TLC $(200 \times$ $200 \mathrm{~mm}$, acetone/hexane 3:8, 2 runs) to give [(S)-(3-Acp)Ala(Npys) $\left.{ }^{7}\right]-M O M-3(7.1 \mathrm{mg}, 64 \%)$ as an yellow foam. $R_{\mathrm{f}}=0.09$ acetone/hexane $3: 8 ;[\alpha]_{20}^{D} 103.0\left(c=0.60, \mathrm{CHCl}_{3}\right) ;{ }^{1} \mathrm{H}$ NMR $(600$ $\left.\mathrm{MHz}, \mathrm{CDCl}_{3}\right): \delta=-0.33--0.19\left[\mathrm{~m}, 1 \mathrm{H}, 3^{\prime}-\mathrm{H}_{\mathrm{a}},(3-N c p)\right.$ Ala $], 0.02-0.15\left[\mathrm{~m}, 1 \mathrm{H}, 3-\mathrm{H}_{\mathrm{a}}\right.$, (3Ncp)Ala], 0.38 [ddd, $J=6.0,6.0,6.0 \mathrm{~Hz}, 1 \mathrm{H}, 3^{\prime}-\mathrm{H}_{\mathrm{a}},(3-$ Acp)Ala $], 0.53-0.62$ [m, $1 \mathrm{H}, 1^{\prime}-\mathrm{H},(3-$ Ncp)Ala $], 0.68-0.76$ [m, $\left.1 \mathrm{H}, 3-\mathrm{H}_{\mathrm{b}},(3-N c p) A l a\right], 0.86-0.90\left[\mathrm{~m}, 1 \mathrm{H}, 3^{\prime}-\mathrm{H}_{\mathrm{b}},(3-\right.$ Acp)Ala $], 0.92$ (t, $J=7.2 \mathrm{~Hz}, 3 \mathrm{H}, 5-\mathrm{H}$, Ile), 0.95-1.01 [m, $1 \mathrm{H}, 1^{\prime}-\mathrm{H},(3-$ Acp)Ala $], 1.07-1.15$ [m, $1 \mathrm{H}, 3^{\prime}-\mathrm{H}_{\mathrm{b}},(3-$ Ncp)Ala], 1.09 (d, J =6.6 Hz, $3 \mathrm{H}, 1^{\prime}-\mathrm{H}$, Ile), 1.27-1.32 (m, $1 \mathrm{H}, 4-\mathrm{H}_{\mathrm{a}}$, Ile), 1.28 [d, J=7.2 Hz, $3 \mathrm{H}, 4-\mathrm{H},(\beta M e) P h e], 1.35-1.46\left[\mathrm{~m}, 1 \mathrm{H}, 3-\mathrm{H}_{\mathrm{a}},(3-A c p) A l a\right] 1.41[\mathrm{~d}, J=7.8 \mathrm{~Hz}, 3 \mathrm{H}, 4-\mathrm{H}$, ( $\beta M e) P h e], 1.56(\mathrm{~d}, J=6.6 \mathrm{~Hz}, 3 \mathrm{H}, 4-\mathrm{H}, a-T h r), 1.56-1.62\left(\mathrm{~m}, 1 \mathrm{H}, 4-\mathrm{H}_{\mathrm{b}}\right.$, Ile $), 1.62-1.68$ [m, $1 \mathrm{H}, 3-\mathrm{H}_{\mathrm{b}},\left(3-\right.$ Acp)Ala] 1.69 [dd, $J=6.6,1.8 \mathrm{~Hz}, 3 \mathrm{H}, 3^{\prime}-\mathrm{H}$, (4-PE)-Pro], 1.81 [ddd, $J=12.0$, 12.0, $\left.12.0 \mathrm{~Hz}, 1 \mathrm{H}, 3-\mathrm{H}_{\mathrm{a}},(4-P E) P r o\right], 1.82-1.87$ (m, $1 \mathrm{H}, 3-\mathrm{H}$, Ile), 2.38 [ddd, $J=12.0,6.0$, $\left.6.0 \mathrm{~Hz}, 1 \mathrm{H}, 3-\mathrm{H}_{\mathrm{b}},(4-P E)-P r o\right], 2.61\left[\mathrm{ddd}, J=6.0,2.5,2.5 \mathrm{~Hz}, 1 \mathrm{H}, 1^{\prime}-\mathrm{H},(3-\right.$ Acp)Ala], 2.82-2.95 [m, $1 \mathrm{H}, 3-\mathrm{H},(\beta M e) P h e], 3.09-3.18\left[\mathrm{~m}, 1 \mathrm{H}, 2^{\prime}-\mathrm{H},(3-N c p) A l a\right], 3.21-3.33$ [m, $2 \mathrm{H}, 4-\mathrm{H}, 5-\mathrm{H}_{\mathrm{a}}$,

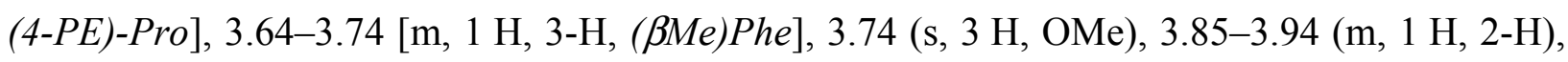
3.94-4.00 [m, $\left.1 \mathrm{H}, 5-\mathrm{H}_{\mathrm{b}},(4-P E)-P r o\right], 4.10-4.17$ (br, $\left.1 \mathrm{H}, \mathrm{NH}, N p y s\right), 4.25$ (dd, J = 9.6, 9.6 Hz, $1 \mathrm{H}, 2-\mathrm{H}), 4.28(\mathrm{dd}, J=10.8,6.0 \mathrm{~Hz}, 1 \mathrm{H}, 2-\mathrm{H}), 4.57(\mathrm{dd}, J=9.6,1.8 \mathrm{~Hz}, 1 \mathrm{H}, 2-\mathrm{H}), 4.60-4.69$ (m, $1 \mathrm{H}, 2-\mathrm{H}), 4.67$ [ddd, $J=8.4,8.4,8.4 \mathrm{~Hz}, 1 \mathrm{H}, 2-\mathrm{H},(3-$ Acp)Ala], 5.01-5.12 (m, $1 \mathrm{H}, 2-\mathrm{H})$, 5.24-5.29 [m, 1 H, 1'-H, (4-PE)-Pro], 5.38 (q, J=7.2 Hz, $1 \mathrm{H}, 3-\mathrm{H}, a-T h r), 5.46$ (d, J=6.0 Hz, $\left.1 \mathrm{H}, \mathrm{H}_{\mathrm{a}}, \mathrm{OCH}_{2} \mathrm{O}\right), 5.51\left(\mathrm{~d}, J=6.0 \mathrm{~Hz}, 1 \mathrm{H}, \mathrm{H}_{\mathrm{b}}, \mathrm{OCH}_{2} \mathrm{O}\right), 5.63\left[\mathrm{dq}, J=10.8,6.6 \mathrm{~Hz}, 1 \mathrm{H}, 2^{\prime}-\mathrm{H}\right.$, (4-PE)-Pro], 5.74 (d, $J=7.2 \mathrm{~Hz}, 1 \mathrm{H}, \mathrm{NH}), 6.12$ (d, $J=4.8 \mathrm{~Hz}, 1 \mathrm{H}, 4-\mathrm{H}$, Chpca), 6.79 (d, $J=$ $4.5 \mathrm{~Hz}, 1 \mathrm{H}, 3-\mathrm{H}, \mathrm{Chpca}), 6.81$ (d, $J=9.6 \mathrm{~Hz}, 1 \mathrm{H}, \mathrm{NH}), 7.03-7.11$ (m, $4 \mathrm{H}$, Ar-H), 7.12-7.18 (m, 4 H, Ar-H, 5-H, Npys), 7.21-7.30 (m, 4 H, Ar-H, NH), 7.37 (d, J=9.0 Hz, 1 H, NH), 7.507.61 (br, $1 \mathrm{H}, \mathrm{NH}), 7.52$ (d, $J=10.2 \mathrm{~Hz}, 1 \mathrm{H}, \mathrm{NH}), 8.48$ (dd, $J=8.4,1.8 \mathrm{~Hz}, 1 \mathrm{H}, 4-\mathrm{H}, N p y s)$, 
$8.75(\mathrm{~d}, J=3.6 \mathrm{~Hz}, 1 \mathrm{H}, 6-\mathrm{H}, \mathrm{Npys}), 8.78-8.88(\mathrm{br}, 1 \mathrm{H}, \mathrm{NH}) ;{ }^{13} \mathrm{C} \mathrm{NMR}\left(150.8 \mathrm{MHz}, \mathrm{CDCl}_{3}\right): \delta$ $=10.8(+, \mathrm{C}-5$, Ile $), 13.3\left[+, \mathrm{C}-4,(\beta M e) P h e, \mathrm{C}-3^{\prime},(4-P E)-P r o\right], 14.5\left[-, \mathrm{C}-3^{\prime},(3-A c p)\right.$ Ala $], 15.2$ (+, C-1', Ile), 17.4 (+, C-4, a-Thr), 17.6 [-, C-3', (3-Ncp)Ala], 18.2 [+, C-1', (3-Acp)Ala $], 18.3$ $[+, \mathrm{C}-4,(\beta M e) P h e], 20.8\left[+, \mathrm{C}-1^{\prime},(3-N c p) A l a\right], 25.0(-, \mathrm{C}-4$, Ile $), 34.1[-, \mathrm{C}-3,(3-N c p)$ Ala $], 35.5$ [-, C-3, (4-PE)-Pro], 36.7 [+, C-4, (4-PE)-Pro], 38.5 [-, C-3, (3-Acp)Ala], 38.7 [+, C-2', (3-

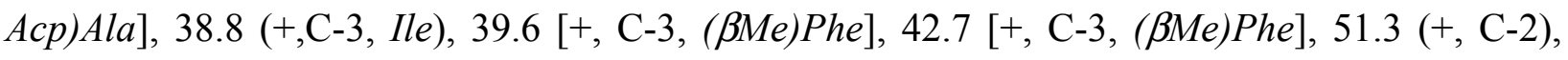
51.5 (+, C-2), 52.8 [-, C-5, (4-PE)-Pro], 54.7 [+, C-2, (3-Acp)Ala], 54.8 (+, C-2), 58.5 [+, C-2', (3-Ncp)Ala], 59.3 (+, OMe, C-2), 59.5 (+, C-2), $60.1(+, \mathrm{C}-2), 61.4(+, \mathrm{C}-2), 69.8(+, \mathrm{C}-3, a-$ Thr), 104.3 (+, C-4, Chpca), 106.1 (-, $\left.\mathrm{OCH}_{2} \mathrm{O}\right), 111.0$ (+, C-3, Chpca), 119.1 (+, C-5, Npys) $119.3\left(\mathrm{C}_{\text {quat }}, \mathrm{C}-2\right.$, Chpca $), 122.0$ (C $\mathrm{C}_{\text {quat }}, \mathrm{C}-2$, Chpca $), 126.8,127.0,127.5,127.6,128.5,128.6(+$, Ar-C), 128.3 [+, C-2', (4-PE)-Pro], 133.5 (+, C-4, Npys), $140.0\left(\mathrm{C}_{\text {quat }}, \mathrm{C}-3\right.$, Npys), 142.3, 142.4

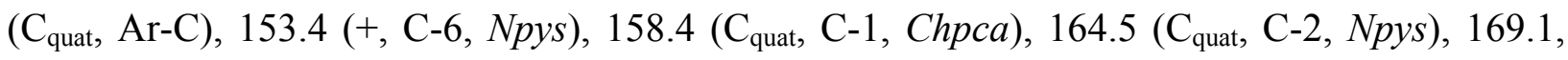
170.0, $170.1(\times 2), 171.3(\times 2), 172.4\left(\mathrm{C}_{\text {quat }}, \mathrm{C}-1\right)$; Peak $127.6(+$, Ar-C) masked absorption for [+, C-1', (4-PE)-Pro]; MS (ESI): positive mode, $m / z(\%)=1320(100)\left[\mathrm{M}+\mathrm{Na}^{+}\right]$; negative mode, $m / z(\%)=1296(100)\left[\mathrm{M}-\mathrm{H}^{+}\right]$.

Attempt of the deprotection of [(3-Acp)Ala(Npys) $\left.{ }^{7}\right]-\mathrm{MOM}-3$ : A solution of the protected depsipeptide $(6.0 \mathrm{mg}, 4.62 \mu \mathrm{mol})$ in $\mathrm{CH}_{2} \mathrm{Cl}_{2}(4 \mathrm{~mL})$ was treated with

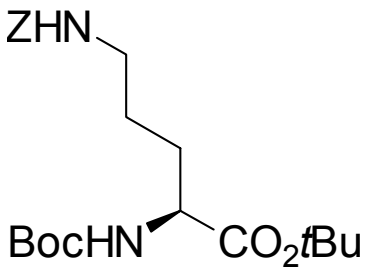
$\mathrm{MgBr}_{2} \cdot \mathrm{Et}_{2} \mathrm{O}(48 \mathrm{mg}, 18.59 \mu \mathrm{mol})$ and $\mathrm{EtSH}(14 \mu \mathrm{L}, 18.90 \mu \mathrm{mol})$ according to GP 10 for $4 \mathrm{~h}$. The crude product after usual aqueous workup (GP 10) was dried, dissolved in $\mathrm{CH}_{2} \mathrm{Cl}_{2}(0.5 \mathrm{~mL})$ and treated with 0.1 $\mathrm{M} \mathrm{PPh}$ in $\mathrm{CH}_{2} \mathrm{Cl}_{2}(0.4 \mathrm{~mL})$ and $0.1 \mathrm{M} \mathrm{Py} \cdot \mathrm{HCl}$ in $\mathrm{CH}_{2} \mathrm{Cl}_{2}(0.4 \mathrm{~mL})$ for $10 \mathrm{~min}$. The mixture, containing (according to TLC) two Erlich reagent active compounds, was then placed on the top of the column with Sephadex LH-20. Elution with $\mathrm{CH}_{2} \mathrm{Cl}_{2}$, then $\mathrm{CH}_{2} \mathrm{Cl}_{2} / \mathrm{MeOH} 10: 1$, then $\mathrm{CH}_{2} \mathrm{Cl}_{2} / \mathrm{MeOH}$ 3:1, $\mathrm{CH}_{2} \mathrm{Cl}_{2} / \mathrm{MeOH}$ 1:1 and finally with pure $\mathrm{MeOH}$ didn't give the desired product.Boc-(S)-Orn(Z)-OtBu (206): To a solution of Boc-(S)-Orn(Z)-OH 205 (1.50 g, 4.09 
mmol) in DMA (31 mL) tert-butyl bromide (22.2 mL, $197 \mathrm{mmol}), \mathrm{K}_{2} \mathrm{CO}_{3}(14.71 \mathrm{~g}, 106.4 \mathrm{mmol})$ and benzyltriethylammonium chloride $(0.932 \mathrm{~g}, 4.09 \mathrm{mmol})$ were added and the mixture was vigorously stirred at $55-60{ }^{\circ} \mathrm{C}$ for $48 \mathrm{~h}$. The reaction mixture was then poured in water $(0.71)$ and the resultant emulsion was extracted with $\mathrm{Et}_{2} \mathrm{O}(2 \times 200 \mathrm{~mL})$. The organic layer was washed with water $(14 \times 50 \mathrm{~mL})$, brine $(2 \times 50 \mathrm{~mL})$, dried, filtered and concentrated to give $\mathbf{2 0 6}(1.63 \mathrm{~g}$, 94\%) as a colorless oil. $R_{\mathrm{f}}=0.21$ EtOAc/hexane 1:4.

Boc-(S)-(NO 2$) N v a-O t B u$ (207): Ester 206 (1.63 g, $3.86 \mathrm{mmol})$ was hydrogenated on 10\% Pd on charcoal $(0.28 \mathrm{~g})$ in EtOAc $(15 \mathrm{~mL})$ under ambient pressure of hydrogen for $3 \mathrm{~h}$. The mixture was then filtered and concentrated under reduced pressure to give the crude monoprotected diamino ester $\left(R_{\mathrm{f}}=0.20 \mathrm{CHCl}_{3} / \mathrm{MeOH} 10: 1\right)$ which was immediately used without any purification for the next step. This material was dissolved in 1,2-dichloroethane $(50 \mathrm{~mL})$ and added to the vigorously stirred solution MCPBA (7.397 g, 90\% purity, $42.86 \mathrm{mmol})$ in 1,2dichloroethane $(200 \mathrm{~mL})$ under intensive reflux for $2 \mathrm{~min}$. Stirring and refluxing continued for an additional $20 \mathrm{~min}$ and the reaction flask was then placed in a water/ice bath. After 15 min the reaction mixture was concentrated under reduced pressure to ca. $120 \mathrm{~mL}$, diluted with $\mathrm{Et}_{2} \mathrm{O}(400$ $\mathrm{mL})$, washed with aqueous saturated $\mathrm{Na}_{2} \mathrm{SO}_{3}(2 \times 100 \mathrm{~mL})$, saturated aqueous $\mathrm{NaHCO}_{3}(3 \times 100$ $\mathrm{mL})$, water $(2 \times 100 \mathrm{~mL})$, brine $(2 \times 50 \mathrm{~mL})$, dried, filtered and concentrated under reduced pressure. The residual brown oil was purified by column chromatography (EtOAc/hexane 1:6, $R_{\mathrm{f}}$ $=0.36)$ to give $207(0.73 \mathrm{~g}, 58 \%)$ as a faint yellow oil. ${ }^{1} \mathrm{H} \mathrm{NMR}\left(250 \mathrm{MHz}, \mathrm{CDCl}_{3}\right): \delta=1.44[\mathrm{~s}$, $\left.9 \mathrm{H}, \mathrm{C}\left(\mathrm{CH}_{3}\right)_{3}\right], 1.46\left[\mathrm{~s}, 9 \mathrm{H}, \mathrm{C}\left(\mathrm{CH}_{3}\right)_{3}\right], 1.63-1.80\left(\mathrm{~m}, 1 \mathrm{H}, 4-\mathrm{H}_{\mathrm{a}}\right), 1.80-1.95\left(\mathrm{~m}, 1 \mathrm{H}, 4-\mathrm{H}_{\mathrm{b}}\right), 1.95-$ $2.05(\mathrm{~m}, 2 \mathrm{H}, 3-\mathrm{H}), 4.10-4.28(\mathrm{~m}, 1 \mathrm{H}, 2-\mathrm{H}), 4.43$ (t, $J=6.6 \mathrm{~Hz}, 2 \mathrm{H}, 5-\mathrm{H}), 5.12$ (d, $J=7.5 \mathrm{~Hz}$, $1 \mathrm{H}, \mathrm{NH}) ;{ }^{13} \mathrm{C} \mathrm{NMR}\left(62.9 \mathrm{MHz}, \mathrm{CDCl}_{3}\right): \delta=23.0(-, \mathrm{C}-4), 27.8\left[+, \mathrm{C}\left(\mathrm{CH}_{3}\right)_{3}\right], 28.1[+$, $\left.\mathrm{C}\left(\mathrm{CH}_{3}\right)_{3}\right], 29.6(-, \mathrm{C}-3), 52.9(+, \mathrm{C}-2), 74.8(-, \mathrm{C}-5), 79.8\left[\mathrm{C}_{\text {quat }}, \mathrm{C}\left(\mathrm{CH}_{3}\right)_{3}\right], 82.4\left[\mathrm{C}_{\text {quat }}, \mathrm{C}\left(\mathrm{CH}_{3}\right)_{3}\right]$, $155.3\left(\mathrm{C}_{\text {quat }}, \mathrm{NCO}_{2}\right), 170.9\left(\mathrm{C}_{\text {quat }}, \mathrm{C}-1\right)$; IR (film): nu(tilde) $=3443 \mathrm{~cm}^{-1}, 3008,2980,2868,1743$, 1556, 1499, 1456, 1393, 1369, 1253, 1149, 1055; MS (ESI): positive mode, $m / z=341$ (100) [M 
$+\mathrm{Na}^{+}$]; elemental analysis calcd (\%) for $\mathrm{C}_{14} \mathrm{H}_{26} \mathrm{~N}_{2} \mathrm{O}_{6}$ (318.4): C 52.82, H 8.23, N 8.80; found C 52.56, H 8.57, N 8.84.

$\mathrm{Z}-(\mathrm{S})-\left(\mathrm{NO}_{2}\right) \mathrm{Nva}-\mathrm{OtBu}$ : This compound was obtained from Z-(S)-( $\left.\mathrm{NO}_{2}\right) \mathrm{Nva-O} t \mathrm{Bu}$ in a similar manner as compound $\mathbf{2 0 7}$ in $41 \%$ yield as a faint yellow oil. $R_{\mathrm{f}}=0.21$ EtOAc/hexane 1:4; $[\alpha]_{20}^{D}$ $10.7\left(c=0.60, \mathrm{CHCl}_{3}\right) ;{ }^{1} \mathrm{H} \mathrm{NMR}\left(250 \mathrm{MHz}, \mathrm{CDCl}_{3}\right): \delta=1.47\left[\mathrm{~s}, 9 \mathrm{H}, \mathrm{C}\left(\mathrm{CH}_{3}\right)_{3}\right], 1.65-1.82(\mathrm{~m}$, $\left.1 \mathrm{H}, 4-\mathrm{H}_{\mathrm{a}}\right), 1.82-1.98\left(\mathrm{~m}, 1 \mathrm{H}, 4-\mathrm{H}_{\mathrm{b}}\right), 1.98-2.12(\mathrm{~m}, 2 \mathrm{H}, 3-\mathrm{H}), 4.13-4.25(\mathrm{~m}, 1 \mathrm{H}, 2-\mathrm{H}), 4.41(\mathrm{t}$, $J=6.6 \mathrm{~Hz}, 2 \mathrm{H}, 5-\mathrm{H}), 5.11(\mathrm{~s}, 2 \mathrm{H}, \mathrm{Bzl}-\mathrm{H}), 5.32$ (d, $J=7.5 \mathrm{~Hz}, 1 \mathrm{H}, \mathrm{NH}), 7.26-7.39$ (m, $5 \mathrm{H}$, $\mathrm{Ar}-\mathrm{H}) ;{ }^{13} \mathrm{C} \mathrm{NMR}\left(62.9 \mathrm{MHz}, \mathrm{CDCl}_{3}\right): \delta=22.8(-, \mathrm{C}-4), 27.7\left[+, \mathrm{C}\left(\mathrm{CH}_{3}\right)_{3}\right], 29.3(-, \mathrm{C}-3), 53.3$ (+, C-2), 66.7 (-, Bzl-C), $74.6(-, \mathrm{C}-5), 82.5\left[\mathrm{C}_{\text {quat }}, \mathrm{C}\left(\mathrm{CH}_{3}\right)_{3}\right], 127.9,128.0,128.3(+, \mathrm{Ar}-\mathrm{H})$, $136.0\left(\mathrm{C}_{\text {quat }}, \mathrm{Ar}-\mathrm{C}\right), 155.7\left(\mathrm{C}_{\text {quat }}, \mathrm{NCO}_{2}\right), 170.5$ ( $\left.\mathrm{C}_{\text {quat }}, \mathrm{C}-1\right)$; IR (film): nu(tilde) $=3440 \mathrm{~cm}^{-1}$, $3034,2980,2935,1742,1555,1500,1451,1371,1349,1257,1218,1153,1058 ; \mathrm{MS}$ (CI), $m / z$ (\%):370 (100) $\left[\mathrm{M}+\mathrm{NH}_{4}{ }^{+}\right]$; elemental analysis calcd (\%) for $\mathrm{C}_{17} \mathrm{H}_{24} \mathrm{~N}_{2} \mathrm{O}_{6}$ (352.4): C 57.94, $\mathrm{H}$ 6.86, N 7.95; found C 57.75, H 6.99, N 8.10.

(S)-5-Nitronorvaline hydrochloride (208): Compound 207 (1.20 g, $3.77 \mathrm{mmol})$ was deprotected

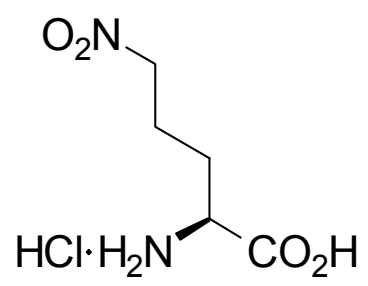
with TFA $(5 \mathrm{~mL})$ for $1 \mathrm{~h}$. All volatiles were then removed under reduced pressure and the residue was taken up with toluene $(2 \times 20 \mathrm{~mL})$ which was then distilled off to remove the last traces of TFA. To transform the resulting trifluoroacetate into hydrochloride it was taken up with $2 \mathrm{M}$

$\mathrm{HCl}$ in EtOAc $(3 \times 20 \mathrm{~mL})$ which was then distilled off. The resulting oil was triturated with acetone to give light brown precipitate. $\mathrm{Et}_{2} \mathrm{O}$ was then added to complete precipitation. The crude product was filtered off and washed with acetone/ $\mathrm{Et}_{2} \mathrm{O} 1: 1(200 \mathrm{~mL})$ to give $\mathrm{HCl} \cdot \mathbf{2 0 8}$ $(0.420 \mathrm{~g}, 56 \%)$ as an off-white solid. M.p $132-133{ }^{\circ} \mathrm{C}($ decomp. $) ;[\alpha]_{20}^{D} 16.8(c=0.22,0.1 \mathrm{M}$ $\mathrm{HCl})\left[\right.$ lit. (for free base): ${ }^{[144]}[\alpha]_{20}^{D} 22.8(c=0.56$, dil. $\left.\mathrm{HCl})\right] ;{ }^{1} \mathrm{H}$ NMR $\left(300 \mathrm{MHz}, \mathrm{D}_{2} \mathrm{O}\right): \delta=$ 
1.89-2.24 (m, $4 \mathrm{H}, 3-\mathrm{H}, 4-\mathrm{H}), 4.06(\mathrm{t}, J=5.0 \mathrm{~Hz}, 1 \mathrm{H}, 2-\mathrm{H}), 4.56(\mathrm{t}, J=5.4 \mathrm{~Hz}, 2 \mathrm{H}, 5-\mathrm{H}) ;{ }^{13} \mathrm{C}$

NMR (50.3 MHz, D $2 \mathrm{O}): \delta=22.4(-$, C-4), $26.8(-$, C-3), $52.5(+$, C-2), $74.5(-$, C-5), 171.7 $\left(\mathrm{C}_{\text {quat }}, \mathrm{C}-1\right)$; IR $(\mathrm{KBr}): \mathrm{nu}($ tilde $)=3750-1800 \mathrm{~cm}^{-1}, 2933,1735,1552,1500,1379,1349,1244$; MS (ESI): positive mode, $m / z=229(5)\left[\mathrm{M}-2 \mathrm{H}^{+}+3 \mathrm{Na}^{+}\right], 207(8)\left[\mathrm{M}-\mathrm{H}^{+}+2 \mathrm{Na}^{+}\right], 185$ (12) $\left[\mathrm{M}+\mathrm{Na}^{+}\right]$; negative mode, $m / z=345(100)\left[2 \mathrm{M}-2 \mathrm{H}^{+}+\mathrm{Na}^{+}\right]$; elemental analysis calcd $(\%)$ for $\mathrm{C}_{5} \mathrm{H}_{11} \mathrm{~N}_{2} \mathrm{O}_{4} \mathrm{Cl}(198.6)$ : C 30.24, H 5.58, N 14.10; found C 30.50, H 5.30, N 13.97.

Teoc-(S)-(NO $\left.\mathrm{N}_{2}\right) \mathrm{Nva-OH}$ (209) from 207: Compound 207 (0.220 g, $\left.0.691 \mathrm{mmol}\right)$ was deprotected

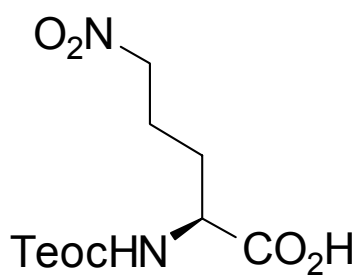
with TFA $(1 \mathrm{~mL})$ for $1 \mathrm{~h}$. All volatiles were then removed under reduced pressure and the residue was taken up with toluene $(2 \times 20 \mathrm{~mL})$ which was then distilled off to remove the last traces of TFA. The crude product was filtered off and washed with acetone/ $\operatorname{Et}_{2} \mathrm{O} 1: 1(50 \mathrm{~mL})$ to give TFA.208 $(0.105 \mathrm{~g})$ as a white solid. A solution of TeocOSu $(0.180 \mathrm{~g}, 0.694 \mathrm{mmol})$ in acetone $(5 \mathrm{~mL})$ was added to a vigorously stirred solution of this material and $\mathrm{NaHCO}_{3}(0.159 \mathrm{~g}$, $1.893 \mathrm{mmol}$ ) in water $(5 \mathrm{~mL}$ ) (if emulsion formed acetone and/or water were added to obtain homogeneous solution) and stirring continued for a further 2 h. N,N-dimethylaminopropylamine $(0.07 \mathrm{~mL}, 0.56 \mathrm{mmol})$ was then added. After an additional $10 \mathrm{~min}$ acetone was removed under reduced pressure and $\mathrm{pH}$ of the residual water solution was adjusted to 2-3 with $1 \mathrm{M} \mathrm{NaHSO}_{4}$. The resulting emulsion was extracted with $\mathrm{Et}_{2} \mathrm{O}(50 \mathrm{~mL})$ and the ethereal layer was washed with $1 \mathrm{M} \mathrm{KHSO}_{4}(3 \times 10 \mathrm{~mL})$, water $(10 \times 10 \mathrm{~mL})$, brine $(2 \times 5 \mathrm{~mL})$, dried, filtered and concentrated under reduced pressure. The residual oil solidified on seeding and the crude product was crystallized from $\mathrm{Et}_{2} \mathrm{O} /$ pentane to give $209(0.108 \mathrm{~g}, 51 \%$ on 2 steps $)$ as a white solid. $R_{\mathrm{f}}=0.24$ EtOAc/hexane 1:2 (1.5\% AcOH) (staining with ninhidrin); M.p 70-72 ${ }^{\circ} \mathrm{C}$ (decomp.); $[\alpha]_{20}^{D} 23.7$ $\left(c=0.30, \mathrm{CHCl}_{3}\right) ;{ }^{1} \mathrm{H} \mathrm{NMR}\left(250 \mathrm{MHz}, \mathrm{CDCl}_{3}\right): \delta=0.04\left[\mathrm{~s}, 9 \mathrm{H}, \mathrm{Si}\left(\mathrm{CH}_{3}\right)_{3}\right], 1.00(\mathrm{t}, J=8.5 \mathrm{~Hz}$, $2 \mathrm{H}, 2-\mathrm{H}$, Tеос), 1.69-1.96 (m, $\left.1 \mathrm{H}, 4-\mathrm{H}_{\mathrm{a}}\right), 1.96-2.22\left(\mathrm{~m}, 3 \mathrm{H}, 4-\mathrm{H}_{\mathrm{b}}, 3-\mathrm{H}\right), 4.18(\mathrm{t}, J=8.3 \mathrm{~Hz}$, 2 H, 1-H, Teoc), 4.20-4.45 (m, 1 H, 2-H), 4.44 (t, J=6.1 Hz, 2 H, 5-H), 5.18-5.26, 6.95-7.07 (2 
$\times$ br, $1 \mathrm{H}, \mathrm{NH}) ;{ }^{13} \mathrm{C} \mathrm{NMR}\left(62.9 \mathrm{MHz}, \mathrm{CDCl}_{3}\right): \delta=-1.6\left[+, \mathrm{Si}\left(\mathrm{CH}_{3}\right)_{3}\right], 17.6(-, \mathrm{C}-2$, Teoc $), 22.9$, $23.2(-$, C-4), 29.2 (-, C-3), 52.8, 53.3 (+, C-2), 64.0, 65.0 (-, C-1, Teoc), 74.7 (-, C-5), 156.6, $157.6\left(\mathrm{C}_{\text {quat }}, \mathrm{NCO}_{2}\right), 175.0,175.7\left(\mathrm{C}_{\text {quat }}, \mathrm{C}-1\right)$; IR $(\mathrm{KBr}): \mathrm{nu}($ tilde $)=3750-2800 \mathrm{~cm}^{-1}, 2957$, $2898,1754,1731,1657,1556,1249,1205,1182,1070 ;$ MS (ESI): positive mode, $m / z=329$ (100) $\left[\mathrm{M}+\mathrm{Na}^{+}\right]$; negative mode, $m / z=305(100)\left[2 \mathrm{M}-\mathrm{H}^{+}\right]$; elemental analysis calcd $(\%)$ for $\mathrm{C}_{11} \mathrm{H}_{22} \mathrm{~N}_{2} \mathrm{O}_{6} \mathrm{Si}$ (306.4): C 43.12, H 7.24, N 9.14; found C 43.07, H 7.55, N 9.03.

Teoc- $(S)-\left(\mathrm{NO}_{2}\right) N v a-O H$ (38) from Z-(S)-(NO 2$) N v a-O t B u$ : Z-(S)-( $\left.\mathrm{NO}_{2}\right) \mathrm{Nva}-\mathrm{O} t \mathrm{Bu}(0.300 \mathrm{~g}, 0.851$ mmol) was deprotected with $3.4 \mathrm{M} \mathrm{HBr}$ in $\mathrm{AcOH}(1.5 \mathrm{~mL})$ for $40 \mathrm{~min}$. Ether $(50 \mathrm{~mL})$ was then added to the reaction mixture and the precipitating red oil was washed with $\mathrm{Et}_{2} \mathrm{O}(3 \times 50 \mathrm{~mL})$, dried and used without further purification for the next step. The treatment with TeocOSu $(1.033$ $\mathrm{mmol})$ and $\mathrm{NaHCO}_{3}(0.315 \mathrm{~g}, 3.750 \mathrm{mmol})$ in a water/acetone mixture gave after usual work-up (see above) the crude product as a red oil which was further purified by column chromatography $\left(\right.$ EtOAc/hexane $1: 2(1.5 \% \mathrm{AcOH}), R_{\mathrm{f}}=0.24$ (staining with ninhidrin)) and finally by trituration with hexane to give $\mathbf{3 8}(0.111 \mathrm{~g}, 43 \%$ on 2 steps $)$ as a white solid.

Chpca(MOM)-(NO $\left.\mathrm{N}_{2}\right) \mathrm{Nva-Phe-Val-OMe} \mathrm{(211):} \mathrm{Dipeptide} \mathrm{Boc-Phe-Val-OMe} 117$ (10 mg, 26.42 $\mu \mathrm{mol})$ was deprotected with TFA $(1 \mathrm{~mL})$ for $20 \mathrm{~min}$. All volatiles were then removed and the residue was taken up with toluene $(2 \times 15 \mathrm{~mL})$ which was distilled off to remove the last traces of TFA to give corresponding trifluoroacetate as a white solid which was further coupled with $N$ Teoc protected nitronorvaline 209 (16 mg, $52.22 \mu \mathrm{mol})$ using HATU (20.1 mg, $52.86 \mu \mathrm{mol})$, HOAt $(7.1 \mathrm{mg}, 52.54 \mu \mathrm{mol})$, DIEA $(3.4 \mathrm{mg}, 26.31 \mu \mathrm{mol})$ and TMP $(19.2 \mathrm{mg}, 158.5 \mu \mathrm{mol})$ in $\mathrm{CH}_{2} \mathrm{Cl}_{2}(3 \mathrm{~mL})$ according to GP 7 for $16 \mathrm{~h}$. The crude product obtained after usual aqueous work-up (GP 5) was further purified by preparative TLC $(200 \times 200 \mathrm{~mm}$, EtOAc/hexane 1:3, 2 runs, then EtOAc/hexane 3:7, 1 run) to give Teoc-( $\left.\mathrm{NO}_{2}\right)$ Nva-Phe-Val-OMe 210 (8.9 mg, 59\%) as a colorless glass. $R_{\mathrm{f}}=0.27 \mathrm{EtOAc} /$ hexane $1: 1.5 ;{ }^{1} \mathrm{H} \mathrm{NMR}\left(250 \mathrm{MHz}, \mathrm{CDCl}_{3}\right): \delta=0.02[\mathrm{~s}$, 
$\left.9 \mathrm{H}, \mathrm{Si}\left(\mathrm{CH}_{3}\right)_{3}\right], 0.81\left(\mathrm{~d}, J=6.8 \mathrm{~Hz}, 3 \mathrm{H}, 4-\mathrm{H}_{\mathrm{a}}, V a l\right), 0.85$ (d, $\left.J=6.8 \mathrm{~Hz}, 3 \mathrm{H}, 4-\mathrm{H}_{\mathrm{b}}, V a l\right), 0.97$ (dd, $J=8.8,8.8 \mathrm{~Hz}, 2 \mathrm{H}, 2-\mathrm{H}, \mathrm{Teoc}), 1.53-2.02\left[\mathrm{~m}, 4 \mathrm{H}, 3-\mathrm{H}, 4-\mathrm{H},\left(\mathrm{NO}_{2}\right) \mathrm{Nva}\right], 2.10$ (octet, $J=$ $6.8 \mathrm{~Hz}, 1 \mathrm{H}, 3-\mathrm{H}, V a l), 3.06$ (d, J = 7.3 Hz, $2 \mathrm{H}, 3-\mathrm{H}, P h e), 3.70$ (s, $3 \mathrm{H}, \mathrm{OMe}$ ), 4.01-4.40 (m, $3 \mathrm{H}, 2-\mathrm{H}, \mathrm{Val}, 1-\mathrm{H}, T e o c), 4.35\left[\mathrm{t}, J=6.6 \mathrm{~Hz}, 2 \mathrm{H}, 5-\mathrm{H},\left(\mathrm{NO}_{2}\right) N v a\right], 4.43[\mathrm{dd}, J=8.5,5.3 \mathrm{~Hz}$, $\left.1 \mathrm{H}, 2-\mathrm{H},\left(\mathrm{NO}_{2}\right) \mathrm{Nva}\right], 4.71(\mathrm{dd}, J=7.3,7.3 \mathrm{~Hz}, 1 \mathrm{H}, 2-\mathrm{H}, P h e), 5.28(\mathrm{~d}, J=8.5 \mathrm{~Hz}, 1 \mathrm{H}, \mathrm{NH})$, $6.46(\mathrm{~d}, J=8.8 \mathrm{~Hz}, 1 \mathrm{H}, \mathrm{NH}), 6.79(\mathrm{~d}, J=7.8 \mathrm{~Hz}, 1 \mathrm{H}, \mathrm{NH}), 7.13-7.32(\mathrm{~m}, 5 \mathrm{H}$, Ar-H). This compound $(8.6 \mathrm{mg}, 15.17 \mu \mathrm{mol})$ was deprotected with TFA $(1 \mathrm{~mL})$ for $1 \mathrm{~h}$. All volatiles were removed under reduced pressure, the residue was taken up with toluene $(2 \times 15 \mathrm{~mL})$ which was then distilled off to remove the last traces of TFA to give trifluoroacetate of the deprotected tripeptide as a colorless solid which was coupled with acid 109 (4.1 mg, $19.94 \mu \mathrm{mol})$ using HATU $(7.2 \mathrm{mg}, 18.93 \mu \mathrm{mol})$, DIEA $(2.0 \mathrm{mg}, 15.47 \mu \mathrm{mol})$ and TMP $(7.3 \mathrm{mg}, 60.24 \mu \mathrm{mol})$ in $\mathrm{CH}_{2} \mathrm{Cl}_{2}(3 \mathrm{~mL})$ according GP 7 for $2 \mathrm{~h}$. The crude product obtained after usual aqueous work-up (GP 5) was finally purified by preparative TLC $(200 \times 200 \mathrm{~mm}$, EtOAc/hexane 1:3, 3 runs $)$ to give the acylated tripeptide $211(7.3 \mathrm{mg}, 79 \%) . R_{\mathrm{f}}=0.10 \mathrm{EtOAc} /$ hexane $1: 3 ;[\alpha]_{20}^{D}-32.3(c=$ $\left.0.50, \mathrm{CHCl}_{3}\right) ;{ }^{1} \mathrm{H} \mathrm{NMR}\left(600 \mathrm{MHz}, \mathrm{CDCl}_{3}\right): \delta=0.83\left(\mathrm{~d}, J=6.6 \mathrm{~Hz}, 3 \mathrm{H}, 4-\mathrm{H}_{\mathrm{a}}, \mathrm{Val}\right), 0.86(\mathrm{~d}, J=$ $\left.6.6 \mathrm{~Hz}, 3 \mathrm{H}, 4-\mathrm{H}_{\mathrm{b}}, V a l\right), 1.68-1.75\left[\mathrm{~m}, 1 \mathrm{H}, 3-\mathrm{H}_{\mathrm{a}},\left(N O_{2}\right) N v a\right], 1.86-1.94\left[\mathrm{~m}, 1 \mathrm{H}, 3-\mathrm{H}_{\mathrm{a}}\right.$, $\left(\mathrm{NO}_{2}\right) \mathrm{Nva}$ ], 2.09 (octet, $\left.J=6.6 \mathrm{~Hz}, 1 \mathrm{H}, 3-\mathrm{H}, \mathrm{Val}\right), 2.99$ (dd, $\left.J=13.8,7.8 \mathrm{~Hz}, 1 \mathrm{H}, 3-\mathrm{H}_{\mathrm{a}}, P h e\right)$, $3.09\left(\mathrm{dd}, J=13.8,6.6 \mathrm{~Hz}, 2 \mathrm{H}, 3-\mathrm{H}_{\mathrm{b}}\right.$, Phe), 3.53 (s, $\left.3 \mathrm{H}, \mathrm{OMe}, M O M\right), 3.69$ (s, $\left.3 \mathrm{H}, \mathrm{OMe}\right), 4.363$ $\left[\mathrm{dd}, J=6.6,6.6 \mathrm{~Hz}, 2 \mathrm{H}, 5-\mathrm{H},\left(N O_{2}\right) N v a\right], 4.35(\mathrm{dd}, J=8.4,5.4 \mathrm{~Hz}, 1 \mathrm{H}, 2-\mathrm{H}, V a l), 4.70(\mathrm{dd}, J=$ 13.8, 8.4 Hz, $1 \mathrm{H}, 2-\mathrm{H}, P h e), 4.72\left[\mathrm{dd}, J=7.2,7.2 \mathrm{~Hz}, 1 \mathrm{H}, 2-\mathrm{H},\left(\mathrm{NO}_{2}\right) \mathrm{Nva}\right], 5.19$ (dd, $J=12.6$, $\left.12.6 \mathrm{~Hz}, 2 \mathrm{H}, \mathrm{OCH}_{2} \mathrm{O}\right), 6.05(\mathrm{~d}, J=4.8 \mathrm{~Hz}, 1 \mathrm{H}, 4-\mathrm{H}, C h p c a), 6.63(\mathrm{~d}, J=8.4 \mathrm{~Hz}, 1 \mathrm{H}, \mathrm{NH})$, $6.74(\mathrm{~d}, J=4.8 \mathrm{~Hz}, 1 \mathrm{H}, 3-\mathrm{H}$, Chpca), 7.12-7.20 (m, $5 \mathrm{H}, \mathrm{Ar}-\mathrm{H}), 7.21(\mathrm{~d}, J=7.8 \mathrm{~Hz}, 1 \mathrm{H}, \mathrm{NH})$; ${ }^{13} \mathrm{C} \mathrm{NMR}\left(150.8 \mathrm{MHz}, \mathrm{CDCl}_{3}\right): \delta=17.8,18.8(+, \mathrm{C}-4, \mathrm{Val}), 23.2\left[-, \mathrm{C}-4,\left(\mathrm{NO}_{2}\right) \mathrm{Nva}\right], 29.1[-, \mathrm{C}-$ 3, $\left(\mathrm{NO}_{2}\right) \mathrm{Nva}$, $31.1(+, \mathrm{C}-3, \mathrm{Val}), 37.9$ (-, C-3, Phe), $51.9(+, \mathrm{C}-2), 52.1(+, \mathrm{C}-2), 54.7(+, \mathrm{C}-2)$, $57.4(+, \mathrm{OMe}, \mathrm{MOM}), 59.7$ (+, OMe), $74.8\left[-, \mathrm{C}-5,\left(\mathrm{NO}_{2}\right) \mathrm{Nva}\right], 104.4(+, \mathrm{C}-4, \mathrm{Chpca}), 104.8(-$, $\left.\mathrm{OCH}_{2} \mathrm{O}\right), 111.6(+, \mathrm{C}-3$, Chpca $), 118.8\left(\mathrm{C}_{\text {quat }}, \mathrm{C}-2\right.$, Chpca $), 121.9\left(\mathrm{C}_{\text {quat }}, \mathrm{C}-5\right.$, Chpca $), 126.9$, 
128.5, 129.2130 .4 (+, Ar-C), 136.3 (C quat $_{\text {, }}$ Ar-C), 158.6 ( $\mathrm{C}_{\text {quat }}, \mathrm{C}-1$, Chpca $), 170.6,171.0,171.9$ $\left(\mathrm{C}_{\text {quat }}, \mathrm{C}-1\right)$; $\mathrm{MS}(\mathrm{ESI})$ : positive, $m / z=632(48)\left[\mathrm{M}+\mathrm{Na}^{+}\right]$; negative, $m / z=608(100)\left[\mathrm{M}-\mathrm{H}^{+}\right]$.

$\left[(\mathrm{S})-\left(\mathrm{NO}_{2}\right) \mathrm{Nva}^{7}\right]-$ Hormaomycin $\left[(\mathrm{S})-\left(\mathrm{NO}_{2}\right) \mathrm{Nva}^{7}\right]-(3)$ : Cyclodepsipeptide 171c $(19.0 \mathrm{mg}, 19.42$ $\mu \mathrm{mol})$ was deprotected with $10 \%$ anisole in TFA $(1.1 \mathrm{~mL})$ according to GP 11 for $2 \mathrm{~h}$ and the resultant deprotected depsipeptide was coupled with $N$-Teoc protected amino acid 209 (16.9 mg, $55.16 \mu \mathrm{mol})$ using HATU $(21.0 \mathrm{mg}, 55.23 \mu \mathrm{mol})$, HOAt $(7.5 \mathrm{mg}, 55.50 \mu \mathrm{mol})$, DIEA $(2.86 \mathrm{mg}$, $22.09 \mu \mathrm{mol})$ and TMP $(24.12 \mathrm{mg}, 199.04 \mu \mathrm{mol})$ in $\mathrm{CH}_{2} \mathrm{Cl}_{2}(2 \mathrm{~mL})$ according to $\mathrm{GP} 7$ for $15 \mathrm{~h}$. The mixture was then diluted with $\mathrm{Et}_{2} \mathrm{O}(20 \mathrm{~mL})$ and the crude product obtained after usual aqueous work-up (GP 5) was purified by preparative TLC $(200 \times 200 \mathrm{~mm}$, acetone/hexane 1:3, 3 runs) to give Teoc- $(S)-\left(\mathrm{NO}_{2}\right) \mathrm{Nva}$-cyclo-hexadepsipeptide $\left(19.0 \mathrm{mg}, 88 \%, \quad R_{\mathrm{f}}=0.06\right.$ acetone/hexane 1:3, 3 runs) as a colorless glass which was used for the next step without any characterization. Teoc group was cleaved from this substance $(19.0 \mathrm{mg}, 16.99 \mu \mathrm{mol})$ with TFA $(1.0 \mathrm{~mL})$ for $1 \mathrm{~h}$. The mixture was concentrated under reduced pressure at $20^{\circ} \mathrm{C}$ and then taken up with toluene $(3 \times 15 \mathrm{~mL})$ which was distilled off to remove the last traces of TFA. The resultant deprotected depsipeptide was coupled with Chpca(MOM)-OH 109 (7.0 mg, 34.04 $\mu \mathrm{mol})$ using HATU $(12.9 \mathrm{mg}, 33.93 \mu \mathrm{mol})$, DIEA $(2.85 \mathrm{mg}, 22.08 \mu \mathrm{mol})$ and TMP (16.06 mg, $132.49 \mu \mathrm{mol})$ in $\mathrm{CH}_{2} \mathrm{Cl}_{2}(2 \mathrm{~mL})$ according to $\mathrm{GP} 7$ for $2.5 \mathrm{~h}$. The mixture was then diluted with $\mathrm{Et}_{2} \mathrm{O}(20 \mathrm{~mL})$ and the crude product obtained after usual aqueous work-up (GP 5) was purified by preparative TLC $(200 \times 200 \mathrm{~mm}$, acetone/hexane 1:2.7) to give $O$-MOM protected $[(S)$ $\left.\left(\mathrm{NO}_{2}\right) \mathrm{Nva}^{7}\right]-3\left(15.0 \mathrm{mg}, 76 \%, R_{\mathrm{f}}=0.17\right.$ acetone/hexane $\left.1: 2.5\right)$ as a colorless glass which was used for the next step without any characterization. This substance $(15.0 \mathrm{mg}, 12.91 \mu \mathrm{mol})$ was deprotected using $\mathrm{MgBr}_{2} \cdot \mathrm{Et}_{2} \mathrm{O}$ (178 $\left.\mathrm{mg}, 0.688 \mathrm{mmol}\right)$ and $\mathrm{EtSH}(0.03 \mathrm{~mL}, 0.405 \mathrm{mmol})$ in $\mathrm{CH}_{2} \mathrm{Cl}_{2}(10 \mathrm{~mL})$ according to GP 10 for $3 \mathrm{~h}$. The mixture was taken up with $\mathrm{Et}_{2} \mathrm{O}$ and the crude product obtained after usual aqueous work-up (GP 10) was crystallized from $\mathrm{CH}_{2} \mathrm{Cl}_{2} /$ pentane. The mother liquor was concentrated under reduced and crystallized from $\mathrm{Et}_{2} \mathrm{O} /$ pentane. This 
second crop of the desired product was combined with first one to give $\left[(S)-\left(\mathrm{NO}_{2}\right) \mathrm{Nva}^{7}\right]-3(12.9$ $\mathrm{mg}, 90 \%, 60 \%$ on 5 steps from 24$)$ as a white solid. $R_{\mathrm{f}}=0.11$ acetone/hexane 1:2.5; analytical HPLC: column $\mathrm{B}$, gradient $25 \% \rightarrow 85 \% \mathrm{MeCN}$ in $0.15 \%$ ammonium acetate buffer $(\mathrm{pH}=5.5)$ for $25 \mathrm{~min}$, flow rate $=0.5 \mathrm{~mL} / \mathrm{min}, t_{\mathrm{R}}=20.22 \mathrm{~min}$, purity $>93 \% ;[\alpha]_{20}^{D} 13.0\left(c=0.1, \mathrm{CHCl}_{3}\right)$; ${ }^{1} \mathrm{H}$ NMR $\left(600 \mathrm{MHz}, \mathrm{CDCl}_{3}\right): \delta=-0.72--0.59\left[\mathrm{~m}, 1 \mathrm{H}, 3^{\prime}-\mathrm{H}_{\mathrm{a}},(3-N c p)\right.$ Ala $],-0.19--0.06[\mathrm{~m}$, $\left.1 \mathrm{H}, 3-\mathrm{H}_{\mathrm{a}},(3-N c p) A l a\right], 0.25-0.34$ [m, $\left.1 \mathrm{H}, 1^{\prime}-\mathrm{H},(3-N c p) A l a\right], 0.55$ [ddd, J = 14.4, 4.8, $4.8 \mathrm{~Hz}$, $\left.1 \mathrm{H}, 3-\mathrm{H}_{\mathrm{b}},(3-N c p) A l a\right], 0.92\left(\mathrm{t}, J=7.2 \mathrm{~Hz}, 3 \mathrm{H}, 5-\mathrm{H}\right.$, Ile), 0.97-1.04 [m, $1 \mathrm{H}, 3^{\prime}-\mathrm{H}_{\mathrm{b}},(3-N c p)$ Ala], $1.06\left(\mathrm{~d}, J=7.2 \mathrm{~Hz}, 3 \mathrm{H}, 1^{\prime}-\mathrm{H}\right.$, Ile $), 1.24-1.32\left(\mathrm{~m}, 1 \mathrm{H}, 4-\mathrm{H}_{\mathrm{a}}\right.$, Ile $), 1.30[\mathrm{~d}, J=7.2 \mathrm{~Hz}, 3 \mathrm{H}, 4-\mathrm{H}$, ( $\beta M e) P h e], 1.42$ [d, J=7.2 Hz, $3 \mathrm{H}, 4-\mathrm{H},(\beta M e) P h e], 1.53$ (d, $J=6.6 \mathrm{~Hz}, 3 \mathrm{H}, a-T h r), 1.58-1.65$ (m, $1 \mathrm{H}, 4-\mathrm{H}_{\mathrm{b}}$, Ile $), 1.67-1.76\left[\mathrm{~m}, 1 \mathrm{H}, 3-\mathrm{H}_{\mathrm{a}},\left(N O_{2}\right) N v a\right], 1.69$ [dd, J = 7.2, $1.8 \mathrm{~Hz}, 3 \mathrm{H}, 3^{\prime}-\mathrm{H},(4-$ PE)Pro], 1.76-1.84 [m, $2 \mathrm{H}, 3-\mathrm{H}_{\mathrm{b}},\left(\mathrm{NO}_{2}\right) N v a, 3-\mathrm{H}_{\mathrm{a}}$, (4-PE)Pro], 1.85-1.92 (m, $1 \mathrm{H}, 3-\mathrm{H}$, Ile), 1.92-2.09 [m, $2 \mathrm{H}, 4-\mathrm{H},\left(\mathrm{NO}_{2}\right) \mathrm{Nva}$ ], 2.38 [ddd, $J=12.0,5.4,5.4 \mathrm{~Hz}, 1 \mathrm{H}, 4-\mathrm{H}_{\mathrm{b}},(4-P E)$ Pro], 2.91 $\left[\mathrm{ddd}, J=6.6,3.0,3.0 \mathrm{~Hz}, 1 \mathrm{H}, 2^{\prime}-\mathrm{H},(3-N c p) A l a\right], 3.04[\mathrm{dq}, J=11.1,7.2 \mathrm{~Hz}, 1 \mathrm{H}, 3-\mathrm{H}$, (ßMe)Phe], 3.24-3.34 [m, $\left.2 \mathrm{H}, 4-\mathrm{H}, 5-\mathrm{H}_{\mathrm{a}},(4-P E) P r o\right], 3.45-3.52$ [m, $1 \mathrm{H}, 2-\mathrm{H},(3-N c p)$ Ala $]$, $3.69[\mathrm{dq}, J=4.2,7.2 \mathrm{~Hz}, 1 \mathrm{H}, 3-\mathrm{H},(\beta M e) P h e], 3.95-4.03\left[\mathrm{~m}, 1 \mathrm{H}, 5-\mathrm{H}_{\mathrm{b}},(4-P E)\right.$ Pro $], 4.26$ [dd, $J$ $=11.7,6.0 \mathrm{~Hz}, 1 \mathrm{H}, 2-\mathrm{H},(4-P E) P r o], 4.36\left[\mathrm{t}, J=6.6 \mathrm{~Hz}, 2 \mathrm{H}, 5-\mathrm{H},\left(N O_{2}\right) N v a\right], 4.37[\mathrm{dd}, J=$ 11.0, $11.0 \mathrm{~Hz}, 1 \mathrm{H}, 2-\mathrm{H},(\beta M e) P h e], 4.49[\mathrm{dd}, \mathrm{J}=9.3,4.2 \mathrm{~Hz}, 1 \mathrm{H}, 2-\mathrm{H},(\beta M e) P h e], 4.56(\mathrm{dd}, J$ $=9.0,2.4 \mathrm{~Hz}, 1 \mathrm{H}, 2-\mathrm{H}, a-T h r), 4.66(\mathrm{dd}, J=9.0,9.0 \mathrm{~Hz}, 1 \mathrm{H}, 2-\mathrm{H}$, Ile $), 5.04$ [ddd, $J=9.0,9.0$, $\left.7.2 \mathrm{~Hz}, 1 \mathrm{H}, 2-\mathrm{H},\left(\mathrm{NO}_{2}\right) \mathrm{Nva}\right], 5.24-5.31\left[\mathrm{~m}, 1 \mathrm{H}, 1^{\prime}-\mathrm{H},(4-P E)\right.$ Pro], 5.38 (dq, $J=7.2,2.4 \mathrm{~Hz}$, $1 \mathrm{H}, 3-\mathrm{H}, a-T h r), 5.64\left[\mathrm{dq}, J=10.8,7.2 \mathrm{~Hz}, 1 \mathrm{H}, 2^{\prime}-\mathrm{H},(4-P E) P r o\right], 6.14$ (d, $J=4.8 \mathrm{~Hz}, 1 \mathrm{H}, 4-$ H, Chpca), 6.46 (d, J=6.6 Hz, $1 \mathrm{H}, \mathrm{NH}), 6.75-6.84(\mathrm{br}, 1 \mathrm{H}, \mathrm{NH}), 6.82$ (d, J=4.8 Hz, $1 \mathrm{H}, 3-\mathrm{H}$, Chpca), $6.88(\mathrm{~d}, J=9.6 \mathrm{~Hz}, 1 \mathrm{H}, \mathrm{NH}), 7.02-7.05(\mathrm{~m}, 1 \mathrm{H}, \mathrm{Ar}-\mathrm{H}), 7.10-7.20(\mathrm{~m}, 5 \mathrm{H}, \mathrm{Ar}-\mathrm{H})$, 7.21-7.27 (m, 5 H, Ar-H, NH), 7.33 (d, $J=9.0$ Hz, 1 H, NH), 7.94-8.09 (br, $1 \mathrm{H}, \mathrm{NH}), 9.08$ (d, $J$ $=9.0 \mathrm{~Hz}, 1 \mathrm{H}, \mathrm{NH}) ;{ }^{13} \mathrm{C} \mathrm{NMR}\left(150.8 \mathrm{MHz}, \mathrm{CDCl}_{3}\right): \delta=10.5(+, \mathrm{C}-5$, Ile $), 13.3\left[+, \mathrm{C}-3^{\prime},(4-\right.$ PE)Pro, C-4, (BMe)Phe], 14.8 (+, C-1', Ile), 17.1 (+, C-4, a-Thr), 17.1 [-, C-3', (3-Ncp)Ala], 
$17.7[+, \mathrm{C}-4,(\beta M e) P h e], 20.1\left[+, \mathrm{C}-1^{\prime},(3-N c p) A l a\right], 23.7\left[-, \mathrm{C}-4,\left(\mathrm{NO}_{2}\right) \mathrm{Nva}\right], 25.1(-, \mathrm{C}-3, \mathrm{Ile})$, $30.7\left[-, \mathrm{C}-3,\left(\mathrm{NO}_{2}\right) \mathrm{Nva}\right], 33.0[-, \mathrm{C}-3,(3-\mathrm{Ncp}) \mathrm{Ala}], 35.5$ [-, C-3, (4-PE)Pro], 36.7 [+, C-4, (4-

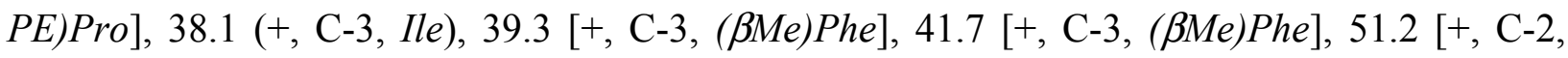
$\left.\left(\mathrm{NO}_{2}\right) \mathrm{Nva}\right], 51.8$ [+, C-2, (3-Ncp)Ala], 52.8 [-, C-5, (4-PE)Pro], 54.5 (+, C-2, Ile), $54.9(+, \mathrm{C}-2$,

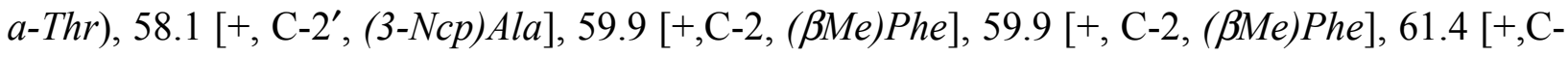
5, (4-PE)Pro], 69.3 (+, C-3, a-Thr), 75.0 [-, C-5, (NO2)-Nva], 103.5 (+, C-4, Chpca), $109.6(+$, C-3, Chpca), 119.8 ( $\mathrm{C}_{\text {quat }}, \mathrm{C}-2$, Chpca $), 121.7$ (C quat, $\mathrm{C}-5$, Chpca $), 127.0,127.2,127.4,127.7$, 128.5, 128.6 (+, Ar-C), 127.5 [+, C-1', (4-PE)Pro], 128.3 [+, C-2', (4-PE)Pro], 141.6, 142.1 $\left(\mathrm{C}_{\text {quat }}, \mathrm{Ar}-\mathrm{C}\right), 159.3$ ( $\left.\mathrm{C}_{\text {quat }}, \mathrm{C}-1, \mathrm{Chpca}\right), 168.5,168.7,170.7,171.3,171.6,172.3\left(\mathrm{C}_{\text {quat }}, \mathrm{C}-1\right)$; UV $(\mathrm{MeOH})$ : neutral: $\lambda_{\max }(\varepsilon)=278\left(1.2 \times 10^{4}\right) \mathrm{nm}$; basic: $282\left(1.0 \times 10^{4}\right), 205\left(5.7 \times 10^{4}\right) \mathrm{nm}$;

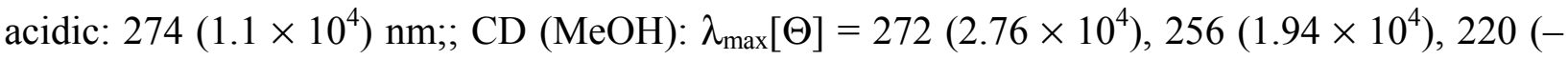
$\left.5.50 \times 10^{4}\right) \mathrm{nm}\left(c=6.63 \times 10^{-6} \mathrm{M}\right) ; \mathrm{MS}(\mathrm{ESI}):$ positive, $m / z=1162(40)\left[\mathrm{M}-\mathrm{H}^{+}+2 \mathrm{Na}^{+}\right], 1140$ (100) $\left[\mathrm{M}+\mathrm{Na}^{+}\right]$; negative, $m / z=1115$ (100) $\left[\mathrm{M}-\mathrm{H}^{+}\right]$; HRMS (ESI): calcd for $\left[\mathrm{C}_{54} \mathrm{H}_{70} \mathrm{~N}_{10} \mathrm{O}_{14} \mathrm{Cl}^{+}\right]$: 1117.4756 ; found 1117.4752 .

MeZ-a-Dab(Fmoc)-OMe (215): Boc-(R)-a-Dab(Fmoc)-OtBu 214 (0.39 g, 0.785 mmol) was

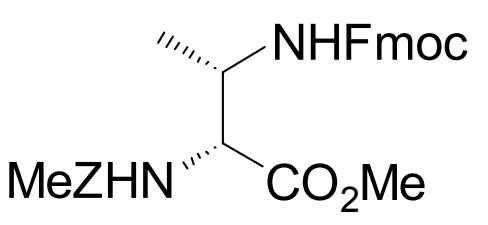
deprotected with TFA $(5 \mathrm{~mL})$ for $1 \mathrm{~h}$. All volatiles were removed under reduced pressure at $20^{\circ} \mathrm{C}$. The solid residue was taken up with $1 \mathrm{M} \mathrm{HCl}(5 \mathrm{~mL})$ and methanol $(20 \mathrm{~mL})$ and after $10 \mathrm{~min}$ the mixture was concentrated and the residue was dried at 0.02 Torr for $16 \mathrm{~h}$ and used for the next step without further purification. $\mathrm{SOCl}_{2}(0.60 \mathrm{~mL}, 8.272 \mathrm{mmol})$ was added dropwise to a solution of the crude hydrochloride of amino acid in anhydrous methanol $(35 \mathrm{~mL})$ at $-20^{\circ} \mathrm{C}$ for $5 \mathrm{~min}$ and stirring continued at the same temperature for a further $15 \mathrm{~min}$. The mixture was then allowed to warm to $20^{\circ} \mathrm{C}$ and after stirring at this temperature for $1 \mathrm{~h}$ the reaction flask was sealed and the mixture was heated to $50{ }^{\circ} \mathrm{C}$ and stirred for an additional $20 \mathrm{~h}$. The reaction mixture was then concentrated under reduced pressure and the residue was triturated with $\mathrm{Et}_{2} \mathrm{O}$ 
to give crude $\mathrm{HCl} \cdot \mathrm{H}-\mathrm{Dab}(\mathrm{Fmoc})$-OMe $(0.29 \mathrm{~g}, 94 \%)$ as a white solid. $R_{\mathrm{f}}=0.30 \mathrm{MeOH} / \mathrm{CHCl}_{3}$ 1:100; ${ }^{1} \mathrm{H}$ NMR $\left(250 \mathrm{MHz}, \mathrm{CD}_{3} \mathrm{OD}\right): \delta=1.36(\mathrm{~d}, J=7 \mathrm{~Hz}, 3 \mathrm{H}, \mathrm{H}-4), 3.97$ (s, $\left.3 \mathrm{H}, \mathrm{OMe}\right)$, 4.16-4.34 (m, 3 H, 2-H and $\left.1^{\prime}-\mathrm{H}, 2^{\prime}-\mathrm{H}_{\mathrm{a}}, F m o c\right), 4.40-4.59(\mathrm{~m}, 2 \mathrm{H}, 3-\mathrm{H}$ and 2'-H, Fmoc), 7.27$7.50(\mathrm{~m}, 4 \mathrm{H}, \mathrm{Ar}-\mathrm{H}), 7.54(\mathrm{~d}, J=6.8 \mathrm{~Hz}, 1 \mathrm{H}, \mathrm{NH}), 7.70(\mathrm{~d}, J=7.3 \mathrm{~Hz}, 2 \mathrm{H}, \mathrm{Ar}-\mathrm{H}), 7.84(\mathrm{~d}, J=$ $7.3 \mathrm{~Hz}, 1 \mathrm{H}, \mathrm{Ar}-\mathrm{H})$. A solution of MeZOSu $(0.244 \mathrm{~g}, 0.927 \mathrm{mmol})$ in acetone $(5 \mathrm{~mL})$ was added to a solution of this material $(0.29 \mathrm{~g}, 0.742 \mathrm{mmol})$ in water $(5 \mathrm{~mL})$ (if emulsion formed acetone and/or water were added to obtain homogeneous solution) and stirring continued for a further 1.5 h. Acetone was then distilled off under reduced pressure and the resultant suspension was filtered. The crude product was washed with $\mathrm{Et}_{2} \mathrm{O} /$ pentane 1:1 $(50 \mathrm{~mL})$, water $(100 \mathrm{~mL}), 3 \%$ $\mathrm{NaHCO}_{3}(50 \mathrm{~mL})$, water $(20 \mathrm{~mL}), 1 \mathrm{M} \mathrm{HCl}$, water $(50 \mathrm{~mL})$, pentane $(50 \mathrm{~mL})$ dried and finally crystallized from $\mathrm{CH}_{2} \mathrm{Cl}_{2}$ /hexane to give 215 ( $0.272 \mathrm{~g}, 69 \%$ over 3 steps) as a colorless solid. M.p. $167-168{ }^{\circ} \mathrm{C} ;[\alpha]_{20}^{D} 8.5(c=0.40, \mathrm{THF}) ;{ }^{1} \mathrm{H}$ NMR $\left(250 \mathrm{MHz}, \mathrm{CDCl}_{3}\right): \delta=1.47(\mathrm{~d}, J=$ $6.8 \mathrm{~Hz}, 3 \mathrm{H}, 4-\mathrm{H}), 2.34$ (s, $\left.3 \mathrm{H}, 1^{\prime}-\mathrm{H}, M e Z\right), 3.77$ (s, $\left.3 \mathrm{H}, \mathrm{OMe}\right), 4.10-4.51$ (m, $4 \mathrm{H}, 3-\mathrm{H}$ and 1'H, 2'-H, Fmoc), 4.60 (dd, $J=8.1,3.1 \mathrm{~Hz}, 1 \mathrm{H}, 2-\mathrm{H}), 5.08$ (s, $2 \mathrm{H}, \mathrm{Bzl}-\mathrm{H}), 5.32$ (d, $J=8.0 \mathrm{~Hz}$, $1 \mathrm{H}, \mathrm{NH}), 5.67(\mathrm{~d}, J=6.8 \mathrm{~Hz}, 1 \mathrm{H}, \mathrm{NH}), 7.16(\mathrm{~d}, J=7.3 \mathrm{~Hz}, 2 \mathrm{H}, \mathrm{Ar}-\mathrm{H}), 7.21-7.46(\mathrm{~m}, 6 \mathrm{H}, \mathrm{Ar}-$ H), $7.61(\mathrm{~d}, J=6.8 \mathrm{~Hz}, 2 \mathrm{H}, \mathrm{Ar}-\mathrm{H}), 7.77(\mathrm{~d}, J=7.3 \mathrm{~Hz}, 2 \mathrm{H}, \mathrm{Ar}-\mathrm{H}) ;{ }^{13} \mathrm{C} \mathrm{NMR}(62.9 \mathrm{MHz}$, $\left.\mathrm{CDCl}_{3}\right): \delta=16.3(+, \mathrm{C}-4), 21.0\left(+, \mathrm{C}-1^{\prime}, \mathrm{MeZ}\right), 47.0(+, \mathrm{C}-3), 48.9\left(+, \mathrm{C}-1^{\prime}\right.$, Fmoc $), 52.5(+$, OMe), 57.8 (+, C-2), 66.8 (-, Bzl-H, MeZ), 67.1 (-, C-2', Fmoc), 119.8, 125.0, 126.9, 127.5, 128.3, $129.1(+, \mathrm{Ar}-\mathrm{C}), 132.8\left(\mathrm{C}_{\text {quat }}, \mathrm{Ar}-\mathrm{C}\right), 137.9\left(\mathrm{C}_{\text {quat }}, \mathrm{Ar}-\mathrm{C}\right), 141.1\left(\mathrm{C}_{\text {quat }}, \mathrm{Ar}-\mathrm{C}\right), 143.7,143.9$ $\left(\mathrm{C}_{\text {quat }}, \mathrm{Ar}-\mathrm{C}\right), 155.8,156.4\left(\mathrm{C}_{\text {quat }}, \mathrm{NCO}_{2}\right), 170.7\left(\mathrm{C}_{\text {quat }}, \mathrm{C}-1\right)$; IR $(\mathrm{KBr}): \mathrm{nu}($ tilde $)=3316 \mathrm{~cm}^{-1}$, $3067,2948,1748,1691,1542,1450,1338,1316,1282,1231,1169 ;$ MS (EI, $70 \mathrm{eV}), \mathrm{m} / z(\%)=$ $502(1)\left[\mathrm{M}^{+}\right], 266(10)\left[\mathrm{C}_{14} \mathrm{H}_{20} \mathrm{NO}_{4}^{+}\right], 178(100)\left[\mathrm{C}_{14} \mathrm{H}_{10}{ }^{+}\right], 165$ (5), 105 (22) $\left[\mathrm{C}_{8} \mathrm{H}_{9}^{+}\right], 44(11)$ $\left[\mathrm{CO}_{2}{ }^{+}\right]$; HRMS (EI): calcd for $\mathrm{C}_{29} \mathrm{H}_{30} \mathrm{~N}_{2} \mathrm{O}_{6}$ : 502.2104; found 502.2104; elemental analysis calcd (\%) for $\mathrm{C}_{29} \mathrm{H}_{30} \mathrm{~N}_{2} \mathrm{O}_{6}(502.6)$ : C 69.31, H 6.02, N 5.57; found C 69.08, H 5.88, N 5.38. 
MeZ-a-Dab[Boc-(4-PE)Pro]-OMe (216): The compound 215 (0.191 g, 0.380 mmol) was deprotected according to GP 4 and the resultant crude $N_{\alpha}$-protected diamino ester was coupled with $N$-Boc protected (4-propenyl)proline $16(0.100 \mathrm{~g}, 0.392 \mathrm{mmol})$ using EDC (0.077 g, 0.402 mmol), HOAt $(0.055 \mathrm{~g}, 0.407 \mathrm{mmol})$ and TMP $(0.142 \mathrm{~g}, 1.172 \mathrm{mmol})$ in $\mathrm{CH}_{2} \mathrm{Cl}_{2}(4 \mathrm{~mL})$ according to GP 5 for $6 \mathrm{~h}$. The crude product obtained after usual aqueous work-up (GP 5) was finally purified by column chromatography (EtOAc/hexane $\left.1: 1.5, R_{\mathrm{f}}=0.35\right)$ to give $216(0.163$ $\mathrm{g}, 83 \%$ ) as a turbid oil, which solidified during drying at $60^{\circ} \mathrm{C}$ and 0.02 Torr in a white solid. M.p. $94-95^{\circ} \mathrm{C} ;[\alpha]_{20}^{D}-41.6\left(c=0.32, \mathrm{CHCl}_{3}\right) ;{ }^{1} \mathrm{H}$ NMR $\left(250 \mathrm{MHz}, \mathrm{CDCl}_{3}\right): \delta=1.04-1.19(\mathrm{~m}$, $3 \mathrm{H}, 4-\mathrm{H}, a-D a b), 1.41\left[\mathrm{~s}, 9 \mathrm{H}, \mathrm{C}\left(\mathrm{CH}_{3}\right)_{3}\right], 1.64\left[\mathrm{dd}, J=7.0,1.5 \mathrm{~Hz}, 3 \mathrm{H}, 3^{\prime}-\mathrm{H},(4-\right.$ PE)Pro], $1.72-$ 2.00 [m, $1 \mathrm{H}, 3-\mathrm{H}_{\mathrm{a}},(4-P E)$ Pro], 2.34 (s, $\left.3 \mathrm{H}, 1^{\prime}-\mathrm{H}, M e Z\right), 2.34-2.54$ [m, $1 \mathrm{H}, 3-\mathrm{H}_{\mathrm{b}}$, (4-PE)Pro], 2.92-3.15 [m, 2 H, 4-H, 5- $\mathrm{H}_{\mathrm{a}}$, (4-PE)Pro], 3.76 (s, $\left.3 \mathrm{H}, \mathrm{OMe}\right), 3.80-3.96$ [m, $1 \mathrm{H}, 5-\mathrm{H}_{\mathrm{b}}$, (4PE)Pro], 4.03-4.22 (m, $1 \mathrm{H}, 3-\mathrm{H}, a-D a b), 4.35-4.57(\mathrm{~m}, 2 \mathrm{H}, 2-\mathrm{H}), 5.01(\mathrm{~d}, J=12.3 \mathrm{~Hz}, 1 \mathrm{H}$, Bzl-H $\left.\mathrm{a}_{\mathrm{a}}\right), 5.09\left(\mathrm{~d}, J=12.3 \mathrm{~Hz}, 1 \mathrm{H}, \mathrm{Bzl}-\mathrm{H}_{\mathrm{b}}\right), 5.20-5.37$ [m, $1 \mathrm{H}, 1^{\prime}-\mathrm{H},(4-P E)$ Pro], 5.54 [dq, $J=$ 10.0, 7.0 Hz, $1 \mathrm{H}, 2^{\prime}-\mathrm{H},(4-P E)$ Pro], 5.59-5.77, 6.20-6.40 (2× m, $\left.1 \mathrm{H}, \mathrm{NH}\right), 6.81(\mathrm{~d}, J=8.8 \mathrm{~Hz}$, $1 \mathrm{H}, \mathrm{NH}), 7.15(\mathrm{~d}, J=7.8 \mathrm{~Hz}, 2 \mathrm{H}, \mathrm{Ar}-\mathrm{H}), 7.25$ (d, $J=7.8 \mathrm{~Hz}, 2 \mathrm{H}, \mathrm{Ar}-\mathrm{H}) ;{ }^{13} \mathrm{C} \mathrm{NMR}(62.9 \mathrm{MHz}$, $\left.\mathrm{CDCl}_{3}\right): \delta=13.0\left[+, \mathrm{C}-3^{\prime},(4-P E)\right.$ Pro $], 15.3,16.8(+, \mathrm{C}-4, a-T h r), 21.0\left(+, \mathrm{C}-1^{\prime}, \mathrm{MeZ}\right), 28.1[+$, $\left.\mathrm{C}\left(\mathrm{CH}_{3}\right)_{3}\right], 35.8$ [+, C-4, (4-PE)Pro], 37.8 [-, C-3, (4-PE)Pro $], 46.6,47.1$ (+, C-3, a-Dab), 52.0 [, C-5, (4-PE)Pro], 52.4 (+, OMe), 57.5 (+, C-2, a-Dab), 60.9, 61.5 [+, C-2, (4-PE)Pro], 66.8, 67.2 (-, Bzl-H, MeZ), $80.2\left[\mathrm{C}_{\text {quat }}, \mathrm{C}\left(\mathrm{CH}_{3}\right)_{3}\right] 126.4$ [+, C-2', (4-PE)Pro], 128.2, 129.0 (+, Ar-C), 129.4 [+,C-1', (4-PE)Pro], 132.7, 137.8 ( $\left.\mathrm{C}_{\text {quat }}, \mathrm{Ar}-\mathrm{C}\right), 154.2,156.5\left(\mathrm{C}_{\text {quat }}, \mathrm{NCO}_{2}\right), 170.2,171.0$ $\left(\mathrm{C}_{\text {quat }}, \mathrm{C}-1\right), 172.0,172.3\left(\mathrm{C}_{\text {quat }}, \mathrm{C}-1\right)$; IR $(\mathrm{KBr}): \mathrm{nu}($ tilde $)=3012 \mathrm{~cm}^{-1}, 2978,2929,2869,1728$, $1703,1678,1541,1519,1394,1368,1259,1212,1162$; MS (EI, $70 \mathrm{eV}), m / z(\%)=517(1)\left[\mathrm{M}^{+}\right]$, $444(3)\left[\mathrm{M}^{+}-\mathrm{C}_{4} \mathrm{H}_{9} \mathrm{O}\right], 416(6)\left[\mathrm{M}^{+}-\mathrm{C}_{5} \mathrm{H}_{9} \mathrm{O}_{2}\right], 281$ (52) $\left[\mathrm{C}_{15} \mathrm{H}_{24} \mathrm{~N}_{2} \mathrm{O}_{3}{ }^{+}\right], 238$ (15) $\left[\mathrm{C}_{13} \mathrm{H}_{20} \mathrm{~N}_{2} \mathrm{O}_{3}{ }^{+}\right]$, 225 (32) $\left[\mathrm{C}_{11} \mathrm{H}_{16} \mathrm{~N}_{2} \mathrm{O}_{3}^{+}\right], 182$ (11), $154(100)\left[\mathrm{C}_{8} \mathrm{H}_{12} \mathrm{NO}_{2}^{+}\right], 110$ (88) $\left[\mathrm{C}_{7} \mathrm{H}_{12} \mathrm{~N}^{+}\right], 105$ (70) $\left[\mathrm{C}_{8} \mathrm{H}_{9}^{+}\right], 57(49)\left[\mathrm{C}_{4} \mathrm{H}_{9}{ }^{+}\right], 44$ (68) $\left[\mathrm{CO}_{2}^{+}\right]$; HRMS (EI): calcd for $\mathrm{C}_{27} \mathrm{H}_{39} \mathrm{~N}_{3} \mathrm{O}_{7}$ : 517.2788; found 
517.2788; elemental analysis calcd (\%) for $\mathrm{C}_{27} \mathrm{H}_{39} \mathrm{~N}_{3} \mathrm{O}_{7}$ (517.6): C 62.65, H 7.59, N 8.12; found C 62.48, H 7.35, N 7.90.

MeZ-a-Dab[Boc-(4-PE)Pro]-OH (217): A 40\% aqueous solution of tetra- $n$-butylammonium hydroxide $(0.545 \mathrm{~g}, 0.840 \mathrm{mmol})$ was added dropwise to an ice-cold solution of ester $216(0.145$ $\mathrm{g}, 0.280 \mathrm{mmol})$ in THF $(1.8 \mathrm{~mL})$ and stirring continued at the same temperature for a further 45 min. A $1 \mathrm{M}$ aqueous $\mathrm{H}_{2} \mathrm{SO}_{4}(2.1 \mathrm{~mL})$ was then added and the mixture was diluted with $\mathrm{Et}_{2} \mathrm{O}(50$ $\mathrm{mL})$ and washed with $1 \mathrm{M} \mathrm{KHSO}_{4}(2 \times 10 \mathrm{~mL})$, water $(5 \times 10 \mathrm{~mL})$, brine $(2 \times 5 \mathrm{~mL})$, dried, filtered and concentrated under reduced pressure. The residue was recrystallized from $\mathrm{Et}_{2} \mathrm{O} /$ hexane to give the first crop of the acid $217(0.108 \mathrm{~g})$ as a colorless solid. The mother liquor was concentrated under reduced pressure and the residue was recrystallized twice from $\mathrm{Et}_{2} \mathrm{O} /$ hexane to give the second crop of the title compound $(0.012 \mathrm{~g}, 85 \%$ overall yield $) . R_{\mathrm{f}}=$ $0.06 \mathrm{EtOAc} /$ hexane 1:2 (1.5\% AcOH); ${ }^{1} \mathrm{H}$ NMR $\left(250 \mathrm{MHz}, \mathrm{CDCl}_{3}\right): \delta=1.13-1.47(\mathrm{~m}, 3 \mathrm{H}, 4-$ $\mathrm{H}, a-D a b), 1.35,1.39\left[2 \times \mathrm{s}, 9 \mathrm{H}, \mathrm{C}\left(\mathrm{CH}_{3}\right)_{3}\right], 1.64\left[\mathrm{~d}, J=5.8 \mathrm{~Hz}, 3 \mathrm{H}, 3^{\prime}-\mathrm{H},(4-P E)\right.$ Pro $], 1.73-$ 2.00 [m, $1 \mathrm{H}, 3-\mathrm{H}_{\mathrm{a}},(4-P E)$ Pro], 2.33 (s, $\left.3 \mathrm{H}, 1^{\prime}-\mathrm{H}, M e Z\right), 2.33-2.57$ [m, $1 \mathrm{H}, 3-\mathrm{H}_{\mathrm{b}}$, (4-PE)Pro], 2.87-3.20 [m, $2 \mathrm{H}, 4-\mathrm{H}, 5-\mathrm{H}_{\mathrm{a}}$, (4-PE)Pro], 3.48-3.73, 3.73-3.95 [2 × m, $1 \mathrm{H}, 5-\mathrm{H}_{\mathrm{b}},(4-P E)$ Pro], 4.07-4.29 (m, $1 \mathrm{H}, 3-\mathrm{H}, a-D a b), 4.41$ (d, J=6.5 Hz, $1 \mathrm{H}, 2-\mathrm{H}), 4.43-4.69$ (m, $1 \mathrm{H}, 2-\mathrm{H}), 5.03$ (s, $2 \mathrm{H}$, Bzl-H), 5.18-5.33 [m, $1 \mathrm{H}, 1^{\prime}-\mathrm{H}$, (4-PE)Pro], 5.53 [dq, $J=10.8,7.0 \mathrm{~Hz}, 1 \mathrm{H}, 2^{\prime}-\mathrm{H}$, (4PE)Pro], 5.83-6.02, 6.31-6.48 (2 $\times \mathrm{m}, 1 \mathrm{H}, \mathrm{NH}), 6.81(\mathrm{~d}, J=8.8 \mathrm{~Hz}, 1 \mathrm{H}, \mathrm{NH}), 7.14(\mathrm{~d}, J=$ 8.0 Hz, $2 \mathrm{H}, \mathrm{Ar}-\mathrm{H}), 7.21-7.38$ (br, $\left.1 \mathrm{H}, \mathrm{NH}, \mathrm{CO}_{2} \mathrm{H}\right), 7.23$ (d, J=8.0 Hz, $\left.2 \mathrm{H}, \mathrm{Ar}-\mathrm{H}\right)$.

\section{MeZ-a-Dab[Boc-(4-PE)Pro]-( $\beta M e) P h e-(R)-(3-N c p) A l a-(\beta M e) P h e-I l e-O D C P M(\mathbf{2 1 8})$ :}

Tetrapeptide $188 \mathrm{~b}(0.172 \mathrm{~g}, 0.186 \mathrm{mmol})$, after removal of the Fmoc-group according to GP 4, was coupled with ester acid 216 (0.104 g, $0.207 \mathrm{mmol})$ using HATU (79 mg, $0.208 \mathrm{mmol})$, HOAt (30 mg, $0.222 \mathrm{mmol})$ and TMP (75 mg, $0.619 \mathrm{mmol})$ in $\mathrm{CH}_{2} \mathrm{Cl}_{2}(5 \mathrm{~mL})$ according to GP 7 for $15 \mathrm{~h}$. The mixture was then diluted with $\mathrm{Et}_{2} \mathrm{O}(50 \mathrm{~mL})$, subjected to the usual aqueous work- 
up (GP 5), dried, filtered and concentrated under reduced pressure. The resulting oil was recrystallized from hexane, then purified by column chromatography (EtOAc/hexane 4:3, $R_{\mathrm{f}}=$ 0.34) and finally recrystallized from hexane again to give the branched hexapeptide $218(0.176$ g, 80\%) as a white solid. m.p. $101-103{ }^{\circ} \mathrm{C}$ (decomp.), $[\alpha]_{20}^{D} 52.8\left(c=0.29\right.$, THF); ${ }^{1} \mathrm{H}$ NMR $(600$ $\left.\mathrm{MHz}, \mathrm{CDCl}_{3}\right): \delta=0.34\left(\mathrm{dddd}, J=4.8,4.8,4.8,4.8 \mathrm{~Hz}, 1 \mathrm{H}, 2^{\prime}-\mathrm{H}, D C P M\right), 0.40(\mathrm{dddd}, J=4.8$, 4.8, 4.8, 4.8 Hz, $\left.1 \mathrm{H}, 2^{\prime}-\mathrm{H}, D C P M\right), 0.43$ (dddd, $J=4.8,4.8,4.8,4.8 \mathrm{~Hz}, 1 \mathrm{H}, 2^{\prime}-\mathrm{H}, D C P M$ ), 0.45-0.54 (m, 2 H, 2'-H, DCPM), 0.54-0.59 (m, 2 H, 2'-H, DCPM), 0.66 (ddddd, $J=4.2,4.2$, 4.2, 4.2, 4.2 Hz, $1 \mathrm{H}, 2^{\prime}-\mathrm{H}$, DCPM), 0.79 (d, J=6.6 Hz, $3 \mathrm{H}, 1^{\prime}-\mathrm{H}$, Ile), 0.84-0.92 [m, $1 \mathrm{H}, 1^{\prime}-\mathrm{H}$, (3-Ncp)Ala] 0.90 (t, $J=7.2 \mathrm{~Hz}, 3 \mathrm{H}, 5-\mathrm{H}$, Ile), 1.04 [ddd, $J=6.0,7.2,7.2 \mathrm{~Hz}, 1 \mathrm{H}, 3^{\prime}-\mathrm{H}_{\mathrm{a}}$, (3Ncp)Ala $], 1.06-1.14\left(\mathrm{~m}, 1 \mathrm{H}, 1^{\prime}-\mathrm{H}_{\mathrm{a}}, D C P M\right), 1.14-1.23\left(\mathrm{~m}, 1 \mathrm{H}, 1^{\prime}-\mathrm{H}_{\mathrm{b}}, D C P M\right), 1.23[\mathrm{~d}, J=$ 6.6 Hz, $3 \mathrm{H}, 4-\mathrm{H},(\beta M e) P h e], 1.25$ [d, J=6.6 Hz, $3 \mathrm{H}, 4-\mathrm{H},(\beta M e) P h e], 1.28-1.39\left[\mathrm{~m}, 3 \mathrm{H}, 4-\mathrm{H}_{\mathrm{b}}\right.$, Ile, 3-H, (3-Ncp)Ala], $1.33(\mathrm{~d}, J=7.2 \mathrm{~Hz}, 3 \mathrm{H}, 4-\mathrm{H}, a-\mathrm{Dab}), 1.34$ [s, $\left.9 \mathrm{H}, \mathrm{C}\left(\mathrm{CH}_{3}\right)_{3}\right], 1.45-1.53$ [m, $\left.1 \mathrm{H}, 3^{\prime}-\mathrm{H}_{\mathrm{b}},(3-N c p) A l a\right], 1.69$ [dd, $J=6.6,1.2 \mathrm{~Hz}, 3^{\prime}-\mathrm{H},(4-P E)$ Pro], 1.81 [ddd, $J=12.0$, 12.0, $12.0 \mathrm{~Hz}, 1 \mathrm{H}, 3-\mathrm{H}_{\mathrm{a}},(4-P E)$ Pro], 1.85-1.93 (m, $1 \mathrm{H}, 3-\mathrm{H}$, Ile), 2.32 (s, $\left.3 \mathrm{H}, 1^{\prime}-\mathrm{H}, M e Z\right)$, $2.36\left[\mathrm{ddd}, J=12.0,6.0,6.0 \mathrm{~Hz}, 3-\mathrm{H}_{\mathrm{b}},(4-P E)\right.$ Pro $], 3.12-3.23\left[\mathrm{~m}, 2 \mathrm{H}, 3-\mathrm{H},(\beta M e) P h e, 5-\mathrm{H}_{\mathrm{a}},(4-\right.$

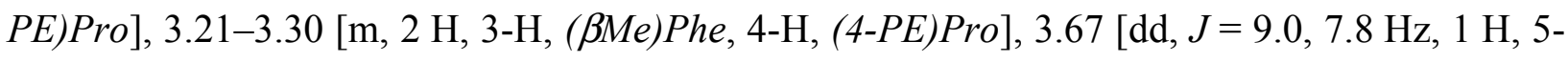
$\mathrm{H}_{\mathrm{b}}$, (4-PE)Pro], 3.89 [ddd, $\left.J=7.2,3.0,3.0 \mathrm{~Hz}, 1 \mathrm{H}, 2^{\prime}-\mathrm{H},(3-N c p) A l a\right], 4.14$ (t, $J=7.8 \mathrm{~Hz}, 1 \mathrm{H}$, 1-H, DCPM), 4.22 [dd, $J=4.8,2.4 \mathrm{~Hz}, 1 \mathrm{H}, 2-\mathrm{H},(4-P E)$ Pro], 4.24 (dd, $J=9.6,6.6 \mathrm{~Hz}, 1 \mathrm{H}, 2-$ $\mathrm{H}, a-D a b), 4.30[\mathrm{dd}, J=10.8,6.0 \mathrm{~Hz}, 1 \mathrm{H}, 2-\mathrm{H},(\beta M e) P h e], 4.34(\mathrm{dd}, J=9.3,4.5 \mathrm{~Hz}, 1 \mathrm{H}, 2-\mathrm{H}$, Ile), $4.60[\mathrm{ddd}, J=10.5,5.4,5.4 \mathrm{~Hz}, 1 \mathrm{H}, 2-\mathrm{H},(3-N c p) A l a], 4.62-4.70$ [m, $2 \mathrm{H}, 2-\mathrm{H},(\beta M e) P h e$, 3-H, $a-D a b], 5.00\left(\mathrm{~d}, J=12.0 \mathrm{~Hz}, \mathrm{Bzl}-\mathrm{H}_{\mathrm{a}}\right), 5.06$ (d, $\left.J=12.0 \mathrm{~Hz}, \mathrm{Bzl}-\mathrm{H}_{\mathrm{b}}\right), 5.26-5.33$ [m, $1 \mathrm{H}, 1^{\prime}-$ H, (4-PE)Pro], 5.56 [dq, $J=11.1,6.6 \mathrm{~Hz}, 1 \mathrm{H}, 2^{\prime}-\mathrm{H},(4-P E)$ Pro], 6.61 (d, $\left.J=6.6 \mathrm{~Hz}, 1 \mathrm{H}, \mathrm{NH}\right)$, $6.97(\mathrm{~d}, J=10.2 \mathrm{~Hz}, 1 \mathrm{H}, \mathrm{NH}), 7.01(\mathrm{~d}, J=9.0 \mathrm{~Hz}, 1 \mathrm{H}, \mathrm{NH}), 7.10(\mathrm{~d}, J=8.4 \mathrm{~Hz}, 2 \mathrm{H}, \mathrm{Ar}-\mathrm{H})$, 7.16-7.32 (m, $13 \mathrm{H}, \mathrm{Ar}-\mathrm{H}, \mathrm{NH}), 7.49$ (d, $J=9.6 \mathrm{~Hz}, 1 \mathrm{H}, \mathrm{NH}), 7.60$ (d, $J=9.6 \mathrm{~Hz}, 1 \mathrm{H}, \mathrm{NH})$; The absorptions of 4-H, $(\beta M e)$ Phe masked the peak of $4-\mathrm{H}_{\mathrm{a}}, I l e ;{ }^{13} \mathrm{C} \mathrm{NMR}\left(150.8 \mathrm{MHz}, \mathrm{CDCl}_{3}\right)$ : 
$\delta=2.5,2.8,2.8,3.0\left(-, \mathrm{C}-2^{\prime}\right.$, DCPM $), 11.6(+, \mathrm{C}-5$, Ile $), 13.2\left[+, \mathrm{C}-3^{\prime},(4-P E)\right.$ Pro $], 14.1,14.6(+$, C-1' $\left.{ }^{\prime}, D C P M\right), 15.7\left(+, \mathrm{C}-1^{\prime}\right.$, Ile $), 18.5[+, \mathrm{C}-4,(\beta M e) P h e], 18.6\left[-, \mathrm{C}-3^{\prime},(3-N c p)\right.$ Ala $], 19.7$ [+, C4, (ßMe)Phe], 19.9 (+, C-4, a-Dab), 21.1 (+, C-1', MeZ), 21.6 [+,C-1', (3-Ncp)Ala ], $25.2(-, \mathrm{C}-4$, Ile), $28.3\left[+, \mathrm{C}\left(\mathrm{CH}_{3}\right)_{3}\right], 30.8$ [-, C-3, (3-Ncp)Ala $], 36.2$ [-, C-3, (4-PE)Pro], 36.5 (+, C-3, Ile), $37.1[+, \mathrm{C}-4,(4-P E)$ Pro $], 40.2[+, \mathrm{C}-3,(\beta M e) P h e], 41.9[+, \mathrm{C}-3,(\beta M e) P h e], 46.3(+, \mathrm{C}-3, a-$ Dab), 50.8 [+, C-2, (3-Ncp)Ala], 52.5 [-, C-5, (4-PE)Pro], 56.6 (+, C-2, Ile), 59.5 [+, C-2', (3Ncp)Ala], 60.9 (+,C-2, a-Dab), 61.5 [+, C-2, (ßMe)Phe], 63.3 [+, C-2, (4-PE)Pro], 63.4 [+, C-2, (BMe)Phe], 66.7 (-, Bzl-C), $80.2\left[\mathrm{C}_{\text {quat }}, \mathrm{C}\left(\mathrm{CH}_{3}\right)_{3}\right], 83.1$ (+, C-1, DCPM), 126.7, 127.4, 127.6, 127.7, 128.4, 128.4, 128.9, 128.9, (+, Ar-C), 127.0 [+, C-2', (4-PE)Pro], $129.2\left[+, \mathrm{C}^{\prime} 1^{\prime},(4-\right.$ PE)Pro], 133.5, 137.7, 141.6, 141.7 ( $\left.\mathrm{C}_{\text {quat }}, \mathrm{Ar}-\mathrm{C}\right), 154.4,155.9\left(\mathrm{C}_{\text {quat }}, \mathrm{NCO}_{2}\right), 169.7,170.9$, 173.6, 173.6, 174.1, $174.1\left(\mathrm{C}_{\text {quat }}, \mathrm{C}-1\right)$; IR $(\mathrm{KBr}): v=3374 \mathrm{~cm}^{-1}, 3087,3010,2973,2934,2876$, $1730,1673,1545,1513,1390,1368,1162 ; \mathrm{MS}(\mathrm{ESI}):$ positive mode, $m / z(\%)=1212(100)[\mathrm{M}+$ $\left.\mathrm{Na}^{+}\right]$; negative mode, $m / z(\%)=1188(100)\left[\mathrm{M}-\mathrm{H}^{+}\right]$; elemental analysis calcd $(\%)$ for $\mathrm{C}_{65} \mathrm{H}_{88} \mathrm{~N}_{8} \mathrm{O}_{13}$ (1189.5): C 65.64, H 7.46, N 9.42; found C 65.63, H 7.22, N 9.26.

MeZ-Protected branched cyclohexapeptide (219a) and its epimer (219b): To the branched hexapeptide 218 (0.188 g, $0.165 \mathrm{mmol})$ was added freshly prepared $2 \mathrm{M} \mathrm{HCl}$ in EtOAc $(3 \mathrm{~mL})$. The reaction mixture was stirred for $1 \mathrm{~h}$ at $20^{\circ} \mathrm{C}$ in the dark room and then was concentrated under reduced pressure at $20^{\circ} \mathrm{C}$. The residue was recrystallized from $\mathrm{Et}_{2} \mathrm{O} /$ hexane to give the deprotected peptide as a white solid (0.145 g; MS (ESI): positive mode, $m / z(\%)=996(100)[\mathrm{M}$ $\left.+\mathrm{H}^{+}\right]$; negative mode, $\left.m / z(\%)=994(100)\left[\mathrm{M}-\mathrm{H}^{+}\right]\right)$, which was taken up with anhydrous $\mathrm{CH}_{2} \mathrm{Cl}_{2}$ (1.5 l) and cyclizated employing HATU $(2 \times 61 \mathrm{mg}, 2 \times 0.160 \mathrm{mmol})$ and HOAt $(2 \times 18$ $\mathrm{mg}, 2 \times 0.133 \mathrm{mmol})$ and solution of DIEA $(2 \times 55 \mathrm{mg}, 2 \times 0.426 \mathrm{mmol})$ in $\mathrm{CH}_{2} \mathrm{Cl}_{2}(2 \times 20 \mathrm{~mL})$ according to GP 8 for $16 \mathrm{~h}$. After this, the solvent was removed under reduced pressure, the residue was taken up with $\mathrm{Et}_{2} \mathrm{O}(50 \mathrm{~mL})$, and after usual aqueous work-up (GP 5), drying and filtration, the organic layer was concentrated under reduced pressure. The residue was purified 
first by column chromatography (acetone/hexane $1: 1.75, R_{\mathrm{f}}=0.29$ ) and then by recrystallization $\left(\mathrm{Et}_{2} \mathrm{O} /\right.$ pentane $)$ to give crude product $(0.081 \mathrm{~g})$, which contained two components according to analytical HPLC. The mixture was separated by preparative HPLC to give cyclodepsipeptide 219a (41 mg, 28\% on 2 steps) and its epimer 219b (28 mg, 19\% on 2 steps) as white solids. Preparative HPLC: isocratic, $75 \% \mathrm{MeCN}$ in $\mathrm{H}_{2} \mathrm{O}(0.1 \%$ TFA).

219a: analytical HPLC 1: column $B$, isocratic, $60 \% \mathrm{MeCN}$ in $\mathrm{H}_{2} \mathrm{O}(0.1 \%$ TFA), flow rate $=0.5$ $\mathrm{mL} / \min , t_{\mathrm{R}}=20.18 \mathrm{~min}$, purity $>99 \%$; analytical HPLC 2 : column $\mathrm{C}$, isocratic, $75 \% \mathrm{MeCN}$ in $\mathrm{H}_{2} \mathrm{O}(0.1 \% \mathrm{TFA})$, flow rate $=0.8 \mathrm{~mL} / \mathrm{min}, t_{\mathrm{R}}=14.83 \mathrm{~min}$, purity $>99 \%$; $[\alpha]_{20}^{D} 8.6(c=0.21$, $\left.\mathrm{CHCl}_{3}\right) ;{ }^{1} \mathrm{H} \mathrm{NMR}\left(600 \mathrm{MHz}, \mathrm{CDCl}_{3}\right): \delta=0.15-0.31\left[\mathrm{~m}, 1 \mathrm{H}, 3^{\prime}-\mathrm{H}_{\mathrm{a}},(3-N c p)\right.$ Ala $], 0.32-0.47[\mathrm{~m}$, $\left.1 \mathrm{H}, 3-\mathrm{H}_{\mathrm{a}},(3-N c p) A l a\right], 0.78\left(\mathrm{t}, J=7.2 \mathrm{~Hz}, 3 \mathrm{H}, 5-\mathrm{H}\right.$, Ile), 0.84 (d, J =6.6 Hz, $3 \mathrm{H}, 1^{\prime}-\mathrm{H}$, Ile), 0.88-0.97 [m, $1 \mathrm{H}, 1^{\prime}-\mathrm{H},(3-N c p)$ Ala], 0.97-1.06 (m, $1 \mathrm{H}, 4-\mathrm{H}_{\mathrm{a}}$, Ile), $1.08-1.25$ [m, $1 \mathrm{H}, 3-\mathrm{H}_{\mathrm{b}},(3-$ Ncp)Ala], $1.25[\mathrm{~d}, J=7.2 \mathrm{~Hz}, 3 \mathrm{H}, 4-\mathrm{H},(\beta M e) P h e], 1.26-1.34\left(\mathrm{~m}, 1 \mathrm{H}, 4-\mathrm{H}_{\mathrm{b}}\right.$, Ile $), 1.29[\mathrm{~d}, J=$ 7.2 Hz, $3 \mathrm{H}, 4-\mathrm{H},(\beta M e) P h e], 1.34-1.44\left[\mathrm{~m}, 1 \mathrm{H}, 3^{\prime}-\mathrm{H}_{\mathrm{b}},(3-N c p) A l a\right], 1.40(\mathrm{~d}, J=7.2 \mathrm{~Hz}, 3 \mathrm{H}, 4-$ $\mathrm{H}, a-D a b), 1.63-1.73(\mathrm{~m}, 1 \mathrm{H}, 3-\mathrm{H}$, Ile $), 1.66\left[\mathrm{~d}, J=6.6 \mathrm{~Hz}, 3^{\prime}-\mathrm{H},(4-P E)\right.$ Pro], 1.87 [ddd, $J=$ 11.4, 11.4, 11.4 Hz, 1 H, 3- $\mathrm{H}_{\mathrm{a}}$, (4-PE)Pro], 2.18 [ddd, $J=11.4,6.0,6.0 \mathrm{~Hz}, 1 \mathrm{H}, 3-\mathrm{H}_{\mathrm{b}}$, (4PE)Pro], 2.38 (s, $\left.3 \mathrm{H}, 1^{\prime}-\mathrm{H}, M e Z\right), 3.01$ [dq, $\left.J=1.2,7.2 \mathrm{~Hz}, 1 \mathrm{H}, 3-\mathrm{H},(\beta M e) P h e\right], 3.07-3.19$ [m, $1 \mathrm{H}, 4-\mathrm{H},(4-P E)$ Pro], 3.23 [dd, $J=9.6,9.6 \mathrm{~Hz}, 1 \mathrm{H}, 5-\mathrm{H}_{\mathrm{a}},(4-P E)$ Pro], 3.40-3.51 [m, $1 \mathrm{H}, 2-\mathrm{H}$, (3-Ncp)Ala], 3.64-3.71 [m, $1 \mathrm{H}, 3-\mathrm{H},(\beta M e) P h e], 3.71-3.79$ (m, $1 \mathrm{H}, 2-\mathrm{H}), 3.89$ [dd, $J=8.4$, 8.4 Hz, $1 \mathrm{H}$, 5-Hb , (4-PE)Pro], 3.89-3.98 (m, $1 \mathrm{H}, 2-\mathrm{H}), 4.28-4.34$ [m, 2 H, 2'-H, (3-Ncp)Ala, 3$\mathrm{H}, a-D a b], 4.34-4.50(\mathrm{~m}, 1 \mathrm{H}, 2-\mathrm{H}), 4.50-4.60(\mathrm{~m}, 2 \mathrm{H}, 2-\mathrm{H}), 4.97$ (d, J=12.0 Hz, Bzl-H $\left.{ }_{\mathrm{a}}\right), 5.19$ (d, $J=12.0 \mathrm{~Hz}, \mathrm{Bzl}-\mathrm{H}_{\mathrm{b}}$ ), 5.25 [dd, $J=9.6,9.6 \mathrm{~Hz}, 1 \mathrm{H}, 1^{\prime}-\mathrm{H},(4-P E)$ Pro], 5.58 [dq, $J=9.6$, 7.2 Hz, $1 \mathrm{H}, 2^{\prime}-\mathrm{H},(4-P E)$ Pro], 5.63-5.80 (br, $\left.1 \mathrm{H}, \mathrm{NH}\right), 6.04-6.37$ (br, $\left.1 \mathrm{H}, \mathrm{NH}\right), 6.51-6.67$ (br, $1 \mathrm{H}, \mathrm{NH}), 6.86-7.02$ (br, $1 \mathrm{H}, \mathrm{NH}), 7.02-7.12$ (m, $1 \mathrm{H}, \mathrm{NH}), 7.14-7.31$ (m, $15 \mathrm{H}, \mathrm{Ar}-\mathrm{H}, \mathrm{NH})$; ${ }^{13} \mathrm{C} \mathrm{NMR}\left(150.8 \mathrm{MHz}, \mathrm{CDCl}_{3}\right): \delta=10.5(+, \mathrm{C}-5$, Ile $), 13.2[+, \mathrm{C}-3$ ', (4-PE)Pro $], 13.5$ [+, C-4, (ßMe)Phe $], 14.9\left(+, \mathrm{C}-1^{\prime}\right.$, Ile $), 17.8$ [-, C-3', (3-Ncp)Ala], $18.0[+, \mathrm{C}-4,(\beta M e) P h e], 18.7(+, \mathrm{C}-4$, 
a-Dab), $21.0\left[+, \mathrm{C}-1^{\prime},(3-N c p) A l a\right], 21.1$ (+, C-1', MeZ), 24.3 (-, C-4, Ile), 32.7 [-, C-3, (3Ncp)Ala], 35.2 [-, C-3, (4-PE)Pro], 36.6 [+, C-4, (4-PE)Pro], 37.3 (+, C-3, Ile), 38.8 [+, C-3, (ßMe)Phe], $43.6[+, \mathrm{C}-3,(\beta M e) P h e], 47.5(+, \mathrm{C}-3, a-D a b), 52.5[+, \mathrm{C}-2,(3-N c p) A l a], 52.7$ [-, C-5, (4-PE)Pro], 54.5 (+, C-2), 58.9 [+, C-2 and C-2', (3-Ncp)Ala], $59.3(+, \mathrm{C}-2), 59.5$ (+, C-2), 62.3 [+, C-2, (4-PE)Pro], 67.0 (-, Bzl-C), 126.8, 127.1, 127.2, 127.5, 127.7, 128.0, 128.4, 128.5, 128.6, 129.1 [+, Ar-C, C-1', C-2', (4-PE)Pro], 133.4, 137.8, 141.4, 142.3 ( $\mathrm{C}_{\text {quat, }}$ Ar-C), 157.3 $\left(\mathrm{C}_{\text {quat }}, \mathrm{NCO}_{2}\right), 169.1,170.5(\times 2), 170.9(\times 2), 172.0\left(\mathrm{C}_{\text {quat }}, \mathrm{C}-1\right) ; \mathrm{IR}(\mathrm{KBr}): \mathrm{v}=3406 \mathrm{~cm}^{-1}, 3060$, 3029, 2969, 2936, 2877, 1670, 1634, 1542, 1517, 1452, 1369, 1205; MS (ESI): positive mode, $m / z(\%)=1000(100)\left[\mathrm{M}+\mathrm{Na}^{+}\right]$; negative mode, $m / z(\%)=976(100)\left[\mathrm{M}-\mathrm{H}^{+}\right] ;$HRMS (ESI): calcd for $\left[\mathrm{C}_{53} \mathrm{H}_{68} \mathrm{~N}_{8} \mathrm{O}_{10} \mathrm{Na}^{+}\right]$: 999.4951; found 999.4959.

219b: analytical HPLC 1: column $\mathrm{B}$, isocratic, $60 \% \mathrm{MeCN}$ in $\mathrm{H}_{2} \mathrm{O}(0.1 \% \mathrm{TFA})$, flow rate $=0.5$ $\mathrm{mL} / \min , t_{\mathrm{R}}=17.25 \mathrm{~min}$, purity $>97 \%$; analytical HPLC 2 : column $\mathrm{C}$, isocratic, $75 \% \mathrm{MeCN}$ in $\mathrm{H}_{2} \mathrm{O}(0.1 \%$ TFA $)$, flow rate $=0.8 \mathrm{~mL} / \mathrm{min}, t_{\mathrm{R}}=10.12 \mathrm{~min}$, purity $>97 \% ;[\alpha]_{20}^{D}-42.68 .6(c=$ $\left.0.27, \mathrm{CHCl}_{3}\right) ;{ }^{1} \mathrm{H}$ NMR $\left(600 \mathrm{MHz}, \mathrm{CDCl}_{3}\right): \delta=0.65-0.73\left(\mathrm{~m}, 3 \mathrm{H}, 1^{\prime}-\mathrm{H}, a-I l e\right), 0.84(\mathrm{t}, J=$ $7.2 \mathrm{~Hz}, 3 \mathrm{H}, 5-\mathrm{H}$, a-Ile), 0.89 [ddd, $J=5.4,5.4,5.4 \mathrm{~Hz}, 1 \mathrm{H}, 3^{\prime}-\mathrm{H}_{\mathrm{a}},(3-N c p)$ Ala], 1.10 [d, $J=$ $5.4 \mathrm{~Hz}, 1 \mathrm{H}, 4-\mathrm{H},(\beta M e) P h e], 1.10-1.15\left[\mathrm{~m}, 1 \mathrm{H}, 3-\mathrm{H}_{\mathrm{a}}{ }^{*},(3-N c p) A l a\right], 1.17-1.26[\mathrm{~m}, 4 \mathrm{H}, 4-\mathrm{H}$, (BMe)Phe, 4- $\left.\mathrm{H}_{\mathrm{a}}{ }^{*}, a-I l e\right], 1.40-1.48\left[\mathrm{~m}, 1 \mathrm{H}, 3-\mathrm{H}_{\mathrm{b}}{ }^{*},(3-N c p) A l a\right], 1.44(\mathrm{~d}, J=7.8 \mathrm{~Hz}, 3 \mathrm{H}, 4-\mathrm{H}$, $a-D a b), 1.50-1.59\left[\mathrm{~m}, 1 \mathrm{H}, 1^{\prime}-\mathrm{H},(3-N c p) A l a\right], 1.59-1.63$ [m, $2 \mathrm{H}, 3^{\prime}-\mathrm{H}_{\mathrm{b}},(3-N c p)$ Ala $\left., 3-\mathrm{H}, a-I l e\right]$, $1.63\left[\mathrm{~d}, J=6.6 \mathrm{~Hz}, 3^{\prime}-\mathrm{H},(4-P E)\right.$ Pro], 2.08-2.22 [m, $1 \mathrm{H}, 3-\mathrm{H}_{\mathrm{a}}$, (4-PE)Pro], 2.28 (s, $3 \mathrm{H}, 1^{\prime}-\mathrm{H}$, MeZ), 2.25-2.33 [m, $1 \mathrm{H}, 3-\mathrm{H}_{\mathrm{b}},(4-P E)$ Pro], 3.06 [ddddd, $J=7.8,7.8,7.8,7.8,7.8 \mathrm{~Hz}, 1 \mathrm{H}, 4-\mathrm{H}$,

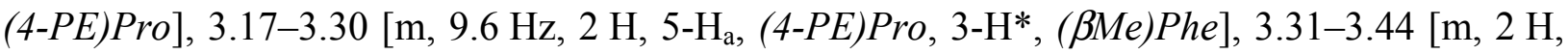

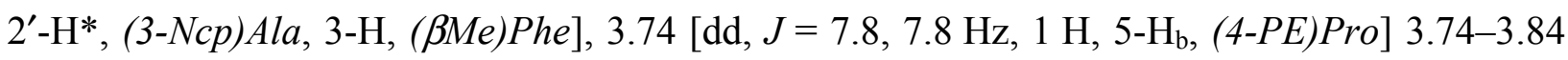
(m, 1 H, 2-H), 4.08-4.20 (m, 1 H, 3-H, $a-D a b), ~ 4.31-4.84(\mathrm{~m}, 2$ H, 2-H), 4.48-4.62 (m, 2 H, 2H), 4.73 (dd, $J=7.8,7.8 \mathrm{~Hz}, 1 \mathrm{H}, 2-\mathrm{H}), 4.98$ (d, $\left.J=12.0 \mathrm{~Hz}, \mathrm{Bzl}-\mathrm{H}_{\mathrm{a}}\right), 5.09$ (d, $J=12.0 \mathrm{~Hz}, \mathrm{Bzl}-$ $\left.\mathrm{H}_{\mathrm{b}}\right), 5.29\left[\mathrm{dd}, J=9.0,9.0 \mathrm{~Hz}, 1 \mathrm{H}, 1^{\prime}-\mathrm{H},(4-P E)\right.$ Pro], 5.52 [dq, $J=10.8,6.6 \mathrm{~Hz}, 1 \mathrm{H}, 2^{\prime}-\mathrm{H},(4-$ 
PE)Pro], 5.92-6.04 (br, $1 \mathrm{H}, \mathrm{NH}), 6.74$ (d, $J=7.2 \mathrm{~Hz}, 1 \mathrm{H}, \mathrm{NH}), 7.05-7.12$ (br, $1 \mathrm{H}, \mathrm{NH}), 7.07$ (d, $J=7.8 \mathrm{~Hz}, 2 \mathrm{H}, \mathrm{Ar}-\mathrm{H}), 7.16-7.29(\mathrm{~m}, 10 \mathrm{H}, \mathrm{Ar}-\mathrm{H}, \mathrm{NH}), 7.30-7.36(\mathrm{~m}, 4 \mathrm{H}, \mathrm{Ar}-\mathrm{H}, \mathrm{NH})$, 7.44-7.51 (br, $1 \mathrm{H}, \mathrm{NH})$; The absorption of $4-\mathrm{H}_{\mathrm{b}}$, a-Ile is masked by the signal of $3^{\prime}-\mathrm{H}$, (4PE)Pro; ${ }^{13} \mathrm{C}$ NMR (150.8 MHz, $\left.\mathrm{CDCl}_{3}\right): \delta=11.7$ (+, C-5, a-Ile), $13.2\left[+, \mathrm{C}-3^{\prime},(4-P E)\right.$ Pro $], 14.0$ (+, C-1', a-Ile), $17.3\left[{ }^{+}, \mathrm{C}-4,(\beta M e) P h e\right], 17.5(+, \mathrm{C}-4, a-D a b), 17.6\left[-, \mathrm{C}-3^{\prime},(3-N c p)\right.$ Ala $], 17.6$ [+,C-4, (BMe)Phe], $21.1\left(+, \mathrm{C}-1^{\prime}, \mathrm{MeZ}\right), 21.9$ [+,C-1', (3-Ncp)Ala], 26.3 (-, C-4, a-Ile), 31.8 [-, C-3, (3-Ncp)Ala], 34.2 [-, C-3, (4-PE)Pro], 36.1 [+, C-4, (4-PE)Pro], 36.9 (+, C-3, a-Ile), 40.4 [+, $2 \times \mathrm{C}-3,(\beta M e) P h e], 49.3(+, \mathrm{C}-3, a-D a b), 51.0(+, \mathrm{C}-2), 52.9$ [-, C-5, (4-PE)Pro $], 54.1(+$, C-2), $58.8\left[+, \mathrm{C}-2^{*},(3-N c p)\right.$ Ala $], 59.3\left(+, \mathrm{C}-2^{*}\right), 59.4\left(+, \mathrm{C}-2^{*}\right), 60.3(+, 2 \times \mathrm{C}-2), 67.2$ (-, BzlC), $126.7,127.0,127.6,127.7,128.2,128.3,128.5,128.7,129.0,129.1$ [+, Ar-C, C-1', C-2', (4PE)Pro], 132.8, 138.0, 142.0, $142.6\left(\mathrm{C}_{\text {quat }}, \mathrm{Ar}-\mathrm{C}\right), 156.2\left(\mathrm{C}_{\text {quat }}, \mathrm{NCO}_{2}\right), 170.3(\times 2), 170.8(\times 2)$, 170.9, $171.0\left(\mathrm{C}_{\text {quat }}, \mathrm{C}-1\right)$; IR (KBr): $v=3420 \mathrm{~cm}^{-1}, 3061,3030,2969,2934,2877,1654,1540$, 1453, 1369, 1270; MS (ESI): positive mode, $m / z(\%)=1000(100)\left[\mathrm{M}+\mathrm{Na}^{+}\right]$; negative mode, $m / z(\%)=976(100)\left[\mathrm{M}-\mathrm{H}^{+}\right]$.

Comparison of amino acid composition of cyclopeptides 219a and 219b: Aliquots of 219a and 219b (0.5 mg each) were hydrolyzed according to GP 1 and the resultant hydrolyzates were after concentration under reduced pressure treated with (S)-FDVA according to GP 2 and analyzed by HPLC/MS using the mixtures of (S)-FDVA derivatives of synthetic amino acids as standards. These experiments showed the only difference between these two products: cyclopeptide 219a contained $(R)$ - $a$-Ile residue and cyclopeptide $219 b$ contained Ile moiety.

$\left[a-D a b^{1}\right]-H o r m a o m y c i n\left[a-D a b^{1}\right]-(3)$ : An ethereal solution $(50 \mathrm{~mL})$ of the CHA salt of 197 (26.6 $\mathrm{mg}, 63.75 \mu \mathrm{mol})$ was washed with $1 \mathrm{M} \mathrm{H}_{2} \mathrm{SO}_{4}(3 \times 5 \mathrm{~mL}), 1 \mathrm{M} \mathrm{KHSO}_{4}(2 \times 5 \mathrm{~mL})$, water $(3 \times 5$ $\mathrm{mL})$, brine $(2 \times 5 \mathrm{~mL})$, dried, filtered and concentrated under reduced pressure. The resultant $N$ protected amino acid was dried at 0.02 Torr for $2 \mathrm{~h}$ and then coupled with the depsipeptide, 
obtained after deprotection of 219a $(19.5 \mathrm{mg}, 19.96 \mu \mathrm{mol})$ with $10 \%$ anisole in TFA $(1.1 \mathrm{~mL})$ according to GP 11 for $2 \mathrm{~h}$, using HATU (22.8 mg, $59.96 \mu \mathrm{mol})$, HOAt $(8.1 \mathrm{mg}, 59.94 \mu \mathrm{mol})$, DIEA $(2.57 \mathrm{mg}, 19.88 \mu \mathrm{mol})$ and TMP $(21.8 \mathrm{mg}, 179.00 \mu \mathrm{mol})$ in $\mathrm{CH}_{2} \mathrm{Cl}_{2}(3 \mathrm{~mL})$ according to GP 7 for $15 \mathrm{~h}$. The mixture was then diluted with $\mathrm{Et}_{2} \mathrm{O}(40 \mathrm{~mL})$ and the crude product obtained after usual aqueous work-up (GP 5) was purified by preparative TLC $(200 \times 200 \mathrm{~mm}$, acetone/hexane 1:2.7) to give Teoc-(S)-(3-Ncp)Ala-cyclo-hexapeptide $\left(21.6 \mathrm{mg}, 96 \%, R_{\mathrm{f}}=0.18\right.$ acetone/hexane 1:2.5) as a colorless glass which was used for the next step without any characterization. This substance $(21.6 \mathrm{mg}, 19.13 \mu \mathrm{mol})$ was deprotected with TFA $(2.0 \mathrm{~mL})$ for $1 \mathrm{~h}$. The mixture was concentrated under reduced pressure at $20^{\circ} \mathrm{C}$ and then taken up with toluene $(3 \times 15 \mathrm{~mL})$ which was distilled off to remove the last traces of TFA. The resultant deprotected branched peptide was coupled with $\mathrm{Chpca}(\mathrm{MOM})-\mathrm{OH} 109$ (7.0 mg, $34.04 \mu \mathrm{mol})$ using HATU (12.9 mg, $33.93 \mu \mathrm{mol})$, DIEA $(2.47 \mathrm{mg}, 19.13 \mu \mathrm{mol})$ and TMP (12.37 mg, 102.08 $\mu \mathrm{mol})$ in $\mathrm{CH}_{2} \mathrm{Cl}_{2}(3 \mathrm{~mL})$ according to $\mathrm{GP} 7$ for $2.5 \mathrm{~h}$. The mixture was then diluted with $\mathrm{Et}_{2} \mathrm{O}$ (40 mL) and the crude product obtained after usual aqueous work-up (GP 5) was purified by preparative TLC $(200 \times 200 \mathrm{~mm}$, acetone/hexane 1:2.7, 2 runs $)$ and finally by recrystallization from $\mathrm{Et}_{2} \mathrm{O} /$ hexane to give MOM-[a-Dab $\left.{ }^{1}\right]-3\left(20.2 \mathrm{mg}, 90 \%, R_{\mathrm{f}}=0.09\right.$ acetone/hexane $\left.1: 3\right)$ as a colorless solid which was used for the next step without any characterization. MOM- $\left[a-\mathrm{Dab}^{1}\right]-3$ (19.1 mg, $16.92 \mu \mathrm{mol})$ was deprotected using $\mathrm{MgBr}_{2} \cdot \mathrm{Et}_{2} \mathrm{O}(164 \mathrm{mg}, 633.89 \mu \mathrm{mol})$ and EtSH $(0.018 \mathrm{~mL}, 243.07 \mu \mathrm{mol})$ in $\mathrm{CH}_{2} \mathrm{Cl}_{2}(10 \mathrm{~mL})$ according to $\mathrm{GP} 10$ for $3 \mathrm{~h}$. The mixture was diluted $\mathrm{Et}_{2} \mathrm{O}(50 \mathrm{~mL})$ and the crude product obtained after usual aqueous work-up (GP 10) was recrystallized from $\mathrm{Et}_{2} \mathrm{O}$ /pentane and then from $\mathrm{CH}_{2} \mathrm{Cl}_{2} /$ pentane to give $\left[a-\mathrm{Dab}^{1}\right]-3$ (15.4 mg, $84 \%, 68 \%$ on 5 steps from 219 a) as a colorless solid. $R_{\mathrm{f}}=0.14$ acetone/hexane 1:2.5; analytical HPLC: column $\mathrm{B}$, gradient $25 \% \rightarrow 85 \% \mathrm{MeCN}$ in $0.15 \%$ ammonium acetate buffer $(\mathrm{pH}=5.5)$ for $25 \mathrm{~min}$, flow rate $=0.5 \mathrm{~mL} / \mathrm{min}, t_{\mathrm{R}}=21.72 \mathrm{~min}$, purity $>99 \% ;[\alpha]_{20}^{D} 23.0\left(c=0.1, \mathrm{CHCl}_{3}\right)$; ${ }^{1} \mathrm{H}$ NMR $\left(600 \mathrm{MHz}, \mathrm{CDCl}_{3}\right): \delta=-0.69\left[\mathrm{ddd}, J=6.6,6.6,6.6 \mathrm{~Hz}, 1 \mathrm{H}, 3{ }^{\prime}-\mathrm{H}_{\mathrm{a}},(3-N c p) A l a\right],-$ 
$0.17--0.07\left[\mathrm{~m}, 1 \mathrm{H}, 3-\mathrm{H}_{\mathrm{a}},(3-N c p) A l a\right], 0.20-0.27\left[\mathrm{~m}, 1 \mathrm{H}, 1^{\prime}-\mathrm{H},(3-N c p) A l a\right], 0.54$ [ddd, $J=$ 14.4, 4.8, 4.8 Hz, $\left.1 \mathrm{H}, 3-\mathrm{H}_{\mathrm{b}},(3-N c p) A l a\right], 0.90$ (t, $J=7.2 \mathrm{~Hz}, 3 \mathrm{H}, 5-\mathrm{H}$, Ile), 0.98-1.05 [m, $1 \mathrm{H}$, 3'- $\left.\mathrm{H}_{\mathrm{b}},(3-N c p) A l a\right], 1.03\left[\mathrm{ddd}, J=7.2,7.2,7.2 \mathrm{~Hz}, 1 \mathrm{H}, 3^{\prime}-\mathrm{H}_{\mathrm{a}},(3-N c p) A l a\right], 1.07$ (d, J= 7.2 Hz, $\left.3 \mathrm{H}, 1^{\prime}-\mathrm{H}, I l e\right), 1.32[\mathrm{~d}, J=7.2 \mathrm{~Hz}, 3 \mathrm{H}, 4-\mathrm{H},(\beta M e) P h e], 1.35[\mathrm{~d}, J=7.2 \mathrm{~Hz}, 3 \mathrm{H}, 4-\mathrm{H}$, ( $\beta M e) P h e], 1.41(\mathrm{~d}, J=7.8 \mathrm{~Hz}, 3 \mathrm{H}, a-D a b), 1.53-1.59\left(\mathrm{~m}, 1 \mathrm{H}, 4-\mathrm{H}_{\mathrm{b}}\right.$, Ile), 1.63-1.75 [m, $2 \mathrm{H}$, 3-H, (3-Ncp)Ala], 1.69 [dd, $J=7.2,1.8$ Hz, 3 H, 3'-H, (4-PE)Pro], 1.84-1.95 [m, 3 H, 3'- $\mathrm{H}_{\mathrm{b}}$, (3Ncp)Ala, 3-H, Ile, 3- $\mathrm{H}_{\mathrm{a}}$, (4-PE)Pro], 1.95-2.01 [m, $1 \mathrm{H}, 3-\mathrm{H}_{\mathrm{a}}$, (4-PE)Pro], 2.27 [ddd, J = 12.0, 6.0, 6.0 Hz, $1 \mathrm{H}, 4-\mathrm{H}_{\mathrm{b}},(4-P E)$ Pro], 2.85 [ddd, $J=6.6,4.8,4.8 \mathrm{~Hz}, 1 \mathrm{H}, 2^{\prime}-\mathrm{H},(3-N c p)$ Ala $], 3.02$ [dq, $J=11.4,7.2 \mathrm{~Hz}, 1 \mathrm{H}, 3-\mathrm{H},(\beta M e) P h e], 3.22-3.32$ [m, $\left.2 \mathrm{H}, 4-\mathrm{H}, 5-\mathrm{H}_{\mathrm{a}},(4-P E) P r o\right], 3.46-3.52$ [m, $1 \mathrm{H}, 2-\mathrm{H},(3-N c p) A l a], 3.70$ [dq, $J=3.6,7.2 \mathrm{~Hz}, 1 \mathrm{H}, 3-\mathrm{H},(\beta M e) P h e], 3.92[\mathrm{dd}, J=11.4$, $5.4 \mathrm{~Hz}, 2-\mathrm{H},(4-P E) P r o], 3.95-3.99$ [m, $1 \mathrm{H}, 5-\mathrm{H}_{\mathrm{b}},(4-P E)$ Pro], 4.03 [ddd, J = 7.2, 3.6, $3.6 \mathrm{~Hz}$, $\left.1 \mathrm{H}, 2^{\prime}-\mathrm{H},(3-N c p) A l a\right], 4.35(\mathrm{dd}, J=10.8,10.8 \mathrm{~Hz}, 1 \mathrm{H}, 2-\mathrm{H}), 4.42-4.48$ [m, $\left.1 \mathrm{H}, 3-\mathrm{H}, a-D a b\right]$, $4.45(\mathrm{dd}, J=10.2,4.2 \mathrm{~Hz}, 1 \mathrm{H}, 2-\mathrm{H}), 4.50(\mathrm{dd}, J=9.0,3.0 \mathrm{~Hz}, 1 \mathrm{H}, 2-\mathrm{H}), 4.66(\mathrm{dd}, J=9.0$, $9.0 \mathrm{~Hz}, 1 \mathrm{H}, 2-\mathrm{H}), 5.11-5.16$ [m, $1 \mathrm{H}, 2-\mathrm{H},(3-N c p) A l a], 5.26-5.31$ [m, $1 \mathrm{H}, 1^{\prime}-\mathrm{H},(4-P E)$ Pro], $5.63\left[\mathrm{dq}, J=10.8,6.6 \mathrm{~Hz}, 1 \mathrm{H}, 2^{\prime}-\mathrm{H},(4-P E) P r o\right], 6.15$ (d, J = 4.2 Hz, $1 \mathrm{H}, 4-\mathrm{H}$, Chpca), 6.43 (d, $J=7.8 \mathrm{~Hz}, 1 \mathrm{H}, \mathrm{NH}), 6.77-6.86$ (br, $1 \mathrm{H}, \mathrm{NH}), 6.85$ (d, J=4.2 Hz, $1 \mathrm{H}, 3-\mathrm{H}$, Chpca), 7.02-7.06 (m, $2 \mathrm{H}, \mathrm{Ar}-\mathrm{H}), 7.12-7.19(\mathrm{~m}, 6 \mathrm{H}, \mathrm{Ar}-\mathrm{H}, \mathrm{NH}), 7.21-7.30(\mathrm{~m}, 4 \mathrm{H}, \mathrm{Ar}-\mathrm{H}, \mathrm{NH}), 7.44(\mathrm{~d}, J=$ $10.8 \mathrm{~Hz}, 1 \mathrm{H}, \mathrm{NH}), 8.87$ (d, $J=9.0 \mathrm{~Hz}, 1 \mathrm{H}, \mathrm{NH}), 10.70-11.00(\mathrm{br}, 1 \mathrm{H}, \mathrm{OH})$; The signal of 4$\mathrm{H}_{\mathrm{a}}$, Ile was masked by absorptions of $\mathrm{C}-4,(\beta M e) P h e ;{ }^{13} \mathrm{C} \mathrm{NMR}\left(150.8 \mathrm{MHz}, \mathrm{CDCl}_{3}\right): \delta=10.4$ (+, C-5, Ile), $13.1\left[+, \mathrm{C}-3^{\prime},(4-P E)\right.$ Pro $], 13.3$ [+, C-4, a-Dab], 14.9 (+, C-1', Ile), 17.0 [-, C-3',

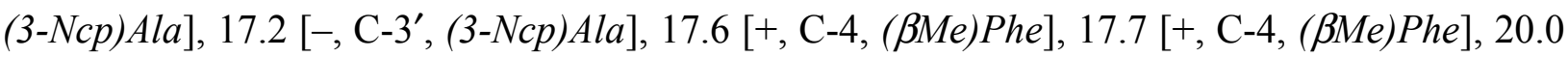
[+, C-1', (3-Ncp)Ala], 21.7 [+, C-1', (3-Ncp)Ala], 25.1 (-, C-3, Ile), 32.8 [-, C-3, (3-Ncp)Ala], 35.2 [-, C-3, (3-Ncp)Ala], 35.9 [-, C-3, (4-PE)Pro], 36.4 [+, C-4, (4-PE)Pro], 38.0 (+, C-3, Ile), $39.2[+, \mathrm{C}-3,(\beta M e) P h e], 41.2[+, \mathrm{C}-3,(\beta M e) P h e], 45.3(+, \mathrm{C}-3, a-D a b), 51.0[+, \mathrm{C}-2,(3-$ Ncp)Ala, 51.8 [+, C-2, (3-Ncp)Ala ], 53.1 [-, C-5, (4-PE)Pro], $54.6(+, \mathrm{C}-2), 55.2(+, \mathrm{C}-2), 58.1$ 
$\left[+, \mathrm{C}-2^{\prime},(3-N c p)\right.$ Ala $], 59.3\left[+, \mathrm{C}-2^{\prime},(3-N c p)\right.$ Ala $], 59.8(+, \mathrm{C}-2), 60.0(+, \mathrm{C}-2), 63.8[+, \mathrm{C}-2,(4-$

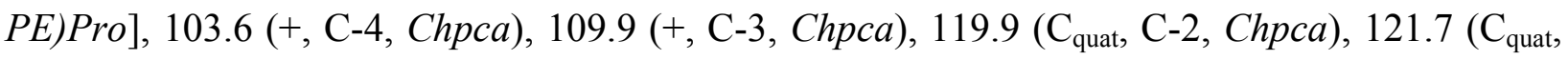
C-5, Chpca), 127.0, 127.3, $127.4(\times 2), 127.7,127.9,128.5(\times 2), 128.7$ [+, Ar-C, C-1', C-2',$(4-$ PE)Pro], 141.3, 142.2 (C $\left.\mathrm{C}_{\text {quat }}, \mathrm{Ar}-\mathrm{C}\right), 159.3$ (C $\mathrm{C}_{\text {quat }}, \mathrm{C}-1$, Chpca), 168.3, 169.6, 170.0, 170.2, 171.6 $(\times 2), 171.9\left(\mathrm{C}_{\text {quat }}, \mathrm{C}-1\right)$; UV $(\mathrm{MeOH})$ : neutral: $\lambda_{\max }(\varepsilon)=277\left(1.6 \times 10^{4}\right) \mathrm{nm}$; basic: $281(1.5 \times$ $\left.10^{4}\right), 205\left(7.0 \times 10^{4}\right) \mathrm{nm}$; acidic: $272\left(1.4 \times 10^{4}\right) \mathrm{nm} ; \mathrm{CD}(\mathrm{MeOH}): \lambda_{\max }[\Theta]=280.2\left(2.01 \times 10^{4}\right)$; $276.5\left(2.05 \times 10^{4}\right), 225.6\left(-4.55 \times 10^{-4}\right), 221.5\left(-5.06 \times 10^{4}\right) \mathrm{nm}\left(c=1.45 \times 10^{-5} \mathrm{M}\right) ; \mathrm{MS}(\mathrm{ESI})$ : positive, $m / z=1151(100)\left[\mathrm{M}+\mathrm{Na}^{+}\right]$; negative, $m / z=1127(100)\left[\mathrm{M}-\mathrm{H}^{+}\right]$; HRMS (ESI): calcd for $\left[\mathrm{C}_{55} \mathrm{H}_{70} \mathrm{~N}_{11} \mathrm{O}_{13} \mathrm{ClNa}^{+}\right]$: 1128.4916 ; found 1128.4921 .

$\left[a-D a b^{1}, a-I l e^{5}\right]-H o r m a o m y c i n\left[a-\mathrm{Dab}^{1}, a-\mathrm{Ile}^{5}\right]-(\mathbf{3})$ : An ethereal solution $(50 \mathrm{~mL})$ of the CHA salt of $197(27.3 \mathrm{mg}, 65.39 \mu \mathrm{mol})$ was washed with $1 \mathrm{M} \mathrm{H}_{2} \mathrm{SO}_{4}(3 \times 5 \mathrm{~mL}), 1 \mathrm{M} \mathrm{KHSO}_{4}(2 \times 5 \mathrm{~mL})$, water $(3 \times 5 \mathrm{~mL})$, brine $(2 \times 5 \mathrm{~mL})$, dried, filtered and concentrated under reduced pressure. The resultant $\mathrm{N}$-protected amino acid was dried at 0.02 Torr for $2 \mathrm{~h}$ and then coupled with the depsipeptide, obtained after deprotection of $\mathbf{2 1 9 b}(20.0 \mathrm{mg}, 20.47 \mu \mathrm{mol})$ with $10 \%$ anisole in TFA (1.1 mL) according to GP 11 for $2 \mathrm{~h}$, using HATU (23.4 mg, $61.54 \mu \mathrm{mol})$, HOAt (8.3 mg, $61.42 \mu \mathrm{mol})$, DIEA $(2.64 \mathrm{mg}, 20.39 \mu \mathrm{mol})$ and TMP $(22.36 \mathrm{mg}, 183.6 \mu \mathrm{mol})$ in $\mathrm{CH}_{2} \mathrm{Cl}_{2}(3 \mathrm{~mL})$ according to GP 7 for $15 \mathrm{~h}$. The mixture was then diluted with $\mathrm{Et}_{2} \mathrm{O}(40 \mathrm{~mL})$ and the crude product obtained after usual aqueous work-up (GP 5) was purified by two crystallizations from $\mathrm{Et}_{2} \mathrm{O} /$ hexane to give Teoc-(S)-(3-Ncp)Ala-epi-cyclo-hexapeptide $\left(17.0 \mathrm{mg}, 74 \%, R_{\mathrm{f}}=0.19\right.$ acetone/hexane 1:2.5) as a colorless glass which was used for the next step without any characterization. This substance $(17.0 \mathrm{mg}, 14.50 \mu \mathrm{mol})$ was deprotected with TFA $(2.0 \mathrm{~mL})$ for $1 \mathrm{~h}$. The mixture was concentrated under reduced pressure at $20^{\circ} \mathrm{C}$ and then taken up with toluene $(3 \times 15 \mathrm{~mL})$ which was distilled off to remove the last traces of TFA. The resultant deprotected branched peptide was coupled with $\mathrm{Chpca}(\mathrm{MOM})-\mathrm{OH} 109$ (5.5 mg, $26.74 \mu \mathrm{mol})$ 
using HATU (10.15 mg, $26.69 \mu \mathrm{mol})$, DIEA (1.95 mg, $15.09 \mu \mathrm{mol})$ and TMP (12.37 mg, 102.08 $\mu \mathrm{mol})$ in $\mathrm{CH}_{2} \mathrm{Cl}_{2}(3 \mathrm{~mL})$ according to $\mathrm{GP} 7$ for $2.5 \mathrm{~h}$. The mixture was then diluted with $\mathrm{Et}_{2} \mathrm{O}$ (40 mL) and the crude product obtained after usual aqueous work-up (GP 5) was crystallized twice from $\mathrm{CH}_{2} \mathrm{Cl}_{2} /$ hexane to give MOM- $\left[a-\mathrm{Dab}^{1}, a-\mathrm{Ile}^{5}\right]-3 \quad\left(14.2 \mathrm{mg}, 80 \%, R_{\mathrm{f}}=0.11\right.$ acetone/hexane 1:3) as a colorless solid which was used for the next step without any characterization. MOM- $\left[a-\mathrm{Dab}^{1}, a-\mathrm{Ile}^{5}\right]-3 \quad(14.2 \mathrm{mg}, 12.11 \mu \mathrm{mol})$ was deprotected using $\mathrm{MgBr}_{2} \cdot \mathrm{Et}_{2} \mathrm{O}(110 \mathrm{mg}, 425.17 \mu \mathrm{mol})$ and $\mathrm{EtSH}(0.018 \mathrm{~mL}, 243.07 \mu \mathrm{mol})$ in $\mathrm{CH}_{2} \mathrm{Cl}_{2}(10 \mathrm{~mL})$ according to GP 10 for $3 \mathrm{~h}$. The mixture was taken up with $\mathrm{Et}_{2} \mathrm{O}(50 \mathrm{~mL})$ and the crude product obtained after usual aqueous work-up (GP 10) was recrystallized from $\mathrm{Et}_{2} \mathrm{O}$ /pentane and then from $\mathrm{CH}_{2} \mathrm{Cl}_{2}$ /pentane to give the crude product $(13.1 \mathrm{mg})$, which was finally purified by preparative HPLC to give $\left[a-\mathrm{Dab}^{1}, a-\mathrm{Ile}^{5}\right]-3(9.0 \mathrm{mg}, 39 \%$ on 5 steps from 219b)as a colorless solid. $R_{\mathrm{f}}=0.14$ acetone/hexane 1:2.5; preparative HPLC: Nucleosil RP 18, JASCO, $250 \times 8 \mathrm{~mm}$, $62 \% \mathrm{MeCN}$ in $\mathrm{H}_{2} \mathrm{O}(0.07 \% \mathrm{TFA})$, flow rate $=2.5 \mathrm{~mL} / \mathrm{min}$; analytical HPLC: the same column, the same conditions, $t_{\mathrm{R}}=17.72 \mathrm{~min}$, purity $>99 \%$; MS (ESI): positive, $m / z=1151(100)[\mathrm{M}+$ $\left.\mathrm{Na}^{+}\right] ; 1129\left[\mathrm{M}+\mathrm{H}^{+}\right]$; negative, $m / z=1127(100)\left[\mathrm{M}-\mathrm{H}^{+}\right]$.

MeZ-N $N_{\beta} M e-a-D a b(F m o c)-O M e$ (222): $O$-TBDMS Derivate of $N M e-Z$ protected tert-Butyl ester

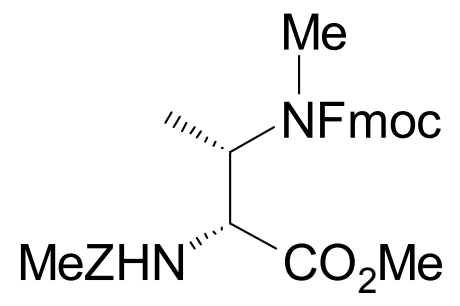
of (S)-isothreonine $220(0.73 \mathrm{~g}, 1.67 \mathrm{mmol})$ was dissolved in $\mathrm{MeCN}(38 \mathrm{~mL})$ and treated with $5 \%$ aqueous $\mathrm{HF}(40 \mathrm{~mL})$ at $4{ }^{\circ} \mathrm{C}$ for $10 \mathrm{~min}$. The mixture was allowed to warm to $20^{\circ} \mathrm{C}$ and stirring continued for a further $4 \mathrm{~h}$. A saturated aqueous $\mathrm{NaHCO}_{3}$ was then carefully added to adjust $\mathrm{pH}$ to ca. 8 and the mixture was extracted with $\mathrm{Et}_{2} \mathrm{O}$ $(2 \times 50 \mathrm{~mL})$. The organic fraction was washed with water $(5 \times 20 \mathrm{~mL})$, brine $(2 \times 10 \mathrm{~mL})$, dried, filtered and concentrated under reduced pressure. The resultant crude alcohol was dried under 0.02 Torr for $2 \mathrm{~h}$ and then deprotected with TFA $(6 \mathrm{~mL})$. After $1 \mathrm{~h}$, all volatiles were removed under reduced pressure, the residue was dissolved in toluene $(2 \times 20 \mathrm{~mL})$ which was distilled off 
to remove the last traces of TFA. The residue was dried under 0.02 Torr for $2 \mathrm{~h}$ and then taken up with $\mathrm{Et}_{2} \mathrm{O}$ (10 mL; some quantity of methanol was added to obtain homogeneous solution) and treated with excess of diazomethane till the steady yellow coloration of the mixture was obtained. The mixture was then concentrated under reduced pressure and the residue was purified by column chromatography to give $N \mathrm{Me}-\mathrm{Z}$ protected $(S)$-isothreonine methyl ester $\left(0.361 \mathrm{~g}, 71 \%, \mathrm{R}_{\mathrm{f}}=0.22 \mathrm{EtOAc} /\right.$ hexane $\left.1: 3\right)$ as a turbid oil which was directly used for the next step. Mesyl chloride $(0.14 \mathrm{~mL}, 1.81 \mathrm{mmol})$ as added dropwise to a solution of this substance $(0.36 \mathrm{~g}, 1.28 \mathrm{mmol})$ and TEA $(0.254 \mathrm{~mL}, 1.81 \mathrm{mmol})$ in $\mathrm{CH}_{2} \mathrm{Cl}_{2}(7 \mathrm{~mL})$ at $-30{ }^{\circ} \mathrm{C}$ for $3 \mathrm{~min}$ and stirring continued at the same temperature for $1 \mathrm{~h}$. The mixture was then allowed to warm to $4{ }^{\circ} \mathrm{C}$ and it was stirred at this temperature for a further $1 \mathrm{~h}$. Finally, the cooling bath was removed and stirring continued at $20^{\circ} \mathrm{C}$ for an additional $3 \mathrm{~h}$. Saturated aqueous $\mathrm{NaHCO}_{3}(3$ $\mathrm{mL})$ was then added and the mixture was taken up with $\mathrm{Et}_{2} \mathrm{O}(50 \mathrm{~mL})$. After usual aqueous work-up (GP 5) the organic layer was dried, filtered and concentrated under reduced pressure to give crude mesylate of $\mathrm{NMe}-\mathrm{Z}$ protected $(S)$-isothreonine methyl ester $(0.46 \mathrm{~g}, 100 \%$ crude yield, $R_{\mathrm{f}}=0.11$, EtOAc/hexane 1:6) as a colorless oil. $\mathrm{NaN}_{3}(0.086 \mathrm{~g}, 1.32 \mathrm{mmol})$ was added to a solution of the mesylate $(0.46 \mathrm{~g}, 1.28 \mathrm{mmol})$ in DMF $(8 \mathrm{~mL})$ and stirring continued at $70{ }^{\circ} \mathrm{C}$ for 14.5 h. The mixture was then cooled, concentrated under reduced pressure and the residue was taken up with $\mathrm{Et}_{2} \mathrm{O}(50 \mathrm{~mL})$. After usual aqueous work-up (GP 5) the organic layer was dried, filtered and concentrated under reduced pressure. The resultant crude product was purified by column chromatography (EtOAc/hexane $\left.1: 6, \mathrm{R}_{\mathrm{f}}=0.19\right)$ to give methyl $(2 R, 3 R)-N \mathrm{Me}-\mathrm{Z}$ protected 2-azido-butanoate $(0.191 \mathrm{~g}, 49 \%)$ as a mobile colorless oil. This substance $(0.191 \mathrm{~g}$, $0.62 \mathrm{mmol})$ was dissolved in $\mathrm{THF} / \mathrm{H}_{2} \mathrm{O}(20: 1)(15.8 \mathrm{~mL}), \mathrm{Ph}_{3} \mathrm{P}(0.262 \mathrm{~g}, 1.00 \mathrm{mmol})$ was added and stirring continued for $24 \mathrm{~h}$. $\mathrm{Boc}_{2} \mathrm{O}(0.272 \mathrm{~g}, 1.25 \mathrm{mmol})$ was then added and stirring continued for a further $24 \mathrm{~h}$. The mixture was then concentrated and the residue was purified by column chromatography (twice, hexane $1: 4, \mathrm{R}_{\mathrm{f}}=0.22$ ) to give $\mathrm{N}_{\beta}$-methylated Boc- $a$-Dab(Z)OMe $(0.135 \mathrm{~g}, 57 \%)$ as a viscous colorless oil. This material $(0.135 \mathrm{~g}, 0.35 \mathrm{mmol})$ was 
hydrogenated on $10 \% \mathrm{Pd}$ on charcoal $(0.07 \mathrm{~g})$ under ambient pressure of hydrogen in EtOAc (7 $\mathrm{mL}$ ) for $3 \mathrm{~h}$. The mixture was then filtered and concentrated under reduced pressure to give crude Boc- $a-\mathrm{N}_{\beta}$ MeDab-OMe. FmocOPfp $(0.159 \mathrm{~g}, 0.39 \mathrm{mmol})$ was added to a solution of this material, TMP (43 mg, $0.35 \mathrm{mmol})$ and a little bit of HOAt in EtOAc $(5 \mathrm{~mL})$ and stirring continued for $15 \mathrm{~h}$. The mixture was then taken up with $\mathrm{Et}_{2} \mathrm{O}(50 \mathrm{~mL})$ and subjected to usual aqueous work-up (GP 5). The organic layer was dried, filtered and concentrated under reduced pressure. The residue was purified by column chromatography (EtOAc/hexane 1:4, $\left.\mathrm{R}_{\mathrm{f}}=0.22\right)$ to give $N_{\beta}$-methylated Boc- $a$-Dab(Fmoc)-OMe $(0.135 \mathrm{~g}, 81 \%)$ as a colorless oil. This material $(0.135 \mathrm{~g}, 0.29 \mathrm{mmol})$ was deprotected with $2 \mathrm{M} \mathrm{HCl}$ in EtOAc $(4 \mathrm{~mL})$ for $1 \mathrm{~h}$. The mixture was then concentrated under reduced pressure and the residue was dissolved in $\mathrm{MeCN}(4 \mathrm{~mL}), \mathrm{TMP}$ (45 mg, $0.37 \mathrm{mmol}$ ), DIEA (37 mg, $0.29 \mathrm{mmol})$ and finally MeZOSu (83 mg, $0.32 \mathrm{mmol}$ ) were added to this solution and stirring continued for a further 16 h. $N, N$-Dimethylaminopropylamine (20 $\mathrm{mg}, 0.20 \mathrm{mmol}$ ) was then added and after $10 \mathrm{~min}$ the mixture was concentrated under reduced pressure. The residue was taken up with $\mathrm{Et}_{2} \mathrm{O}$ and subjected to usual aqueous work-up (GP 5). The organic layer was dried, filtered and concentrated under reduced pressure. The resultant crude product was purified by column chromatography (EtOAc/hexane 1:3, $R_{\mathrm{f}}=0.30$ ) to give $222(0.122 \mathrm{~g}, 13 \%$ overall yield on 10 steps $)$ as a turbid glass. $[\alpha]_{20}^{D} 9.2(c=0.25$, $\left.\mathrm{CHCl}_{3}\right) ;{ }^{1} \mathrm{H}$ NMR $\left(300 \mathrm{MHz}, \mathrm{CDCl}_{3}\right): \delta=1.16,1.25(2 \times \mathrm{d}, J=6.9 \mathrm{~Hz}, 3 \mathrm{H}, 4-\mathrm{H}), 2.33(\mathrm{~s}, 3 \mathrm{H}$, 1'-H, MeZ), 2.81 (s, 3 H, NMe), 3.66 (s, 3 H, OMe), 4.12-4.61 (m, 5 H, 2-H, 3-H and 1'-H, 2'Fmoc), $5.06(\mathrm{~d}, J=5.1 \mathrm{~Hz}, 2 \mathrm{H}, \mathrm{Bzl}-\mathrm{H}), 5.31,5.60(2 \times \mathrm{d}, J=7.5 \mathrm{~Hz}, 1 \mathrm{H}, \mathrm{NH}), 7.15(\mathrm{~d}, J=$ $7.8 \mathrm{~Hz}, 2 \mathrm{H}, 2-\mathrm{H}, M e Z), 7.24$ (d, J=7.8 Hz, $2 \mathrm{H}, 3-\mathrm{H}, M e Z), 7.31$ (dd, J= 7.5, 7.5 Hz, $2 \mathrm{H}, 3-\mathrm{H}$, Fmoc), 7.40 (dd, $J=7.5,7.5 \mathrm{~Hz}, 2 \mathrm{H}, 4-\mathrm{H}, F m o c), 7.58-7.64(\mathrm{~m}, 2 \mathrm{H}, 2-\mathrm{H}, F m o c), 7.76(\mathrm{~d}, J=$ $7.2 \mathrm{~Hz}, 5-\mathrm{H}, \mathrm{Fmoc}) ;{ }^{13} \mathrm{C}$ NMR $\left(50.3 \mathrm{MHz}, \mathrm{CDCl}_{3}\right): \delta=14.3(+, \mathrm{C}-4), 21.0\left(+, \mathrm{C}-1^{\prime}, \mathrm{MeZ}\right), 28.9$ (+, NMe), 47.1 (+, C-3), 52.4 (+, C-2), 52.9 (+, C-1', Fmoc), $56.6(+, \mathrm{OMe}), 67.0$ (-, Bzl-H, MeZ), 67.5 (-, C-2', Fmoc), 119.8, 124.9, 126.9, 127.5, 128.2, 129.1 (+, Ar-C), 133.0 (C quat ArC), $137.9\left(\mathrm{C}_{\text {quat }}, \mathrm{Ar}-\mathrm{C}\right), 141.2\left(\mathrm{C}_{\text {quat }}, \mathrm{Ar}-\mathrm{C}\right), 143.8,143.9\left(\mathrm{C}_{\text {quat }}, \mathrm{Ar}-\mathrm{C}\right), 155.9,156.4\left(\mathrm{C}_{\text {quat, }}\right.$, 
$\left.\mathrm{NCO}_{2}\right), 170.9\left(\mathrm{C}_{\text {quat }}, \mathrm{C}-1\right)$; IR $(\mathrm{KBr}): \mathrm{nu}($ tilde $)=2951 \mathrm{~cm}^{-1}, 1751,1725,1700,1521,1451,1320$, 1273, 1242, 1204, 1018; MS (ESI): positive, $m / z=539(100)\left[\mathrm{M}+\mathrm{Na}^{+}\right]$.

$M e Z-N_{\beta} M e-a-D a b[B o c-(4-P E) P r o]-O M e$ (223): Compound 222 (0.108 g, $\left.0.21 \mathrm{mmol}\right)$ was deprotected according to GP 4 and the resultant crude monodeprotected diamino ester was coupled with $N$-Boc protected 4-(Z)-propenylproline 16 (64 mg, $0.25 \mathrm{mmol}$ ) using EDC (48 mg, $0.25 \mathrm{mmol})$, HOAt $(34 \mathrm{mg}, 0.25 \mathrm{mmol})$ and TMP (76 mg, $0.63 \mathrm{mmol})$ in $\mathrm{CH}_{2} \mathrm{Cl}_{2}(3 \mathrm{~mL})$ according to GP 5 for $6 \mathrm{~h}$. The crude product, obtained after usual aqueous work-up (GP 5), was finally purified by column chromatography (EtOAc/hexane $\left.1: 1.5, R_{\mathrm{f}}=0.35\right)$ to give $\mathbf{2 2 3}(88 \mathrm{mg}$, $79 \%)$ as a turbid oil. Analytical HPLC: column B, gradient $20 \% \rightarrow 90 \% \mathrm{MeCN}$ in water $(0.1 \%$ $\mathrm{TFA})$ for $35 \mathrm{~min}$, flow rate $=0.5 \mathrm{~mL} / \mathrm{min}, t_{\mathrm{R}}=26.01 \mathrm{~min}$, purity $>97 \% ;[\alpha]_{20}^{D} 12.0(c=0.35$, $\left.\mathrm{CHCl}_{3}\right) ;{ }^{1} \mathrm{H} \mathrm{NMR}\left(300 \mathrm{MHz}, \mathrm{C}_{2} \mathrm{D}_{2} \mathrm{Cl}_{4}, 373 \mathrm{~K}\right): \delta=1.31(\mathrm{~d}, J=7.2 \mathrm{~Hz}, 3 \mathrm{H}, 4-\mathrm{H}$, NMe- $a-D a b)$, $1.42\left[\mathrm{~s}, 9 \mathrm{H}, \mathrm{C}\left(\mathrm{CH}_{3}\right)_{3}\right], 1.50-1.74\left[\mathrm{~m}, 1 \mathrm{H}, 3-\mathrm{H}_{\mathrm{a}},(4-P E)\right.$ Pro], $1.67\left[\mathrm{dd}, J=7.2,1.8 \mathrm{~Hz}, 3 \mathrm{H}, 3^{\prime}-\mathrm{H}\right.$, (4-PE)Pro], 2.26-2.44 [m, 1 H, 3-Hb (4-PE)Pro], 2.36 (s, 3 H, 1'-H, MeZ), 2.90 (s, 3 H, NMe), 3.01-3.21 [m, $2 \mathrm{H}, 4-\mathrm{H}, 5-\mathrm{H}_{\mathrm{a}},(4-P E)$ Pro], 3.23-3.48 [m, 0.5 H, 5-H,$\left.(4-P E) P r o\right], 3.74$ (s, $3 \mathrm{H}$, OMe), 4.47 (dd, $J=7.8,7.8 \mathrm{~Hz}, 1 \mathrm{H}, 3-\mathrm{H}, N M e-a-D a b), 4.57$ (dd, $J=7.8,7.8 \mathrm{~Hz}, 1 \mathrm{H}, 2-\mathrm{H}$, NMe-a-Dab), 4.50-4.95 [m, 1 H, C-2, (4-PE)Pro], 5.08 (s, 2 H, Bzl-H), 5.25-5.36 [m, 1 H, 1'-H, (4-PE)Pro], 5.50-5.85 (br, $1 \mathrm{H}, \mathrm{NH}), 5.54$ [dq, $\left.J=11.4,7.2 \mathrm{~Hz}, 1 \mathrm{H}, 2^{\prime}-\mathrm{H},(4-P E) P r o\right], 7.15$ (d, $J=7.8 \mathrm{~Hz}, 2 \mathrm{H}, \mathrm{Ar}-\mathrm{H}), 7.23(\mathrm{~d}, J=7.8 \mathrm{~Hz}, 2 \mathrm{H}, \mathrm{Ar}-\mathrm{H})$; The peak of OMe masked absorption of $0.5 \mathrm{H}, 5-\mathrm{H}_{\mathrm{b}}$ of $(4-P E)$ Pro $;{ }^{13} \mathrm{C} \mathrm{NMR}\left(75.5 \mathrm{MHz}, \mathrm{C}_{2} \mathrm{D}_{2} \mathrm{Cl}_{4}, 373 \mathrm{~K}\right): \delta=12.7$ [+, C-3', (4-PE)Pro], $14.5(+, \mathrm{C}-4, \mathrm{NMe}-a-D a b), 20.7\left(+, \mathrm{C}-1^{\prime}, \mathrm{MeZ}\right), 28.2\left(+, \mathrm{C}\left(\mathrm{CH}_{3}\right)_{3}, \mathrm{NMe}\right), 36.0[+, \mathrm{C}-4$, (4PE)Pro], 51.9 [-, C-5, (4-PE)Pro], 52.1 (+, C-2, NMe-a-Dab), 57.2 (+, OMe), 66.7 (-, Bzl-H, $M e Z), 79.3\left[\mathrm{C}_{\text {quat }}, \mathrm{C}\left(\mathrm{CH}_{3}\right)_{3}\right], 125.8\left[+, \mathrm{C}-2^{\prime},(4-P E)\right.$ Pro $], 127.7,128.8(+, \mathrm{Ar}-\mathrm{C}), 130.0\left[+, \mathrm{C}-1^{\prime}\right.$, (4-PE)Pro], 133.3, $137.5\left(\mathrm{C}_{\text {quat }}, \mathrm{Ar}-\mathrm{C}\right), 153.5,155.6\left(\mathrm{C}_{\text {quat, }}, \mathrm{NCO}_{2}\right), 170.6,173.2\left(\mathrm{C}_{\text {quat }}, \mathrm{C}-1\right)$; The signals of C-2, C-3 of (4-PE)Pro and C-3 of NMe- $a$-Dab were unobservable because of their low 
intensity; IR (KBr): nu(tilde) $=2977 \mathrm{~cm}^{-1}, 1751$ 1728, 1700, 1521, 1402, 1281, 1163; MS (ESI): positive, $m / z=554(100)\left[\mathrm{M}+\mathrm{Na}^{+}\right]$. 


\section{Summary}

This work has been focused on the synthesis of hormaomycin $\mathbf{3}$ and analogs, and pror to that, on the assignment of the absolute structure of unusual amino acids, which comprise hormaomycin, their stereoselective synthesis, and development of methodology of the fine "tuning" of protecting groups applicable towards the synthesis of complicated objects and particularly cyclic peptides with very rare amino acids.

$N$-Boc protected cis- and trans-4-(Z)-propenylprolines, cis-16 and trans-16b, were obtained after HPLC separation of the mixture of diastereomers, prepared in high yield by reaction between tosylate 15 and lithium di-(Z)-propenylcuprate. After removal of the Boc groups and derivatization with $p$-dimethylaminoazobenzenesulfonyl chloride, they, together with derivatizated total hydrolyzate of hormaomycin 3, were used for HPLC experiments, which showed that the (4-PE)Pro moiety of natural $\mathbf{3}$ had cis-configuration.

The results of LC/MS experiments according to the advanced Marfey method with the total hydrolizate of the hydrogenated hormaomycin allowed one to assign the $(2 S, 4 R)$ absolute configuration of the (4-PE)Pro fragment unambigously. Synthetic 4-propylprolines, $(2 S, 4 R)-29$ and $(2 R, 4 S)-29$, were used as standards in this set of experiments.

The developed stereoselective synthesis of the $N$-Boc-protected $(2 S, 4 R)-4-(Z)$-propenylproline $(2 S, 4 R)-16$, starting from $(2 S, 4 R)-\mathrm{Boc}-\mathrm{Hyp}-\mathrm{OH} \mathbf{4 3}$, allowed to prepare this compound on a multigramm scale in $16 \%$ yield over 8 steps. 
Based on this protocol, labelled $(2 S, 4 R)-\left(4,1^{\prime}\right)$-dideutero-4-(Z)-propenylproline $(2 S, 4 R)-\mathrm{D}_{2}-17$ was synthesized. A feeding experiment with this amino acid was successful and confirmed the previously proposed absolute configuration of the (4-PE)Pro fragment of $\mathbf{3}$.

A preparation of the enantiomerically pure $\left(2 S, 1^{\prime} R S, 2^{\prime} R S\right)$-2-deutero-3-(2'-nitrocyclopropyl)alanine D-(2S, $\left.1^{\prime} R S, 2^{\prime} R S\right)-\mathbf{8 3}$ by alkylation of the Belokon' glycine chiral equivalent became feasible after the deuterium-labelled nickel complex $(S)-\mathrm{D}_{2}-\mathbf{8 1}$ had been synthesized. Alkylation of the latter with racemic trans-(2'-nitrocyclopropyl)methyl iodide $\mathbf{8 0}$ was followed by acidolytic decomposition of the intermediate product and gave D-(2S,1'RS,2'RS)-83 in $48 \%$ yield over 2 steps. It is noteworthy, that deuteration at C-2' of the nitrocyclopropyl ring also occurred. The corresponding feeding experiment allowed one to assign the configuration at C-2 of the (3Ncp)Ala residue in the cyclic part of hormaomycin as $R$ and that in the side chain of $\mathbf{3}$ as $S$.

The O-MOM protected 5-chloro-1-hydroxypyrrol-2-carboxylic acid 109 was prepared from known 2,6-dichloropyridine- $N$-oxide 100, using thermolysis of the azide $\mathbf{1 0 2}$ to the nitrile $\mathbf{1 0 3}$ according to protocol of Abramovitch and Cue as a key step, in 9\% yield over 7 steps.

The new 4-methylbenzyloxycarbonyl $N$-protecting group (MeZ) had to be applied in the course of assembling of the backbone of hormaomycin $\mathbf{3}$, since all other convenient $N$-protective groups orthogonal to the Boc group turned out to be incompatible with the restrictions imposed on the deprotection conditions due to the nitro group on the (3-Ncp)Ala moiety, the double bond in the (4-PE)Pro residue as well as the base-sensitive ester bond between the (4-PE)Pro and $a$-Thr fragments.

The linear precursor 191a, prepared by sequential elongation of the growing peptide chain, was cyclized under high dilution conditions to give the cyclic intermediate 171c in moderate yield 
(53\% over 2 steps). Assembly of $\mathbf{3}$ was completed in a stepwise manner and final removal of the MOM group gave the artificial hormaomycin.

Starting from 173c, the branched acyl depsipeptide [(3-Acp)-Ala(Npys $\left.)^{7}\right]-M O M-3$ was prepared. However, an attempted removal of the protecting groups failed to give the target hormaomycin analog.

The analog $\left[\mathrm{NO}_{2} \mathrm{Nva}^{7}\right]-3$ was successfully synthesized, employing the same methodology. Feeding experiment, performed with $(S)$-5-nitronorvaline 208, was successful and $\left[\mathrm{NO}_{2} \mathrm{Nva}^{3}\right]-\mathbf{3}$ as well as $\left[\mathrm{NO}_{2} \mathrm{Nva}^{7}, \mathrm{NO}_{2} \mathrm{Nva}^{3}\right]-3$ were isolated.

After removal of the Boc and DCPM groups, the branched peptide 218 was cyclized to give the mixture of epimeric 219a and 219b, which was separated by HPLC to give individual diastereomers. They were further used for a successful preparation of [ $\left.\mathrm{Dab}^{1}\right]-\mathbf{3}$ and $\left[\mathrm{Dab}^{1}, a-\mathrm{Ile}\right]-$ 3 analogs.

Plate diffusion tests with hormaomycin 3, its analogs as well as with some intermediates and model compounds clearly showed that the side chain of $\mathbf{3}$ defines its specific antibiotic activity.

Among other results, some observations not directly connected with chemistry of hormaomycin, deserve to be mentioned: deconjugative isomerisation of $(S)-(Z)-\mathbf{3 9}$ in $(2 S)-(Z)-\mathbf{4 0}$, which proceeds with full retention of the configuration of the double bond, zinc mediated elimination of iodide $(S)-41$ in the $\mathrm{N}, \mathrm{O}$-protected allylglycine $(\mathrm{S})-\mathbf{4 2}$ and the successful alkylation of the tritylprotected alcohol $\left(1^{\prime} S, 2^{\prime} S\right)-87$ at $\mathrm{C}-2^{\prime}$ of the cyclopropyl ring, and deuteration of several nitrocyclopropyl-containing species at the same position. 

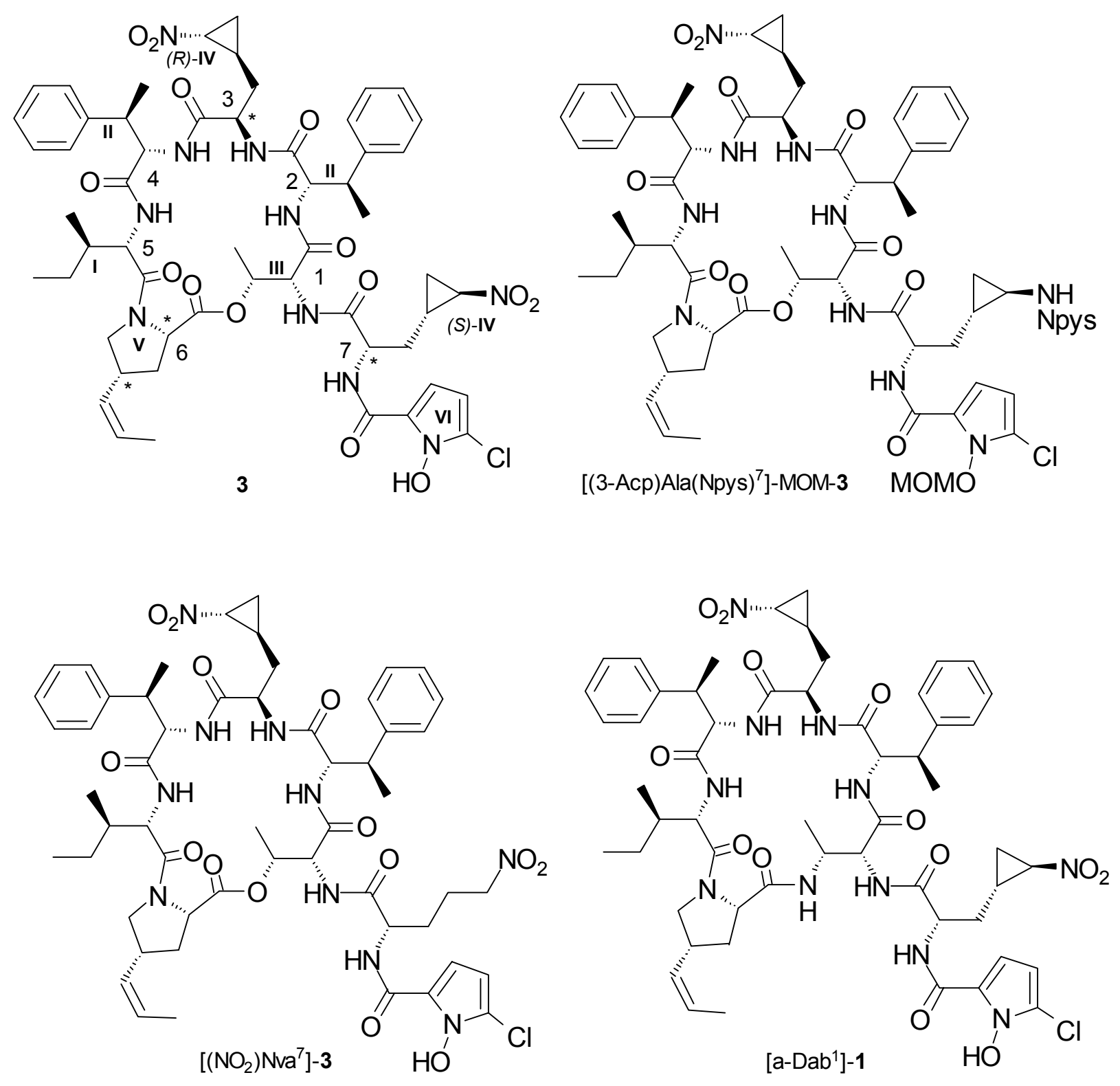
<smiles>C/C=C\C1CN[C@H](C(=O)O)C1</smiles>

trans-16

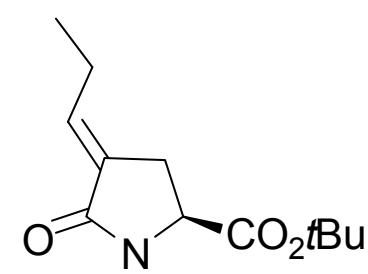

Boc

(S)-39<smiles>C=CCC(NC(=O)OCc1ccccc1)C(OC)OC</smiles>

(2S)-42

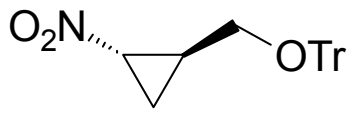

(1'S,2'S)-87<smiles></smiles>

100<smiles>C/C=C\C1CNC(C(=O)O)C1</smiles>

cis-16<smiles>C/C=C/[C@H]1C[C@H](C(=O)OCCC)NC1=O</smiles>

Boc

(2S,4S)-40<smiles>O=[N+](O)C1CC1CI</smiles>

80<smiles>[2H][C@]1([N+](=O)[O-])CC1C[C@@]([2H])(N)C(=O)O</smiles>

(85\%)

D-(2S, $\left.1^{\prime} R S, 2^{\prime} R S\right)-83$<smiles>C/C([18OH])=C\[C@@]1([18OH])CN[C@H](C(=O)O)C1</smiles>

$\mathrm{D}_{2}-(2 S, 4 R)-17$

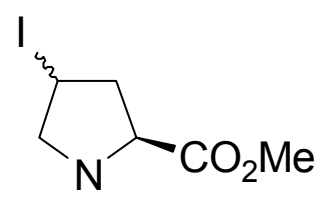

Boc

(2S)-41

(2S)- $D_{2}-81$<smiles>Nc1cccc(Cl)[n+]1O</smiles>

102<smiles>N#Cc1ccc(Cl)n1O</smiles><smiles></smiles>

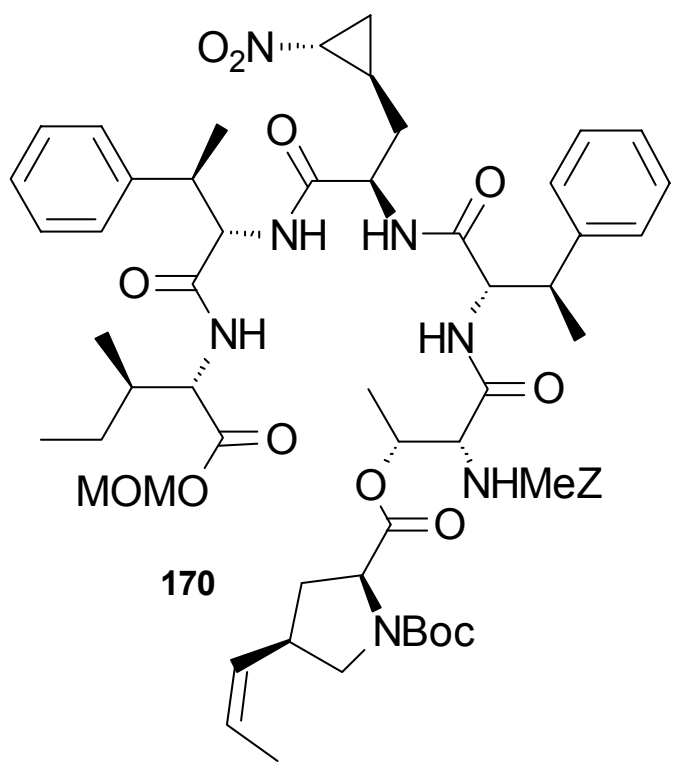

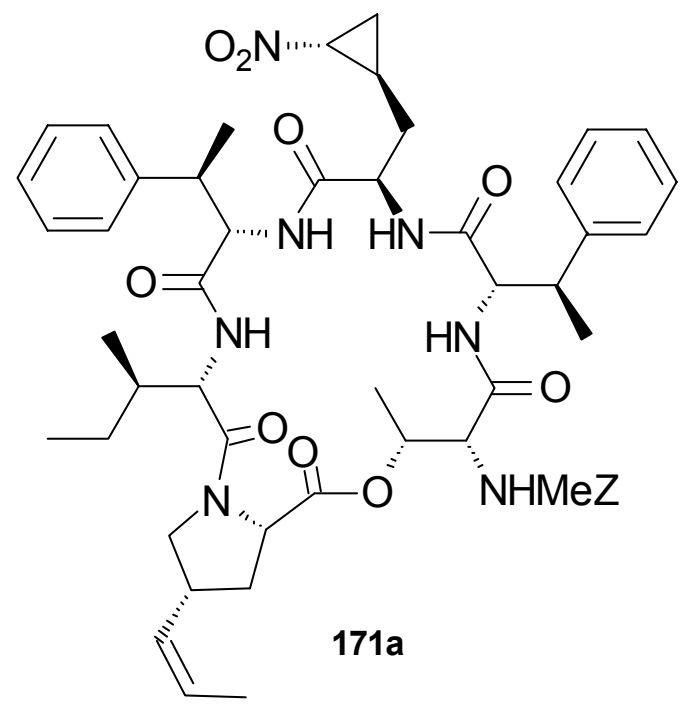


MeZ-a-Dab[Boc-(4-PE)Pro]-( $\beta M e) P h e-(R)-(3-N c p) A l a-(\beta M e) P h e-l l e-O D C P M$ 218
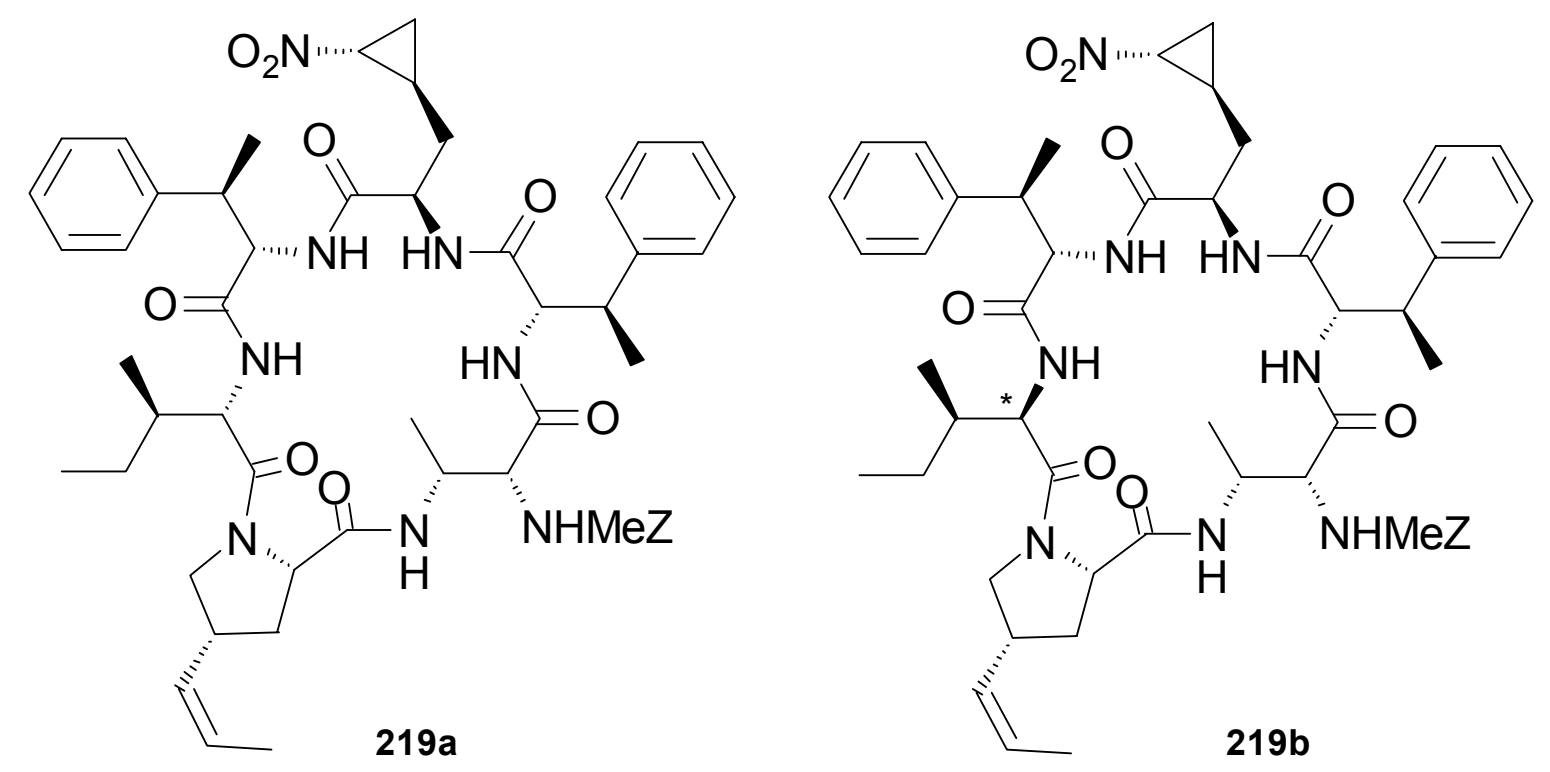


\section{E. References}

[1] M. F. Vincente, A. Basilio, A. Cabello, F. Peläez, Clinical Microbiology and Infection 2003, 9, 15-32.

[2] K. Stephens, CRC Crit. Rev. Microbiol. 1986, 13, 309-334.

[3] J. Engebrecht, K. Nealson, M. Silverman, Cell 1983, 32, 773-781. M. Perego, J. A. Hoch, Proc. Natl. Acad. Sci. USA 1996, 93, 1549-1553. H. B. Kaplan, L. Plamam, FEMS Microbiol. Lett. 1996, 139, 89-95.

[4] a) A. S. Khokhlow in Frontiers of bioorganic chemistry and molecular biology (S. N. Ananchenko, ed.), Pergamon Press, Oxford 1980, 201-210. b) K. Shigeru, K. Hiroshi, N. Takuya, Y. Yasuhiro, J. Bacteriol. 1999, 181, 5081-5084.

[5] A.L. Demain, Internatl. Microbiol. 1998, 1, 259-264.

[6] N. Andres, H. Wolf, H. Zähner, Z. Naturforsch. 1990, 45, 851-855.

[7] N. Andres, H. Wolf, H. Zähner, E. Rössner, A. Zeeck, W. A. König, V. Sinnwell, Helv. Chim. Acta 1989, 426-437.

[8] E. Rössner, Dissertation, Universität Göttingen, 1989.

[9] E. Rössner, A. Zeeck, W. A. König, Angew. Chem. 1990, 102, 84-85; Angew. Chem. Int. Ed. Engl. 1990, 29, 64-65.

[10] a) J. Zindel, A. de Meijere, J. Org. Chem. 1995, 60, 2968-2973. b) J. Zindel, A. Zeeck, W. A. König, A. de Meijere, Tetrahedron Lett. 1993, 34, 1917-1920.

[11] K. Loscha, Diplomarbeit, Universität Göttingen, 2001.

[12] P. Alvermann, Dissertation, Universität Göttingen, 2001.

[13] J. Zindel, Dissertation, Universität Göttingen, 1993.

[14] M. Brandl, S. I. Kozhushkov, K. Loscha, O. V. Kokoreva, D. S. Yufit, J. A. K. Howard, A. de Meijere, Synlett 2000, 12, 1741-1744

[15] K. Osugi Yakugaki Zasshi 1958, 78, 1338-1342; C. A. 53, 8110e. 
[16] E. Melotto, Dissertation, Universität Göttingen, 1999.

[17] M. Ritzau, Dissertation, Universität Göttingen, 1992.

[18] M. Brandl, Dissertation, Universität Göttingen, 2000.

[19] K. Wagner-Gillen, Diplomarbeit, Universität Göttingen, 1998.

[20] K. Ohba, H. Nakayama, K. Furihata, A. Shimazu, T. Endo, U. Seto, N. Otake, J. Antibiot. 1987, 40, 709-713.

[21] S. Hanessian, M. Bayrakdarian, X. Luo, J. Am. Chem. Soc. 2002, 124, 4716-4721.

[22] J. Thottathil, J. Moniot, Tetrahedron Lett. 1986, 27, 151-154.

[23] Although known, this compound was not characterized even a melting point.

[24] An noticeable (three-fold) excess of the alkylating agent should be used because of its low reactivity and instability at the reaction temperature.

[25] This and all other HPLC experiments, described in this work with several exceptions, were performed by H.-P. Kroll and P. Alvermann in Prof. A. Zeeck group (Göttingen).

[26] H. Brückner, C. Keller-Hoehl, Chromatographia 1990, 30, 621-629.

[27] P. Marfey, Carlsberg Res. Communications 1984, 49, 591-596.

[28] R. Knecht, J.-Y. Chang, Anal. Chem., 1986, 58, 2375-2379.

[29] K. Hashimoto, Y. Shima, H. Shirahama, Heterocycles 1996, 42, 489-492.

[30] M. M. Bowers-Nemia, M. M. Joullie, Heterocycles 1983, 20, 817-828.

[31] P. S. Portogese, J. G. Turcotte, Tetrahedron 1971, 27, 961-967.

[32] P. G. M. Wuts, L. E. Pruitt, Synthesis 1989, 622-623.

[33] a) A. Koskinen, H. Rapoport, J. Org. Chem. 1989, 54, 1859-1866. b) C. Moody; D. Young, J. Chem. Soc. Perkin Trans.1 1997, 3519-3530.

[34] This compound was prepared as it was described for its $(S)$-isomer: E. Morera, F. Pinnen, G. Lucente, Org. Lett. 2002, 4, 1139-1142.

[35] M. Steger, D. Young, Tetrahedron 1999, 55, 7935-7956. 
[36] J. Ezquerra, A. Escribano, A. Rubio, M. J. Remuinan, J. J. Vaquero, Tetrahedron Lett. 1995, 36, 6149-6152.

[37] A sample of this compound and was kindly provided by P. Alvermann, Prof. A. Zeeck group (Göttingen).

[38] Cf. a) K. Fujii, Y. Ikai, H. Oka, M. Suzuki, K. Harada, Anal. Chem. 1997, 69, 3346-3352.

b) Y. Suzuki, M. Ojika, Y. Sakagami, K. Kaida, R. Fudou, T. Kameyama, J. Antibiot. 2001, 54, 22-28.

[39] A. F. Coudert, R. Azerad, Synthesis 1997, 863-865.

[40] $(2 S, 4 R)-33,(2 S, 4 R)-34$ and $(2 S, 4 R)-35$ were also prepared.

[41] For a review see: Negishi, E.-i. In: Metal-Catalyzed Cross-Coupling Reactions (F. Diederich, P. J. Stang, Eds.) Wiley-VCH: New York, 1998, Chapter 1.

${ }^{[42]}$ P. Galatsis, J. J. Manwell, S. D. Millan, Tetrahedron Lett. 1996, 37, 5261-5264 and references cited therein.

[43] J. Ezquerra, C. Pedregal, B. Yruretagoyena, A. Rubio, M. C. Carreno, J. Org. Chem. 1995, 60, 2925-2930.

[44] J. E. Baldwin, T. Miranda, M. Moloney, T. Hokelek, Tetrahedron 1989, 45, 7459-7468.

[45] J.-D. Charrier, J. E. S. Duffy, P. B. Hitchcock, D. W. Young, Tetrahedron Lett. 1998, 39, 2199-2204.

[46] P. Merino, S. Anoro, S. Franco, F. L. Merchan, T. Tejero, V. Tunon, J. Org. Chem. 2000, $65,1590-1596$.

[47] J.-R. Dormoy, Synthesis 1982, 753-756.

${ }^{[48]}$ P. Knochel, M. C. P. Yeh, S. C. Berk, J. Talbert, J. Org. Chem. 1988, 53, 2390-2392.

${ }^{[49]}$ P. N. Collier, A. D. Campbell, I. Patel, R. J. K. Taylor, Tetrahedron Lett. 2000, 41, 71157120.

[50] Review on the chemistry of DIBAH: E. Winterfeld, Synthesis 1975, 617-630. 
[51] Cf. H. Ishiyama, T. Takemura, M. Tsuda, J. Kobayashi Tetrahedron 1999, 55, 45834594.

[52] a) R. J. Bridges, M. S. Stanley, M. W. Anderson, C. W. Cotman, A. R. Chamberlin, J. Med. Chem. 1991, 34, 717-725. b) T. R. Webb, C. Eigenbrot, J. Org. Chem. 1991, 56, 3009-3016.

[53] M. Shigeyasu, M. Kuwahara, M. Sisido, T. Ishikawa, Chem. Lett. 2001, 7, 634-635.

[54] P. Chevallet, P. Garrouste, B. Malawska, J. Martinez, Tetrahedron Lett. 1993, 74097412.

[55] K.-i. Tanaka, H. Sawanishi, Tetrahedron: Asymmetry 1998, 9, 71-78.

[56] S. Hagishita, K. Seno, Chem. Pharm. Bull. 1989, 37, 327-335.

[57] Y. Iso, T. Irie, T. Iwaki, M. Kii, Y. Sendo, K. Motokawa, Y. Nishitani, J. Antibiot. 1996, 49, 478-484.

[58] a) H. J. Bestmann, W. Stransky, O. Vostrowsky, Chem. Ber. 1976, 109, 1694-1700. b) C. A. Henrick, Tetrahedron 1977, 33, 1845-1889. c) A. Albeck, N. Friedman, M. Sheves, M. Ottolenghi, J. Am. Chem. Soc. 1986, 108, 4614-4618.

[59] T. Katoh, Y. Nagata, Y. Kobayashi, K. Arai, J. Minami, S. Terashima, Tetrahedron 1994, 50, 6221-6238.

[60] E. J. Corey, P. L. Fuchs, Tetrahedron Lett. 1972, 36, 3769-3772.

[61] C. A. Brown, V. K. Ahuja, J. Org. Chem. 1973, 38, 2226-2230.

[62] Y. Aoyagi, R. M. Williams, Tetrahedron 1998, 54, 13045-13058.

[63] When the synthesis of the $N$-Boc protected 4-(Z)-propenylproline had already been completed, a very similar procedure for the preparation of allyl 4-cyano-2(\{[dimethyl(1,1,2-trimethylpropyl)silyl]oxy $\}$ methyl)pyrrolidine-1-carboxylate was published: M. Sunagawa, M. Itoh, K. Kubota, A. Sasaki, Y. Ueda, P. Angehrn, A. Bourson, E. Goetschi, P. Hebeisen, R. L. Then, J. Antibiot. 2002, 55, 722-757. 
[64] B. Zheng, H. Maeda, M. Mori, S. Kusaka, O. Yonemitsu, T. Matsushima, N. Nakajima, J. Uenishi Chem. Pharm. Bull. 1999, 47, 1288-1296.

[65] a) Several other ways to oxidize the alcohol $\mathbf{5 4}$ to the acid $\mathbf{1 6}$ were tried, but all of them (PDC in DMF, first Swern or Dess-Martin oxidation and then the oxidation of the intermediate alcohol with sodium chlorite in tert-butanylic alcohol/phosphate buffer) gave the product in lower yield and purity than the oxidation with Jones reagent. b) A. Barco, S. Benetti, G. Spalluto, A. Casolari, G. P. Pollini, V. Zanirato, J. Org. Chem. $1992,57,6279-6286$.

[66] a) The peak of the epimer $(2 S, 4 S)$-16 was unobservable. b) Enantiomeric excess was determined according to method of Merfey for the corresponding $(S)$-FDVA derivative, obtained after removal of the Boc group from $(2 S, 4 R)-\mathbf{1 6}$.

[67] T. Weber, D. Seebach, Helv. Chim. Acta 1985, 68, 155-161.

${ }^{[68]}$ R. Annunziata, M. Ferrari, G. Papeo, M. Resmini, M. Sisti, Synth. Commun. 1997, 27, $23-38$.

[69] H. Wang, J. P. Germanas, Synlett. 1999, 33-36.

[70] S. Takano, W. Uchida, S. Hatakeyama, K. Ogasawara, Chem. Lett. 1982, 733-736.

${ }^{[71]}$ For runs on a small scale $(0.5-1.5 \mathrm{mmol})$ yields were ca. $10 \%$ higher.

[72] H. Heimgartner, H. Schmid, H. J. Hansen, Helv. Chim. Acta 1972, 55, 1385-1401.

[73] T. W. Green, P. G. M.Wuts, Protecting Groups in Organic Synthesis , $3^{\text {nd }}$ Ed., Wiley, New York, 1999.

[74] The nondeuterated $(2 S, 4 R)-\mathbf{1 6}$ was also prepared. See experimental section for details.

[75] a) D. F. Elliot J. Chem. Soc. 1950, 62. b) P. G. Anderson, D. Guijaro, D. Tanner J. Org. Chem. 1997, 62, 7364-7375. c) For several examples of other syntheses of (R)-allothreonine see: a) D. Pons, M. Savignac, J.-P. Genet, Tetrahedron Lett. 1990, 31, 5023- 
5026. b) P. J. Maurer, H. Takahata, H. Rapoport, J. Am. Chem. Soc. 1984, 106, 10951098. c) H. Shao, M. Goodman, J. Org. Chem. 1996, 61, 2582-2583.

[76] Selected examples see: a) R. Fitzi, D. Seebach, Tetrahedron 1988, 17, 5277-5292. b) M. Pasto, A. Moyano, M. A. Pericas, A. Riera, J. Org. Chem. 1997, 62, 8425-8431. c) F. A. Davis, H. Liu, P. Zhou, T. Fang, G. V. Reddy, Y. Zhang, J. Org. Chem. 1999, 64, 75597567.

[77] V. J. Hruby, R. Dharanipragada, K. VanHulle, A. Bannister, S. Bear, L. Kennedy, Tetrahedron 1992, 48, 4733-4748.

${ }^{[78]}$ D. A. Evans, T. C. Britton, J. A. Ellman, R. L. Dorrow, J. Am. Chem. Soc. 1990, 112, 4011-4030.

[79] G. Li, D. Patel, V. J. Hruby, J. Chem. Soc. Perkin Trans. 1 1994, 3057-3059.

[80] This compound was prepared from $(R)$-phenylglycine: $(R)$-phenylglycinol, which was obtained by reduction of $(R)$-phenylglycine with $\mathrm{NaBH}_{4} / \mathrm{H}_{2} \mathrm{SO}_{4}$ in THF ( $c f:$ : A. Abiko, $\mathrm{S}$. Masamune, Tetrahedron Lett. 1992, 33, 5517-5518), was cyclized by heating in dimethylcarbonate with $\mathrm{K}_{2} \mathrm{CO}_{3}(c f:$ V. I. Tararov, T. F. Savel'eva, Yu. T. Struchkov, A. P. Pisarevskii, N. I. Raevskii, Yu. N. Belokon', Russ. Chem. Bull. 1996, 45, 600-609, Izv. Akad. Nauk Ser. Khim. 1996,3, 640-648).

[81] E. Nicolas, K. C. Russel, V. J. Hruby J. Org. Chem. 1993, 58, 766-770.

[82] H. Josien, A. Martin, G. Chassaing, Tetrahedron Lett. 1991, 32, 6547-6550.

[83] For a review on thise chiral auxiliary see: Yu. N. Belokon', Janssen Chim. Acta 1992, 10, $4-12$.

${ }^{[84]}$ O. V. Larionov, T. F. Savelieva, K. A. Kochetkov, N. S. Ikonnikov, S. I. Kozhushkov, D. S. Yufit, J. A. K. Howard, V. N. Khrustalev, Yu. N. Belokon, A. de Meijere, Eur. J. Org. Chem. 2003, 869-877.

[85] L. L. Gordon, C. Gottardo, Synth. Commun. 1990, 20, 1473-1479. 
[86] Yu. N. Belokon', V. I. Taranov, V. I. Maleev, T. F. Savel'eva, M. G. Ryzhov, Tetrahedron: Asymetry 1998, 9, 4249-4252.

[87] a) Y. Elemes, U. Ragnarsson, J. Chem. Soc. Perkin Trans.1 1996, 537-540. b) To the best of our knowledge no procedure for the preparation of deuterated $\mathbf{8 1}$ was published before.

[88] If nondeuterated acetonitrile was used, ca. $50 \%$ loss of deuterium occurred.

[89] For the full assignment of ${ }^{1} \mathrm{H}$ - and ${ }^{13} \mathrm{C}-\mathrm{NMR}$ spectra of $\mathbf{3}$, see: P. Henne, Dissertation, Universität Göttingen, 1994.

${ }^{[90]}$ Y. Kai, P. Knochel, S. Kwiatkowski, J. D. Dunitz, J. F. M. Oth, D. Seebach, H. O. Kalinowski, Helv. Chim. Acta 1982, 65, 137-161.

[91] A similar experiment with $\left(2 S, 2^{\prime} S, 1^{\prime \prime} R, 2^{\prime \prime} S\right)-16$ was unsuccessful because of solubility problems.

[92] This compound was generously donated by Oleg V. Larionov, Göttingen.

[93] P. E. O'Bannon, W. P. Dailey, J. Amer. Chem. Soc. 1989, 111, 9244-9245.

[94] P. A. Wade, P. A. Kondracki, P. A. Carroll, J. Am. Chem. Soc. 1991, 113, 8807-8811.

[95] A. Thomas, S. Rajappa, Tetrahedron 1995, 51, 10571-10580.

[96] a) F. Effenberger, W. Mueller, H. Isak, Chem. Ber. 1987, 120, 45-54. b) P. Netchitailo, M. Othman, A. Daich, B. Decroix, Tetrahedron Lett. 1997, 38, 3227-3230.

[97] G. Zinner, R. Moll, Chem. Ber. 1966, 99, 1292-1298.

[98] R. A. Abramovitch, B. W. Cue, J. Am. Chem. Soc. 1976, 98, 1478-1486.

[99] T. Ikemoto, T. Kawamoto, H. Wada, T. Ishida, T. Ito, Y. Isogami, Y. Miyano, Y. Mizuno, K. Tomimatsu, K. Hamamura, M. Takatani, M. Wakimasu, Tetrahedron 2002, $58,489-494$.

${ }^{[100]}$ L. Pentimalli, Gazz. Chim. Ital. 1964, 94, 458. 
${ }^{[101]}$ L. P. Ferrarini, G. Biagi, O. Livi, G. Primofiore, M. Carpene J. Heterocycl. Chem. 1981, $18,1007-1010$.

${ }^{[102]}$ S. V. D'Andrea, D. Bonner, J. J. Bronson, J. Clark, K. Denbleyker, J. Fung-Tome, S. E. Höft, T. W. Hudyma, J. D. Matiskella, R. F. Miller, P. F. Misco, M. Pucci, R. Sterzycki, Y. Tsai, Y. Ueda, J. A. Wichtowski, J. Singh, T. P. Kissick, J. T. North, A. Pullockaran, M. Humora, B. Boyhan, T. Vu, A. Fritz, J. Heikes, R. Fox, J. D. Godfrey, R. Perrone, M. Kaplan, D. Kronenthal, R. H. Müller, Tetrahedron 2000, 56, 5687-5698.

${ }^{[103]}$ S. Cacchi, D. Misiti, P. La Torre, Synthesis 1980, 243-244.

${ }^{[104]}$ S. K. Davidsen, P. D. May, J. B. Summers, J. Org. Chem. 1991, 56, 5482-5485.

${ }^{[105]}$ S. A. M. Merette, A. P. Burd, J. J. Deadman, Tetrahedron Lett. 1999, 40, 753-754.

${ }^{[106]}$ S. Kim, I. S. Kee, Y. H. Park, J. H. Park, Synlett 1991, 3, 183-184.

${ }^{[107]}$ L. A. Carpino, J. Am. Chem. Soc. 1993, 115, 4397-4398.

${ }^{[108]}$ J. Bernstein, B. Stearns, E. Shaw, W. A. Lott, J. Am. Chem. Soc. 1947, 69, 1151-1158.

${ }^{[109]}$ This compound was also obtained according to P. L. Ferrarini, C. Mori, C. Manera, F. Mori, V. Calderone, E. Martinotti, J. Heterocycl. Chem. 1999, 36, 1123-1128 in 33\% yield.

${ }^{[110]}$ For a review on peptide coupling agents see: F. Albericio, R. Chinchilla, D. J. Dodsworth, C. Najera, Org. Prep. Proc. Int. 2001, 33, 203-303.

${ }^{[111]}$ L. A. Carpino, A. El-Faham, Tetrahedron 1999, 55, 6813-6830.

${ }^{[112]}$ W. R. Kobertz, J. M. Essigmann, J. Am. Chem. Soc. 1996, 118, 7101-7107.

${ }^{[113]}$ W.-R. Li, W. R. Ewing, B. D. Harris, M. M. Joulie, J. Am. Chem. Soc. 1990, 112, 76597672.

${ }^{[114]}$ S. F. Brady, S. L. Varga, R. M. Freidinger, D. A. Schwenk, M. Mendlowski, F. W. Holly, D. F. Veber, J. Org. Chem. 1979, 44, 3101-3105.

${ }^{[115]}$ L. Zervas, D. Borovas, E. Gazis, J. Am. Chem. Soc. 1963, 85, 3660-3667. 
${ }^{[116]}$ R. E. Vegner, L. K. Polevaja, I. A. Vosekalna, Izv. AN LatSSR 1979, 103-106 (in Russian).

${ }^{[117]}$ R. Sharan, B. Kundu, K. B. Mathur, Indian J. Chem. Sect. B 1991, 30, 728-731.

Dichloromethane instead of DMF was used as a solvent.

${ }^{[118]}$ A. F. Abdel-Magid, J. H. Cohen, C. A. Maryanoff, R. D. Shah, F. J. Villiani, F. Zhang, Tetrahedron Lett. 1998, 39, 3391-3394.

${ }^{[119]}$ D. M. Birney, D. C. Cole, C. E. Crosson, B. F. Kahl, B. W. Neff, T. W. Reid, K. Ren, R. D. Walkup, J. Med. Chem. 1995, 38, 2478-2482.

${ }^{[120]}$ J. Robles, M. Beltran, V. Marchan, Y. Perez, I. Travesset, E. Pedroso, A. Grandas, Tetrahedron 1999, 55, 13251-13264.

${ }^{[121]}$ E. C. Roos, P. Bernabe, H. Hiemstra, W. N. Speckamp, B. Kaptein, W. H. J. Bösten, J. Org. Chem. 1995, 60, 1733-1740.

${ }^{[122]}$ P. Sieber, Helv. Chim. Acta 1977, 60, 2711-2716.

${ }^{[123]}$ B. H. Lipshutz, J. J. Pegram, Tetrahedron Lett. 1980, 21, 3343-3346.

${ }^{[124]}$ W.-C. Chen, M. D. Vera, M. M. Joullie, Tetrahedron Lett. 1997, 38, 4025-4028.

${ }^{[125]}$ A. Ehrlich, H. U. Heyne, R. Winter, M. Beyermann, H. Haber, L. A. Carpino, M. Bienert, J. Org. Chem. 1996, 61, 8831-8838.

[126] Y. Kiso, K. Ukawa, T. Akita, J. Chem. Soc. Chem. Commun. 1980, 101-102.

${ }^{[127]}$ a) M. B. Smith, March's Advanced Organic Chemistry: Reactions, Mechanisms and Structure (M. B. Smith, J. March, eds.), Wiley, New-York 2001, 370. b) C. G. Swain, E. C. Lupton, J. Am. Chem. Soc. 1968, 90, 4328-4339.

${ }^{[128]}$ L. A. Carpino, H. G. Chao, S. Ghassemi, E. M. Mansour, C. Riemer, R. Warrass, D. Sadat-Aalaee, G. A. Truran, H. Imazumi, A. El-Faham, D. Ionescu, M. Ismail, T. L. Kowaleski, C. H. Han, H. Wenschuh, M. Beyermann, M. Bienert, H. Shroff, F. Albericio, S. A. Triolo, N. A. Sole, S. A. Kates, J. Org. Chem. 1995, 60, 7718-7719. 
${ }^{[129]}$ O. V. Larionov, S. I. Kozhushkov, M. Brandl, A. de Meijere, Mendeleev Commun., 2003, in press.

${ }^{[130]}$ R. Matsueda, R. Walter, Int. J. Peptide Protein Res. 1980, 16, 392-401.

${ }^{[131]}$ F. Yokokawa, H. Sugiyama, T. Shioiri, N. Katagiri, O. Oda, H. Ogawa, Tetrahedron 2001, 57, 4759-4766.

${ }^{[132]}$ a) K. E. Gilbert, W. T. Borden, J. Org. Chem. 1979, 44, 659-661. b) P. Peterli-Roth, M. P. Maguire, E. Leon, H. Rapoport, J. Org. Chem. 1994, 59, 4186-4193.

${ }^{[133]}$ H. Han, J. Yoon, K. D. Janda, J. Org. Chem. 1998, 63, 2045-2048.

${ }^{[134]}$ K. Wen, H. Han, T. Z. Hoffman, K. D. Janda, L. E. Orgel, Bioorg. Med. Chem. Lett. 2001, 11, 689-692.

${ }^{[135]}$ D. D. Hennings, R. M. Williams, Synthesis 2000, 1310-1314.

${ }^{[136]}$ K. Sakai, J.-P. Anselme, J. Org. Chem. 1971, 36, 2387-2388.

${ }^{[137]}$ Y. Kataoka, Y. Seto, M. Yamamoto, T. Yamada, S. Kuwata, H. Watanabe, Bull. Chem. Soc. Jpn. 1976, 49, 1081-1084.

${ }^{[138]}$ V. Fedij, E. A. Lenoir III, M. J. Suto, J. R. Zeller, J. Wemple, Tetrahedron: Asymmetry 1998, 5, 1131-1134.

${ }^{[139]}$ A. A. Gerschkovich, V. K. Kibirev, Himicheskij syntez peptidov 1992, Kiev, Naukova Dumka (in Russian).

${ }^{[140]}$ M. Ueki, M. Honda, Y. Kazama, T. Katoh, Synthesis 1994, 21-22.

${ }^{[141]}$ L. A. Carpino, US Patent 5,580,981, 1996.

${ }^{[142]}$ R. E. Shute, D. H. Rich, Synthesis 1987, 346-349.

${ }^{[143]}$ I. Ojima, T. Kogure, N. Yoda, J. Org. Chem. 1980, 45, 4728-4739.

${ }^{[144]}$ B. Maurer, W. Keller-Schierlein, Helv. Chim. Acta 1969, 52, 388-396. 


\section{F. Spectral Data}

1. ${ }^{1} \mathrm{H}-\mathrm{NMR}$ Spectra

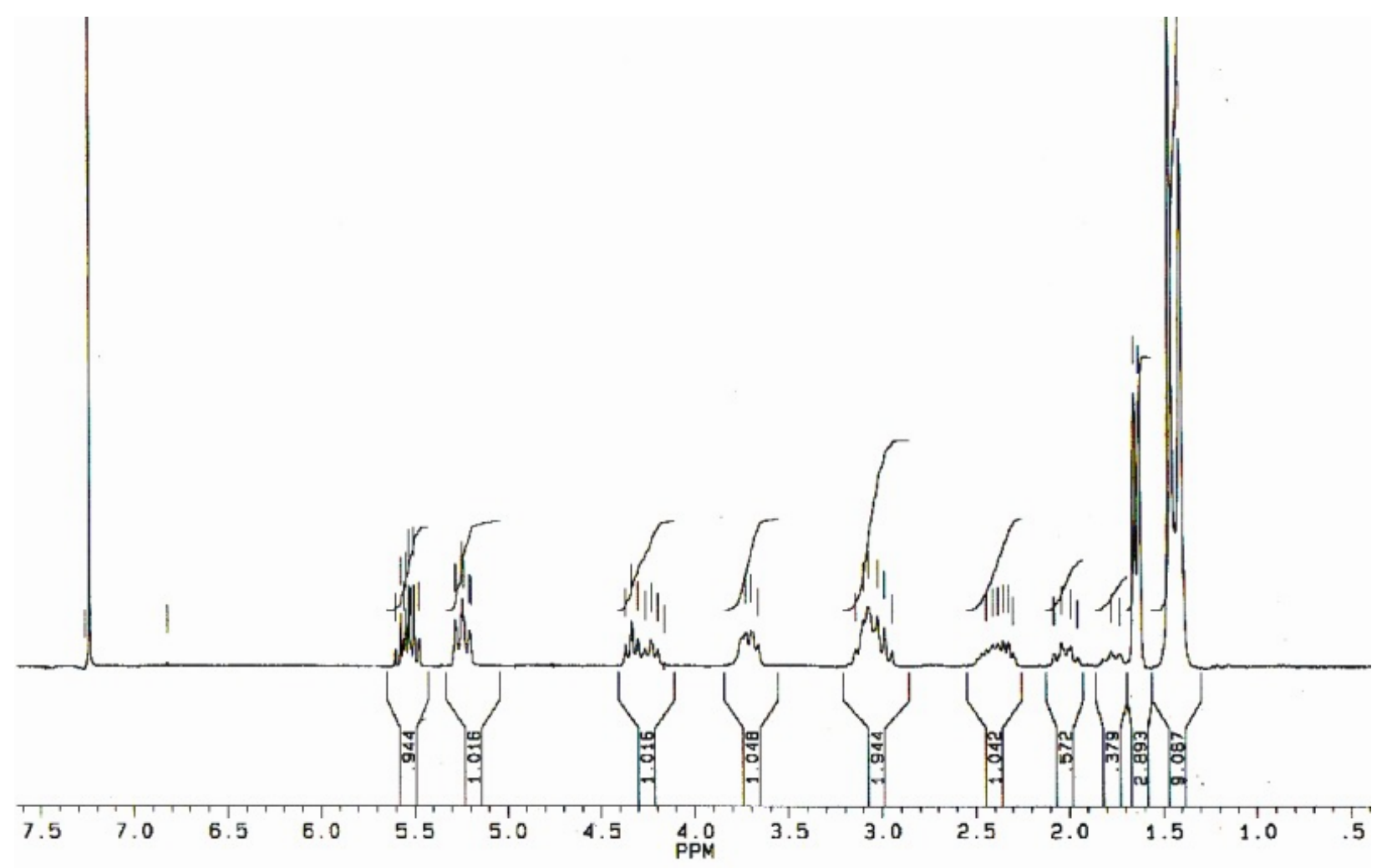

$(2 S, 4 R)-N$-(tert-Butyloxycarbonyl)-4-(Z)-propenylproline (2S,4R)-16 
임

페교

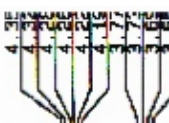

gag

a

13

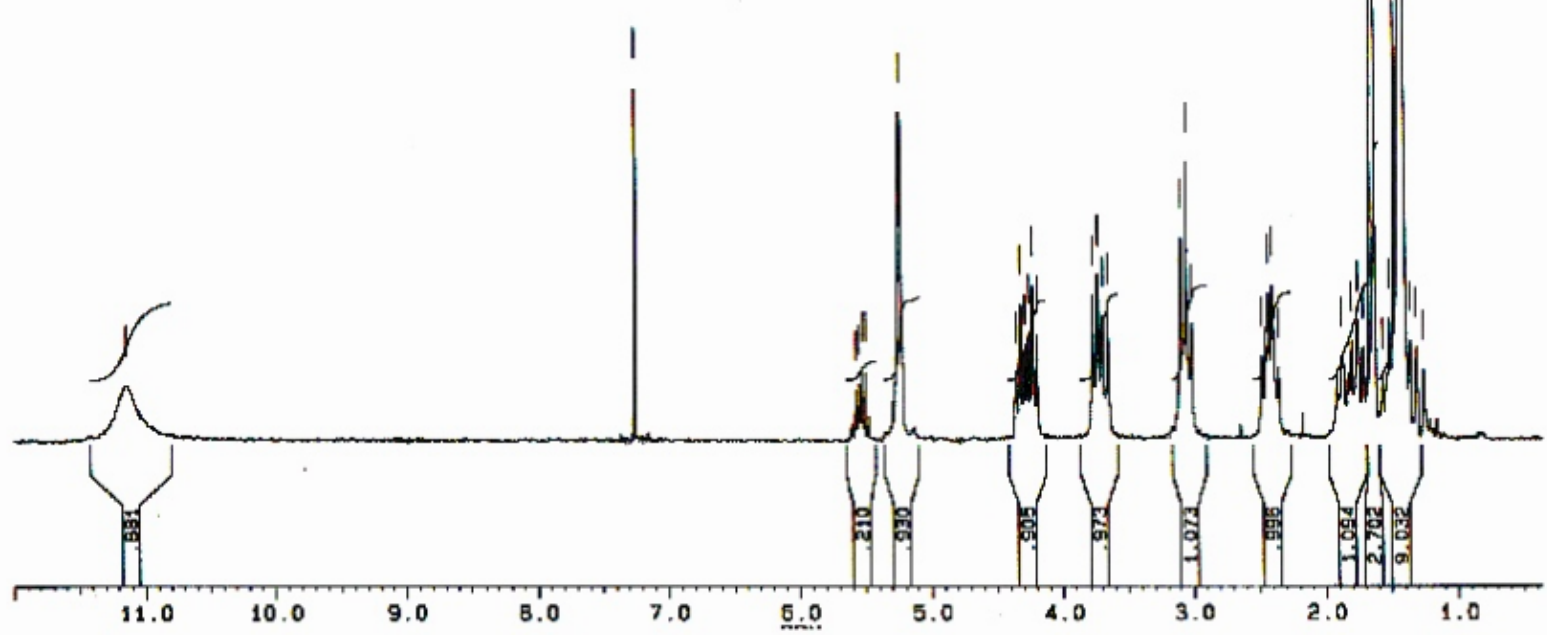

$(2 S, 4 R)-N$-(tert-Butyloxycarbonyl)-4,1'-dideutero-4-( $Z$ )-propenylproline $(2 S, 4 R)-\mathrm{D}_{2}-16$

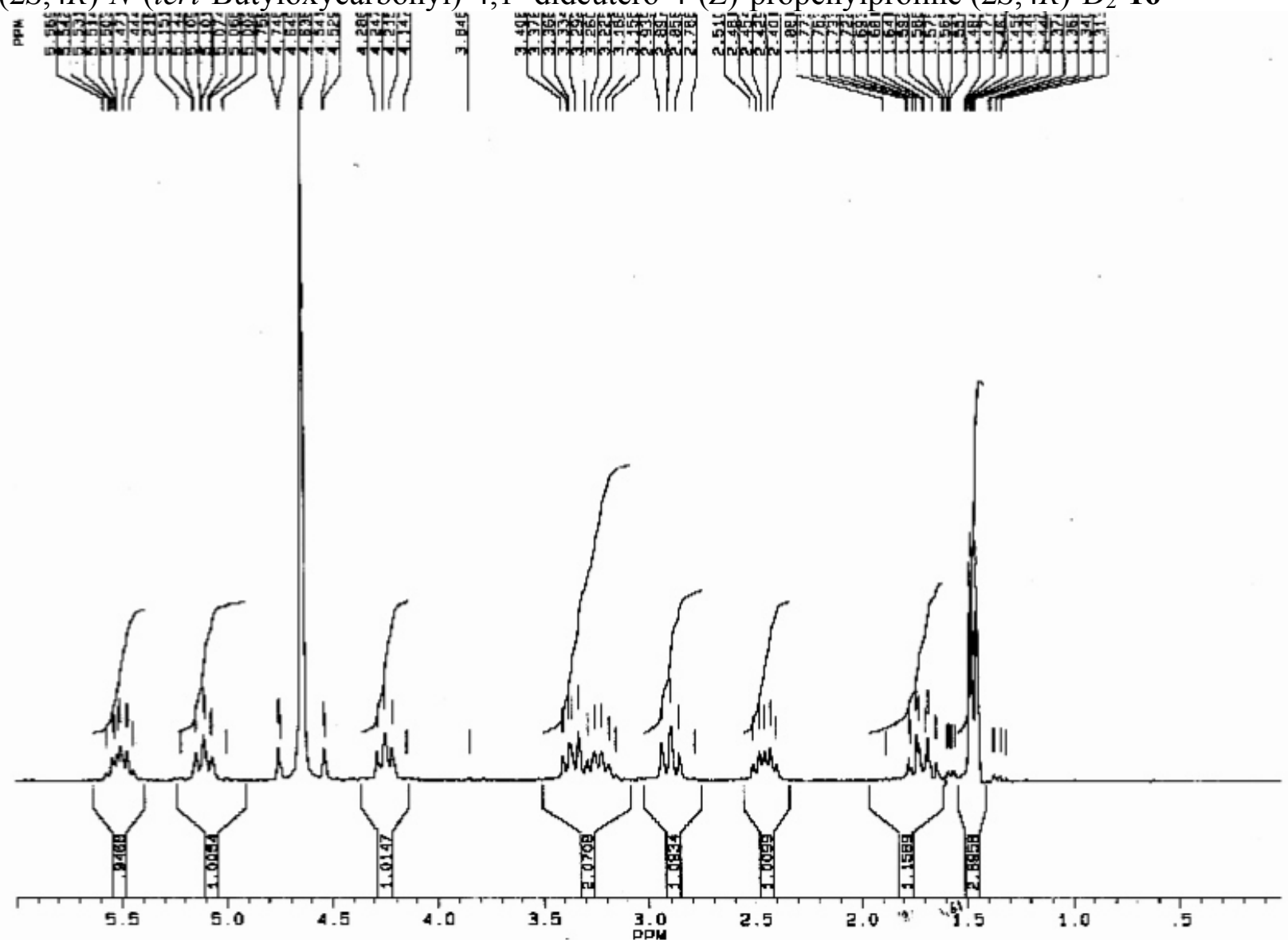

$(2 S, 4 R)-4-(Z)$-propenylproline hydrochloride $(2 S, 4 R)-\mathbf{1 7} \cdot \mathrm{HCl}$ 


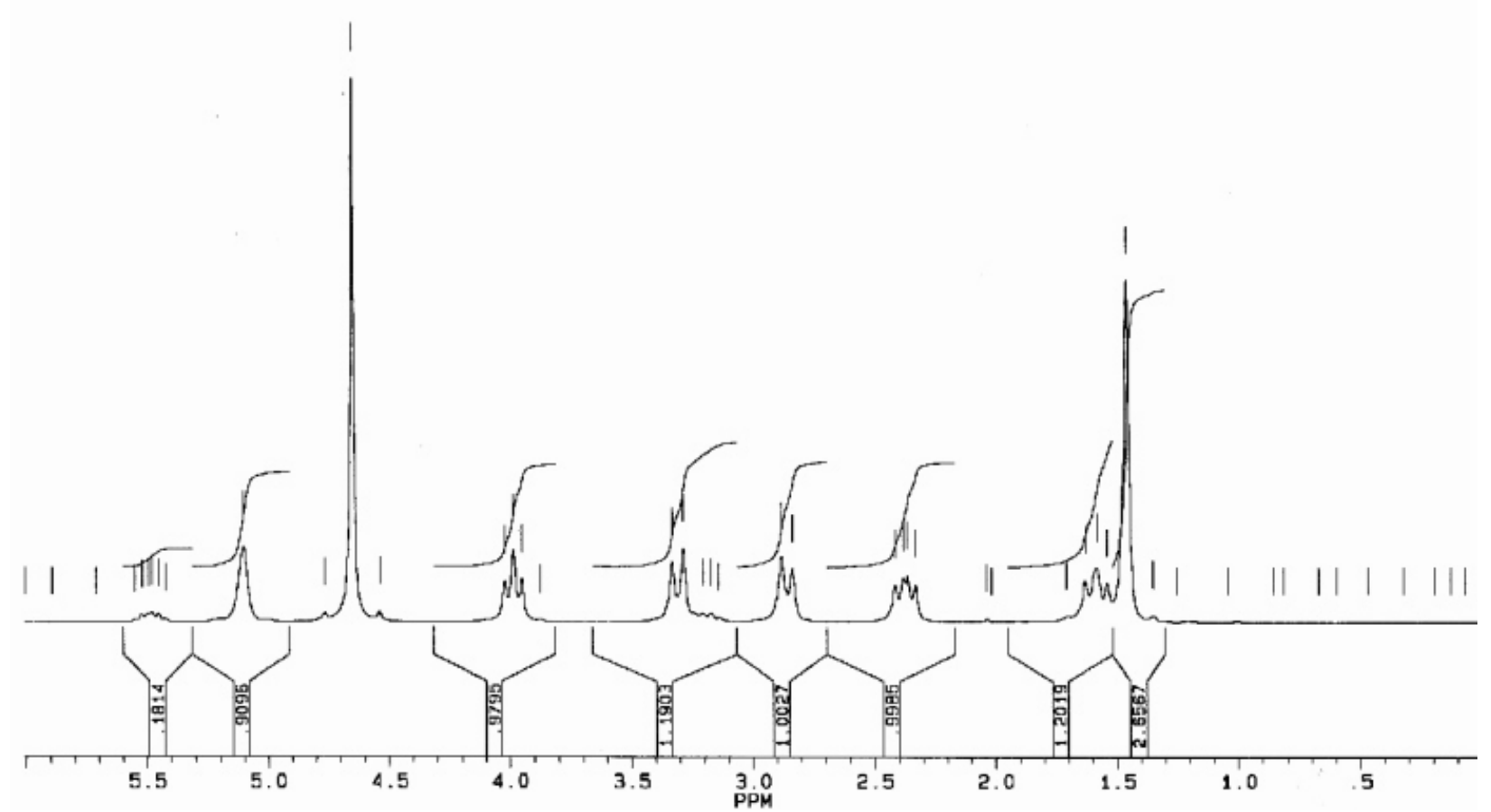

(2S,4R)-4,1'-Dideutero-4-(Z)-propenylproline $(2 S, 4 R)-17$
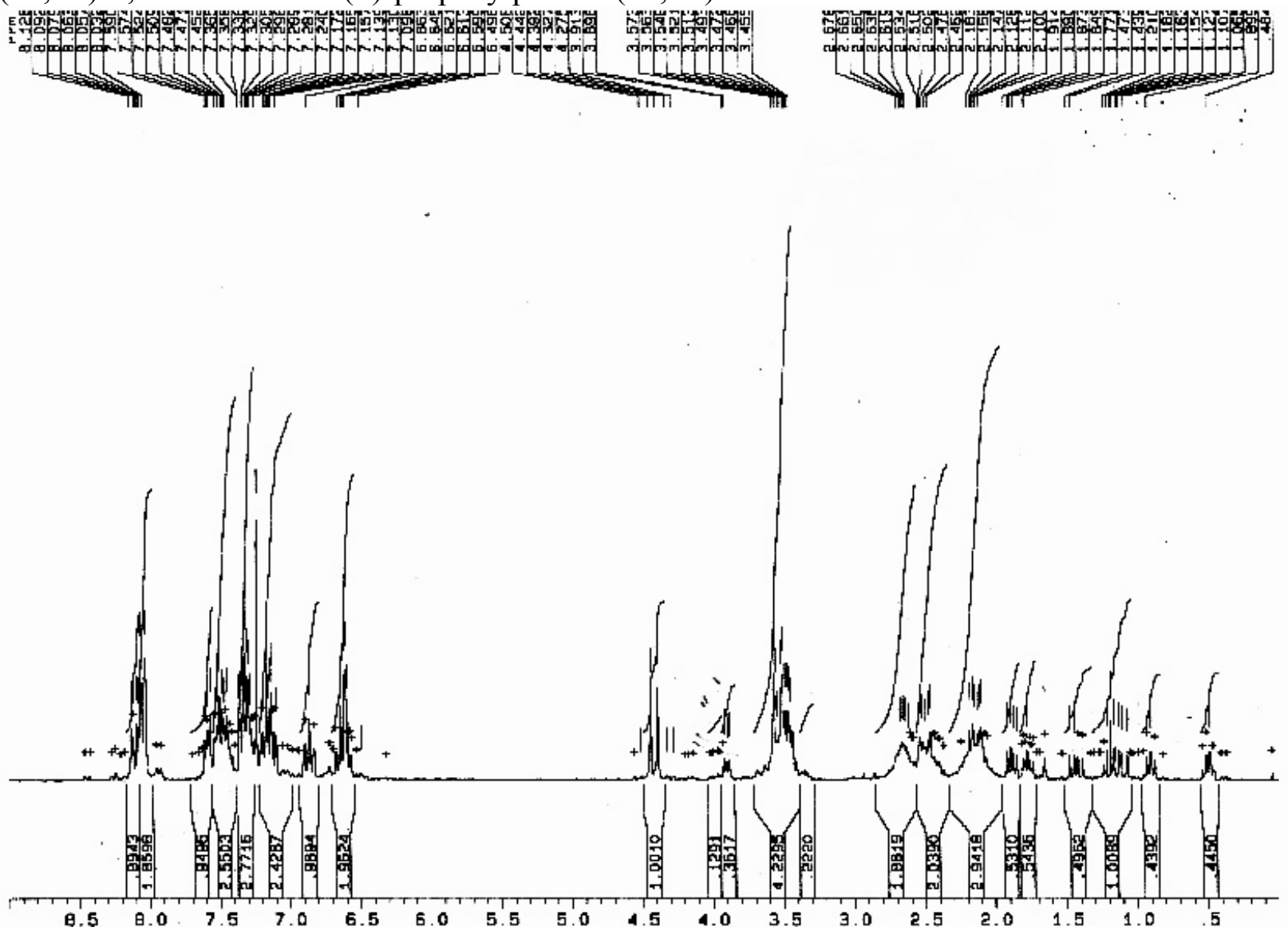

$N i$-complex $\left(2 S, 2^{\prime} S, 1^{\prime \prime} R S, 2^{\prime \prime} R S\right)$-D-82 


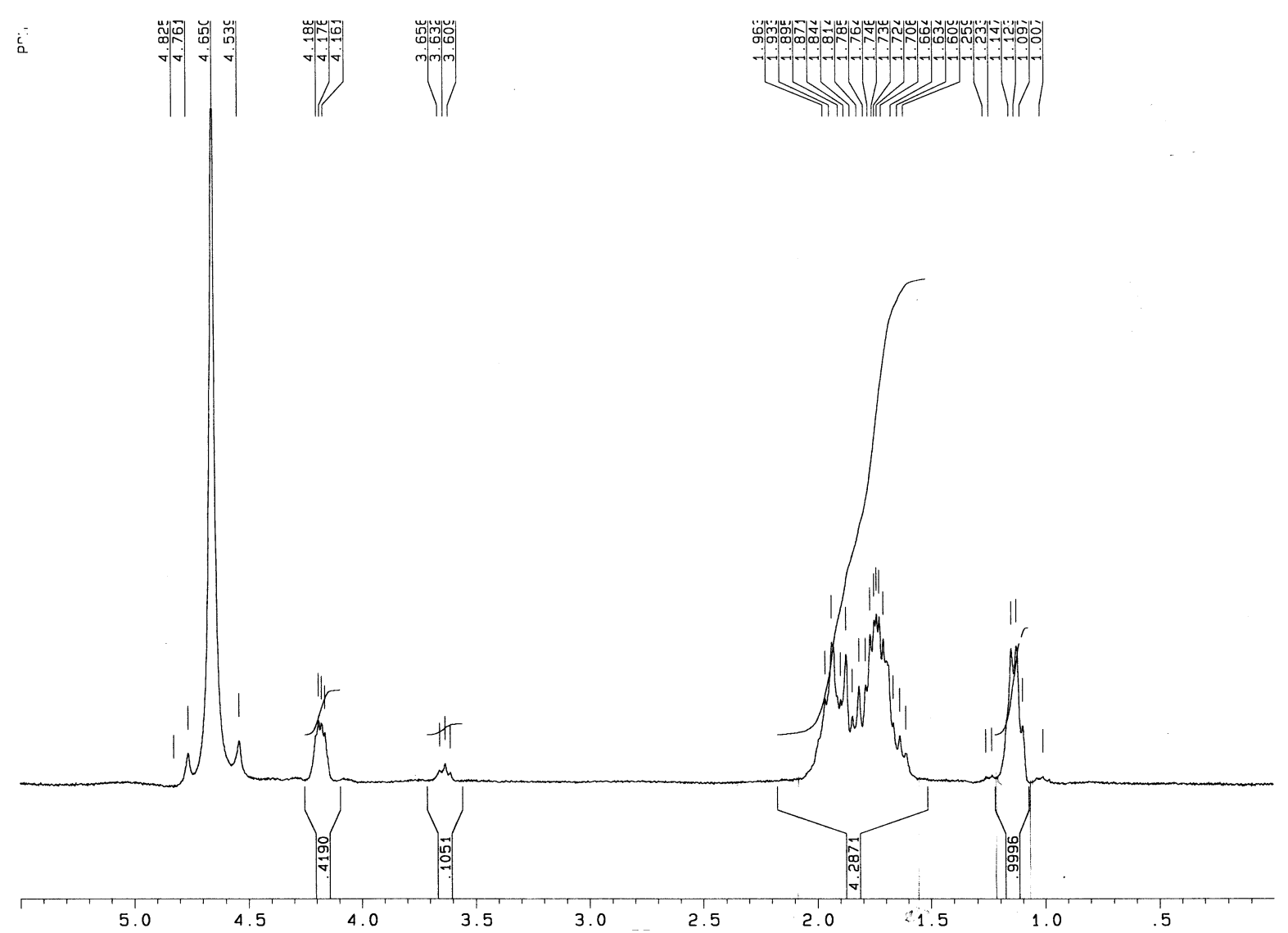

( $\left.2 S, 1^{\prime} R S, 2^{\prime} R S\right)-2$-Deutero-3-(2'-nitrocyclopropyl)alanine $\left(2 S, 1^{\prime} R S, 2^{\prime} R S\right)$-D-(83)
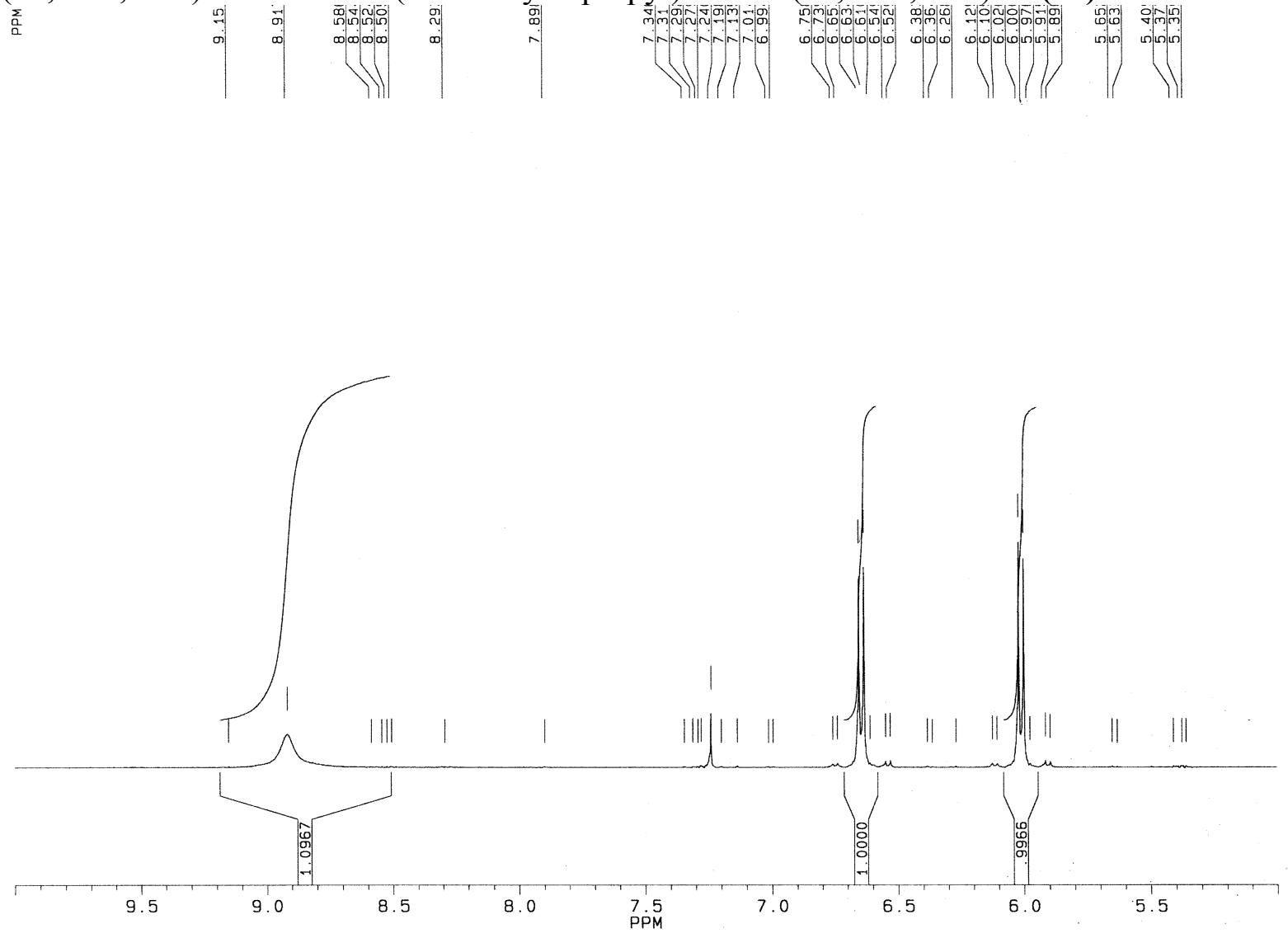

5-Chloro-1-hydroxypyrrole-2-carbonitrile (103) 


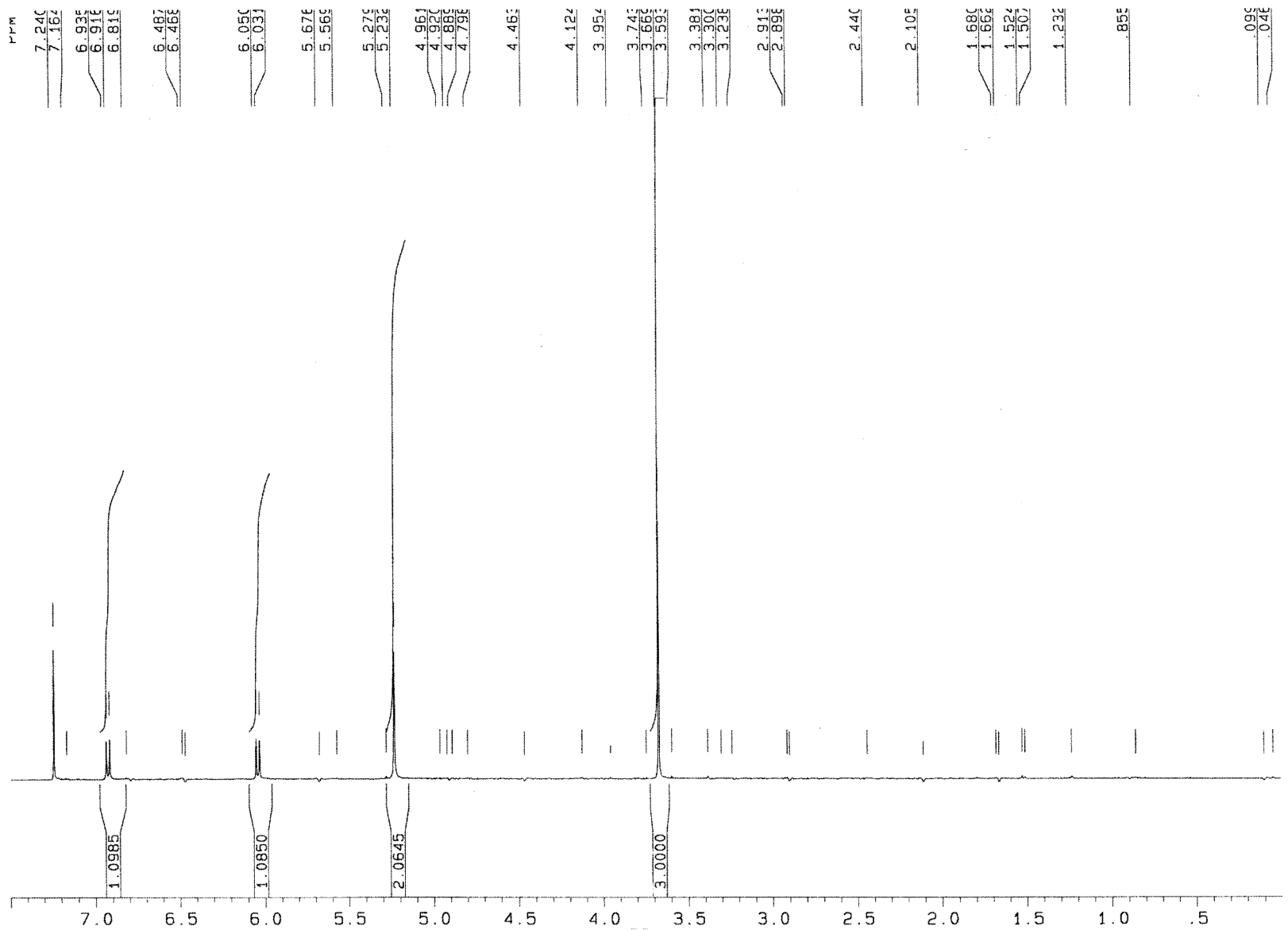

5-Chloro-1-methoxymethoxypyrrole-2-carboxylic acid (109)

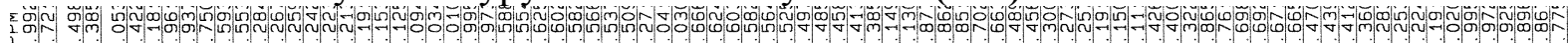

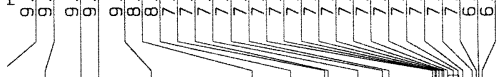

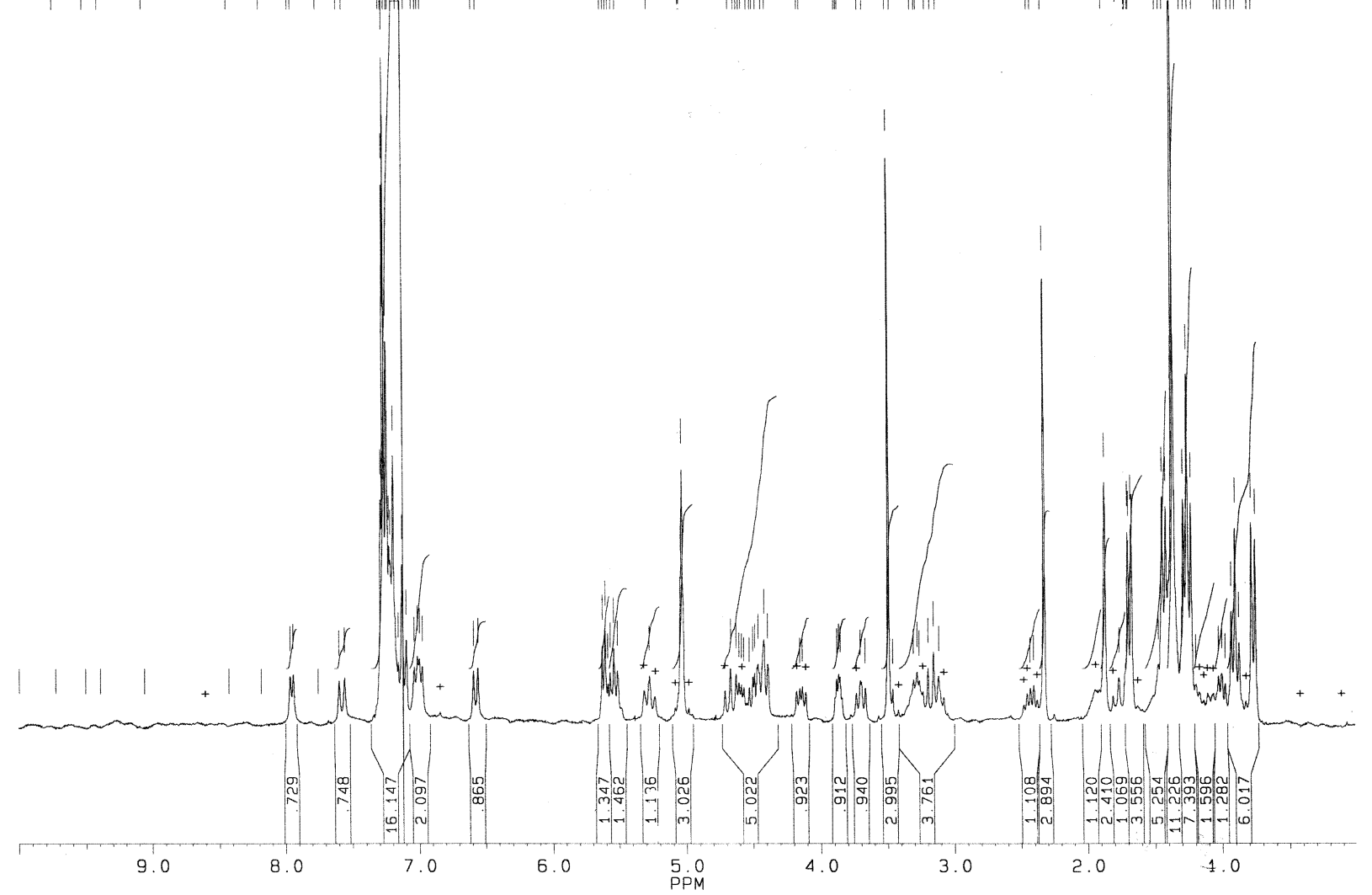

MeZ- $a$-Thr[Boc-(4-PE)Pro]-( $\beta M e) P h e-(3-N c p) A l a-(\beta M e) P h e-I l e-O M O M ~(191 a)$ 


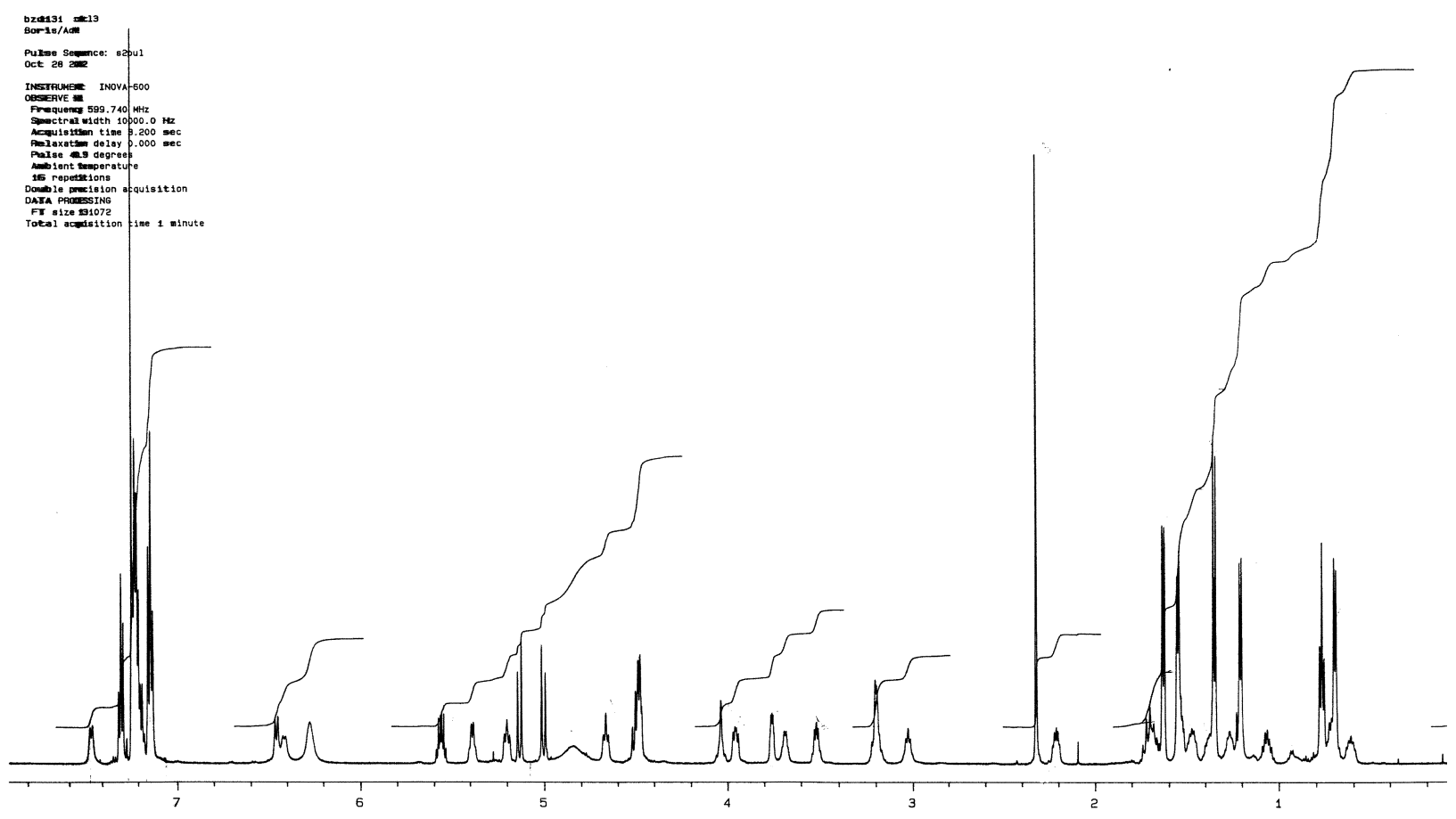

$N$-MeZ protected cyclohexadepsipeptide (171c)

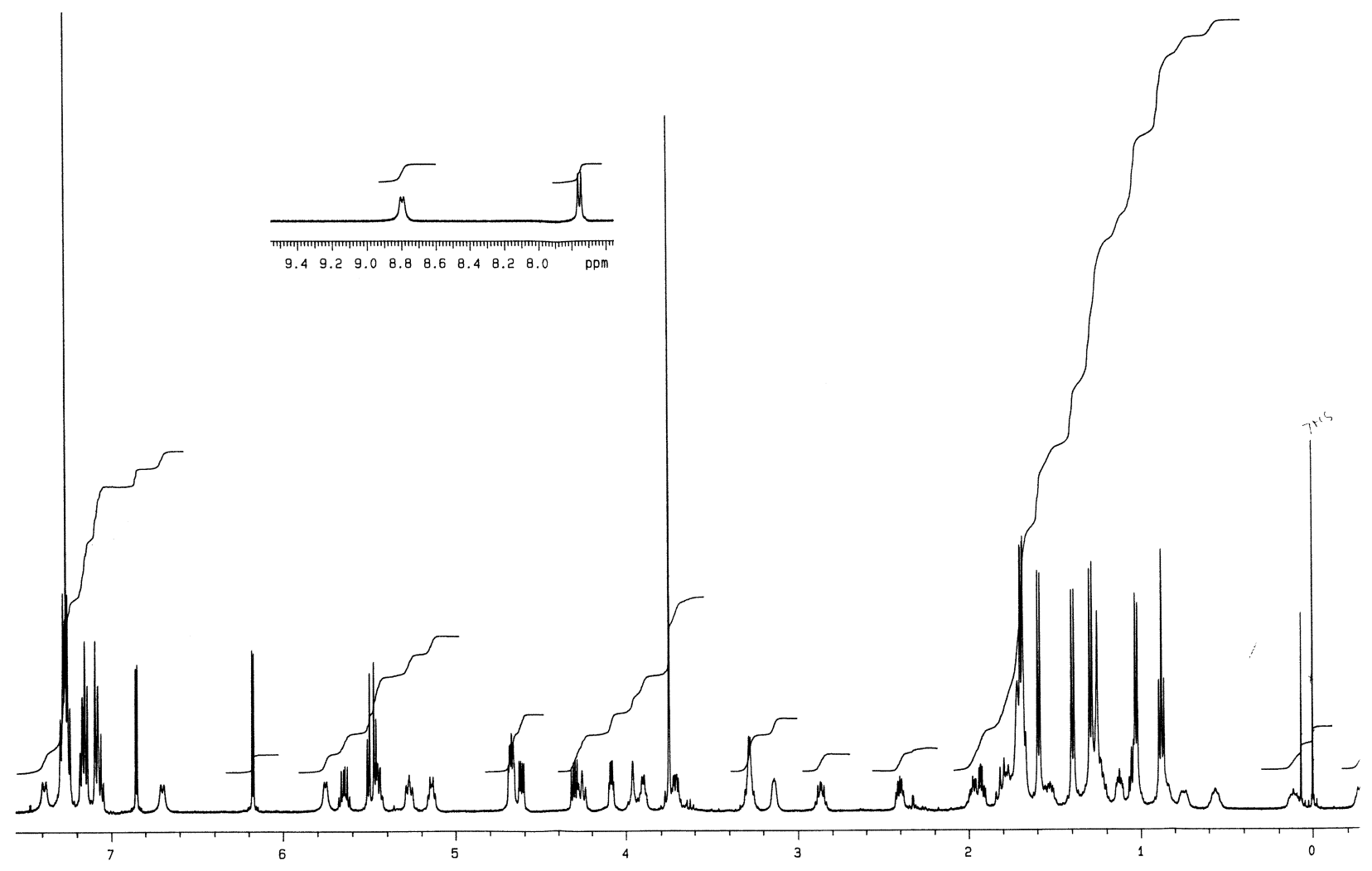

$O$-MOM protected hormaomycin $O$-MOM-(3) 


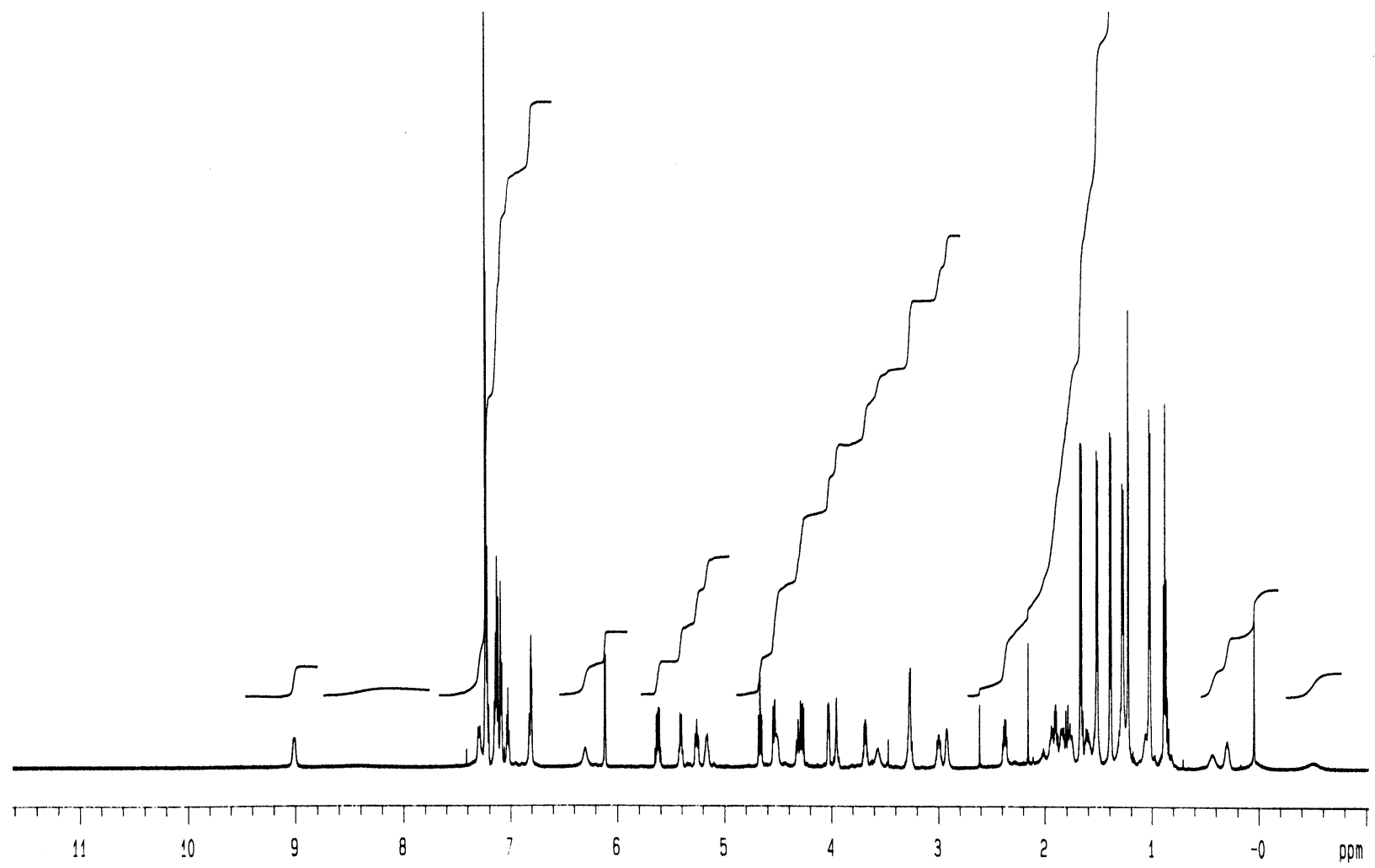

\section{Hormaomycin (3)}

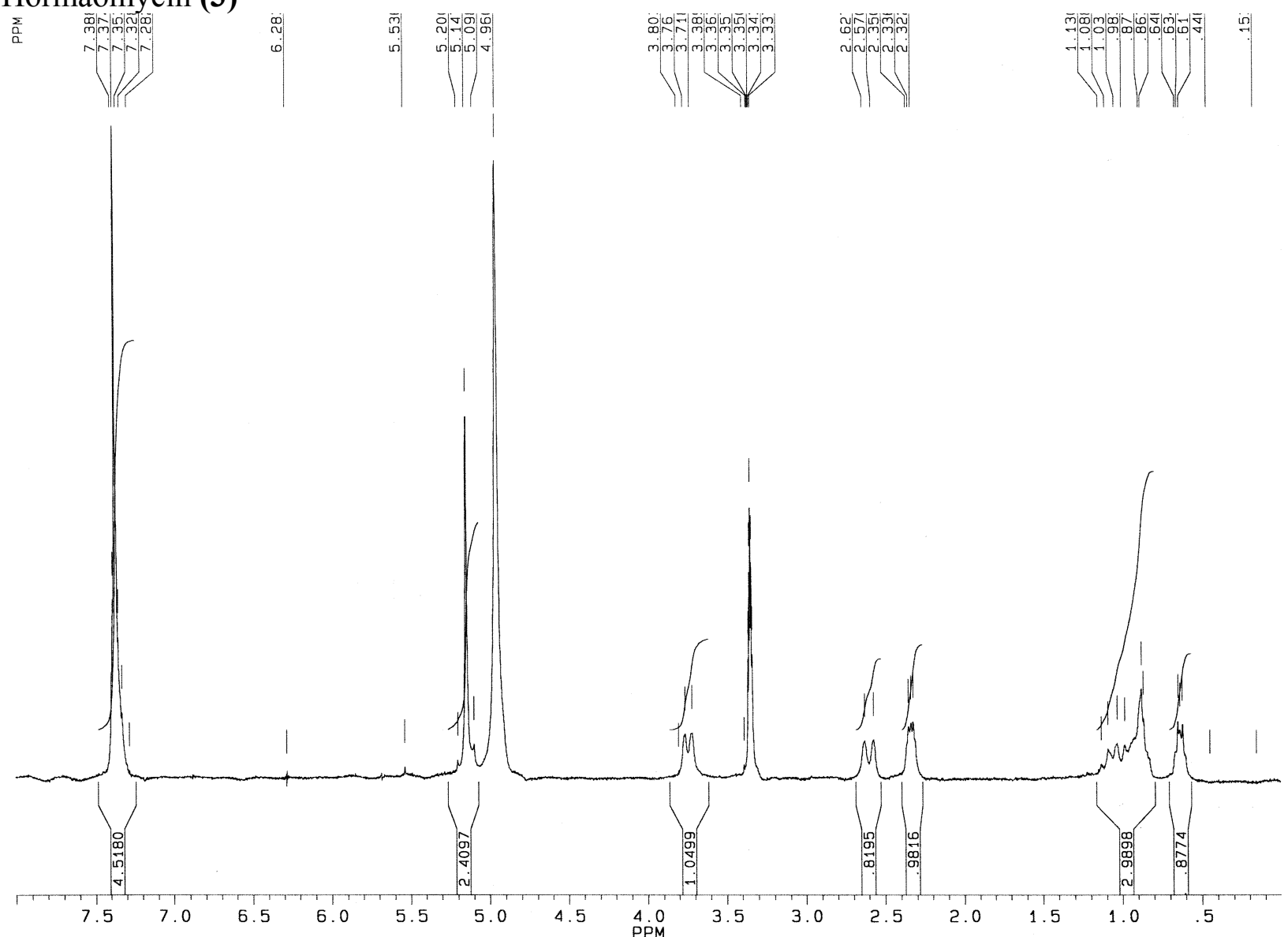

$N_{\omega^{-}} \mathrm{Z}$ protected (2S, 1'S, $\left.2^{\prime} \mathrm{R}\right)-3-\left(2^{\prime}\right.$-Aminocyclopropyl)alanine (S)-(199) 


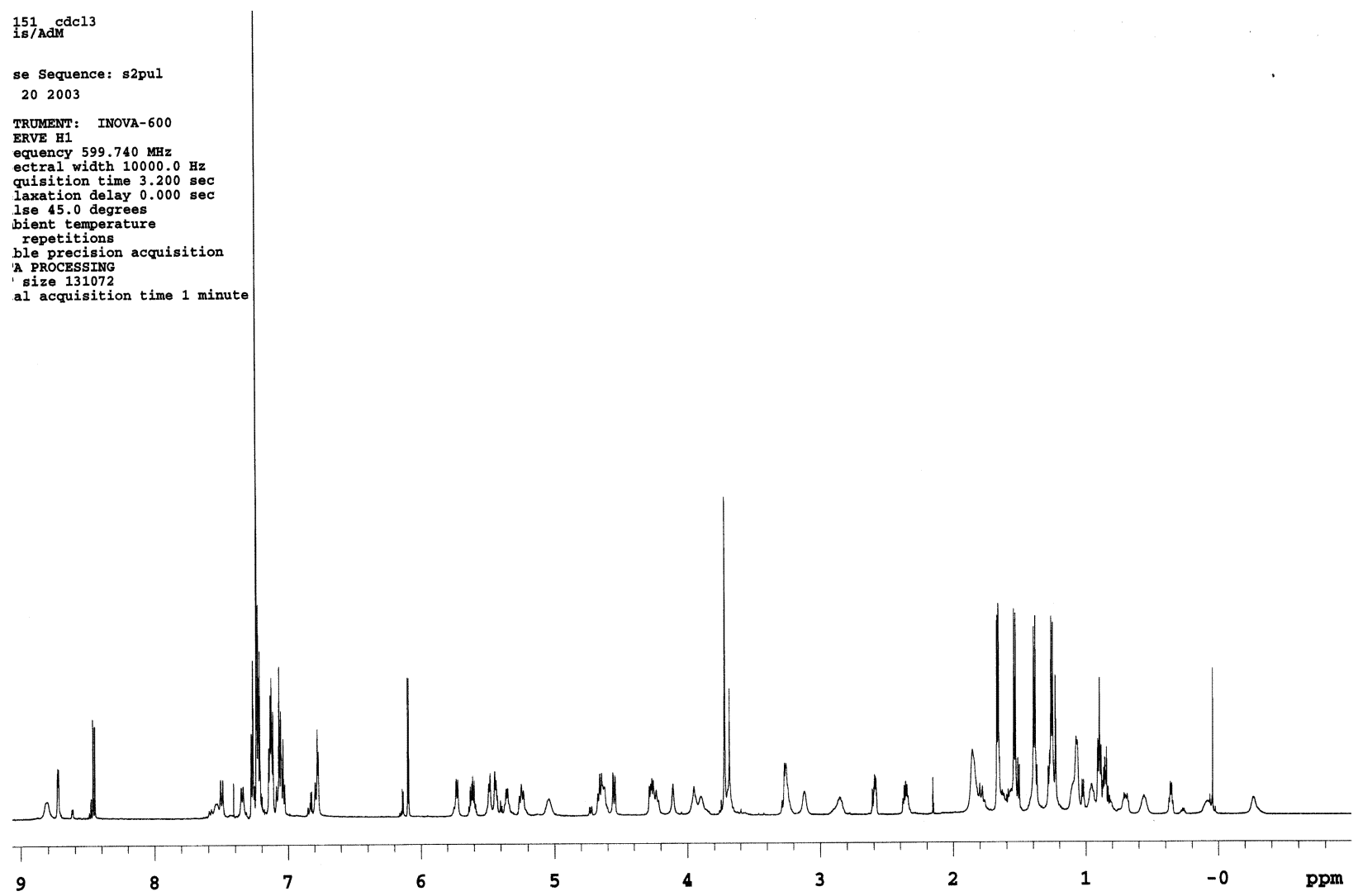

$\left[(S)-(3-A c p) A l a(N p y s)^{7}\right]-M O M-3$

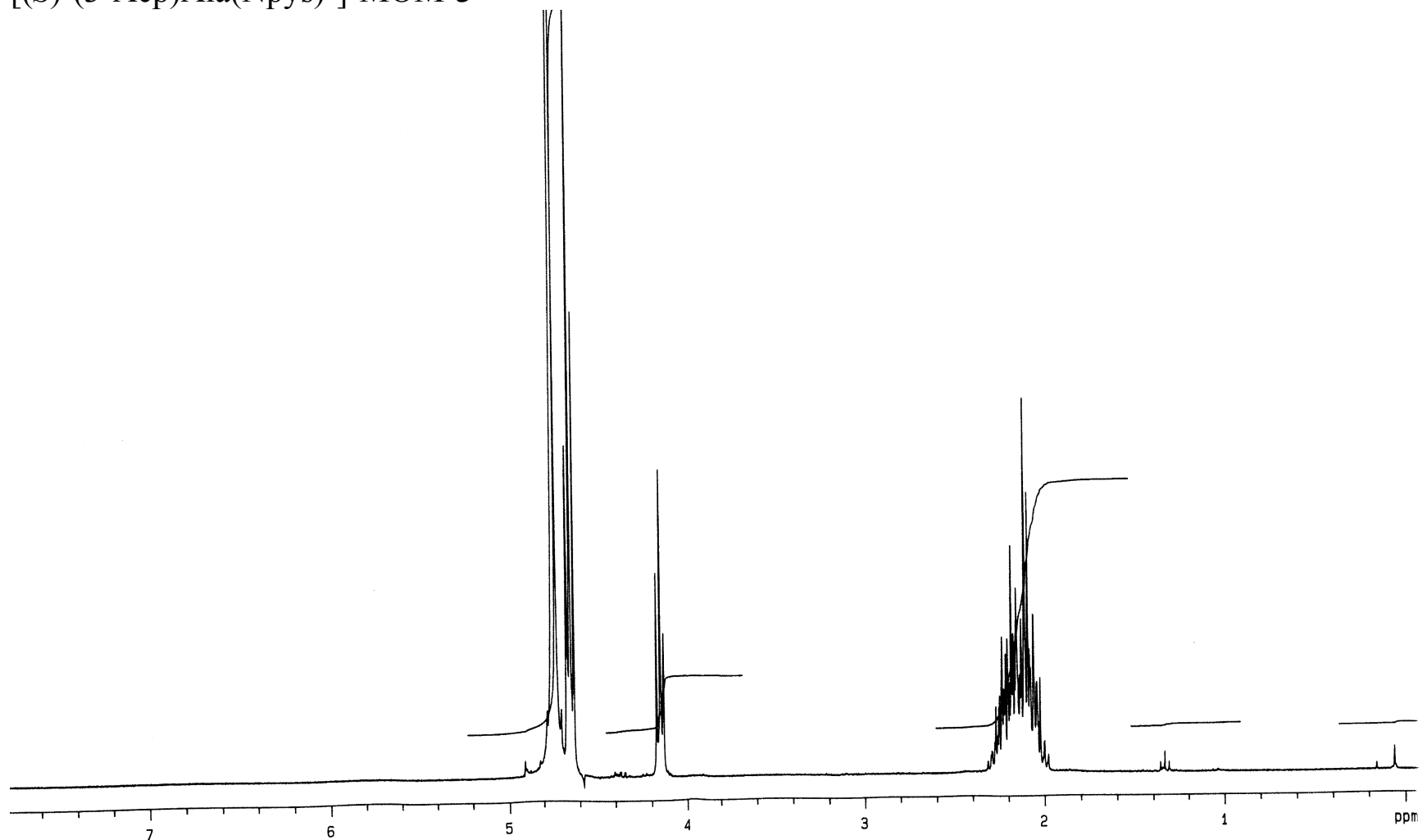

(S)-5-Nitronorvaline hydrochloride (208) 


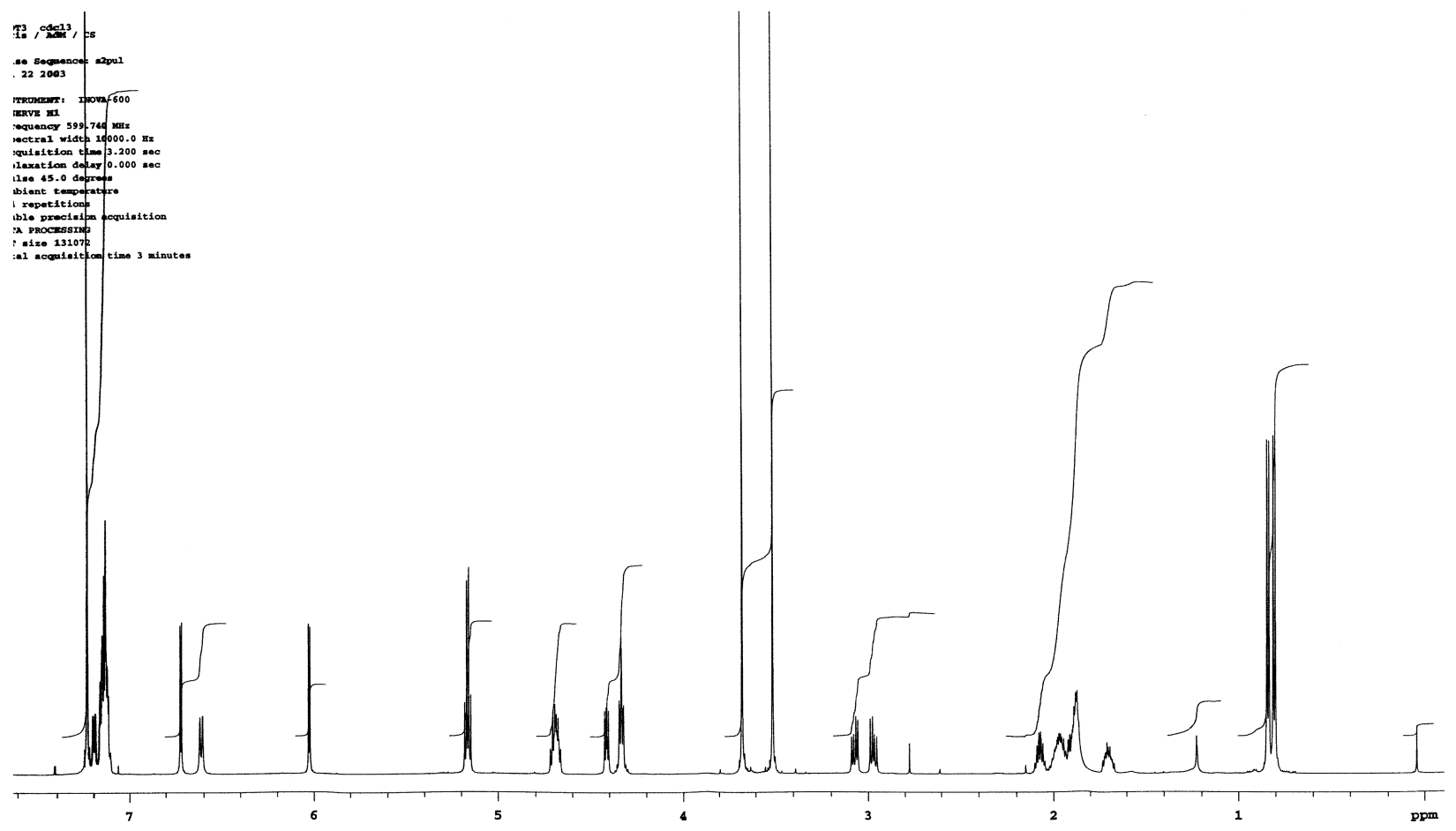

Chpca(MOM)-( $\left.\mathrm{NO}_{2}\right)$ Nva-Phe-Val-OMe (211)

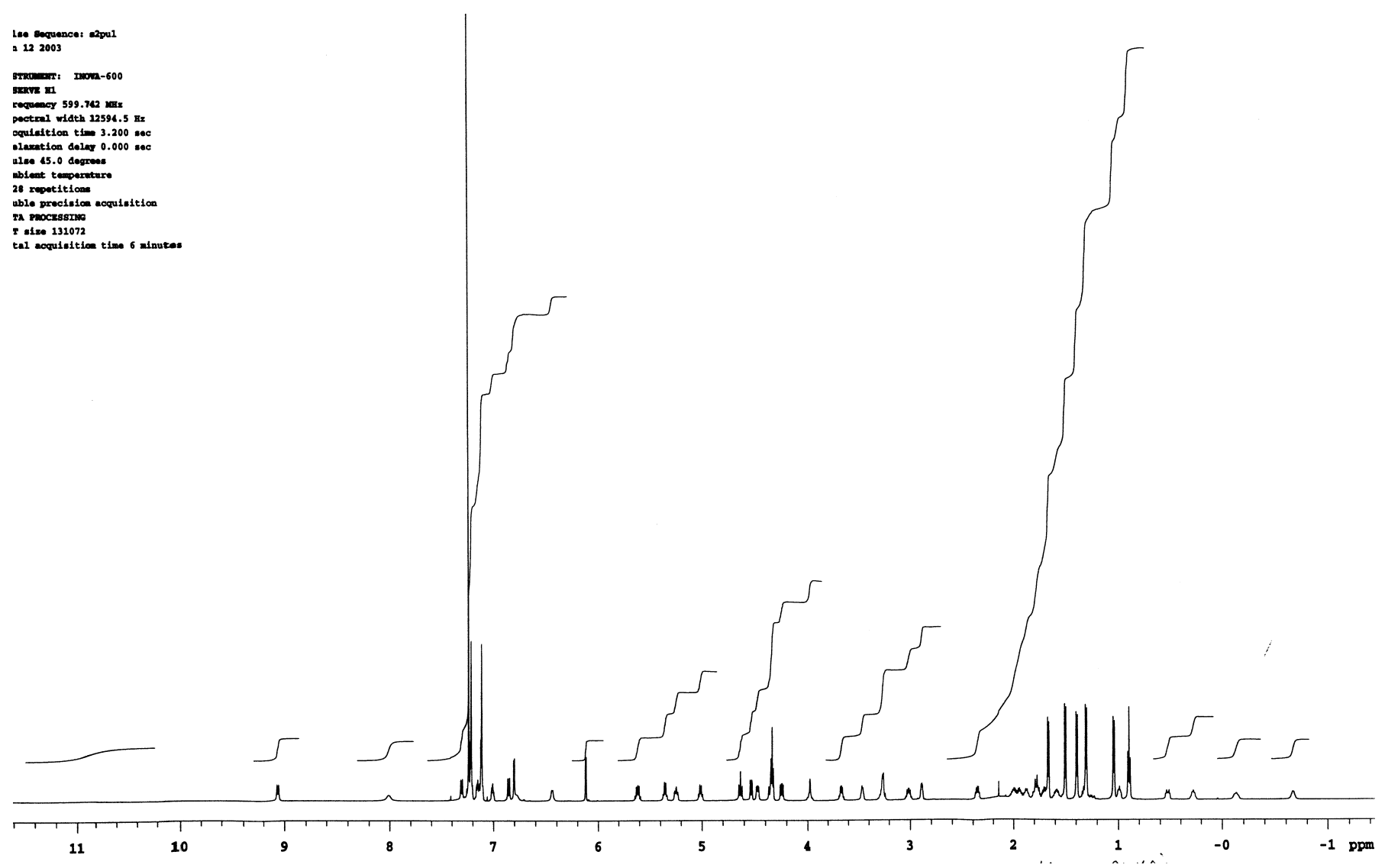

$\left[(\mathrm{S})-\left(\mathrm{NO}_{2}\right) \mathrm{Nva}^{7}\right]-$ Hormaomycin $\left[(S)-\left(\mathrm{NO}_{2}\right) \mathrm{Nva}^{7}\right]-(\mathbf{3})$ 


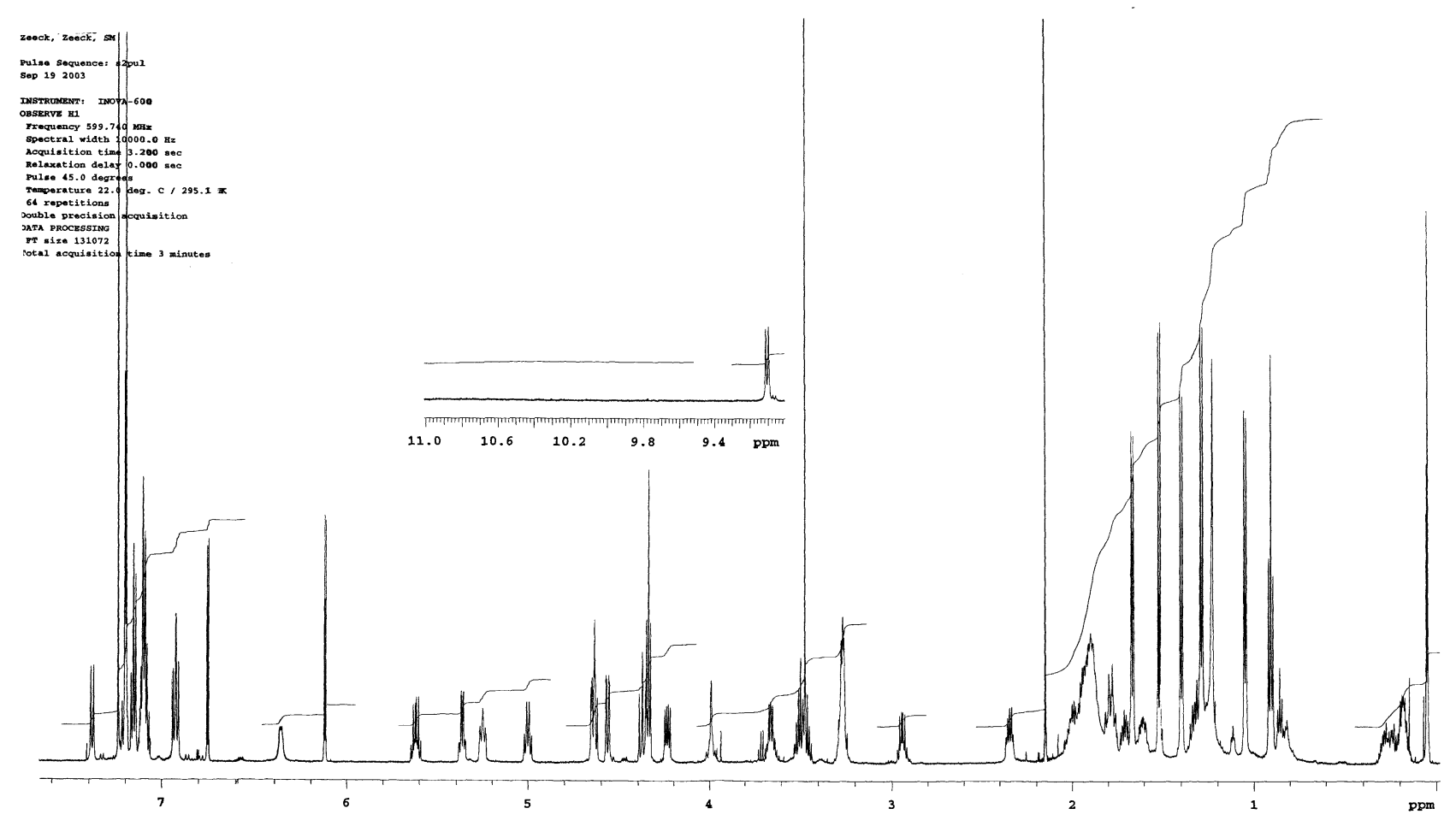

$\left[(\mathrm{S})-\left(\mathrm{NO}_{2}\right) \mathrm{Nva}^{3},(\mathrm{~S})-\left(\mathrm{NO}_{2}\right) \mathrm{Nva}^{7}\right]-$ Hormaomycin $\left[(\mathrm{S})-\left(\mathrm{NO}_{2}\right) \mathrm{Nva}^{3},(\mathrm{~S})-\left(\mathrm{NO}_{2}\right) \mathrm{Nva}^{7}\right]-(\mathbf{3})$

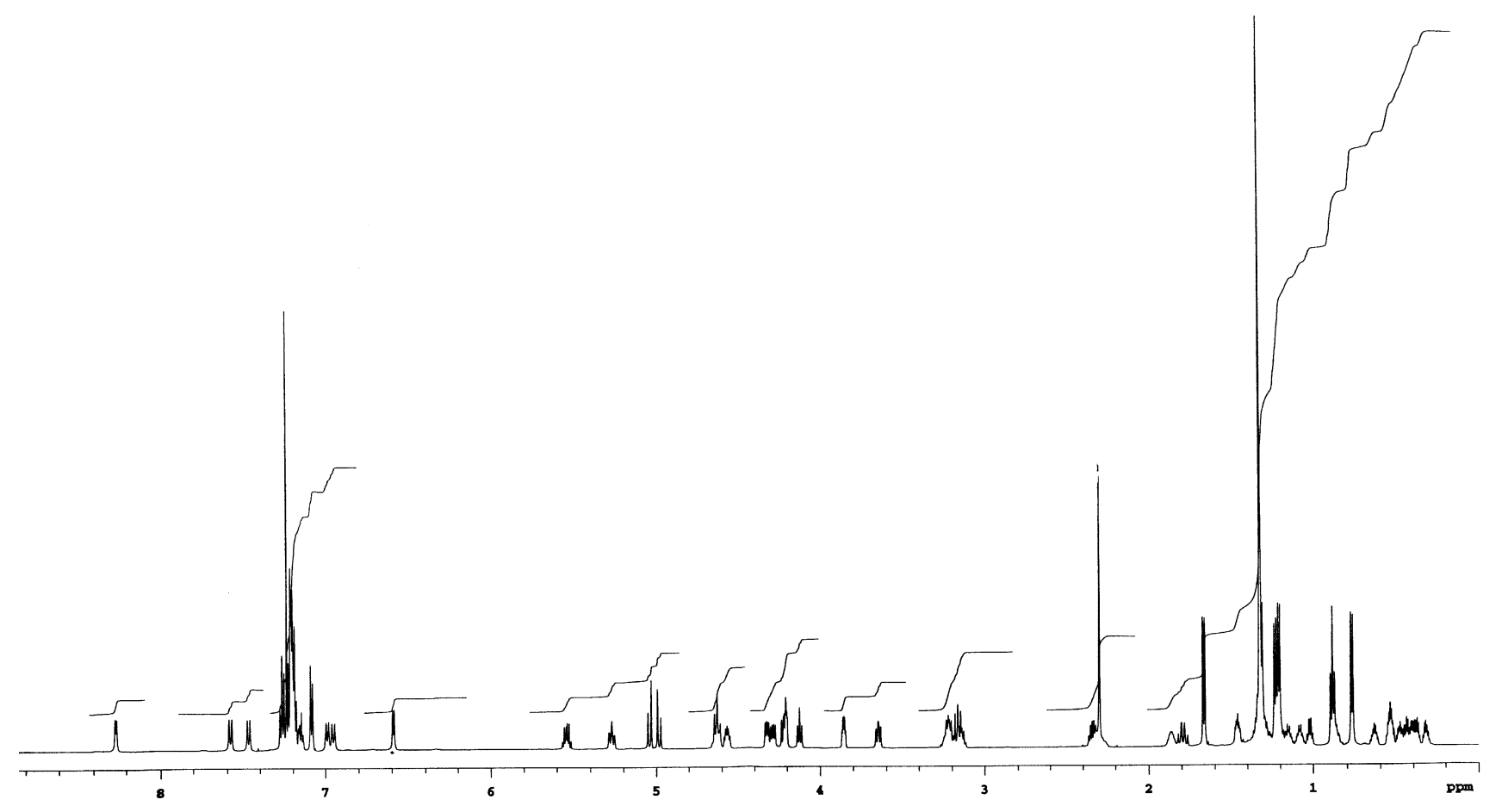

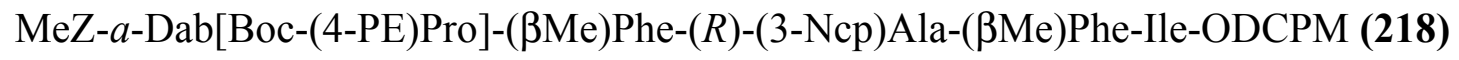




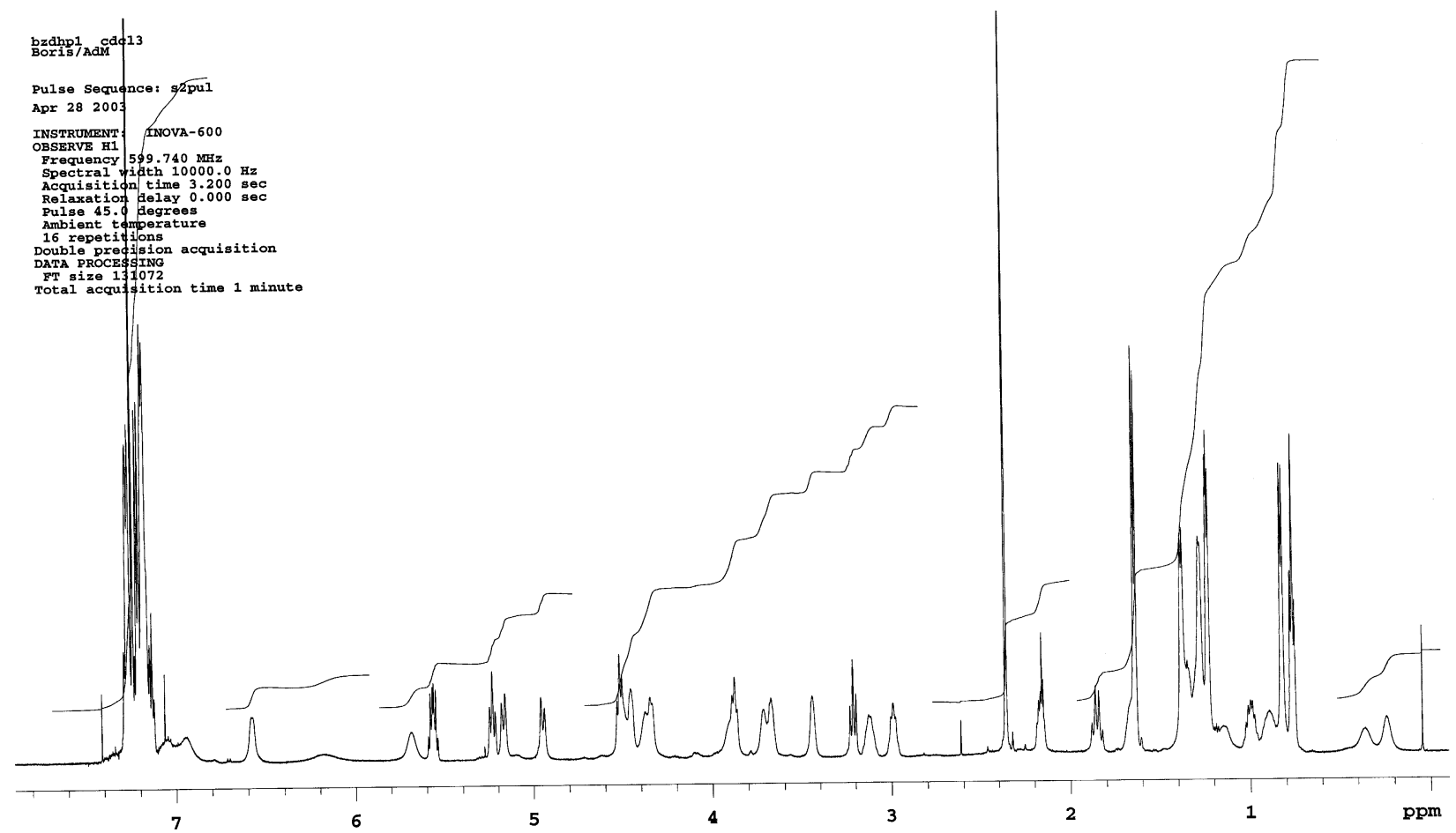

MeZ-Protected branched cyclohexapeptide (219a)

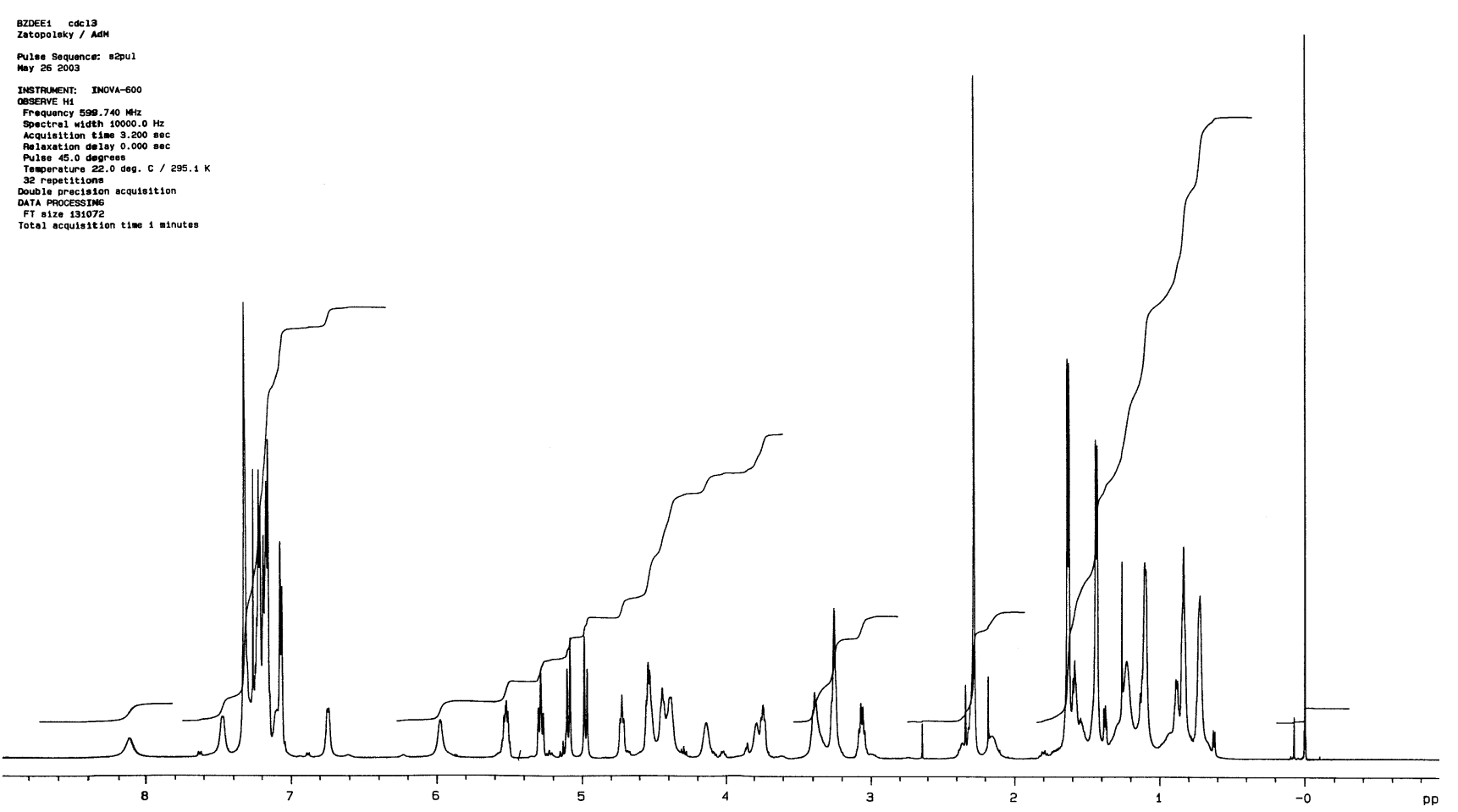

219b 


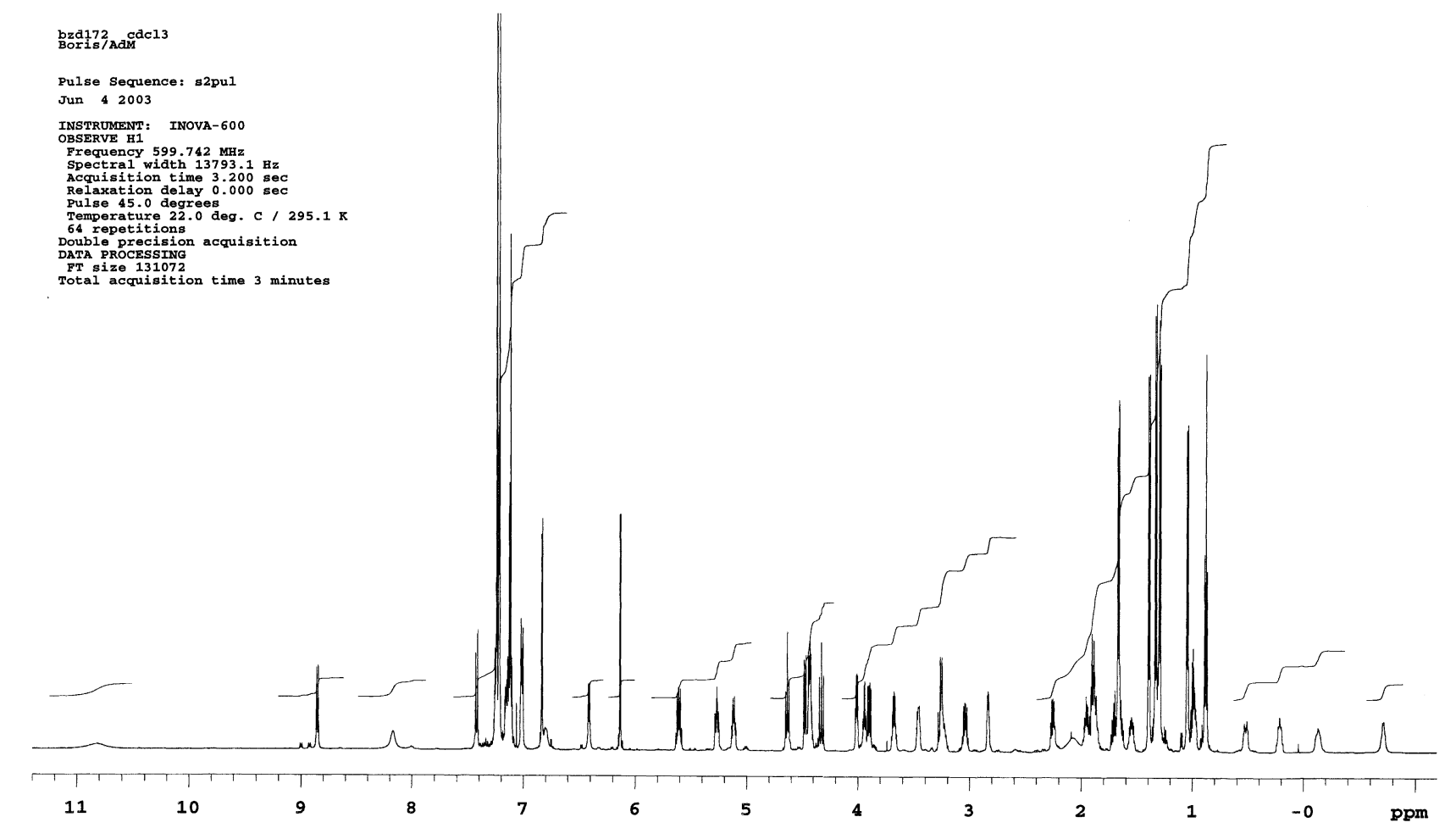

$\left[a-\right.$ Dab $\left.^{1}\right]$-Hormaomycin $\left[a-\right.$ Dab $\left.^{1}\right]-\mathbf{3}$
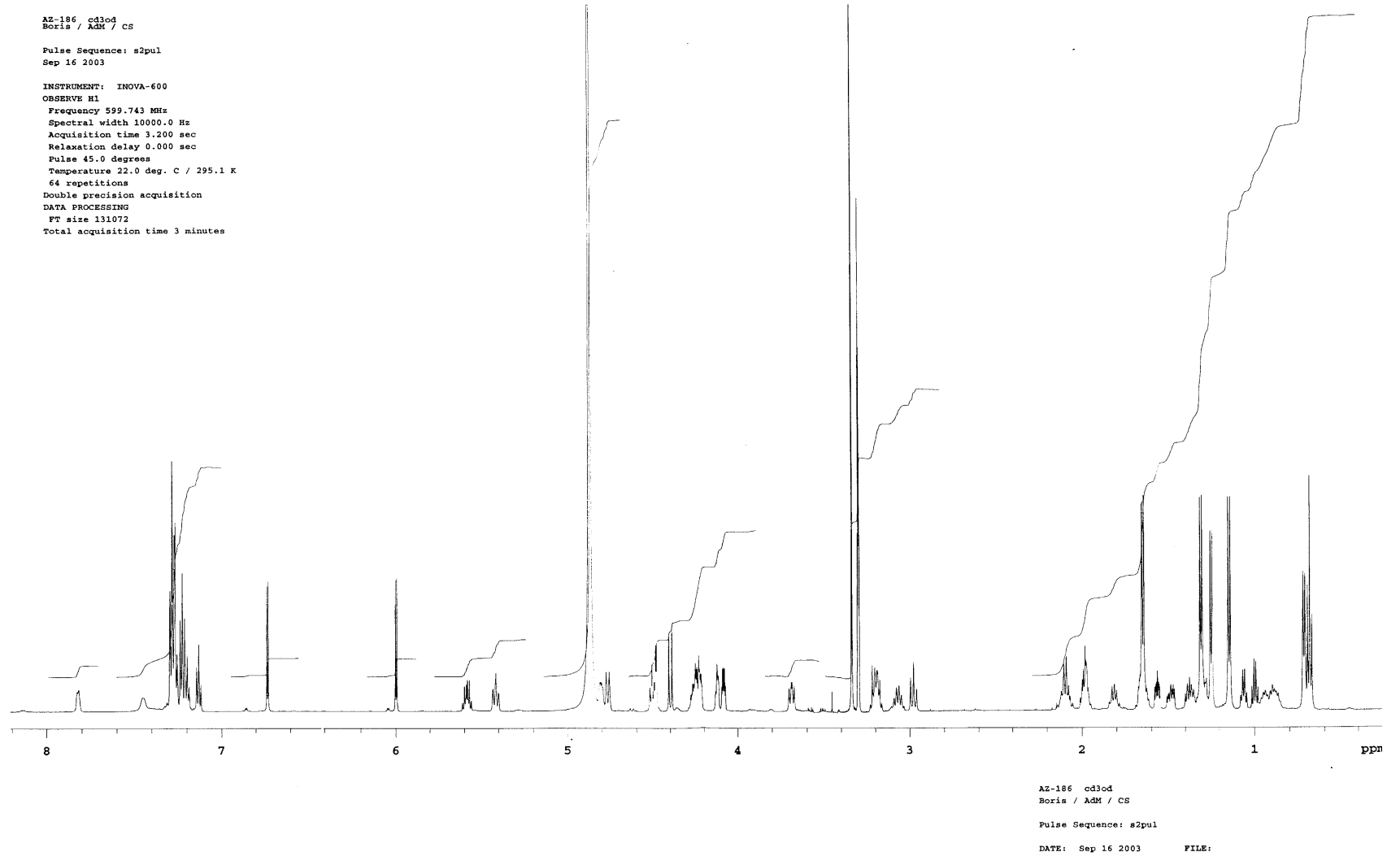

$\left[a-\mathrm{Dab}^{1}, a-\mathrm{Ile}^{5}\right]$-Hormaomycin $\left[a-\mathrm{Dab}^{1}, a-\mathrm{Ile}^{5}\right]-(\mathbf{3})$ 


\section{2. ${ }^{13}$ C-NMR Data}

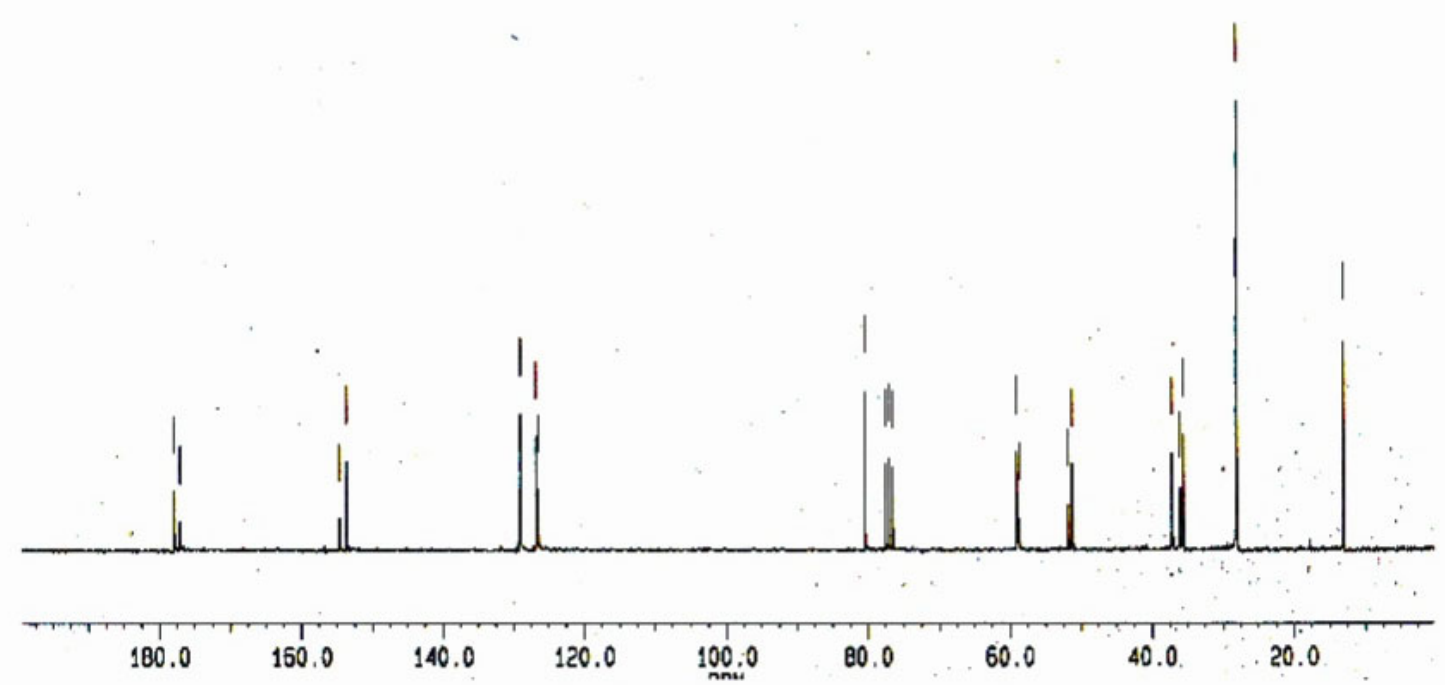

(2S,4R)-N-(tert-Butyloxycarbonyl)-4-(Z)-propenylproline $(2 S, 4 R)-16$

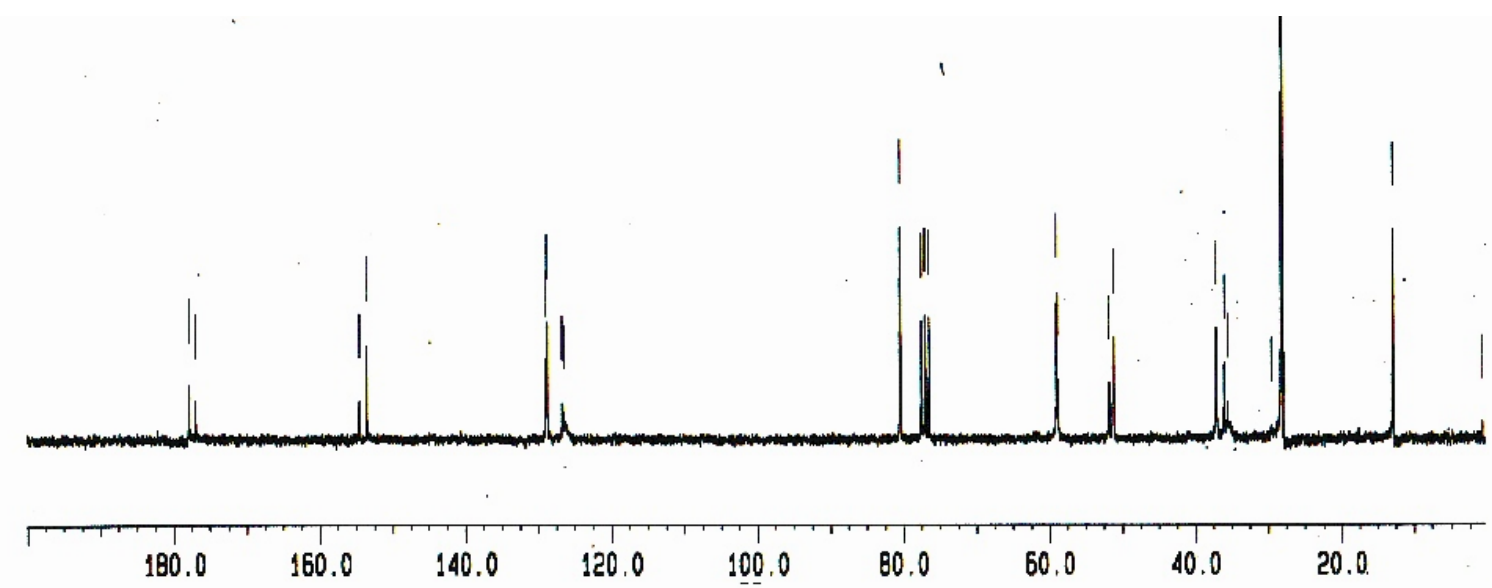

(2S,4R)- $N$-(tert-Butyloxycarbonyl)-4,1'-dideutero-4-(Z)-propenylproline $(2 S, 4 R)-\mathrm{D}_{2}-16$ 


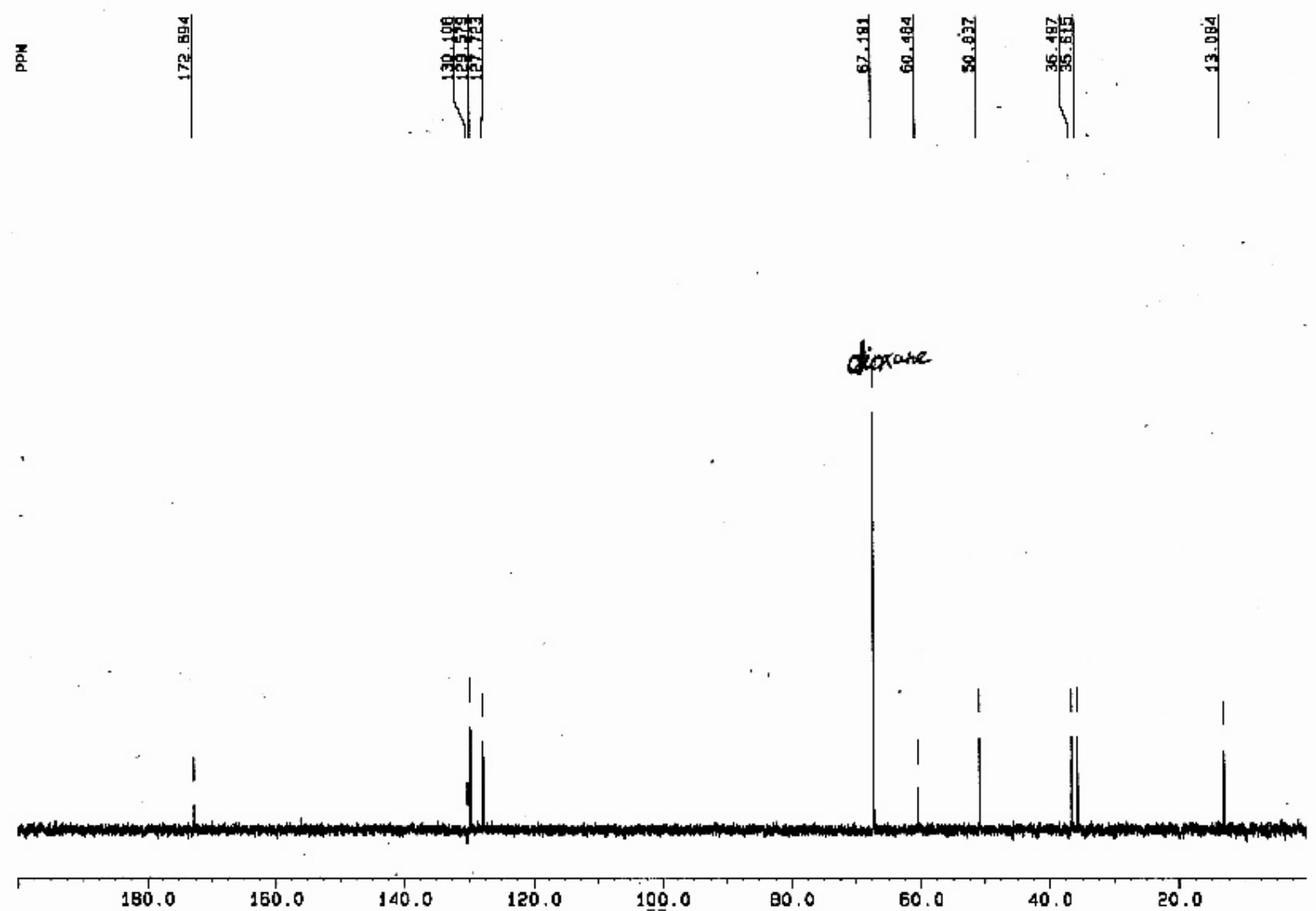

(2S,4R)-4-(Z)-propenylproline hydrochloride $(2 S, 4 R)-\mathbf{1 7} \cdot \mathrm{HCl}$
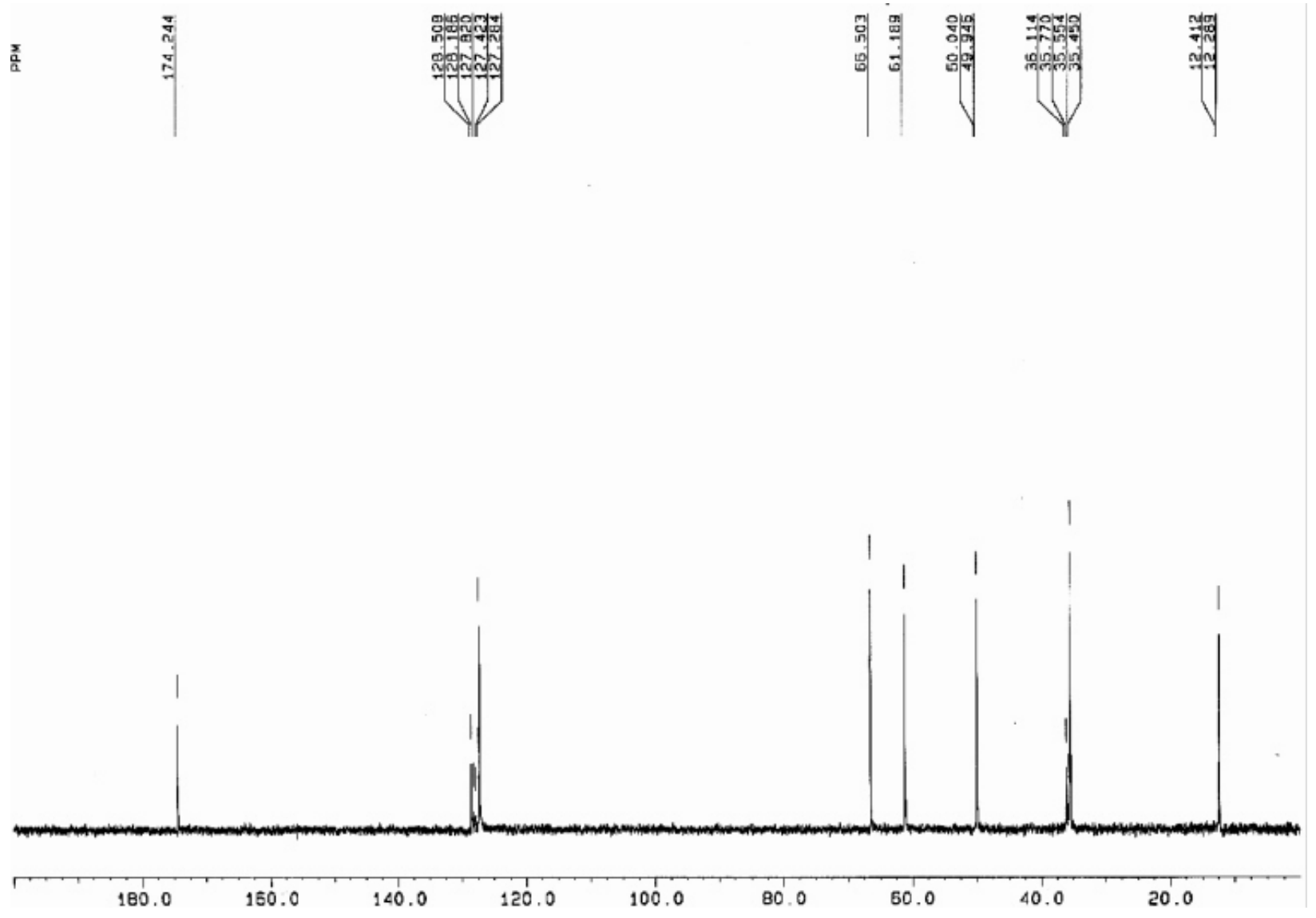

(2S,4R)-4,1'-Dideutero-4-(Z)-propenylproline (2S,4R)-17 (66.5 ppm - dioxane) 

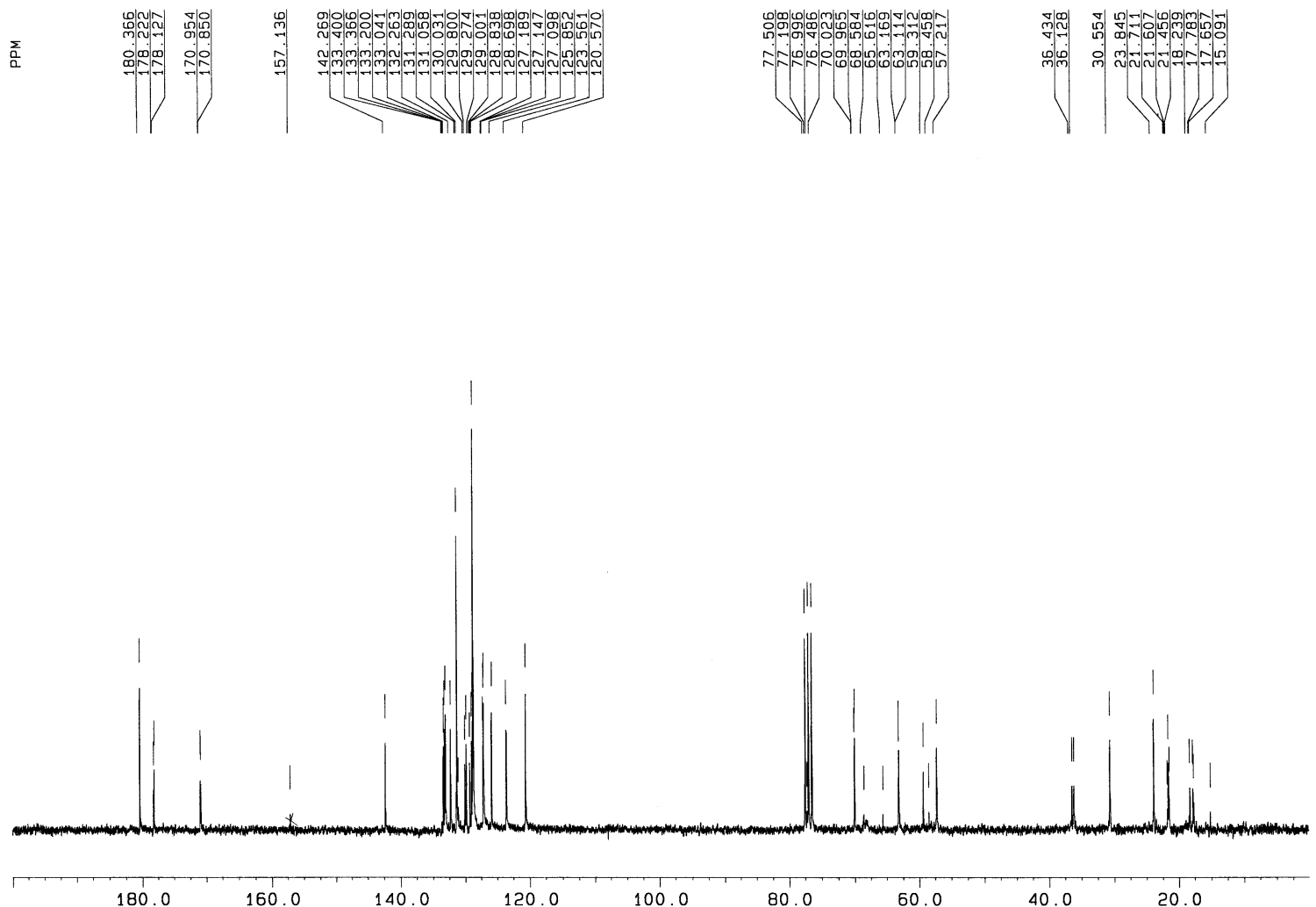

$N i$-complex $\left(2 S, 2^{\prime} S, 1^{\prime \prime} R S, 2^{\prime \prime} R S\right)$-D-82
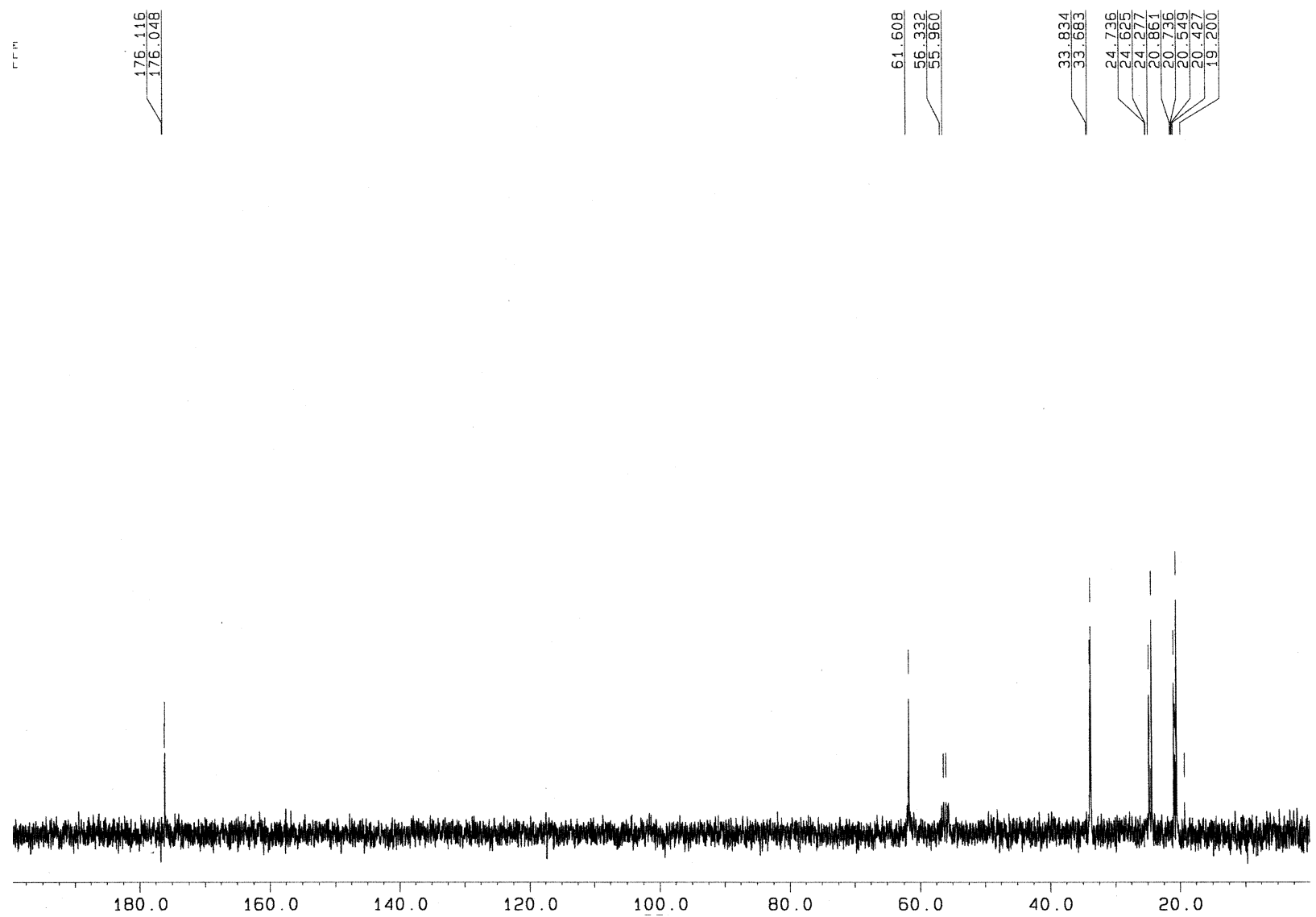

(2S,1' $\left.R S, 2^{\prime} R S\right)$-2-Deutero-3-(2'-nitrocyclopropyl)alanine $\left(2 S, 1^{\prime} R S, 2^{\prime} R S\right)$-D-(83) 


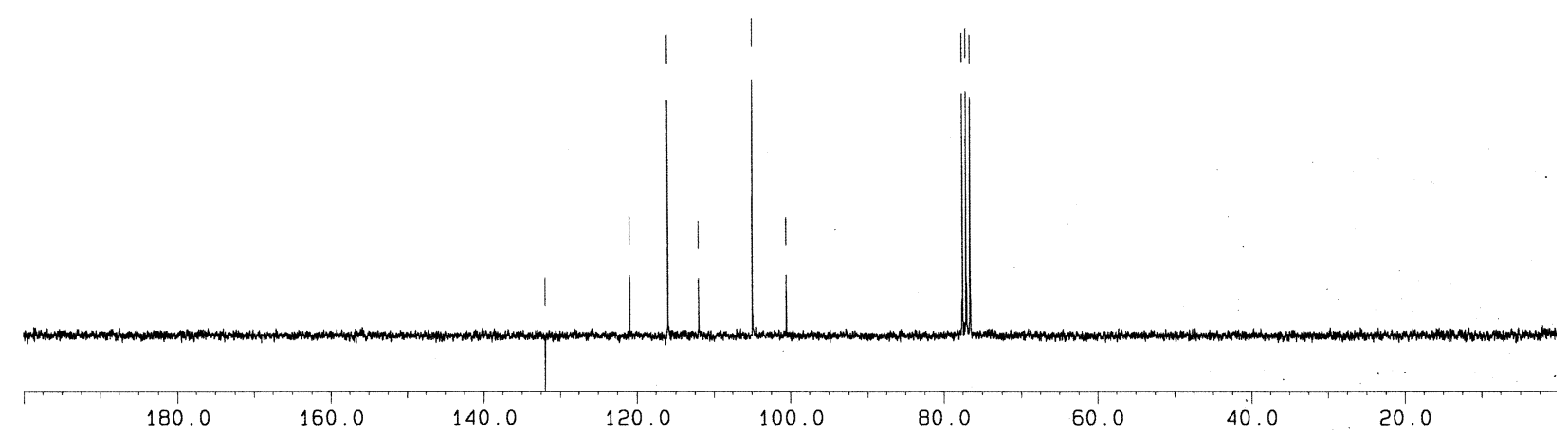

5-Chloro-1-hydroxypyrrole-2-carbonitrile (103) 言
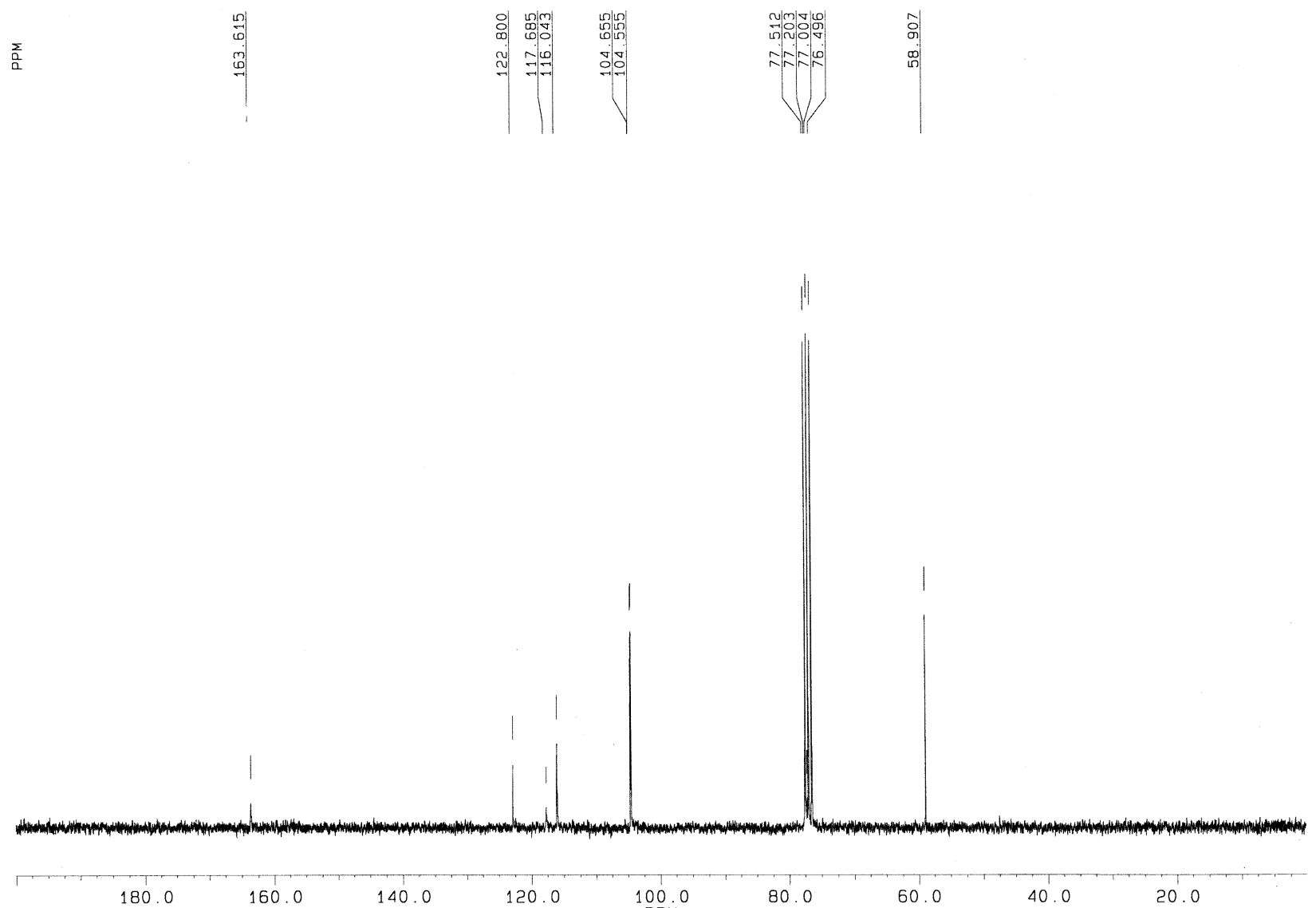

5-Chloro-1-methoxymethoxypyrrole-2-carboxylic acid (109) 

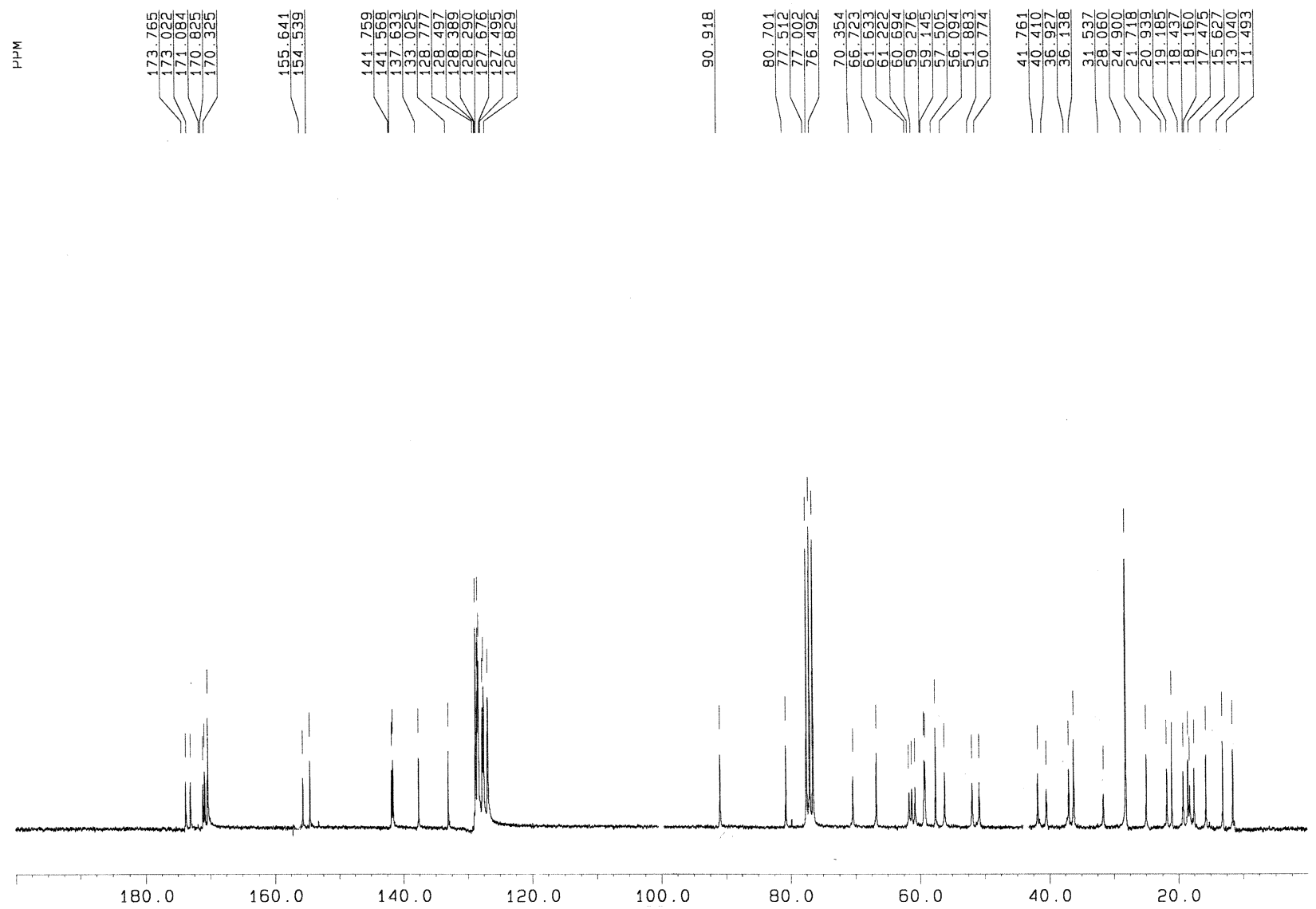

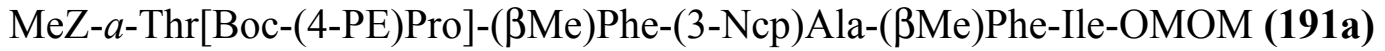
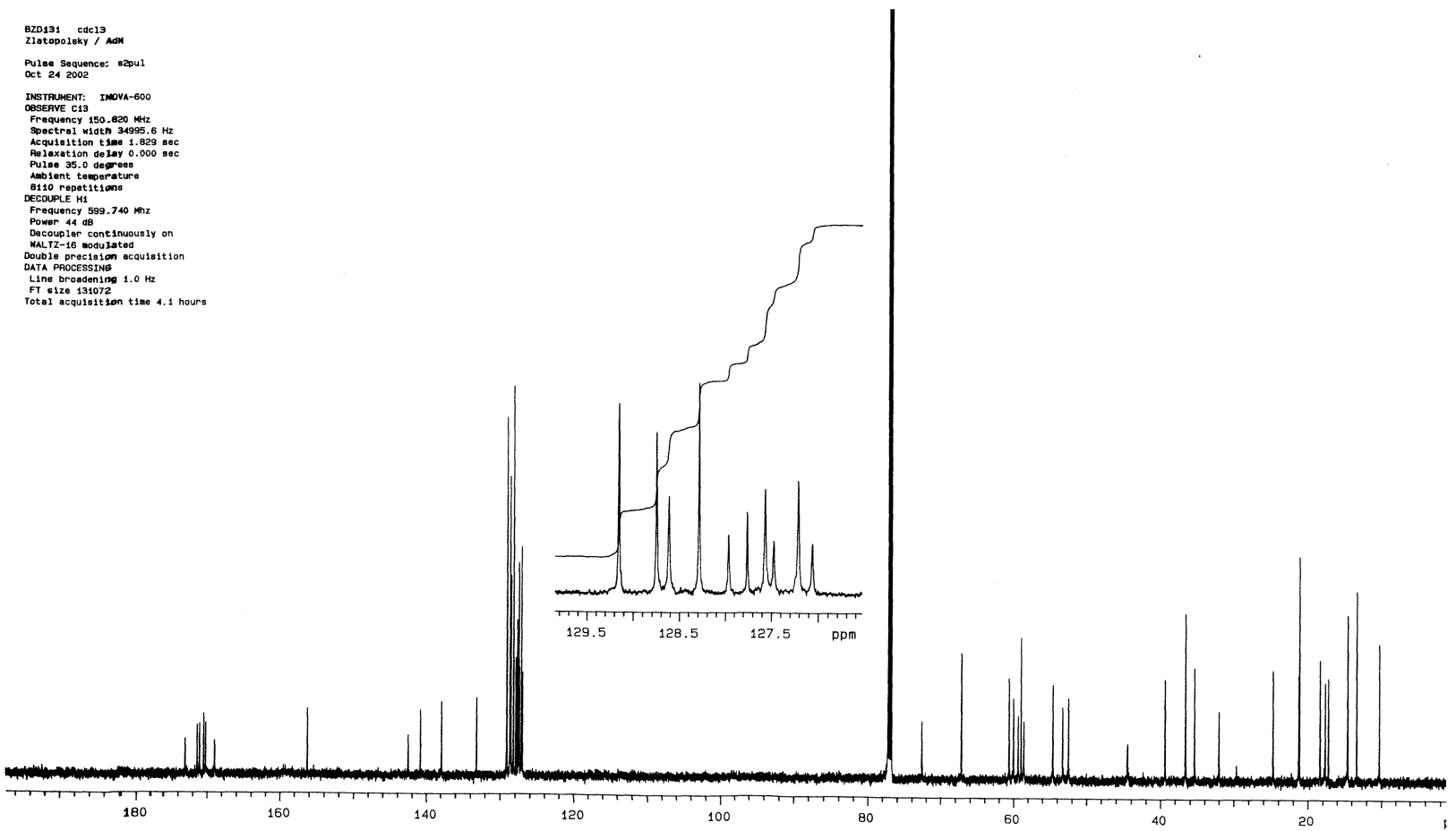

$\mathrm{N}$-MeZ protected cyclohexadepsipeptide (171c) 
Pulse Sequence: s2pul

Nov 112002

INSTRUMENT: INOVA-600

Frequency 150.820 MHz
Spectral width $34995.6 \mathrm{~Hz}$

Spectral width $34995.6 \mathrm{~Hz}$
Acquisition time $1.829 \mathrm{sec}$
Relaxation delay 0.000

Relaxation delay 0.000
Pulse 35 . 0 degrees

Ambient temperature
23504 repetitions

Frequency $599.740 \mathrm{Mhz}$

Power $44 \mathrm{~dB}$ d
Decoupler continuously on

WALTZ-16 modulated $1 Y$ on

DATA PROCESSING
Line broadening $1.0 \mathrm{~Hz}$

Total acquisition time 11.9 hours

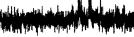

111

140

120

100

80

60

40

20

ppm

$O$-MOM protected hormaomycin $O$-MOM-(3)

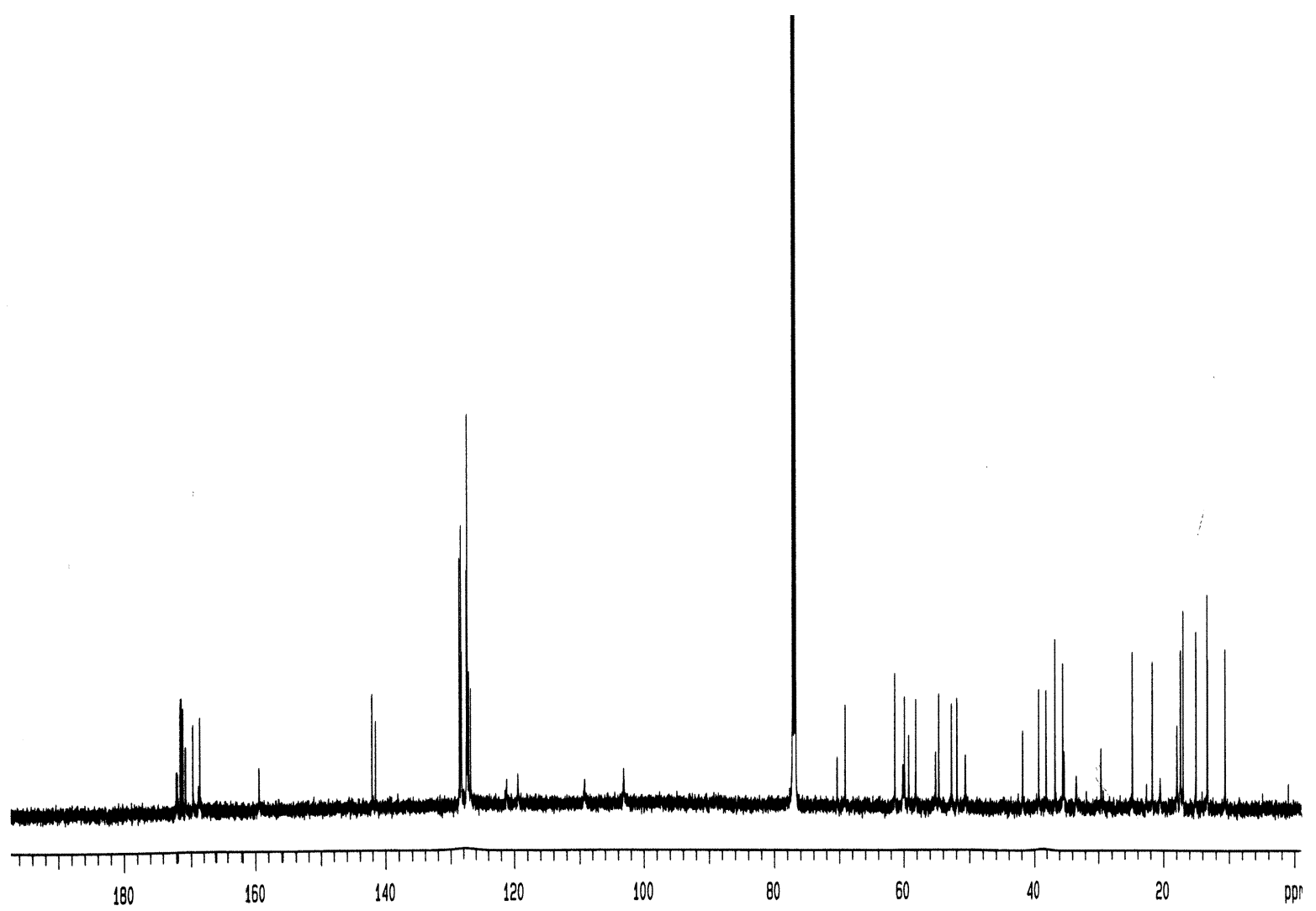

Hormaomycin (3) 


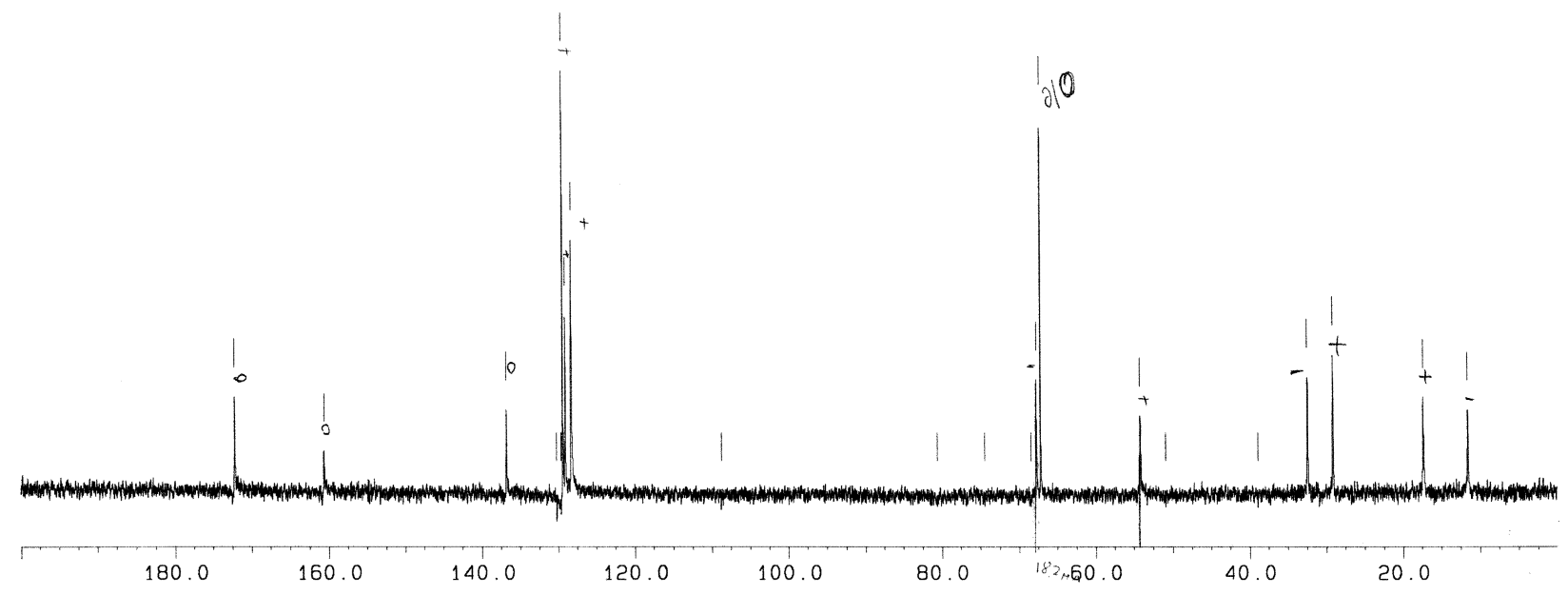

$N_{\omega}$-Z protected (2S, $\left.1^{\prime} S, 2^{\prime} R\right)-3-\left(2^{\prime}\right.$-Aminocyclopropyl)alanine $(S)-(199)$

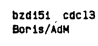

Pulse Sequence: 82001
Feb 272003

INSTRUAET: INOYA-600

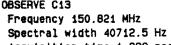

Acquisition time 1.829 see
helaxation dealay 0.000 sec

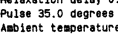

24000 repat it ions
DECOUPLE H1
H

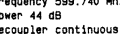

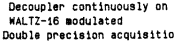

DATA Processing
Line broadenting 1.0

FT 912 12e 131072
Total acquist ition time 12.2 hour

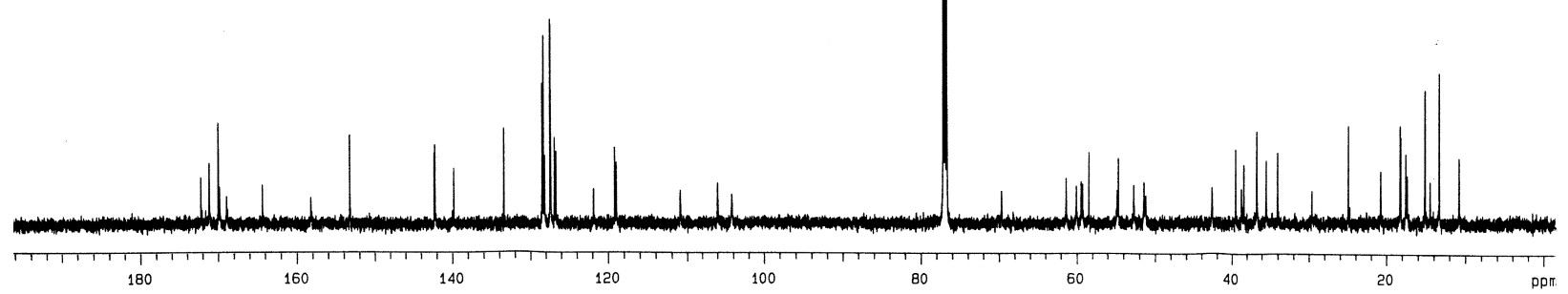

$\left[(S)-(3-A c p) A l a(N p y s)^{7}\right]-M O M-3$ 
DLuido aco
Zlatopolski / AdM

Jun 272003

INSTRUMENT: UNITY-200

OBSEAVE C13

requency $50.293 \mathrm{MHz}$

Frisition time $1.365 \mathrm{~Hz}$

Relaxation delay $0.000 \mathrm{sec}$

Pulse 35.0 degrees
Temperature 35.0 deg. c / $308.1 \mathrm{~K}$

Temperature 35.0 co.

12800 repetitions

Frequency $199.992 \mathrm{MHz}$

Power $40 \mathrm{~dB}$

Decoupler continuously

WALTZ-16 modulated

Double precision acquisition

FT size 65536

Total acquisition time 4.9 hours
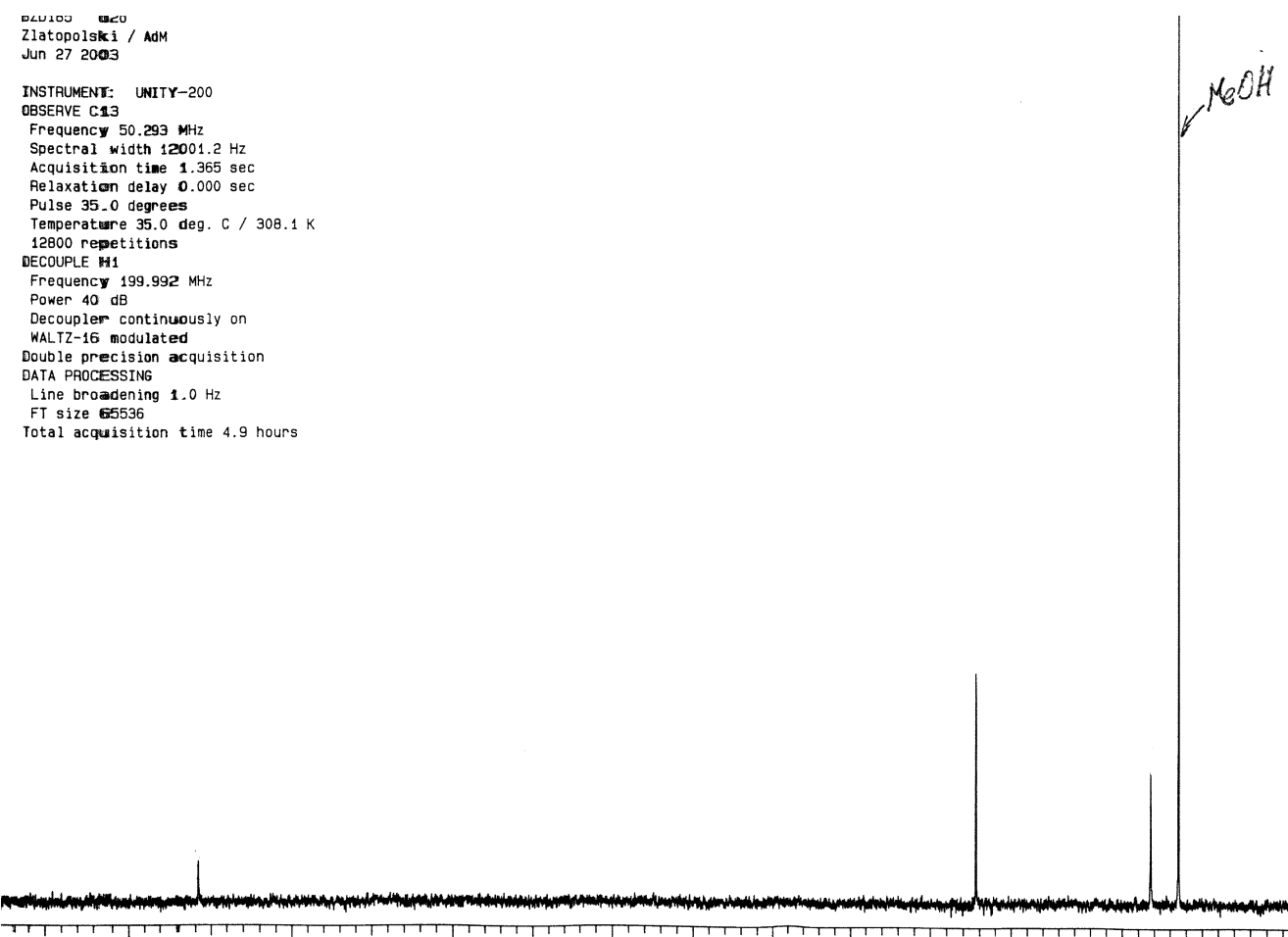

180

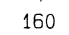

140

120

100

80

60

40

20

ppm

(S)-5-Nitronorvaline hydrochloride (208) (methanol as a standard)
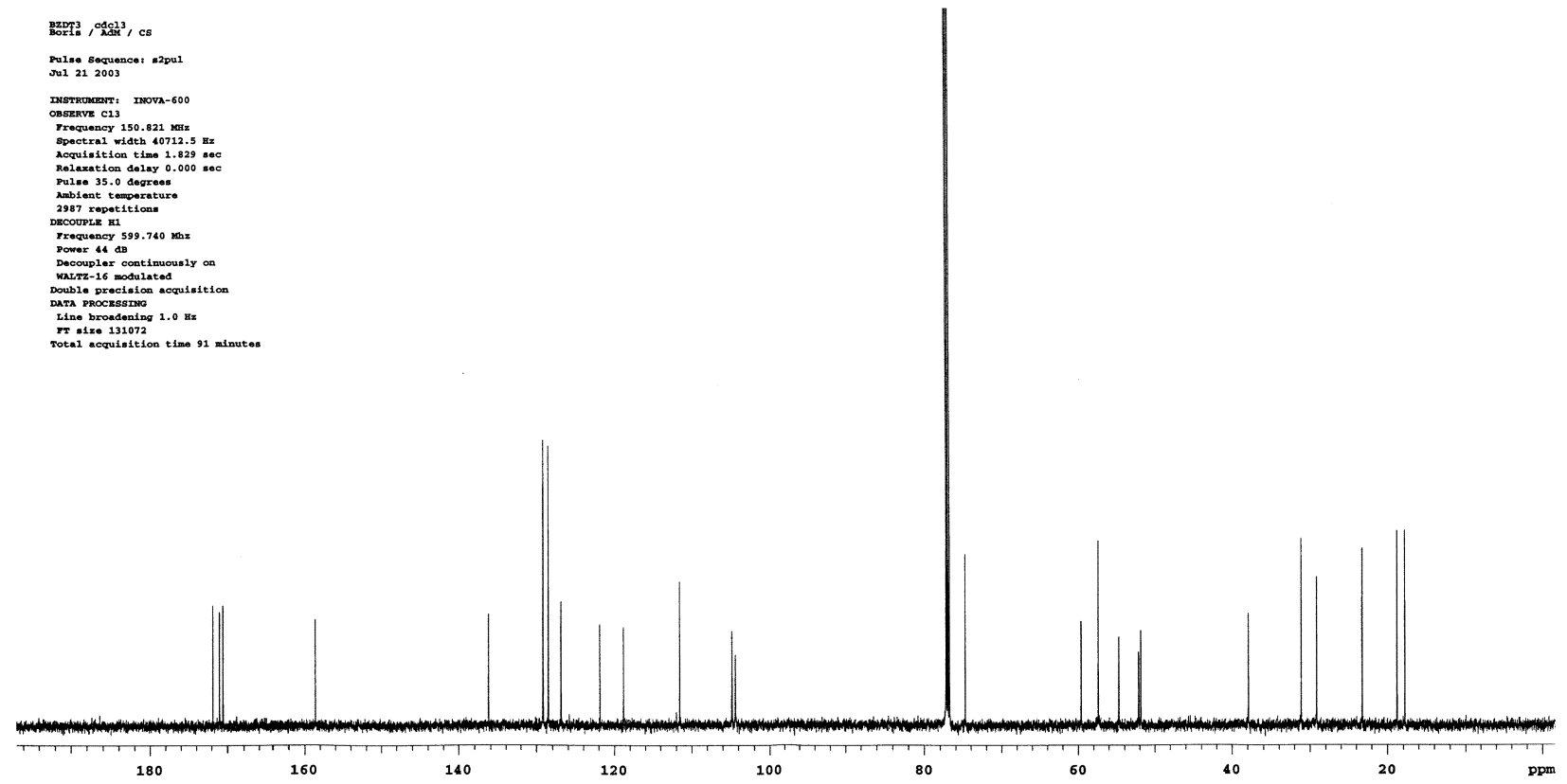

Chpca(MOM)-( $\left.\mathrm{NO}_{2}\right)$ Nva-Phe-Val-OMe (211) 


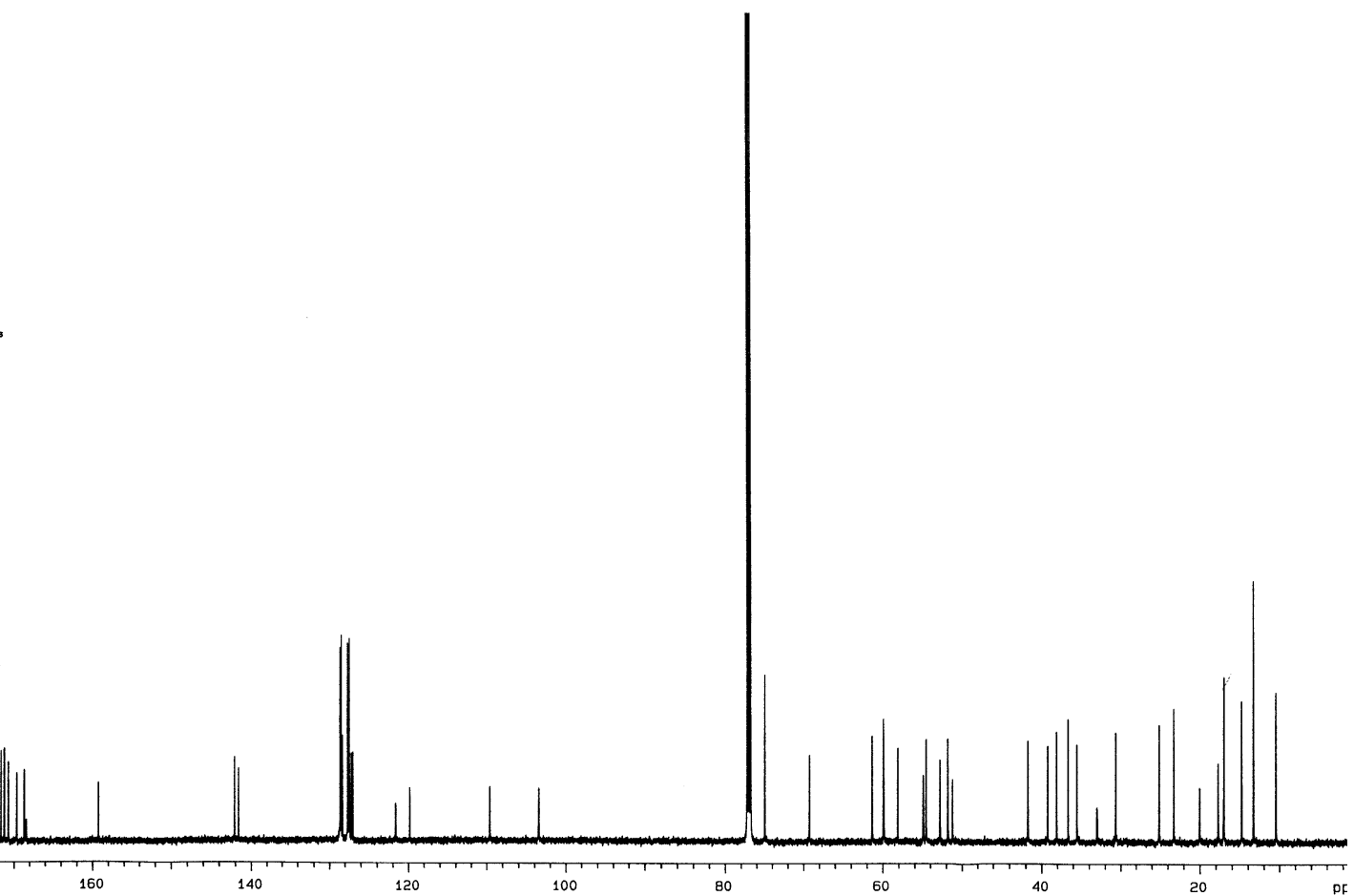

$\left[(S)-\left(\mathrm{NO}_{2}\right) \mathrm{Nva}^{7}\right]$-Hormaomycin $\left[(S)-\left(\mathrm{NO}_{2}\right) \mathrm{Nva}^{7}\right]-(\mathbf{3})$

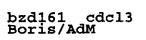

Pu1se Sequence: s2pul

Mar 172003

INSTRUMENT: INOVA-600

Frequency $150.820 \mathrm{MHz}$
Spectral width $34995.6 \mathrm{~Hz}$
Acquisition time $1.829 \mathrm{sec}$
Relagation telay $0.000 \mathrm{sec}$

Relaxation delay 0.000

Pulse 35.0 degrees
Ambient temperature
2496 repetitions

Frequency $599.740 \mathrm{maz}$

Prower 44 dB $59.740 \mathrm{Mhz}$

Decoupler continuous $1 y$ on
WALTZ-16 modulated

ouble precision acquisition

Line broadening $1.0 \mathrm{~Hz}$

Total accuisition time 76 minutes

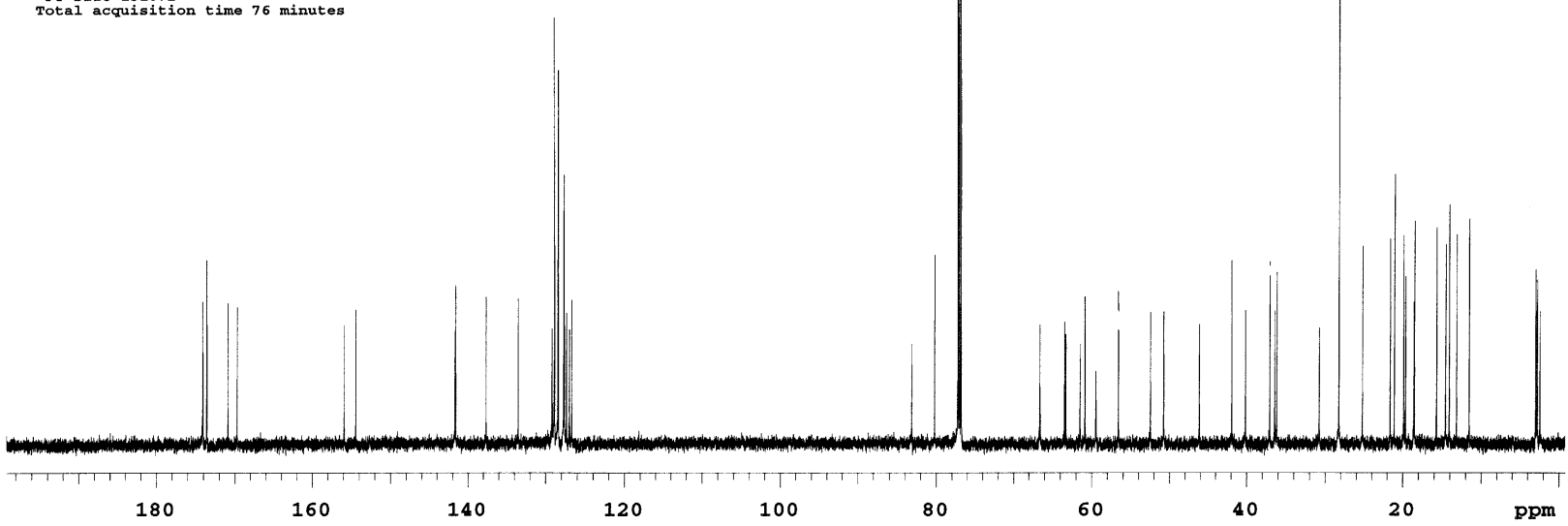

MeZ- $a$-Dab[Boc-(4-PE)Pro]-( $\beta M e) P h e-(R)-(3-N c p) A l a-(\beta M e) P h e-I l e-O D C P M ~(218)$ 


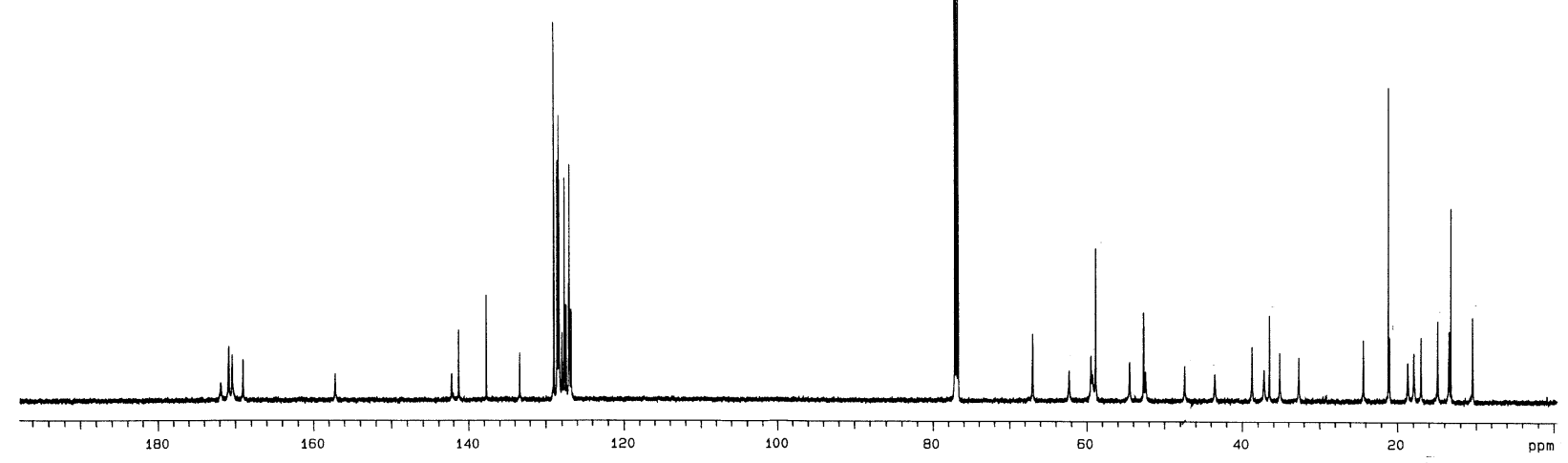

MeZ-Protected branched cyclohexapeptide (219a)

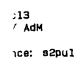

rnoyn-600

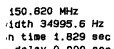

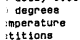

5 . 59.740 .472

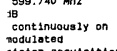

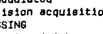

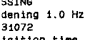

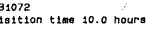




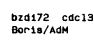

Pulse Sequance:
Sun 3 2003

INSSRMMENT: INOVNaA-600

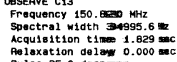

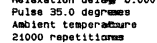

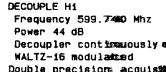

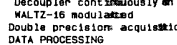

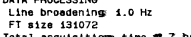

Total acqu1 19t1 tiom t time $m$. 7 hours

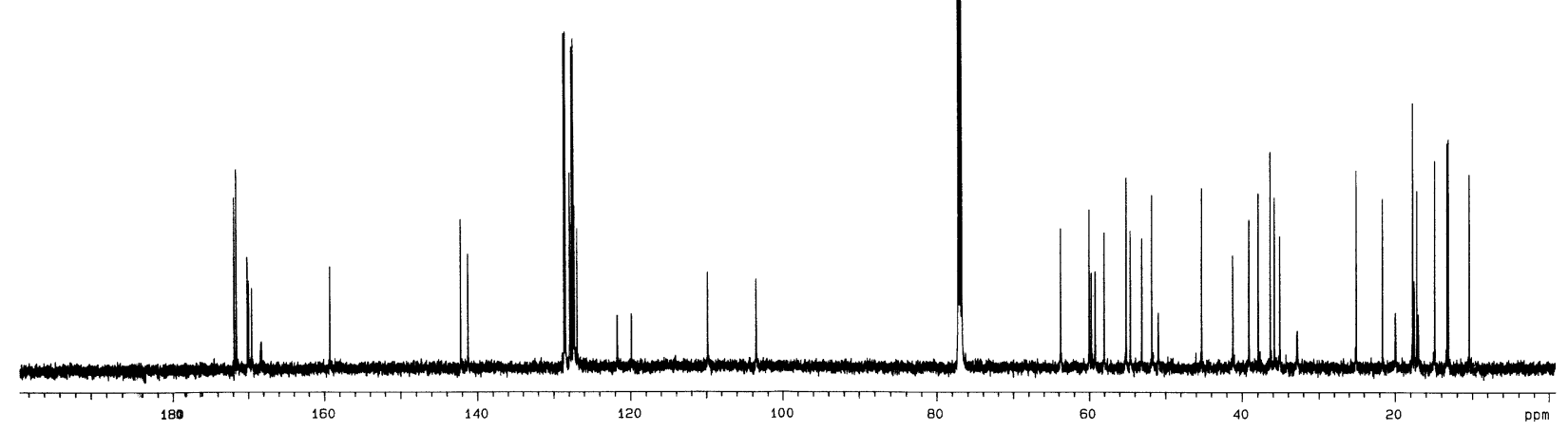

$\left[a-\mathrm{Dab}^{1}\right]$-Hormaomycin $\left[a-\mathrm{Dab}^{1}\right]-\mathbf{( 3 )}$ 


\section{Lebenslauf}

Am 07.03.1970 wurde ich als Sohn des Ingenieurs Dmitrij Zlatopolski und der Konstrukteurin Galina Zlatopolskaja, geborene Avdeeva, in Leningrad (Russische Föderation) geboren.

Von September 1976 bis Juli 1986 besuchte ich die Grund- und Mittelschule N²34 in Leningrad, an der ich im Juli 1986 mein Abitur absolvierte.

Zum Herbstsemester 1986 begann ich das Studium der Chemie an der Leningrader (St.-Petersburger) Staatsuniversität. Nach dem Abschluss des 4. Semesters wurde ich in die Sowjetarmee einberufen, in der ich von Juni 1988 bis September 1989 meinen Grundwehrdienst leistete. Zum Herbstsemester 1989 nahm ich das Studium wieder auf, absolvierte den vollen Studiengang und fertigte unter der wissenschaftlichen Anleitung von Herrn Dr. S. V. Nikolaev meine Diplomarbeit zu dem Thema „Synthese der in sechster Position modifizierten LHRH Analoga“ an. Am 22. Juni 1992 bestand ich meine Diplomhauptprüfung vor der Staatlichen Prüfungskommision, wobei mir die Qualifizierung des Chemikers verliehen wurde.

Im Zeitraum von September 1992 bis Oktober 1995 war ich in der Forschungsabteilung der „SPARK $\mathrm{GmbH}^{\prime \prime}$ angestellt.

Von Oktober 1995 bis Juni 2000 arbeitete ich im Arbeitskreis von Prof. Dr. A. G. Shavva zum Thema „Synthetische Untersuchung amphiphiler Peptidantibiotika“.

Im Dezember 1999 heiratete ich Elizaveta Ouroussova.

Im August 2000 kam ich als Gastwissenschaftler nach Deutschland und arbeite seit Oktober 2000 im Arbeitskreis von Prof. Dr. A. de Meijere an meiner Dissertation „Endgültige Strukturauklärung des Hormaomycins und Totalsynthese von Hormaomycin sowie Analoga“.

Mit Erfolg absolvierte ich im Wintersemester 2002/2003 den Kurs zur Allgemeinen Pharmakologie und Toxikologie.

Im September 2003 wurde mir das an der St.-Petersburger Universität ausgegebene Diplom als Äquivalent zu einem Diplom in Chemie an einer deutschen Universität anerkannt. 



\section{Danksagungen}

Für die Aufnahme in seinen Arbeitkreis und sein Bemühen, mir eine finanzielle Unterstützung zu sichern, möchte ich ganz herzlich Herrn Prof. Dr. A. de Meijere danken.

Mein allergrößter Dank gehört meiner Frau Elizaveta, die mir von Anfang an in jedem schwierigen Moment geholfen hat.

Herrn Prof. A. Zeeck danke ich für viele wertvolle Diskussionen und wichtige Tips rund um die Chemie und Biochemie des Hormaomycins.

Für die sehr gute Ausführung zahlreicher biologischer und HPLC-Experimente sowie für die Aufnahme der UV- und CD-Spektren gilt mein Dank Herrn H.-P. Kroll und Frau M. Klingiebiel. Ohne ihre Zusammenarbeit hätte diese Arbeit nicht durchgeführt werden können.

Ein großes Dankeschön an Herrn Dr. S. V. Nikolaev (Sankt-Petersburg) für die schnelle und exzellente Durchführung sehr wichtiger HPLC-Experimente sowie Herrn Prof. Dr. W. A. König (Hamburg) für sehr wichtige GC-Experimente.

Für die finanzielle Unterstützung im Rahmen des Sonderforschungsbereiches SFB 416 danke ich der Deutschen Forschungsgemeinschaft.

Für die Aufnahme der NMR-Spekten danke ich den Mitarbeitern der NMR-Abteilung und besonders Herrn Dipl. Chem. R. Machinek, Herrn M. Weitemeyer und Frau C. Zolke für die stete Hilfsbereitschaft und die anregenden Diskussionen.

Herrn Hambloch danke ich für die Durchführung der Elementaranalysen, Frau G. Udvarnoki und Herrn Dr. H. Frauendorf für die aufgenommenen Massenspektren und durchgeführten HPLC/MSExperimente, und Herrn Dipl. Chem. O. Senge für die Ausführung präparativer HPLC-Trennungen.

Frau G. Keil-Knepel verdient für ihre Unterstützung bei den verschiedensten Problemen Anerkennung und Dank.

Für stetige Hilfs- und Diskussionsbereitschaft sowie zahlreiche chemische Tips und Tricks bedanke ich mich bei den Herren Dr. S. I. Kozhushkov, V. N. Belov, V. V. Sokolov, den Herren Dipl. Chem. O. V. Larionov, V. Bagutski, der Frau Dr. L. A. Maslovskaja und ganz besonders den Herren Dr. A. I. Savchenko, A. Leonov und S. Nizamov.

Mein besonderer Dank gilt Frau Dipl. Chem. F. Brackmann und Herrn Dipl. Chem. H. Schill für ihre Hilfe in meinem schwierigen „Kampf“ mit der deutschen Orthographie und Grammatik.

Für das sorgfältige Korrekturlesen dieser Arbeit bedanke ich mich bei den Herren Dipl. Chem. I. Freifeld, O. Larionov, Herrn Dr. S. Nizamov und in besonderem Maße bei den Herrn Dipl. Chem. D. Ovchinnikov, Dr. V. N. Belov und Prof. Dr. S. L. Mironov.

Ich möchte allen Mitarbeitern und Gastwissenschaftlern des Arbeitkreises danken, die mir in diesen Jahren ihre Freundschaft erwiesen haben: Farina, Martina, Björn, Gerd, Steffi, Gabriel, Michael (×2), 
Heiko, Mario, Daniel, Karsten, Andrej (× 2), Shamil, Oleg, Victor, Hans, Alessandra, Regina, Jelena, Markus und Stefan $(\times 2)$. Auch den restlichen Mitarbeitern des Arbeitskreises danke ich für ihre stete Diskussions- und Hilfsbereitschaft. 